Old Dominion University

ODU Digital Commons

Mechanical \& Aerospace Engineering Theses \&

Dissertations

Mechanical \& Aerospace Engineering

Summer 2016

\title{
Design of Experiments Enhanced Statistical Process Control for Wind Tunnel Check Standard Testing
}

Ben D. Phillips

Old Dominion University, bdphillips11@gmail.com

Follow this and additional works at: https://digitalcommons.odu.edu/mae_etds

Part of the Aerospace Engineering Commons

\section{Recommended Citation}

Phillips, Ben D.. "Design of Experiments Enhanced Statistical Process Control for Wind Tunnel Check Standard Testing" (2016). Doctor of Philosophy (PhD), Dissertation, Mechanical \& Aerospace Engineering, Old Dominion University, DOI: 10.25777/j5we-6t64

https://digitalcommons.odu.edu/mae_etds/15

This Dissertation is brought to you for free and open access by the Mechanical \& Aerospace Engineering at ODU Digital Commons. It has been accepted for inclusion in Mechanical \& Aerospace Engineering Theses \& Dissertations by an authorized administrator of ODU Digital Commons. For more information, please contact digitalcommons@odu.edu. 
DESIGN OF EXPERIMENTS ENHANCED STATISTICAL PROCESS CONTROL FOR WIND

TUNNEL CHECK STANDARD TESTING

by

Ben D. Phillips

B.S. May 2011, University of Maryland Eastern Shore

M.S. December 2013, Old Dominion University

A Dissertation Submitted to the Faculty of

Old Dominion University in Partial Fulfillment of the

Requirements for the Degree of

DOCTOR OF PHILOSOPHY

AEROSPACE ENGINEERING

OLD DOMINION UNIVERSITY

August 2016

Approved by:

Drew Landman (Director)

Kenneth Toro (Member)

Resit Unal (Member)

Colin Britcher (Member) 


\begin{abstract}
DESIGN OF EXPERIMENTS ENHANCED STATISTICAL PROCESS CONTROL FOR WIND TUNNEL CHECK STANDARD TESTING
\end{abstract}

Ben D. Phillips

Old Dominion University, 2016

Director: Drew Landman

The current wind tunnel check standard testing program at NASA Langley Research Center is focused on increasing data quality, uncertainty quantification and overall control and improvement of wind tunnel measurement processes. The statistical process control (SPC) methodology employed in the check standard testing program allows for the tracking of variations in measurements over time as well as an overall assessment of facility health. While the SPC approach can and does provide researchers with valuable information, it has certain limitations in the areas of process improvement and uncertainty quantification. It is thought by utilizing design of experiments methodology in conjunction with the current SPC practices that one can efficiently and more robustly characterize uncertainties and develop enhanced process improvement procedures. In this research, methodologies were developed to generate regression models for wind tunnel calibration coefficients, balance force coefficients and wind tunnel flow angularities. The coefficients of these regression models were then tracked in statistical process control charts, giving a higher level of understanding of the processes. The methodology outlined is sufficiently generic such that this research can be applicable to any wind tunnel check standard testing program. 


\section{Nomenclature}

\begin{tabular}{|c|c|}
\hline$A$ & $=$ Reference Area \\
\hline$A T$ & $=$ Across-test \\
\hline$C^{\prime}$ & $=$ Wind Tunnel Calibration Coefficient \\
\hline$C_{A}$ & $=$ Axial Force Coefficient \\
\hline$C_{N}$ & $=$ Normal Force Coefficient \\
\hline$C_{M}$ & $=$ Pitching Moment Coefficient \\
\hline$c$ & $=$ Chord Length \\
\hline$D O E$ & $=$ Design of Experiments \\
\hline$F A$ & $=$ Flow Angularity \\
\hline HTC & $=$ Hard to Change Factor \\
\hline LSWT & $=$ Low Speed Wind Tunnel \\
\hline LaRC & $=$ Langley Research Center \\
\hline$m R$ & $=$ Moving Range \\
\hline$\widetilde{m R}$ & $=$ Median of the Moving Range \\
\hline ODU & $=$ Old Dominion University \\
\hline$R$ & $=$ Range \\
\hline$\tilde{R}$ & $=$ Median of the Range \\
\hline$R E M L$ & $=$ Residual Maximum Likelihood \\
\hline$S P C$ & $=$ Statistical Process Control \\
\hline$s$ & $=$ Wing Span \\
\hline$S$ & $=$ Reference Area \\
\hline WG & $=$ Within-group \\
\hline WT & $=$ Within-test \\
\hline$\alpha$ & $=$ Angle of Attack \\
\hline$\phi$ & $=$ Roll Angle \\
\hline$\sigma$ & $=$ Standard Deviation \\
\hline$\sigma^{2}$ & $=$ Variance \\
\hline$\hat{\sigma}$ & $=$ Estimate of the Standard Deviation \\
\hline$\hat{\sigma}^{2}$ & $=$ Estimate of the Variance \\
\hline$q$ & $=$ Dynamic Pressure \\
\hline$\Delta P$ & $=$ Differential Pressure \\
\hline
\end{tabular}




\section{TABLE OF CONTENTS}

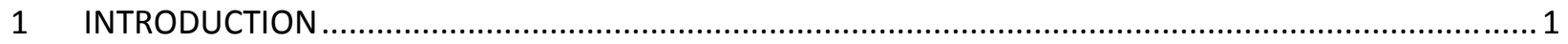

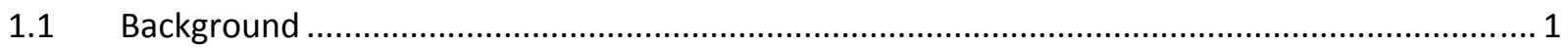

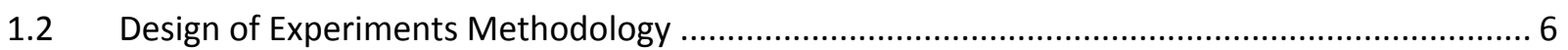

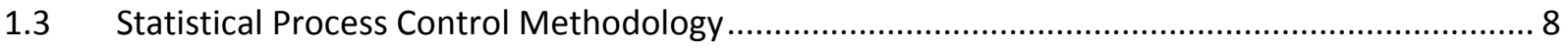

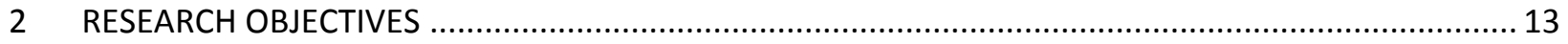

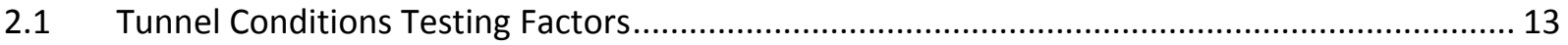

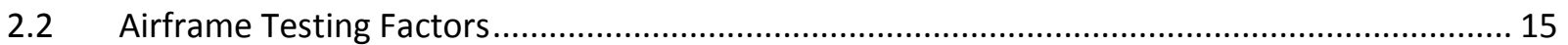

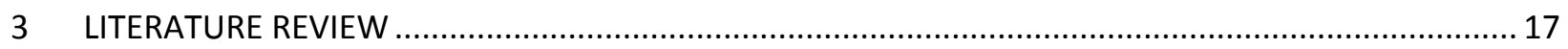

3.1 Wind Tunnel Facility Calibrations and Experimental Uncertainty ........................................ 19

3.2 Check Standard Testing Across Multiple Transonic Wind Tunnels with the Modern Design of

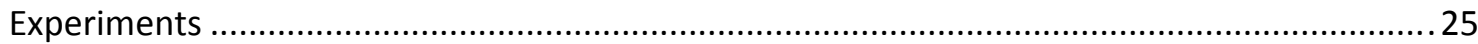

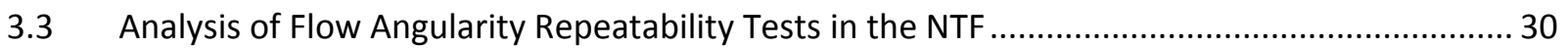

3.4 Repeatability Modeling for Wind-Tunnel Measurements: Results for Three Langley Facilities 42

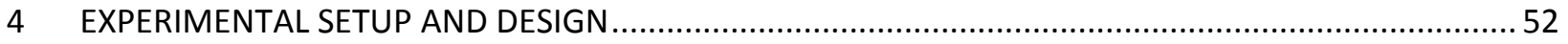

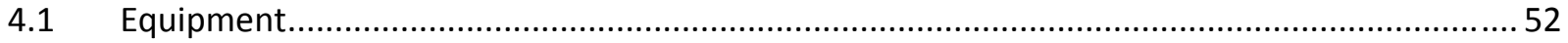

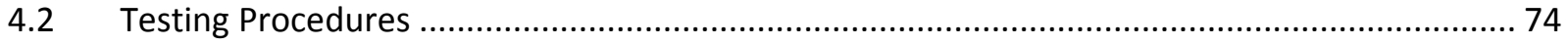

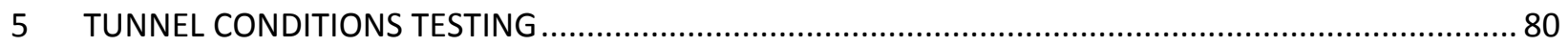

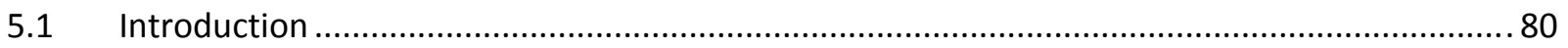

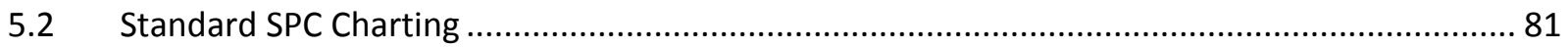

$5.3 \quad$ Use of Design of Experiments Methodology …....................................................................... 93

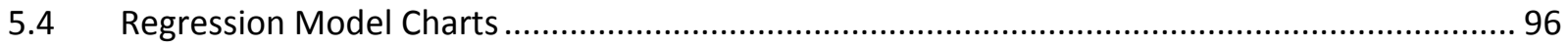

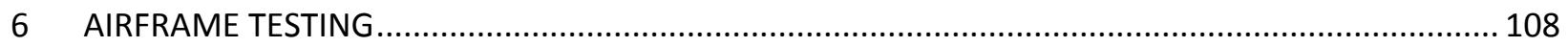

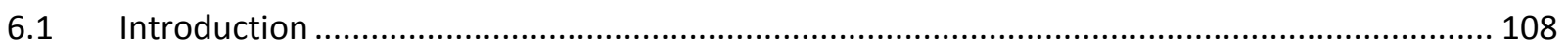




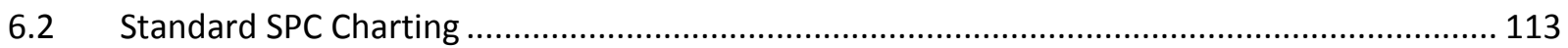

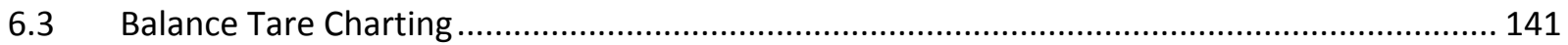

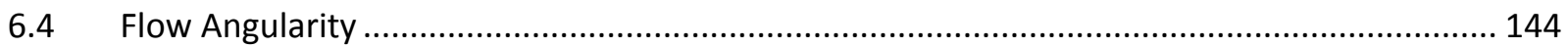

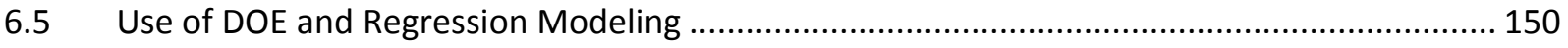

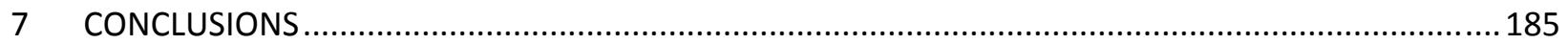

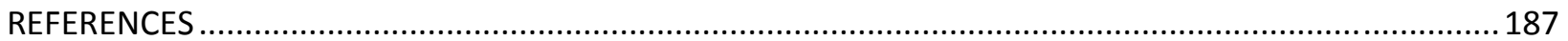

APPENDICES

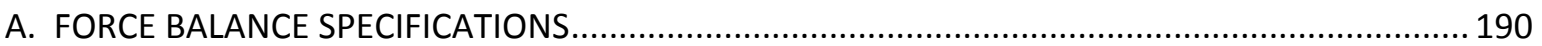

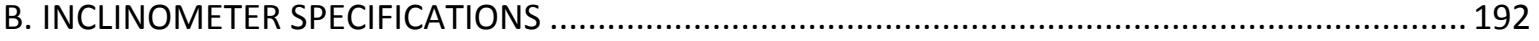

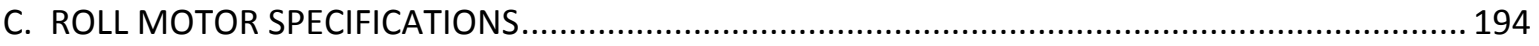

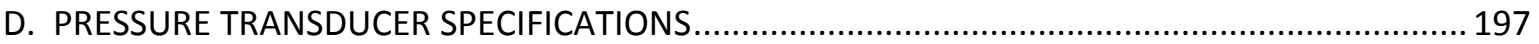

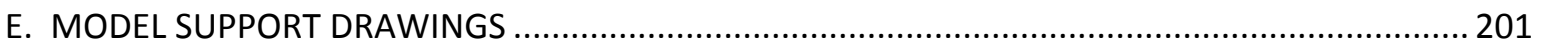

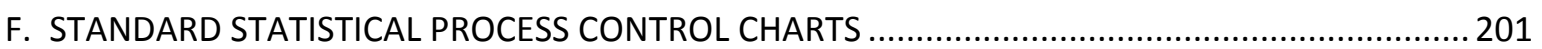

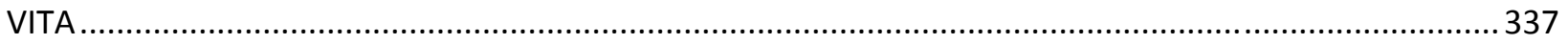




\section{LIST OF TABLES}

Table

Page

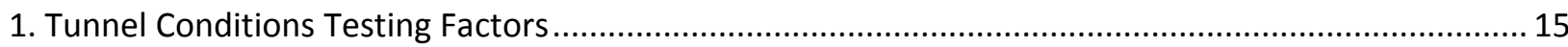

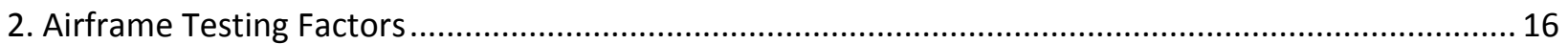

3. Uncertainties from Kammeyer's Supersonic Pressure Coefficient Measurement ...............................20

4. Values for Uncertainty Calculation in Transonic Pressure Coefficient Measurement..........................23

5. Replicated Drag Polars for "Tunnel D" Configuration 0, Roll = $0^{\circ}$, Re 4.5E06, Mach 0.85...................27

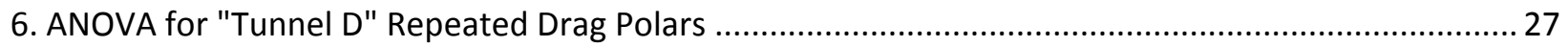

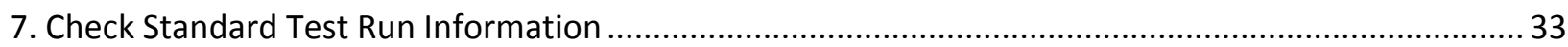

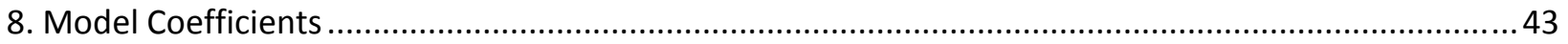

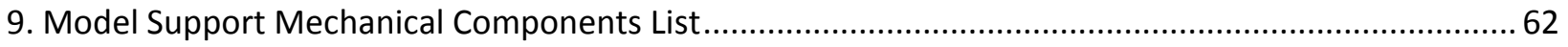

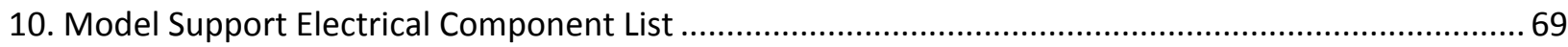

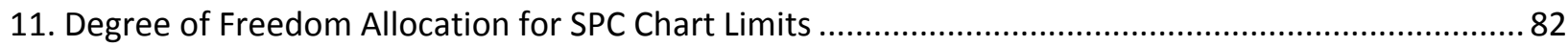

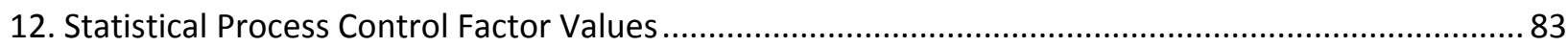

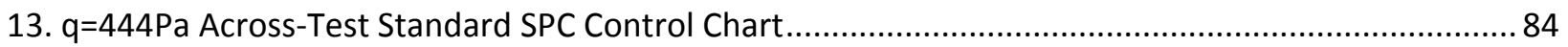

14. Values of $d 4$ for use in Estimation of the Standard Deviation ...................................................... 92

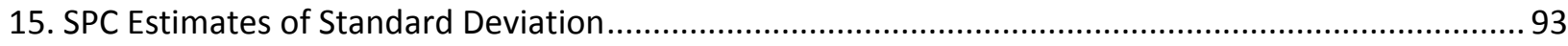

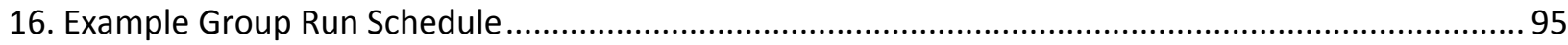

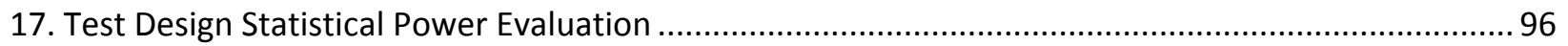

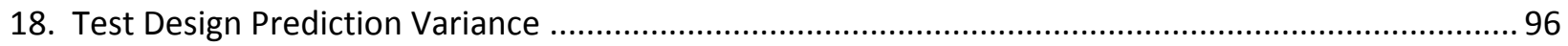

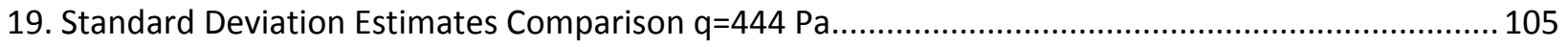

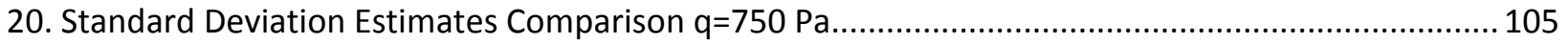

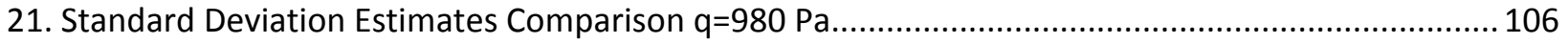

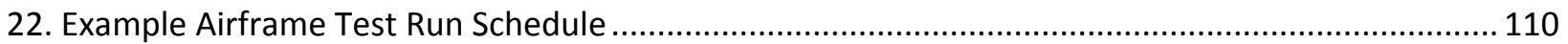


23. Statistical Evaluation of Test Design

24. Power to Estimate Dynamic Pressure Terms - Whole Plot Replication Statistics

25. Common Optimal Designs

26. Comparison of Statistical Evaluation of Candidate Designs

27. Degree of Freedom Allocation for SPC Charts 113

28. SPC Factor Values 114

29. Values of $d 4$ for use in Estimation of the Standard Deviation 128

30. SPC Estimates of the Standard Deviation for $C_{N}$ 128

31. SPC Estimates of the Standard Deviation for $C_{M}$ 128

32. SPC Estimates of the Standard Deviation for $C_{A}$ 129

33. Number of Points out of Statistical Control 130

34. Comparison of Estimates Standard Deviations for $C_{N}$ 132

35. Comparison of Estimates of Standard Deviations for $C_{A}$ 132

36. Comparison of Estimates of Standard Deviations for $C_{M}$ 132

37. Normalized Estimates of Total Variance 140

38. Test 9 Pitch Flow Angularity Calculations 145

39. Example Cross Flow Angularity Run Schedule 147

40. Test 12 Cross Flow Angularity Calculations 148

41. JMP REML Analysis for the Normal Force Coefficient from Test 18 153

42. Normal Force Coefficient Regression Local Coefficients 167

43. Comparison of Model Building Technique's Regression Intercepts 169

44. Comparison of Model Building Technique's Angle of Attack Regression Coefficient. 169

45. Comparison of Model Predicted Values of Confirmation Points 10-13

46. Comparison of Model Predicted Values of Confirmation Points from Tests 14-17 
47. Prediction Interval Comparison for Confirmation Points from Tests $10-13$.................................... 172

48. Prediction Interval Comparison for Confirmation Points from Tests test $14-17$........................... 172

49. Global Prediction Interval Sample Calculation............................................................................ 173

50. Normalized Residuals from Angle of Attack Error Injection ........................................................ 184 


\section{List of Figures}

Figure

1. 14x22 Check Standard Probe (left) and NTF Check Standard Model (right)....................................... 1

2. Check Standard Testing Data from the FAVOR Test ................................................................. 3

3. Example Control Chart with Out of Control Points .................................................................... 4

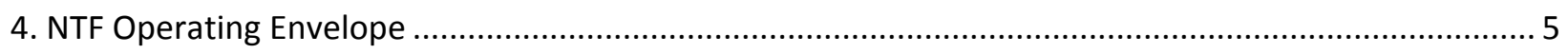

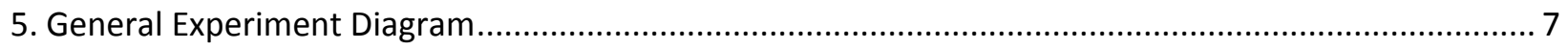

6. Example Two Factor Factorial Experiment …......................................................................... 7

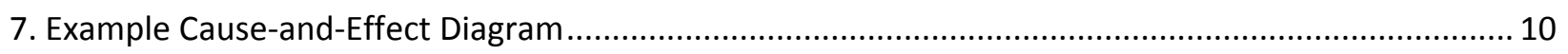

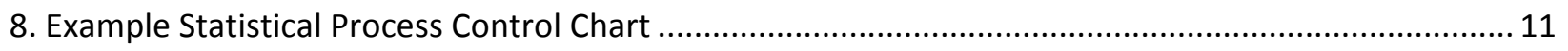

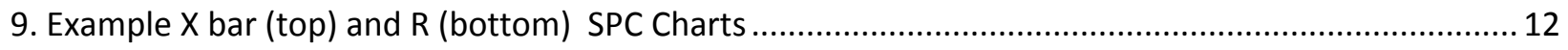

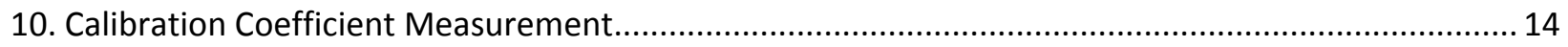

11. Contributions to the Overall Uncertainty in the Supersonic Pressure Coefficient ............................ 21

12. Example Static Pipe Calibration Data from the Boeing PSWT ….................................................. 22

13. Contributions to the Overall Uncertainty in the Transonic Pressure Coefficient ................................23

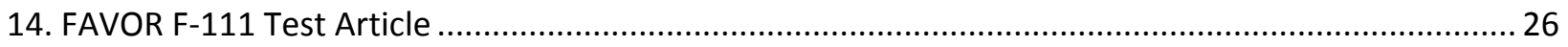

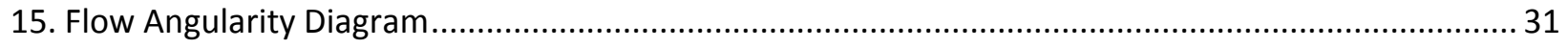

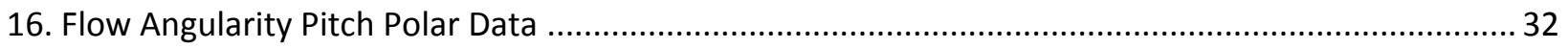

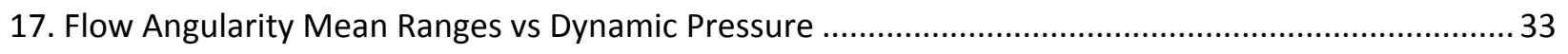

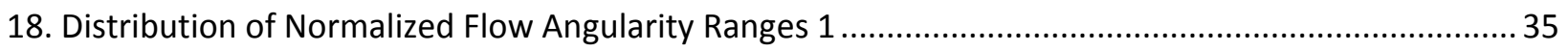

19. Distribution of Normalized Flow Angularity Ranges 2 ................................................................ 36

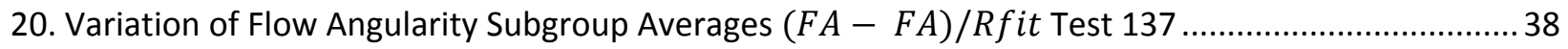

21. Flow Angularity Ranges from Customer Test............................................................................. 41

22. Model of $\sigma W G$ for $C^{\prime}$ from $14 \times 22$ with Control Limits for 90 Degrees of Freedom ......................... 45 
Figure $\quad$ Page

23. Model of $\sigma W T$ for $C^{\prime}$ from $14 \times 22$ with Control Limits for 22 Degrees of Freedom......................... 46

24. Model of $\sigma A T$ for $C^{\prime}$ from $14 \times 22$ with Control Limits for 13 Degrees of Freedom ..........................47

25. 14x22 Elliptical Wing Check Standard Model ................................................................................ 48

26. Model of $\sigma W G$ for Normal for Coefficient from $14 \times 22$ with Control Limits for 82 Degrees of Freedom

27. Model of $\sigma W T$ for Normal for Coefficient from $14 \times 22$ with Control Limits for 22 Degrees of Freedom

28. Model of $\sigma A T$ for Normal for Coefficient from $14 \times 22$ with Control Limits for 12 Degrees of Freedom 50

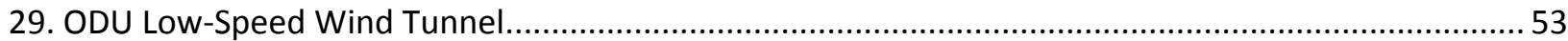

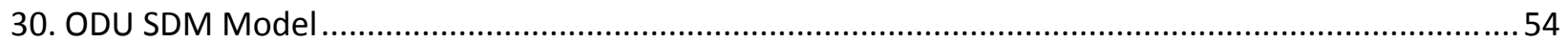

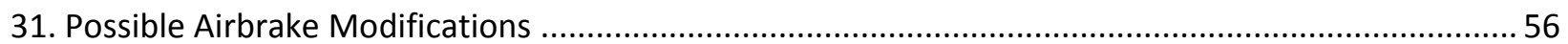

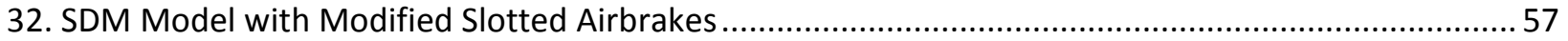

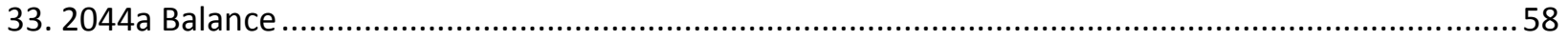

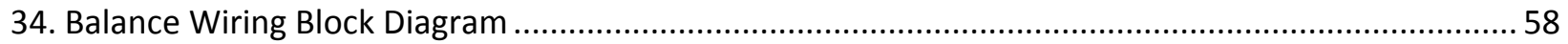

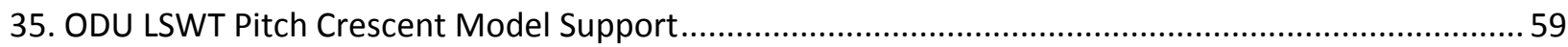

36. NTF Model Support (Left) and TDT Model Support (Right) ......................................................... 60

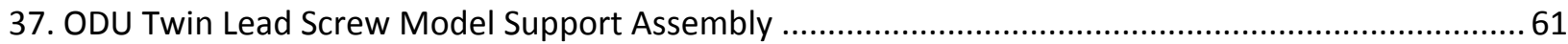

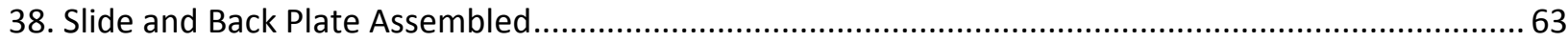

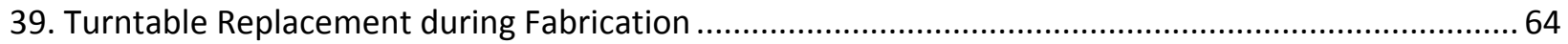

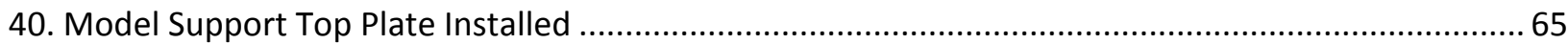

41. ODU Twin Lead Screw Model Support, Arm Section View ................................................................ 66

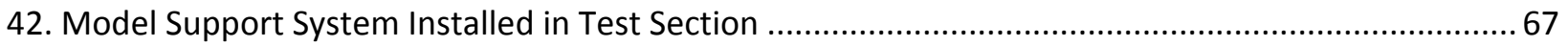

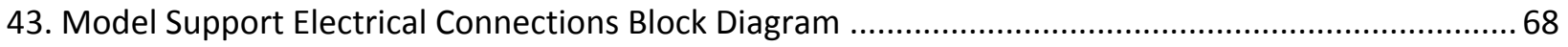


Figure $\quad$ Page

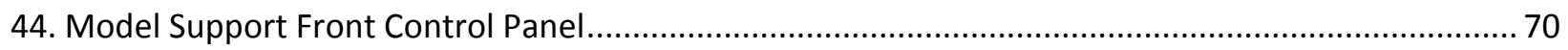

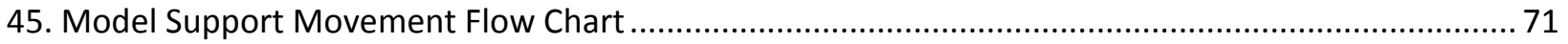

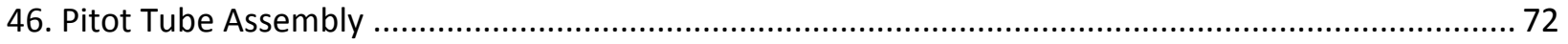

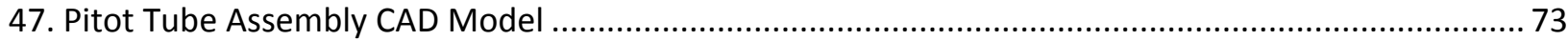

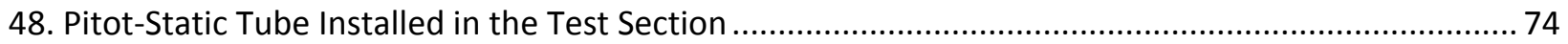

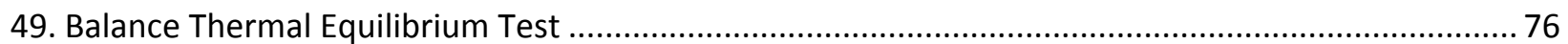

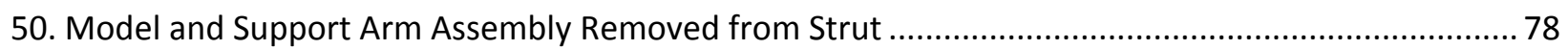

51. Reference Angle Procedure (Roll Angle Left, Angle of Attack Right) .............................................. 79

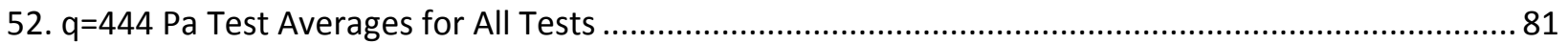

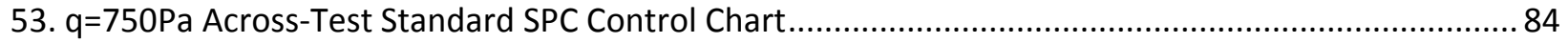

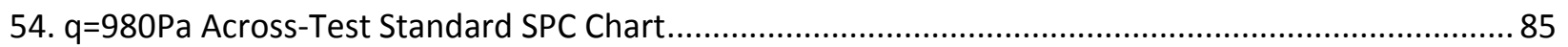

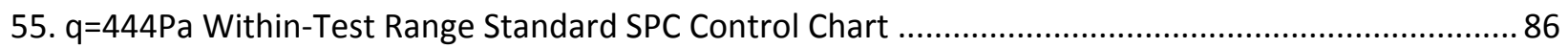

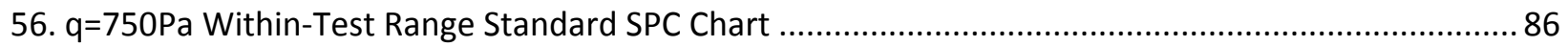

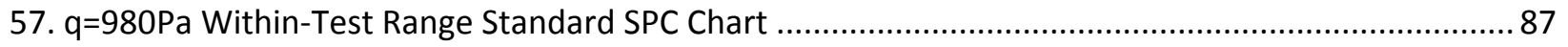

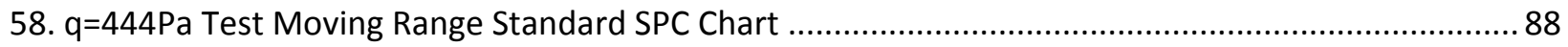

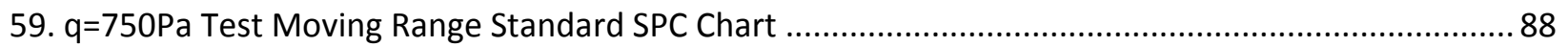

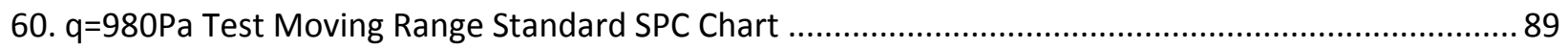

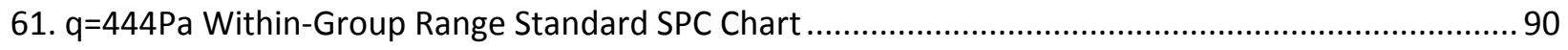

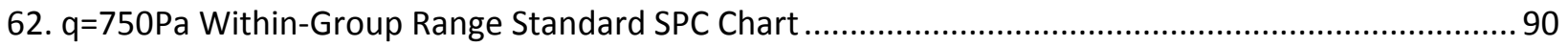

63. q=980Pa Within-Group Range Standard SPC Control Chart .......................................................... 91

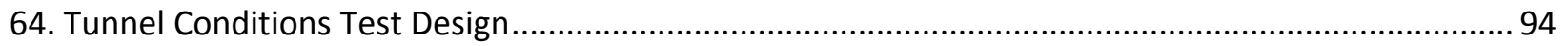

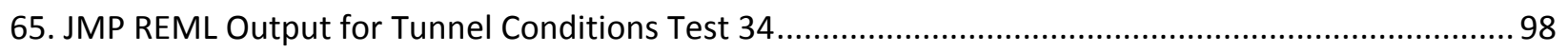

66. JMP REML Output for Tunnel Conditions Global Regression Model ...............................................99

67. Tunnel Calibration Coefficient Global Regression Model Residuals vs Dynamic Pressure ..................100 
Figure $\quad$ Page

68. Tunnel Calibration Coefficient Global Regression Model Residuals vs Temperature .......................100

69. Tunnel Calibration Coefficient Global Regression Model Residuals vs Predicted ............................ 101

70. Tunnel Calibration Coefficient Global Regression Model Residuals vs Test.................................... 102

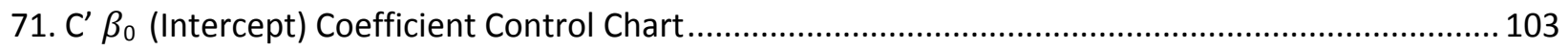

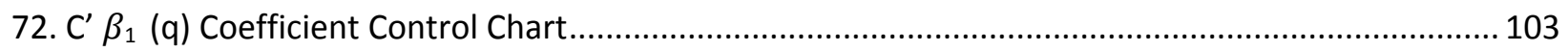

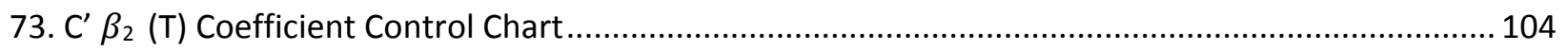

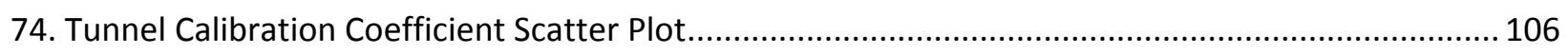

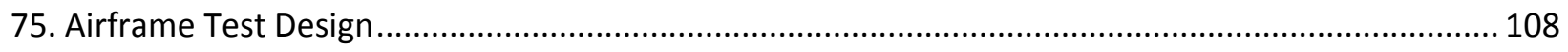

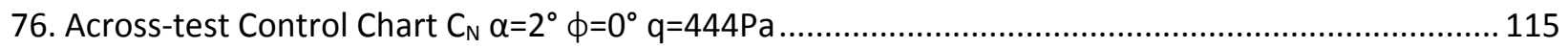

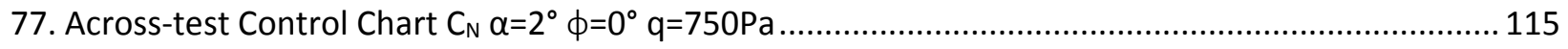

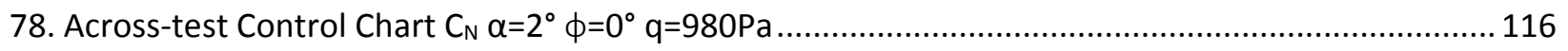

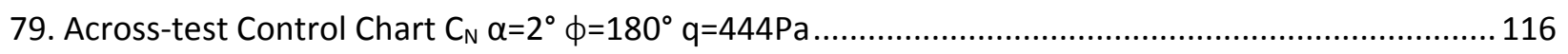

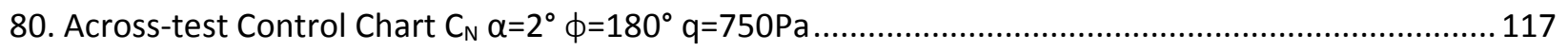

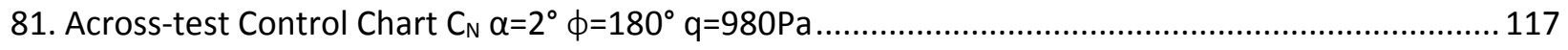

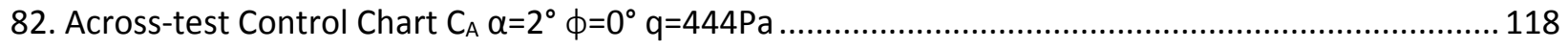

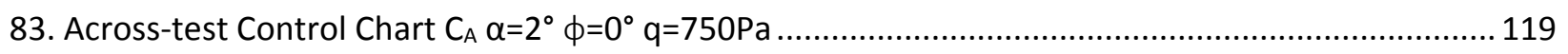

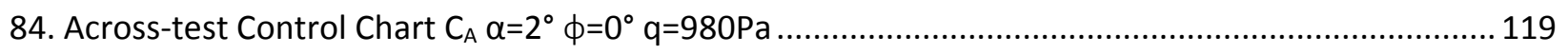

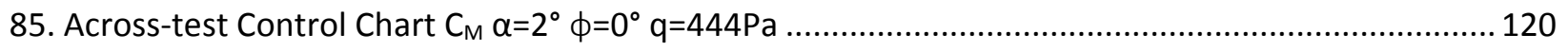

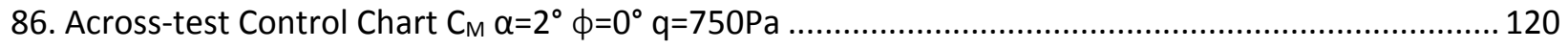

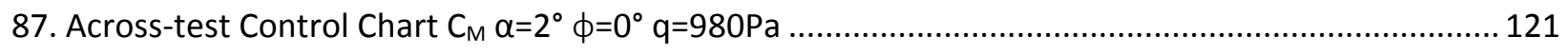

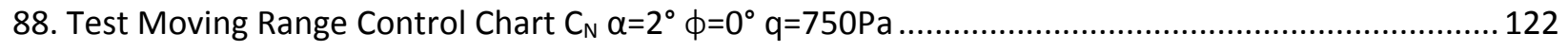

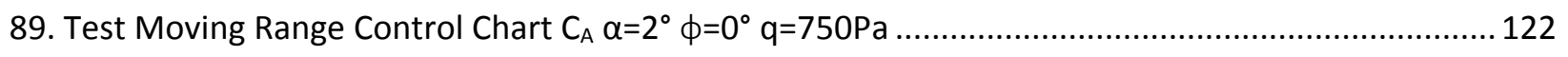

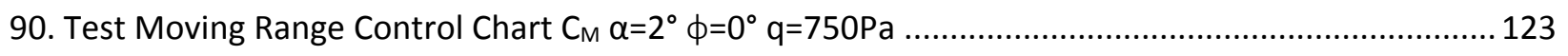

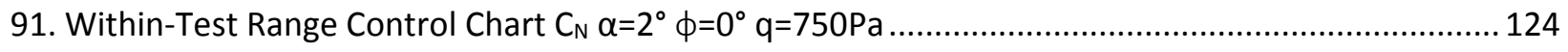


Figure $\quad$ Page

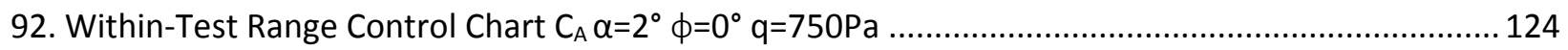

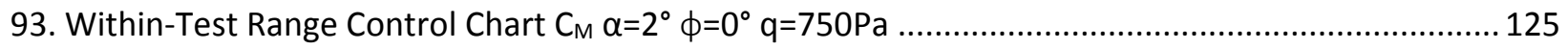

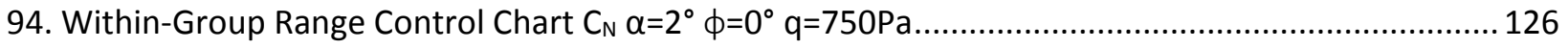

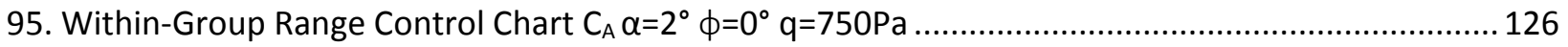

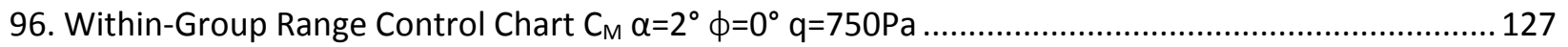

97. REML Estimate of the Total Normal Force Coefficient Standard Deviation ...................................... 134

98. REML Estimate of the Total Pitching Moment Coefficient Standard Deviation ................................. 134

99. REML Estimate of the Total Axial Force Coefficient Standard Deviation......................................... 135

100. Estimate of the Deviation of the Normal Force Coefficient from Check Standard Testing at the

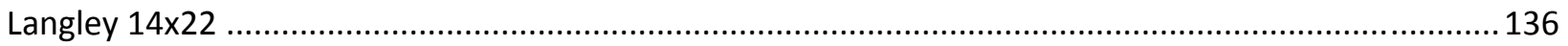

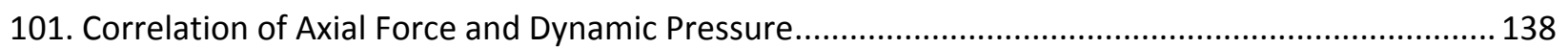

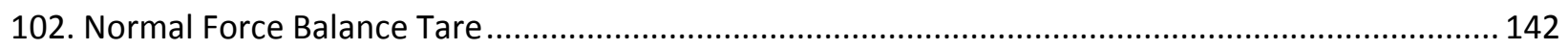

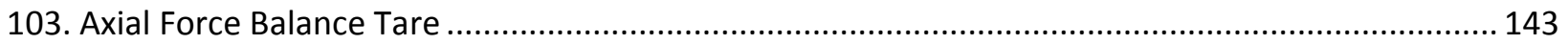

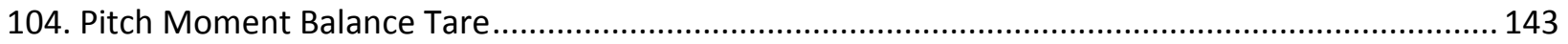

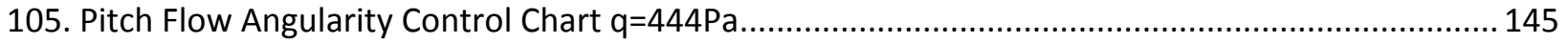

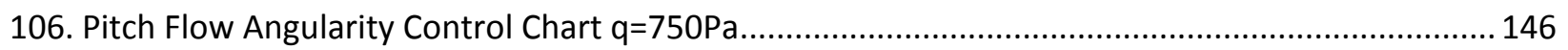

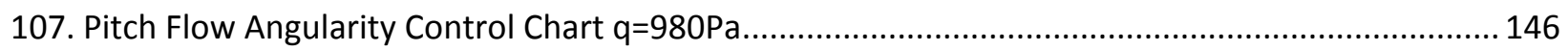

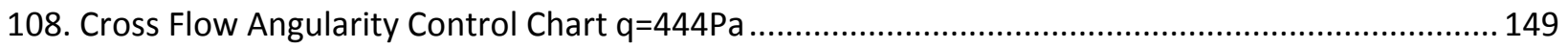

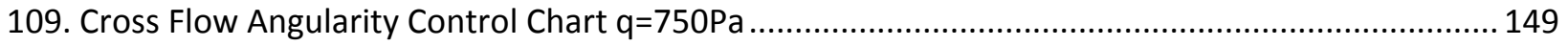

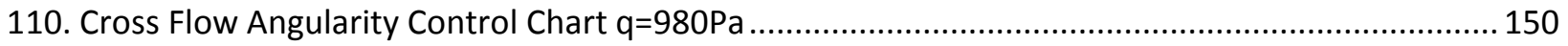

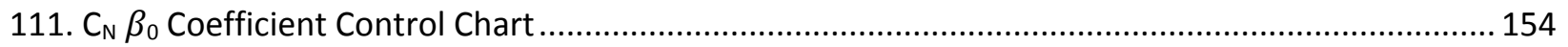

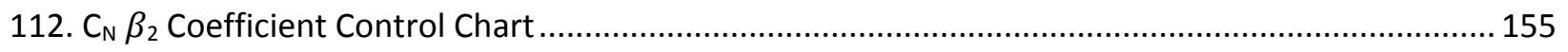

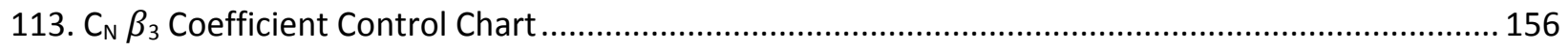

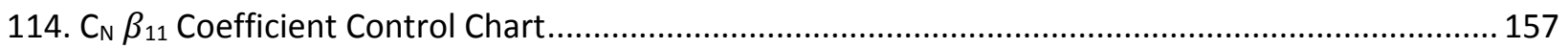


Figure $\quad$ Page

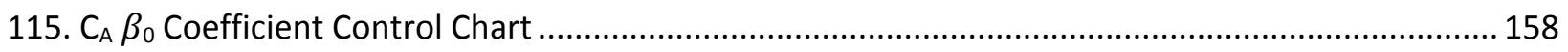

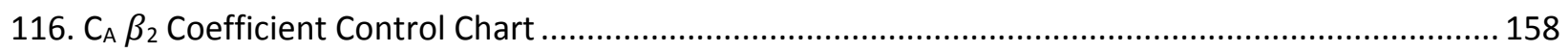

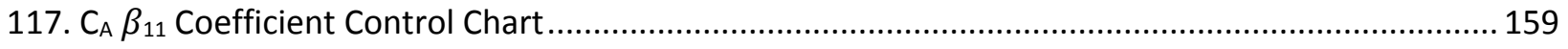

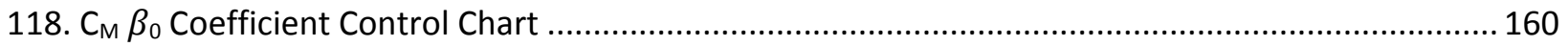

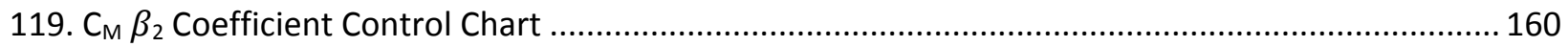

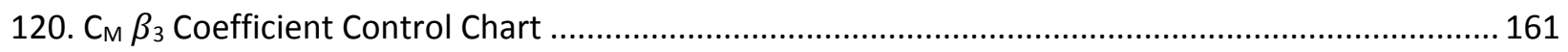

121. Normal Force Root Mean Square Error Confirmation Points .......................................................... 162

122. Test 18 Normal Force Coefficient Regression Model Residuals vs Dynamic Pressure ....................164

123. Test 18 Normal Force Coefficient Regression Model Residuals vs Angle of Attack........................ 164

124. Test 18 Normal Force Coefficient Regression Model Normal Plot of Residuals ............................. 165

125. Test 18 Normal Force Coefficient Regression Model Residuals vs Run ........................................ 165

126. Test 18 Normal Force Coefficient Regression Model Residuals vs Predicted.................................166

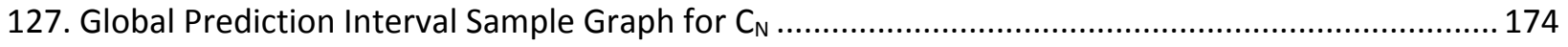

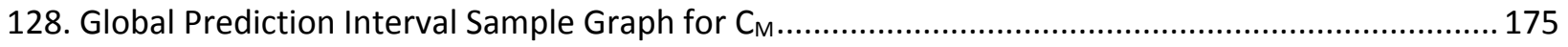

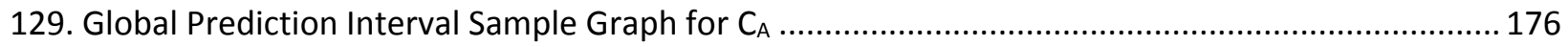

130. Normal Force Coefficient Global Regression Model Normalized Residuals .................................. 178

131. Normal Force Coefficient Global Regression Model Residuals vs Test........................................ 178

132. Normal Force Coefficient Global Regression Model Residuals vs Angle of Attack.........................179

133. Normal Force Coefficient Global Regression Model Residuals vs Dynamic Pressure.......................179

134. Normal Force Coefficient Global Regression Model Residuals vs Roll .......................................... 180

135. Normal Force Coefficient Global Regression Model Residuals vs Roll .......................................... 180

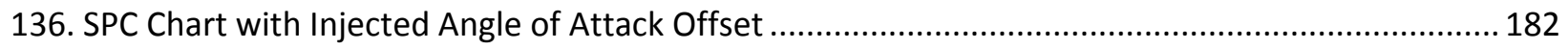

137. Regression Coefficient Control Chart with Injected Angle of attack Offset .................................. 183 


\section{INTRODUCTION}

This chapter will introduce the background of current check standard testing at NASA wind tunnel facilities, as well as the benefits and limitations of the practice. The fundamental principles and applications of Design of Experiments (DOE) and Statistical Process Control (SPC) methodologies will be introduced. The detailed discussion of the check standard practices and procedures is left for later chapters.

\subsection{Background}

The current wind tunnel check standard testing program at NASA Langley Research Center is focused on increasing data quality, uncertainty quantification and overall control and improvement of wind tunnel measurement processes. The implementation of the check standard testing generally involves the testing of a stable, representative test article in various facilities over time. Examples of these test articles include a specialized Pitot-static probe which measures tunnel conditions and a generic transport aircraft model. Two examples of these test artifacts from the $14 \times 22$ low-speed wind tunnel and the National Transonic Facility are shown below ${ }^{1,2}$.

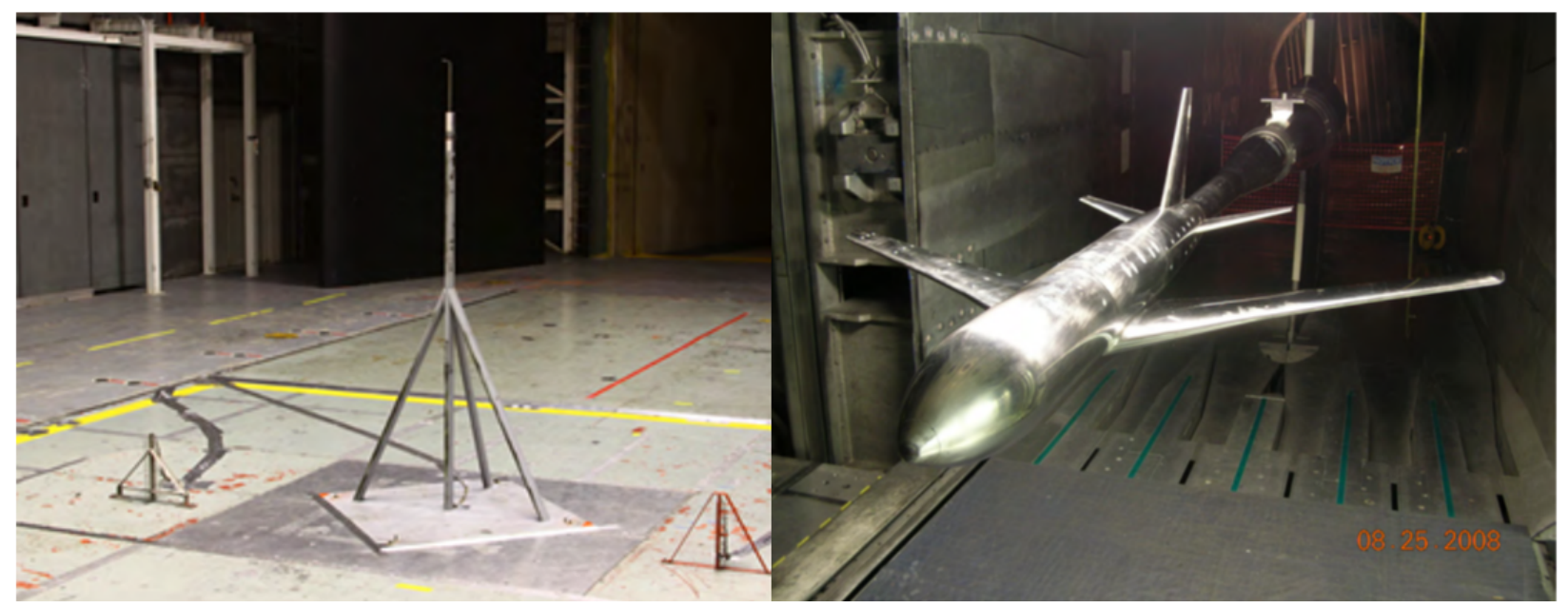

Figure 1 14x22 Check Standard Probe (left) and NTF Check Standard Model (right) 
The current practice for developing uncertainty models and tracking variance in the measurements of the check standard testing is based in statistical process control (SPC) first developed by Shewhart in the 1930's. Shewhart, whose work focused on manufacturing, summarized the reasoning behind the development of his methodology eloquently when describing quality control in a manufacturing process $^{3}$.

"He (the manufacturer) sets up a standard for the quality of a given kind of product. He then tries to make all the pieces of product conform with this standard. Here his troubles begin. For him standard quality is like a marksman shooting at a bull's-eye, he often misses. As is the case in everything we do, unknown or chances causes exert their influence. The problem then is: how much may the quality of a product vary and yet be controlled? In other words how much variation should we leave to chance?"

Check standard testing with SPC methodology allows researchers and facility managers to develop estimates of uncertainty for wind tunnel testing processes as well as understand the long-term stability of measurements in the wind tunnel. This information is not only useful to researchers, but to wind tunnel customers as well, as they will have some level of confidence in the data they receive.

While the SPC approach can and does provide researchers with valuable information, it has certain limitations in the areas of process improvement and uncertainty quantification. For example, when developing uncertainty estimates utilizing statistical process control, the only statistically robust estimates are those at specific points within the tunnel's operating envelope where data have been taken. In Figure 2, the Mach - Reynolds number operating envelope of wind tunnels data are shown with check standard data points taken from multiple tests, across multiple facilities. ${ }^{4}$ With the current practices in check standard testing, researchers are only able to robustly estimate uncertainties at the points in which the data has been gathered. From inspection of Figure 2, it is clear that there are large areas within the tunnel's operating envelope in which no statistically defensible estimates of uncertainty are available. This can become problematic for a number of reasons. First, if a customer wants to run a wind tunnel test in areas of the design space (tunnel operating envelope) that were not part of the check standard testing program, no statistically defensible estimates of uncertainty are available for the researcher's data. Second, if the check standard testing and statistical process control methodology indicate that there are no problems with the process of obtaining wind tunnel measurements, facility managers could potentially falsely conclude that the process is in statistical control. With current 
practices, the researchers are only able to conclude with statistical rigor the statistical control status of the specific data points which have been gathered.

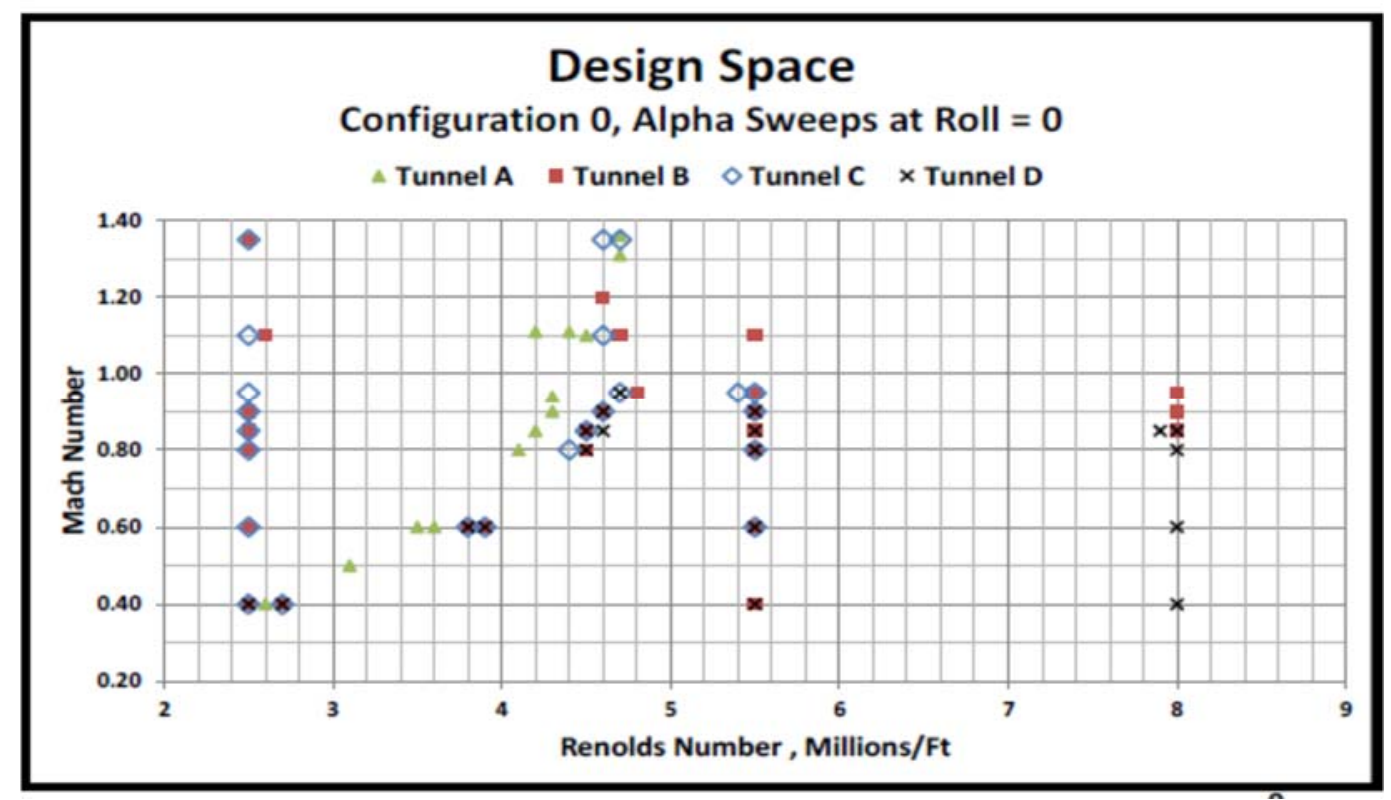

Figure 2 Check Standard Testing Data from the FAVOR Test

Also, while the charting procedures of SPC can identify when the process is no longer in statistical control, SPC struggles when establishing an "assignable cause", that is a reason for the "out of control" statistical control state. Statistical process control, through the establishment of process limits, performs very well as an initial data quality control metric. However, the methodology, as it is currently employed does not give the researcher any further insight into the causes of the unexplained variability in the process. This is evident in Figure 3 which is a SPC across-test control chart of the pitching moment coefficient from a check standard model. Clearly, tests 18 and 19 are out of the established process control limits but that is all the information the researcher is able to gain. 
$C_{M} \alpha=2^{\circ} \phi=0^{\circ} q=980 P a$

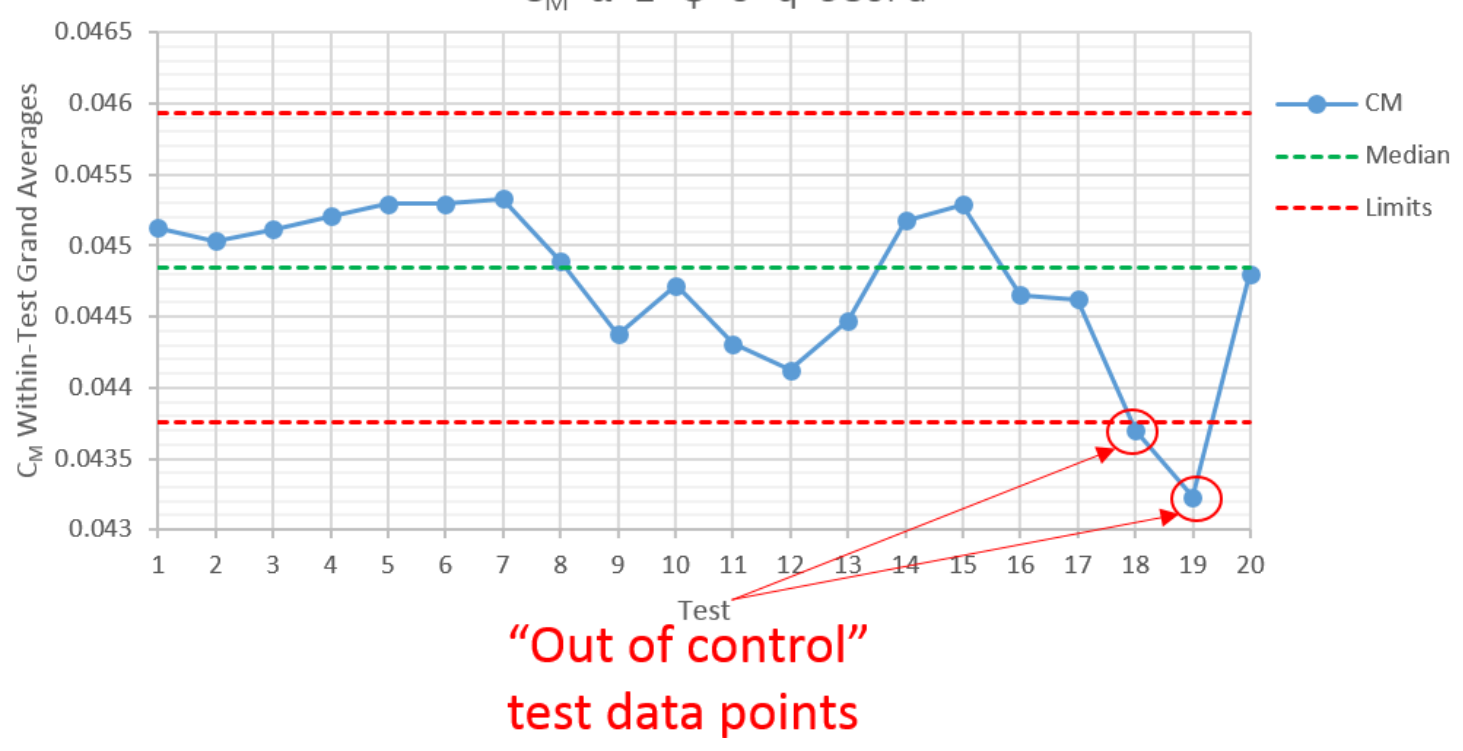

Figure 3 Example Control Chart with Out of Control Points

Furthermore, in certain facilities like the National Transonic Facility (NTF) ${ }^{5}$, gathering data at certain locations within the tunnel's operating envelope can be expensive relative to other locations. In Figure 4, the Reynolds - Mach number operating envelope of NTF is given. To achieve high Reynolds numbers, the facility runs at cryogenic temperatures by injecting nitrogen into the flow. This is extremely expensive and very rarely will check standard testing occur in this region. This can lead to an uneven spatial distribution of data points (information) for the check standard program. 


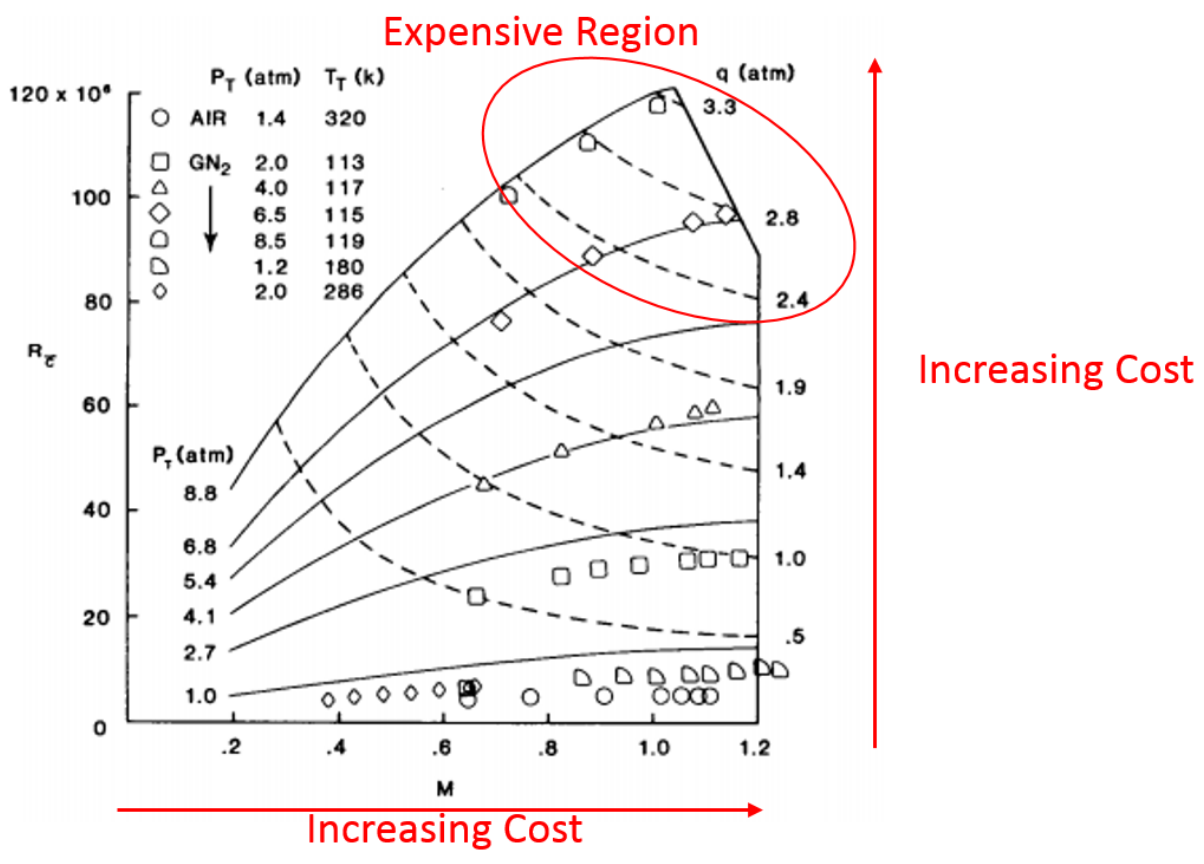

Figure 4 NTF Operating Envelope

Overall, the current wind tunnel check standard program and implementation of statistical process control methodology is inefficient with respect to the information gained and resources expended. An effective statistical process control program for wind tunnel check standard testing should be able to successfully address each of the following questions.

- How much variation is present in the data?

- What level of variation in the data would be considered excessive?

- How are we able to tell if the level of acceptable variation has been exceeded?

- What are the causes of increased or unexpected variation?

- What corrective actions are taken to reduce the undesirable variation?

It is thought by utilizing design of experiments methodology (DOE) in conjunction with the current SPC practices that one can efficiently and more robustly characterize uncertainties and develop enhanced process improvement procedures. 


\subsection{Design of Experiments Methodology}

Although design of experiments methodology is a relatively new technique to the aerospace community, specifically the ground testing community, it has its origins in the agricultural industry dating back to the early 1900's. Initial work in the field was championed by the English statistician Ronald Fisher. ${ }^{6}$ Box and Wilson ${ }^{7}$ further expanded the methodology throughout the 1950's and 1960's in the chemical and manufacturing industries, developing the foundations of response surface methodology (RSM). In the 1980's Taguchi ${ }^{8}$ investigated quality improvement in industrial engineering focusing on robust parameter design and overall process robustness. More recently (1990's to present) design of experiments has been expanded to use in computer programs, greatly simplifying the analysis of complex designs.

The overarching goals of any experimentation is to understand how the experimental factors affect the observed response. The most simplistic experimental technique is the best-guess or trial and error approach. In this approach, the experimenter chooses experimental factor levels and combinations that are thought to produce a desired response. The experiment is run followed by a successive experiment with different factor levels or combinations based on the results of the first experiment. This technique, as ad hoc as it may appear, is often times fairly successful. However this approach requires significant knowledge of the entire process. Also the experimental procedure can continue on infinitely as there is no statistical basis for determining if the "best" solution has been found. Another experimental technique that is widely used across all fields is the one factor at a time (OFAT) approach (sometimes mistakenly referred to as the "Scientific Method"). In this method, one factor is sequentially changed while all other factors are held constant. Once the first factor has been sequentially changed to all desirable levels, the second factor is subjected to the same procedure with the first factor held at a nominal level, and so forth for the other remaining factors. Although a step up in robustness from the best-guess technique, the OFAT approach still has a few flaws. If the experimental factors are not varied simultaneously, the interaction between factors cannot be determined with any statistical rigor and the variance estimates are often biased. Also, this approach is very inefficient due to the number of runs required to exercise all factor combinations. 


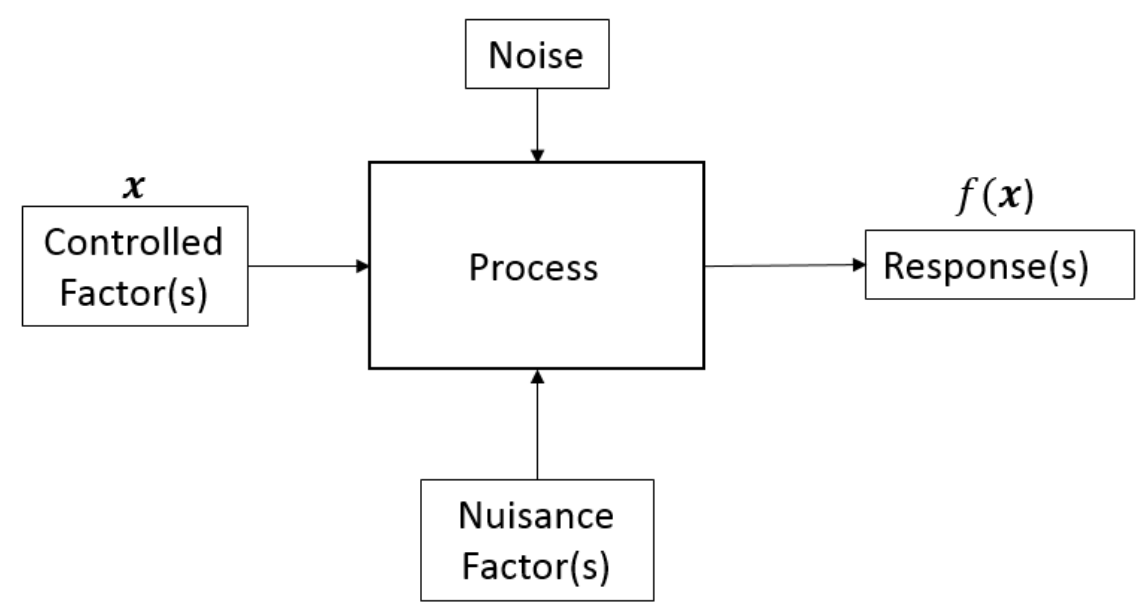

Figure 5 General Experiment Diagram

DOE methods are utilized in both setting up a controlled experiment and the statistical evaluation of the experiment. In comparison to the other experimental techniques introduced, it is a systematic, rigorous, efficient approach to solving complex engineering problems. One of the fundamental experiment designs in DOE is the factorial experiment. In the factorial experiment, the levels of the factors are changed simultaneously instead of one at a time. Two-level factorial experiments are referred to as $2^{k}$ ("two to the $k^{\prime \prime}$ ), where $k$ is the number of factors in the experiment and 2 represents the number of levels of each factor. Figure 6 depicts a $2^{2}$ design consisting of two factors and two levels of each factor.

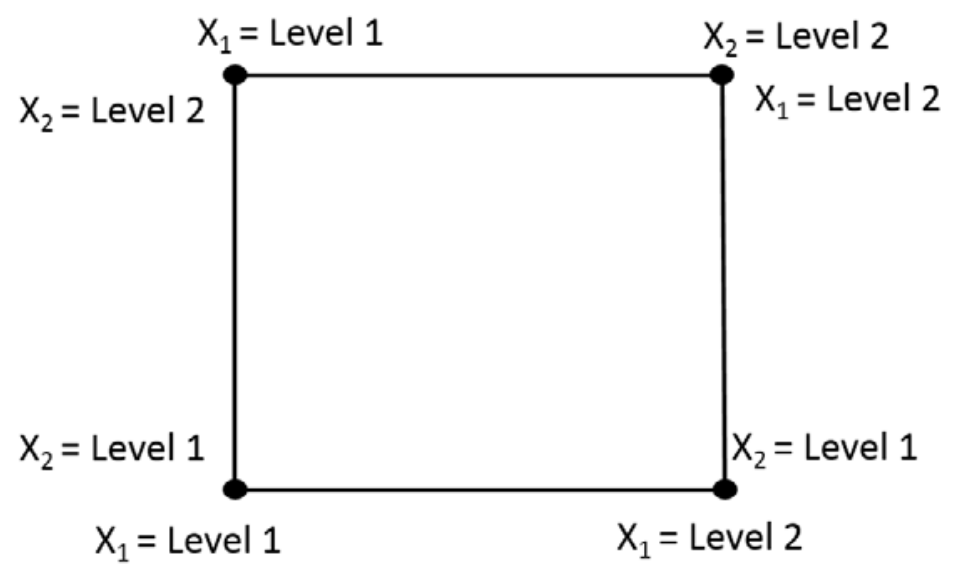

Figure 6 Example Two Factor Factorial Experiment 
The naming convention of two-level factorial experimental designs refers to the number of runs necessary to execute the experiment. For example, without replication, a $2^{2}$ test would require 4 runs. However, one of the fundamental principles of DOE is replication of runs. Repeating a run in an experiment allows for the estimation of the "pure error," or systematic error due to random factors in the experimental process. For example, from Figure 6 , if the run with $X_{1}=$ Level 1 and $X_{2}=$ Level 1 was replicated, a different response could be observed even though the experimental factors were identical.

Another fundamental principle of design of experiments is the randomization of runs, which differs from the sequential approach to experimentation in OFAT. An underlying assumption in DOE is that the observations (or errors) be independently distributed random normal variables. ${ }^{9}$ Randomization of the test order gives the ability to guard against systematic bias in the experiment. For example if a nonrandomized designed experiment was conducted in the presence of a systematic bias such as a time varying bias, there could potentially be correlation between a factor and the time varying bias. In this scenario, the researcher could incorrectly conclude that changes in a factor caused the observed change in the response.

In DOE, once the experimental runs have been completed, a regression model is built using the analysis of variance (ANOVA) where the effects of the experimental factors are evaluated for significance using F-tests. ${ }^{10}$ For the experiment design shown in Figure 6, the regression model could take the form

$$
y=\beta_{1} x_{1}+\beta_{2} x_{2}+\beta_{12} x_{1} x_{2}
$$

where $y$ is the response, $x_{1}$ and $x_{2}$ are the levels of the factors 1 and 2 respectively, and the $\beta^{\prime} s$ are the regression coefficients. To estimate terms of higher order in the regression model, such as $\beta_{22} x_{2}^{2}$, additional runs must be made at additional factor level combinations.

\subsection{Statistical Process Control Methodology}

Statistical process control (SPC) is a methodology focused on increasing the quality of a process through the implementation of statistical methods. SPC was first developed by Walter Shewhart in the 1920's while he was working at Bell Telephone Laboratories. Shewhart was tasked with implementing a quality control program for the production of telephone components. He later published his groundbreaking work, "Economic Control of Quality of Manufactured Product" in $1931 .{ }^{11}$ In implementing a quality 
control program, the term "quality" must be well defined. However, defining quality may not be as straightforward as one might expect. Quality could be defined based on a certain physical property of a product such as weight, length, or color. It could also be defined based on a process outcome such as time or cost to manufacture a product. In defining quality, it is easy to see that the definition, whatever it may be, cannot be complete without the mention of variability. For example, the quality variable of concern in the manufacturing of a ruler might be the overall length. The target value, for a 1-foot ruler of course, is twelve inches. However, how much variability in the overall length of the ruler is acceptable? Can we accept a ruler that is 12.1 inches long; how about 11.99 inches long? A definition of quality that can be applied in any scenario is simply that "quality is inversely proportional to variability." ${ }^{12}$ Therefore, the higher the variability in the process or product, the lower the quality.

Broadly speaking, SPC refers to a set of problem solving tools utilized to achieve process stability and process improvement through the quantification and reduction of variability. Some of these tools include cause-and-effect diagrams, defect concentration diagrams, scatter diagrams, Pareto charts, histograms and control charts. An example cause-and-effect diagram for defects on a $\operatorname{tank}^{12}$ is shown in Figure 7. A cause-and-effect-diagram lists and categorizes the potential causes of defects on the output of the process with the attempt to identify the driving factors of variability. 


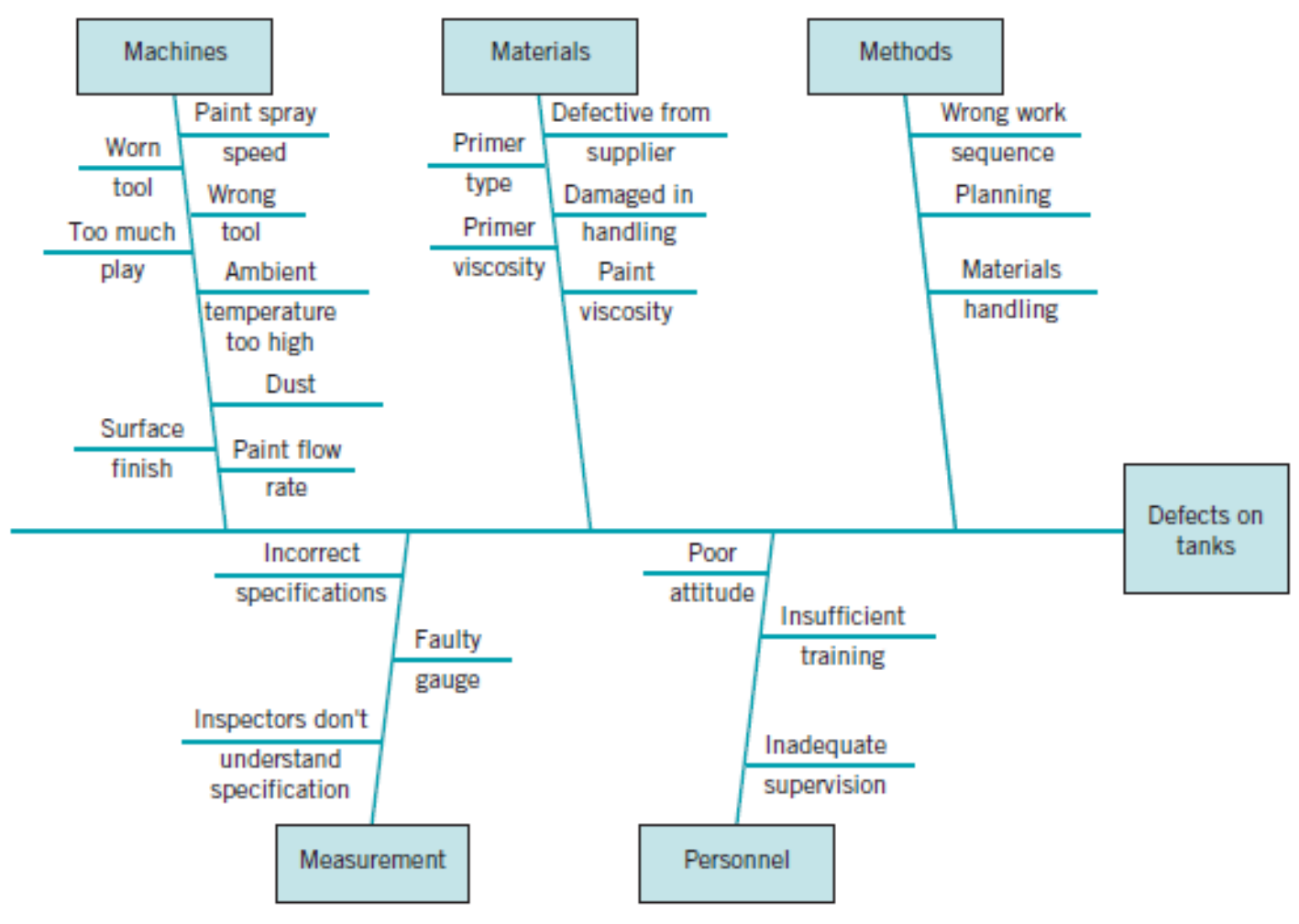

Figure 7 Example Cause-and-Effect Diagram

One of the fundamental tools of SPC is the process control chart. The control chart estimates and tracks product or process variability in samples over time. An example control chart is shown in Figure $8 .{ }^{12}$ Most SPC charts consist of quality characteristics sampled over time, which are usually averages of multiple values. A centerline and an upper and lower control limit based on the estimate of variability are used to plot results. 


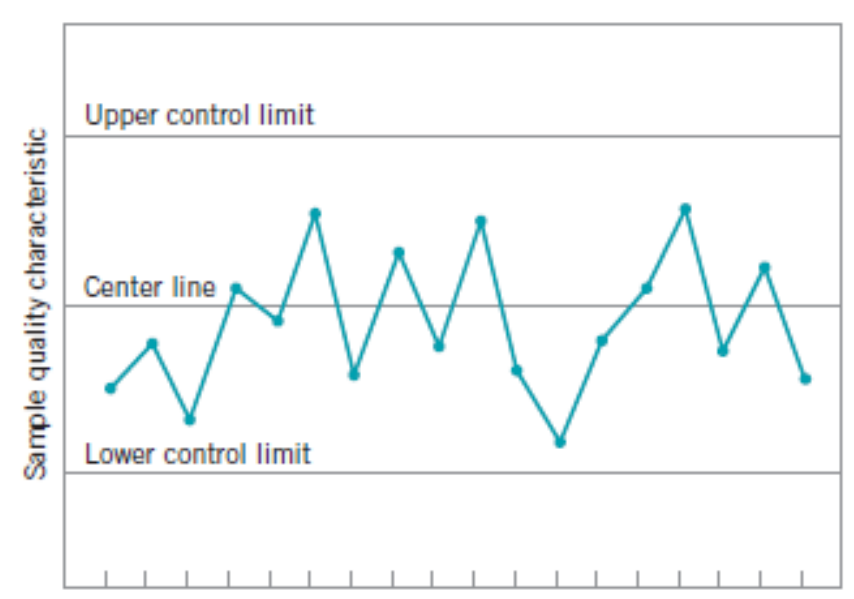

Sample number or time

Figure 8 Example Statistical Process Control Chart

Two common specific types of control charts are known as the "X-bar" and "R" charts. In this type of charting, it is assumed that each individual sample is an averaged value made up of multiple samples (subgroup). In these charts, the estimation of the process variability is based on the range of the subgroups. That is, the difference between the largest and smallest value in each subgroup. The centerline on the "X-bar" chart is the grand mean, $\overline{\bar{x}}$ which is the average of all of the samples. The upper control limit (UCL) and the lower control limit ( $(\mathrm{CL})$ are given by ${ }^{3}$ :

$$
\begin{aligned}
& U C L=\overline{\bar{x}}+\mathrm{A}_{2} \bar{R} \\
& L C L=\overline{\bar{x}}-\mathrm{A}_{2} \bar{R}
\end{aligned}
$$

where $A_{2}$ is an SPC constant which is a function of degrees of freedom (i.e. the number of samples) and $\bar{R}$ is the average of the subgroup ranges. The centerline of the " $\mathrm{R}$ " chart is $\bar{R}$ and the upper control limit is given $b^{3}$ :

$$
U C L=D_{4} \bar{R}
$$

where $D_{4}$ is an SPC constant which is a function of degrees of freedom. Example SPCX bar and R charts are shown in Figure $9 .^{12}$ 


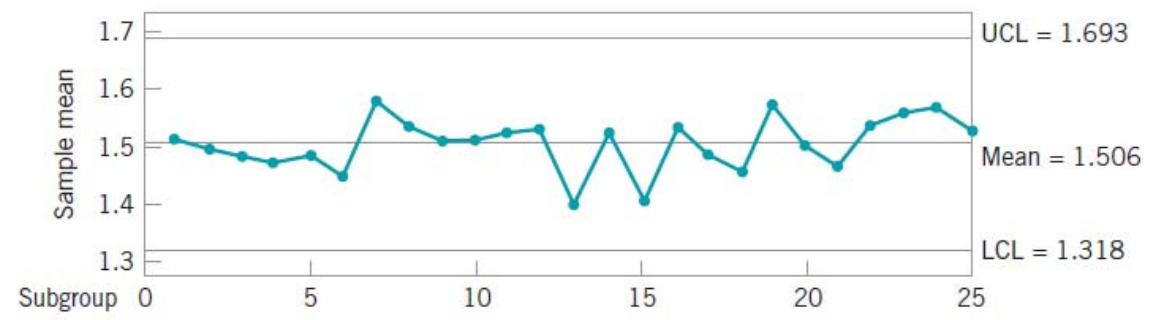

(a)

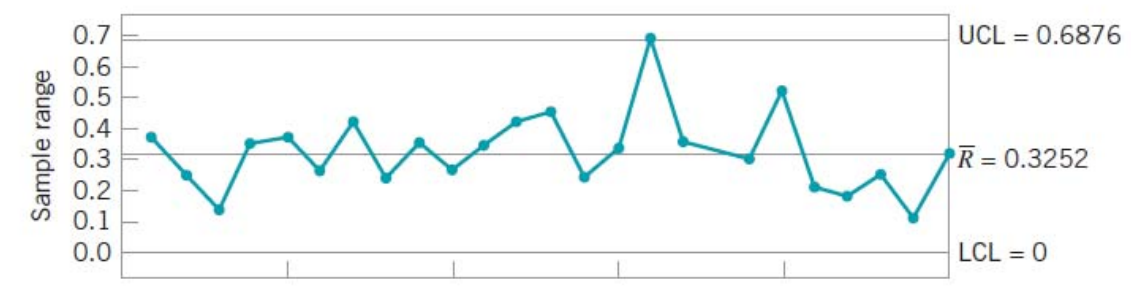

(b)

Figure 9 Example $X$ bar (top) and $R$ (bottom) SPC Charts

Under the assumption of a normally distributed variable, the estimation of the standard deviation of the process is given by ${ }^{3}$ :

$$
\hat{\sigma}=\frac{\bar{R}}{d_{2}}
$$

where $d_{2}$ is a SPC constant which is a function of degrees of freedom. In the example given of the X bar and $\mathrm{R}$ charts, SPC gives the ability to track quality variables over time and estimate process variability which are the first steps toward overall process improvement. There are many different types of SPC charts and rules for determining the control status of the process, the reader is referred to the references of Montgomery ${ }^{12}$ and Wheeler ${ }^{3}$ for more detailed discussion of SPC charts. 


\section{RESEARCH OBJECTIVES}

This chapter will discuss the overall scope of the research including objectives and limitations. The factors of interest as well as the responses to be studied will be detailed for both the tunnel conditions and airframe testing. Broadly speaking the overall research objectives were to:

- Develop and demonstrate methodology to combine Design of Experiments and Statistical Process Control methodology for check standard wind tunnel tests at NASA Langley Research Center (LaRC)

- Expand the classic SPC methodology by evaluating regression models for desired responses of the check standard tests

More specifically, the initial goals associated with this research were to:

- Use current LaRC check standard practices at Old Dominion University Low-Speed Wind Tunnel to generate a baseline for further research

- Use Design of Experiments to efficiently generate check standard data and allow for an expanded envelope to be studied

- Focus on two check standard tests, tunnel conditions and airframe

- Use the combination of DOE and SPC to create a higher level of understanding of the "control status"

- Use data from DOE runs for a dispersion study to locate areas within the design space with higher variance

- Use DOE methodology to develop a baseline check standard regression model in which statistically defensible limits can be derived for any desired point within the design space

The limitations of this research were:

- Due to contractual obligations, all preparation for testing, testing, data reduction and report formulation were to be completed within two years

- Relative to other low-speed wind tunnel facilities at LaRC (eg. 14×22), the dynamic pressure range of the ODU LSWT is limited

- The ranges and limits associated with the force balance provided, although functional, were not ideal for this specific research (larger than desired full scale ranges)

\subsection{Tunnel Conditions Testing Factors}

The response of interest in the tunnel conditions portion of the check standard testing is the tunnel calibration coefficient, $C^{\prime}$. The tunnel calibration coefficient is used to correct the measured differential 
pressure on the static rings to the test section dynamic pressure during testing. The tunnel calibration coefficient is defined by Equation 6, below.

$$
C^{\prime}=\frac{\Delta P_{\text {Pitot }}}{\Delta P_{\text {Static Ring }}}
$$

The differential pressure in the numerator is measured by a Pitot-static tube in the center of the test section and the differential pressure in the denominator is measured by two static rings located on the upstream and downstream ends of the contraction nozzle leading into the test section as depicted in Figure 10. Note that in some LaRC wind tunnel facilities, a Pitot probe is used to measure the reference pressure upstream of the contraction nozzle. The process of determining the calibration coefficient does not change.

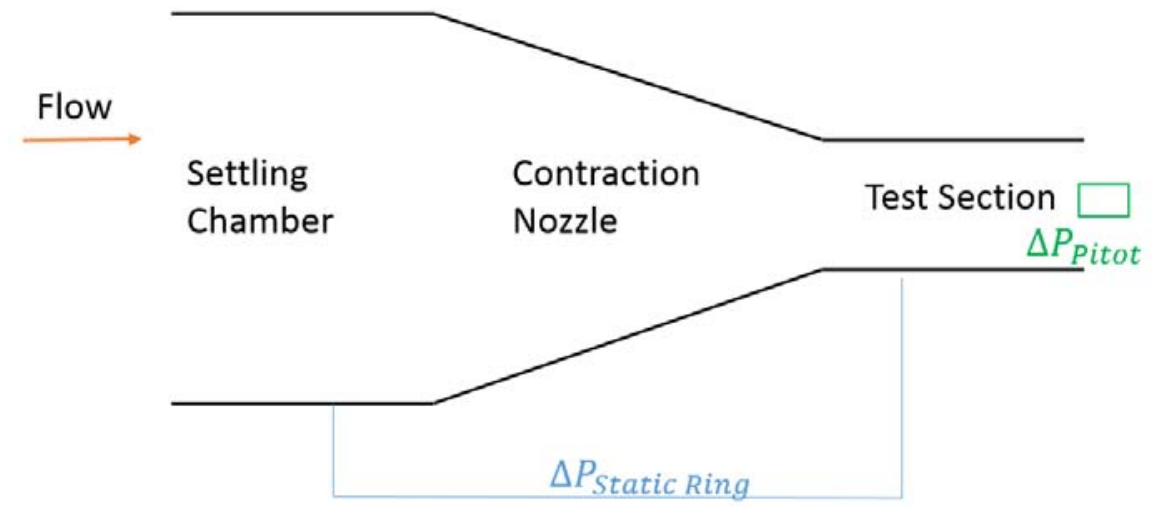

Figure 10 Calibration Coefficient Measurement

The factors which influence the tunnel calibration coefficient of the ODU LSWT are shown in Table 1 along with the ranges and levels studied. 


\begin{tabular}{|l|c|l|l|l|l|}
\hline Tunnel Conditions Factors \\
\hline Factor & Symbol & Units & Range Low & Range High & Factor Levels \\
\hline Tunnel Dynamic Pressure & $q$ & Pascals (Pa) & 444 & 980 & $444,750,980$ \\
\hline Tunnel Temperature & $T$ & Celsius (C) & 18 & 30 & Uncontrolled \\
\hline
\end{tabular}

Table 1 Tunnel Conditions Testing Factors

The range of the tunnel dynamic pressure studied is representative of the ranges in which testing is routinely conducted in the ODU LSWT and not necessarily the total capability of the facility. Due to the fact the ODU LSWT is an atmospheric wind tunnel without any temperature control, the temperature factor was uncontrolled. Refer to Chapter Error! Reference source not found. for an in-depth discussion of the treatment of the factors.

To achieve the goals set forth for the check standard tunnel conditions testing, the overarching philosophy was to build a regression model for $C^{\prime}$ as a function of dynamic pressure and temperature, $C^{\prime}=f(q, T)$. This was to be achieved by taking data points at three levels of $q$ with multiple replicates and confirmation points for model verification. The testing procedure for the tunnel followed a nested design structure which consisted of fifteen tests, each containing three groups. Within each group, each data point was taken five times. This nested design structure allows for the estimation of different time frame dependent variances as well as the implementation of DOE methodology. For a detailed discussion of the test matrix and testing schedule for the tunnel conditions testing see Section 5.3.

\subsection{Airframe Testing Factors}

The responses of interest in the airframe testing portion of the check standard testing were the balance force and moment coefficients, axial, normal and pitching moment, $C_{A}, C_{N}$ and $C_{M}$ respectively. The coefficients are given by Equation 7.

$$
C_{A}=\frac{F_{A}}{q S} \quad C_{N}=\frac{F_{N}}{q S} \quad C_{M}=\frac{M}{q c S}
$$


where $F_{A}, F_{N}$ and $M$ are the axial force, normal force, and pitching moment respectively and $S$ and $c$ represent the reference area and chord respectively. The factors studied which influence the balance force and moment coefficients are given in Table 2.

\begin{tabular}{|l|c|l|l|l|l|}
\hline \multicolumn{7}{|c|}{ Airframe Testing Factors } \\
\hline Factor & Symbol & Units & Range Low & Range High & Factor Levels \\
\hline Angle of Attack & $\alpha$ & Degrees $\left(^{\circ}\right)$ & -2 & 6 & $-2,2,6$ \\
\hline Roll Angle & $\phi$ & Degrees $\left({ }^{\circ}\right)$ & -90 & 180 & $-90,0,90,180$ \\
\hline Tunnel Dynamic Pressure & $q$ & Pascals (Pa) & 444 & 980 & $444,750,980$ \\
\hline
\end{tabular}

Table 2 Airframe Testing Factors

Both the angle of attack and the tunnel dynamic pressure were treated as continuous factors while the roll angle was treated as a categorical factor (Upright/Inverted and Right/Left). Chapter Error!

Reference source not found. presents an in-depth discussion of the treatment of the factors. It is important to note that these factors and associated levels are specific to the Old Dominion Low Speed Wind Tunnel. When implementing the proposed procedures in this research, facility managers should independently verify the factors and levels that influence their respective calibrations and airframe check standard testing.

To achieve the goals set forth for the check standard airframe testing, the overarching philosophy was to build a regression model for the balance force and moment coefficients as a function of angle of attack, roll angle and dynamic pressure, $C_{N}=f(\alpha, \phi, q)$. This was to be achieved through the use of DOE methodology, and similar to the tunnel conditions testing, a nested design structure. The airframe testing consisted of twenty total tests with each test containing two groups for each level of dynamic pressure. Within each group, each data point was taken twice. For a detailed discussion of the test matrix, and testing schedule of the tunnel conditions testing, see Section 6.5. 


\section{LITERATURE REVIEW}

The research being conducted is related to the use of statistical methodologies employed in the calibration and uncertainty quantification in wind tunnel testing. This research will enable wind tunnel users and facility managers to develop robust estimates of uncertainty for wind tunnel testing processes as well as understand the long-term stability of measurements in the wind tunnel. Facility calibrations and uncertainty quantification have long been the focus of extensive research. Technical standards and comparative testing procedures from the American Institute for Aeronautics and Astronautics (AIAA) ${ }^{13}$ and the Advisory Group for Aeronautical Research and Development (AGARD) ${ }^{14}$ give guidance on best practices for wind tunnel research. This research, however, will be more specifically focused in the application of Design of Experiments and Statistical Process Control methodologies and their applications to uncertainty quantification and reduction in wind tunnel testing.

Design of experiments methodology, while initially developed for agricultural research, has since been applied in numerous engineering fields as well as non-engineering disciplines such as economics $\left(\right.$ Starmer ${ }^{15}$, Aldrich ${ }^{16}$, Müller et al. $\left.{ }^{17}\right)$, psychology (Brunswik ${ }^{18,19}$, Campbell ${ }^{20}$, Rosnow \& Rosenthal ${ }^{21}$ ) and medicine (Collins et al. ${ }^{22}$, George et al. ${ }^{23}$, Sonnaert et. al. ${ }^{24}$, Knight $^{25}$ ). Applications of Statistical Process Control have been primarily in industrial engineering and manufacturing (including automotive and aerospace), however, the methodology has been employed in other fields such as ecology (Elskens et al. ${ }^{26}$ ) and medicine (Bartfai et al. ${ }^{27}$, Rutman et al. ${ }^{28}$ )

Recently applications of statistical process control in process monitoring referred to as profile monitoring have been investigated. In general, profile monitoring refers to observing some quality characteristic that is dependent on multiple input or process variables. The dependency or statistical model is then tracked over time. The first mention of profile monitoring or the integration of DOE and SPC methodologies was found in Kang and Albin's work ${ }^{29}$. They monitored a process in which the quality control variable, the amount of a sweetener that was dissolvable in water, was a linear function of multiple process variables such as temperature, pressure and gas type. Kang and Albin monitored the deviation in the linear regression model over time by observing model residuals. While regression models were estimated using ordinary least squares (OLS), there was no discussion on experimental design or data collection in their work. 
Although some practitioners insist on designed experiments being performed in a state of statistical control, which would prevent the implementation of the methodologies, Bisgaard ${ }^{30}$ persuasively refutes this notion. Bisgaard's work is the foundation for the justification of employing design of experiments in a continual improvement process. He concludes, "Design of experiments is perhaps the most potent tool available for the quality engineer to get a process in control."

Callahan et al. ${ }^{31}$ demonstrated the use of statistical process control to monitor the sensitivity coefficients of wind tunnel force balances over multiple calibrations. In their work, Callahan performed the same calibration procedure for the force balances and attempted to identify potential problems in the process of calibration or with the balance itself. Deficiencies in the force balance were able to be identified by monitoring the balance calibration regression model coefficients. Noorossana et al. ${ }^{32}$ also investigated the use of statistical process control to detect shifts in the process parameters of a multiple linear regression profile of force balances.

Zhang et al..$^{33}$ investigated profile monitoring of a linear process where the detection of undesired second order effects was of importance. In their application, the nature of the second order effect gave insight into the potential deficiencies (or assignable cause of the deficiency) in the quality of the product. Hypothesis testing on the second order regression model coefficients was used along with exponentially weighted moving average (EWMA) charts to monitor the process profile.

Nikoo and Noorossana ${ }^{34}$ discussed the monitoring of a nonlinear profile variance using nonparametric regression with wavelet functions. They demonstrated the use of multivariate (multiple factors) control charts formed from regression coefficients in addition to variance control charts to monitor the stability of the process mean. Examples of the methods were given for the quality characteristic of the vertical density of particleboard.

A critical review and assessment of recent calibration and facility process control methods developed for wind tunnel testing was conducted. Some of the relevant papers by Kammeyer ${ }^{35}$, DeLoach ${ }^{4}$, Hemsch and Houlden ${ }^{1}$, and Hemsch ${ }^{36}$ are discussed in detail in this chapter. This list is not intended to be exhaustive, but rather focus on the most directly relatable current research. 


\subsection{Wind Tunnel Facility Calibrations and Experimental Uncertainty}

\subsubsection{Report Overview}

In his paper "Wind Tunnel Facility Calibrations and Experimental Uncertainty", Kammeyer explores the impact of wind tunnel calibrations on the overall uncertainty in wind tunnel measurements. He was able to show for examples in both transonic and supersonic flow regimes that the uncertainty in wind tunnel calibrations was the "dominant contributor" to the overall measurement uncertainty. Kammeyer further discusses the significance of documenting the full uncertainty analysis for wind tunnel measurements.

\subsubsection{Analysis of Content}

\subsubsection{Supersonic Measurements}

Kammeyer walks through, step-by-step, the calculation of the uncertainty in both a pressure coefficient, $C_{p}$ and a drag coefficient, $C_{D}$ for the Boeing St. Louis Poly Sonic Wind Tunnel (PSWT). The pressure coefficient is defined as

$$
C_{p}=\left(p-p_{s}\right) / q
$$

where $p$ is the measured pressure, $p_{s}$ is the freestream static pressure and $q$ is the freestream dynamic pressure. In the supersonic regime, the static and dynamic pressure are calculated from isentropic flow relations ${ }^{37}$.

$$
\begin{gathered}
p_{s}=p_{0}\left(1+\frac{\gamma-1}{2} M^{2}\right)^{\frac{-\gamma}{\gamma-1}} \\
q=p_{0} \frac{\gamma}{2} M^{2}\left(1+\frac{\gamma-1}{2} M^{2}\right)^{\frac{-\gamma}{\gamma-1}}
\end{gathered}
$$


where $p_{0}$ is the reference total pressure measured in the stilling chamber. The set point Mach number, $M$ is determined from a prior facility calibration, and $\gamma$ is the specific heat ratio (assumed to be constant at a value of 1.4). To determine the uncertainty in the overall measurement of the supersonic pressure coefficient, Kammeyer utilizes a Taylor Series expansion ignoring correlation and uncertainty in the specific heat ratio ${ }^{38}$

$$
U_{C_{p}}^{2}=\left(\frac{\partial C_{p}}{\partial p}\right)^{2} U_{p}{ }^{2}+\left(\frac{\partial C_{p}}{\partial p_{0}}\right)^{2} U_{p_{0}}{ }^{2}+\left(\frac{\partial C_{p}}{\partial M}\right)^{2} U_{M}{ }^{2}
$$

Where $U_{p_{0}}, U_{M}$ and $U_{C_{p}}$ are the uncertainties in the total pressure, Mach number and overall pressure coefficient respectively. Note that Equations 9 and 10 were substituted into Equation 8 so that the freestream static pressure does not show up in Equation 11. An example measurement at tunnel conditions of $p_{0}=33.9$ sia, $M=1.97$ psia and a measured pressure of $p=4.82$ psia yields a $C_{p}=0.0227$. To determine the overall uncertainty in the example measurement, the individual uncertainties must first be defined, then substituted into the evaluated Equation 11 . The individual uncertainties are given in Table 3.

\begin{tabular}{|c|c|}
\hline Variable & Uncertainty \\
\hline $\boldsymbol{p}$ & 0.036 psia \\
\hline $\boldsymbol{p}_{\mathbf{0}}$ & 0.009 psia \\
\hline $\boldsymbol{M}$ & 0.00076 Mach \\
\hline
\end{tabular}

Table 3 Uncertainties from Kammeyer's Supersonic Pressure Coefficient Measurement

In addition to the uncertainty in the measurement of the Mach number listed above, Kammeyer proposes the addition of another component due to the averaging of the Mach number measurement. This additional term, $U_{M_{v a r}}$ is determined using a $95 \%$ confidence interval (t-statistic) which is related back to the calibration of the tunnel. Therefore, the total uncertainty in this example pressure coefficient represented as one standard deviation is $C_{p}=0.0227 \pm 0.00654$. The breakdown of the components contributing to the overall uncertainty is given in Figure 11. 


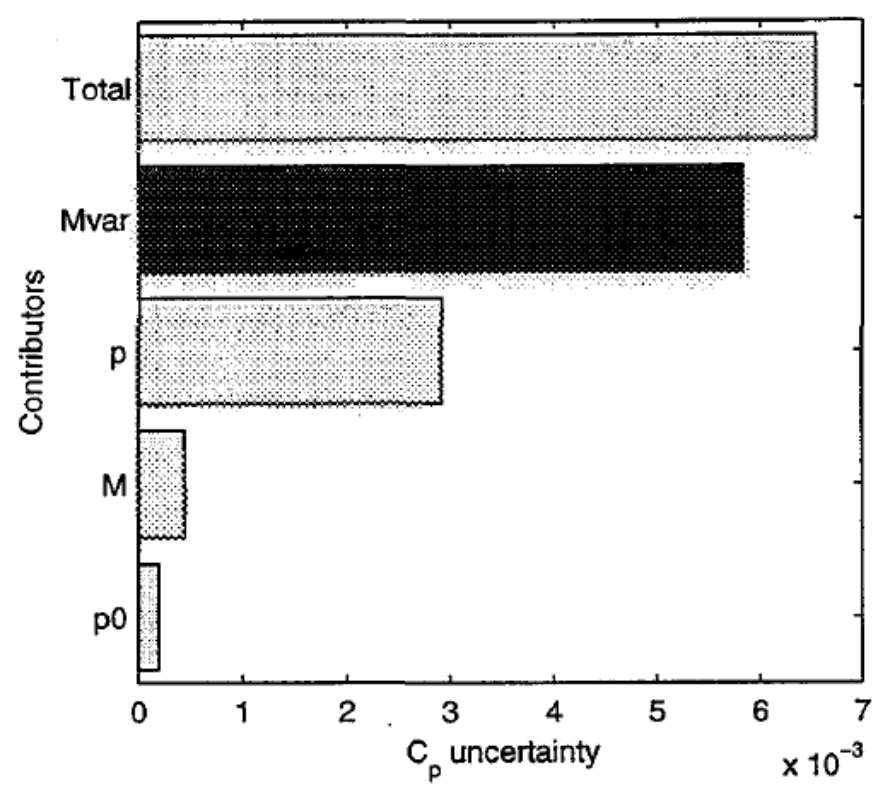

Figure 11 Contributions to the Overall Uncertainty in the Supersonic Pressure Coefficient

From inspection of Figure 11, it is seen for this example that the $U_{M_{v a r}}$ term contributes the majority of the overall uncertainty. This implies that the uncertainty in the facility calibration is by far the highest contribution to overall uncertainty. In his research, Kammeyer went on to show the same analysis for the supersonic measurement of drag coefficient which the same conclusions were drawn.

\subsubsection{Transonic Measurements}

In the PSWT during transonic testing conditions, the test section conditions are determined from the total temperature, $T_{0}$, total pressure, $p_{0}$ and the average static pressure in the plenum chamber, $p_{p}$. The calibration was completed using a static pipe which runs the length of the test section. During the calibration procedure, plenum pressure ratios, $p_{p} / p_{0}$ versus static pressure ratios $p_{s} / p_{0}$ are obtained for the transonic flow regime. Linear interpolation is used when testing conditions are not directly obtained in the calibration. Sample data from a calibration using the static pipe with the pressure ratios and location along length of the test section (axial station) is shown in Figure 12. 


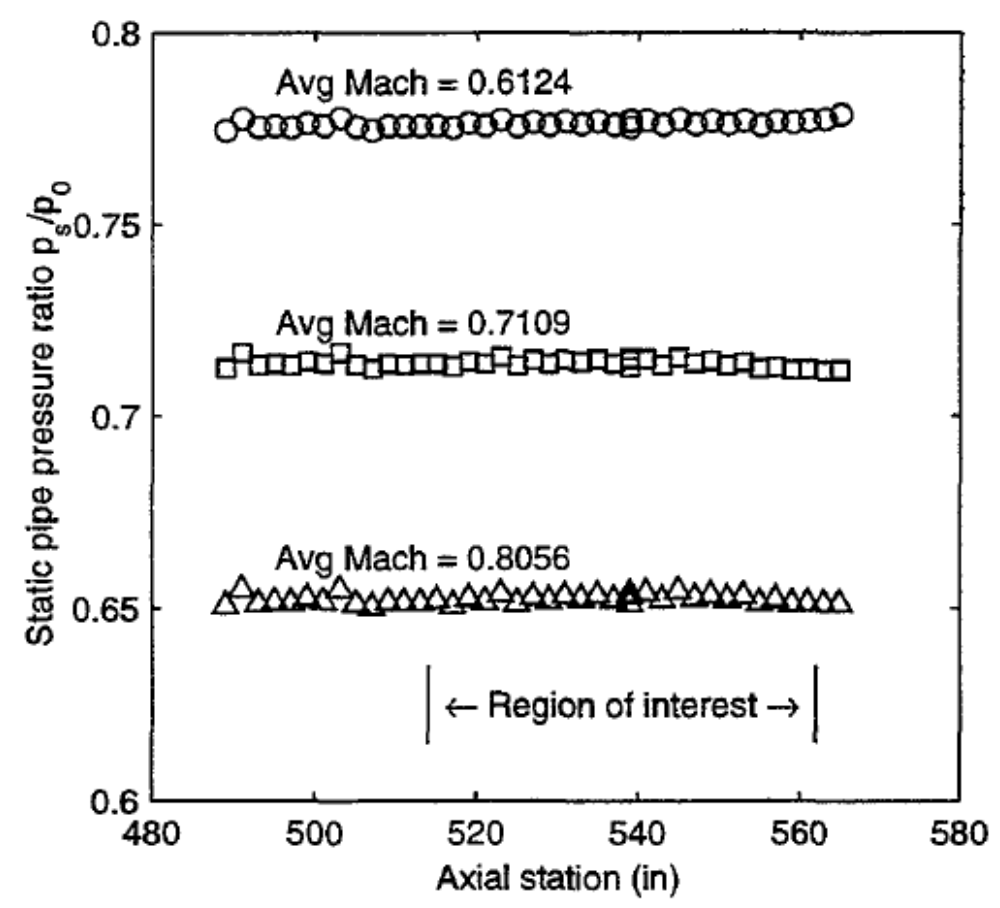

Figure 12 Example Static Pipe Calibration Data from the Boeing PSWT

In contrast to the supersonic testing conditions where the calibration uncertainty was based on the Mach number, the calibration uncertainty in the transonic case must be based on the pressure ratio. Kammeyer develops the estimate of uncertainty in the transonic calibration to be the standard deviation of the mean value of the pressure ratio for the number of static pressure taps in the region of interest (See Figure 12).

$$
U_{p_{c a l}}=t_{95} S_{p_{s} / p_{0}} N^{-1 / 2}
$$

This formulation is valid assuming the bias errors in the individual pressure taps are independent and random. Taking a nominal values from a sample test and their associated uncertainties, the total uncertainty in the pressure coefficient at transonic conditions can be formulated. 


\begin{tabular}{|c|c|c|}
\hline Variable & Value & Uncertainty \\
\hline $\boldsymbol{p}_{\mathbf{0}}$ psia & 31.09 & 0.009 \\
\hline $\boldsymbol{p}_{\boldsymbol{p}}$ psia & 24.33 & 0.01 \\
\hline $\boldsymbol{p}_{\boldsymbol{c a l}}, \boldsymbol{p}_{\boldsymbol{s}} / \boldsymbol{p}_{\mathbf{0}}$ & 0 & 0.000251 \\
\hline
\end{tabular}

Table 4 Values for Uncertainty Calculation in Transonic Pressure Coefficient Measurement

The breakdown of the sources of uncertainty in the transonic pressure coefficient are shown in Figure 13.

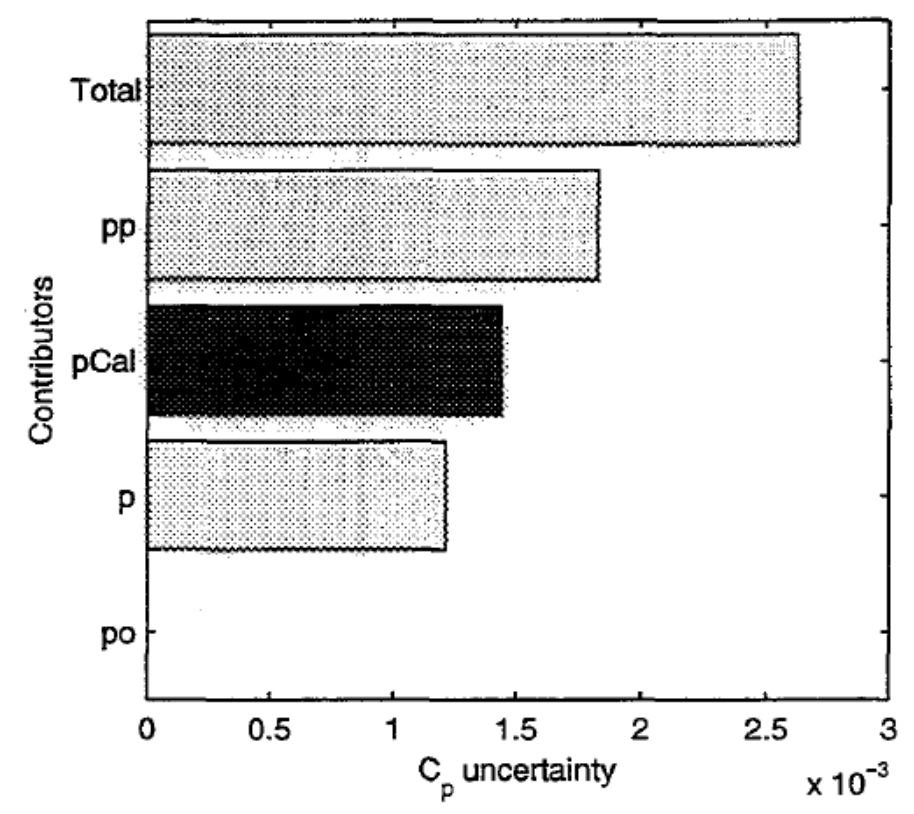

Figure 13 Contributions to the Overall Uncertainty in the Transonic Pressure Coefficient

Although the calibration uncertainty is not the largest source of uncertainty in the overall uncertainty in the pressure coefficient, it is a still a large percentage, indicating the importance of facility calibration.

\subsubsection{Summary}

The importance of statistically rigorous characterization of wind tunnel uncertainty is clearly presented in Kammeyer's work. The step-by-step procedure of breaking down the contributors to the overall variation is extremely insightful. The contribution of the uncertainty in the facility calibration to the 
overall measurements is not unexpected, however, the percentages are very high (over half in most cases). With the methods to determine the overall uncertainty well established, more scrutiny should now be placed on how the individual uncertainties are determined. At times, these values are determined in an ad-hoc manner. For example, Kammeyer sets one uncertainty as "a value observed in the past." Adopting a design of experiments based calibration could eliminate most of the ambiguity in assigning uncertainties and could lead further the goal of incorporating measurement uncertainty into standard test data package. 


\subsection{Check Standard Testing Across Multiple Transonic Wind Tunnels with the Modern Design of Experiments}

\subsubsection{Report Overview}

In his paper, "Check-Standard Testing Across Multiple Transonic Wind Tunnels with the Modern Design of Experiments," DeLoach presents the results from the analysis of wind tunnel data acquired from the Facility Analysis Verification and Operational Reliability (FAVOR) project. The premise of the FAVOR project was to conduct very similar, if not identical, wind tunnel tests at multiple transonic facilities with the same test article, force balance and sting, and analyze the data with what DeLoach refers to as the "Modern Design of Experiments" (MDOE). With the design of experiments and analysis of variance, DeLoach attempted to quantify the variance in the data and partition it into "explained" and "unexplained" components. The unexplained variance was to be further subdivided into random and systematic variance. These partitioned variances at each facility were then compared.

\subsubsection{Analysis of Content}

\subsubsection{Experimental Equipment}

The four participating wind tunnel facilities in the FAVOR project were the National Transonic Facility (NTF) at NASA Langley Research Center (LaRC), the 8x6 Foot supersonic wind tunnel at NASA Glenn Research Center (GRC), the 11 Foot Unitary Plan wind tunnel at NASA Ames Research Center (ARC) and the 16T wind tunnel at the Arnold Engineering Development Center (AEDC). The test article utilized for the FAVOR project testing was the AEDC check standard model which is a $5 \%$ scale model of the F- 111 . Due to the different test section sizes, the model was modified to give a 48in wing span at a fixed wing sweep angle of $35^{\circ}$. While not explicitly stated, it is assumed that data presented and analyzed in the paper was independently "corrected" at each facility for wall interference and other effects as the percent blockage in each tunnel will vary. Trip dots were located on the model nose, upper and lower surfaces of the wing strake, wing and horizontal and vertical tails. The two control surface configurations tested were a zero (Configuration 0 ) and ten degree (Configuration 1 ) deflection of the horizontal tail. For all four tests, the same balance (NTF-115), balance calibration, sting and instrumentation were used. 


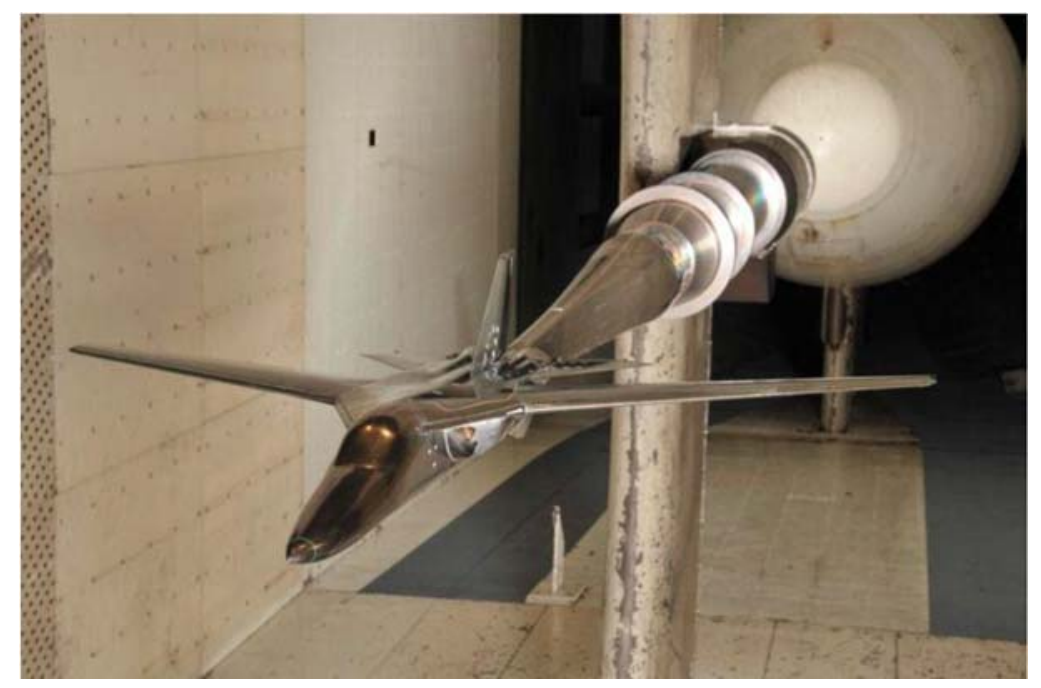

Figure 14 FAVOR F-111 Test Article

\subsubsection{Data Collection and Reduction}

It is important to note that the test designs were developed and executed as OFAT designs and not with design of experiments methodologies, however, DOE concepts were employed in the analysis of the data. This post-test use of DOE to analyze data is not ideal as the full benefits of the methodology cannot be exploited. However, the realities of complex ground testing sometimes do not permit the ideal testing schedules.

Nearly 40,000 force, moment and pressure polars (both angle of attack and sideslip) were collected across all four facilities for the FAVOR experiment (In this paper, the analysis of the data was limited to only the force and moment data). However, so few polars were collected at "identical" design space locations that DeLoach was unable to quantify the across-facilities variance. The focus of the analysis was thus shifted to characterize the within-test variance for each facility and compare these variances across facilities. A representation of the design space for zero degree roll, angle of attack sweeps at Configuration 0 was shown previously in Figure 2.

\subsubsection{Data Analysis}

The author goes into great detail in delineating the differences between "explained" and "unexplained" variances. In short, explained variances are what aerodynamicists are interested in such as how the independent variable angle of attack affects the aerodynamic forces. Facility managers are more 
concerned with unexplained variances; that is the "residual" variance that cannot be attributed to changes in the independent variables.

The first question DeLoach attempts to answer relates to the replicated data within each facility, specifically, are there statistical differences within the replicated polars at each of the four facilities. The methodology employed by the author to characterize the differences within repeated data at each facility is Analysis of Variance (ANOVA). Repeated drag polars from "Tunnel D" obtained over the course of multiple days are given in Table 5 and the ANOVA calculations are given in Table 6.

\begin{tabular}{|c|c|c|c|c|c|c|c|c|}
\hline AoA & \multicolumn{10}{|c|}{ CD: 1CDS_SLC } \\
\cline { 2 - 9 } Set-Point & Run 1 & Run 2 & Run 3 & Run 4 & Run 5 & Run 6 & Run 7 & Run 8 \\
\hline-2.8 & 0.0354 & 0.0353 & 0.0355 & 0.0353 & 0.0354 & 0.0351 & 0.0352 & 0.0350 \\
\hline-1.8 & 0.0303 & 0.0304 & 0.0303 & 0.0304 & 0.0304 & 0.0303 & 0.0302 & 0.0303 \\
\hline-0.8 & 0.0289 & 0.0289 & 0.0289 & 0.0289 & 0.0290 & 0.0289 & 0.0288 & 0.0288 \\
\hline 0.2 & 0.0300 & 0.0299 & 0.0299 & 0.0299 & 0.0299 & 0.0298 & 0.0299 & 0.0298 \\
\hline 1.2 & 0.0337 & 0.0337 & 0.0336 & 0.0337 & 0.0336 & 0.0337 & 0.0337 & 0.0338 \\
\hline 2.2 & 0.0412 & 0.0415 & 0.0412 & 0.0411 & 0.0410 & 0.0410 & 0.0414 & 0.0411 \\
\hline 3.2 & 0.0526 & 0.0528 & 0.0524 & 0.0526 & 0.0527 & 0.0526 & 0.0528 & 0.0526 \\
\hline
\end{tabular}

Table 5 Replicated Drag Polars for "Tunnel D" Configuration 0, Roll = 0\%, Re 4.5E06, Mach 0.85

\begin{tabular}{|l|c|c|c|c|c|c|}
\hline Source & SS & $d f$ & MS & $\boldsymbol{F}$ & P-value & F crit \\
\hline Rows & 0.003435 & 6 & $5.73 \mathrm{E}-04$ & 51648 & $1.5 \mathrm{E}-79$ & 2.324 \\
\hline Columns & $1.45 \mathrm{E}-07$ & 7 & $2.06 \mathrm{E}-08$ & 1.863 & 0.1004 & 2.237 \\
\hline Error & $4.66 \mathrm{E}-07$ & 42 & $1.11 \mathrm{E}-08$ & & & \\
\hline & & & & & & \\
\hline Total & 0.003436 & 55 & & & & \\
\hline
\end{tabular}

Table 6 ANOVA for "Tunnel D" Repeated Drag Polars

The row sum of squares (SS) is given by

$$
S S_{R}=n\left(\bar{y}_{l} \cdot-\overline{y \cdot \cdot}\right)^{2}
$$


where $n$ is the number of runs, $\overline{y_{l}}$. is the row average (average at one set angle of attack) and $\bar{y}$.. is the grand average (average of all data points). The column sum of squares is similarly calculated by using the column averages and number of angle of attack entries (7). The degrees of freedom (df) are given as $n-1$ for each of the rows and columns. The total degrees of freedom are given by total data points minus one, and the sum of squares error is given by $S S_{T}-S S_{R}-S S_{C}$. The mean square (MS) is given by $S S / d f$ and the $\mathrm{F}$ value is given by $M S / M S_{E}$. The test statistic, $F_{C r i t}$ is determined from the $\mathrm{F}$ distribution with given degrees of freedom and the desired significance level ( 0.05 in this analysis). If the F value is larger than the test statistic the null hypothesis can be rejected, in this case that there is no statistical difference between the columns (runs) or separately the rows (angle of attack). From inspection of Table 6 it is seen that the null hypothesis, that there is no statistical difference between levels of angle of attack, can be rejected, which is not surprising. From first aerodynamic principles, one would expect changes in angle of attack to cause changes in drag coefficient. However inspecting the $\mathrm{F}$ test for the runs, the null hypothesis that there is no statistical difference between the runs was not able to be rejected. The wording of the previous statement is important; the fact that we are unable to reject the null hypothesis does not mean there is no difference between the runs. Clearly there are runto-run differences, but given the data, it was not able to be determine that there was a statistical difference between runs at the chosen level of significance of $5 \%$.

To further illustrate this point, assume that 19 replicated runs were completed for the data given in Table 5 with the ratio of $M S_{C} / M S_{E}$. In this fictitious case, the degrees of freedom would have increased to 18 , giving a test statistic of 1.855 which would have been less than the $F$ value. In turn, it could have been determined that there was a statistical difference between the runs. This fabricated scenario summarizes dilemmas often encountered in ground testing. That is, inferences or hypotheses may be anecdotally supported by the data, but a large and usually expensive data set is sometimes necessary to statistically accept or reject the hypotheses.

DeLoach examined all replicated polars, both angle of attack and sideslip, within each facility with the ANOVA process outlined above and determined that combined in all four facilities that there was a statistical difference in $51.4 \%$ of the replicated data. It should not be surprising that there was such a large percentage of replicated data that was significantly different. It should however underscore the importance of the design, execution and collection of the data. 
With the information derived from the ANOVA analysis, DeLoach outlines the methodology to develop a total uncertainty due to unexplained variance, $u_{c}$.

$$
u_{c}^{2}=M S_{\text {random }}+M S_{\text {systematic }}
$$

Referring again back to the data in Table 5 and analysis in Table 6, the mean square error is designated as the random component and the mean square associated with columns is designated as the systematic component in Equation 14. Evaluating the equation for unexplained variance using the data from the drag coefficient ANOVA gives a $u_{c}=0.00018$ (1.8 drag counts). The author further develops the uncertainty estimate to an expanded uncertainty, $U_{\%}$ which includes a coverage factor based on degrees of freedom and a t-statistic.

$$
U_{\%}=t_{\%} u_{c}
$$

Due to the differing degree of freedom allocation in the columns and error in Table 6, a pooled estimate for degrees of freedom, $v$ is utilized for the t-statistic.

$$
v=\frac{u_{c}^{2}}{\frac{M S_{\text {random }}^{2}}{v_{\text {random }}}+\frac{M S_{\text {systematic }}^{2}}{v_{\text {systematic }}}}
$$

Evaluating Equation 16 with the data in Table 5 gives a pooled estimate of degrees of freedom of 15.8. In turn, evaluating Equation 15 with a significance level of 0.05 , yields an expanded uncertainty of 0.00038 ( 3.8 drag counts). This uncertainty value in the drag coefficient would be considered unacceptably high for the type model and conditions tested. For a civilian transonic transport, it is desired to have the uncertainty in the drag coefficient less than 1 drag count $^{39}$.

\subsubsection{Summary}

The FAVOR experiment was unprecedented in its scope of testing across four facilities utilizing the same experimental equipment (model, balance, sting and instrumentation). However, without the use of 
design of experiments methodology before conducting the testing, the ability to draw statistically defensible conclusions from the across-facility comparisons is limited. The lack of replication and randomization of data points at the same conditions at each of the facilities prevents the across-facility comparison of variances. The author did try to reconcile this by averaging all the variance components, explained and unexplained, at each facility. However the dispersion cannot be assumed to be constant across the entire design space, thus the comparison of within-test dispersions of different facilities must be evaluated at similar design space locations. Therefore comparison of the averages of the variances across the facilities cannot be treated as an "apples-to apples" comparison. It is very likely that the variance levels were not constant across all tunnel operating conditions. These circumstances highlight the potential of the design of experiments methodology, when properly employed, to aid in check standard wind tunnel testing programs.

\subsection{Analysis of Flow Angularity Repeatability Tests in the NTF}

\subsubsection{Report Overview}

In the paper "Analysis of Flow Angularity Repeatability Tests in the NTF", Hemsch establishes the procedure for quantifying the repeatability of flow angularity measurements in wind tunnel testing. Repeatability estimates were primarily formulated through the analysis of ranges (ANOR) and statistical process control (SPC) methodologies. Flow angularities from four check standard tests completed at the NTF were analyzed for statistical consistency and characterization of angle of attack predictions for customer tests. Hemsch also presents methodology for quality assurance of flow angularities during customer tests.

\subsubsection{Analysis of Content}

\subsubsection{Flow Angularity Calculation}

The flow angularity in a wind tunnel test section is generally defined as a measure of the "straightness" of the flow. More specifically, the flow angularity is given by the ratio of "up-flow" or "cross-flow" to the streamwise flow.

$$
F A=\tan \frac{v}{u}
$$


Where $v$ is the velocity of the up-flow and $u$ is the velocity of the streamwise flow.

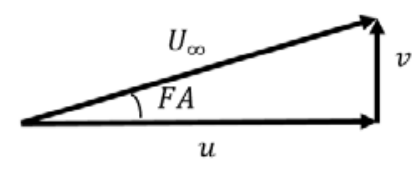

Figure 15 Flow Angularity Diagram

During a wind tunnel facility calibration or characterization, the flow angularity is usually measured in both the pitch and cross flow axis and can be given at a single point (5-hole probe) or as an average (model pitch polars). In Hemsch's paper, the flow angularity discussed is only the measurement of the pitch flow angularity presented as an average.

To determine the test section flow angularity, a generic subsonic transport model was tested in the linear range $\left(-2^{\circ}\right.$ to $\left.2.5^{\circ}\right)$ of the lift curve slope of $C_{N}$ vs $A O A$, both upright ( $0^{\circ}$ roll angle) and inverted $\left(180^{\circ}\right.$ roll angle). If there was no flow angularity present, both the upright and inverted pitch polars would be identical. If a detectable flow angularity was present, a difference would exist in the upright and inverted pitch polars. To solve for the flow angularity, a least squares linear regression was fit to each of the pitch polars.

$$
C_{N}=C_{N_{\alpha}} \alpha+C_{N_{0}}
$$

Then these equations were solved for each of the upright and inverted cases for the angle of attack, $\alpha^{*}$, at the normal force coefficient, $C_{N}^{*}$,equal to 0.2 which was approximately the middle of the $C_{N}$ range. The flow angularity is then given by:

$$
F A=0.5\left(\alpha_{\text {upright }}^{*}-\alpha_{\text {inverted }}^{*}\right)
$$


where

$$
\alpha^{*}=\left(C_{N}^{*}-C_{N_{0}}\right) / C_{N_{\alpha}}
$$

A graphical representation of the calculations is given in Figure 16.

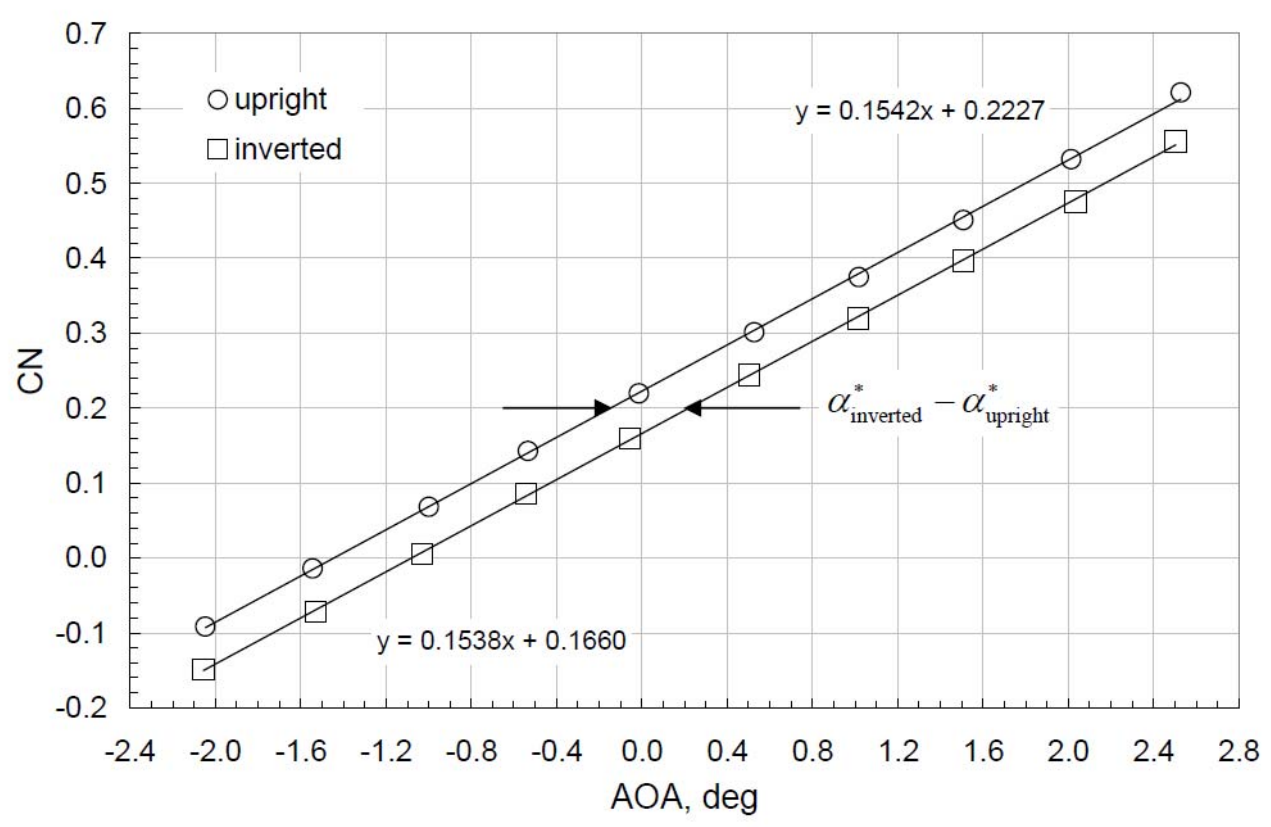

Figure 16 Flow Angularity Pitch Polar Data

For check standard tests 121 and 137, five pitch polars, upright (1), inverted (2), upright (3), upright (4) and inverted (5) were collected five times for a total of 25 polars at each condition (dynamic pressure, total pressure, Mach number). For tests 121 and 137, runs 1 and 2 and runs 4 and 5 gave two estimates of flow angularity forming a subgroup. Check standard test 149 collected only runs at upright (1) inverted (3) and upright (3) each replicated five times. Estimates for flow angularity from test 149 were given from runs 1 and 2 and runs 2 and 3. Check standard test 156 consisted of runs at upright (1), inverted (2), inverted (3) and upright (4). A summary of the test data points is given in Table 7. 


\begin{tabular}{|l|l|l|l|l|l|l|}
\hline Test & Run 1 & Run 2 & Run 3 & Run 4 & Run 5 & FA Calculations \\
\hline $\mathbf{1 2 1}$ & Upright & Inverted & Upright & Upright & Inverted & $1-2$ and 4-5 \\
\hline $\mathbf{1 3 7}$ & Upright & Inverted & Upright & Upright & Inverted & $1-2$ and 4-5 \\
\hline $\mathbf{1 4 9}$ & Upright & Inverted & Upright & N/A & N/A & $1-2$ and 2-3 \\
\hline $\mathbf{1 5 6}$ & Upright & Inverted & Inverted & Upright & N/A & $1-2$ and 3-4 \\
\hline
\end{tabular}

Table 7 Check Standard Test Run Information

\subsubsection{Characterization of Repeatability}

To first characterize the repeatability of the flow angularity data, Hemsch fitted the ranges of the flow angularity as a function of dynamic pressure. Previous check standard and tunnel testing suggested that the repeatability of the normal force coefficient was a function of the tunnel dynamic pressure. Since the measurement of flow angularity is determined via the acquisition of the normal force coefficient, it was reasonable to assume that the repeatability of the flow angularity would display the same tendencies with respect to dynamic pressure. The mean ranges of all the subgroup measurements for each condition in each test as a function of dynamic pressure are shown in Figure 17. Note each data point in Figure 17 represents the mean of the range of a set of 10 flow angularity measurements.

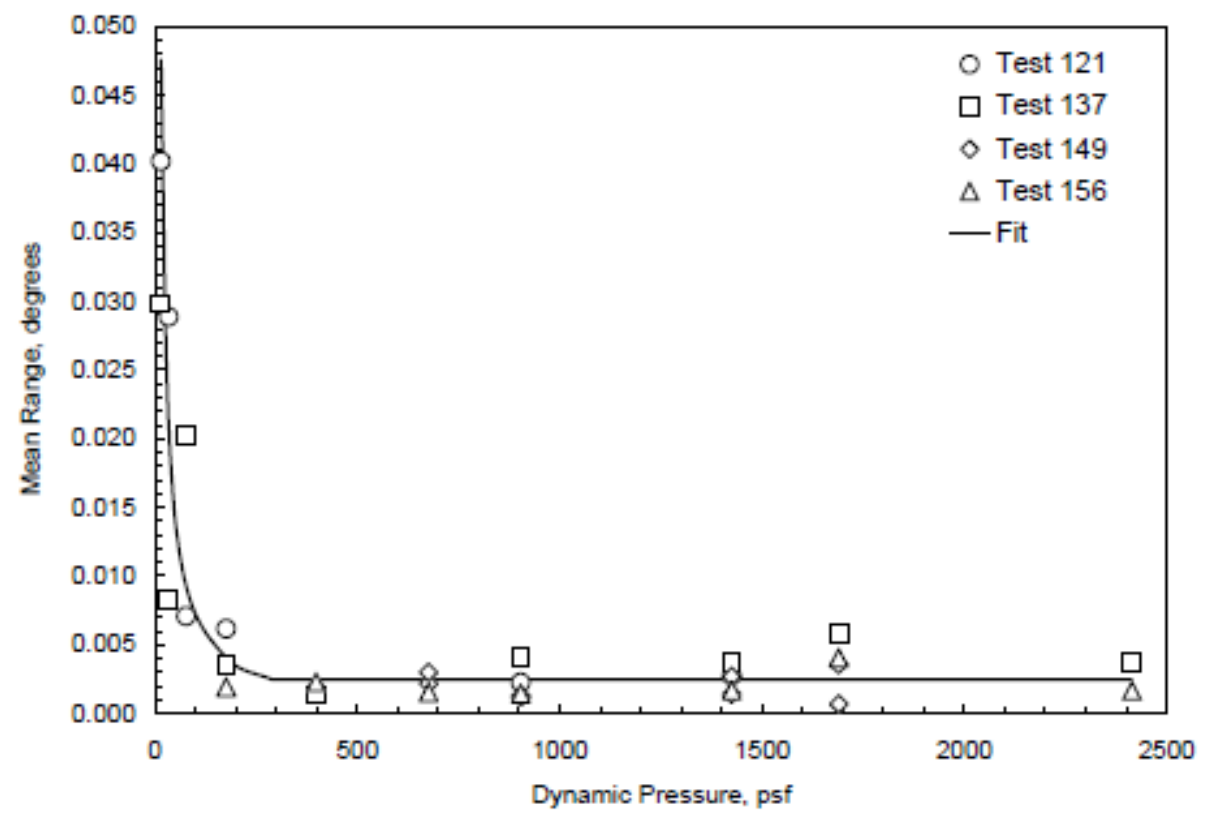

Figure 17 Flow Angularity Mean Ranges vs Dynamic Pressure 
The mean of the range of the flow angularity subgroups were observed to be proportional to the inverse of the tunnel dynamic pressure from zero to approximately $400 \mathrm{psf}$ and constant thereafter. The fit of the mean of the ranges was given as:

$$
\begin{gathered}
R_{f i t}=0.714 / q_{\infty}(\operatorname{deg} / p s f) \quad q_{\infty}<398 p s f \\
R_{f i t}=0.00246 \mathrm{deg} \quad q_{\infty}>380 \mathrm{psf}
\end{gathered}
$$

Hemsch then estimated the flow angularity population standard deviation using the fit for the mean range from Equation 22 citing that the most stringent requirements on flow angularity come from transonic cruise performance testing which occurs in the higher range of dynamic pressure.

$$
\hat{\sigma}_{F A}=R_{f i t} / 1.128=0.00218 \mathrm{deg}
$$

After fitting the ranges, the author attempts to determine if the "scatter" in the ranges fits any frequency distribution. To compare ranges across tunnel conditions, the ranges at each condition are normalized by $R / R_{\text {fit }}$. The frequency of the normalized ranges across all tests are shown in Figure 18 with a normalized range distribution from a standard normal distribution. 


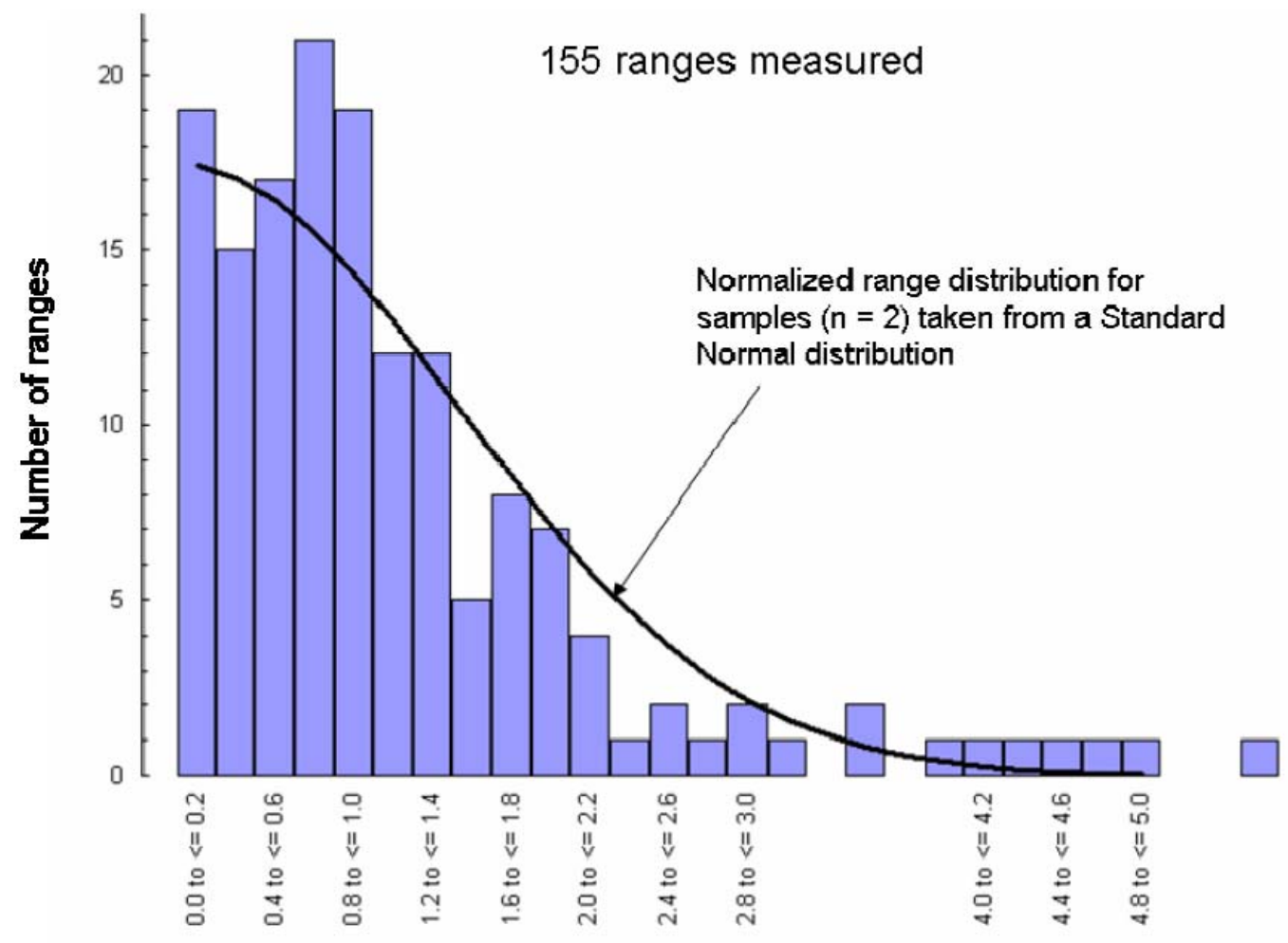

Figure 18 Distribution of Normalized Flow Angularity Ranges 1

Hemsch notes that the distribution reasonably follows the standard normal distribution however a "heavy tail" exists. He further explores the heavy tailed distribution with the analysis of ranges (ANOR). With ANOR, the test for statistical consistency is given by Equation 24.

$$
\frac{R_{0.995}}{R_{\text {fit }}}=\frac{W_{0.995}}{W_{\text {fit }}}=\frac{3.970}{1.128}=3.52
$$

Equation 24 gives the critical value for the normalized mean ranges. For the chosen confidence level of 0.005 , it was expected that only $0.5 \%$ of the normalized ranges $(0.005 * 155 \approx 1$ ) would exceed the critical value by chance for a random sampling from a normal distribution. From inspection of Figure 19, it is seen that the number of normalized ranges larger than the critical value in Equation 24 indicates a heavy tailed, normal distribution. 


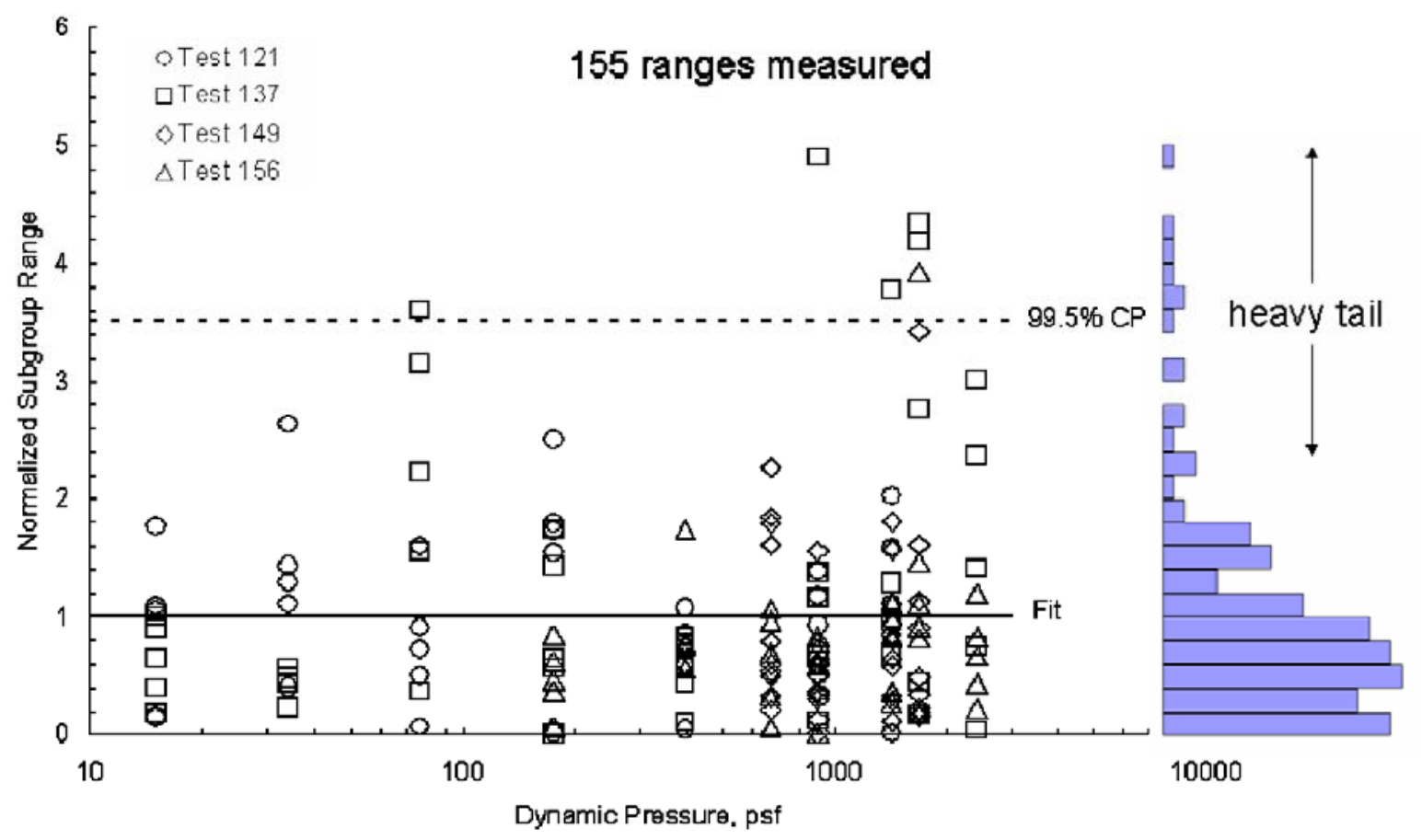

Figure 19 Distribution of Normalized Flow Angularity Ranges 2

The likely cause of the heavy tail, the author theorizes, is the contamination of another normally distributed source of variation coming from a relatively large population standard deviation. However, he points out that fitting the ranges rather than the variances is a more robust estimation process and the presence of the heavy tail in the distribution is not problematic for using the repeatability to estimate the uncertainty.

To characterize the repeatability of the within-test (between subgroup) flow angularity measurements, a differential flow angularity variable is defined.

$$
\Delta F A=(\overline{F A}-\overline{\overline{F A}}) / R_{f i t}
$$

where $\overline{F A}$ is the average of the two flow angularities is obtained in each subgroup and $\overline{\overline{F A}}$ is the flow angularity average for all subgroups in a test for a given condition. To account for the variability attributable to repeatability alone, upper and lower repeatability limits were established via a 99\% coverage range of the normal distribution by 


$$
\Delta F A_{\text {limits }}= \pm 2.576 \hat{\sigma}_{F A}
$$

where the standard deviation of $\triangle F A$ is given by

$$
\hat{\sigma}_{F A}=\hat{\sigma}_{\overline{F A}} / R_{f i t}
$$

Noting that the $\overline{F A}$ is an average and if considering repeatability as the only source of variation gives

$$
\hat{\sigma}_{\overline{F A}}=\sigma_{F A} / \sqrt{2}
$$

Substituting Equations 23, 27 and 28 into Equation 26 and simplifying yields

$$
\Delta F A_{\text {limits }}= \pm 2.576 \frac{\hat{\sigma}_{\overline{F A}}}{R_{\text {fit }}}= \pm 1.615
$$

The limits on the $\triangle F A$ quantity in Equation 29 can be misleading; it does not imply that the limits placed on the flow angularity calculations are $+/-1.615^{\circ}$. Rather, for a $R_{\text {fit }}=0.00246^{\circ}$, the limit on the deviation of a subgroup average flow angularity from the average of all subgroup flow angularities (see Equation 25) was approximately $0.004^{\circ}$. The variation in flow angularity subgroup averages from Test 137 is given in Figure 20. While not necessarily representative of the other three tests, the results from Test 137 give a general representation of the variation of the flow angularities within each group. Test 156 exhibited the lowest within-group flow angularity variation with only $2 / 35$ points outside of the derived limits, while test 149 exhibited systematic trends and had over half of the points outside of the derived limits. 

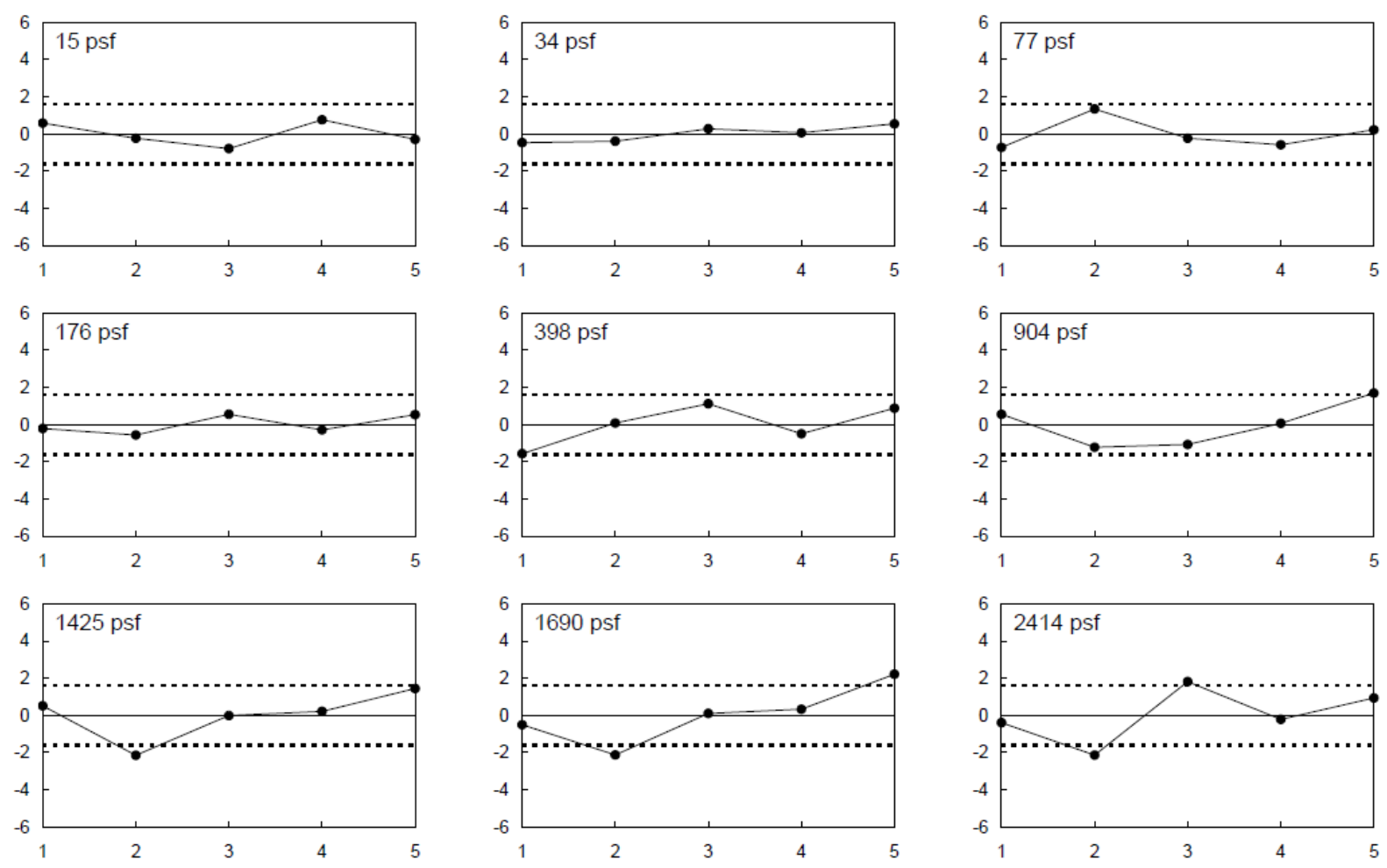

Figure 20 Variation of Flow Angularity Subgroup Averages $(\overline{F A}-\overline{\overline{F A}}) / R_{\text {fit }}$ Test 137

Overall for all four tests, $25 \%$ of the subgroup averages were outside of the derived limits which far exceeds the expected number given the coverage factor of $1 \%$ chosen. Although the largest flow subgroup angularity deviations were only $0.015^{\circ}$, this suggests that other sources of variation were introduced during the acquisition of the flow angularity data causing measureable shifts in the data.

To develop an estimate of the overall uncertainty in the measurement of the flow angularity, the withintest (between-subgroup) variability is examined. The between-subgroup variation is given by removing the variation associated with the repeatability.

$$
\hat{\sigma}_{B G}=\sqrt{\hat{\sigma}^{2}{ }_{\Delta F A, \text { actual }}-\hat{\sigma}^{2}{ }_{\Delta F A, \text { repeatability }}}
$$

Solving for the variation associated with repeatability from Equations 23,27 and 28 gives 


$$
\hat{\sigma}_{\Delta F A, \text { repeatability }}=\frac{\hat{\sigma}_{\overline{F A}}}{R_{\text {fit }}}=0.6269
$$

To solve for the between-subgroup variation, the estimate for the actual $\triangle F A$ was obtained from the averages of all four tests giving $\hat{\sigma}_{\triangle F A \text {,actual }}=1.140$ and $\widehat{\sigma}_{B G}=0.9522$. Carrying out the algebra gives the value for the estimate of the combined variability.

$$
\hat{\sigma}_{\text {combined }}=\sqrt{\hat{\sigma}_{B G}^{2}-\left(\frac{\hat{\sigma}_{\overline{F A}}}{R_{f i t}}\right)^{2}}=1.47 \frac{\hat{\sigma}_{F A}}{R_{f i t}}
$$

Equation 32 indicates that the estimate for the combined variability in the flow angularity measurement is approximately $50 \%$ greater than the estimate of variability for the within-subgroup variability. However, Hemsch notes that this should be taken as a "rule of thumb" due to the fact that the combined uncertainty is likely heavily dependent on testing procedures.

\subsubsection{Verification of Repeatability Predictions}

The methodology for maintaining statistical control over the flow angularity measurements completed during check standard testing can remain unchanged if the testing procedures remain constant. The expected mean range can be predicted utilizing Equations 21 and 22 and the statistical control limit on the normalized subgroup ranges given by Equation 24. However, to adapt the estimation of variability in the flow angularity measurements for different models, modification of the formulation is necessary. By carrying out a simple error propagation analysis and examining the assumptions of the linear regression procedure, Hemsch shows that the variability of the flow angularity is proportional to the variability in the normal force coefficient divided by the lift curve slope.

$$
\sigma_{F A} \sim \sigma_{C_{N}} / C_{N_{\alpha}}
$$

Thus for a customer test, the flow angularity repeatability would be given by

$$
\sigma_{F A, \text { customer }}=\sigma_{F A, P F I}\left(\frac{\sigma_{C_{N, \text { customer }}}}{\sigma_{C_{N, P F I}}}\right)\left(\frac{C_{N_{\alpha, P F I}}}{C_{N_{\alpha, \text { customer }}}}\right)
$$


where $\sigma_{F A, P F I}$ is the variability from the check standard test using the Pathfinder 1 (PFI) model. A further assumption is made that the balance utilized in the customer testing will have similar repeatability as the check standard testing resulting in

$$
\sigma_{F A, \text { customer }}=\sigma_{F A, P F I}\left(\frac{S_{R_{P F I}}}{S_{R, \text { customer }}}\right)\left(\frac{C_{N_{\alpha, P F I}}}{C_{N_{\alpha, \text { customer }}}}\right)
$$

where $S_{R}$ is the model reference area and $C_{N_{\alpha, \text { customer }}}$ must be known or estimated. Similarly the customer fit for the mean range is given by

$$
R_{f i t, \text { customer }}=R_{f i t, P F I}\left(\frac{S_{R_{P F I}}}{S_{R, \text { customer }}}\right)\left(\frac{C_{N_{\alpha, P F I}}}{C_{N_{\alpha, \text { customer }}}}\right)
$$

To verify the formulations proposed in Equations 35 and 36, a data set from a customer with a model area and lift curve slope significantly different from the check standard model was analyzed. The fit for the mean range in the linear range (low dynamic pressure) was given by

$$
R_{f i t, \text { customer }}=\frac{0.0714 \mathrm{deg} / \mathrm{ps} f}{q_{\infty}}\left(\frac{1.988 f \mathrm{ft}^{2}}{5.563 f \mathrm{t}^{2}}\right)\left(\frac{0.140 \mathrm{deg}^{-1}}{0.082 \mathrm{deg}^{-1}}\right)=\frac{0.436 \mathrm{deg} / \mathrm{ps} f}{q_{\infty}}
$$

The fit for the mean range was given by

$$
R_{f i t, \text { customer }}=0.00246 \mathrm{deg}\left(\frac{1.988 f t^{2}}{5.563 f t^{2}}\right)\left(\frac{0.140 \mathrm{deg}^{-1}}{0.082 \mathrm{deg}^{-1}}\right)=0.00150 \mathrm{deg}
$$

The normalized subgroup ranges from the customer test are shown in Figure 21. 


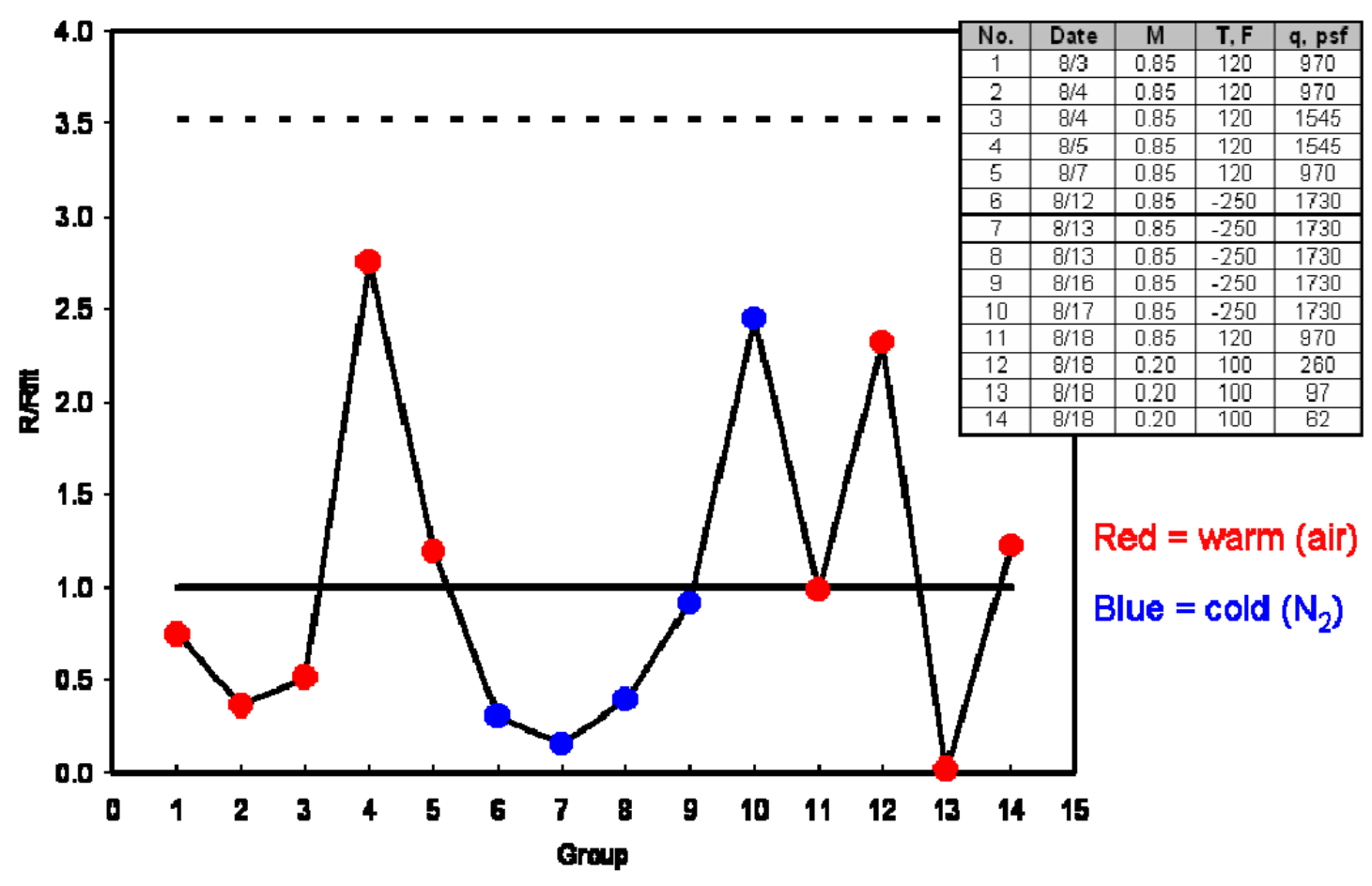

Figure 21 Flow Angularity Ranges from Customer Test

It is seen from the ranges of the flow angularities in the customer test that no ranges exceeded the limit set by Equation 24, the mean of all the subgroup ranges is nominally one, and no apparent difference when operating the tunnel in cryogenic temperatures was observed.

\subsubsection{Summary}

Hemsch was able to demonstrate a comprehensive quality assurance process for wind tunnel flow angularity measurements. It was shown that the confidence interval for flow angularity repeatability for typical transonic cruise conditions at $+/-2 \sigma$ coverage was $+/-0.0044^{\circ}$ (Equation 23 ) which is significantly lower than the required $+/-0.01^{\circ}$. Expanding the confidence interval to account for reproducibility also falls within the required tolerance band. The methodology was applied to a customer test in which the model utilized was significantly different (geometrically, aerodynamically) than the check standard model with promising results. The calculation of the variability in the flow angularity repeatability (within-subgroup) measurements based on the range is computationally straightforward and statistically robust. However, a more robust methodology for the development of the within-test (what Hemsch 
refers to as "reproducibility") is necessary to assure that the required tolerance band is met. Also the author does not attempt to quantify the variability in across-test flow angularity. This variability would be of interest if a full statistical process control program was implemented.

Presumably, it is also of interest to researchers to know how the flow angularity repeatability in the NTF compares to other facilities. This knowledge could potentially lead to process improvement and drive down overall uncertainty in flow angularity measurements.

\subsection{Repeatability Modeling for Wind-Tunnel Measurements: Results for Three Langley Facilities}

\subsubsection{Report Overview}

In their paper "Repeatability Modeling for Wind Tunnel Measurements: Results for Three Langley Facilities", Hemsch and Houlden present check standard data from seven measurement processes across three NASA facilities. The data were analyzed to test models for short-term, within-test and across-test repeatability. Statistical process control was utilized to develop estimates of uncertainty for the three time frames of data collection. The goal of the development of the repeatability models was to support process improvement and development of uncertainty models for tunnel measurements.

\subsubsection{Analysis of Content}

\subsubsection{Development of Estimates of Standard Deviations}

The check standard testing campaign was developed to track the behavior of the wind tunnel's measurement systems over time frames to include short-term (within-group), within-test, and acrosstest variations. This information in addition to estimates of repeatability can give facility managers some objective evaluation of overall tunnel health. In order to verify the derived estimates of variation through statistical process control methods, a model for the estimate of uncertainty (Equation 40) for a given time frame of a generic force or pressure coefficient (Equation 39) was used.

$$
C=\frac{F}{q_{\infty} S_{R e f}}
$$




$$
\hat{\sigma}=\sqrt{\left(\frac{A}{q_{\infty}}\right)^{2}-2 \rho\left(\frac{A}{q_{\infty}}\right) B+B^{2}}
$$

where in Equation 39, $C$ is the generic coefficient, $F$ is the applied force, $q_{\infty}$ is the tunnel dynamic pressure, $S_{R e f}$ is the model reference area. In Equation 40, $A$ is the effective resolution of the instrument(s) divided by the reference area, $B$ is the coefficient of variation of the dynamic pressure $\left(\sigma_{q \infty} / q_{\infty}\right)$ multiplied by the force or pressure coefficient value and $\rho$ is the correlation coefficient for the $A / q_{\infty}$ and $B$ sources of variation. The values for the coefficients for the given facilities, testing conditions and timeframes are given in Table 8.

\begin{tabular}{|c|c|c|c|c|c|}
\hline Facility & Check Standard & Time Frame & A & B & $\rho$ \\
\hline $14 \times 22$ & Pitot-Static Probe & Back-to-Back $q_{\infty}$ Sweeps & 0.00985 & 0.000325 & 0.225 \\
\hline $\mid$ & $\mid$ & Within-Test & 0.0109 & 0.000956 & -0.9 \\
\hline $\mid$ & $\mid$ & Across-Test & 0.0160 & 0.00267 & -0.2 \\
\hline $\mid$ & Elliptical Wing (CN) & Back-to-Back Polars & 0.00919 & 0.000321 & 0.2 \\
\hline $\mid$ & $\mid$ & Within-Test & 0.025 & 0.00067 & -1 \\
\hline $\mid$ & $\mid$ & Across-Test & 0.0163 & 0.00159 & 0 \\
\hline $\mid$ & Elliptical Wing (CA) & Back-to-Back Polars & 0.00266 & 0.000061 & 0.2 \\
\hline $\mid$ & $\mid$ & Within-Test & 0.0050 & 0.00012 & -1 \\
\hline | & Across-Test & 0.0032 & 0.00014 & 0 \\
\hline NTF & Pathfinder I (CN) & Back-to-Back Polars & 0.126 & 0.0009 & 0.2 \\
\hline | & Within-Test & 0.40 & 0.00075 & 0.4 \\
\hline | & Across-Test & 0.50 & 0.00143 & 0.22 \\
\hline | & Pathfinder I (CA) & Back-to-Back Polars & .0149 & 0.00005 & 0.2 \\
\hline | & Within-Test & 0.0390 & 0.00006 & 0.3 \\
\hline | & Across-Test & 0.0500 & 0.000235 & 0.22 \\
\hline LTPT & $\begin{array}{c}\text { Efficient Airfoil } \\
\text { (Balance) }\end{array}$ & Back-to-Back Polars & 0.00306 & 0.0007 & -1 \\
\hline Efficient Airfoil & Integrated Pressure) & | & 0.000826 & 0.0012 & 0 \\
\hline
\end{tabular}

Table 8 Model Coefficients

The three time frame estimates of the standard deviations are denoted as within-group, $\hat{\sigma}_{W G}$ withintest, $\hat{\sigma}_{W T}$ and across-test, $\widehat{\sigma}_{A T}$. The estimates of standard deviations for the within-group and withintest are pooled across all tests. The mathematical formulations for each are given as 


$$
\hat{\sigma}_{W G}=\tilde{R}_{W G} / d_{4}
$$

Equation 41 stares that the estimate of the within-group population standard deviation is equal to the median of all of the within-group ranges divided by the bias correction factor $d_{4}$. The within group measurements are obtained as "back-to-back" measurements, thus representing the shortest possible timeframe for repeatability.

$$
\hat{\sigma}_{W T}=\widetilde{R}_{W T} / d_{4}
$$

Equation 42 states the estimate of the population standard deviation of group averages obtained within a test is equal to the median of all the within-test group average ranges divided by the bias correction factor $d_{4}$.

$$
\hat{\sigma}_{A T}=\widetilde{m R}_{A T} / d_{4}
$$

Equation 43 states the estimate of the population standard deviation of the test grand averages is equal to the median moving range of all the test grand averages divided by the bias correction factor $d_{4}$. The bias correction factor in Equations 41-43 are a function of the degrees of freedom in each equation. The authors note that the development of the estimations of standard deviations through the statistical process control method and the ability to compare these estimates to the derived error propagation model are highly dependent on the degrees of freedom (i.e. the number of tests performed).

\subsubsection{Data Analysis: $14 \times 22$ Test Section Calibration Coefficient}

The NASA Langley $14 \times 22$ subsonic wind tunnel is calibrated through a procedure in which a pitot-static pressure probe is located in the test section and compared to a reference pressure measurement made upstream of the test section. The calibration coefficient, $C^{\prime}$ is defined as

$$
C^{\prime}=\frac{\left(P_{\text {total }}-P_{\text {static }}\right)_{\text {probe }}}{\left(P_{\text {total }}-P_{\text {static }}\right)_{\text {reference }}}=\frac{D P C}{D P I}
$$


It is important to note that the measurement of the calibration coefficient is dependent on two sensors, which were identical to ensure similar performance. The calibration coefficient was obtained in groups during testing of three back-to-back "polars" ranging through the DPI range of 0.5 to 120 psf. The medians from the within-group statistical process control charts (not given) along with the uncertainty propagation based model (Equation 40) are shown in a log-log plot across 14 tests (yielding 90 total degrees of freedom) in Figure 22.

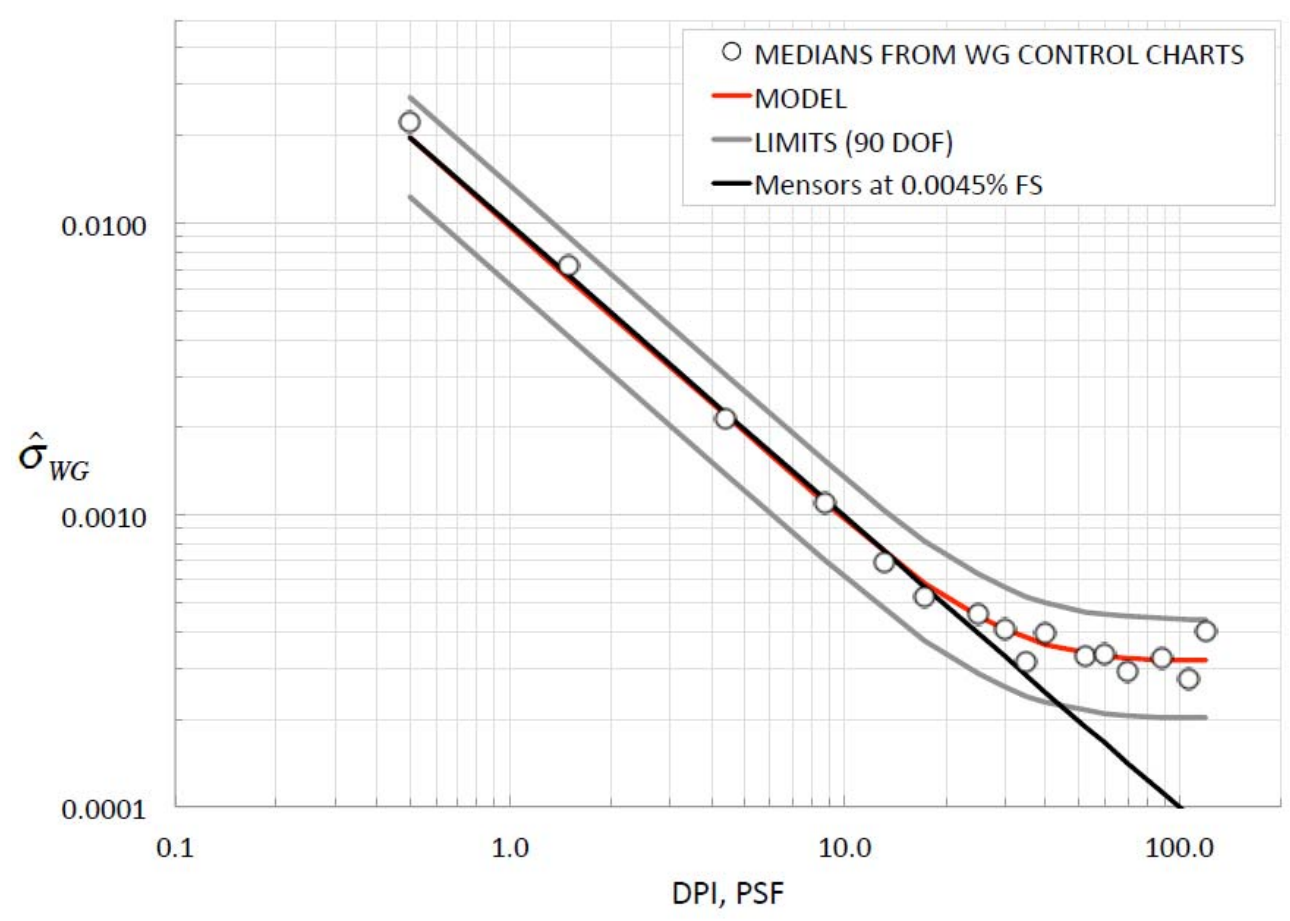

Figure 22 Model of $\widehat{\sigma}_{W G}$ for $C^{\prime}$ from $14 \times 22$ with Control Limits for 90 Degrees of Freedom

To obtain the coefficients in the uncertainty propagation based model (Equation 40), empirical fits and averaging methods were employed. The model coefficient A was obtained by averaging $\sigma *$ DPI over the region for which the 1/DPI dependence holds (low dynamic pressure, approximately DPI = 0.525psf). The model coefficient B was obtained by averaging $\sigma$ over the region where it "seems to be constant" (approximately DPI 25-120psf). The correlation coefficient, $\rho$ was chosen to fit the largest number of data points between the limits. The limits in Figure 22 are given via statistical process control methods for the within-group degrees of freedom (90). Also shown in Figure 22 is the expected result using the manufacturers quoted combined repeatability, nonlinearity and hysteresis. It is seen that the 
uncertainty propagation based model is in very good agreement with the statistical process control derived data as no data points are outside the prescribed limits. Similar figures are shown for the within-test, and across-test estimates of the standard deviations.

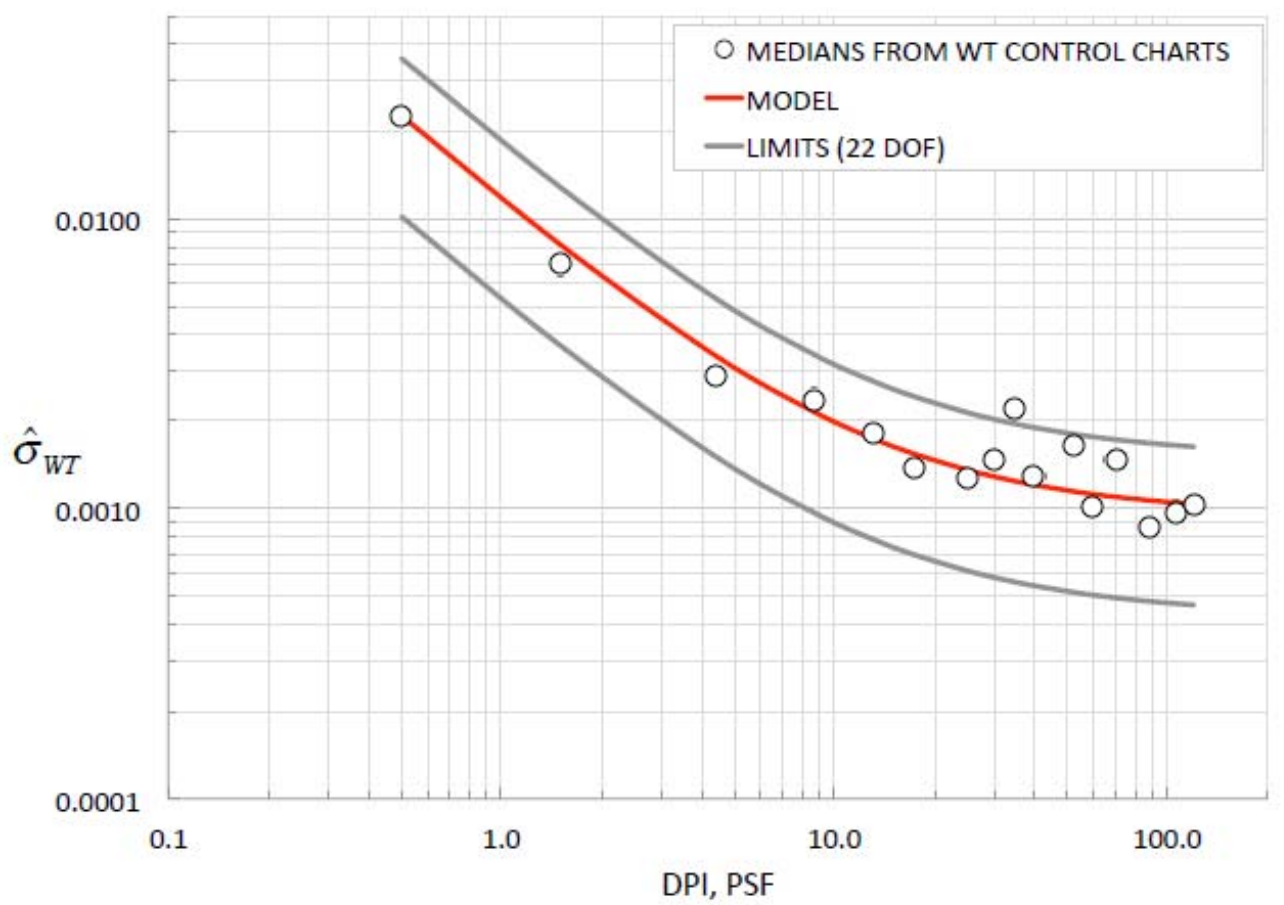

Figure 23 Model of $\widehat{\sigma}_{W T}$ for $C^{\prime}$ from $14 \times 22$ with Control Limits for 22 Degrees of Freedom 


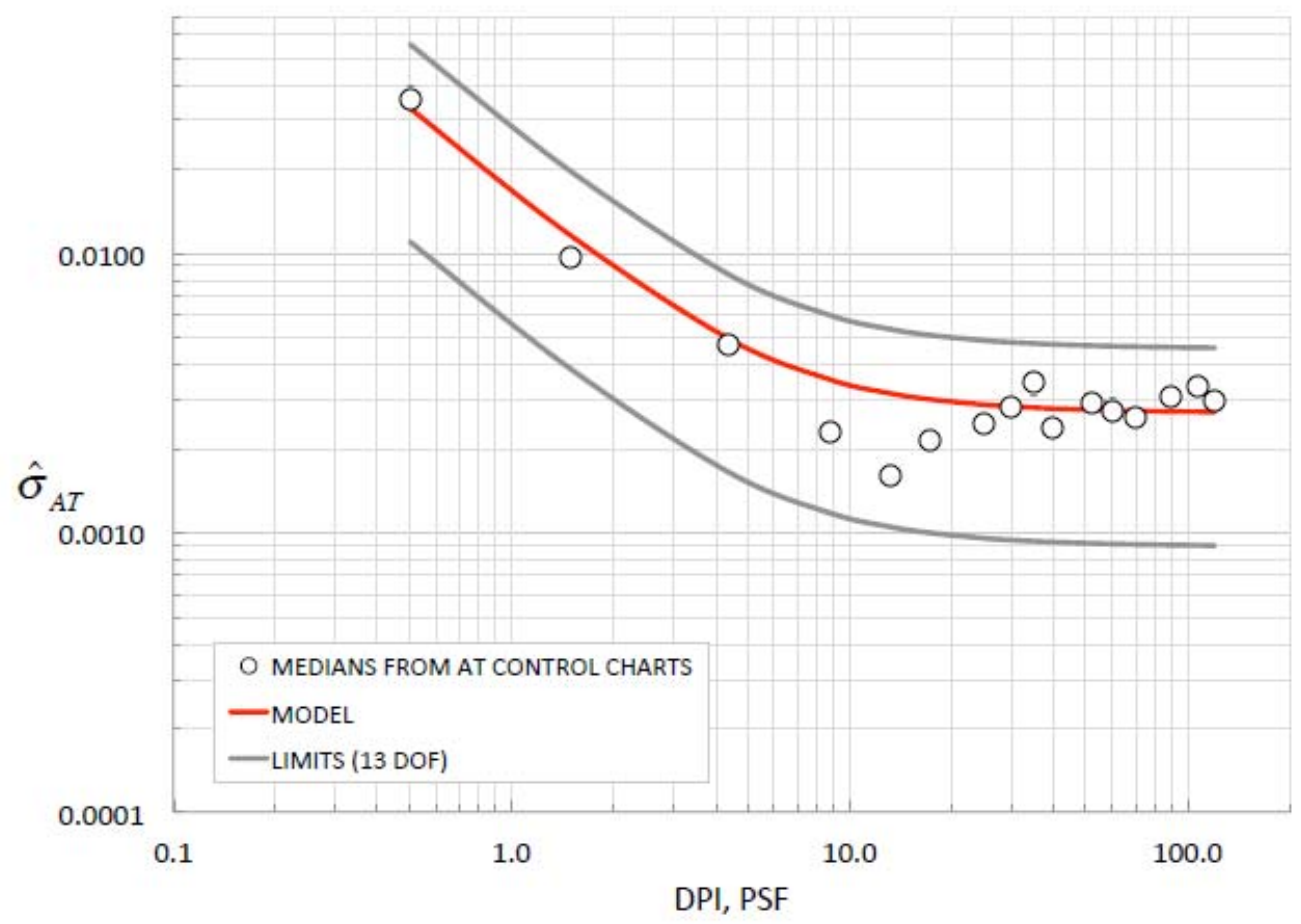

Figure 24 Model of $\widehat{\sigma}_{A T}$ for $C^{\prime}$ from $14 \times 22$ with Control Limits for 13 Degrees of Freedom

Figure 23 and Figure 24 again show very good agreement with the uncertainty propagation based model and the statistical process control based data, as only one data point is outside of the prescribed limits. Note however that the degrees of freedom for the within-test and across-test are significantly lower than the within-group leading to much larger limits. The authors recognize this and state that the agreement between the models should be "considered provisional".

\subsubsection{Data Analysis: 14x22 Normal Force Coefficient}

The check standard model utilized for the $14 \times 22$ is a generic elliptical wing model shown in Figure 25 . It is important to note for the check standard practice the geometry of the model is not crucial. Rather it is important that the geometry and methods of data collection remain unchanged so that the variation observed in the data can be attributed only to the process repeatability. The data and the process of obtaining it for the normal force coefficient was similar to the calibration coefficient. Pitch polars in angle of attack were obtained back-to-back in groups at different levels of tunnel dynamic pressure. The 
analysis of the normal force coefficient conducted by the authors was limited to the $2^{\circ}$ angle of attack case as this condition is most representative of a transport cruise condition.

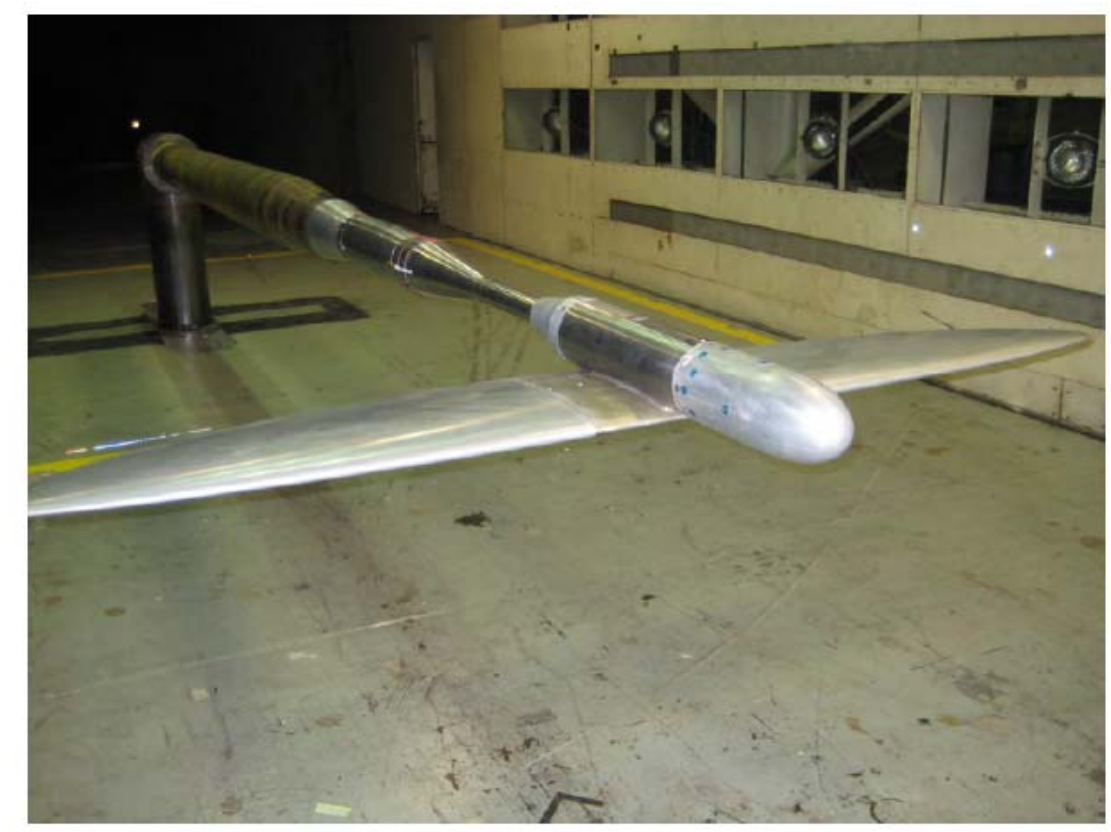

Figure $2514 \times 22$ Elliptical Wing Check Standard Model

The results from the within-group repeatability for the normal force are shown in a log-log plot for data across 11 tests giving 82 degrees of freedom in Figure 26. The medians from the within-group statistical process control charts, their associated limits and the uncertainty propagation based model are shown. Also given is the expected behavior of the balance at its quoted calibration uncertainty of $1 \sigma=0.012 \%$ full scale. The same procedure to determine the constants in the uncertainty propagation based model for the calibration coefficient was also followed. 


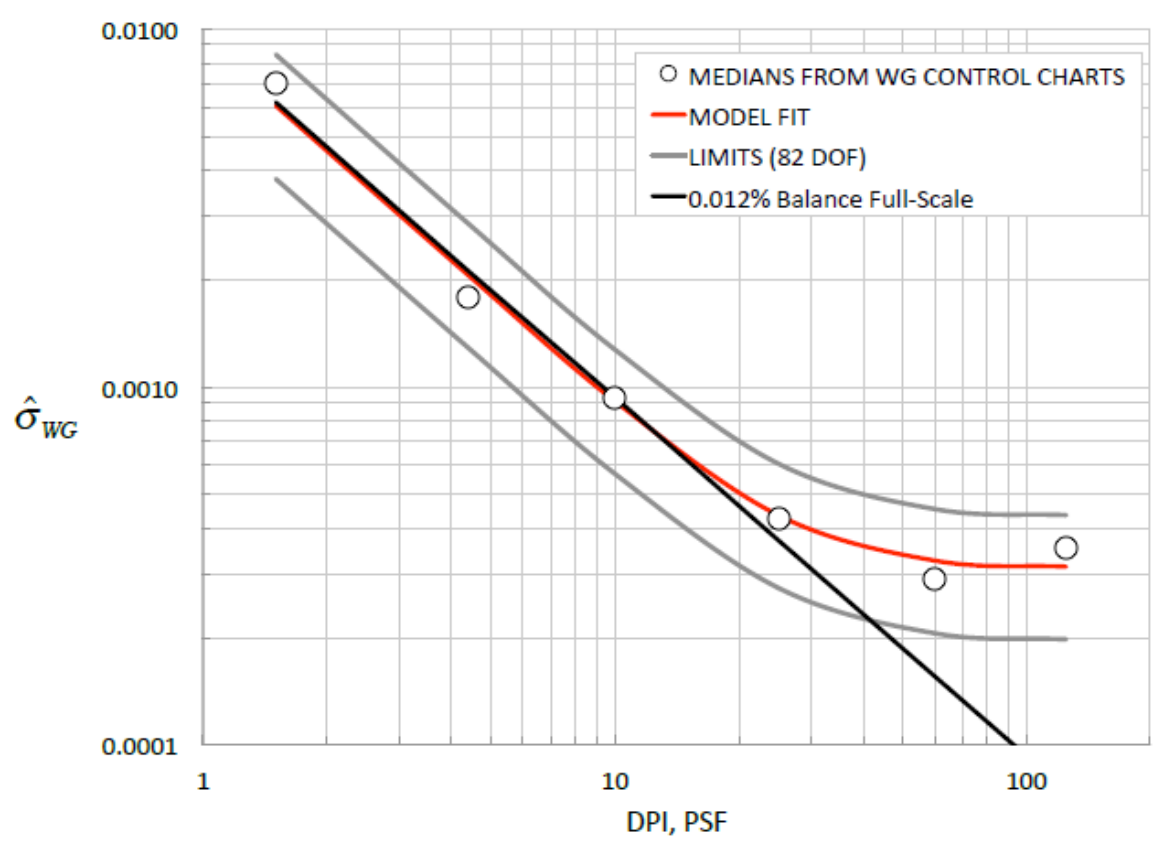

Figure 26 Model of $\widehat{\sigma}_{W G}$ for Normal for Coefficient from 14x22 with Control Limits for 82 Degrees of Freedom

From inspecting Figure 26 it is seen that the model fit from the uncertainty propagation based model is in very good agreement with the statistical process control derived data as no data points are outside the prescribed limits. Similar charts showing the within-test and across-test estimates of the standard deviations are given in Figure 27 and Figure 28 respectively. The same good agreement between the uncertainty propagation based model and the statistical process control derived data exists. As was the case with the calibration coefficient data, lower degrees of freedom at within-test and across-test levels cause the limits to be inflated. 


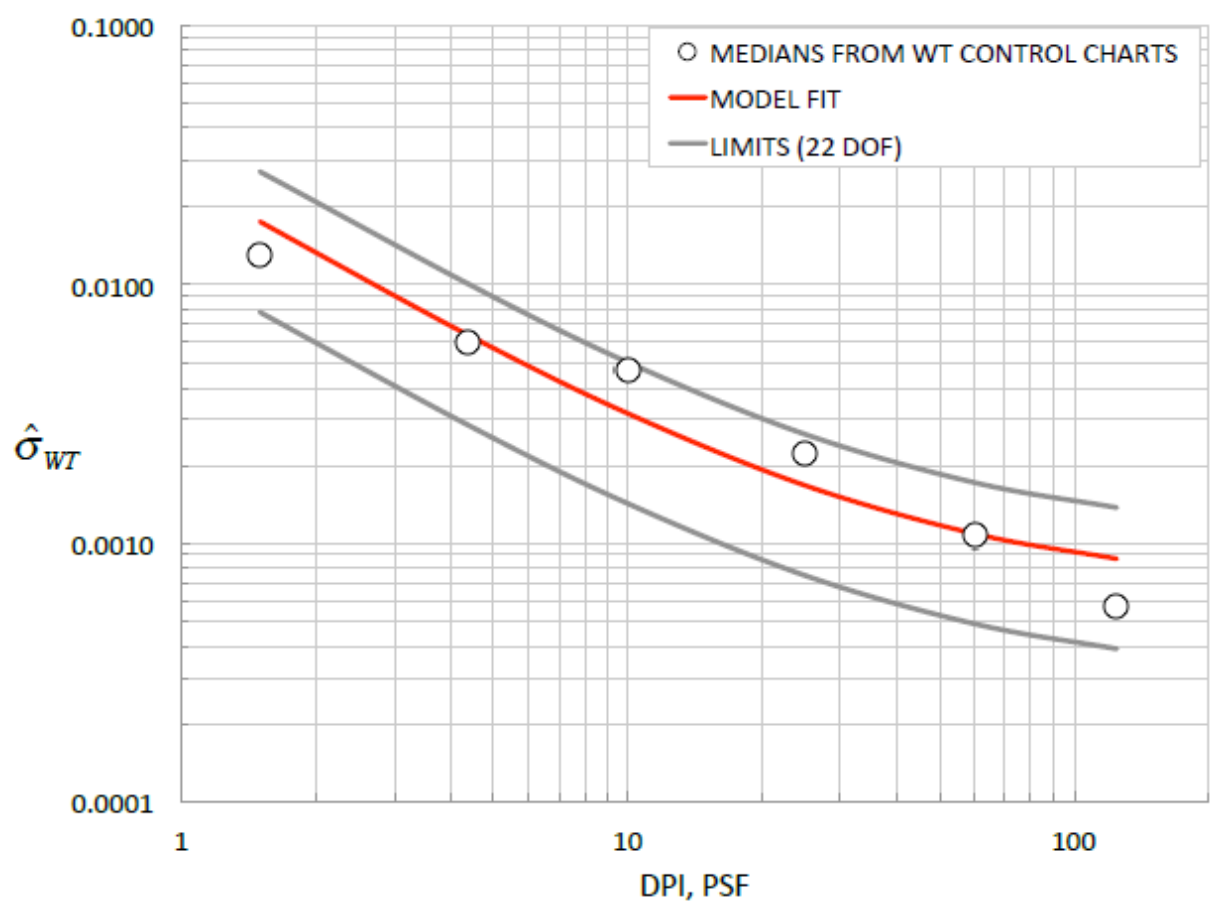

Figure 27 Model of $\widehat{\sigma}_{W T}$ for Normal for Coefficient from 14x22 with Control Limits for 22 Degrees of Freedom

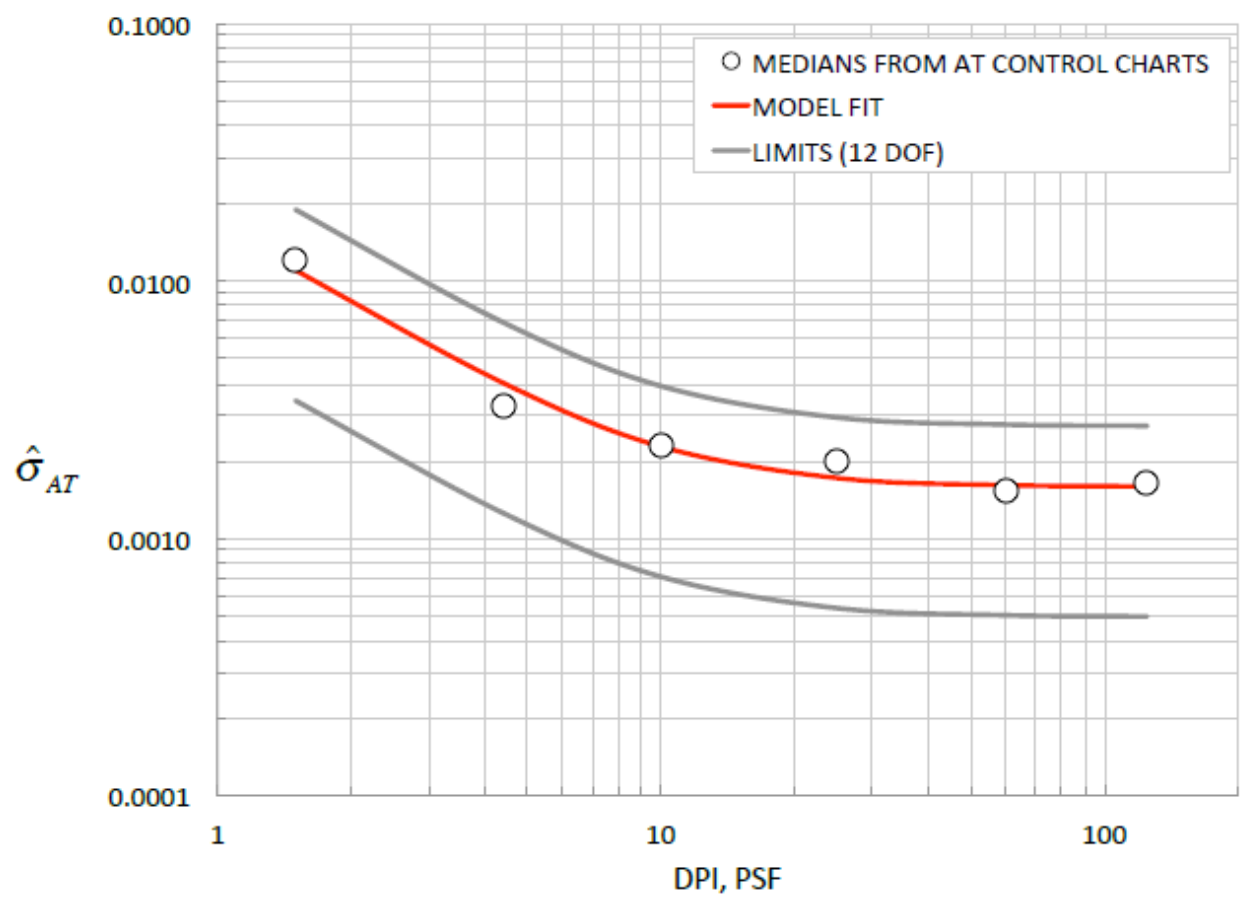

Figure 28 Model of $\widehat{\sigma}_{A T}$ for Normal for Coefficient from 14x22 with Control Limits for 12 Degrees of Freedom 


\subsubsection{Summary}

The authors demonstrated the data derived from the statistical process control methodology can at least, provisionally, be considered to be in very good agreement with uncertainty propagation based models for wind tunnel check standard testing. The uncertainties at the lower levels of tunnel dynamic pressure at the within-group level are driven by the resolution of the measurement devices (both the force balance and pressure sensors). The authors also note that the within-test and across-test variation at all levels of tunnel dynamic pressure "seem unreasonably large" and suggest "significant performance improvement might be possible by examining the measurement process in detail."

One of the major benefits of the application of design of experiments methodology is its ability to reduce the influence of systematic, time based variances on regression models. It is thought that through the implementation of design of experiments methodology in conjunction with traditional check standard procedures, the authors' goals of achieving significant performance improvement can be achieved. 


\section{EXPERIMENTAL SETUP AND DESIGN}

This chapter will detail the experimental setup including both the hardware and software developed for this research. Also covered in this chapter are the experimental design process and procedure.

\subsection{Equipment}

This section will detail the hardware and software utilized in the wind tunnel testing.

\subsubsection{Wind Tunnel Description}

The Old Dominion University Low-Speed Wind Tunnel (ODU LSWT) is an atmospheric, closed circuit tunnel supplied by AeroLab LLC circa 1971. Research conducted at the ODU LSWT has been multidisciplinary in nature and includes studies in aeronautical, automotive and architectural fields. It is powered by a $93 \mathrm{~kW}$ electric motor and is capable of speeds ranging from $10 \mathrm{~m} / \mathrm{s}$ to $50 \mathrm{~m} / \mathrm{s}$ (dynamic pressure range of 61Pa to $1500 \mathrm{~Pa}$ ). The tunnel has two test sections, a high-speed and low-speed section with $2.13 \times 2.44$ meters and $0.91 \times 1.22$ meters cross sectional areas respectively as seen below in Figure 29. Instrumentation includes pressure probes and pressure transducers with up to 80 channels, multiple force balances, as well as particle image velocimetry and hot wire anemometry capabilities. 


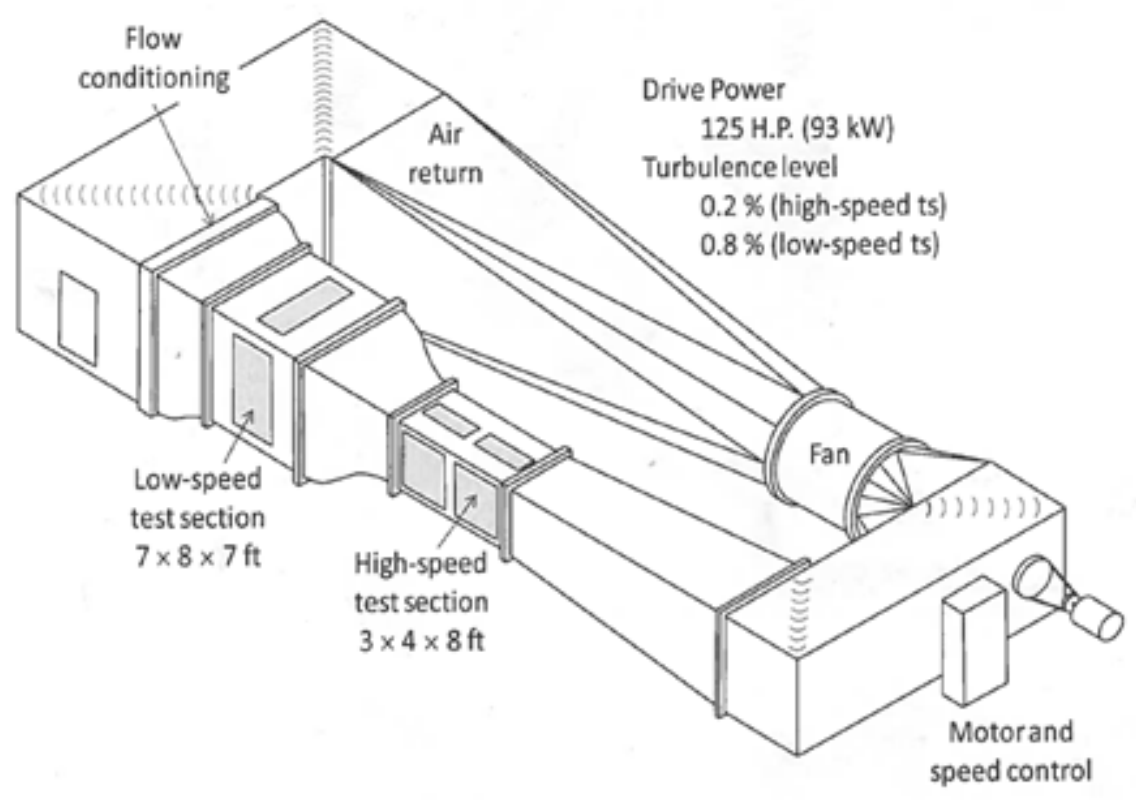

Figure 29 ODU Low-Speed Wind Tunnel

Wind tunnel dynamic pressure is held constant through a fully automated system and measured by a differential pressure transducer connected between two static rings located on the upstream and downstream ends of the contraction nozzle leading into the high speed test section. Test section turbulence intensity is about $0.2 \%$.

\subsubsection{Aircraft Model}

The model utilized in the wind tunnel testing was fabricated in-house at Old Dominion University's machine shop. The model was constructed as part of a previous design project focused on the improvement of research capabilities for the ODU LSWT ${ }^{40}$. The overall configuration was based on the Standard Dynamics Model (SDM) developed in part by the National Research Council (NRC) of Canada in the late 1970's. The SDM was developed by the NRC for dynamic stability research and was loosely based on a generic representation of a fighter aircraft of the time period ${ }^{41}$.

The ODU SDM model has an axisymmetric fuselage and flat tapered lifting surfaces with a wing span of $1 \mathrm{ft}$, a mean aerodynamic chord of $0.333 \mathrm{ft}$, a wing area of $0.333 \mathrm{ft}^{2}$ and some removable control surfaces $^{40}$. Note that for the testing of the SDM model carried out for this research study, the canopy, 
leading edge strakes, and control surfaces were removed. This was done to ensure that the model's geometry was not altered from repeated installations as these components were fragile.

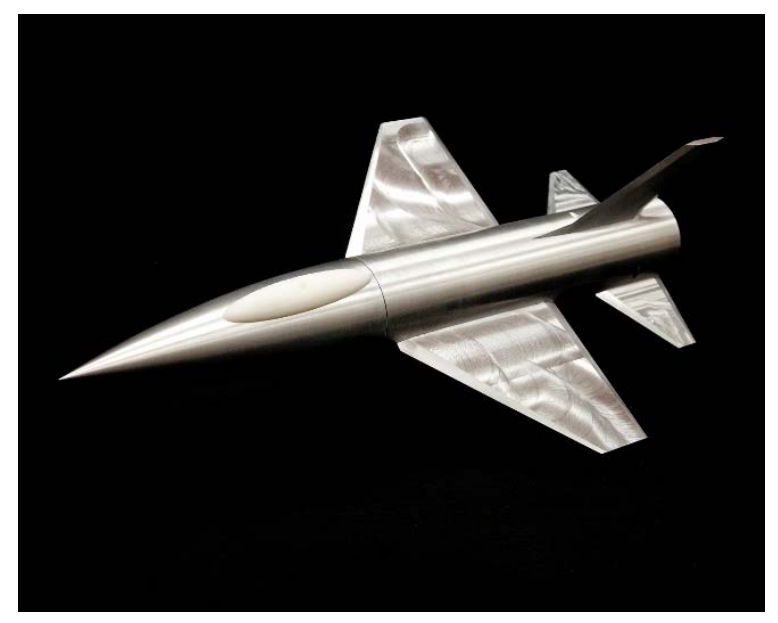

Figure 30 ODU SDM Model

\subsubsection{Model Modification}

During initial verification of the model support system it was observed that the aircraft model did not generate an ample amount of axial force at the desired tunnel conditions and model attitude such that the force measured was significantly larger than the balance uncertainty in the axial direction. Altering the model attitude or tunnel conditions to increase the axial force generated was felt to be not representative of future larger scale testing, so it was necessary to modify the model itself. Four identical airbrakes were fabricated from aluminum angle stock such that both the upper and lower surfaces of each wing maintained symmetry. It is important to note that airbrakes of this magnitude and crude aerodynamic design would never be employed on an aircraft similar to the SDM; the sole purpose of these airbrakes was to increase the axial force generated. Since the motivation of this study is to monitor and characterize forces measured on the SDM and their variation over time, it was not crucial to dedicate a great deal of attention to an "airworthy" design of the airbrakes, but only to assure that the mounting of the airbrakes remains constant throughout the testing process. 


\subsubsection{Airbrake Development}

After installation of the model with the airbrakes, it was observed that the axial force generated significantly exceeded the balance uncertainty. However, the model exhibited an undesirable amount of tail buffeting at higher tunnel speeds and angles of attack. The tail buffeting contributed to unsteady dynamics of the model and potentially higher loadings on the balance and model support system. With the mechanical loadings on the balance and model support system potentially approaching their limits, it was decided to further modify the airbrakes to reduce the tail buffeting and subsequent unsteady dynamics

Extensive research was conducted during the Second World War in airbrake design, primarily for use in dive bombers such as the JU-88. At that time, tail buffeting posed a problem for aircraft with airbrakes deployed. The research compiled by the Aeronautical Research Council ${ }^{42}$ goes into great detail on the methods for reducing tail buffeting caused by airbrakes. One method employed was adding slots or perforations to the airbrake vertical surfaces in an attempt to decrease the width of the wake behind the airbrakes. Of the patterns studied by Davies et al., the recommended choice is the chordwise slotted flap with a $27 \%$ open area ratio (type $C$ in Figure 31 ). This configuration is most effective at reducing tail buffeting and only decreases the overall airbrake drag generating potential by $15 \%$. 


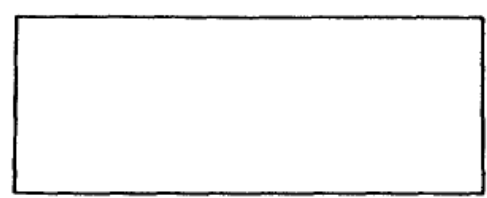

(A) SOLID FLAP

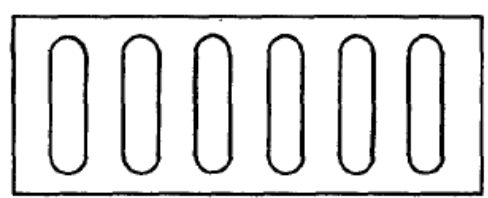

(c) CHORDWISE SLOTTED FLAP SLOTS $7 / 8 \times 33 / 8 \mathrm{iN}$.

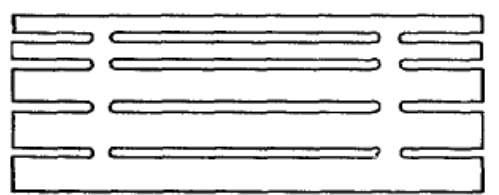

(B) JU 88 TYPE FLAP

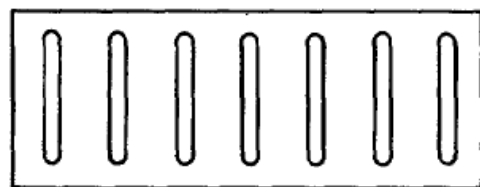

(D) CHORDWISE SLOTTED FLAP SLOTS $7 / 16 \times 3 / 38$ in.

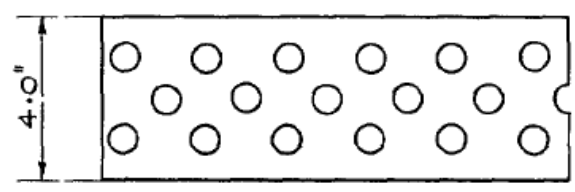

(E) PERFORATED FLAP

PERFORATIONS $3 / 4$ " OIA.

Figure 31 Possible Airbrake Modifications

Chordwise slots were milled into the airbrakes with an open area ratio of approximately $27 \%$ as Davies et al. suggested. The modified airbrakes were subsequently reinstalled and verification testing was again conducted. 


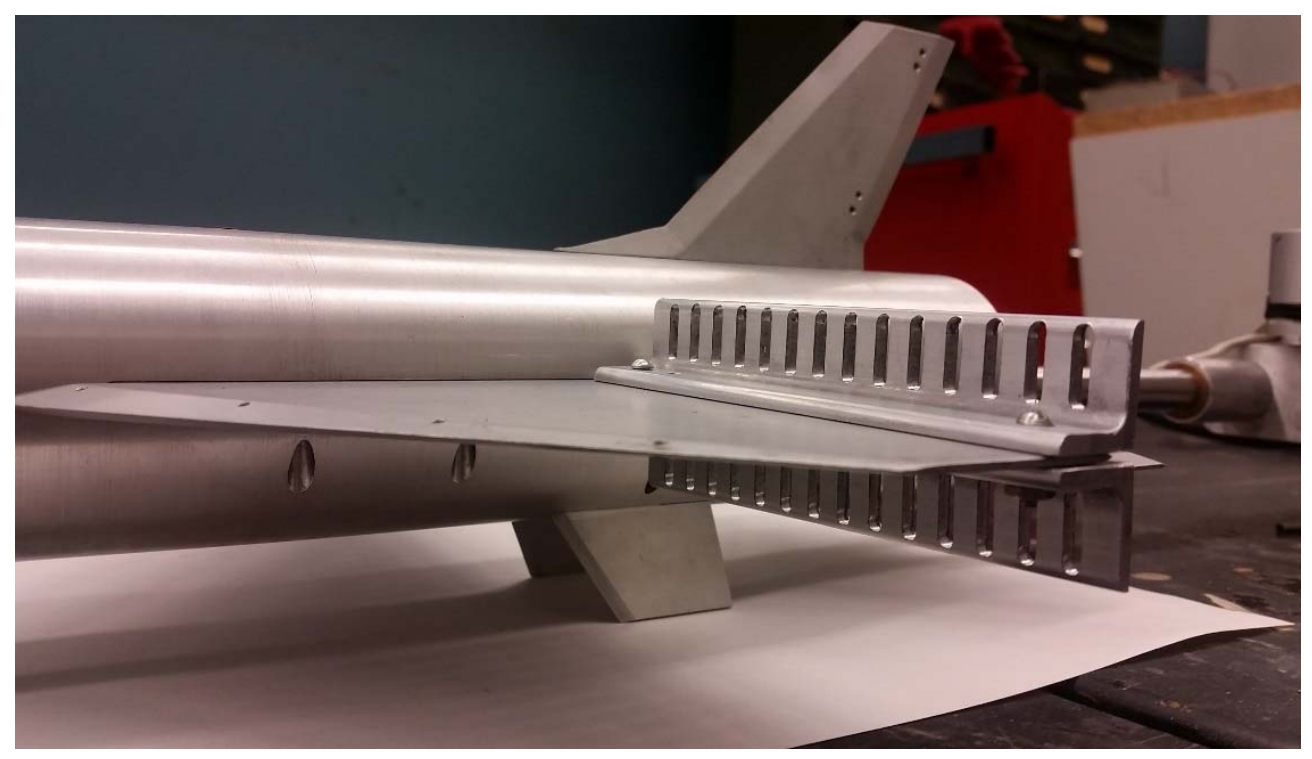

Figure 32 SDM Model with Modified Slotted Airbrakes

It was observed with the modified airbrakes that the appearance of tail buffeting was greatly reduced and the unsteady dynamics attenuated. The drag reduction from the solid airbrakes to the slotted airbrakes was in agreement with the predicted value, measured at $14 \%$

\subsubsection{Force Balance}

The force balance utilized for the wind tunnel testing was the NASA 2044a, which is a 6 degree of freedom strain gage based internal balance. The specifications and calibration information for the 2044a can be found in APPENDIX A. The balance was inserted into the fuselage bulkhead of the model and locked into place by a dowel pin from the top of the aircraft model. The tapered non-metric end of the balance was then fit to a custom sting where the balance would be wired through the sting. The fit between the tapered end of the balance and the sting secures the connection of the model to the model support system and subsequently the tunnel itself. See Section 4.1.4.2 for the details of the mechanical connection of the balance to model and balance to sting. The balance with installed water jacket and sting are shown in Figure 33 for size reference. Although the balance was outfitted with a water jacket, as its initial use was in high speed facilities with elevated temperatures, it was not actively used during this testing. 


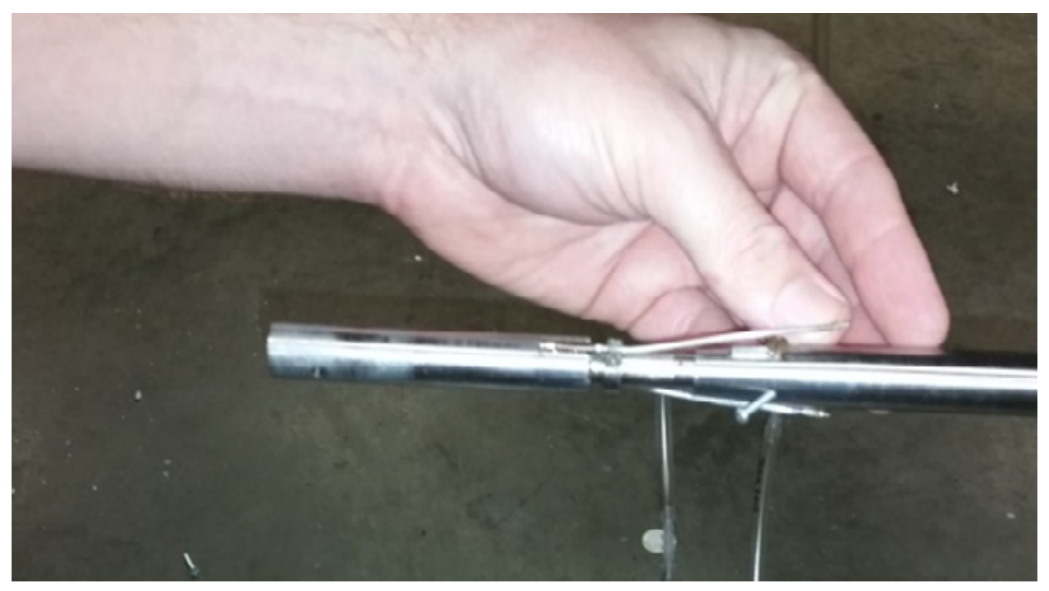

Figure 33 2044a Balance

During the initial verification of the balance, it was observed that there was a substantial amount of electrical noise on the balance signal lines. To mitigate this electrical noise, it was decided to install low pass filters on each channel with a cutoff frequency of $10 \mathrm{~Hz}$. The relatively low frequency was selected due to the quasi-static nature of the testing to be performed. A block diagram of the electrical connections of the balance to the National Instruments data acquisition card is shown in Figure 34.

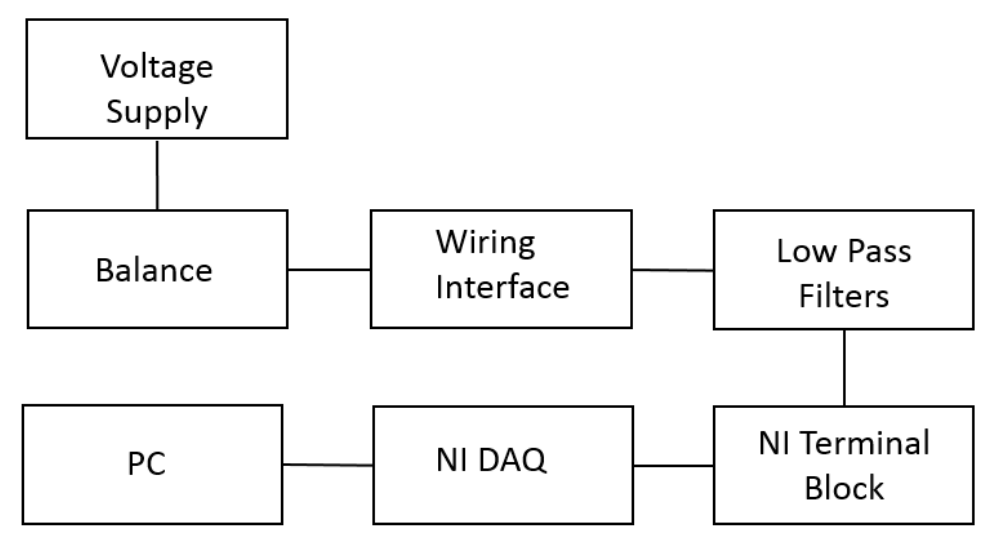

Figure 34 Balance Wiring Block Diagram 


\subsubsection{Model Support Design and Use}

This section will detail the background as well as the design and implementation of the model support system for the tunnel conditions testing.

\subsubsection{Background}

In preparation for conducting testing in the ODU LSWT, the existing capabilities were reviewed and compared to the project requirements. The existing model support system in the high-speed test section was a pitch crescent type design with remote pitch and yaw capabilities. Although more than capable of conducting basic testing, the need for precise, and more importantly, repeatable model positioning along with remote roll capability prompted the development of a new model support system.

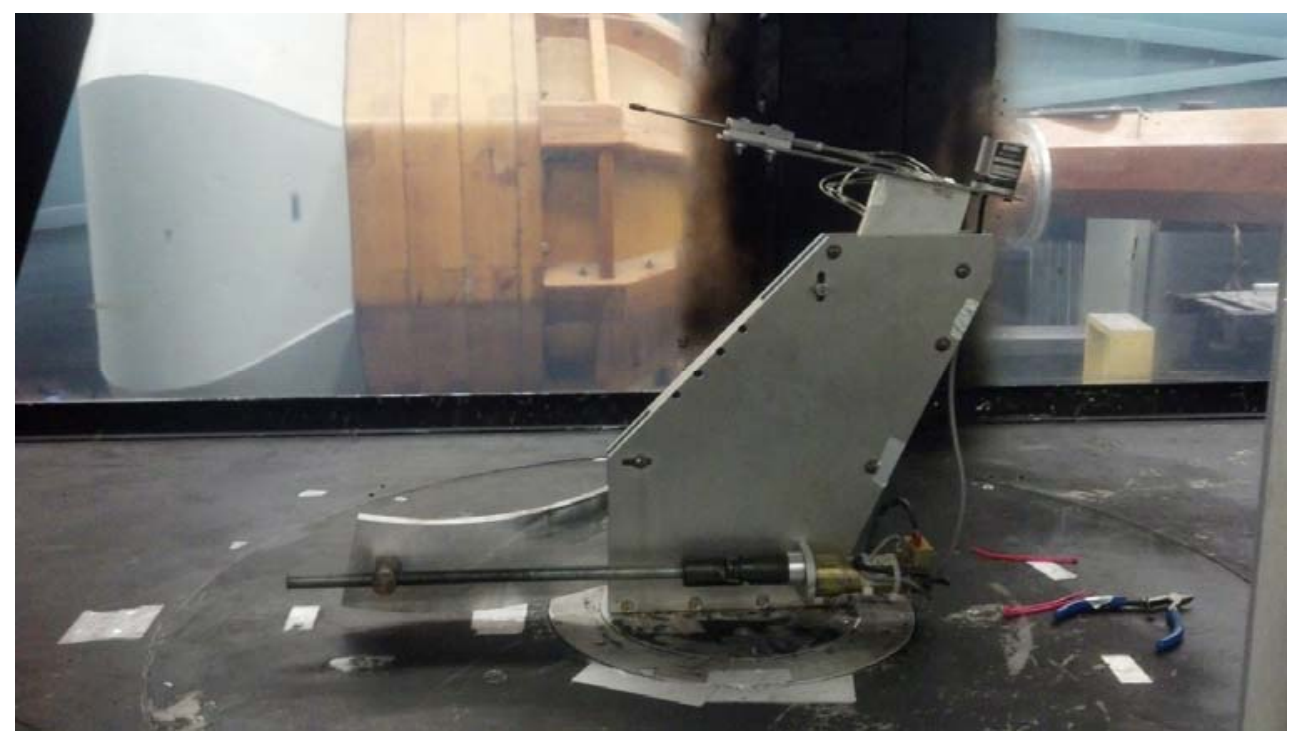

Figure 35 ODU LSWT Pitch Crescent Model Support

The major design considerations for the new model support system included full automation, remote pitch and roll control, ease of construction, minimal cost, and ease of implementation, modularity and adaptability. First, for the automated pitch and roll control, it was of upmost importance that the positioning be both reliable and repeatable. When conducting a study such as this where repeated 
measurements are taken, the researcher needs to have a great deal of confidence that the repeated measurements are in fact taken at the same position. Or more importantly, that the model positioning repeatability must remain constant. Second, the ease of construction and implementation was of great importance due to the overall timeline of the project. Due to contractual obligations, the entire project including the construction of the model support and data collection was not to exceed two years' time. Also the entire fabrication of the model support was to be completed in-house at ODU's machine shop on a limited budget relative to larger tunnel facilities. Thirdly, the model support system was to continue to serve as the primary three-dimensional model support system for NASA projects at the ODU LSWT, following completion of the project. Therefore there had to be a degree of modularity built into the system such that other models and experiments could be accommodated.

With these design considerations in mind, two of the most common model support systems were considered. An arc sector type such as the system in the NASA LaRC National Transonic Facility ${ }^{43}$, and the twin lead screw type model such as the system at the NASA LaRC Transonic Dynamics Tunnel ${ }^{44}$.
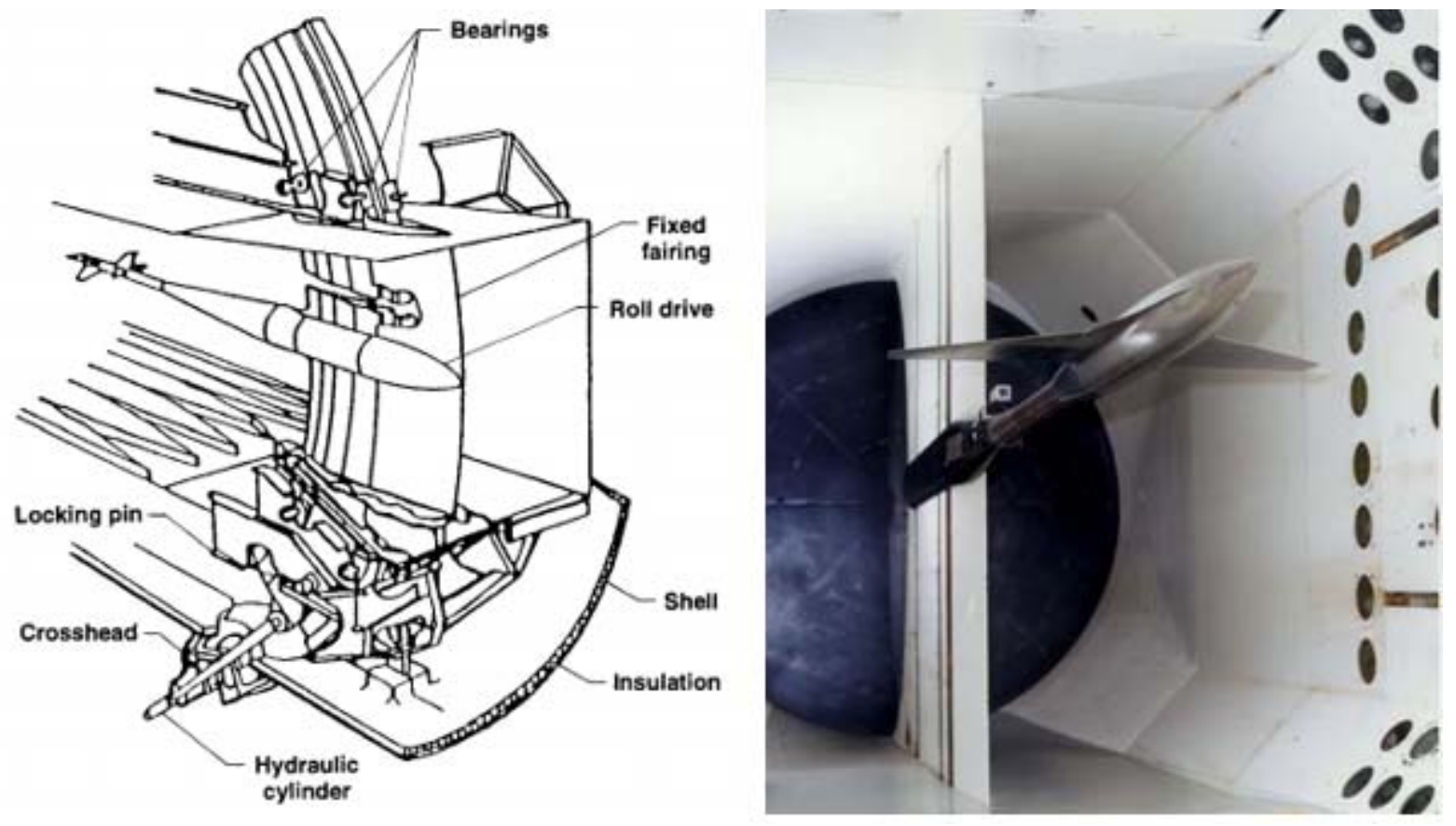

Figure 36 NTF Model Support (Left) and TDT Model Support (Right) 
Both of the model support systems would have been able to satisfy most of the design requirements, however for the intended application, the arc sector design was deficient in a few areas. First, for an arc sector model support, the length of the sting must be fixed to keep the model on tunnel centerline while pitching. This would prevent the model support from being sufficiently adaptable. Second, the fabrication of the arc sector itself posed multiple challenges whereas the vertical struts of the twin lead screw design did not. With these considerations, a twin lead screw model support system based on commercially available lead screw stages was chosen for development.

\subsubsection{Model Support Mechanical Design}

The overall assembly drawing for the twin lead screw model support system is shown in Figure 37 . The following discussion of the components in this section will reference Figure 37 when appropriate.

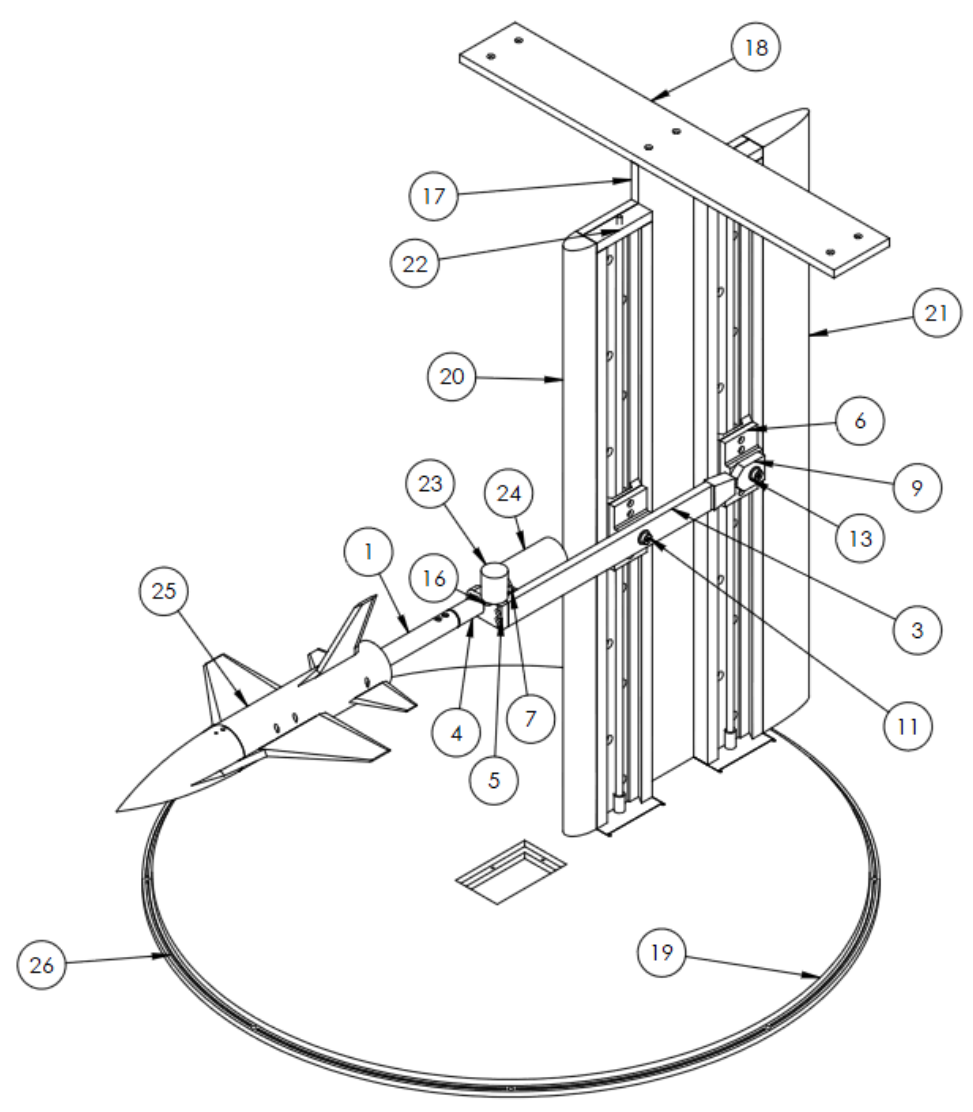

Figure 37 ODU Twin Lead Screw Model Support Assembly 
A complete list of the mechanical components of the model support, with numbering consistent with Figure 37, is found in Table 9. CAD drawings for the individual components can be found in APPENDIX E.

\begin{tabular}{|c|c|}
\hline $\begin{array}{l}\text { Component } \\
\text { Number }\end{array}$ & Part Name \\
\hline 1 & Sting \\
\hline 2 & Sting Shaft \\
\hline 3 & Sting Mount \\
\hline 4 & Sting Revolve Mount \\
\hline 5 & Sting Support Mount \\
\hline 6 & Slide Mount \\
\hline 7 & Motor Mount \\
\hline 8 & Motor Coupling \\
\hline 9 & Rear Dowel Pin Connector \\
\hline 10 & 3/8in x 4in Dowel Rod \\
\hline 11 & $3 / 8$ in $x$ 5/8in Shoulder Screw $(x 2)$ \\
\hline 12 & 3/8in x 1/2in Sleeve Bearing (x2) \\
\hline 13 & $3 / 8$ in $x 1$ /16in Thrust Bearing $(x 4)$ \\
\hline 14 & 3/8in x 1 1/4in Sleeve Bearing \\
\hline 15 & $3 / 8$ in $x$ 1in Flanged Sleeve Bearing $(x 2)$ \\
\hline 16 & Inclinometer Mount \\
\hline 17 & Back Plate \\
\hline 18 & Top Plate \\
\hline 19 & Turntable Replacement \\
\hline 20 & Front Aero Fairing \\
\hline 21 & Rear Aero Fairing \\
\hline 22 & Velmex XY Slides (MA4036) \\
\hline 23 & Inclinometer (LSRP-90) \\
\hline 24 & Motor (GM9232E967-500PPR) \\
\hline 25 & Model \\
\hline 26 & O-Ring \\
\hline 27 & Pitot Tube \\
\hline 28 & Pitot Tube Filler Piece \\
\hline 29 & Pitot Register \\
\hline 30 & Pitot Block Bracket \\
\hline
\end{tabular}

Table 9 Model Support Mechanical Components List

In the development of the twin lead screw model support system, consideration was given to incorporate commercially available products so that the overall design, fabrication and validation time 
could be reduced. The first hardware components chosen were the slide positioning systems, a commercially available product produced by Velmex ${ }^{45}$. Two MA4036 Velmex slides which included integrated stepper motors would function as the twin lead screw drives for the model support system. The stepper motors were driven by an available NF90-3 motor controller produced by Velmex ${ }^{46}$.

The model support system and necessary tunnel modifications would be designed around the chosen slides. A back plate (Part 17, Figure 37) was designed such that the two slides could be fastened together at a set horizontal distance of 4 in. Two slide mounts were designed as tie in points for the model support arm to the slides (Part 6, Figure 37). The slides with the slide mounts assembled to the back plate are shown below in Figure 38.

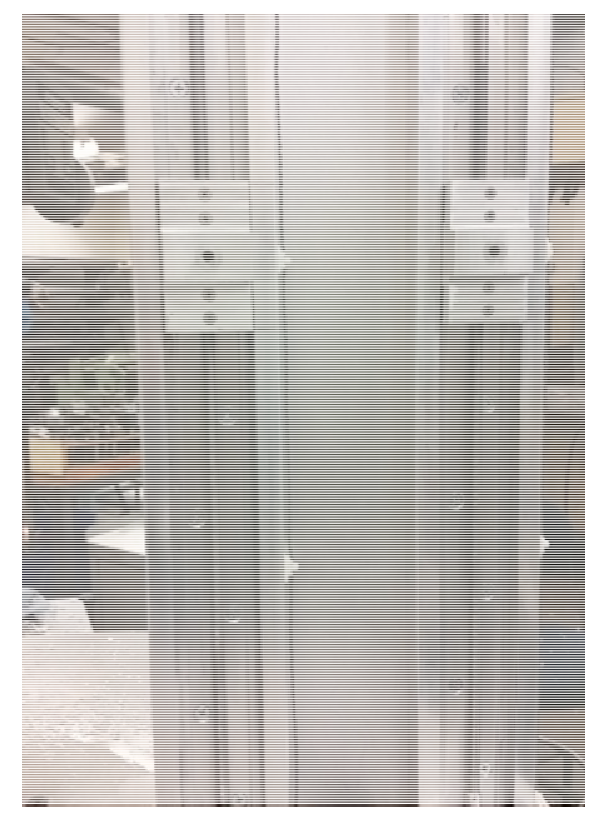

Figure 38 Slide and Back Plate Assembled

To integrate the now joined slides and back plate into the wind tunnel test section, the existing tunnel floor required modification. The previous three-dimensional model support system was tied into the tunnel floor via a turntable (See Figure 35). It was determined that the modification necessary to integrate the new slides and back plate into the turntable would have rendered the rotary function of the table inoperable. Consequently a separate turntable replacement was required. Of all the 
components required, the turntable replacement was by far the largest and most mechanically complex to machine.

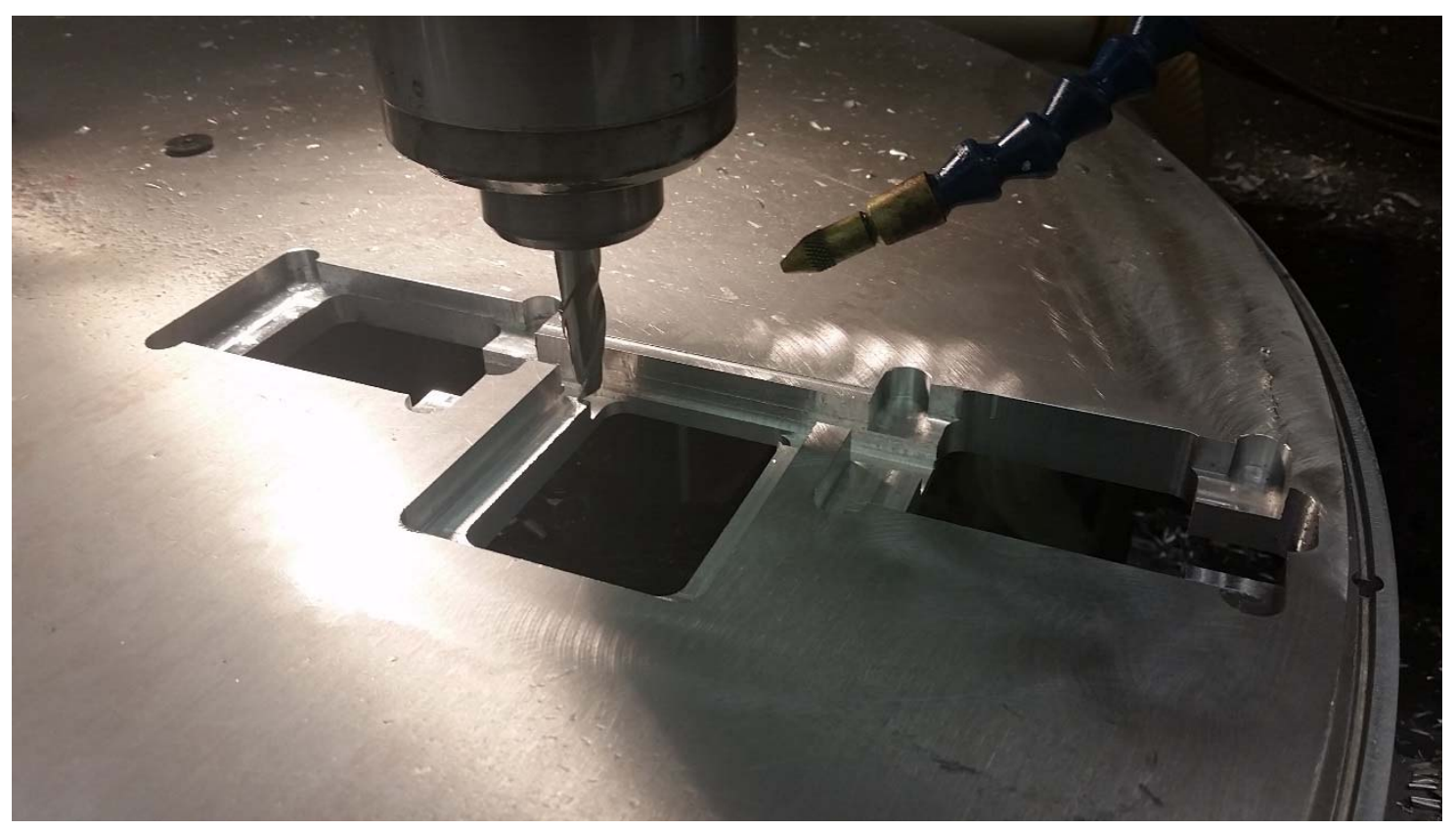

Figure 39 Turntable Replacement during Fabrication

The turntable replacement fastened (Part 19, Figure 37) to the test section via eight mounting holes along the edge of the plate. Pockets were milled to allow for the assembled slides and back plate to sit flush with the test section floor. An access hatch was also designed so that electrical connections from the balance and instrumentation could be passed through the turntable replacement. To allow for the top of the model support to fasten to the test section a top plate was designed (Part 18, Figure 37). This component secured the top of the back plate to structural cross members on the exterior top of the test section as shown in Figure 40. 


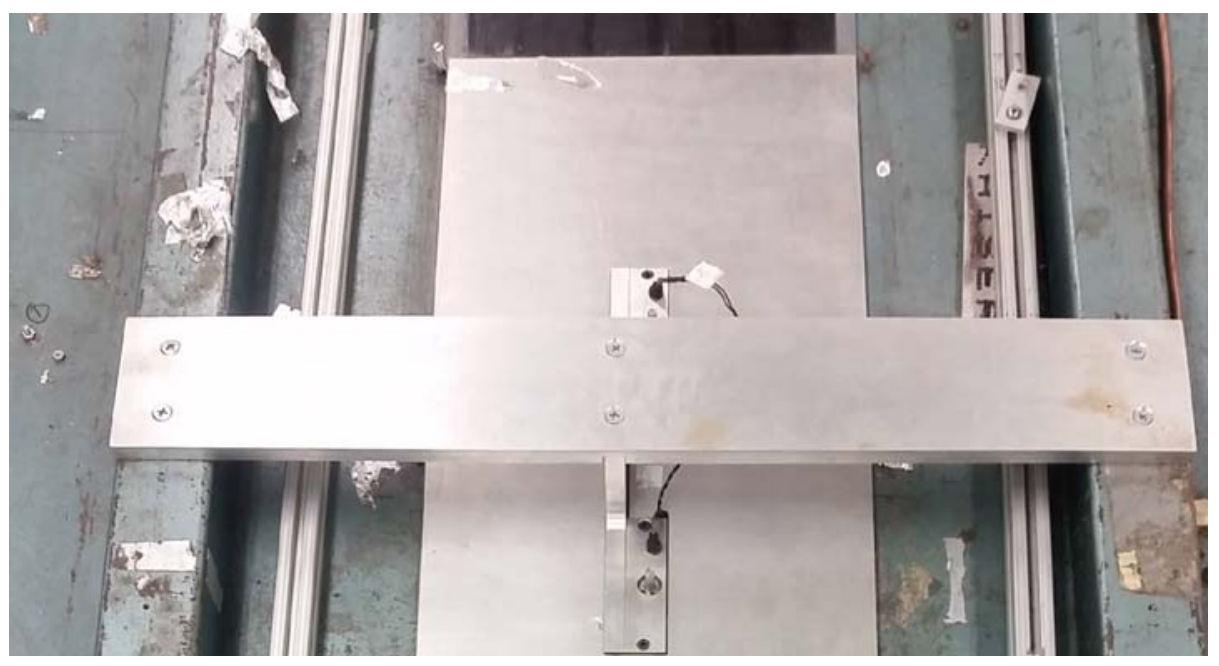

Figure 40 Model Support Top Plate Installed

To complete the strut portion of the model support, half ellipsoid aerodynamic fairings were designed to be fastened to the upstream (Part 20, Figure 37) and downstream (Part 21, Figure 37) faces of the strut. It should be noted that the two aerodynamic fairings were the only components dedicated to streamlining or reducing the model support aerodynamic interference. This was due to the fact that no model support interference corrections were to be made on the aerodynamic force measurements, hence a great deal of time was not dedicated to model support interference reduction.

The next set of components designed comprised the model arm assembly. The sting mount component (Part 3, Figure 37) is connected to the slide mounts via a shoulder screw (Part 11 Figure 37) on the front slide mount and a shoulder screw, dowel pin (Part 10, Figure 41) and sleeve bearing on the rear slide mount (Part 14, Figure 41). A section cut of the entire model arm assembly is shown below in Figure 41. 

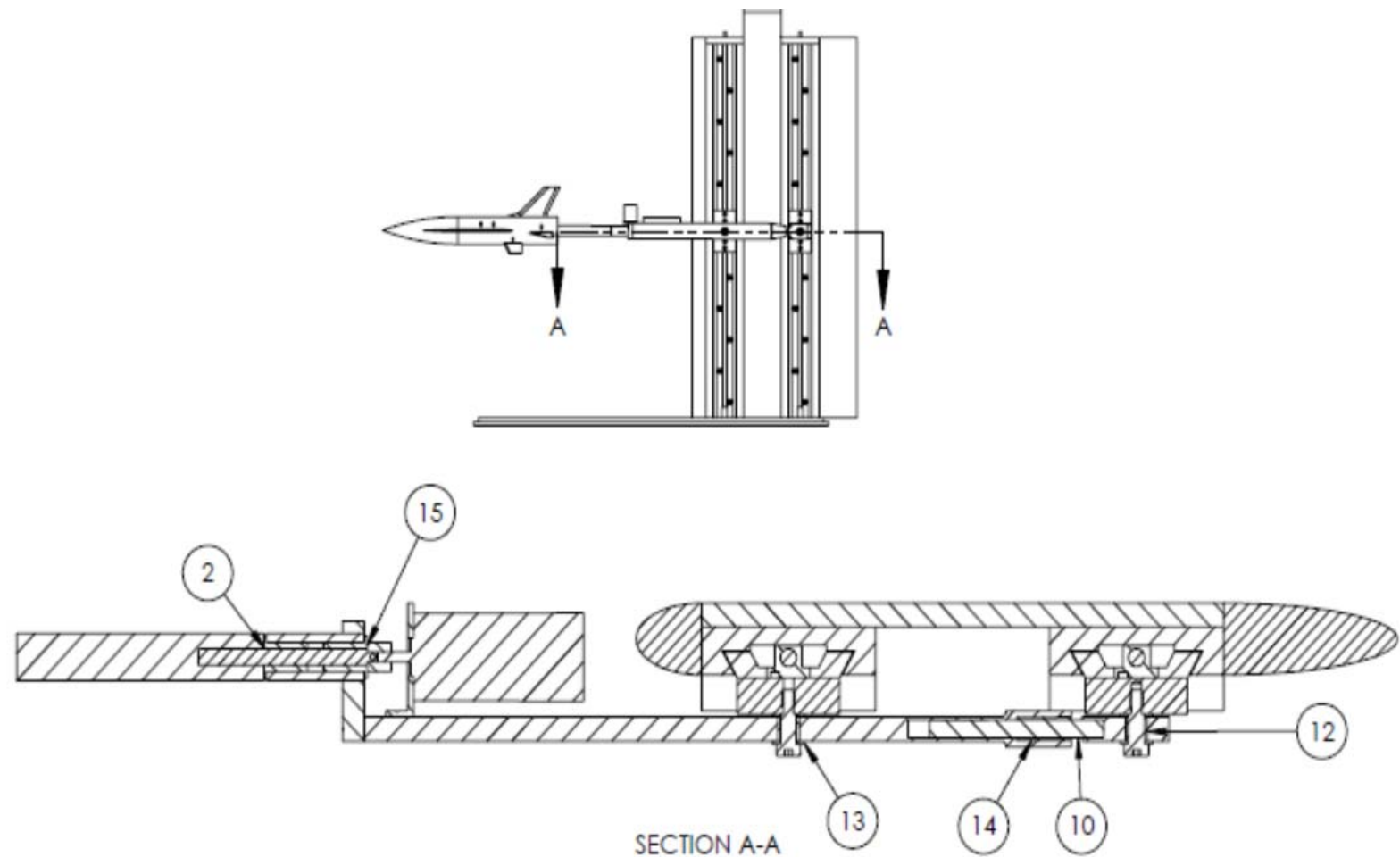

Figure 41 ODU Twin Lead Screw Model Support, Arm Section View

The design of the sting support allows the model arm assembly to pitch up and down when the lead screws are driven, with little movement of the model upstream or downstream in the test section. When the model arm assembly pitches up or down the rear dowel pin (part 10, Figure 37) slides into or out of the sting support (part 5, Figure 37).

The roll motor (Part 24, Figure 37) was mounted to the inside of the sting support by the motor mount bracket (Part 7, Figure 37). To connect the model, balance and sting to the sting support and allow for remote roll functionality, the end of the sting was designed such that it could be fit into a flanged sleeve bearing and coupled to the shaft of the roll motor. The model support fully assembled (without aerodynamic fairings) and installed in the test section shown in Figure 42. 


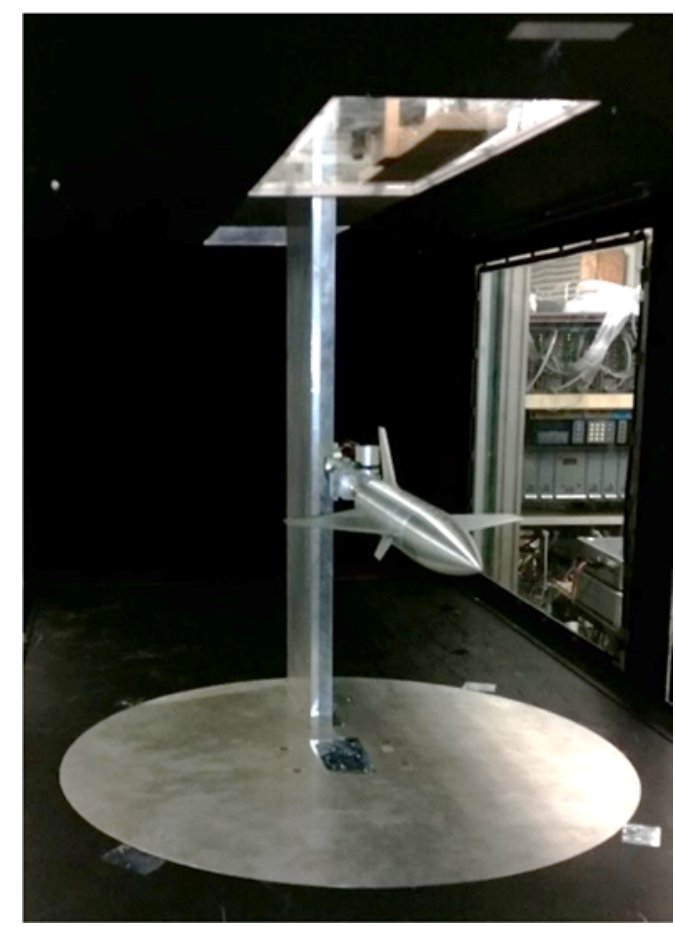

Figure 42 Model Support System Installed in Test Section

\subsubsection{Inclinometer}

The instrument utilized in the airframe portion of the testing to measure the model's angle of attack was a high precision closed loop gravity referenced servo inclinometer, LSRP-90, manufactured by Level Developments ${ }^{47}$ The inclinometer (Part 23, Figure 37) was located on the upstream end of the sting mount, approximately $10 \mathrm{in}$ downstream of the model.

During the early phases of the model support design, an internally mounted, full inertial measurement unit (IMU) was considered, allowing for extremely accurate attitude measurement. However due to the size of the model and other constraints, this option became too cost prohibitive. The positioning of the inclinometer could conceivably lead to small differences in angle of attack reading and the angle of attack seen by the model due to deflection of the sting and balance. Also the location of the inclinometer could potentially cause added model support interference. However, due to the nature of the testing being performed, the aforementioned potential issues are not critically important. Again it is beneficial to mention that the goal of this testing was not to aerodynamically characterize the model, but to obtain repeated measurements over time and to characterize and possibly diagnose the nature 
and causes of the variance in the measurements. Full specifications of the LSRP-90 inclinometer can be found in APPENDIX B.

\subsubsection{Roll Motor}

The motor and integral gear reduction drive utilized to remotely control the roll angle of the aircraft was the Pittman GM9232E967-500PPR DC gear motor (Part 24, Figure 37). The roll motor was positioned such that the rotation of the motor shaft would directly translate to rotation of the model in the roll direction. The positioning of the motor shaft, coincident with the model $x$-body axis, is visible in Figure 41. The motor included an optical encoder which when connected through a counter provides the relative rotation angle to be measured. This allows the PC, through relays, to drive the motor and in turn the model to a specified roll angle. The fact that only the relative and not the absolute roll angle of the model is able to be determined from the encoder will be discussed in Section 4.2. Full specifications of the roll motor and encoder can be found in APPENDIX C.

\subsubsection{Model Support Electrical Design}

A block diagram of the overall model support electrical connections is shown below in Figure 43.

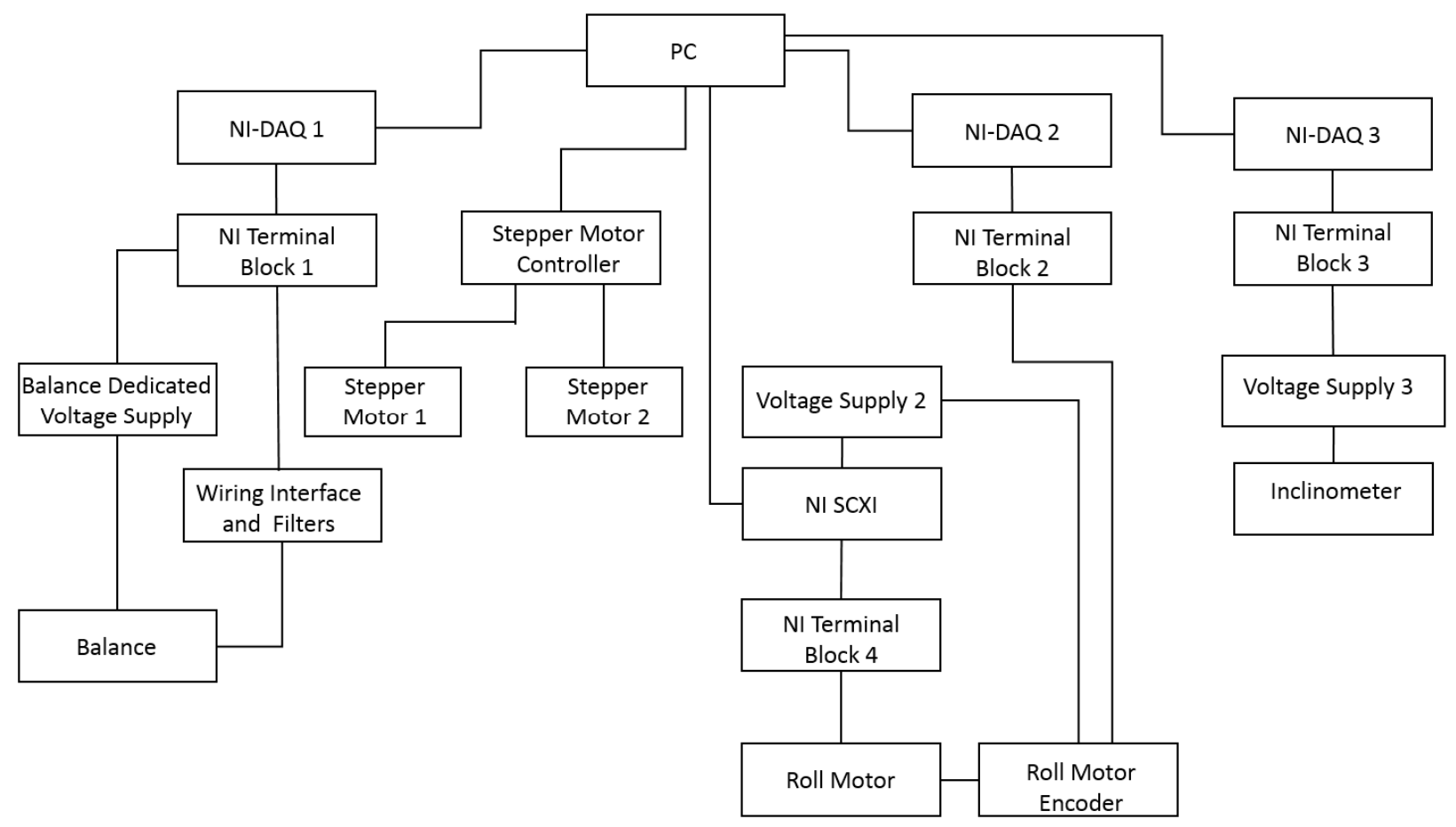

Figure 43 Model Support Electrical Connections Block Diagram 
The balance was excited by a dedicated precision (Part 7, Table 10) power supply where the voltage supplied to the balance is measured and internally corrected to maintain a constant supply voltage. The balance component output cable harness was connected to an interface box where the low pass filters were applied. The connections then entered a National Instruments (NI) terminal block (Component 10, Table 10) before entering the 16 bit NI data acquisition card (Component 16, Table 10) which connected directly to the PC (Component 19, Table 10). The stepper motor controller (Component 15, Table 10) was sent signals from the PC and operated the stepper motors (Components 2 and 3, Table 10) which in turn drove the lead screws on the model support. The roll motor (Component 4, Table 10) received power by a voltage supply (Component 8,Table 10) which was wired into a NI SCXI relay (Component 14, Table 10) and NI terminal block (Component 13, Table 10). The roll motor encoder was also powered by the same power supply and its signal was sent to a NI terminal block (Component 11, Table 10) and NI data acquisition card (Component 17, Table 10) which was connected directly to the PC. The inclinometer (Component 6, Table 10) received power from a power supply (Component 9, Table 10) and its signal was sent to a NI terminal block (Component 12, Table 10) and NI data acquisition card (Component 18, Table 10) which was directly connected to the PC.

\begin{tabular}{|l|l|l|c|}
\hline $\begin{array}{l}\text { Component } \\
\text { Number }\end{array}$ & Component & Manufacturer & Model Number \\
\hline $\mathbf{1}$ & Balance & Modern Machine (NASA) & 2044A \\
\hline $\mathbf{2}$ & Stepper Motor 1 & Velmex & PK 266-03A \\
\hline $\mathbf{3}$ & Stepper Motor 2 & Velmex & PK 266-03A \\
\hline $\mathbf{4}$ & Roll Motor & Pittman & GM9232E967 \\
\hline $\mathbf{5}$ & Roll Motor Encoder & HP & 91-500PPR \\
\hline $\mathbf{6}$ & Inclinometer & Level Developments & LSRP-90 \\
\hline $\mathbf{7}$ & Balance Dedicated Power & HP & \\
\hline $\mathbf{8}$ & Supply & & E3615A \\
\hline $\mathbf{9}$ & Voltage Supply 2 & Agilent & BOM-15/200 \\
\hline $\mathbf{1 0}$ & Voltage Supply 3 & Datel & BNC-2110 \\
\hline $\mathbf{1 1}$ & NI Terminal Block 1 & National Instruments & SCB 68 \\
\hline $\mathbf{1 2}$ & NI Terminal Block 2 & National Instruments & BNC-2110 \\
\hline $\mathbf{1 3}$ & NI Terminal Block 3 & National Instruments & BNC-2110 \\
\hline $\mathbf{1 4}$ & NI Terminal Block 4 & National Instruments & SCXI-1160 \\
\hline $\mathbf{1 5}$ & NI SCXI & National Instruments & NF90-3 \\
\hline $\mathbf{1 6}$ & Stepper Motor Controller & Velmex & PCI-6220 \\
\hline $\mathbf{1 7}$ & NI DAQ 1 & National Instruments & PCI-6220 \\
\hline $\mathbf{1 8}$ & NI DAQ 2 & National Instruments & PCI-6221 \\
\hline $\mathbf{1 9}$ & NI DAQ 3 & National Instruments & Dell T3600 \\
\hline
\end{tabular}

Table 10 Model Support Electrical Component List 


\subsubsection{Model Support Software Design}

The software controlling the model support, wind tunnel, and monitoring of the balance was developed and implemented in LabVIEW. The LabVIEW software is a development environment for creating custom applications or Virtual Instruments (VI's) that interact with signals or real-world data using the $\mathrm{G}$

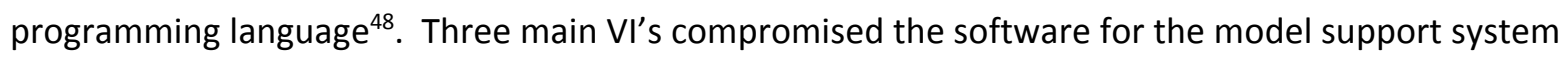
each of which was individually responsible for monitoring the balance and recording data, executing the test matrix and moving the model support, controlling the wind tunnel and interfacing with the user. All three VI's communicated with one another through the use of a global VI. A screenshot of the panel is shown below in Figure 44.

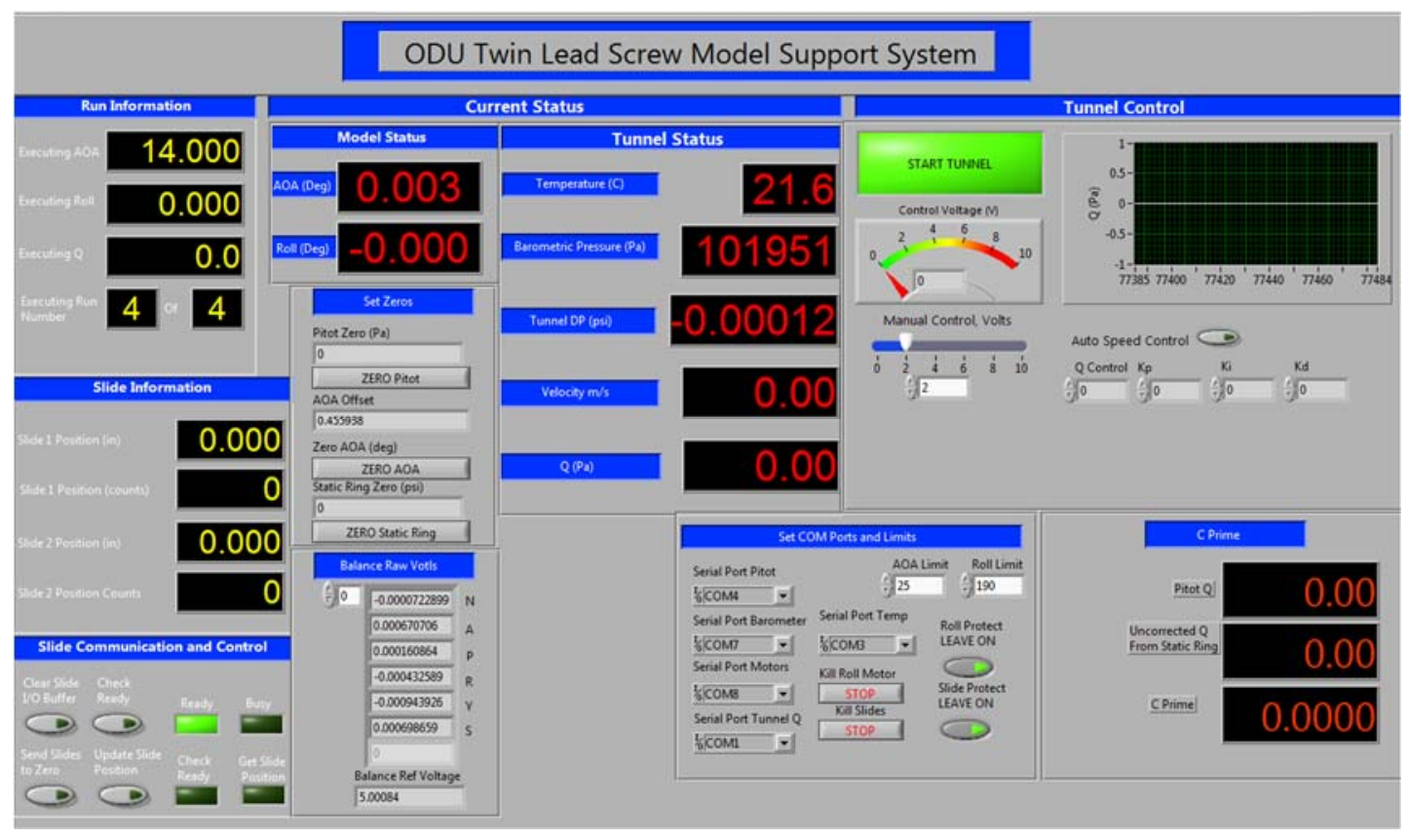

Figure 44 Model Support Front Control Panel 
The process of positioning the model during testing is shown in the flow chart in Figure 45.

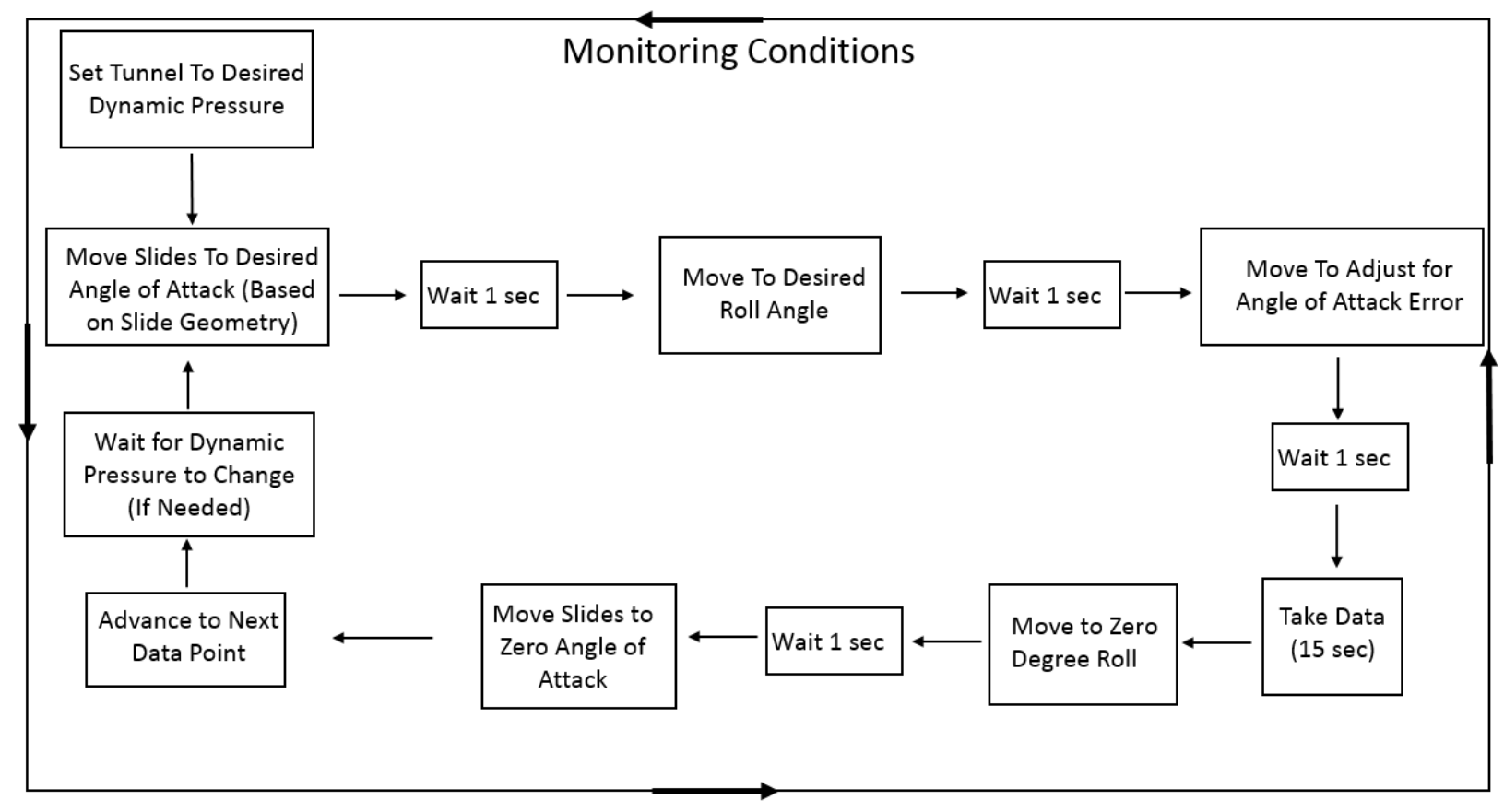

Figure 45 Model Support Movement Flow Chart

All conditions are continually monitored by the software and once the desired test set point is received, the tunnel is brought to the desired dynamic pressure. Then the slides are moved to the locations that would create the desired model angle of attack which is based on the slides and model support arm geometry. Knowing the distance between the centers of the slide mounts and the distance from the slides to the nose of the model, the model can be positioned (roughly) at the desired angle of attack while remaining on the tunnel centerline. Once this movement is complete, the roll motor drives the model to the desired roll angle. Next the inclinometer is read and the model is corrected to the precise angle of attack and data is taken. During the data taking process, the balance signals were sampled at $10 \mathrm{~Hz}$ for 15 seconds (the cut off frequency for the low pass filters) and then averaged over the 15 second sample time. When the data have been recorded the model returns to both zero roll angle and zero angle of attack to avoid aerodynamic hysteretic effects. This entire process is repeated for the duration of the test. During testing, the average time per data point, including model movement and data sampling was approximately 90 seconds. 


\subsubsection{Pressure Probe Assembly}

This section will detail the Pitot-static probe and the pressure transducers utilized for the tunnel conditions testing.

\subsubsection{Pitot-Static Probe Hardware}

The Pitot-static probe utilized in the wind tunnel testing was a 24in long probe manufactured by United Sensor. The Pitot-static probe assembly consisted of four components which are shown in Figure 46 below. The part numbering is consistent with Table 9 .
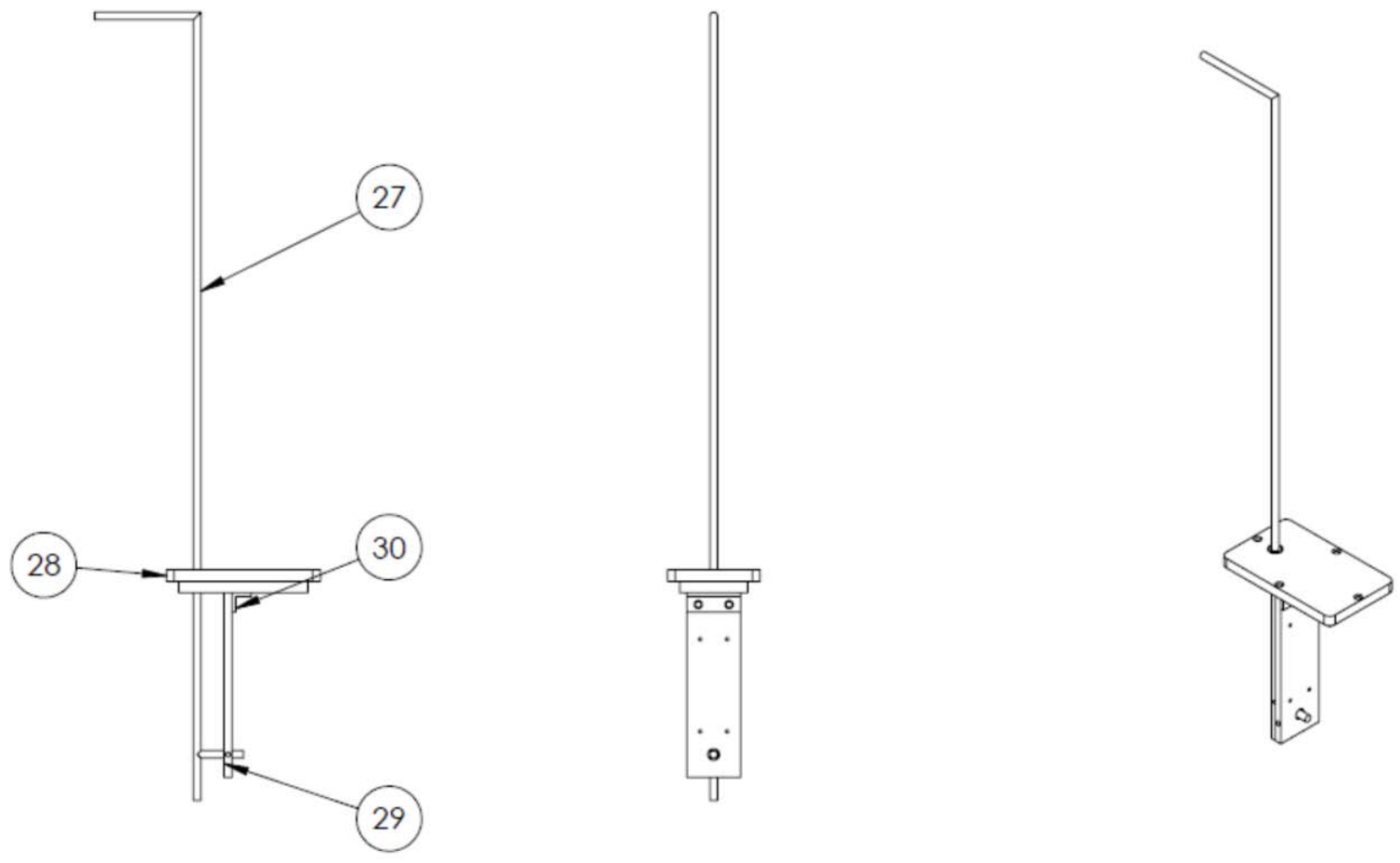

Figure 46 Pitot Tube Assembly

The turntable replacement had a pocket milled out such that the entire Pitot assembly could be easily installed and removed from the test section. A screenshot of the CAD model showing the Pitot tube assembly in the turntable replacement is shown in Figure 47. 


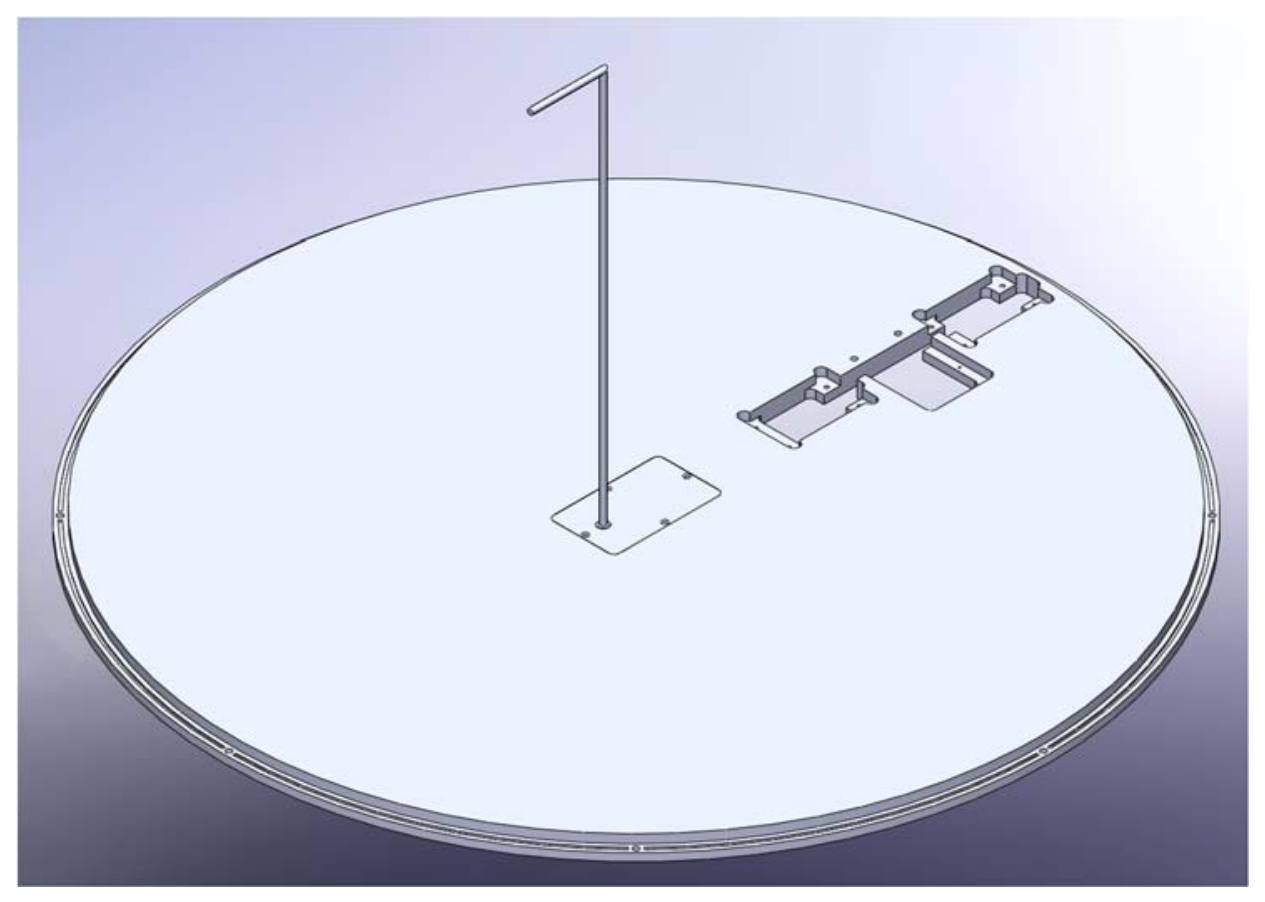

Figure 47 Pitot Tube Assembly CAD Model

The Pitot-static tube (Part 27, Figure 46) was fit to the Pitot tube filler piece (Part 28, Figure 46) via a threaded compression fitting. The Pitot-static tube was then positioned such that the total pressure measurement was at the longitudinal center of the test section (18in above the test section floor). The Pitot-static tube was accurately positioned both in distance from the test section floor and angle with respect to the oncoming flow using the Pitot register (Part 29, Figure 46) and the Pitot block bracket (Part 30, Figure 46). Set screws in the Pitot register locked the Pitot-static tube into position by clamping the static pressure end of the tube. This arrangement allowed for repeatable positioning of the sensor as it was installed and removed multiple times throughout the testing process. The Pitotstatic tube assembly is shown installed (looking downstream) in the test section in Figure 48. 


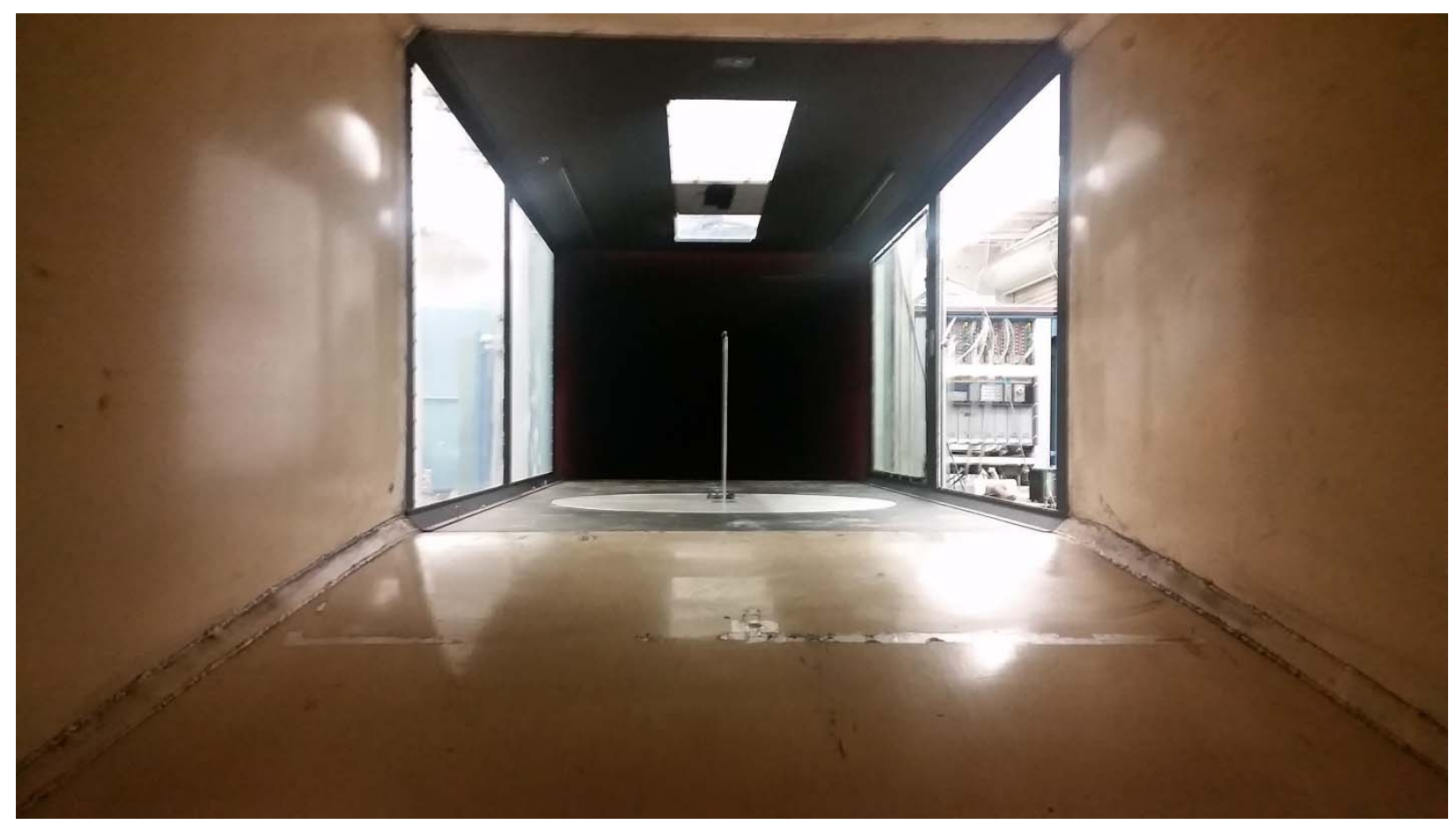

Figure 48 Pitot-Static Tube Installed in the Test Section

\subsubsection{Pressure Transducers}

To calculate the velocity in the test section and complete the tunnel conditions portion of the testing, two pressure transducers were necessary. One measured differential pressure from the Pitot-static tube and the other measured differential pressures between the static rings located on the upstream and downstream ends of the contraction nozzle leading into the high speed test section. Identical Mensor series 6100 pressure transducers with 10 inches of water range were used following a professional ISO:9001 calibration. The complete specifications for the Mensor pressure transducers along with the calibration information can be found in APPENDIX D.

\subsection{Testing Procedures}

This section will outline the practices and procedures utilized for both the airframe and tunnel conditions testing. The specific test schedule and design will be discussed in Sections 5.3 and 6.5. It is necessary to again reiterate the importance of following the exact procedure established each and every 
time when performing a check standard test. Without this rigid adherence to the established procedures it is impossible for the researcher to draw any meaningful conclusions from the test data.

\subsubsection{Pitot-Static Probe Assembly}

During the tunnel conditions portion of the testing, multiple tests were performed with each test compromised of multiple groups. The procedures were established to replicate as closely as possible the actual processes of check standard testing in larger facilities. The following procedures were established for in-between tests:

- At the end of each test, the entire Pitot tube assembly was removed from the test section and reinstalled at the beginning of the next test

- All plumbing was leak checked before the start of the test

- All electrical connections were tested

- Both pressure transducers were zeroed

- If more than one test was completed in a day, the tunnel was allowed to return to the ambient temperature before conducting the next test

The following procedures were followed for in-between groups:

- At the end of the group, the tunnel was brought to zero velocity

- At the end of the group, the test section was opened and hardware was inspected

- Before the start of the next group, the pressure transducers were zeroed

It should also be noted that the physical condition of the tunnel was unchanged throughout the testing. For example if there were mounting holes for a different piece of hardware in the test section, it was taped closed and remained taped closed for the entire duration of testing. In addition, the pressure transducers were always supplied power throughout the course of testing. However, it was necessary to zero the transducers due to the changes in tunnel temperature.

\subsubsection{Aircraft Model, Balance and Model Support}




\subsubsection{Balance Testing Procedure}

The 2044a force balance borrowed from NASA LaRC for this testing was received with a water jacket, a component that allows for the balance temperature to be regulated by pumping cool water through the jacket in high temperature test environments. Employing this feature was overly complex, would require a tube to cross the metric gap, and is typically not used for low speed testing. The balance gages are temperature compensated while it is recognized that temperature change primarily arises from the fact that the resistance based strain gages will exhibit dependencies on temperature and also will serve as heating elements. During initial validation testing it was observed that the balance would increase in temperature for an extended period of time before it appeared to reach a thermal equilibrium. Unable to regulate the balance temperature, the best alternative was to allow the balance to reach its thermal equilibrium before beginning to test.

In an attempt to find the timeframe in which the balance would reach a thermal equilibrium, the balance was supplied power and the force outputs were measured for an extended period of time. Each force channel was sampled approximately every 20 seconds for approximately $24 \mathrm{hrs}$. The result from the axial force response is shown in Figure 49.

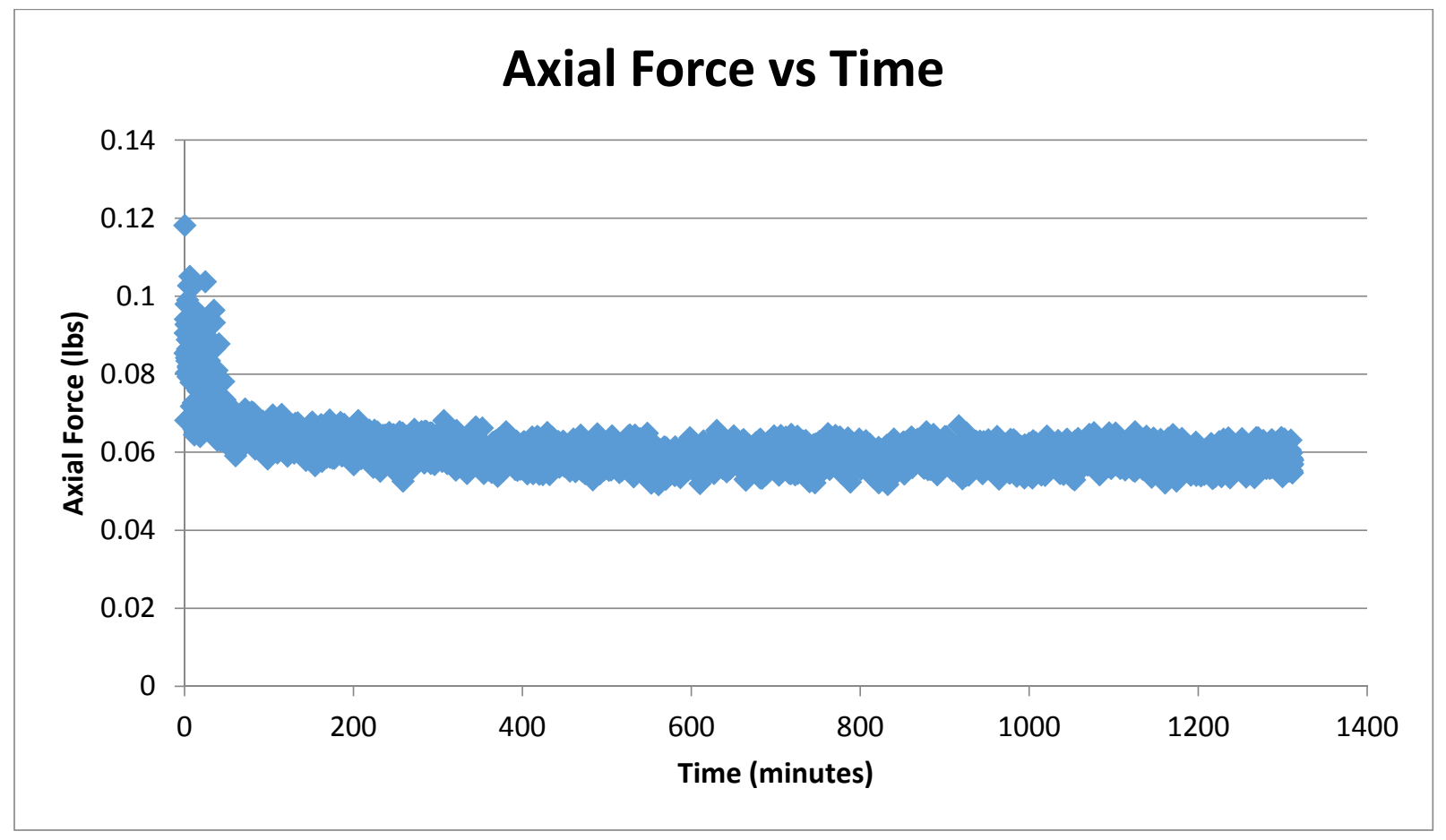

Figure 49 Balance Thermal Equilibrium Test 
It is seen in Figure 49 that roughly around the 400 minute mark, the measurements appear to stabilize. The other channels of the balance exhibited similar responses over the same time period. With this, it was decided to apply power to the balance for a minimum of 7 to 8 hours before testing to allow for the balance to sufficiently reach a thermal equilibrium.

\subsubsection{Model Support Testing Procedure}

As was the case in the tunnel conditions testing, the airframe testing consisted of multiple tests with each test consisting of multiple groups. Again, as was done in the tunnel conditions testing, procedures were established to replicate as closely as possible actual processes of check standard testing in larger facilities. The following testing procedures were followed between tests.

- Only one test per day was conducted

- At the end of each test:

0 the model and support arm assembly were removed from the strut as shown in Figure 50 (The strut was not removed from the test section during testing)

o the balance and all supporting electronic equipment were powered down

- Before the start of the next test:

o the balance and all supporting electronic equipment were powered for at least 8 hours before beginning testing

o the model and model support arm assembly was reattached to the strut

0 the reference zero slide positions were obtained

0 the reference zero degree roll and angle of attack were obtained and a tare was taken 


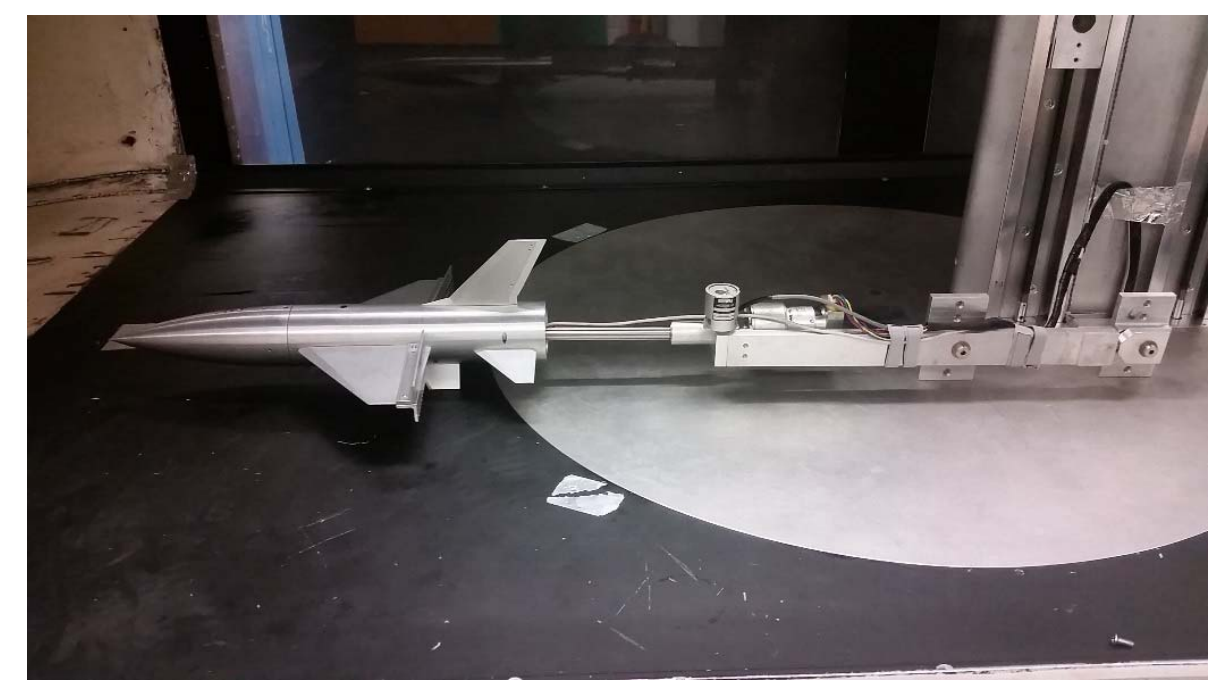

Figure 50 Model and Support Arm Assembly Removed from Strut

Due to the fact that the roll motor encoder only reported relative position, it was necessary to establish the reference zero degree roll angle. This was accomplished by placing two leveling gage blocks of the same exact height and a high precision level on the wing tips of the model and rotating the model until the zero roll angle was reached. A similar process was necessary to establish a reference zero degree angle of attack. Even though the inclinometer reported an absolute angle, the model installation angle and tunnel installation angle did not necessarily coincide with this reference zero degree angle. The same process with the leveling gage blocks and level was followed but this time the blocks were positioned on the leading and trailing edges of the left wing. Once the reference zero slide positions were obtained, only the front slide was moved to obtain the reference zero degree angle of attack and the new position of the front slide was saved as the zero slide reference position. The uncertainty in the initial offset of the roll angle and angle of attack was estimated to be $0.1^{\circ}$. 


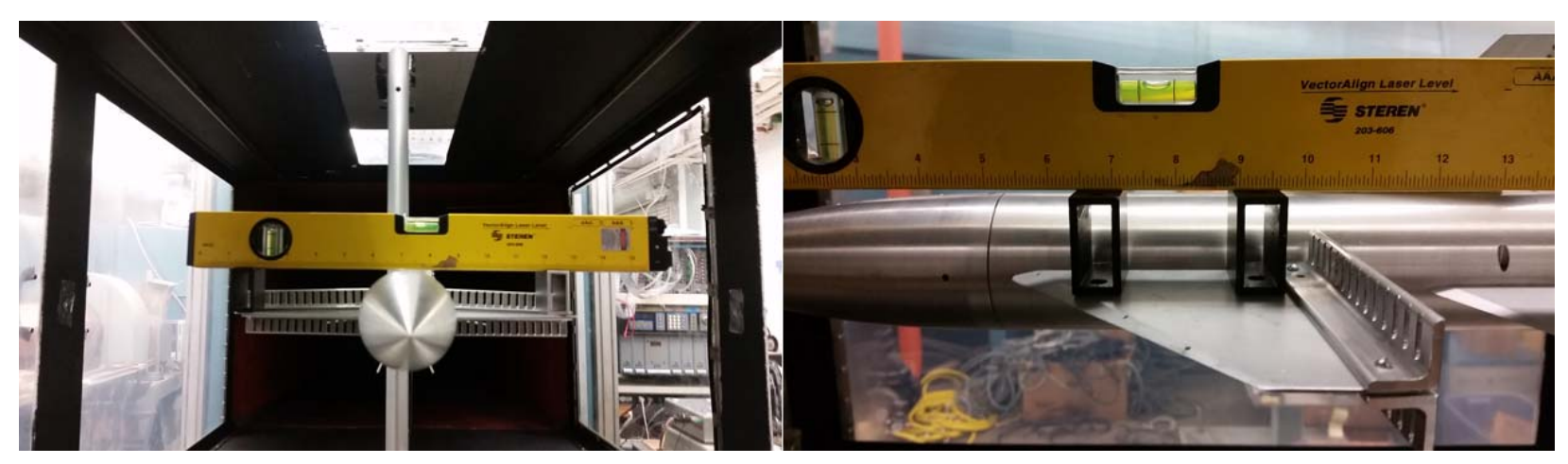

Figure 51 Reference Angle Procedure (Roll Angle Left, Angle of Attack Right)

The original reference zero slide positions were also manually set due to the fact that the stepper motor encoders only reported relative position. This was accomplished by driving each of the stepper motors to the very bottom of their travel limits then driving them up an established distance that would leave them in the direct center of the test section.

The following procedures were followed for in-between groups:

- A tare was taken if the time between tares had exceeded 60mins (This did not necessarily coincide with the end of each group, sometimes one tare was used on more than one group)

- At the end of each group, the reference zero degree roll and angle of attack were reestablished

- At the end of each group, the tunnel was brought to zero velocity

- At the end of each group, the test section was opened and all hardware and electrical connections were inspected 


\section{TUNNEL CONDITIONS TESTING}

This section will detail the research conducted for the tunnel conditions portion of the check standard testing. Standard Statistical Process Control methodology, its applications, and its enhancement by Design of Experiments methodology will be explored.

\subsection{Introduction}

The tunnel conditions portion of the wind tunnel testing took place over the course of six months from September 2014 to February 2015. In total, 44 tests and 132 groups were completed. For the purposes of this testing, establishing a baseline for the SPC charting required 15 to 20 tests $^{*}$ in which the data were in statistical control or reasonably close to statistical control. As the testing progressed, the values obtained for the calibration coefficient were plotted in SPC charts. This allowed trends to be observed and at times problems to be corrected. For example after test 6 , the increasing trends in the calibration coefficient prompted an in-depth investigation of the experimental setup for an assignable cause. This led to the discovery of a crack in the Pitot tube; the failure was corrected and testing resumed. The ability of SPC to identify potential problems in the system was again apparent after test 29 . The trends in the data for a second time prompted investigation into the experimental setup. After this investigation it was discovered there was assignable cause due to the failure in the seals surrounding the test section access door. Again this failure was fixed and testing resumed. It was only after an additional 15 tests were performed (tests 30-44) that it was decided that a sufficient baseline had been established. Therefore the data presented from Section 5.2 through Section 5.4 will only cover the baseline tests for the statistical process control charting, tests 30 through tests 44 . In Figure 52 , the reasoning for using only the last 15 tests for the baseline becomes clearly evident.

\footnotetext{
* There is no universally accepted number of baseline tests necessary to establish the first phase of the SPC charting practice. However, 15-20 tests are generally considered to be sufficient.
} 
$q=444$ Pa Test Averages

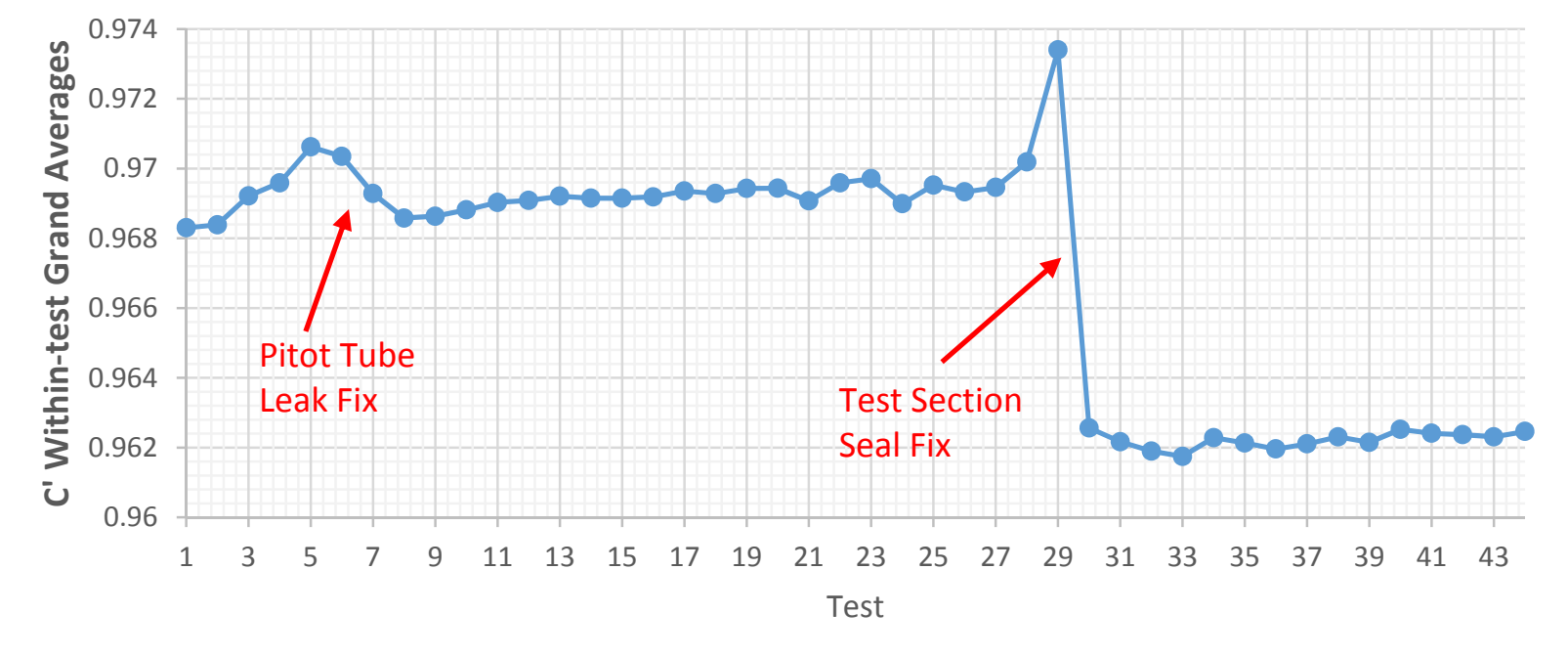

Figure $52 q=444$ Pa Test Averages for All Tests

\subsection{Standard SPC Charting}

This section will detail the methodology and implementation of statistical process control charting as it relates to the tunnel conditions testing and the wind tunnel calibration coefficient. It should be noted that for this research when the term "Standard SPC" chart is used it is not implied that this is the standard or conventional method for all statistical process control charting. This term is used to refer to the standard practice currently utilized at LaRC for implementing SPC charting.

\subsubsection{Methodology}

From Hemsch ${ }^{1}$, the use of statistical process control with the check standard testing allows researchers to characterize the short-term (within-group), within-test and across-test repeatability of wind tunnel measurements. This method of analyzing check standard testing data is intended to support process improvement and development of uncertainty models for measurements.

Four SPC charts are adapted for use with the tunnel conditions testing portion of the check standard testing. The across-test control charts are utilized to estimate the test to test variation of the tunnel calibration coefficient, the longest time frame of variation studied. The within-test range control charts are utilized to estimate the variation within each test of the tunnel calibration coefficient, the 
intermediate time frame of variation. The shortest time of variation in the tunnel calibration coefficient is estimated by the within-group range control charts. The fourth control chart is the test moving range chart and although this chart does not directly give an estimate of variation of the tunnel calibration coefficient, it serves as another metric to visualize the test to test changes. The control limits on each control chart will be presented as the associated chart is presented in Section 5.2.2.

Adapting the conventional statistical process control methods to a low degree of freedom process such as wind tunnel testing can be difficult. In conventional SPC applications focusing on quality control, a worker may randomly select a part from an assembly line for inspection. The quality control variables of interest would be recorded and this process would be repeated for the duration of the production of the parts. The major difference between the application of SPC in the case of the parts and wind tunnel testing is the availability of samples. The part maker has an extensive number of parts available, thus increasing both the ability to estimate variations and the ability to identify when the process is out of statistical control. In the application of SPC to wind tunnel testing, the number of samples (tests) available can be very low in comparison.

\subsubsection{Standard SPC Chart Results}

This section will discuss the results of the tunnel conditions testing in the context of the standard statistical process control charts.

The degree of freedom allocation for the SPC charts is shown below in Table $11^{3}$. (Refer to Section 5.3.2 for a detailed description of the test design and Section 1.3 for the background on SPC)

\begin{tabular}{|l|l|}
\hline Degree of Freedom Allocation \\
\hline Chart & $\mathrm{n}$ \\
\hline Test Control & 2 \\
\hline Test Moving Range Control & 2 \\
\hline Within-group Range Control & 5 \\
\hline Within-test Range Control & 15 \\
\hline
\end{tabular}

Table 11 Degree of Freedom Allocation for SPC Chart Limits 


\begin{tabular}{|l|c|l|}
\hline SPC Factor Values & Coefficient & Value \\
\hline Chart & $d_{4}$ & 0.954 \\
\hline Test Control & $D_{6}$ & 3.865 \\
\hline Test Moving Range Control & $D_{6}$ & 2.179 \\
\hline Within-group Range Control & $D_{6}$ & 1.678 \\
\hline Within-test Range Control & &
\end{tabular}

Table 12 Statistical Process Control Factor Values

\subsubsection{Across-Test Control Charts}

The limits on the across-test control charts are given by the upper and lower control limits defined by Equations 45 and 46 respectively. The equations read the upper or lower control limit is equal to the median of the test averages plus or minus three times the median of the moving range of the test averages divided by the statistical process control factor, $d_{4}$. The value of the factor is given in Table 12 .

$$
\begin{aligned}
& U C L=\tilde{\bar{X}}+\frac{3 m \tilde{R}}{d_{4}} \\
& L C L=\tilde{X}-\frac{3 m \tilde{R}}{d_{4}}
\end{aligned}
$$




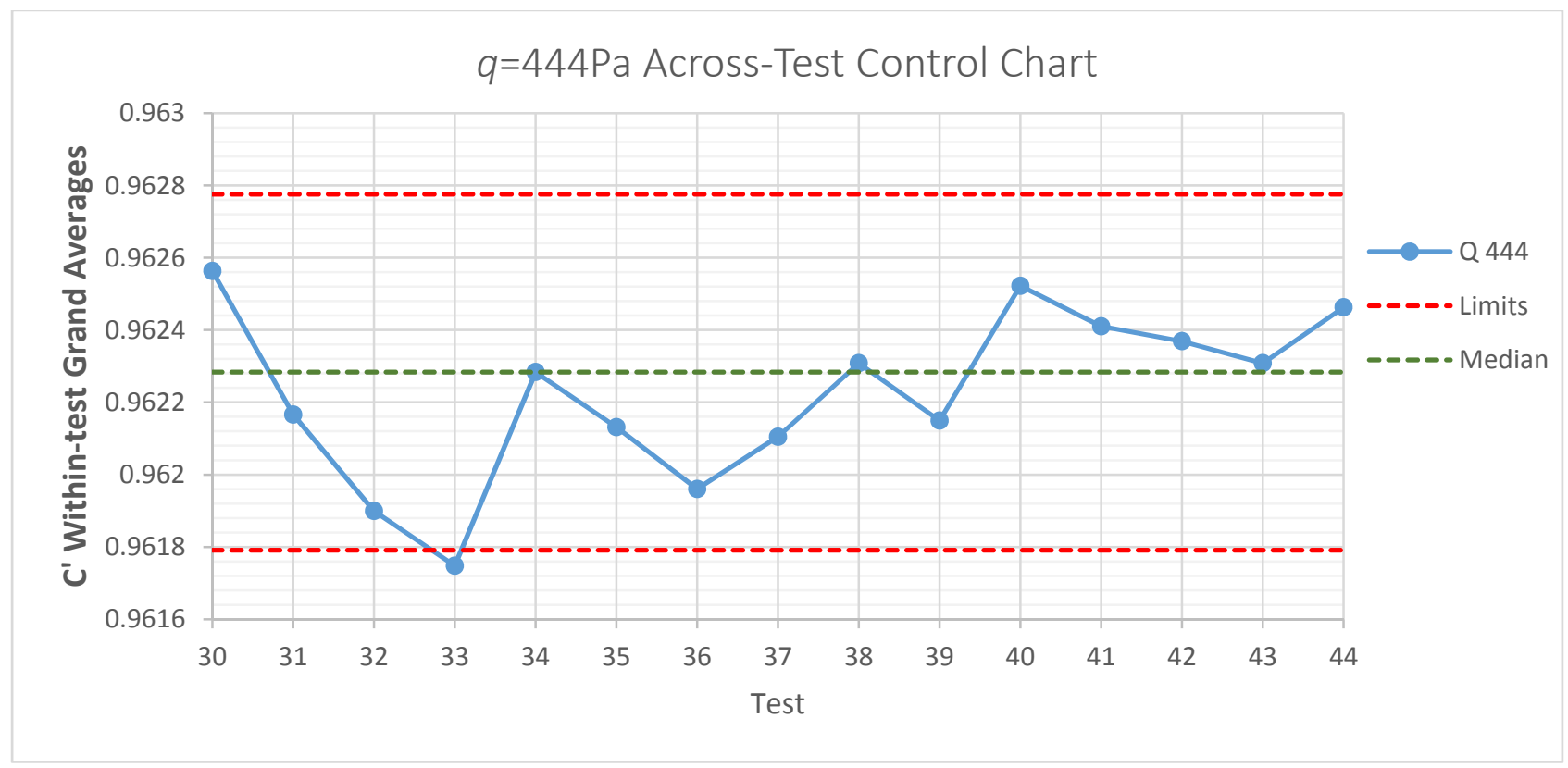

Table $13 q=444 P a$ Across-Test Standard SPC Control Chart

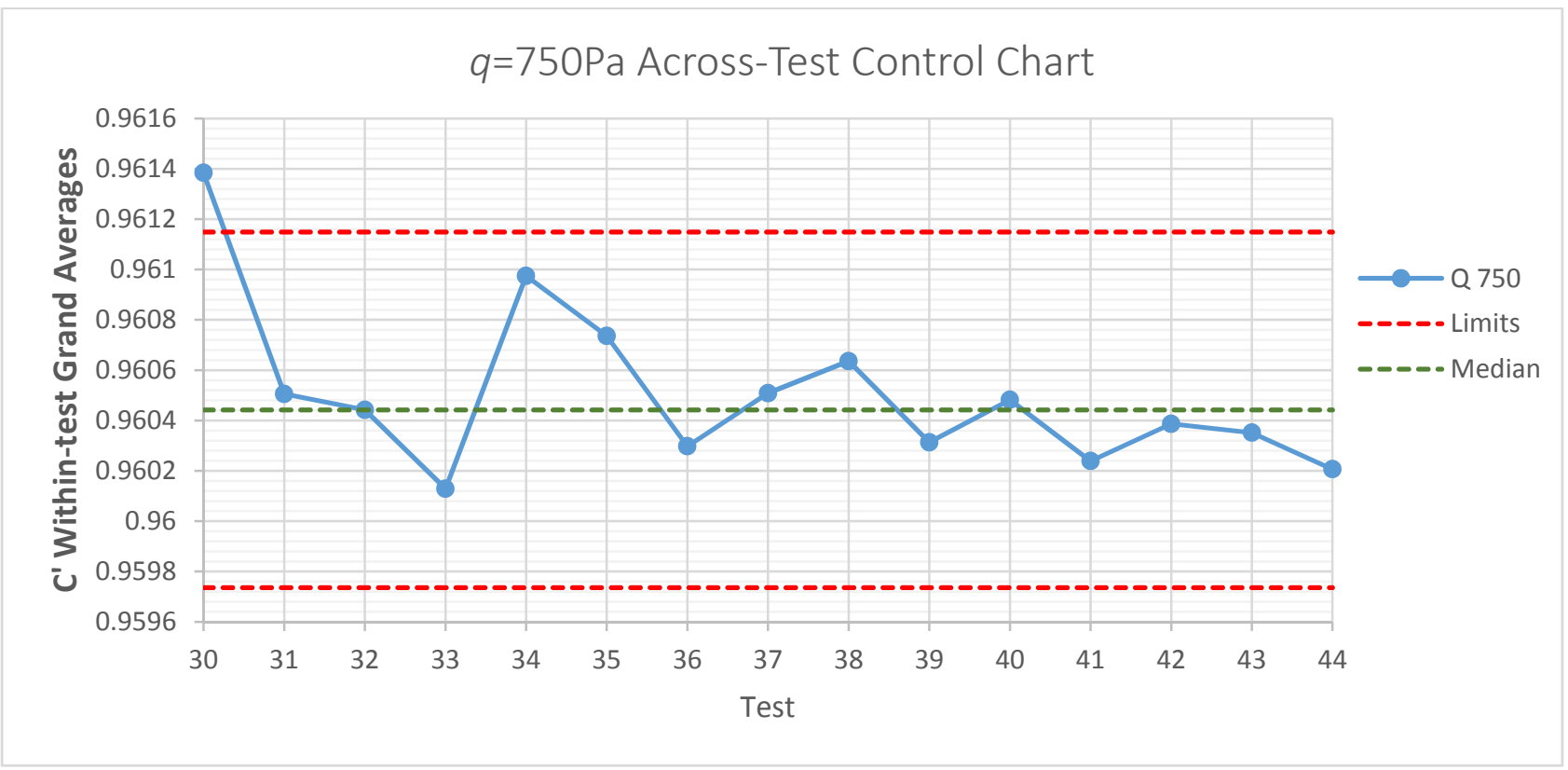

Figure $53 q=750 P a$ Across-Test Standard SPC Control Chart 


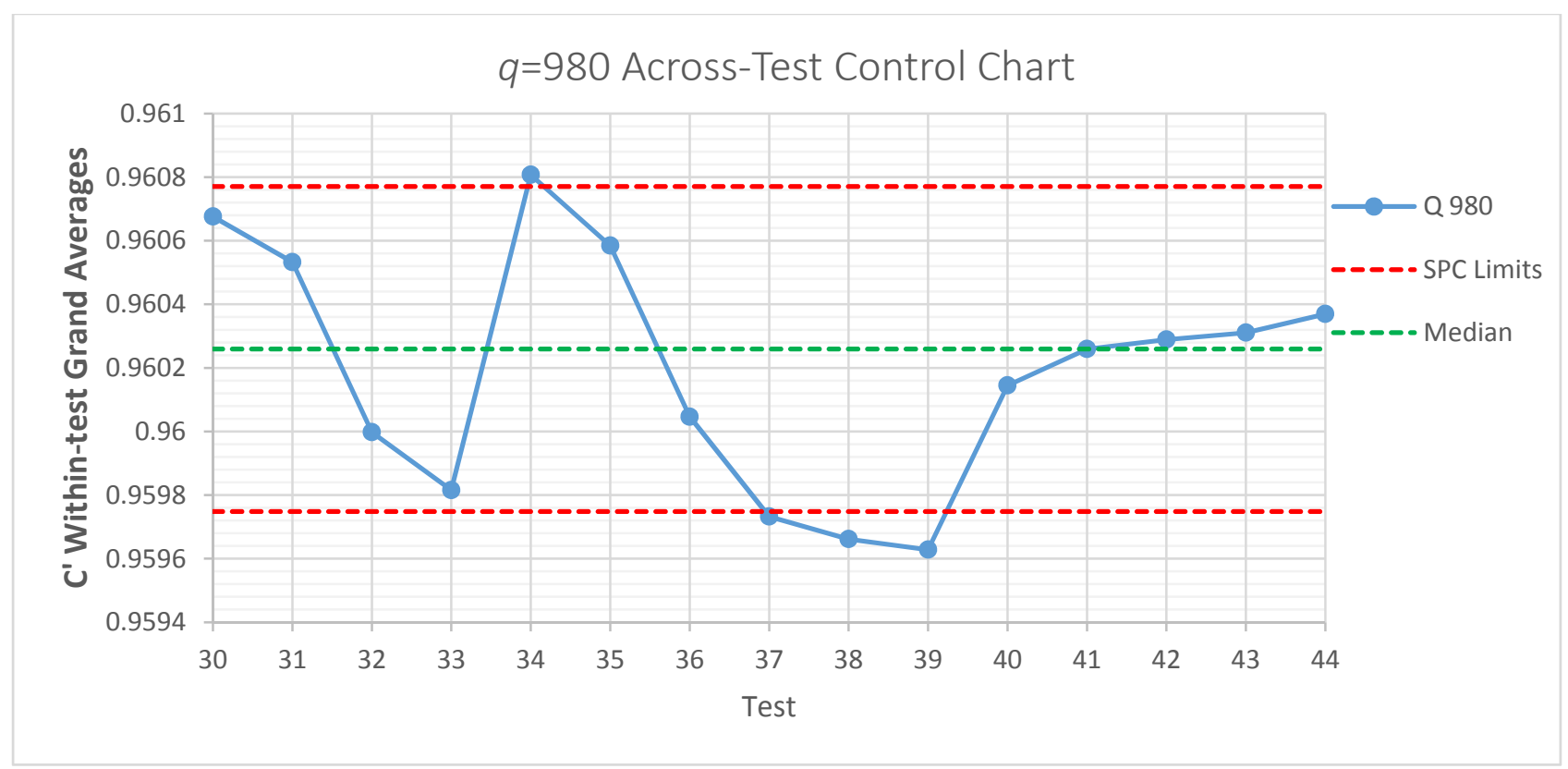

Figure 54 q=980Pa Across-Test Standard SPC Chart

The across-test variation in the tunnel calibration coefficient displayed at the three levels of dynamic pressure indicates that the process is not in strict statistical control however the magnitude of the variations are small and trends are minimal such that the application of the proposed methodology is feasible.

\subsubsection{Within-Test Range Control Chart}

The limit on the within-test range control chart is the upper range limit which is given by Equation 47 below. The equation reads the upper range limit is equal to the statistical process control factor, $D_{6}$, multiplied by the median of the moving range of the test ranges. The value of the coefficient is given in Table $12^{1}$.

$$
U R L=D_{6} * m \tilde{R}
$$




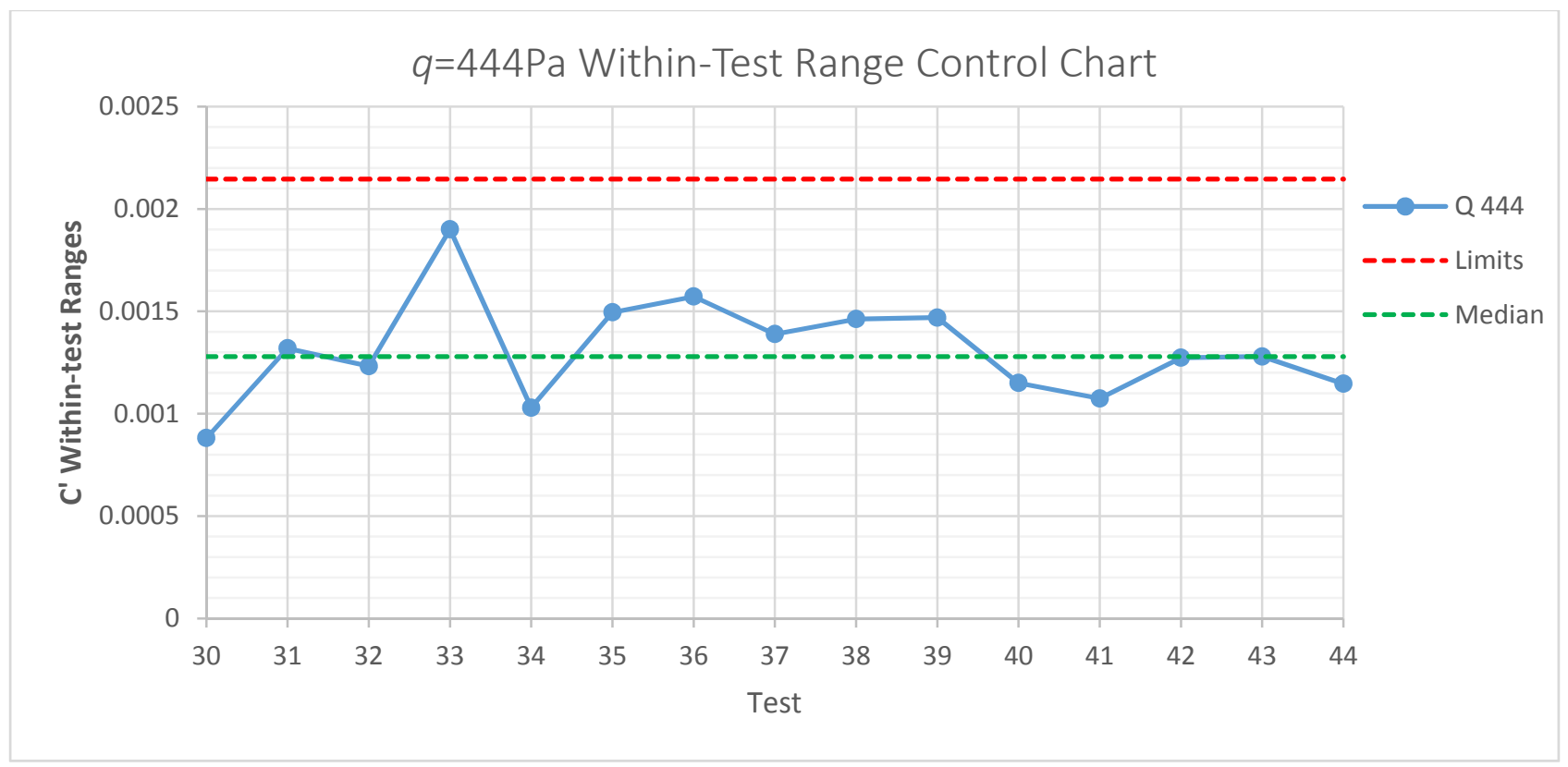

Figure 55 q=444Pa Within-Test Range Standard SPC Control Chart

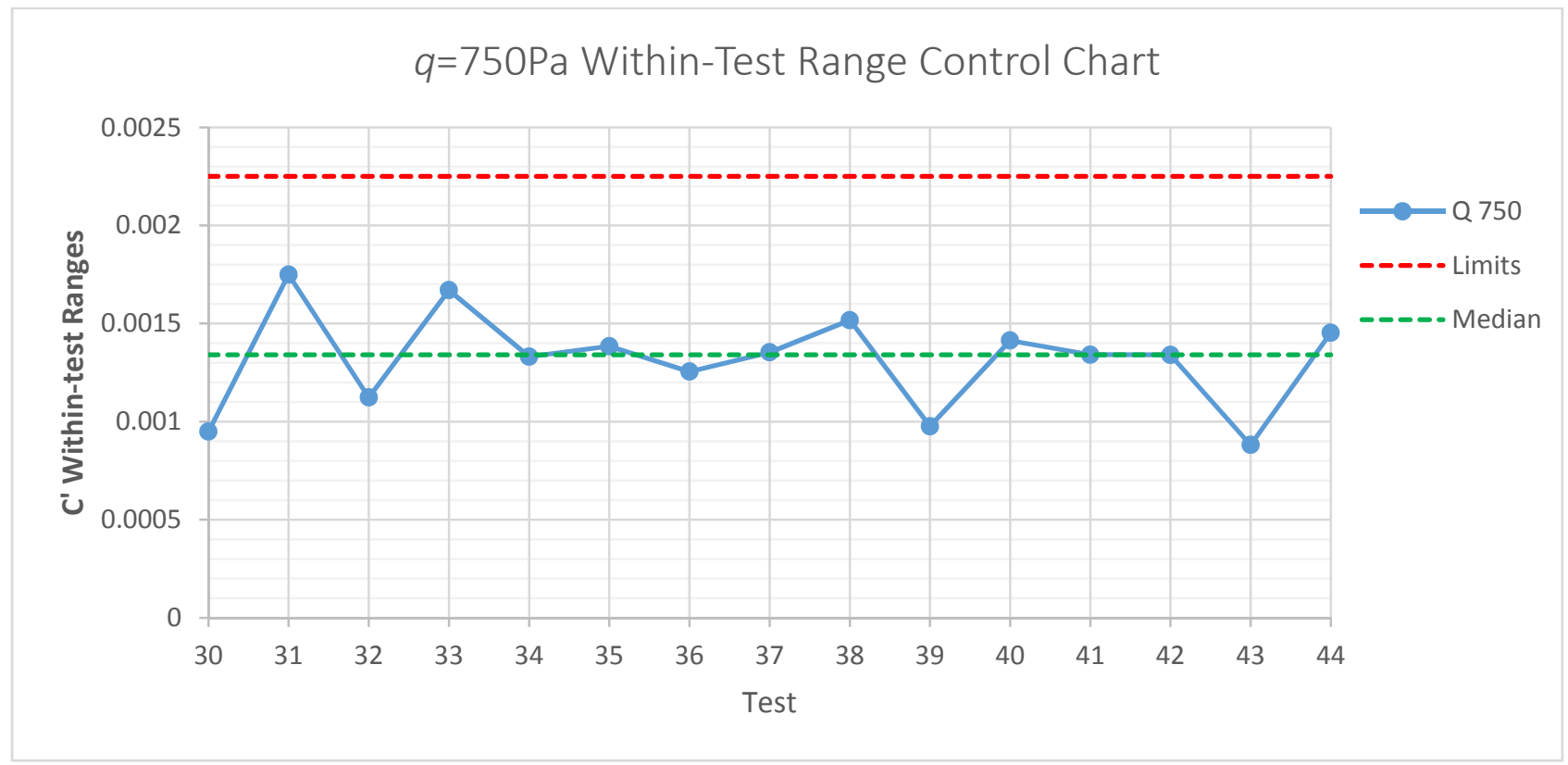

Figure $56 q=750 P a$ Within-Test Range Standard SPC Chart 


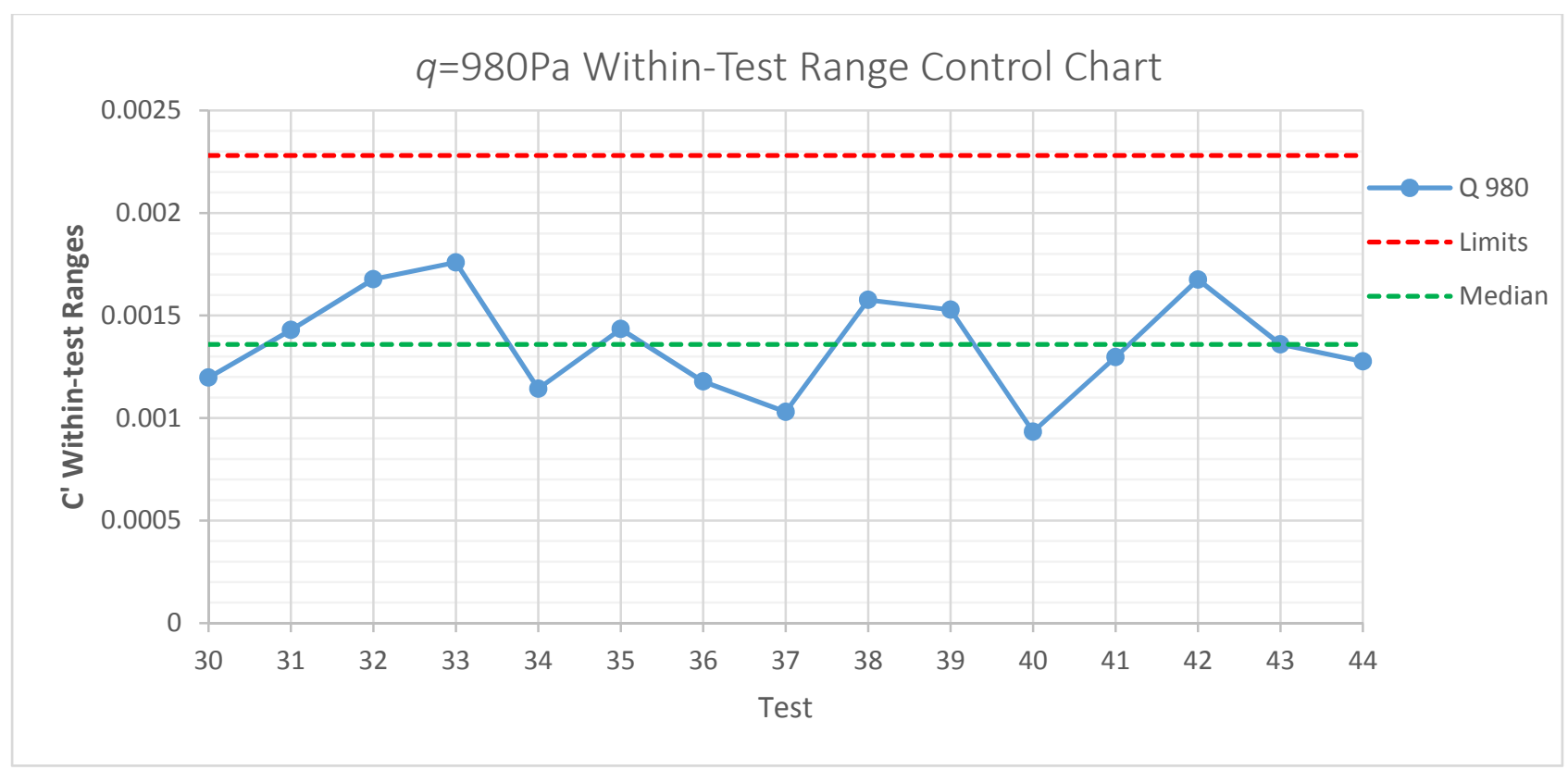

Figure 57 q=980Pa Within-Test Range Standard SPC Chart

The above test range control charts indicate that the within-test variation of the tunnel calibration coefficient is in statistical control and that the proposed methodology can be evaluated since all data points are under the set limits.

\subsubsection{Test Moving Range Control Charts}

The limit on the test moving range control chart is the upper range limit which is given by Equation 47 in Section 5.2.2.2. However the moving range now refers to the moving range of the test averages. The equation reads The upper range limit is equal to the statistical process control factor $D_{6}$, multiplied by the median of the moving range of the test ranges. The value of the coefficient is given in Table 12.

The results for the three levels of dynamic pressure are given in Figure 58 through Figure 60. 


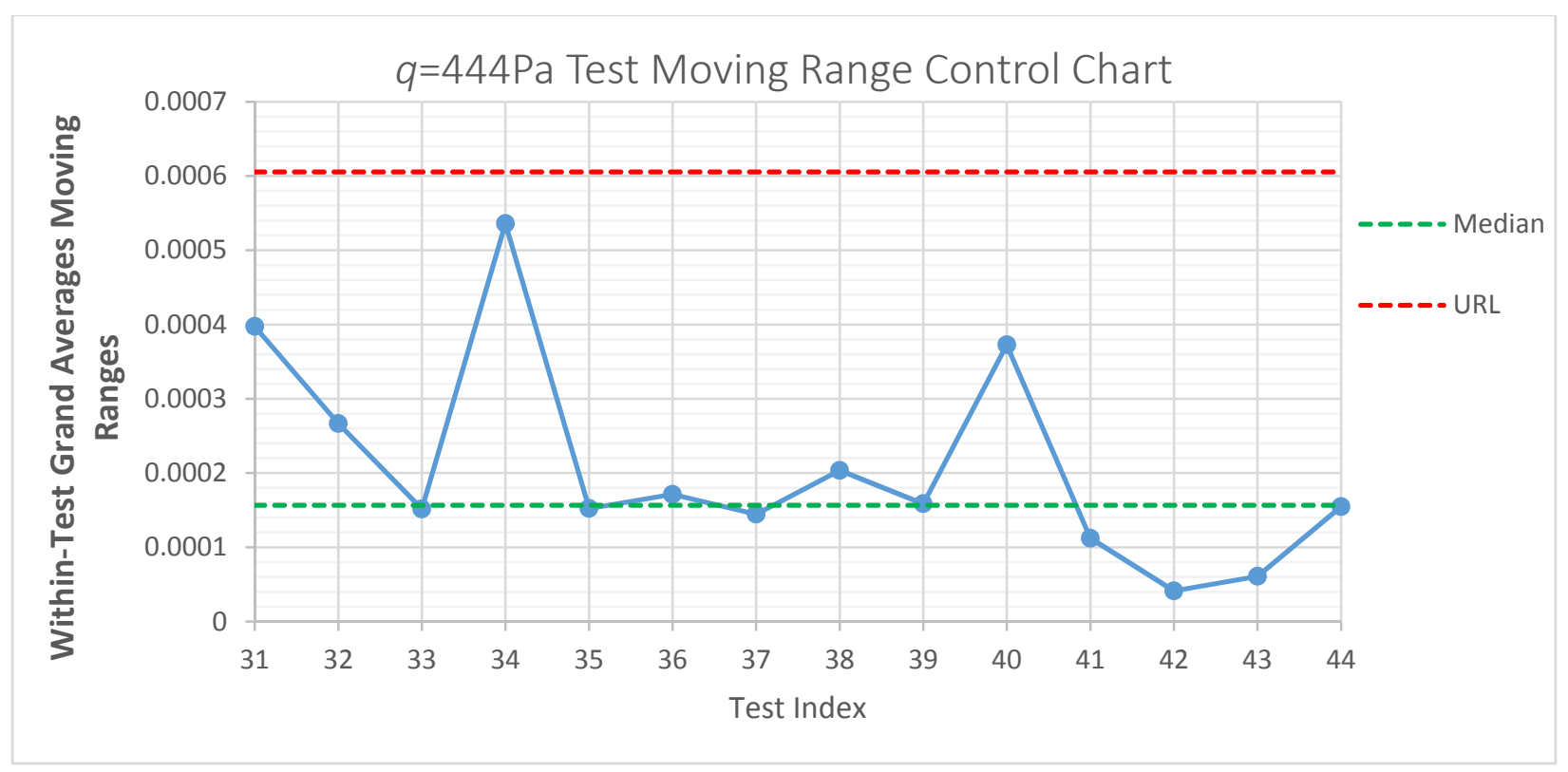

Figure $58 q=444 P a$ Test Moving Range Standard SPC Chart

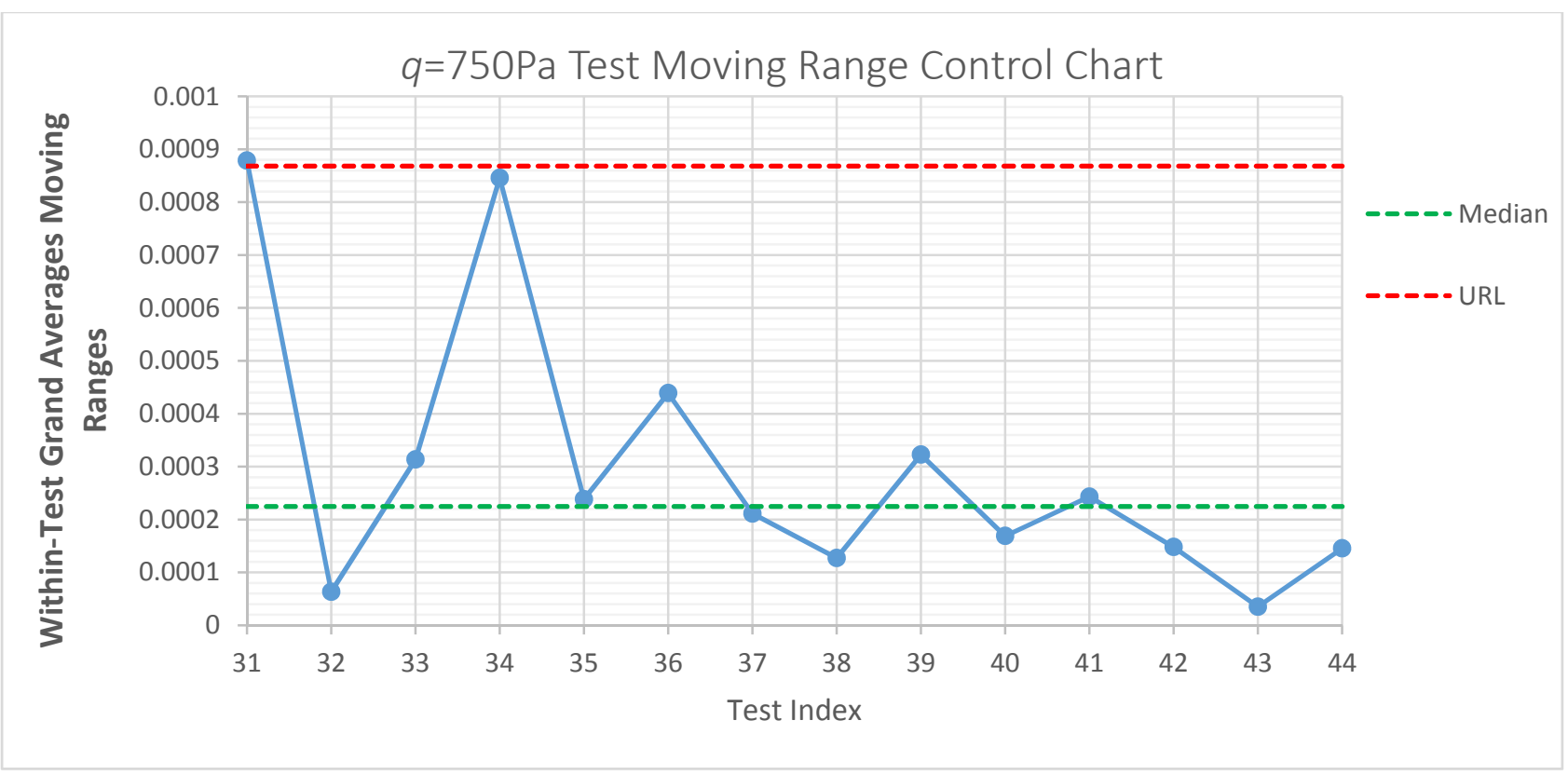

Figure $59 q=750 P a$ Test Moving Range Standard SPC Chart 


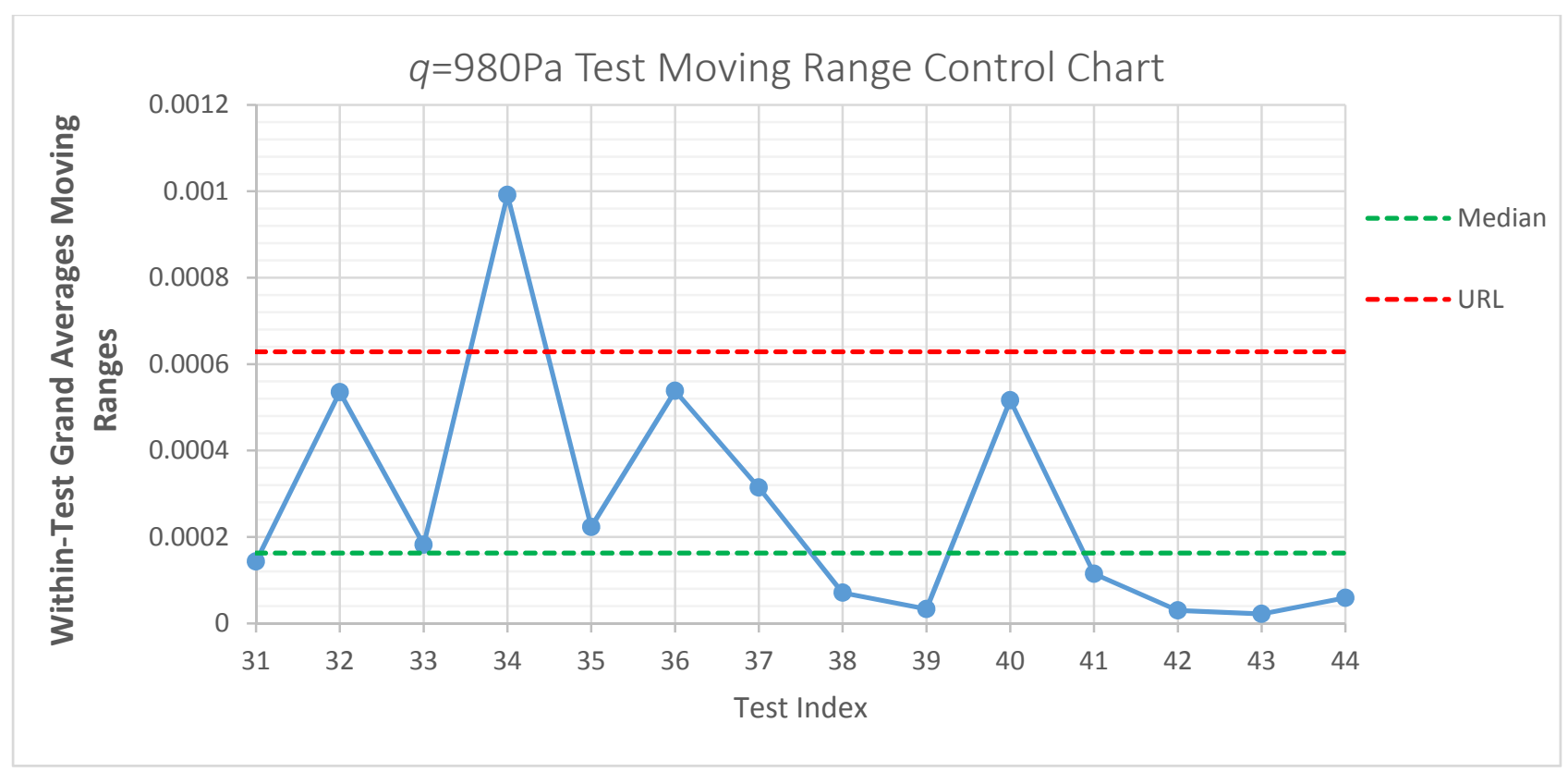

Figure 60 q=980Pa Test Moving Range Standard SPC Chart

With the exception of test index 34 in Figure 60, the moving range control charts could be said to be in statistical control. There was no assignable cause found for the results of test index 34 .

\subsubsection{Within-Group Range Control Charts}

The limit on the within-group control charts is the upper range limit which is given by Equation 47 in Section 5.2.2.2. However the moving range now refers to the moving range of the group ranges. The equation reads the upper range limit is equal to the statistical process control factor, $D_{6}$, multiplied by the median of the moving range of the group ranges. The value of the coefficient is given in Table 12 .

The results for the three levels of dynamic pressure are given in the three charts below. The plots show group numbers for the three groups in each test. For instance test 30 has three groups, 88, 89, and 90. 


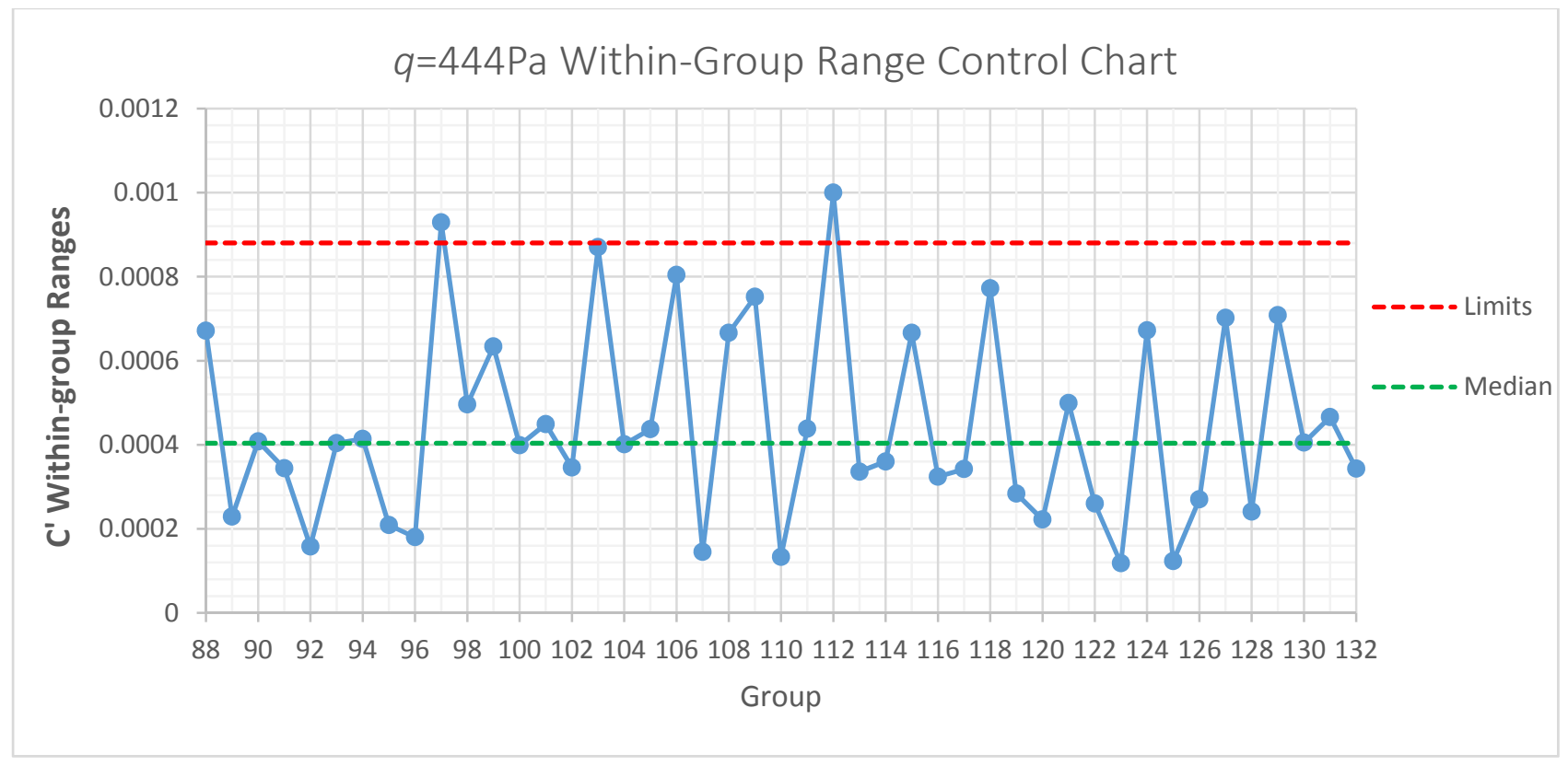

Figure $61 q=444 P a$ Within-Group Range Standard SPC Chart

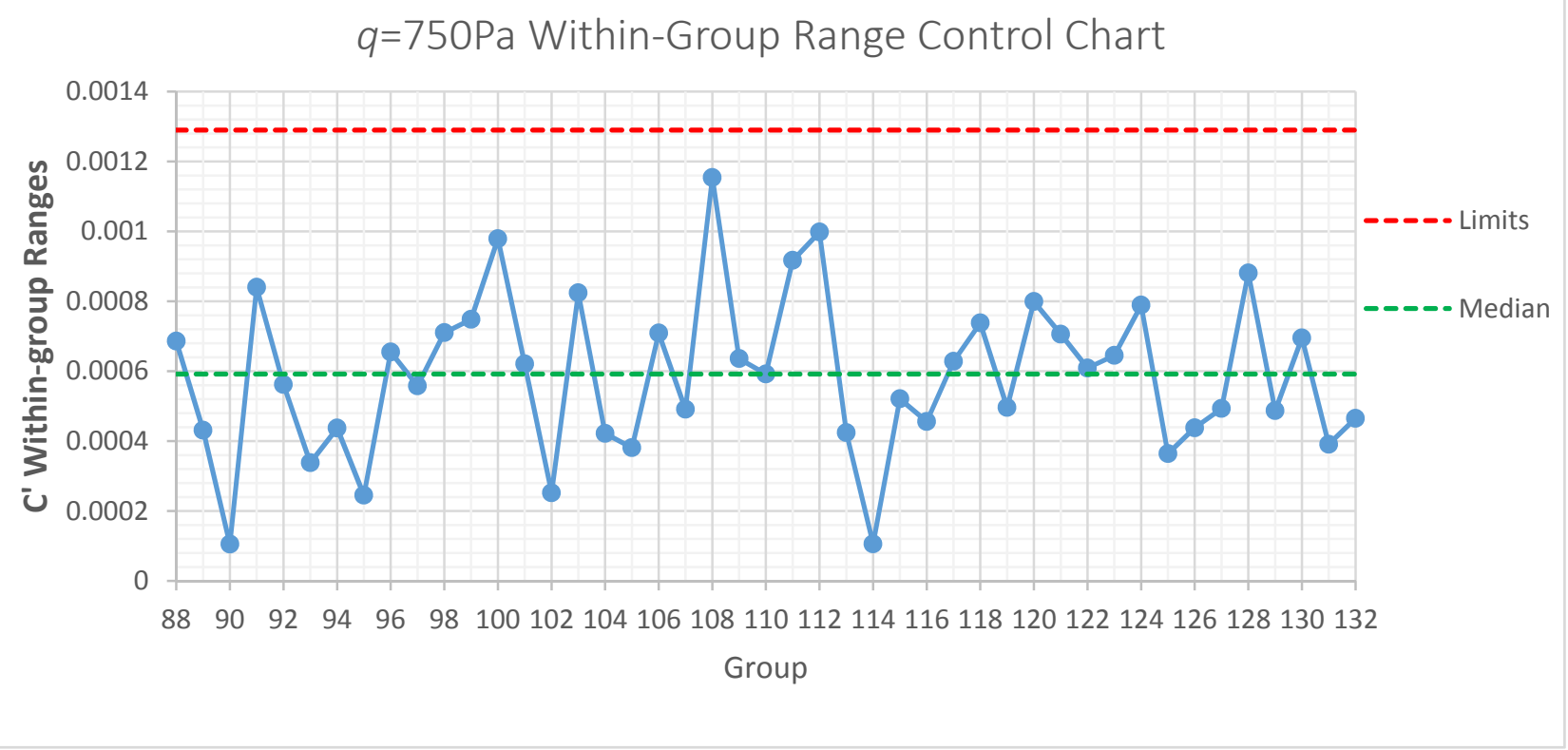

Figure $62 q=750 P a$ Within-Group Range Standard SPC Chart 


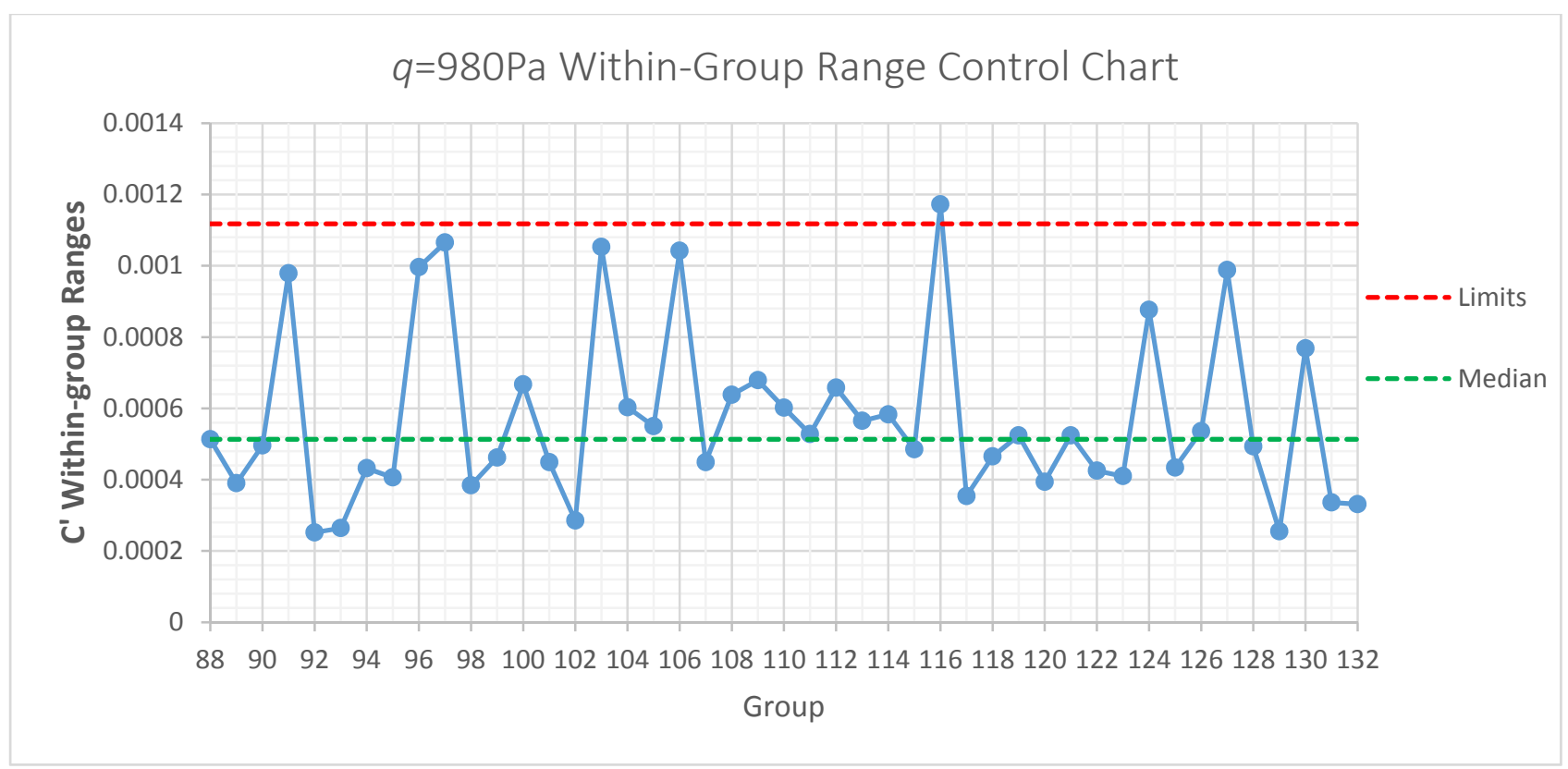

Figure $63 q=980 \mathrm{~Pa}$ Within-Group Range Standard SPC Control Chart

Inspecting the within-group range control charts and the associated short-term variance shows that the process is in or very close to being in statistical control such that the application of the proposed methodology can proceed.

\subsubsection{SPC Estimates of Standard Deviation}

The estimation of the standard deviation, adapted from Hemsch ${ }^{1}$ utilizing the SPC method is shown below. Where $n$ represents the number of observations per group and $b$ represents the number of observations per test.

$$
\hat{\sigma}_{W G}=\tilde{R}_{W G} / d_{4}
$$




$$
\hat{\sigma}_{W T}=\sqrt{\left(\tilde{R}_{W T} / d_{4}\right)^{2}-\frac{\hat{\sigma}^{2} W G}{n}}
$$

$$
\hat{\sigma}_{A T}=\sqrt{\left(\widetilde{m R}_{A T} / d_{4}\right)^{2}-\frac{\hat{\sigma}^{2} W T}{b n}}
$$

The total uncertainty estimate for the tunnel calibration coefficient is given by combining each level of variation.

$$
\hat{\sigma}_{T}=\sqrt{\hat{\sigma}_{A T}^{2}+\hat{\sigma}_{W T}^{2}+\hat{\sigma}_{W G}^{2}}
$$

Where the statistical process control factor $d_{4}$ is a function of degrees of freedom which follows Table 11. The values for $d_{4}$ are given below in Table $14^{3}$.

\begin{tabular}{|l|l|l|}
\hline \multicolumn{3}{|c|}{$\boldsymbol{d}_{\mathbf{4}}$ Values } \\
\hline Level & DOF & Value \\
\hline Across-Test & 2 & 0.954 \\
\hline Within-Test & 3 & 1.588 \\
\hline Within-Group & 5 & 2.257 \\
\hline
\end{tabular}

Table 14 Values of $d_{4}$ for use in Estimation of the Standard Deviation 


\begin{tabular}{|c|l|l|l|}
\hline Level & $\boldsymbol{q}=444 \mathrm{~Pa}$ & $\boldsymbol{q}=\mathbf{7 5 0} \mathrm{Pa}$ & $\boldsymbol{q}=\mathbf{9 8 0} \mathbf{P a}$ \\
\hline$\widehat{\boldsymbol{\sigma}}_{\boldsymbol{A T}}$ & 0.000104 & 0.000213 & 0.000124 \\
\hline$\widehat{\boldsymbol{\sigma}}_{\boldsymbol{W T}}$ & 0.000487 & 0.000374 & 0.000442 \\
\hline$\widehat{\boldsymbol{\sigma}}_{\boldsymbol{W G}}$ & 0.000178 & 0.000262 & 0.000227 \\
\hline$\widehat{\boldsymbol{\sigma}}_{\boldsymbol{T}}$ & 0.000529 & 0.000504 & 0.000512 \\
\hline
\end{tabular}

Table 15 SPC Estimates of Standard Deviation

\subsection{Use of Design of Experiments Methodology}

This section will outline the use of design of experiments as it relates to the tunnel conditions testing including the test design and analysis.

\subsubsection{Split-Plot Designs}

One of the benefits of using Design of Experiments is the ability of the methodology to minimize the possible influence of lurking variables. That is variables that may be present during the experiment that are not necessarily well defined or controllable, but have the potential to alter the response. To take advantage of this feature of DOE, fully randomized, replicated designs are necessary. In certain scenarios the test schedule cannot be fully randomized for a number of different reasons. For example it may be expensive to change the level of a factor, or the changing of a factor level may be time consuming. For this research the factor of dynamic pressure is treated as a Hard-to-Change (HTC) factor $^{\dagger}$. In general when there is a restriction on randomization of the test design, statistical power and overall inference ability are both adversely affected. Also special designs and statistical analysis must be employed to account for this restriction on randomization. One of these designs and statistical analysis combinations that will be utilized in this research is the split-plot design and analysis of variance through Restricted Maximum Likelihood (REML). It is beyond the scope of this dissertation to describe the theory and requirements of split-plot design and analysis; the reader is directed to references by Montgomery et al. ${ }^{9,10}$

\footnotetext{
${ }^{\dagger}$ For the ODU LSWT it is not necessarily difficult to change the dynamic pressure however for purposes of demonstrating this methodology and making the approach adaptable to cases in which HTC factors exist, the dynamic pressure was treated as HTC.
} 


\subsubsection{Test Design and Evaluation}

The primary goals of the test design were to one, create a balanced, nested design to simplify the calculations of standard deviation estimates and two, to create a design with the ability to handle a second order split-plot based regression model. Also of importance was to create a design which was adaptable to any factors of interest including multiple hard-to-change factors. For example, at the ODU LSWT, the tunnel calibration coefficient is only dependent on tunnel dynamic pressure and temperature, however at a more complex facility with more controllable variables (i.e. pressure, Reynolds Number, Mach etc.) the tunnel calibration coefficient could be dependent on multiple factors, some of which could be hard to change. The design structure of the tunnel conditions testing is shown schematically in Figure 64 below.
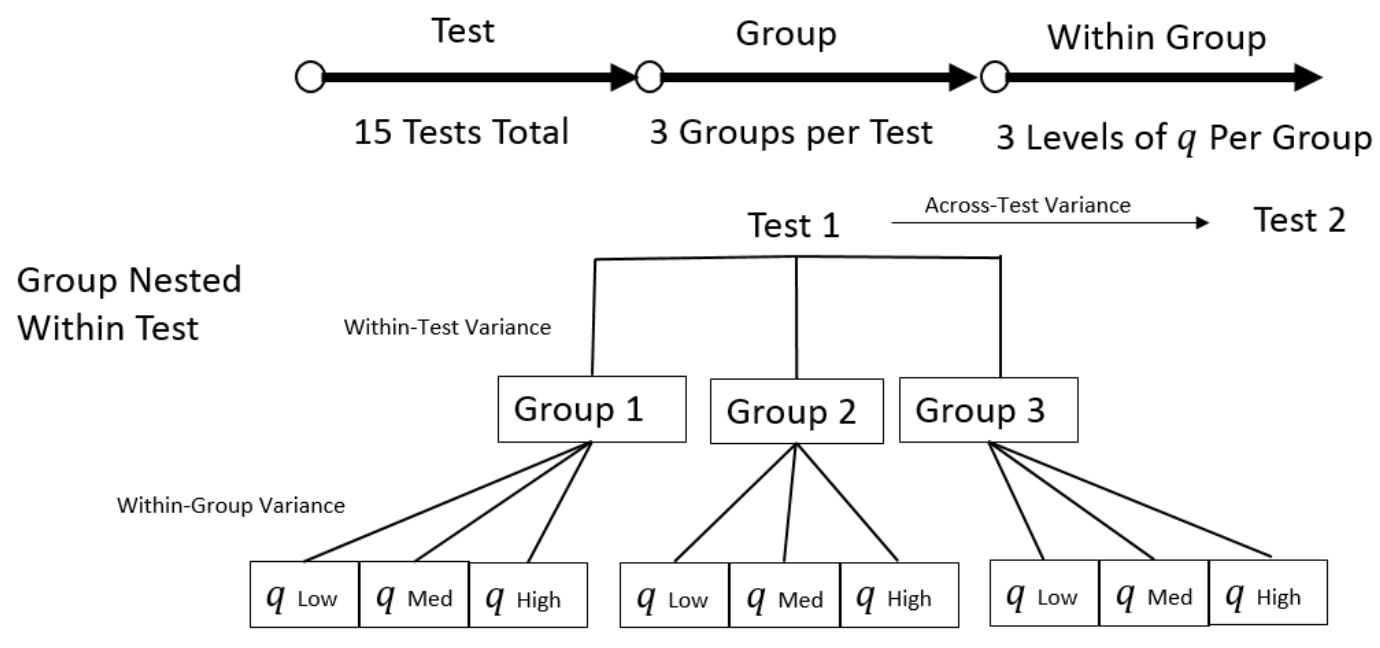

Figure 64 Tunnel Conditions Test Design

Absent from the test structure above is the temperature factor. The ODU LSWT is an atmospheric tunnel and no temperature control is available; therefore, the analysis of temperature will be completed using analysis of covariance.

As was introduced in Section 2.2, the tunnel conditions testing baseline consisted of 15 tests which were performed over the operational range of the tunnel, $20 \mathrm{~m} / \mathrm{s}$ to $40 \mathrm{~m} / \mathrm{s}$ (444Pa to $980 \mathrm{~Pa}$ ). Each test 
consisted of three groups with each group consisting of 17 fully randomized individual data points (runs). Within each group, data points at the three levels of $q(444 \mathrm{~Pa}, 750 \mathrm{~Pa}, 980 \mathrm{~Pa})$ were each taken five times and two confirmation points (600Pa, 865Pa) were also obtained. A sample group run schedule is shown in Table 16. For each test, 51 runs were performed, six of which were confirmation points not used to build the model.

\begin{tabular}{|l|l|}
\hline Run & $\boldsymbol{q}(\mathbf{P a})$ \\
\hline $\mathbf{1}$ & 980 \\
\hline $\mathbf{2}$ & 750 \\
\hline $\mathbf{3}$ & 600 \\
\hline $\mathbf{4}$ & 750 \\
\hline $\mathbf{5}$ & 444 \\
\hline $\mathbf{6}$ & 750 \\
\hline $\mathbf{7}$ & 980 \\
\hline $\mathbf{8}$ & 750 \\
\hline $\mathbf{9}$ & 444 \\
\hline $\mathbf{1 0}$ & 444 \\
\hline $\mathbf{1 1}$ & 750 \\
\hline $\mathbf{1 2}$ & 980 \\
\hline $\mathbf{1 3}$ & 444 \\
\hline $\mathbf{1 4}$ & 865 \\
\hline $\mathbf{1 5}$ & 980 \\
\hline $\mathbf{1 6}$ & 980 \\
\hline $\mathbf{1 7}$ & 444 \\
\hline
\end{tabular}

Table 16 Example Group Run Schedule

Although the levels of dynamic pressure were fully randomized within the design, the analysis of the design still utilized the split-plot methodology where the dynamic pressure was treated as a hard-tochange factor for the purpose of demonstrating the general applicability of the method in accommodating hard to change factors. There was no difference in the regression analysis completed using the split-plot and ordinary least squares.

The statistical power and prediction variance for the model are shown in Table 17 and Table 18. Added to the evaluation is a case for comparison in which the dynamic pressure was treated as an easy-tochange factor, a completely randomized design (CRD). This demonstrates the consequences of loss of statistical power and inference ability associated with a split-plot design. It should be noted that the statistical evaluation for the temperature term was completed by using the average of all test run data. 
The statistical power of the executed design is presented in Table 17 at a $5 \%$ level of significance to detect signal-to-noise ratios of two standard deviations. The fact that temperature was an uncontrolled factor leads to different test matrices and subsequently differing statistical evaluations. Note that 750Pa is not the center of the design space which lowers some of the statistical design parameters (nonorthogonality). If the center of the design $(712 \mathrm{~Pa})$ was used instead of the $750 \mathrm{~Pa}$ factor level, a minimal increase in power and prediction variance would be observed.

\begin{tabular}{|l|l|l|}
\hline \multicolumn{3}{|c|}{ Power to Estimate Terms } \\
\hline & SPD & CRD \\
\hline q & $48.84 \%$ & $99.96 \%$ \\
\hline T & $23.85 \%$ & $59.76 \%$ \\
\hline
\end{tabular}

Table 17 Test Design Statistical Power Evaluation

\begin{tabular}{|l|l|}
\hline \multicolumn{2}{|c|}{ Average Prediction Variance } \\
\hline SPD & CRD \\
\hline 0.4317 & 0.1072 \\
\hline
\end{tabular}

Table 18 Test Design Prediction Variance

\subsection{Regression Model Charts}

This section will detail the development of regression models for the tunnel calibration coefficient through the use of design of experiments.

\subsubsection{Methodology and Applications}

The proposed methodology of integrating design of experiments with statistical process control centers around building a regression model for the tunnel calibration coefficient. This regression model gives a continuous response for the entire design space, which in this case is the entire operating envelope of the wind tunnel. This is in contrast to the current SPC approach where the estimates for the tunnel calibration coefficient are only available at discrete points. It is proposed that a local regression model be built for each tunnel condition test (15 total) as well as one global regression model that encompasses all data obtained from the baseline. As testing progresses and the local regression models are built over time, it is proposed that the coefficients of the regression models be tracked in SPC charts 
in a similar manner to the across-test control charts. By inspecting the regression coefficient control charts, a higher level of understanding of the overall variation in the tunnel calibration coefficient can be gained. This leads to another benefit of the proposed methodology, the diagnostic ability of the regression model charts. In the standard SPC charting methodology, the variation in the tunnel calibration coefficient is easily attainable, however, the sources of that variation are not. In a standard SPC chart a test data point may indicate that the process is out of statistical control; however, there is little more information available and no assignable cause as to why that point was out of control. It is believed that through the regression model charts, an assignable cause for the control status is more easily attainable than current practice allows.

After initial investigations, it was discovered that the wind tunnel calibration coefficient was a function of both tunnel dynamic pressure and temperature. Further investigations showed that this temperature dependence appeared to be linear in nature (it was observed that the interaction term $q * T$ was not significant) thus it was decided to model the wind tunnel calibration coefficient as shown below in Equation 52. If the temperature range studied was expanded significantly, it is possible that other trends in the calibration coefficient with respect to temperature could be observed.

$$
\begin{gathered}
C^{\prime}=f(q, T) \\
C^{\prime}=\beta_{0}+\beta_{1} q+\beta_{2} T
\end{gathered}
$$

A sample output from REML via the $\mathrm{JMP}^{49}$ software package from test 34 is shown below in Figure 65 . 


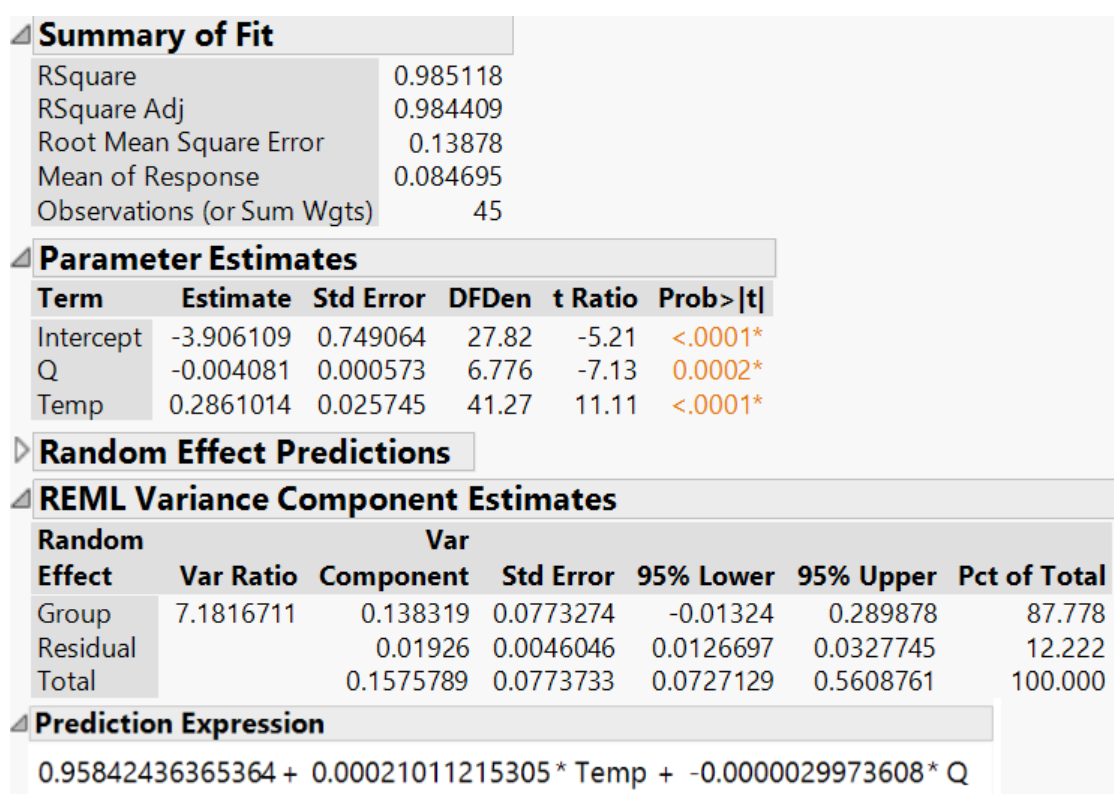

Figure 65 JMP REML Output for Tunnel Conditions Test 34

From inspection of Figure 65, the model for test 34 was fit well with an $R^{2}$ of 0.985 and an adjusted $R^{2}$ of 0.984. Both the dynamic pressure and temperature terms are highly significant as seen under the "Prob $>|t|$ " output line. Note that the figures derived under that tab "Parameter Estimates" were done so using the standardized response of $C^{\prime}$. This allowed JMP to properly calculate the numbers of degrees of freedom for significance testing. The overall prediction expression in terms of engineering units is shown at the bottom of Figure 65. While all tests differed to some degree, the overall analysis of each test was similar to that shown for test 34.

\subsubsection{Development of a Global Regression Model}

After the baseline of 15 tests was completed the entire data set was utilized to build a global regression model. This model was built using the same split-plot and REML analysis as was done in the development of the local regression model. However, in addition, a nested design was also implemented for a global regression model as was outlined in Figure 64. The global model is shown in Equation 54 in terms of engineering units $\left(\mathrm{Pa},{ }^{\circ} \mathrm{C}\right)$.

$$
C^{\prime}=0.9582-4.081 \times 10^{-6} * q+2.329 \times 10^{-4} * T
$$


The output from REML via the JMP software package for the global model is shown in Figure 66. Again note that the figures derived under the tab "Parameter Estimates" were generated using the standardized response of $C^{\prime}$. This allowed JMP to properly calculate the numbers of degrees of freedom for significance testing.

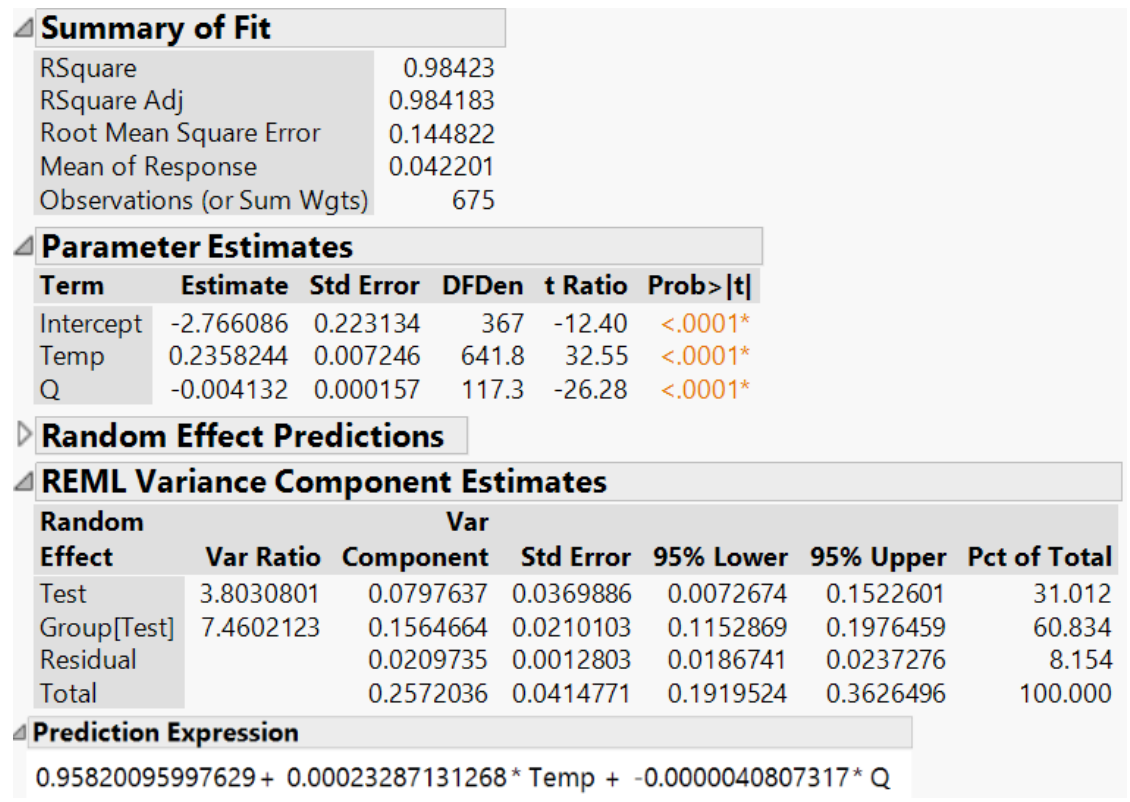

Figure 66 JMP REML Output for Tunnel Conditions Global Regression Model

To evaluate the established global regression model, residual diagnostics are investigated. In Figure 67 through Figure 70, the distribution of residuals should not trend with any factors, predicted values or test number. 


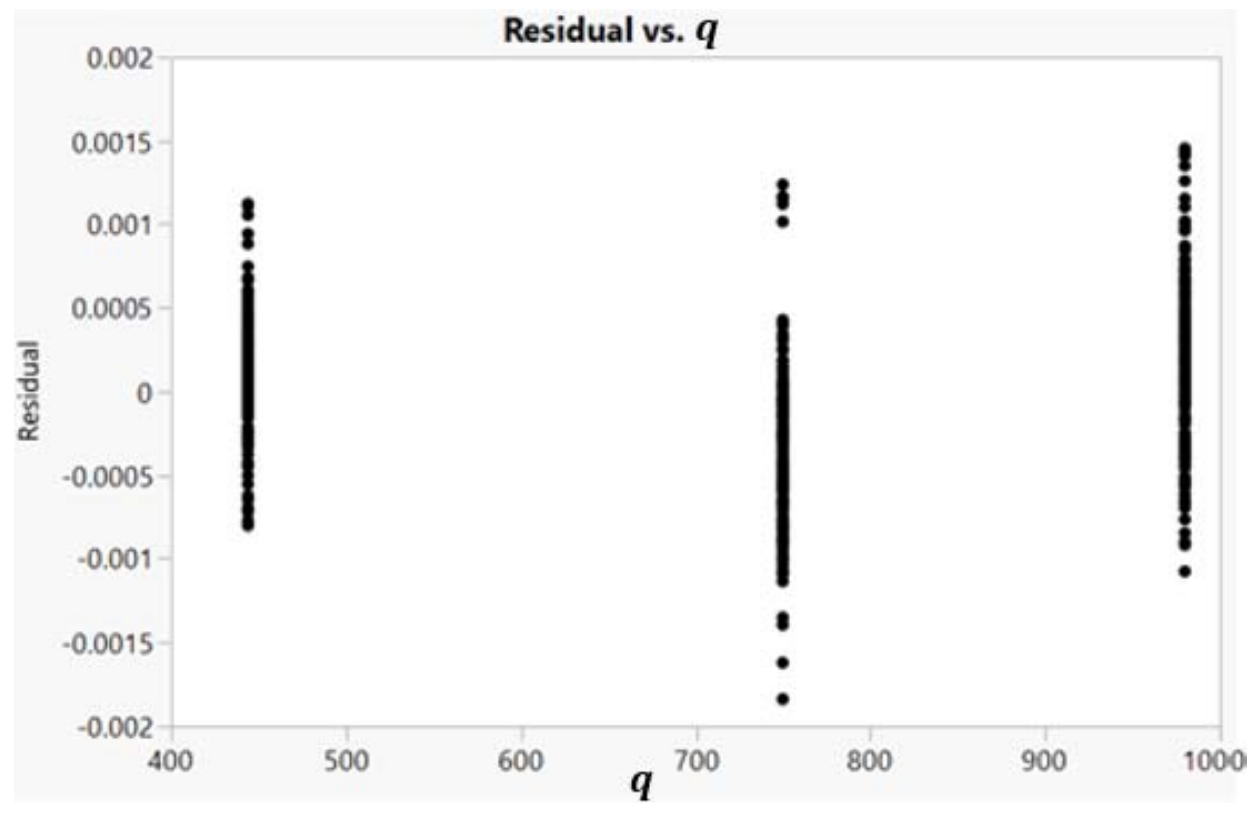

Figure 67 Tunnel Calibration Coefficient Global Regression Model Residuals vs Dynamic Pressure

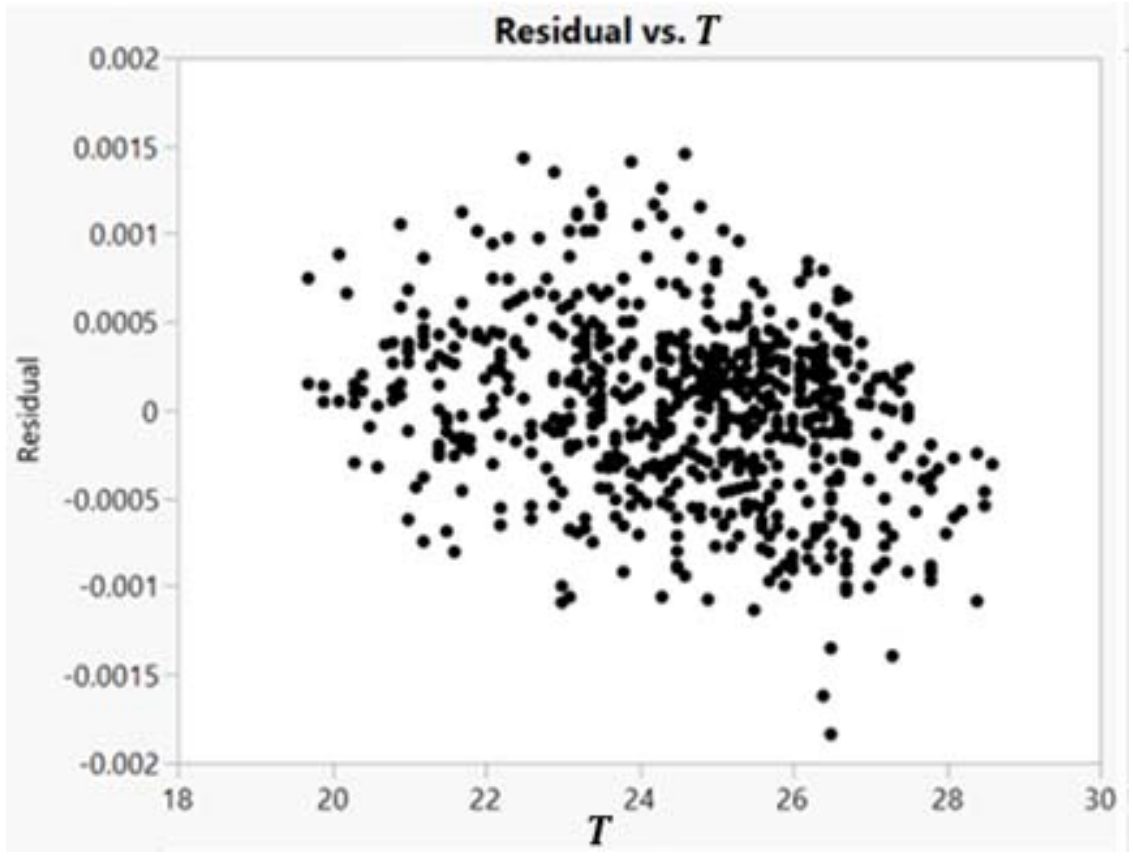

Figure 68 Tunnel Calibration Coefficient Global Regression Model Residuals vs Temperature 


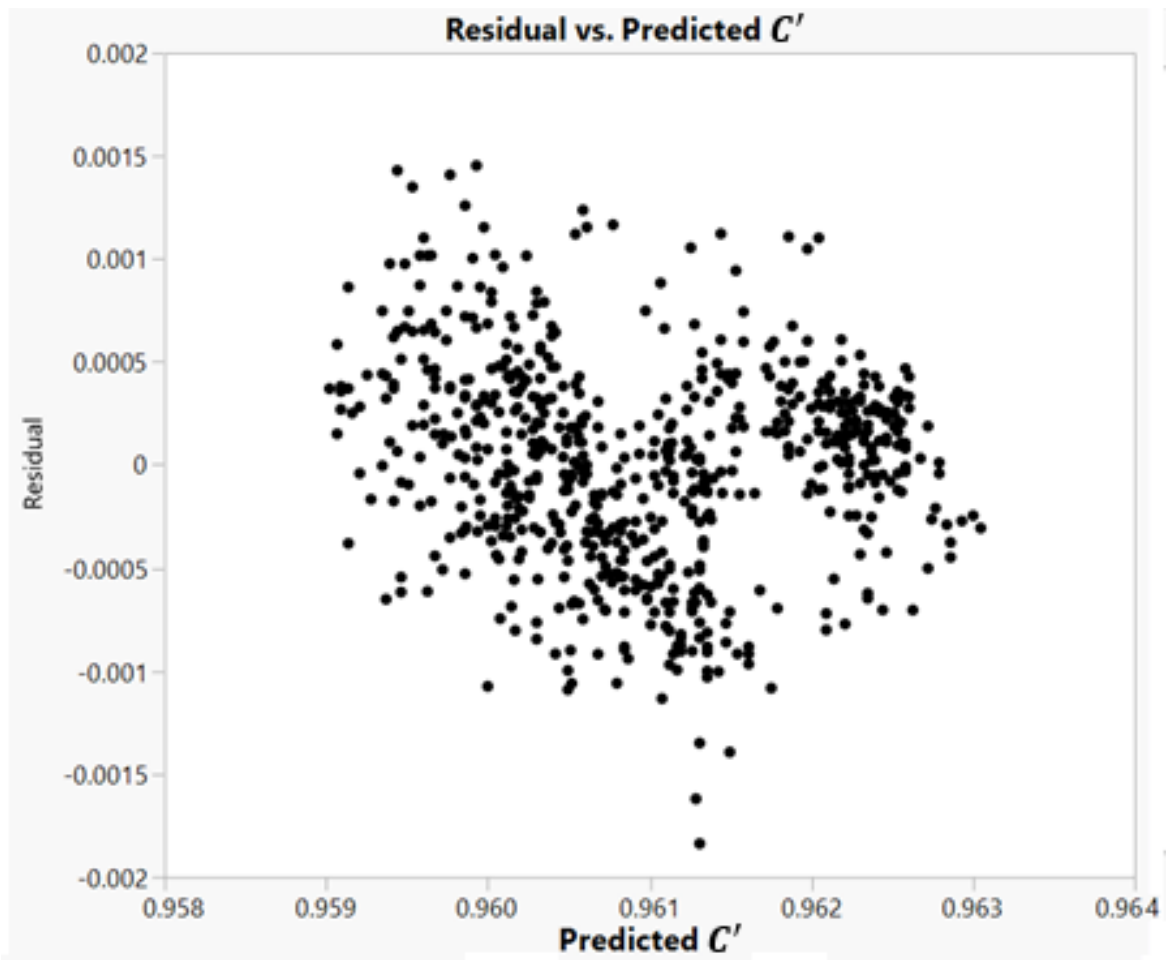

Figure 69 Tunnel Calibration Coefficient Global Regression Model Residuals vs Predicted 


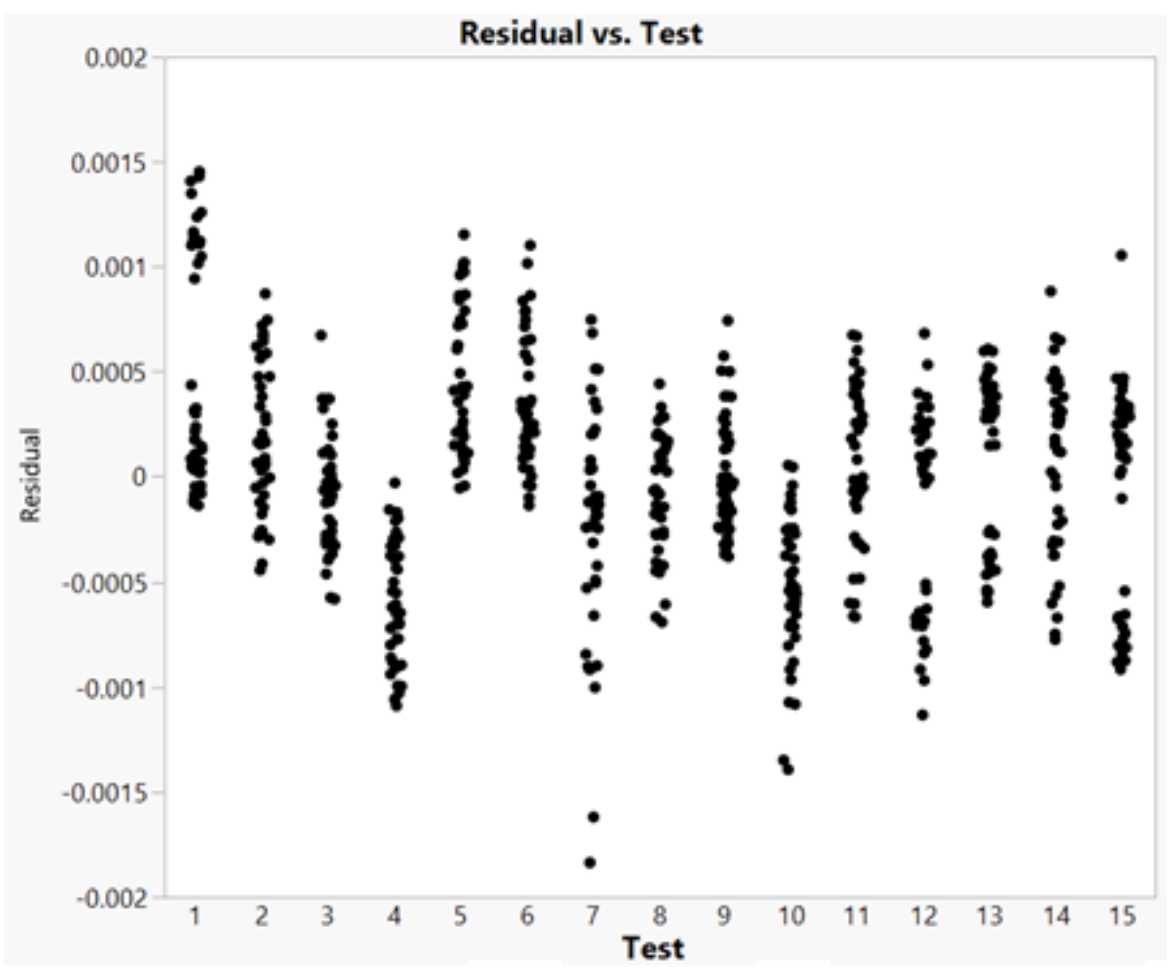

Figure 70 Tunnel Calibration Coefficient Global Regression Model Residuals vs Test

There are moderate trends in the residuals verses test number in Figure 70 , however, they are small in magnitude and did not correlate to any testing procedures or timeframes. From inspection of all the residual diagnostics, it is concluded that the global regression model established for the tunnel calibration coefficient is valid and can serve as the baseline regression model. This will allow for future check standard testing to be treated as confirmation points for the established model. Further general discussion of the application of a global regression model developed through the use of design of experiments methodology will be explored in Chapter Error! Reference source not found..

\subsubsection{Regression Model Chart Results}

The results presented below are the coefficient charts where each test entry represents the coefficient associated with the regression model (Equation 53) built for that test. The limits for these control charts are given by Equations 45 and 46 in Section 5.2.2.1. 


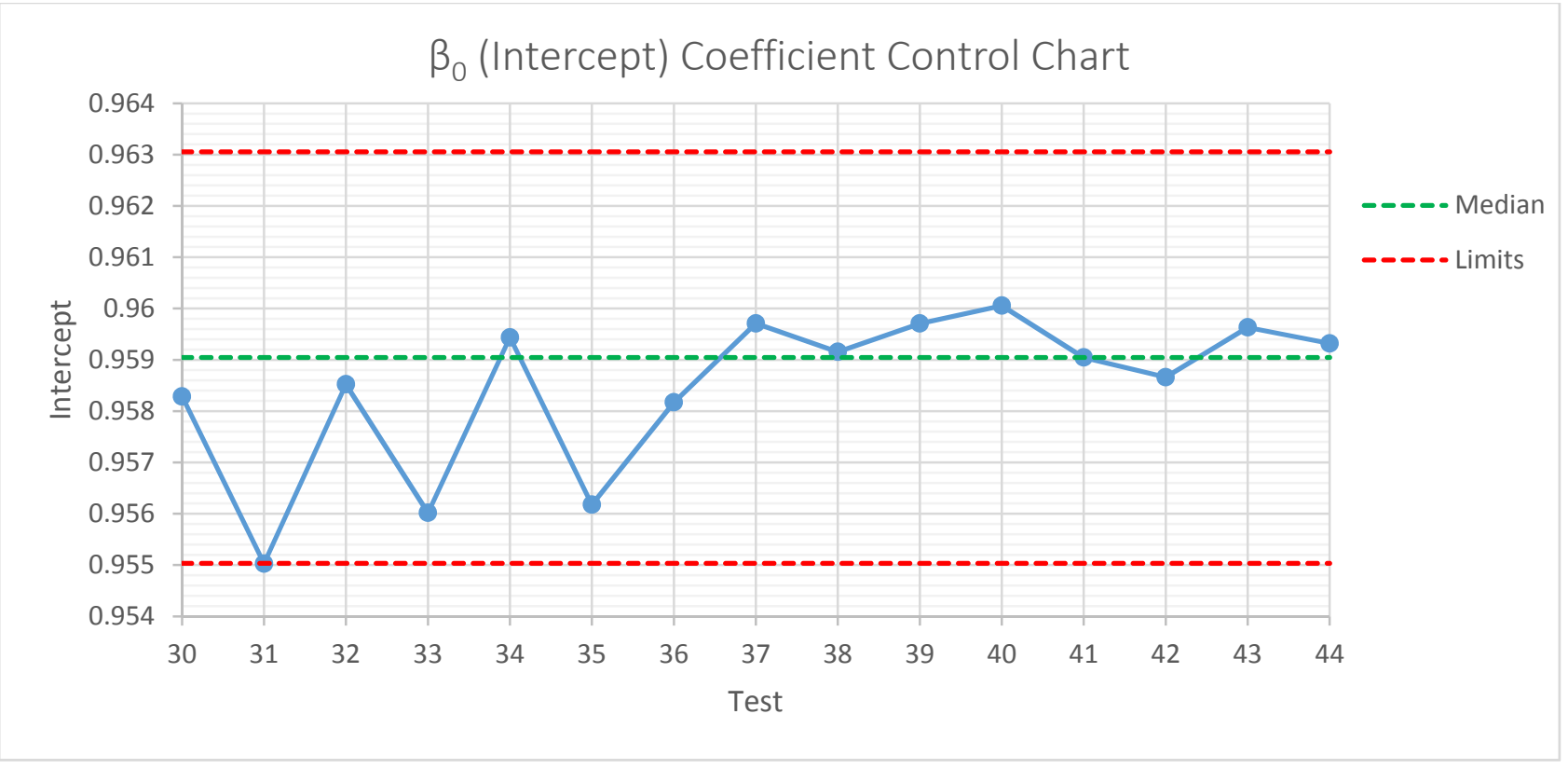

Figure $71 C^{\prime} \beta_{0}$ (Intercept) Coefficient Control Chart

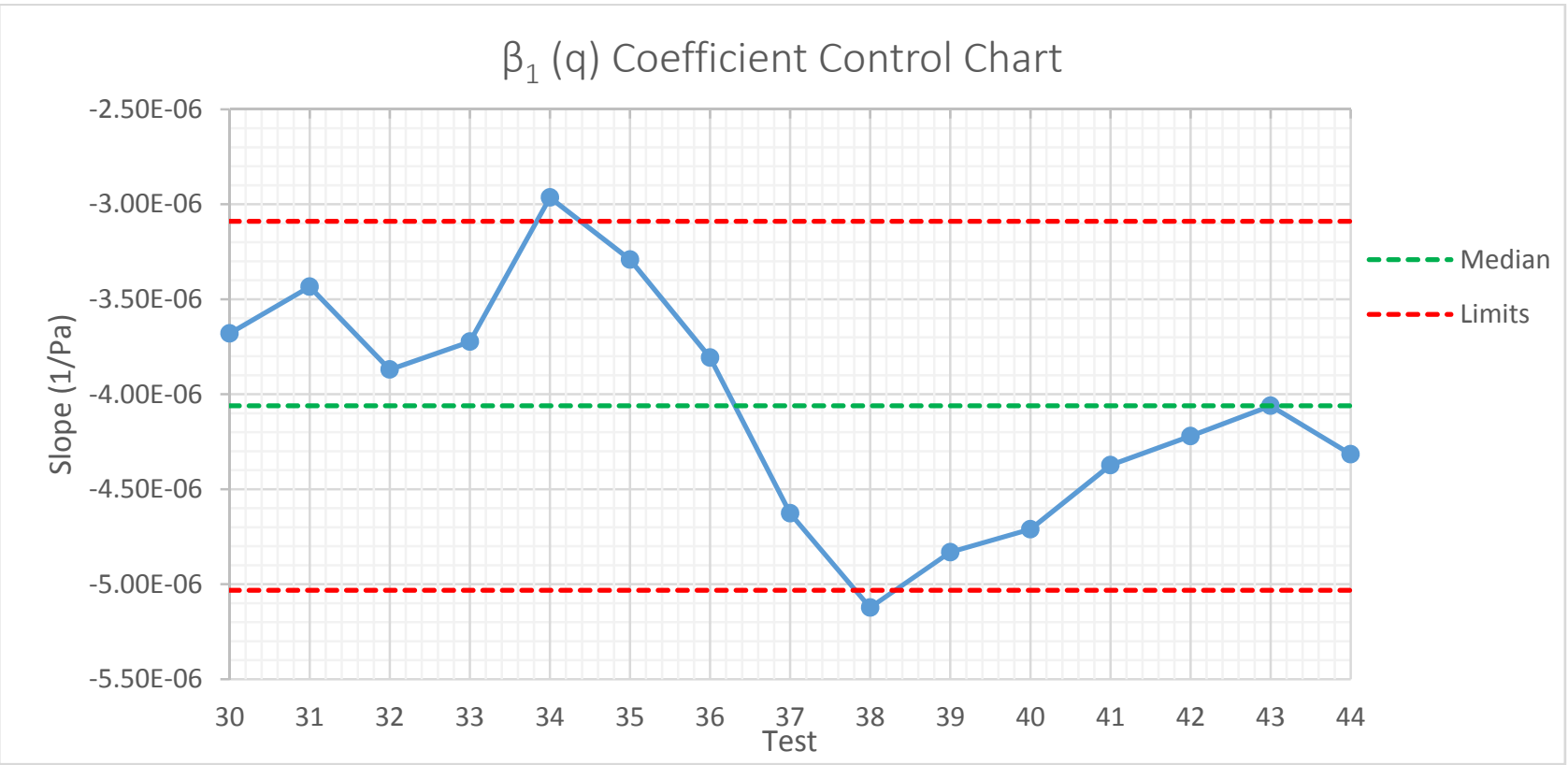

Figure $72 C^{\prime} \beta_{1}(q)$ Coefficient Control Chart 


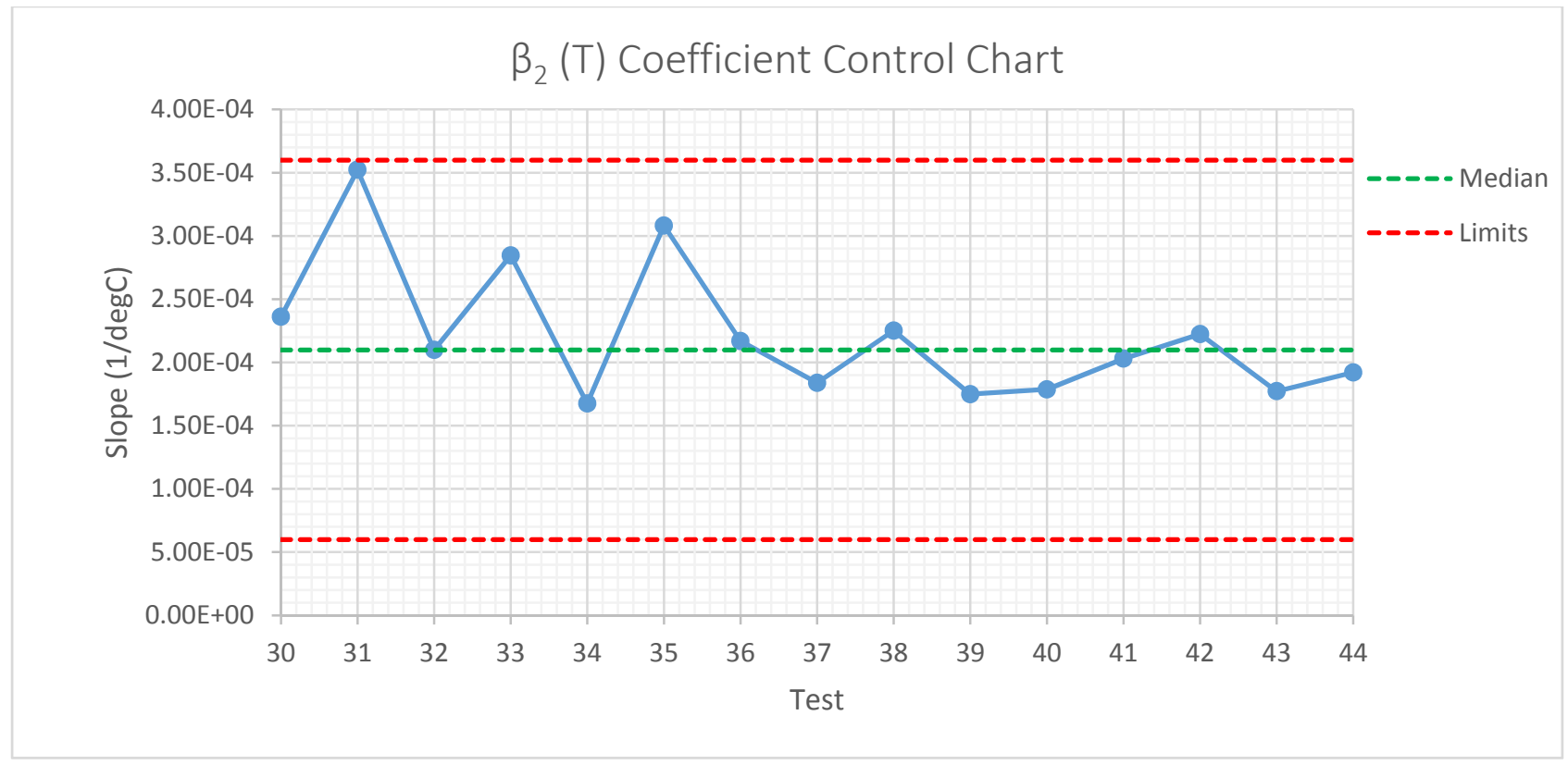

Figure $73 C^{\prime} \beta_{2}(T)$ Coefficient Control Chart

From inspection of the coefficient control charts above, it can be said that the regression intercept and temperature coefficients, $\beta_{0}$ and $\beta_{2}$ are within statistical control and their effects on the response are within a tolerance that could be considered as "natural process variation." However Figure 72 indicates that the variation in the dynamic pressure regression coefficient is out of statistical control due to the downward trend from test 34 to test 38 . This would indicate a problem associated with dynamic pressure. The ability to identify this potential problem and associate it with a specific factor represents a marked improvement in understanding, using the new DOE/SPC hybrid methodology versus the standard SPC approach. Although the standard SPC control charts may not indicate any problems as far as statistical control is concerned, the regression model charts can identify underlying undesirable variances in components which make up the overall tunnel calibration coefficient.

\subsubsection{Comparison of SPC and REML Standard Deviation Estimates}

For a balanced, nested design it can be shown that REML estimates of standard deviation will equal the estimates based on the analysis of variance approach shown below ${ }^{1}$ 


$$
\begin{gathered}
\hat{\sigma}_{W G}=\sqrt{M S_{W G}} \\
\hat{\sigma}_{W T}=\sqrt{\frac{M S_{W T}-M S_{W G}}{n}} \\
\hat{\sigma}_{A T}=\sqrt{\frac{M S_{A T}-M S_{W T}}{b n}}
\end{gathered}
$$

Where there are $a$ levels of treatment A (Tests), $b$ levels of treatment B (Groups) nested within A (Test) and $n$ replicates. For unbalanced designs, REML follows the same principle, but the degree of freedom allocation is altered. The comparison of the REML and SPC estimates of standard deviation are given in Table 19 through Table 21.

\begin{tabular}{|c|l|l|l|}
\hline $\boldsymbol{q}=\mathbf{4 4 4 P a}$ & REML Estimates & SPC Estimates & Percent Difference \\
\hline$\widehat{\boldsymbol{\sigma}}_{\boldsymbol{A T}}$ & 0.0001283 & 0.0001036 & $19.2 \%$ \\
\hline$\widehat{\boldsymbol{\sigma}}_{\boldsymbol{W T}}$ & 0.0003785 & 0.0004867 & $28.6 \%$ \\
\hline$\widehat{\boldsymbol{\sigma}}_{\boldsymbol{W G}}$ & 0.0002097 & 0.0001783 & $15.0 \%$ \\
\hline$\widehat{\boldsymbol{\sigma}}_{\boldsymbol{T}}$ & 0.0004513 & 0.0005286 & $17.1 \%$ \\
\hline
\end{tabular}

Table 19 Standard Deviation Estimates Comparison $q=444 \mathrm{~Pa}$

\begin{tabular}{|c|l|l|l|}
\hline $\boldsymbol{q}=\mathbf{7 5 0 P a}$ & REML Estimates & SPC Estimates & Percent Difference \\
\hline$\widehat{\boldsymbol{\sigma}}_{\boldsymbol{A T}}$ & 0.0002557 & 0.0002126 & $16.9 \%$ \\
\hline$\widehat{\boldsymbol{\sigma}}_{\boldsymbol{W T}}$ & 0.0003302 & 0.0003744 & $13.4 \%$ \\
\hline$\widehat{\boldsymbol{\sigma}}_{\boldsymbol{W G}}$ & 0.0002586 & 0.0002623 & $1.4 \%$ \\
\hline$\widehat{\boldsymbol{\sigma}}_{\boldsymbol{T}}$ & 0.0004913 & 0.0005041 & $2.6 \%$ \\
\hline
\end{tabular}

Table 20 Standard Deviation Estimates Comparison $q=750 \mathrm{~Pa}$ 


\begin{tabular}{|c|l|l|l|}
\hline $\boldsymbol{q}=\mathbf{9 8 0} \boldsymbol{P a}$ & REML Estimates & SPC Estimates & Percent Difference \\
\hline$\widehat{\boldsymbol{\sigma}}_{\boldsymbol{A T}}$ & 0.0002972 & 0.0001238 & $58.4 \%$ \\
\hline$\widehat{\boldsymbol{\sigma}}_{\boldsymbol{W T}}$ & 0.0003757 & 0.0004422 & $17.7 \%$ \\
\hline$\widehat{\boldsymbol{\sigma}}_{\boldsymbol{W G}}$ & 0.0002546 & 0.0002273 & $10.7 \%$ \\
\hline$\widehat{\boldsymbol{\sigma}}_{\boldsymbol{T}}$ & 0.0005425 & 0.0005124 & $5.5 \%$ \\
\hline
\end{tabular}

Table 21 Standard Deviation Estimates Comparison $q=980 \mathrm{~Pa}$

The expected dependency of the standard deviation of the tunnel calibration coefficient on the timeframe in which it was obtained was not experienced. For example it would be expected that the variance levels at the across-test level would be higher than those at the within-test level due to the timeframes associated with each level. The deviation from this expectation is due primarily to the effect of temperature on the calibration coefficient and that the ODU LSWT is an atmospheric tunnel with no temperature control. Within each test, temperature variations (increasing temperatures) drove the overall variation as shown in Figure 74 where the temperature is given in degrees Celsius.

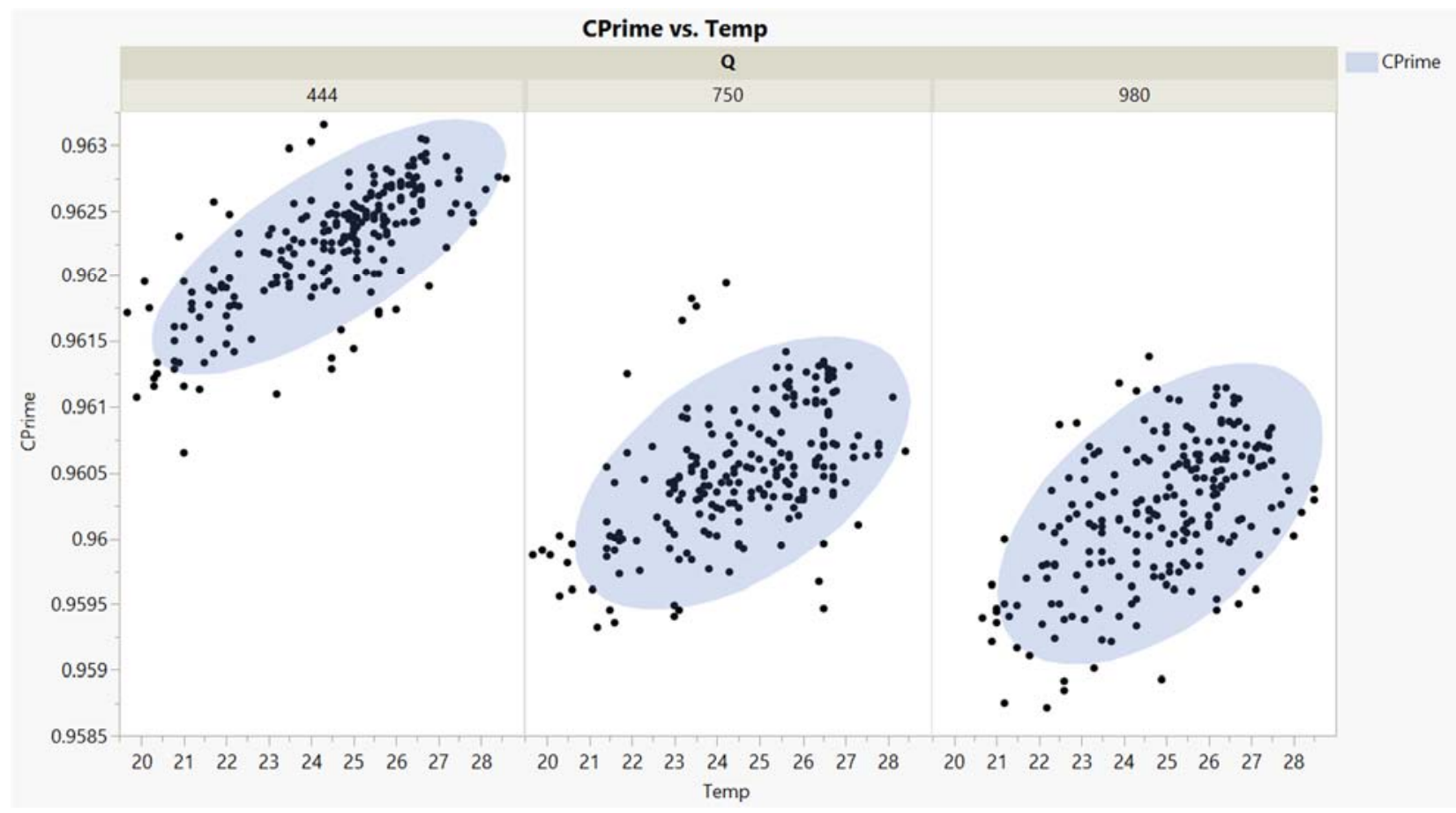

Figure 74 Tunnel Calibration Coefficient Scatter Plot 
While the difference between the SPC and REML estimates differed at each level of variation, (acrosstest, within-test and within-group) the overall standard deviation estimates were in much better agreement. 


\section{AIRFRAME TESTING}

This chapter will detail the research conducted for the airframe testing portion of the check standard testing. Standard statistical process control methodology, its applications and its enhancements by Design of Experiments methodology will be explored.

\subsection{Introduction}

The airframe portion of the check standard testing consisted of 20 tests and took place over 20 consecutive days during July and August 2015. As was the case for the tunnel conditions testing, establishing a baseline for the SPC charting required 15 to 20 tests in which the data were in statistical control or reasonably close to statistical control.

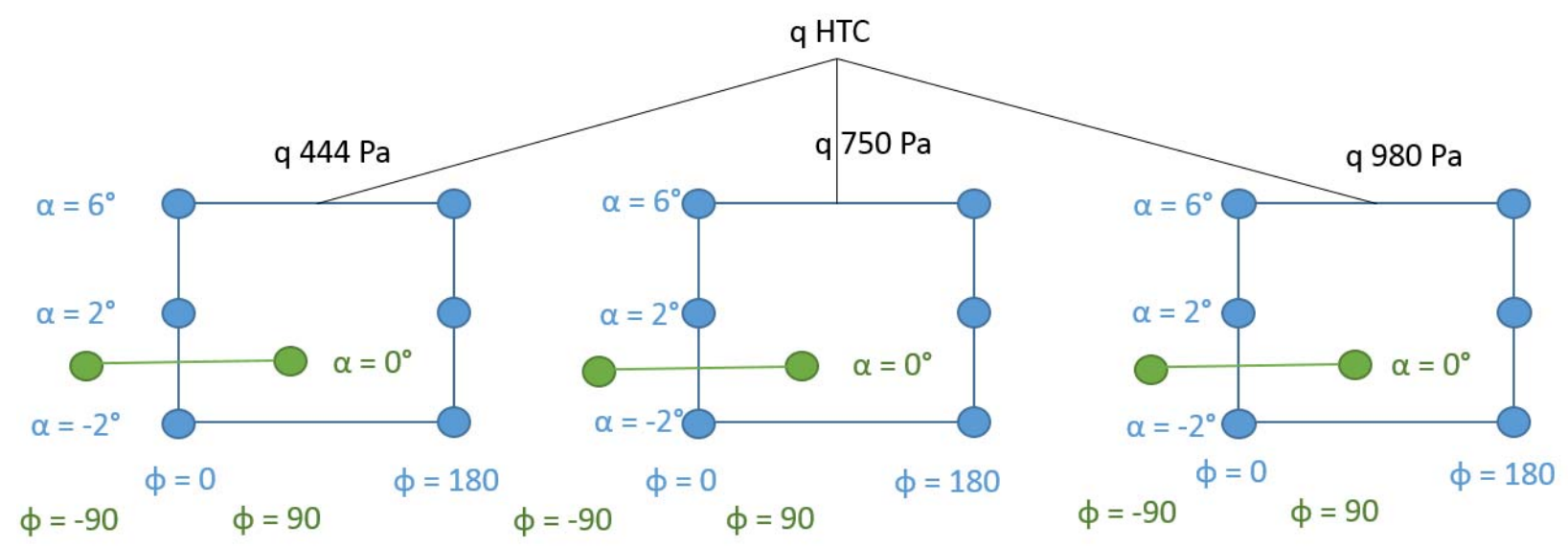

Figure 75 Airframe Test Design

One of the project objectives related to the airframe testing portion of this research was to recreate the standard SPC charts while combining the two methodologies of DOE and SPC. Therefore the test design was centered in angle of attack around $2^{\circ}$ due to the fact that the majority of available check standard data for the aerodynamic force coefficients was at this value and the prediction variance would be lowest in this design at the (pseudo) center point. The airframe test design is shown in Figure 75 Airframe Test Design. The roll angle, $\phi$ was treated as a categorical factor with two levels, upright $(\phi=$ 0 ) and inverted $\left(\phi=180^{\circ}\right)$. Roll angles of $-90^{\circ}$ and $90^{\circ}$ (green design points) were also obtained, but 
were not included in the model build. Instead, they were used to obtain the cross flow angularity that will be discussed in Section 6.4.2.

Again, as was the case with the tunnel conditions testing, the tunnel dynamic pressure, $q$ was treated as a hard-to-change factor (randomization restriction). The levels of $q$ remained the same as the tunnel conditions testing to maintain continuity throughout the entire check standard testing.

Each test consisted of 6 whole plots with 3 of the whole plots serving as replicates (three levels of dynamic pressure, each repeated). The run order of the whole plots was fully randomized. At the subplot level each design point was replicated once. An example test run schedule with added confirmation points (dynamic pressures of 600Pa and $865 \mathrm{~Pa}$ ) but without the cross flow angularity points is shown in Table 22 (See Table 39 for the cross flow angularity runs added). 


\begin{tabular}{|c|c|c|c|c|c|c|c|}
\hline Run & $q$ & $\alpha$ & $\phi$ & Run & $q$ & $\alpha$ & $\phi$ \\
\hline 1 & 444 & -2 & Inverted & 41 & 444 & 6 & Upright \\
\hline 2 & 444 & -2 & Upright & 42 & 444 & -2 & Inverted \\
\hline 3 & 444 & 2 & Upright & 43 & 444 & -2 & Upright \\
\hline 4 & 444 & 2 & Upright & 44 & 444 & 6 & Inverted \\
\hline 5 & 444 & 6 & Upright & 45 & 444 & 2 & Upright \\
\hline 6 & 444 & -2 & Inverted & 46 & 444 & 2 & Inverted \\
\hline 7 & 444 & 6 & Inverted & 47 & 444 & -2 & Inverted \\
\hline 8 & 444 & 6 & Upright & 48 & 444 & 6 & Upright \\
\hline 9 & 444 & 6 & Inverted & 49 & 865 & -1 & Upright \\
\hline 10 & 444 & 2 & Inverted & 50 & 865 & -1 & Inverted \\
\hline 11 & 444 & 2 & Inverted & 51 & 865 & 1 & Inverted \\
\hline 12 & 444 & -2 & Upright & 52 & 865 & 1 & Upright \\
\hline 13 & 980 & 2 & Upright & 53 & 750 & 2 & Upright \\
\hline 14 & 980 & 2 & Inverted & 54 & 750 & -2 & Upright \\
\hline 15 & 980 & 6 & Inverted & 55 & 750 & -2 & Upright \\
\hline 16 & 980 & -2 & Upright & 56 & 750 & 2 & Inverted \\
\hline 17 & 980 & 6 & Upright & 57 & 750 & 6 & Upright \\
\hline 18 & 980 & -2 & Inverted & 58 & 750 & 2 & Inverted \\
\hline 19 & 980 & 6 & Inverted & 59 & 750 & 6 & Upright \\
\hline 20 & 980 & 2 & Upright & 60 & 750 & 2 & Upright \\
\hline 21 & 980 & 6 & Upright & 61 & 750 & 6 & Inverted \\
\hline 22 & 980 & 2 & Inverted & 62 & 750 & -2 & Inverted \\
\hline 23 & 980 & -2 & Upright & 63 & 750 & 6 & Inverted \\
\hline 24 & 980 & -2 & Inverted & 64 & 750 & -2 & Inverted \\
\hline 25 & 980 & 6 & Inverted & 65 & 600 & -1 & Inverted \\
\hline 26 & 980 & 6 & Inverted & 66 & 600 & 1 & Inverted \\
\hline 27 & 980 & -2 & Upright & 67 & 600 & -1 & Upright \\
\hline 28 & 980 & 2 & Upright & 68 & 600 & 1 & Upright \\
\hline 29 & 980 & 2 & Inverted & 69 & 750 & -2 & Inverted \\
\hline 30 & 980 & 2 & Upright & 70 & 750 & 2 & Upright \\
\hline 31 & 980 & -2 & Inverted & 71 & 750 & -2 & Inverted \\
\hline 32 & 980 & 6 & Upright & 72 & 750 & 2 & Inverted \\
\hline 33 & 980 & 6 & Upright & 73 & 750 & 6 & Upright \\
\hline 34 & 980 & 2 & Inverted & 74 & 750 & 2 & Upright \\
\hline 35 & 980 & -2 & Upright & 75 & 750 & 6 & Inverted \\
\hline 36 & 980 & -2 & Inverted & 76 & 750 & -2 & Upright \\
\hline 37 & 444 & 2 & Inverted & 77 & 750 & 6 & Upright \\
\hline 38 & 444 & 2 & Upright & 78 & 750 & 6 & Inverted \\
\hline 39 & 444 & 6 & Inverted & 79 & 750 & 2 & Inverted \\
\hline 40 & 444 & -2 & Upright & 80 & 750 & -2 & Upright \\
\hline
\end{tabular}

Table 22 Example Airframe Test Run Schedule 
The statistical power of the executed design is presented in Table 23 at a $5 \%$ level of significance to detect signal-to-noise ratios of two standard deviations. The variance inflation factors (VIF's) show nearly no correlation in the model terms. Degrees of freedom for error estimation are presented.

\begin{tabular}{|c|l|l|l|l|}
\hline Term & StdErr & Error DoF & VIF & Power \\
\hline $\boldsymbol{q}$ & 0.520416 & 5 & 1.006702 & $34.5 \%$ \\
\hline $\boldsymbol{\alpha}$ & 0.144578 & 58 & 1.003328 & $99.9 \%$ \\
\hline $\boldsymbol{q} \boldsymbol{\alpha}$ & 0.118047 & 58 & 1.003328 & $99.9 \%$ \\
\hline $\boldsymbol{q} \boldsymbol{\phi}$ & 0.176187 & 58 & 1.003328 & $99.9 \%$ \\
\hline $\boldsymbol{\alpha} \boldsymbol{\phi}$ & 0.1443856 & 58 & 1.003328 & $99.9 \%$ \\
\hline $\boldsymbol{q}^{\mathbf{2}}$ & 0.922959 & 5 & 1 & $99.9 \%$ \\
\hline $\boldsymbol{\alpha}^{\mathbf{2}}$ & 0.25 & 58 & 1.006702 & $14.4 \%$ \\
\hline & & & 1 & $97.6 \%$ \\
\hline
\end{tabular}

Table 23 Statistical Evaluation of Test Design

As the case in all restricted randomization designs, the statistical power for the HTC factors is heavily dependent on the number of whole plot replicates. The effect of increasing the whole plot replication on the statistical power is shown below.

\begin{tabular}{|r|l|l|l|}
\hline Term & 9 WP's & 12 WP's & 15 WP's \\
\hline $\boldsymbol{q}$ & $50.6 \%$ & $67.8 \%$ & $79.6 \%$ \\
\hline $\boldsymbol{q}^{\mathbf{2}}$ & $20.2 \%$ & $27.8 \%$ & $35.1 \%$ \\
\hline
\end{tabular}

Table 24 Power to Estimate Dynamic Pressure Terms - Whole Plot Replication Statistics

It is seen that increasing the whole plot replication increases the statistical power for the hard to change factor. However, even in cases with relatively high levels of replication the statistical power still remains low compared to the sub plot factors. Thus, the balance of resources available and desired level of statistical power is one of the main factors that needs to be considered when developing the split-plot test.

Multiple designs were explored before deciding to run the split-plot face centered design. Of those candidate designs, several optimal designs were the most seriously considered. Computer generated optimal designs are one of the most commonly employed methodologies to develop test matrices which satisfy specific requirements. The general premise of optimal designs are to locate the design points 
throughout the design space to satisfy a certain optimum criteria such as minimum prediction variance or higher precision estimates of model terms. Table 25 gives some common optimal designs and the design parameters which are optimized. ${ }^{10}$

\begin{tabular}{|c|c|c|}
\hline \multicolumn{2}{|c|}{ Optimal Designs } \\
\hline Name & Focus & Parameters \\
\hline D & Regression Coefficients & $\operatorname{Min}\left|\left(\boldsymbol{X}^{\prime} \boldsymbol{X}\right)^{-1}\right|$ \\
\hline A & Regression Coefficients & $\operatorname{Min} \operatorname{tr}\left(\boldsymbol{X}^{\prime} \boldsymbol{X}\right)^{-1}$ \\
\hline $\mathbf{V}$ & Prediction Variance & $\operatorname{Min}\left(\boldsymbol{x}_{\mathbf{0}}\left(\boldsymbol{X}^{\prime} \boldsymbol{X}\right)^{-1} \boldsymbol{X}_{\mathbf{0}}\right)$ \\
\hline $\mathbf{I}$ & Integrated Prediction Variance & $\operatorname{Min} \operatorname{tr} \int\left(\boldsymbol{X}^{\prime} \boldsymbol{V}^{-\mathbf{1}} \boldsymbol{X}\right)^{-1} f(\boldsymbol{x}) f^{\prime}(\boldsymbol{x}) d x$ \\
\hline
\end{tabular}

Table 25 Common Optimal Designs

It may be beneficial to utilize an optimal design such as the D-Optimal design when performing the check standard testing outlined in this research. The D-Optimal design would minimize the model dependent variation in the regression coefficients. This would be especially useful when tracking the regression coefficients in control charts. The use of a D-Optimal design was explored for this research however, the benefit of a decrease in model dependent variation in regression coefficients (an increase in Scaled D-Optimality) was negligible in comparison to the standard split-plot FCD design. The statistical evaluation of I-Optimal, D-Optimal and the base split-plot FCD are shown in Table 26. Each of the designs were allocated the same number of whole plots and total runs for comparison purposes.

\begin{tabular}{|l|c|c|c|c|c|c|}
\hline \multicolumn{1}{|c|}{ Design } & $\begin{array}{c}\text { Power } \\
\boldsymbol{q}^{\mathbf{2}}\end{array}$ & $\begin{array}{c}\text { Power } \\
\boldsymbol{q}^{\mathbf{2}}\end{array}$ & $\begin{array}{c}\text { Average Prediction } \\
\text { Variance }\end{array}$ & $\begin{array}{c}\text { Max } \\
\text { VIF }\end{array}$ & $\begin{array}{c}\text { Scaled D- } \\
\text { Optimality }\end{array}$ & $\begin{array}{c}\text { G- } \\
\text { Efficiency }\end{array}$ \\
\hline Split-Plot FCD & $34.5 \%$ & $14.4 \%$ & 1.224 & 1.007 & 4.317 & $18.9 \%$ \\
\hline I-Optimal SPD & $20.0 \%$ & $12.8 \%$ & 1.209 & 1.054 & 4.900 & $10.0 \%$ \\
\hline D-Optimal SPD & $31.3 \%$ & $9.7 \%$ & 1.324 & 1.034 & 4.157 & $10.9 \%$ \\
\hline
\end{tabular}

Table 26 Comparison of Statistical Evaluation of Candidate Designs

From investigation of the comparison of candidate designs, it is seen that the I-Optimal SPD design has the lowest average prediction variance and lowest G-Efficiency. G-Efficiency is a metric for the average prediction variance as a percentage of the maximum prediction variance. ${ }^{10}$ The FCD has the highest G- 
efficiency. All the designs have low correlation of regression terms as seen in the VIF's. The D-Optimal design has the lowest Scaled D-Optimality but relatively high average prediction variance. It should be noted that when any optimal computer generated design is formulated, it is not guaranteed that the design is the "best" based on the desired criteria. The algorithms that generate the designs only can guarantee that the design generated is the best of the candidate points evaluated and there is a random error associated with design generation. Therefore, it is necessary to run the computer generated design algorithms multiple times and then choose the best of the multiple designs created.

For the factors studied and anticipated model build in this research, it was sufficient to use the base split-plot FCD design. However, in a scenario which a more complex model build and or factors of interest are incorporated into a wind tunnel check standard test, there may be more benefit to using computer generated optimal designs. In addition, for split-plot designs, it could be beneficial from the analysis perspective to run a test that is an ordinary least squares (OLS) equivalent. This allows the regression analysis to be completed utilizing the same methodology for completely randomized designs. For more information on OLS equivalent designs, the reader is referred to references by Parker et al. ${ }^{50,51}$

\subsection{Standard SPC Charting}

This section will detail the standard statistical process control charting of the balance force coefficients from the airframe portion of the check standard testing. For the background and methodology of the SPC charting see section 5.2. The test design consists of 18 individual points with each point representing a specific tunnel condition and model attitude (See Figure 75). This allows the tracking and charting of each one of these conditions consistent with the current practice. The degree of freedom allocation for the SPC charts is detailed in Table 27 and the SPC factor values are shown in Table 28.

\begin{tabular}{|l|l|}
\hline Degree of Freedom Allocation \\
\hline Chart & $\mathrm{n}$ \\
\hline Test Control & 2 \\
\hline Test Moving Range Control & 2 \\
\hline Within-group Range Control & 2 \\
\hline Within-test Range Control & 4 \\
\hline
\end{tabular}

Table 27 Degree of Freedom Allocation for SPC Charts 


\begin{tabular}{|l|c|l|}
\hline SPC Factor Values & Coefficient & Value \\
\hline Chart & $d_{4}$ & 0.954 \\
\hline Test Control & $D_{6}$ & 3.865 \\
\hline Test Moving Range Control & $D_{6}$ & 3.865 \\
\hline Within-group Range Control & $D_{6}$ & 2.375 \\
\hline Within-test Range Control & & \\
\hline
\end{tabular}

Table 28 SPC Factor Values

Only the standard SPC charts at $\alpha=2^{\circ}$ are presented here as these charts are the most directly relatable to the check standard airframe data available. The across-test control charts will be presented first.

\subsubsection{Across-test Control Charts}

The across-test range control charts will be presented in this section. The limits on these charts are given by Equations 58 and 59 below which read the upper or lower control limit is equal to the median of the test averages plus or minus three times the median of the moving range of the test averages divided by the statistical process control factor, $d_{4}$. The value of $d_{4}$ is given in Table 28 above.

$$
\begin{aligned}
& U C L=\tilde{\bar{X}}+\frac{3 m \tilde{R}}{d_{4}} \\
& L C L=\tilde{\bar{X}}-\frac{3 m \tilde{R}}{d_{4}}
\end{aligned}
$$




\subsubsection{Normal Force Coefficient Standard SPC Control Charts}

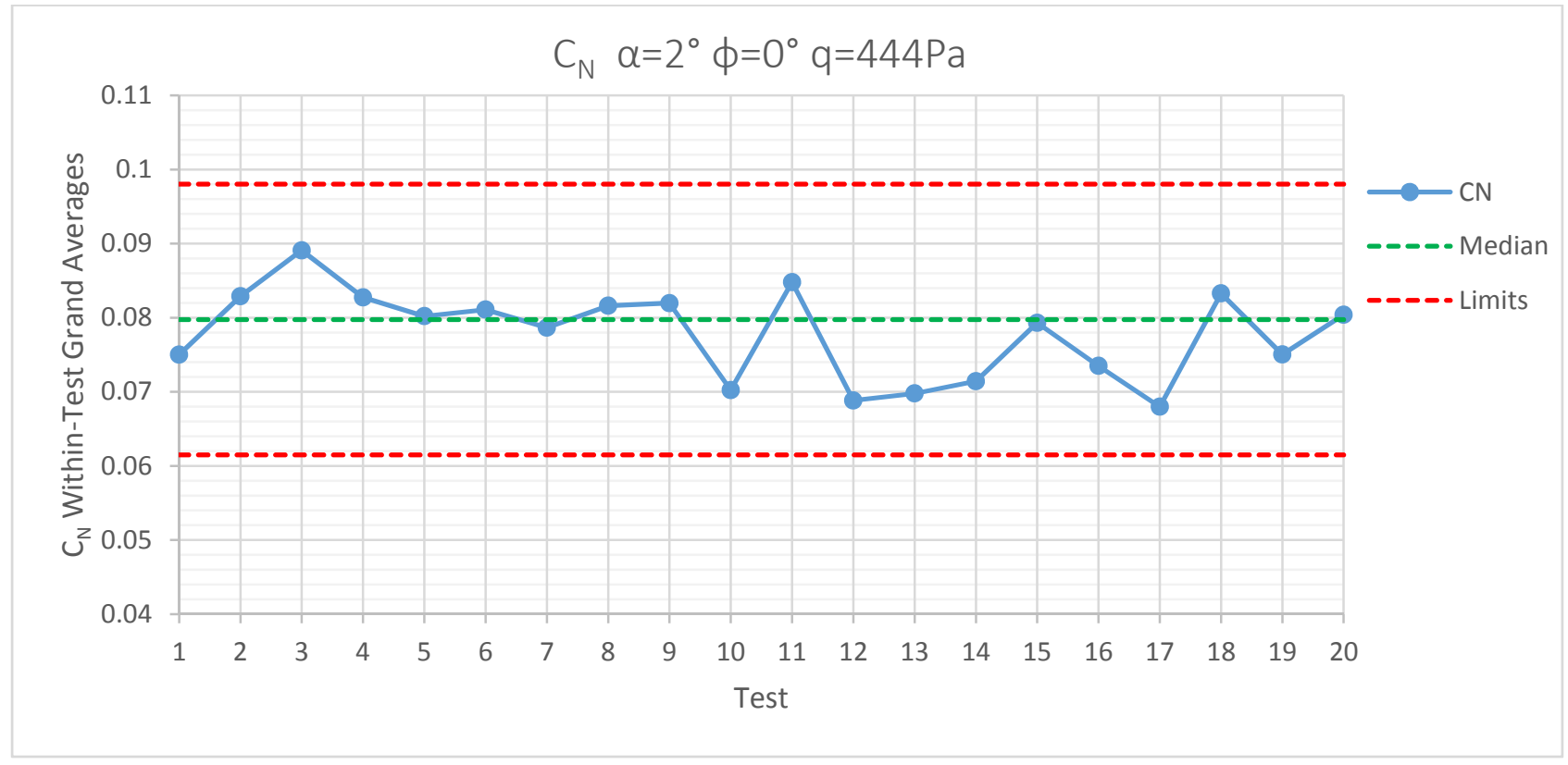

Figure 76 Across-test Control Chart $C_{N} \alpha=2^{\circ} \phi=0^{\circ} q=444 \mathrm{~Pa}$

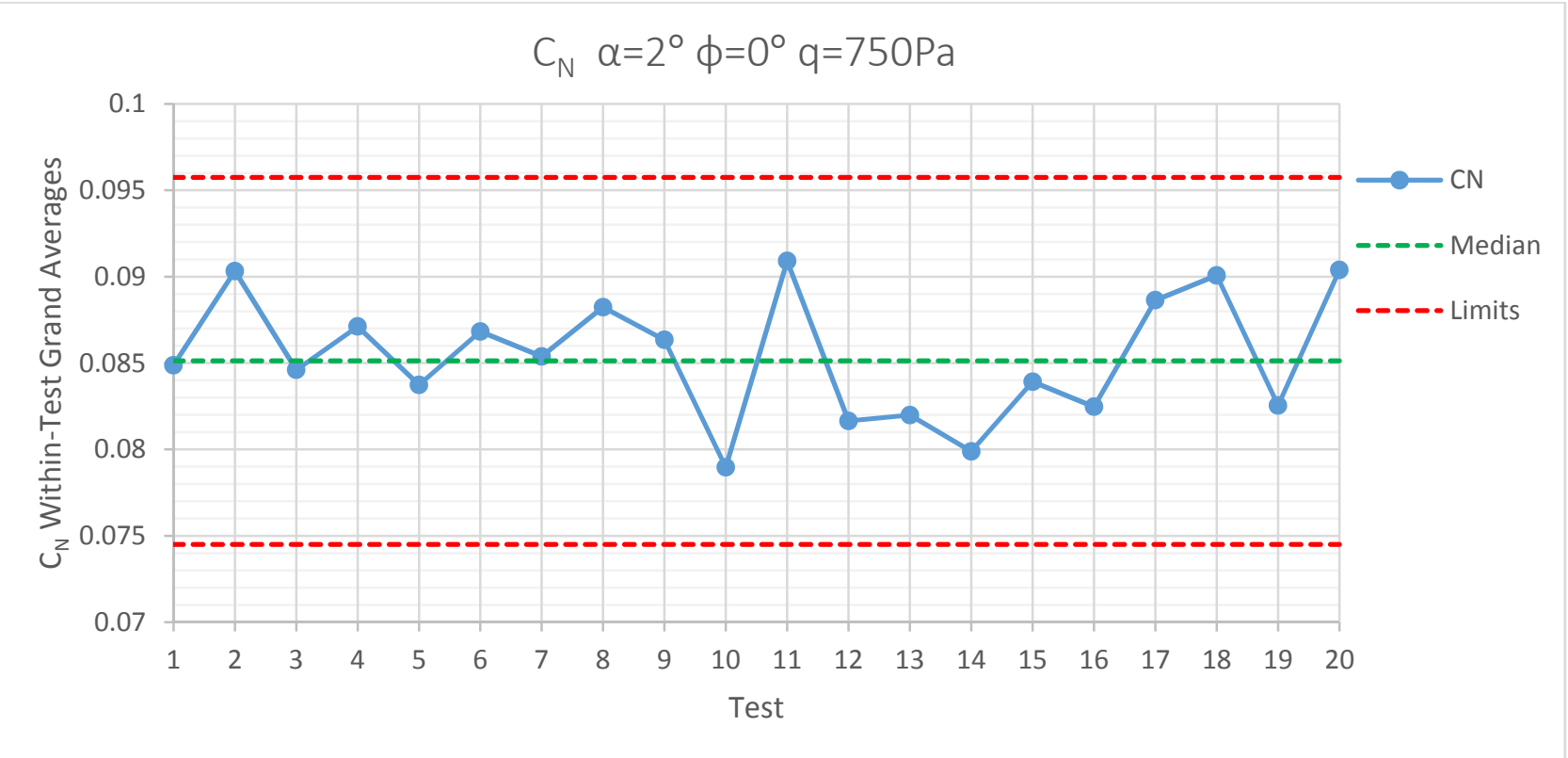

Figure 77 Across-test Control Chart $C_{N} \alpha=2^{\circ} \phi=0^{\circ} q=750 P a$ 


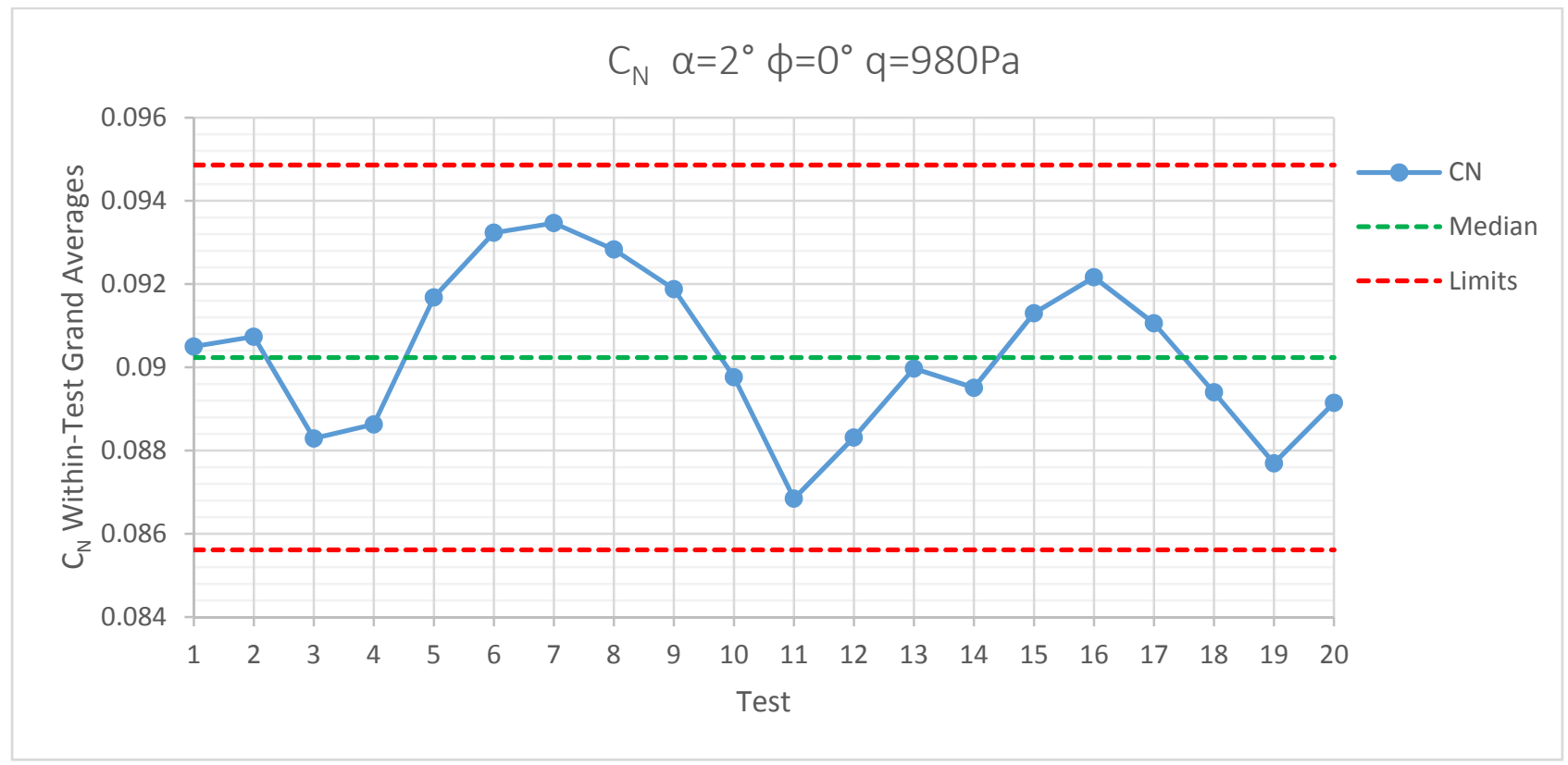

Figure 78 Across-test Control Chart $C_{N} \alpha=2^{\circ} \phi=0^{\circ} q=980 P a$

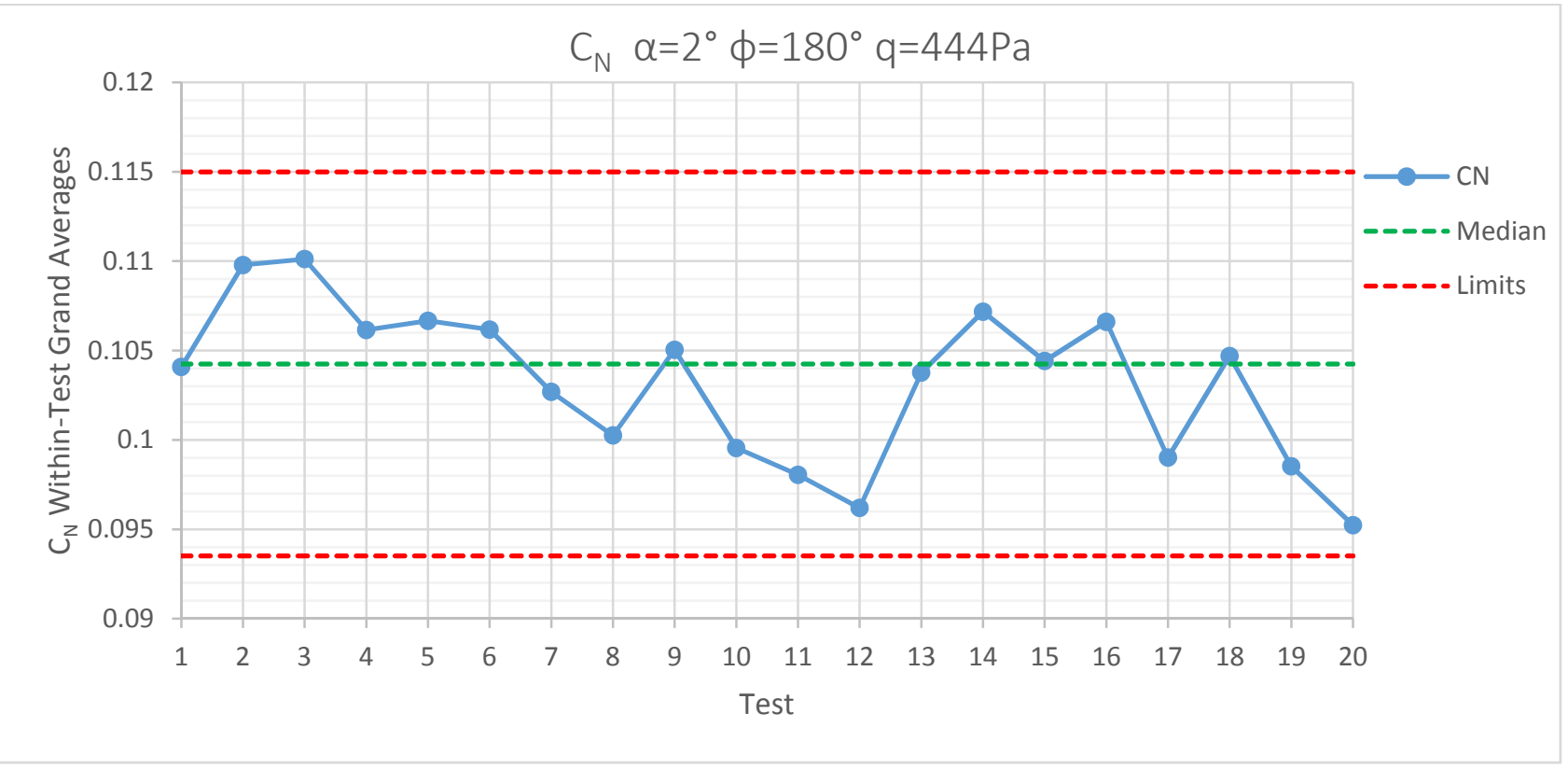

Figure 79 Across-test Control Chart $C_{N} \alpha=2^{\circ} \phi=180^{\circ} q=444 P a$ 


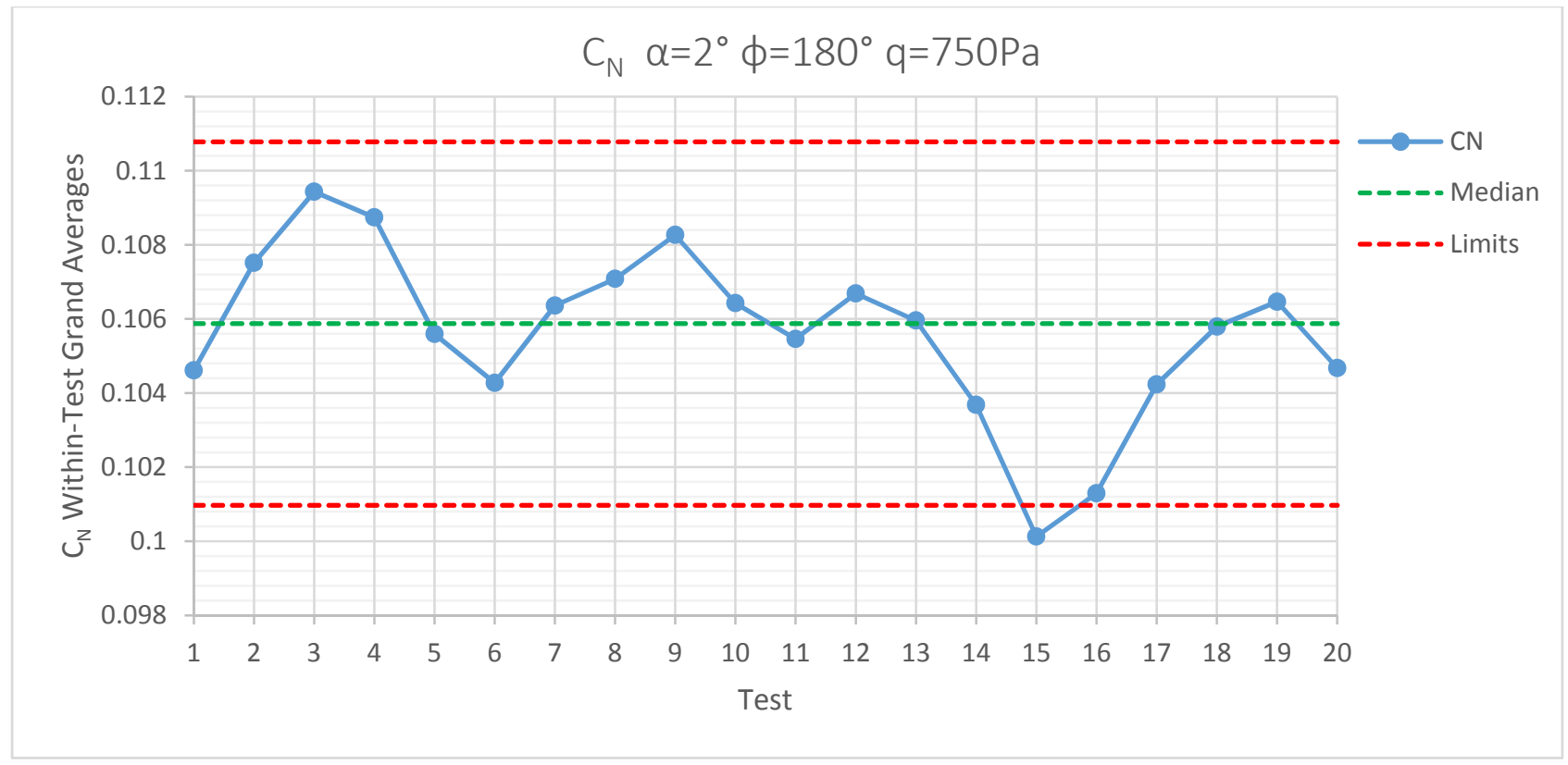

Figure 80 Across-test Control Chart $C_{N} \alpha=2^{\circ} \phi=180^{\circ} q=750 \mathrm{~Pa}$

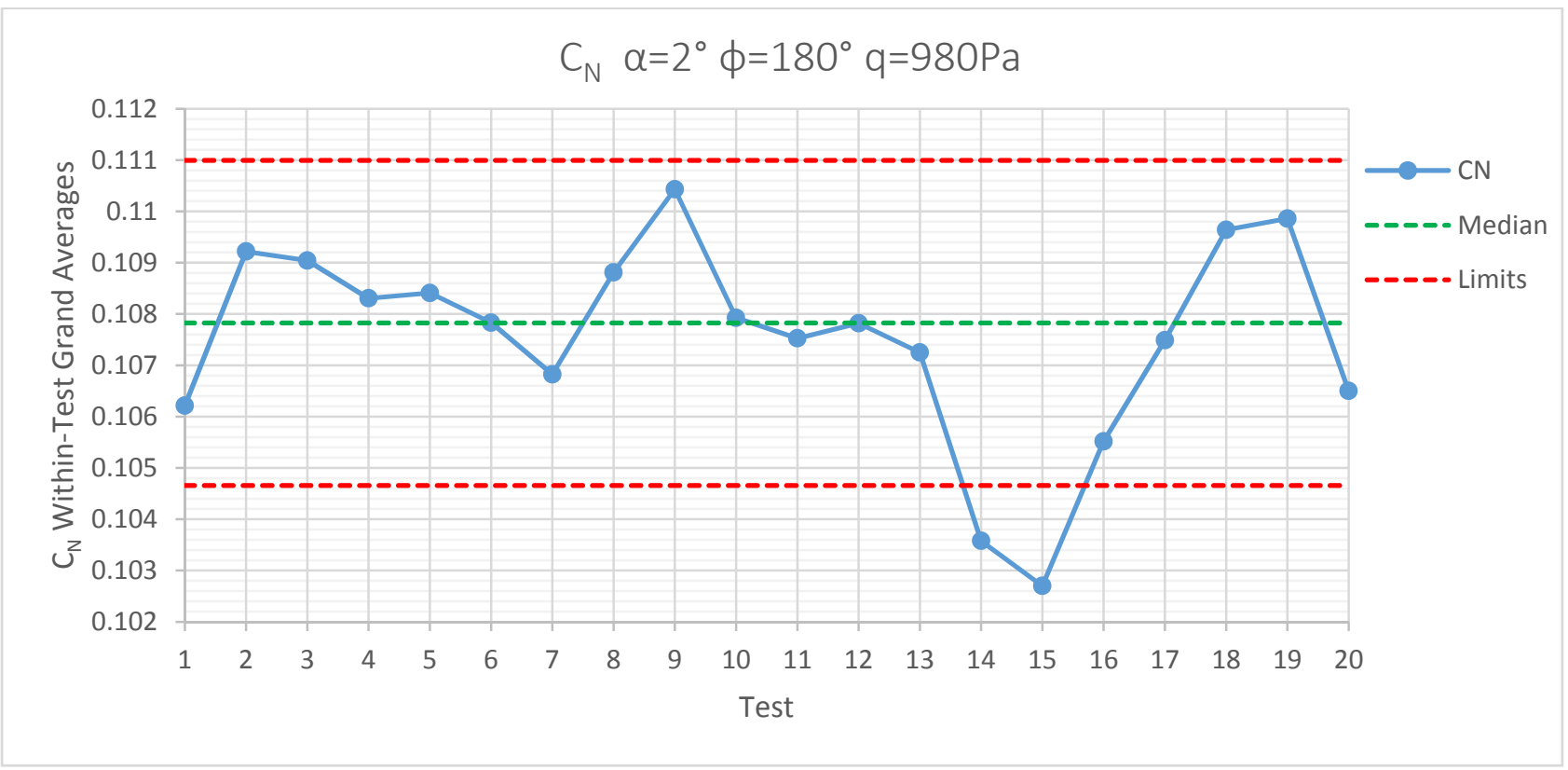

Figure 81 Across-test Control Chart $C_{N} \alpha=2^{\circ} \phi=180^{\circ} q=980 \mathrm{~Pa}$ 


\subsubsection{Axial Force Coefficient Standard SPC Control Charts}

The results for the axial force and pitching moment coefficients will be presented for the upright roll condition $\left(\phi=0^{\circ}\right)$ only.

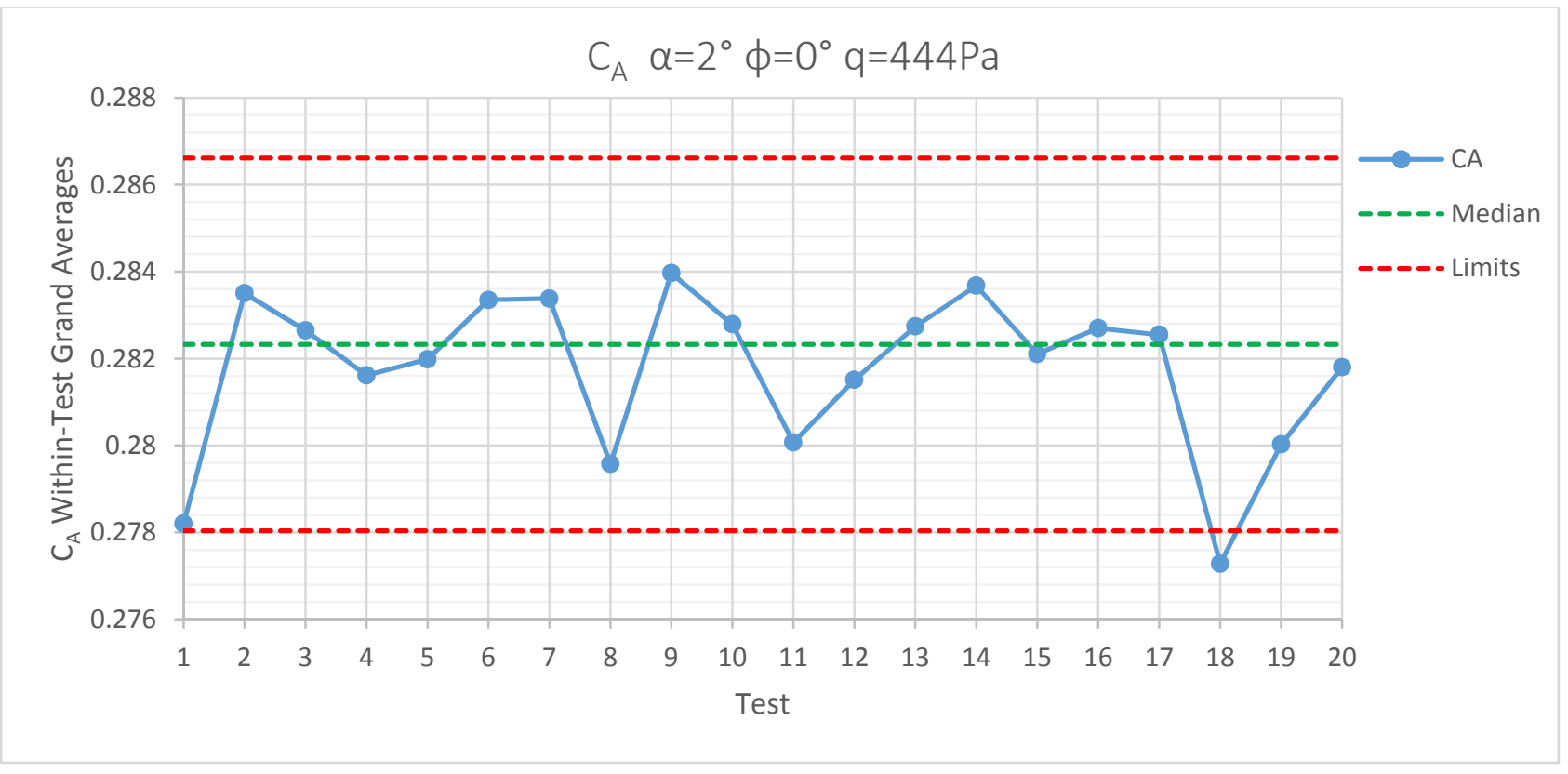

Figure 82 Across-test Control Chart $C_{A} \alpha=2^{\circ} \phi=0^{\circ} q=444 P a$ 


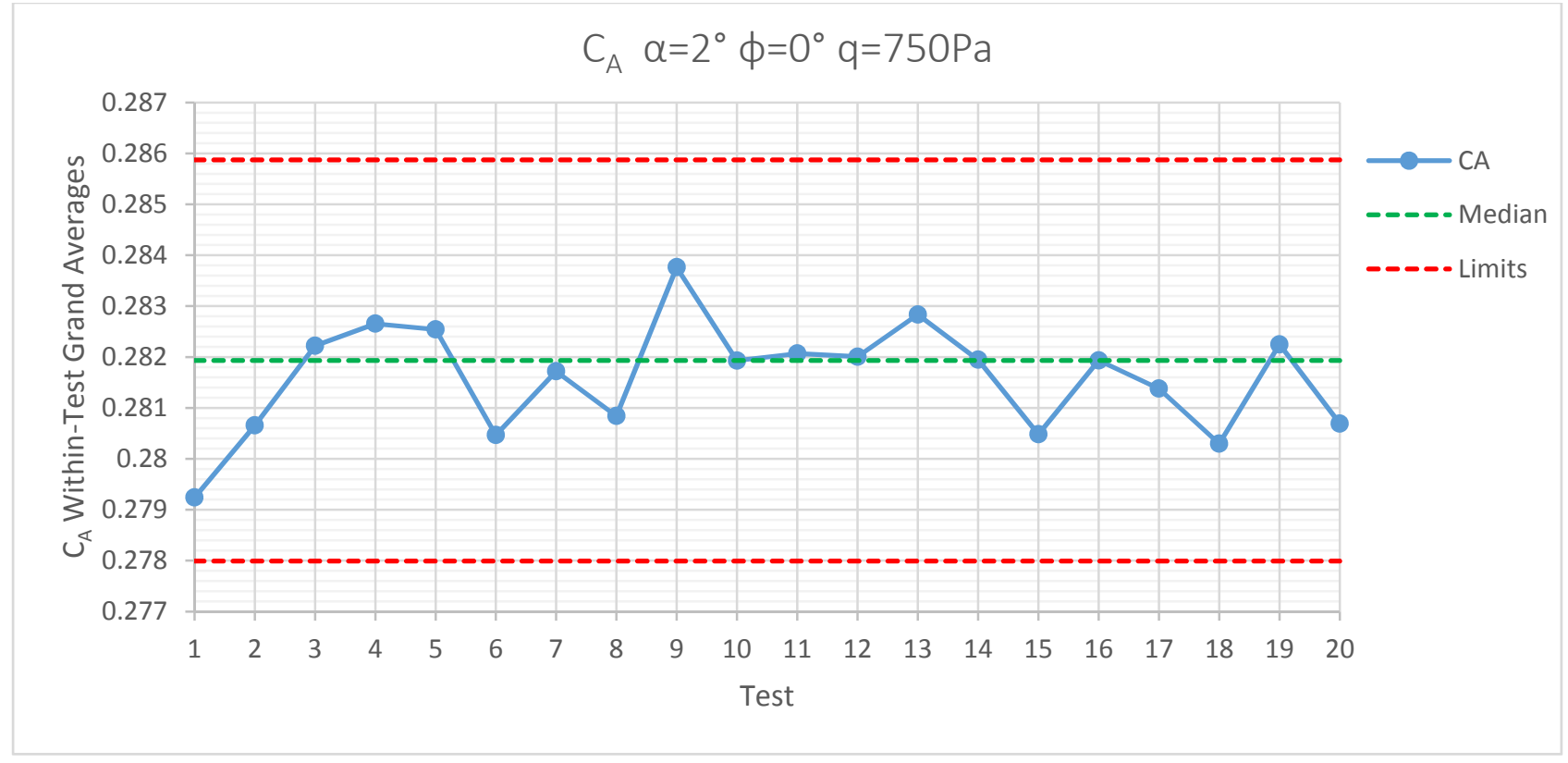

Figure 83 Across-test Control Chart $C_{A} \alpha=2^{\circ} \phi=0^{\circ} q=750 P a$

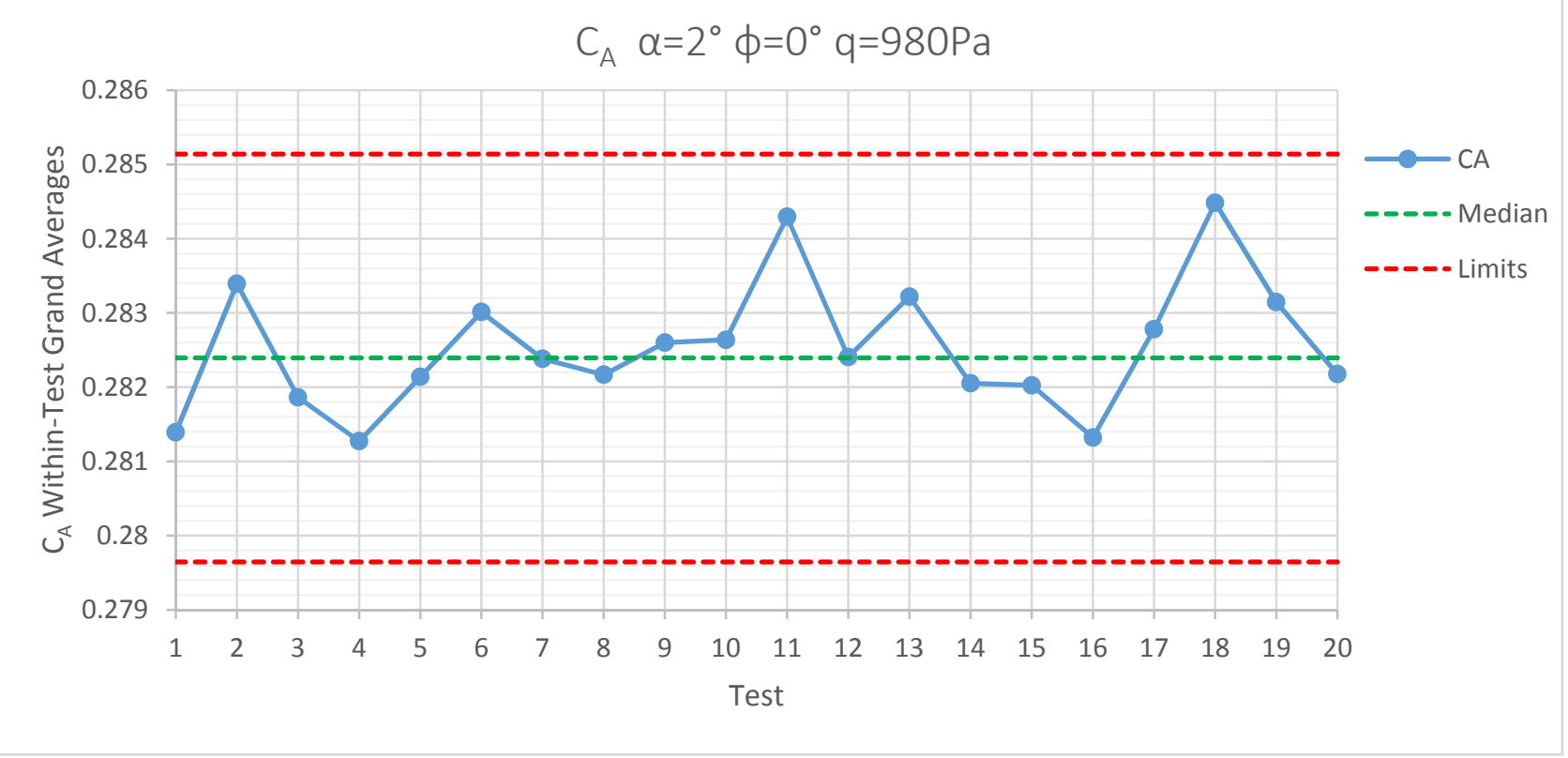

Figure 84 Across-test Control Chart $C_{A} \alpha=2^{\circ} \phi=0^{\circ} q=980 P a$ 


\subsubsection{Pitching Moment Coefficient Standard SPC Control Charts}

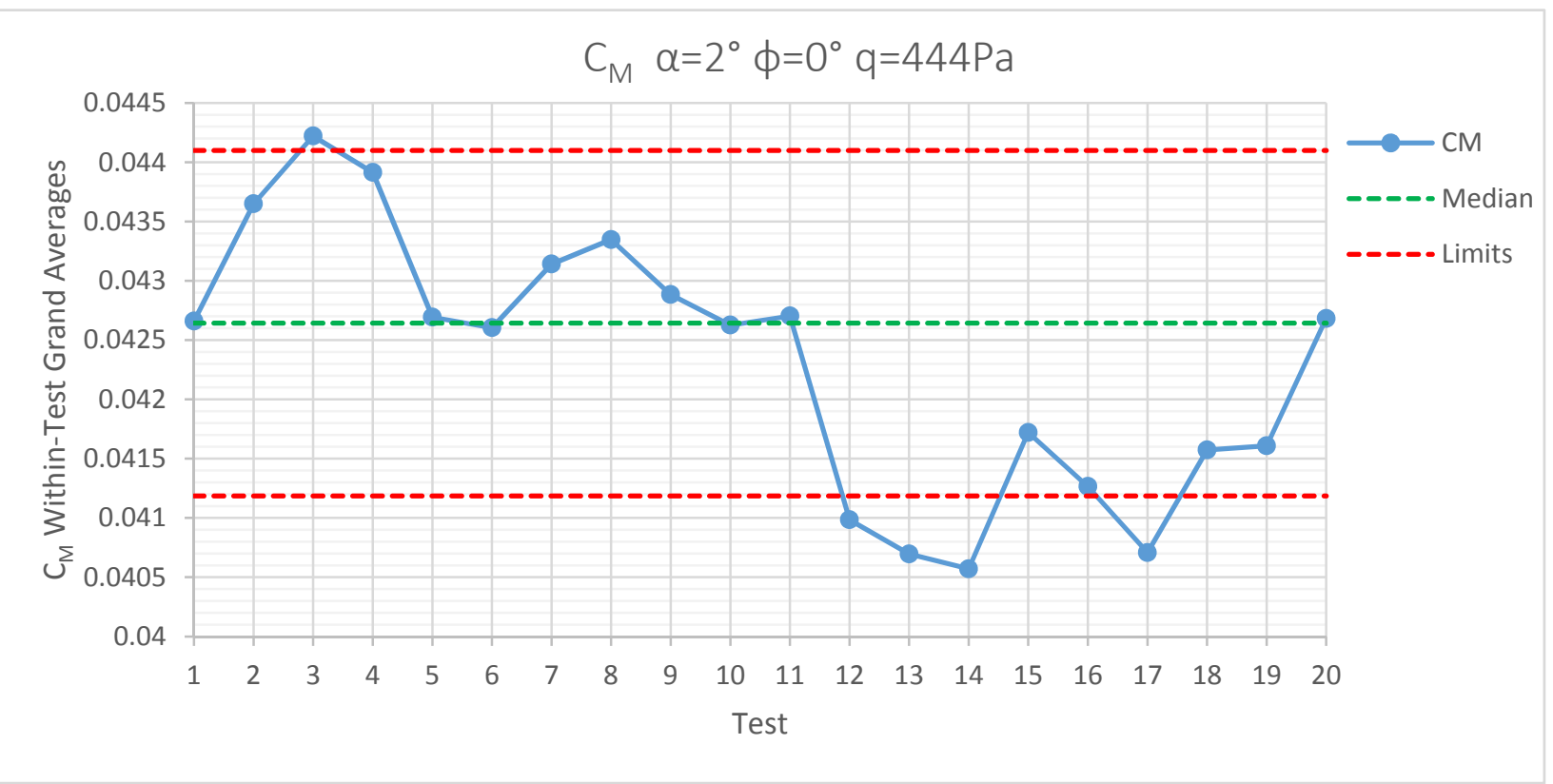

Figure 85 Across-test Control Chart $C_{M} \alpha=2^{\circ} \phi=0^{\circ} q=444 \mathrm{~Pa}$

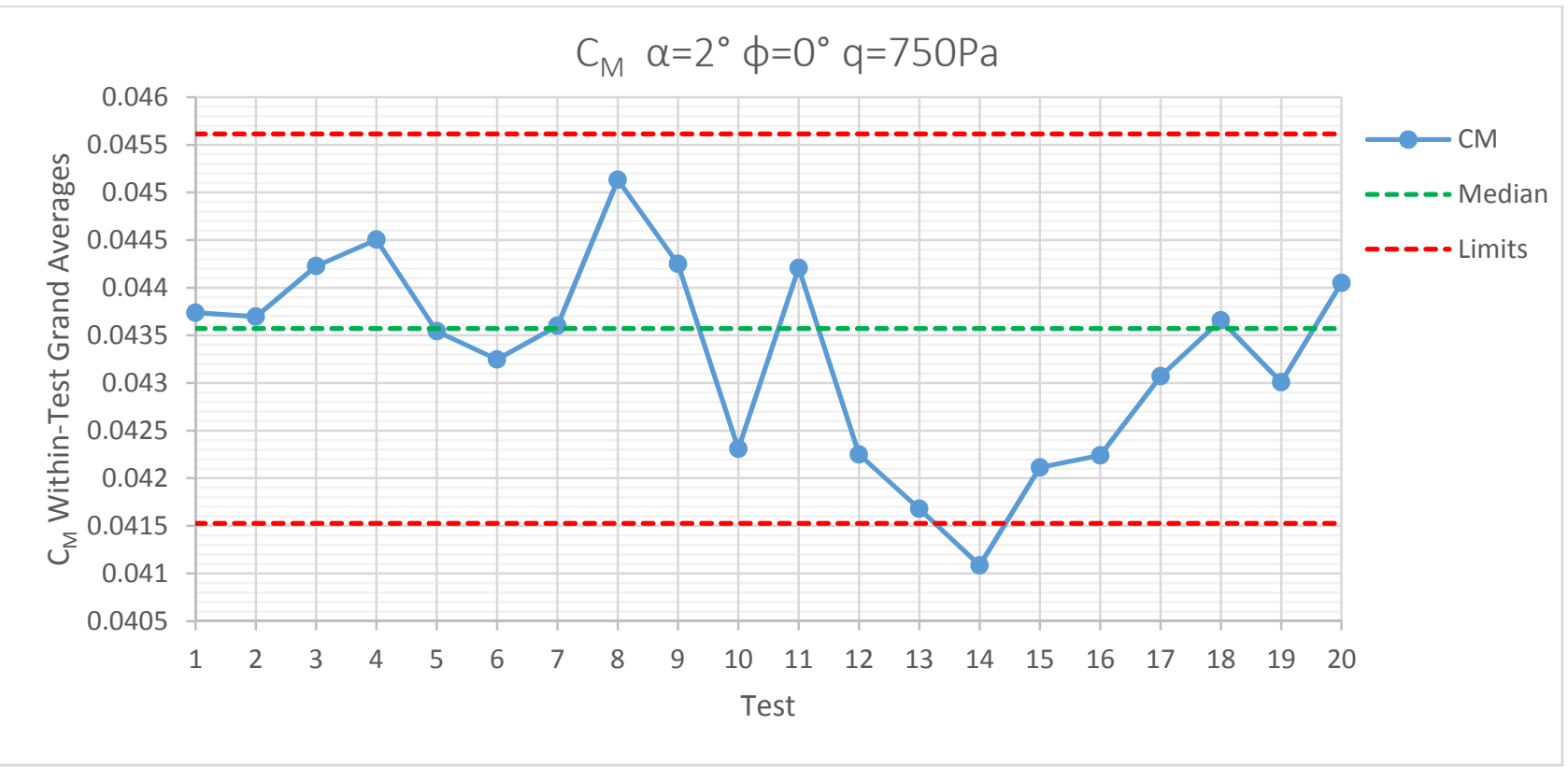

Figure 86 Across-test Control Chart $C_{M} \alpha=2^{\circ} \phi=0^{\circ} q=750 P a$ 


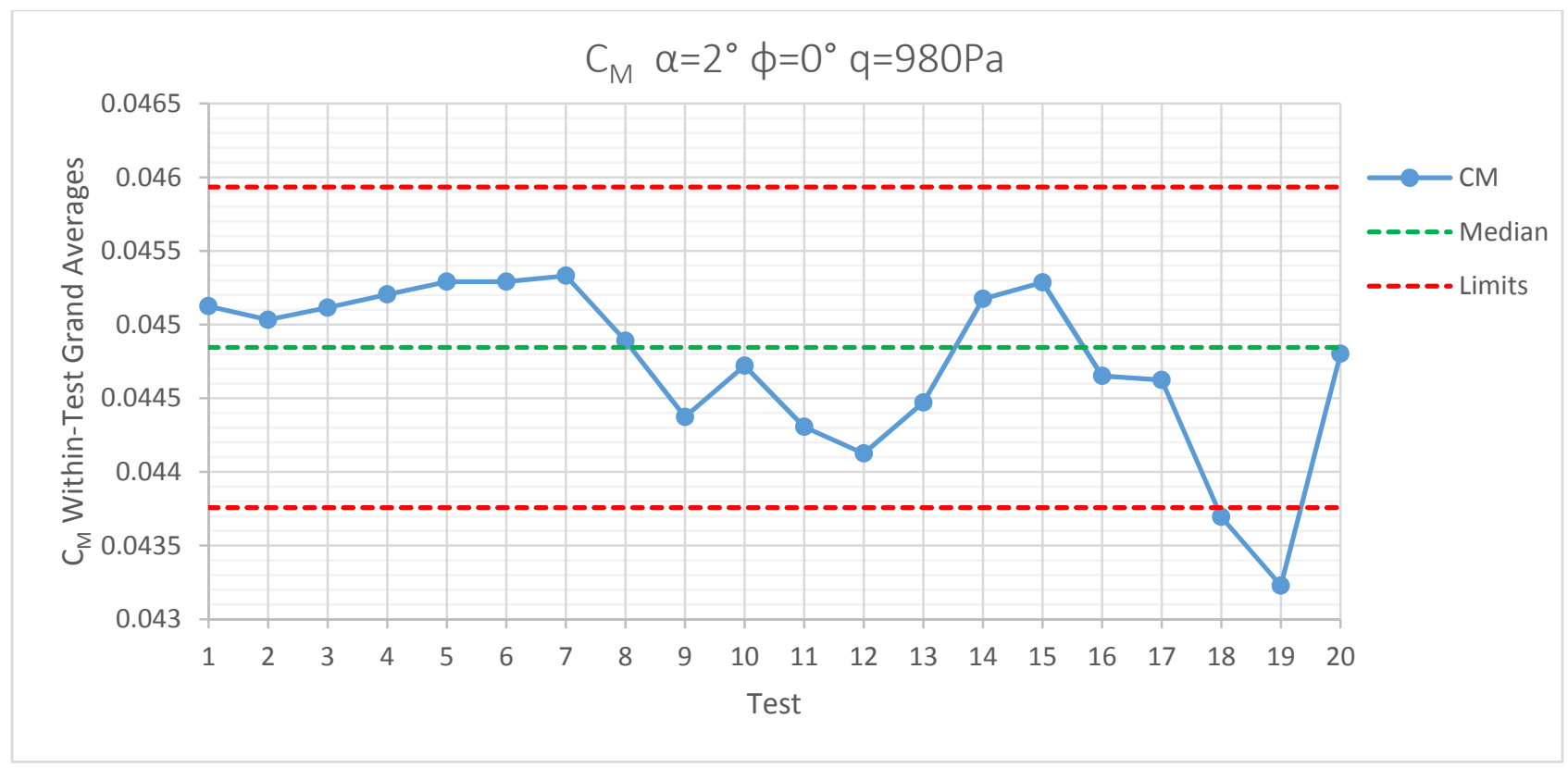

Figure 87 Across-test Control Chart $C_{M} \alpha=2^{\circ} \phi=0^{\circ} q=980 \mathrm{~Pa}$

\subsubsection{Test Moving Range Control Charts}

For brevity, test moving range, the within-test range, and within-group range control charts will be presented only for the design space represented by $\phi=0^{\circ}, \alpha=2^{\circ}, q=750 \mathrm{~Pa}$ for each response. The upper range limit (URL) for the test moving range charts is given by Equation 60 which reads the upper range limit is equal to the statistical process control factor, $D_{6}$, multiplied by the median of the moving range of the baseline test's ranges. The value of $D_{6}$ is given in Table 28.

$$
U R L=D_{6} * m \tilde{R}
$$




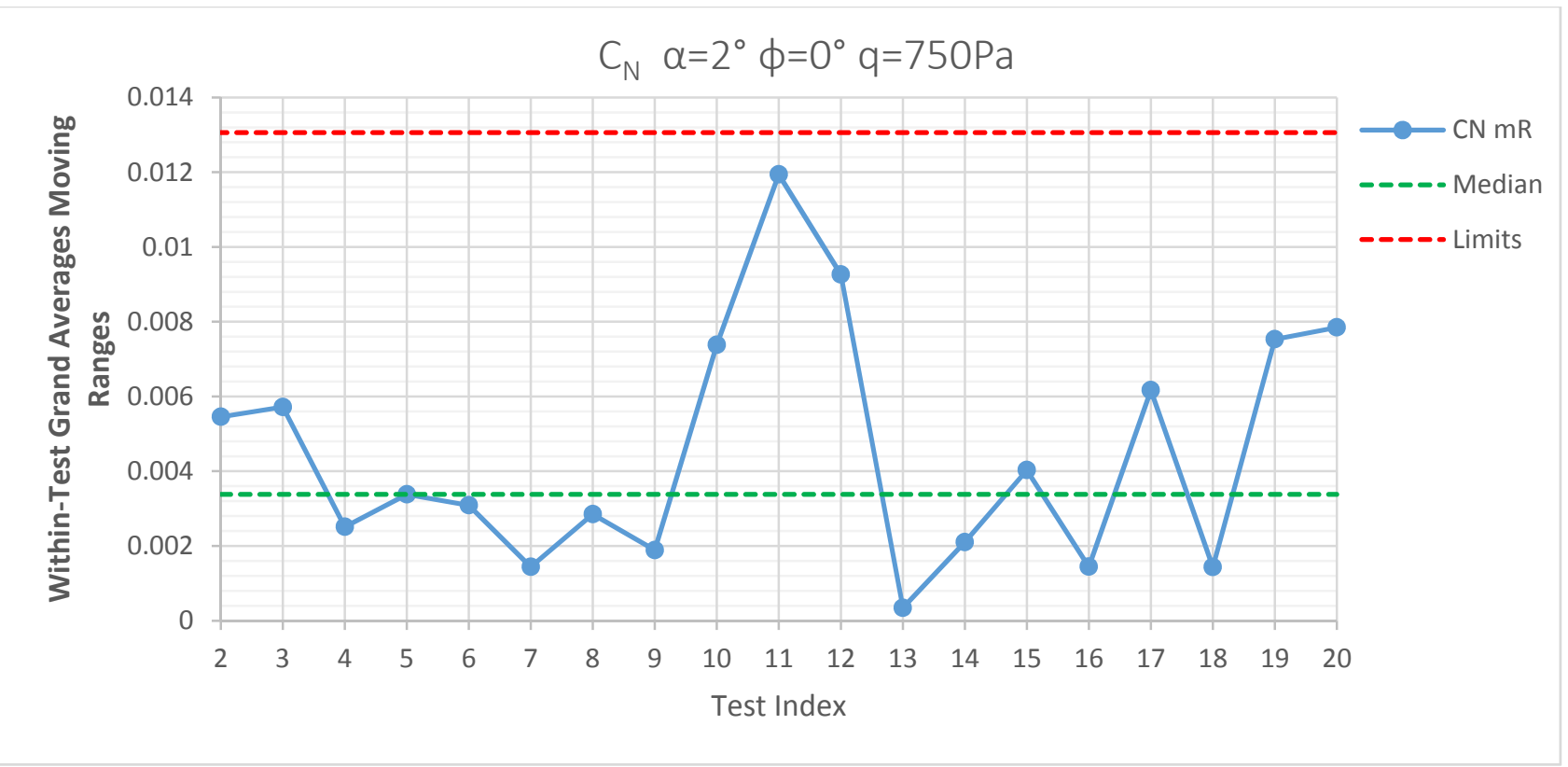

Figure 88 Test Moving Range Control Chart $C_{N} \alpha=2^{\circ} \phi=0^{\circ} q=750 \mathrm{~Pa}$

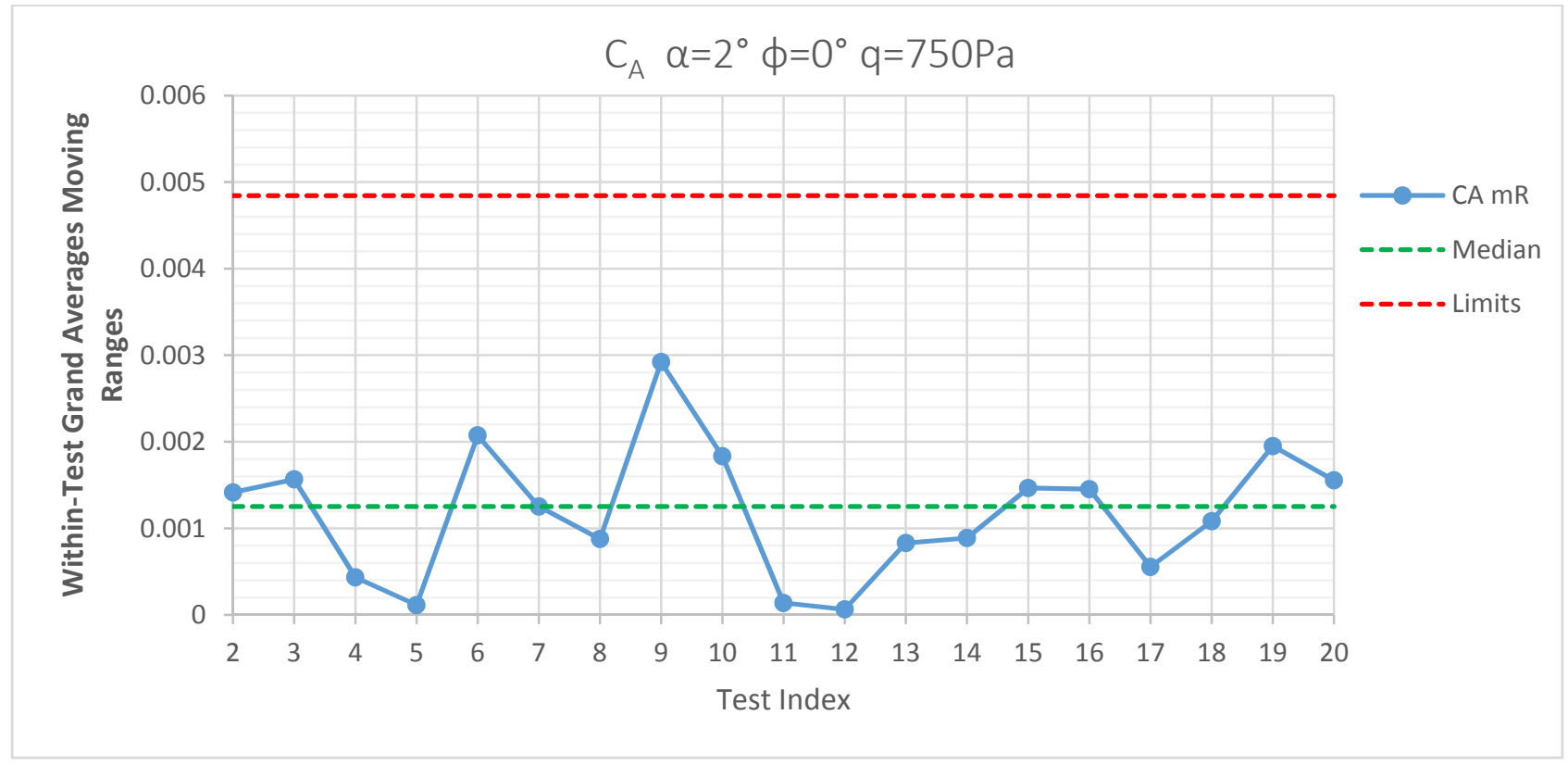

Figure 89 Test Moving Range Control Chart $C_{A} \alpha=2^{\circ} \phi=0^{\circ} q=750 \mathrm{~Pa}$ 


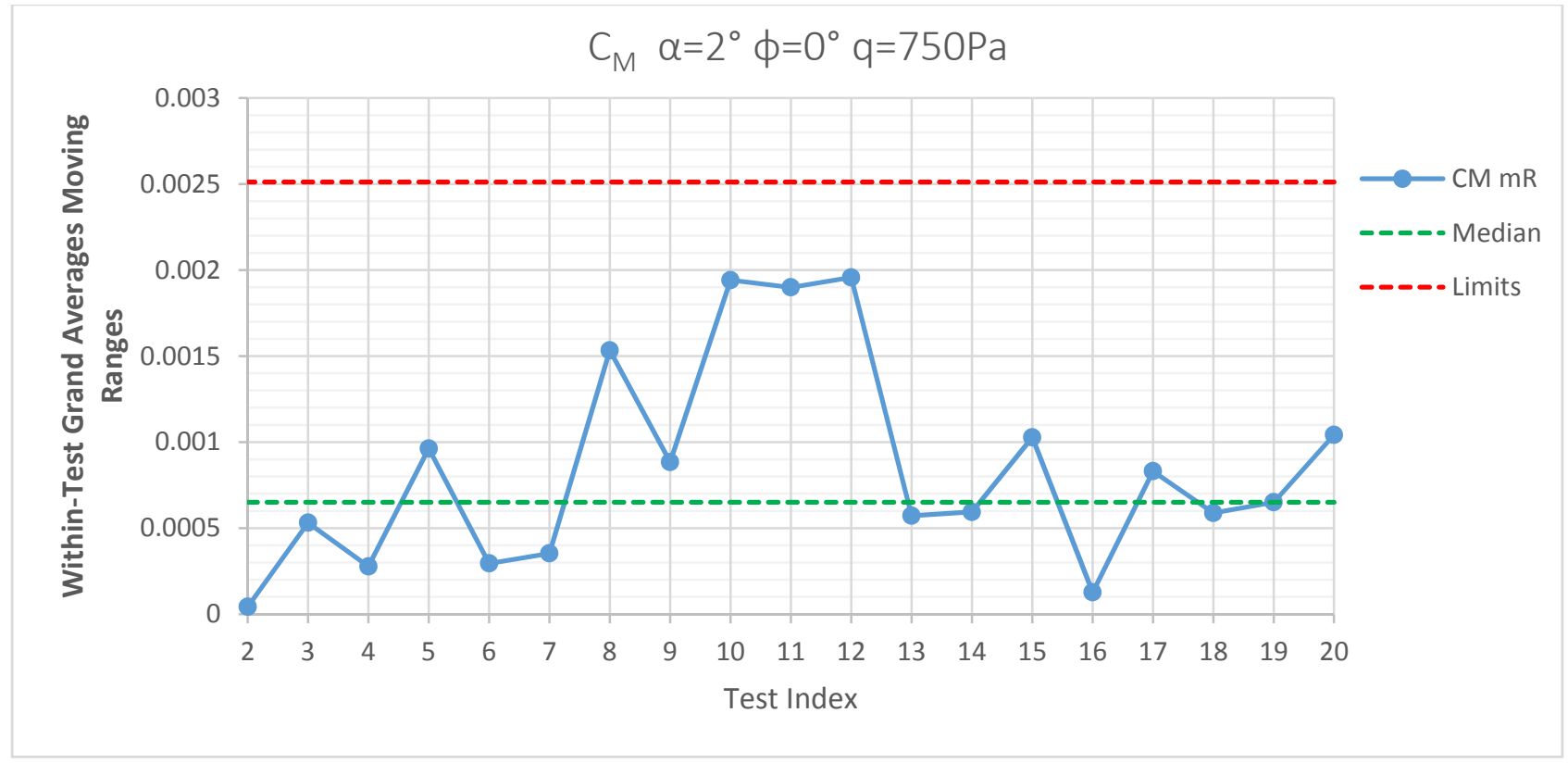

Figure 90 Test Moving Range Control Chart $C_{M} \alpha=2^{\circ} \phi=0^{\circ} q=750 \mathrm{~Pa}$

\subsubsection{Within-Test Range Control Charts}

The within-test range control charts will be presented in this section. The upper range limit (URL) for these charts is given by Equation 61 below which reads the upper range limit is equal to the statistical process control factor $D_{6}$ times the median of the baseline test's ranges. The value of $D_{6}$ is given in Table 28.

$$
U R L=D_{6} * \tilde{R}
$$




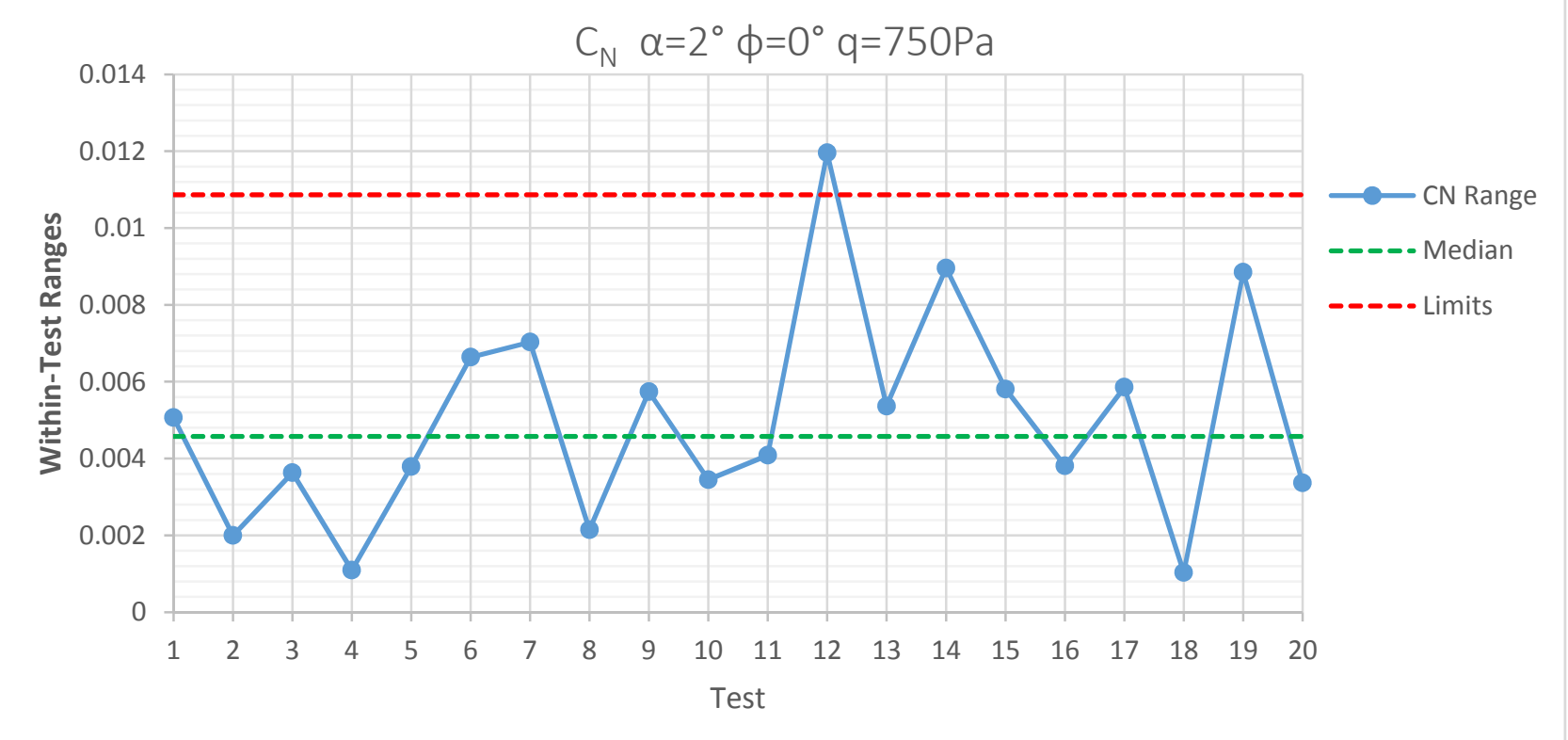

Figure 91 Within-Test Range Control Chart $C_{N} \alpha=2^{\circ} \phi=0^{\circ} q=750 \mathrm{~Pa}$

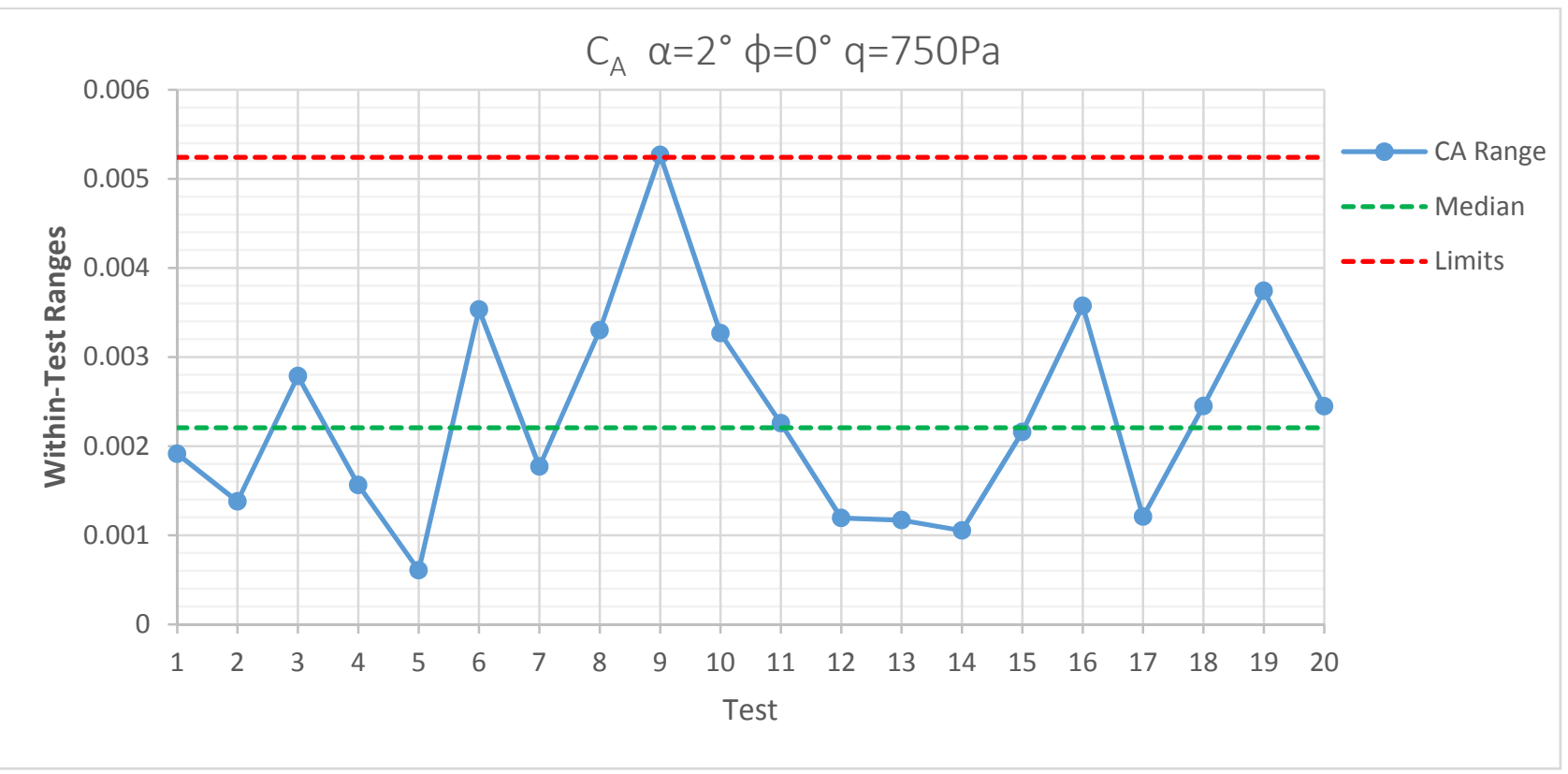

Figure 92 Within-Test Range Control Chart $C_{A} \alpha=2^{\circ} \phi=0^{\circ} q=750 \mathrm{~Pa}$ 


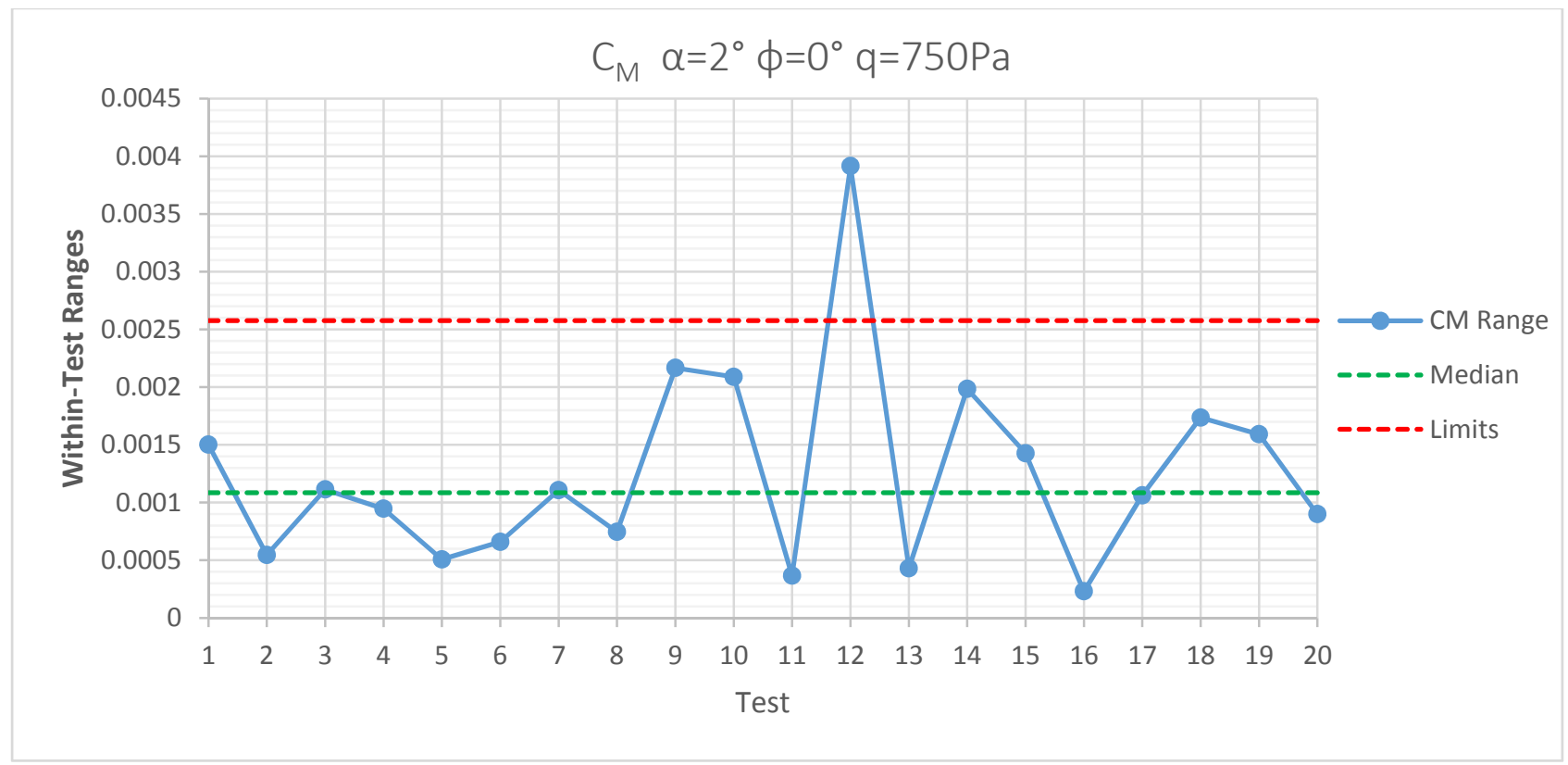

Figure 93 Within-Test Range Control Chart $C_{M} \alpha=2^{\circ} \phi=0^{\circ} q=750 P a$

\subsubsection{Within-Group Range Control Charts}

The within-group range control charts will be presented in this section. The upper range limit (URL) for these charts is given by the same equation for the within-test range control charts, Equation 61, however the median range value now represents the median of the baseline group's ranges. The statistical process control factor also changes to the value given in Table 28. 


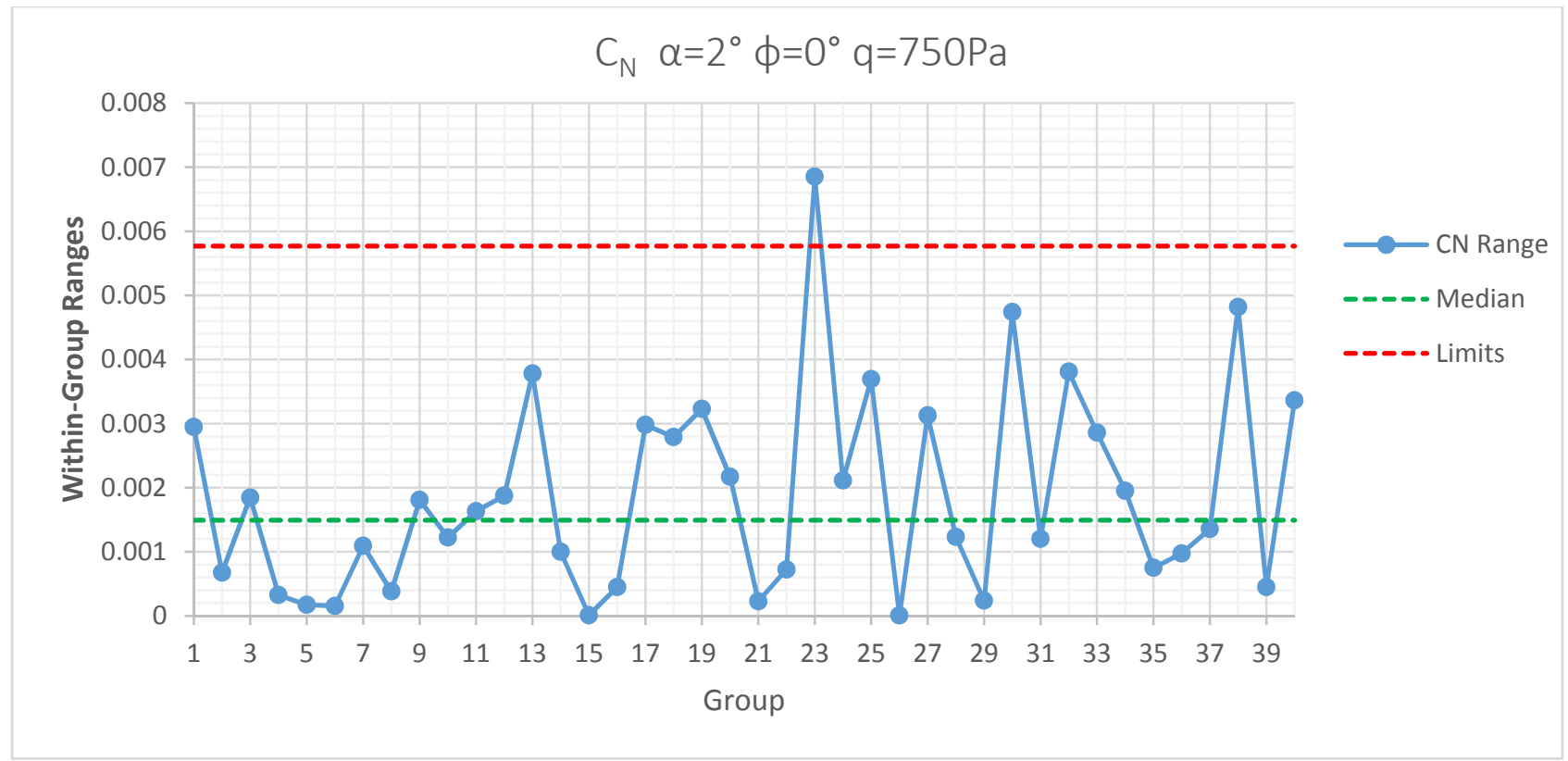

Figure 94 Within-Group Range Control Chart $C_{N} \alpha=2^{\circ} \phi=0^{\circ} q=750 \mathrm{~Pa}$

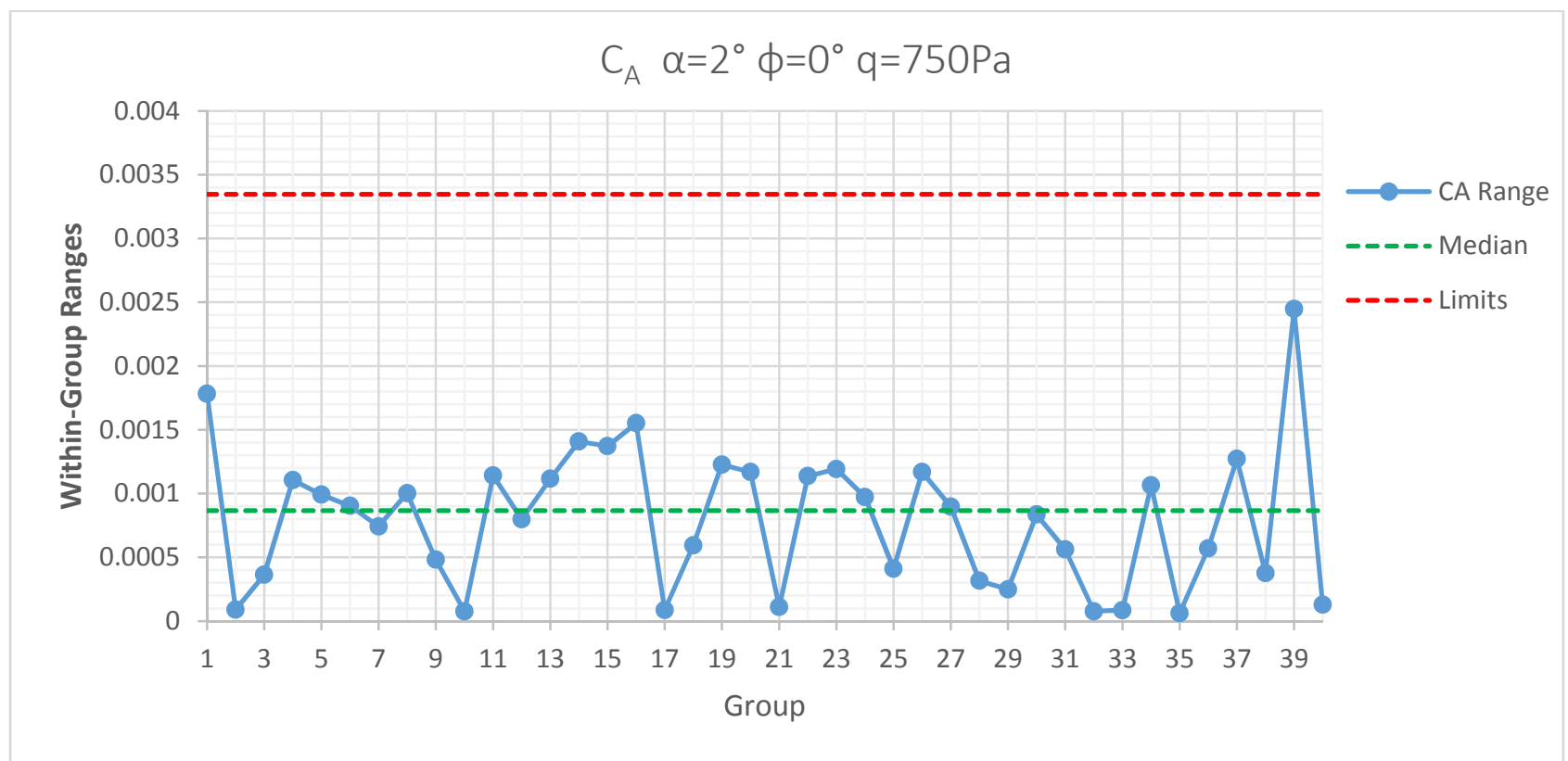

Figure 95 Within-Group Range Control Chart $C_{A} \alpha=2^{\circ} \phi=0^{\circ} q=750 P a$ 


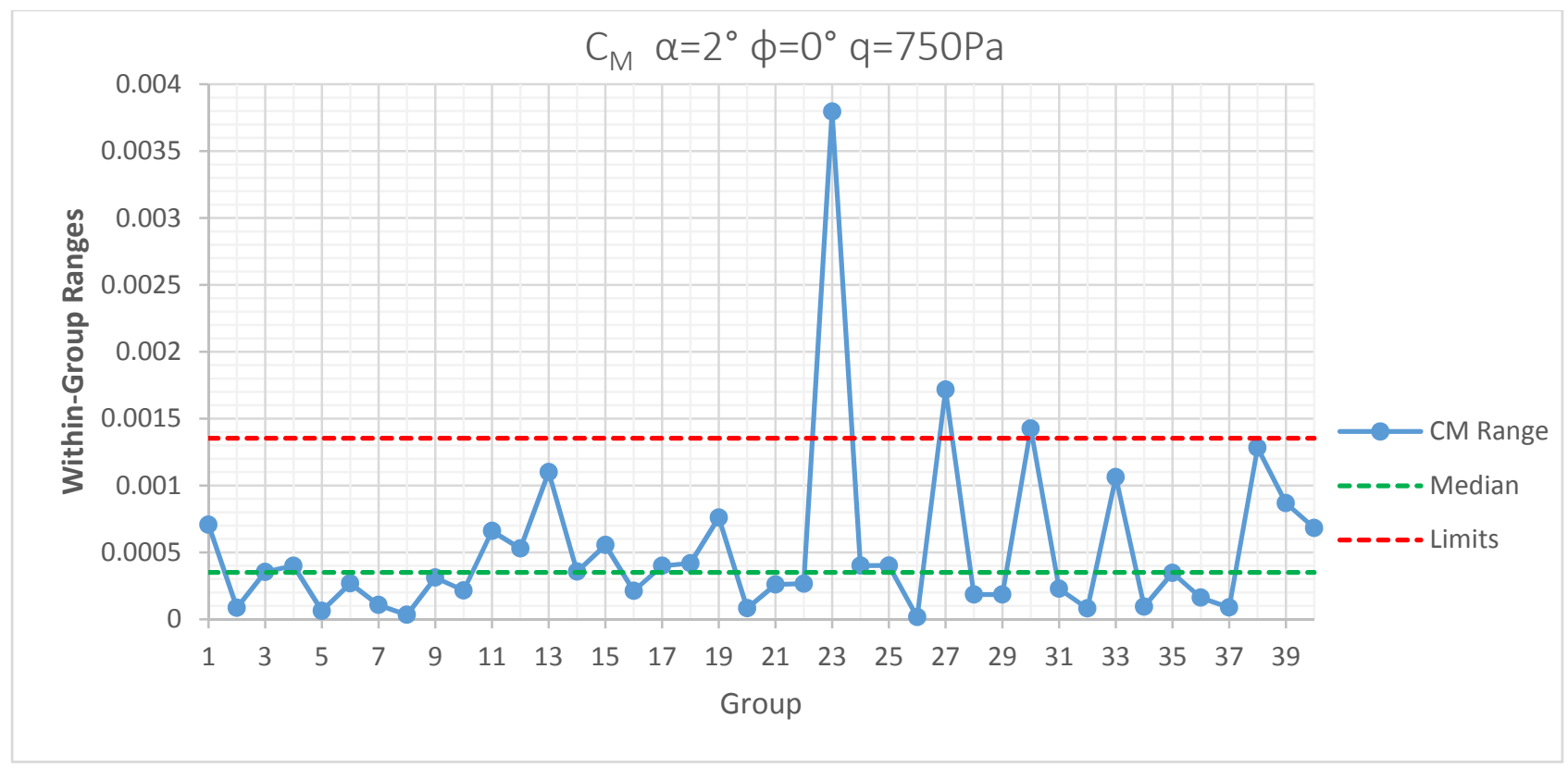

Figure 96 Within-Group Range Control Chart $C_{M} \alpha=2^{\circ} \phi=0^{\circ} q=750 P a$

\subsubsection{SPC Estimates of Standard Deviation}

The statistical process control methodology estimates the standard deviation of the airframe data at three levels, across-test, within-test and within-group, which correlate to long, medium and short-term repeatability respectively. The calculation of these estimates are identical to those completed in the tunnel conditions testing in Section 5.4.4. However, the formulations are presented again below for convenience.

$$
\begin{gathered}
\hat{\sigma}_{W G}=\tilde{R}_{W G} / d_{4} \\
\hat{\sigma}_{W T}=\sqrt{\left(\tilde{R}_{W T} / d_{4}\right)^{2}-\frac{\hat{\sigma}^{2} W G}{n}} \\
\hat{\sigma}_{A T}=\sqrt{\left(\widetilde{m R}_{A T} / d_{4}\right)^{2}-\frac{\hat{\sigma}^{2} W T}{b n}}
\end{gathered}
$$


where $n$ is the number of observations per group and $b$ is the number of observations per test. The statistical process control factor $d_{4}$ is a function of degrees of freedom which follows Table 29 . The total uncertainty estimate for the balance force coefficients is then given by combining each level of variation.

$$
\hat{\sigma}_{T}=\sqrt{\hat{\sigma}_{A T}^{2}+\hat{\sigma}_{W T}^{2}+\hat{\sigma}_{W G}^{2}}
$$

\begin{tabular}{|l|l|l|}
\hline \multicolumn{2}{|l|}{$\boldsymbol{d}_{\mathbf{4}}$ Values } \\
\hline Level & DOF & Value \\
\hline Across-test & 2 & 0.954 \\
\hline Within-test & 4 & 1.588 \\
\hline Within-group & 2 & 0.954 \\
\hline
\end{tabular}

Table 29 Values of $d_{4}$ for use in Estimation of the Standard Deviation

For comparison purposes, the normalized estimates of variation are shown for each dynamic pressure level and each response at the tunnel condition $\phi=0^{\circ}$ and $\alpha=2^{\circ}$. The normalized estimate of the standard deviation is given by the SPC estimate divided by the median of the force coefficient at that design location.

\begin{tabular}{|c|l|l|l|}
\hline \multicolumn{4}{|c|}{ Normalized SPC Estimates of the Standard Deviation for $\mathbf{C}_{\boldsymbol{N}} \boldsymbol{\phi}=\mathbf{0}^{\circ}, \boldsymbol{\alpha}=\mathbf{2}^{\circ}$} \\
\hline Level & \multicolumn{1}{c|}{$q=444 \mathrm{~Pa}$} & $q=750 \mathrm{~Pa}$ & $q=980 \mathrm{~Pa}$ \\
\hline$\widehat{\boldsymbol{\sigma}}_{\boldsymbol{W G}}$ & 0.02940 & 0.018412 & 0.0281 \\
\hline$\widehat{\boldsymbol{\sigma}}_{\boldsymbol{W T}}$ & 0.07465 & 0.023894 & 0.024089 \\
\hline$\widehat{\boldsymbol{\sigma}}_{\boldsymbol{A} T}$ & 0.07144 & 0.040800 & 0.014856 \\
\hline$\widehat{\boldsymbol{\sigma}}_{\boldsymbol{T}}$ & 0.10743 & 0.050741 & 0.039878 \\
\hline
\end{tabular}

Table 30 SPC Estimates of the Standard Deviation for $C_{N}$

\begin{tabular}{|c|l|l|l|}
\hline \multicolumn{4}{|c|}{ Normalized SPC Estimates of the Standard Deviation for $\mathbf{C}_{\mathbf{M}} \boldsymbol{\phi}=\mathbf{0}^{\circ}, \boldsymbol{\alpha}=\mathbf{2}^{\circ}$} \\
\hline Level & $q=444 \mathrm{~Pa}$ & $q=750 \mathrm{~Pa}$ & $q=980 \mathrm{~Pa}$ \\
\hline$\widehat{\boldsymbol{\sigma}}_{\boldsymbol{W G}}$ & 0.013624 & 0.008441 & 0.009222 \\
\hline$\widehat{\boldsymbol{\sigma}}_{\boldsymbol{W T}}$ & 0.024471 & 0.014526 & 0.012978 \\
\hline$\widehat{\boldsymbol{\sigma}}_{\boldsymbol{A T}}$ & 0.007459 & 0.014798 & 0.006622 \\
\hline$\widehat{\boldsymbol{\sigma}}_{\boldsymbol{T}}$ & 0.028988 & 0.022391 & 0.017244 \\
\hline
\end{tabular}

Table 31 SPC Estimates of the Standard Deviation for $C_{M}$ 


\begin{tabular}{|c|c|c|c|}
\hline \multicolumn{4}{|c|}{ Normalized SPC Estimates of the Standard Deviation for $\mathbf{C}_{\mathbf{A}} \boldsymbol{\phi}=\mathbf{0}^{\circ}, \boldsymbol{\alpha}=\mathbf{2}^{\circ}$} \\
\hline Level & $q=444 P a$ & $q=750 P a$ & $q=980 P a$ \\
\hline$\widehat{\boldsymbol{\sigma}}_{\boldsymbol{W G}}$ & 0.001410 & 0.000908 & 0.000905 \\
\hline$\widehat{\boldsymbol{\sigma}}_{\boldsymbol{W T}}$ & 0.003341 & 0.001233 & 0.001088 \\
\hline$\widehat{\boldsymbol{\sigma}}_{\boldsymbol{A} \boldsymbol{T}}$ & 0.000806 & 0.001239 & 0.000831 \\
\hline$\widehat{\boldsymbol{\sigma}}_{\boldsymbol{T}}$ & 0.003715 & 0.001970 & 0.001641 \\
\hline
\end{tabular}

Table 32 SPC Estimates of the Standard Deviation for $C_{A}$

Due to the fact that the estimates of the standard deviation derived from the statistical process control methodology are formulated using the medians of ranges (or moving ranges), the magnitude of the standard deviation is not necessarily an indicator of the process control status. For example, the largest value of the normalized SPC estimate of the standard deviation occurred at across-test level at a dynamic pressure of $444 \mathrm{~Pa}$. However, there were no points out of statistical control at that design point. It is as expected that the largest normalized estimate of the standard deviation occurs at this design space location given it is the lowest total force generated as a function of the balance full scale limits. This will be discussed further in Section 6.2.8. 


\begin{tabular}{|c|c|c|c|c|c|c|c|c|c|c|c|c|}
\hline \multicolumn{12}{|c|}{ Number of Points out of Statistical Control Limits } & \multirow{3}{*}{ Totals } \\
\hline & & & \multicolumn{3}{|c|}{$C_{A}$} & \multicolumn{3}{|c|}{$C_{N}$} & \multicolumn{3}{|c|}{$C_{M}$} & \\
\hline$q(P a)$ & $\alpha(\operatorname{deg})$ & $\phi$ & WG & WT & AT & WG & WT & AT & WG & WT & AT & \\
\hline 444 & -2 & Upright & 0 & 0 & 0 & 1 & 2 & 0 & 0 & 3 & 0 & 6 \\
\hline 444 & -2 & Inverted & 0 & 1 & 0 & 0 & 4 & 0 & 0 & 2 & 0 & 7 \\
\hline 750 & -2 & Upright & 0 & 0 & 1 & 0 & 2 & 0 & 0 & 0 & 0 & 3 \\
\hline 750 & -2 & Inverted & 0 & 0 & 0 & 0 & 1 & 0 & 0 & 1 & 0 & 2 \\
\hline 980 & -2 & Upright & 2 & 0 & 1 & 2 & 0 & 2 & 1 & 1 & 0 & 9 \\
\hline 980 & -2 & Inverted & 0 & 0 & 0 & 0 & 0 & 0 & 1 & 0 & 1 & 2 \\
\hline 444 & 2 & Upright & 0 & 1 & 1 & 2 & 1 & 0 & 3 & 0 & 3 & 11 \\
\hline 444 & 2 & Inverted & 3 & 0 & 0 & 1 & 3 & 0 & 1 & 0 & 0 & 8 \\
\hline 750 & 2 & Upright & 0 & 1 & 0 & 1 & 1 & 0 & 3 & 0 & 1 & 7 \\
\hline 750 & 2 & Inverted & 0 & 0 & 0 & 0 & 0 & 1 & 0 & 0 & 1 & 2 \\
\hline 980 & 2 & Upright & 0 & 2 & 0 & 0 & 1 & 0 & 3 & 0 & 2 & 8 \\
\hline 980 & 2 & Inverted & 0 & 0 & 1 & 0 & 0 & 2 & 0 & 0 & 0 & 3 \\
\hline 444 & 6 & Upright & 0 & 1 & 0 & 1 & 0 & 0 & 1 & 0 & 2 & 5 \\
\hline 444 & 6 & Inverted & 1 & 1 & 0 & 1 & 2 & 0 & 1 & 2 & 1 & 9 \\
\hline 750 & 6 & Upright & 0 & 0 & 0 & 1 & 1 & 0 & 0 & 0 & 2 & 4 \\
\hline 750 & 6 & Inverted & 0 & 1 & 1 & 3 & 0 & 0 & 2 & 0 & 1 & 8 \\
\hline 980 & 6 & Upright & 0 & 0 & 0 & 0 & 0 & 0 & 0 & 0 & 0 & 0 \\
\hline 980 & 6 & Inverted & 0 & 0 & 0 & 4 & 0 & 0 & 1 & 2 & 2 & 9 \\
\hline \multicolumn{3}{|l|}{ Totals } & 6 & 8 & 5 & 17 & 18 & 5 & 17 & 11 & 16 & \\
\hline
\end{tabular}

Table 33 Number of Points out of Statistical Control

From inspecting Table 33, there were no trends with respect to the number of points out of statistical control and the independent variables (dynamic pressure, angle of attack and roll angle). From a wind tunnel check standard perspective, this is a welcome finding. This indicates that the "controllability" of the measurement process is not dependent on or correlated with the independent variables. Again to reinforce this concept, the controllability does not trend with independent variables but the variance does.

\subsubsection{SPC Charting Conclusions}

The following conclusions were drawn from the standard SPC charting:

- The SPC charting practice can certainly provide a valuable quality control mechanism for a wind tunnel facility even in a relatively low degree of freedom environment. 
- For every level of the standard deviation captured by the standard SPC charts, the axial force coefficient remained in statistical control for more tests when compared to the other two responses studied. This could have been due in part to the fact that the axial force measured was a larger percentage of the full scale range of the balance than the other two responses.

- The pitching moment coefficient remained in statistical control at the lowest rate of the three responses. From the existing available data on balance coefficient check standard data in the literature, maintaining statistical control of the pitching moment appears to be difficult.

- While none of the responses remained in strict statistical control at all levels, from an overall perspective the variance in all three responses would not be a cause for alarm in practical application. For example in Figure 85, an across-test control chart for $C_{M}$, the chart indicates a process out of statistical control however the total range of the test averages is only 0.0036 . Thus the SPC charts must be viewed with an "engineering perspective."

From the evaluation of the statistical process control charts, it is concluded that the data set does not deviate substantially from statistical control and the application of the proposed methodology is feasible.

\subsubsection{Comparison of SPC and REML Standard Deviation Estimates}

For a balanced nested design it can be shown that REML estimates of the standard deviation will equal the estimates based on the analysis of variance approach shown below ${ }^{9}$. This is the same formulation as shown in the tunnel conditions testing; it is shown here again for convenience.

$$
\begin{gathered}
\hat{\sigma}_{W G}=\sqrt{M S_{W G}} \\
\hat{\sigma}_{W T}=\sqrt{\frac{M S_{W T}-M S_{W G}}{n}} \\
\hat{\sigma}_{A T}=\sqrt{\frac{M S_{A T}-M S_{W T}}{b n}}
\end{gathered}
$$


Where there are $a$ levels of treatment A (Tests), $b$ levels of treatment B (Groups) nested within A (Test) and $n$ replicates. For unbalanced designs, REML follows the same principle but the degree of freedom allocation is altered. The comparison of estimates of standard deviation will be shown for the case of $\phi=0^{\circ}, \alpha=2^{\circ}, q=750 P a$.

\begin{tabular}{|c|l|l|l|}
\hline \multicolumn{4}{|c|}{ Comparison Standard Deviation Estimates of $\mathbf{C}_{\boldsymbol{N}} \boldsymbol{\phi}=\mathbf{0}^{\circ} \boldsymbol{\alpha}=\mathbf{2}^{\circ} \boldsymbol{q}=\mathbf{7 5 0 P a}$} \\
\hline Level & REML Estimates & SPC Estimates & Percent Difference \\
\hline$\widehat{\boldsymbol{\sigma}}_{\boldsymbol{W G}}$ & 0.00174 & 0.001565 & $10.06 \%$ \\
\hline$\widehat{\boldsymbol{\sigma}}_{\boldsymbol{W T}}$ & 0.00232 & 0.002031 & $12.46 \%$ \\
\hline$\widehat{\boldsymbol{\sigma}}_{\boldsymbol{A T}}$ & 0.00308 & 0.003468 & $12.60 \%$ \\
\hline$\widehat{\boldsymbol{\sigma}}_{\boldsymbol{T}}$ & 0.00423 & 0.00431 & $1.95 \%$ \\
\hline
\end{tabular}

Table 34 Comparison of Estimates Standard Deviations for $C_{N}$

\begin{tabular}{|c|l|l|l|}
\hline \multicolumn{4}{|c|}{ Comparison of Standard Deviation Estimates of $\mathbf{C}_{\boldsymbol{A}} \boldsymbol{\phi}=\mathbf{0}^{\circ} \boldsymbol{\alpha}=\mathbf{2}^{\circ} \boldsymbol{q}=\mathbf{7 5 0 P \boldsymbol { a }}$} \\
\hline Level & REML Estimates & SPC Estimates & Percent Difference \\
\hline$\widehat{\boldsymbol{\sigma}}_{\boldsymbol{W G}}$ & 0.00095 & 0.000644 & $32.24 \%$ \\
\hline$\widehat{\boldsymbol{\sigma}}_{\boldsymbol{W T}}$ & 0.00049 & 0.000632 & $28.96 \%$ \\
\hline$\widehat{\boldsymbol{\sigma}}_{\boldsymbol{A} \boldsymbol{T}}$ & 0.0006 & 0.000367 & $38.80 \%$ \\
\hline$\widehat{\boldsymbol{\sigma}}_{\boldsymbol{T}}$ & 0.00123 & 0.00097 & $20.53 \%$ \\
\hline
\end{tabular}

Table 35 Comparison of Estimates of Standard Deviations for $C_{A}$

\begin{tabular}{|c|l|l|l|}
\hline \multicolumn{4}{|c|}{ Comparison of Standard Deviation Estimates of $\mathbf{C}_{\mathbf{M}} \boldsymbol{\phi}=\mathbf{0}^{\circ} \boldsymbol{\alpha}=\mathbf{2}^{\circ} \boldsymbol{q}=\mathbf{7 5 0 P a}$} \\
\hline Level & REML Estimates & SPC Estimates & Percent Difference \\
\hline$\widehat{\boldsymbol{\sigma}}_{\boldsymbol{W G}}$ & 0.00069 & 0.000908 & $31.59 \%$ \\
\hline$\widehat{\boldsymbol{\sigma}}_{\boldsymbol{W T}}$ & 0.00128 & 0.001233 & $3.67 \%$ \\
\hline$\widehat{\boldsymbol{\sigma}}_{\boldsymbol{A T}}$ & 0.00042 & 0.001239 & $195.00 \%$ \\
\hline$\widehat{\boldsymbol{\sigma}}_{\boldsymbol{T}}$ & 0.00151 & 0.00197 & $30.14 \%$ \\
\hline
\end{tabular}

Table 36 Comparison of Estimates of Standard Deviations for $C_{M}$

From inspection of Table 34, Table 35 and Table 36 above, it is seen that the estimates of standard deviation between REML and SPC vary, and in some instances vary greatly. However the total standard deviation estimates appear to be in better agreement than the individual levels. It is also important to 
note that displayed above is only one design point and it is expected that the estimates and difference in the estimates of the standard deviation will change as a function of the design space location.

\subsubsection{Variance Modeling}

With the estimates of the standard deviation from the REML analysis it is possible to model the variance throughout the design space. For researchers to achieve the goal of the overall reduction of variation in the wind tunnel measurements, the variance must first be very well understood. Modeling the variance throughout the design space is one method to visualize and better understand the variance. To model the variance, the response studied is the natural log of the variance. This is due to the fact that the expected distribution of the variance is chi-squared, not normal. ${ }^{9}$

$$
\operatorname{Ln}\left(\sigma^{2}\right)=f(\alpha, \phi, q)
$$

While it is possible to model each level of variance, that is, within-group, within-test, across test and total, only the total variance will be modeled as it includes each level of variation. The estimates of the force coefficients standard deviations plotted against dynamic pressure, colored by angle of attack and symbolically noted by roll condition are given in Figure 97, Figure 98 and Figure 99. 


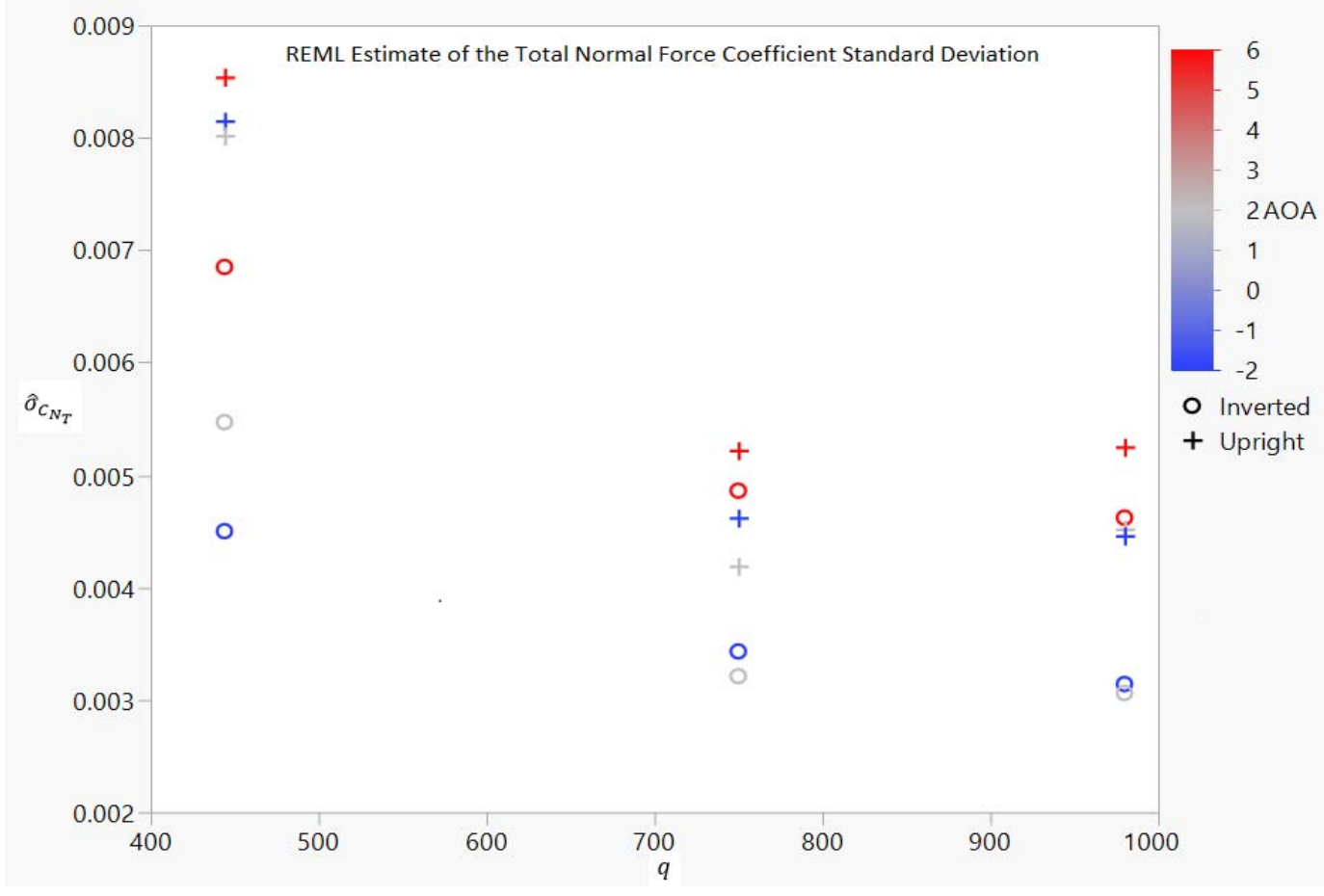

Figure 97 REML Estimate of the Total Normal Force Coefficient Standard Deviation

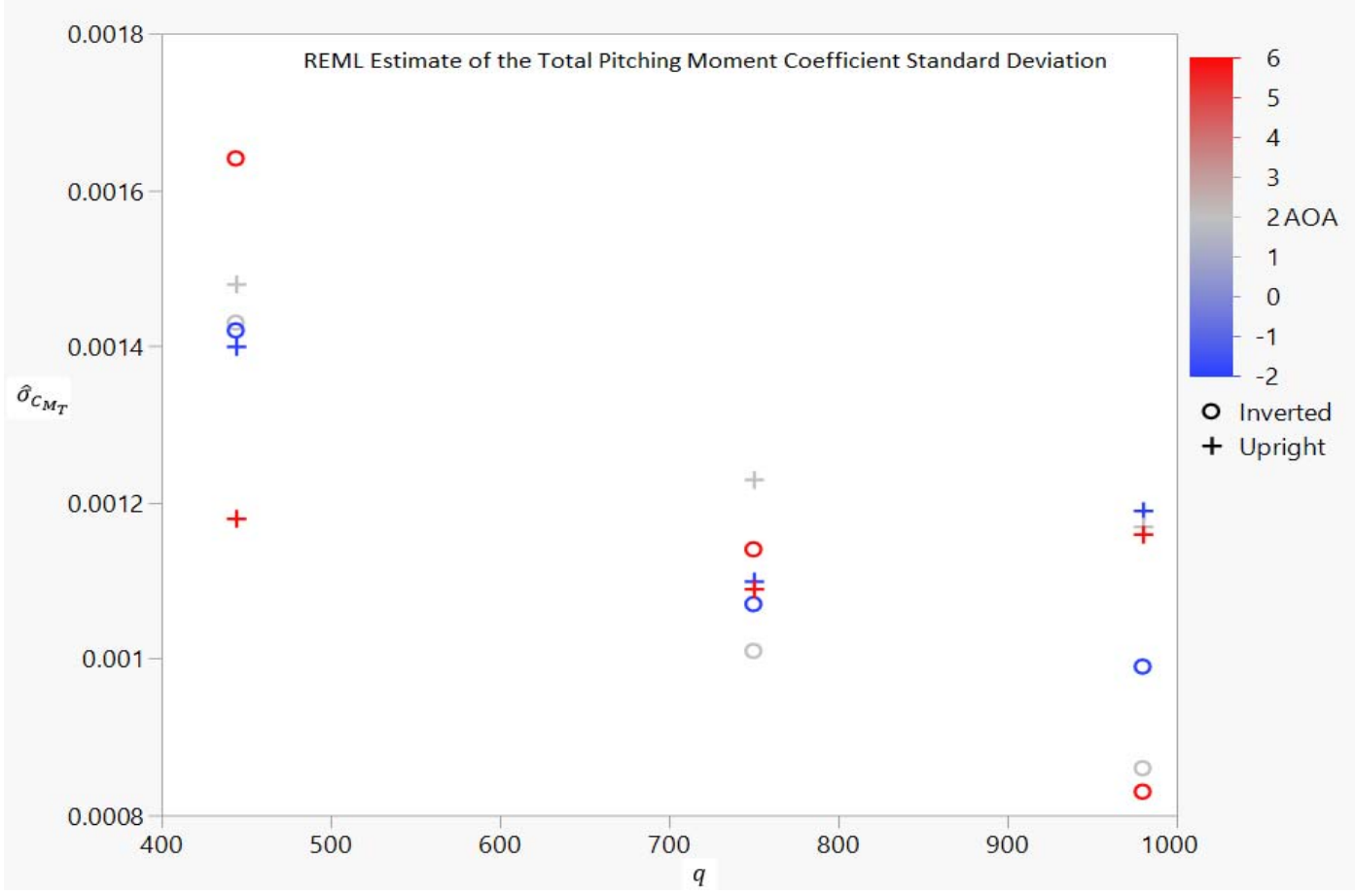

Figure 98 REML Estimate of the Total Pitching Moment Coefficient Standard Deviation 


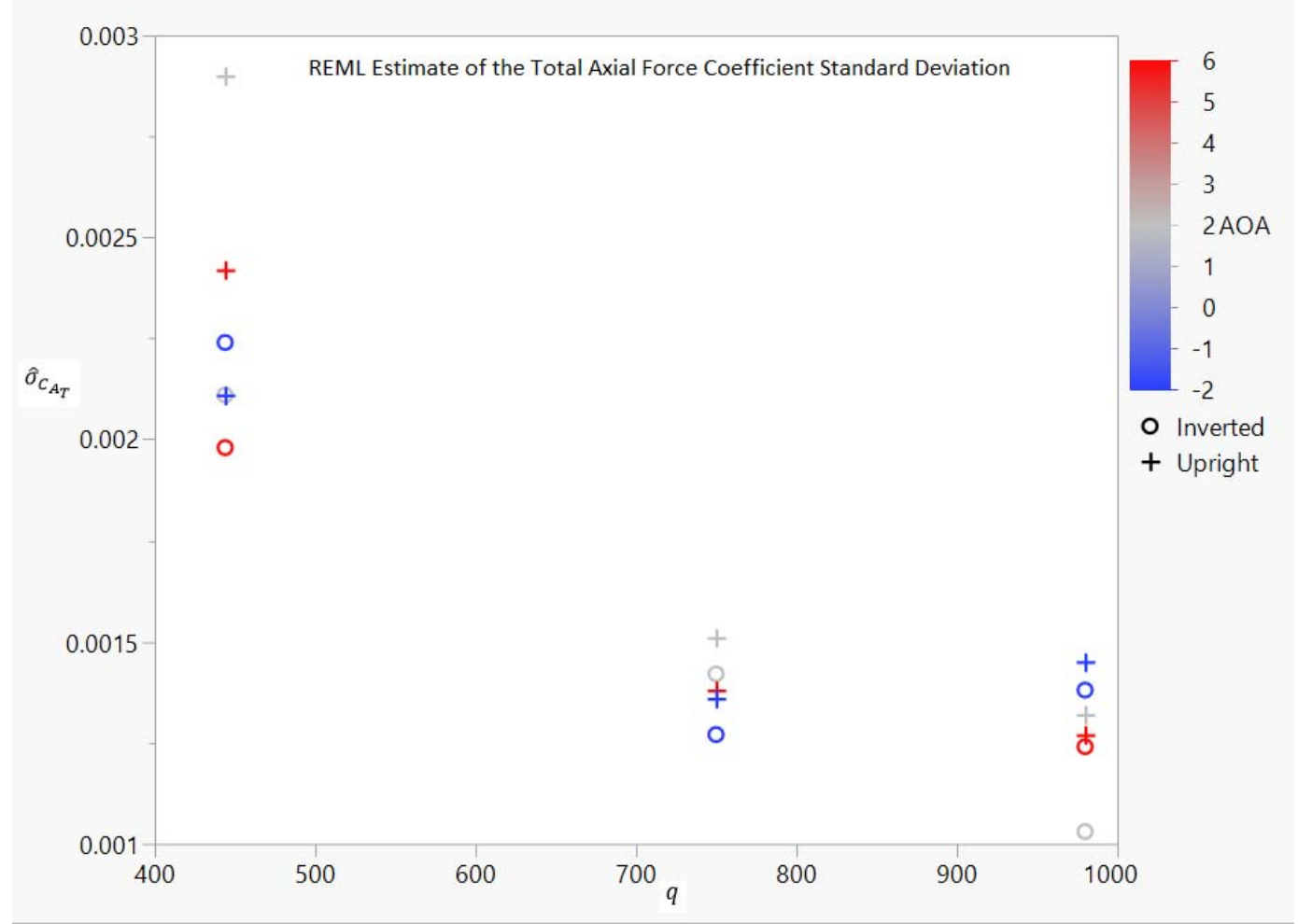

Figure 99 REML Estimate of the Total Axial Force Coefficient Standard Deviation

Previous research by Hemsch ${ }^{1}$ encountered the same trends in the estimates of the standard deviation of the force coefficients found in the tunnel conditions testing which is given as a log-log plot in Figure 100. Note that the dynamic pressure range in Figure 100 is given in pounds per square foot and is not a one-to-one scaling with the data presented above $980 P a \approx 20.5 p s f$. 


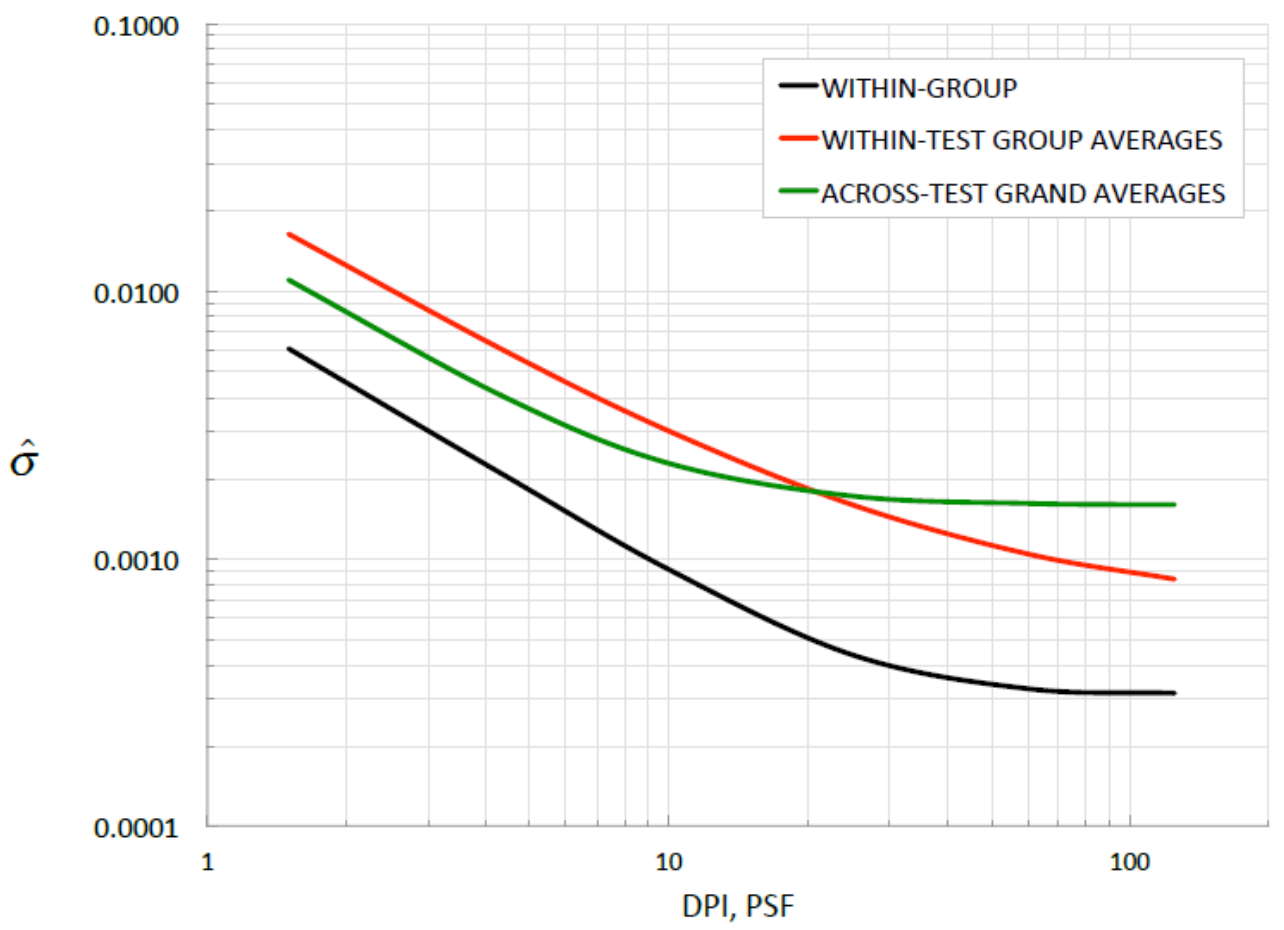

Figure 100 Estimate of the Deviation of the Normal Force Coefficient from Check Standard Testing at the Langley $14 \times 22$

The models for the natural log of the total variance for each of the force and moment responses are given in Equations 67, 68 and 69. The equations are given in terms of coded units so that the relative magnitude of the coefficients can be compared. It is seen that for all of the models, the dominant factor on the variance is dynamic pressure.

$$
\begin{aligned}
& \ln \left(\hat{\sigma}_{C_{A_{T}}}^{2}\right)=- 13.07-0.7458 q-0.03227 \alpha-0.1111 \phi-0.05916 q \alpha+0.3644 q^{2} \\
&-0.07554 \alpha^{2}+0.2495 q \alpha^{2} \\
& \ln \left(\hat{\sigma}_{C_{N_{T}}}^{2}\right)=- 11.05-0.4998 q-0.2474 \alpha-0.2999 \phi+0.1370 \alpha \phi+0.3905 q^{2} \\
&+0.2467 \alpha^{2} \\
& \ln \left(\hat{\sigma}_{C_{M_{T}}}^{2}\right)=-13.57-0.3253 q-0.02597 \alpha-0.1679 \phi-0.04119 q \alpha-0.1860 q \phi \\
&+
\end{aligned}
$$


To further examine the causes of the variance in the wind tunnel measurements during the check standard testing, the known drivers of variance should be removed or accounted for. This will allow the researcher to identify areas within the design space that have higher levels of variance that are not directly attributable to an independent variable. The "known" or attributable variance in the measurements can be derived from an uncertainty propagation for a generic force coefficient, $C_{F}$ and is given by

$$
C_{F}=\frac{F}{q_{\infty} S}
$$

where $F$ is the applied force, $q_{\infty}$ is the freestream reference dynamic pressure and $S$ is the reference area. The generic moment coefficient is given by

$$
C_{M}=\frac{M}{q_{\infty} S c}
$$

where $c$ is the reference chord. The uncertainty in the force coefficient and moment coefficient assuming no uncertainty in reference area are given respectively by ${ }^{1,52}$

$$
\begin{gathered}
\sigma_{C_{F}}^{2}=\left(\frac{\partial C}{\partial F}\right)^{2} \sigma_{F}^{2}+2 \rho \frac{\partial C}{\partial F} \frac{\partial C}{\partial q_{\infty}} \sigma_{F} \sigma_{q_{\infty}}+\left(\frac{\partial C}{\partial q_{\infty}}\right)^{2} \sigma_{q_{\infty}}^{2} \\
\sigma_{C_{M}}^{2}=\left(\frac{\partial C}{\partial M}\right)^{2} \sigma_{M}{ }^{2}+2 \rho \frac{\partial C}{\partial M} \frac{\partial C}{\partial q_{\infty}} \sigma_{M} \sigma_{q_{\infty}}+\left(\frac{\partial C}{\partial q_{\infty}}\right)^{2} \sigma_{q_{\infty}}^{2}
\end{gathered}
$$

Where $\rho$ is the correlation coefficient between the measured force or moment and reference dynamic pressure given by Equations $75^{53}$.

$$
\rho\left(x_{1}, x_{2}\right)=\frac{\operatorname{cov}\left(x_{1}, x_{2},\right)}{\sigma_{x_{1}} \sigma_{x_{2}}}
$$




$$
\rho\left(x_{1}, x_{2}\right)=\frac{1}{n-1} \sum_{i=1}^{n} \frac{x_{1}-\mu_{x_{1}}}{\sigma_{x_{1}}} \frac{x_{2}-\mu_{x_{2}}}{\sigma_{x_{2}}}
$$

The correlation coefficient will differ for each force (axial, normal, pitch moment). The correlation of axial force and dynamic pressure is shown in Figure 101. Due to the relatively low angle of attack values in this testing, there is a very strong correlation between axial force generated and the dynamic pressure, $\rho=0.992$.

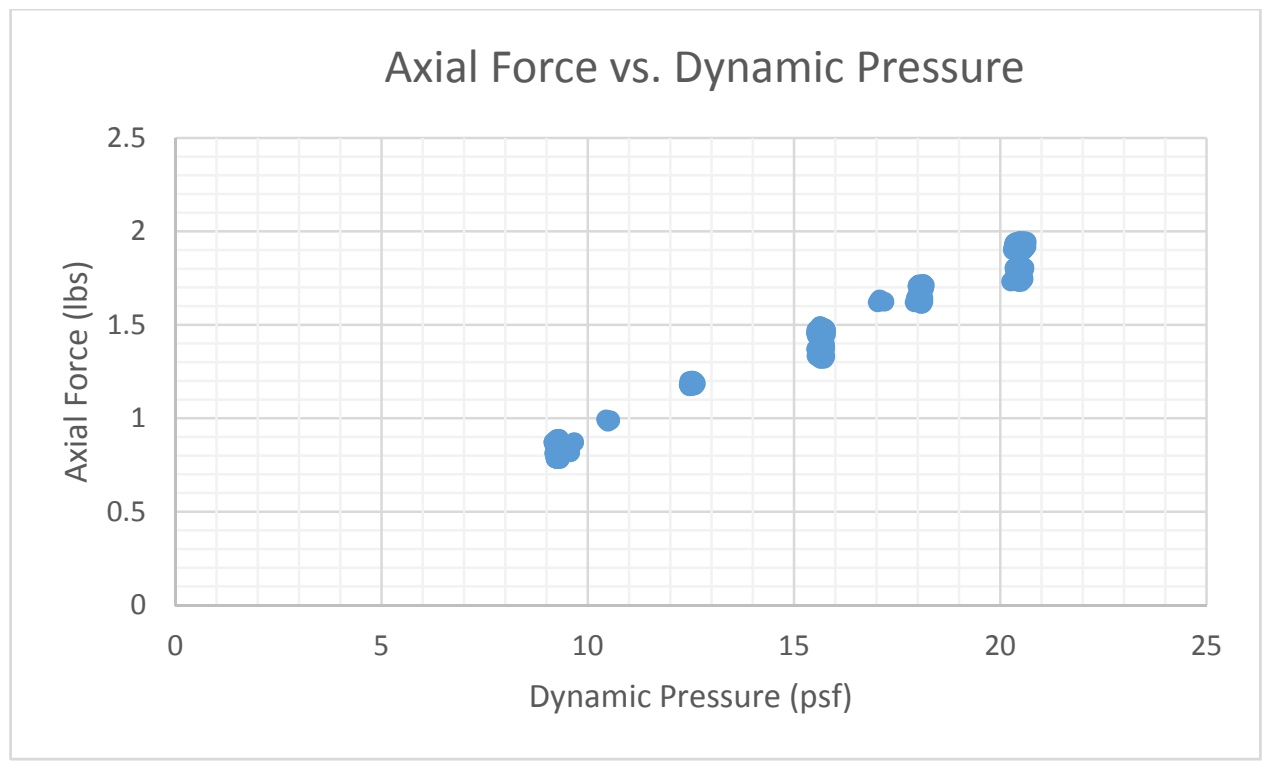

Figure 101 Correlation of Axial Force and Dynamic Pressure

From Equation 70 the partial derivative of the force coefficient with respect to the applied force, moment and the partial derivative of the force coefficient with respect to the freestream dynamic pressure are given respectively as

$$
\frac{\partial C_{F}}{\partial F}=\frac{1}{q_{\infty} S}
$$




$$
\frac{\partial C_{M}}{\partial M}=\frac{1}{q_{\infty} S C}
$$

$$
\frac{\partial C_{F}}{\partial q_{\infty}}=\frac{-F}{q_{\infty}^{2} S}
$$

$$
\frac{\partial C_{M}}{\partial q_{\infty}}=\frac{-M}{q_{\infty}^{2} S}
$$

Substituting Equations 76 and 78 into the uncertainty propagation in Equation 73 gives the "known" or attributable variance.

$$
\sigma_{C_{F}}^{2}=\left(\frac{1}{q_{\infty} S}\right)^{2} \sigma_{F}{ }^{2}+2 \rho \frac{1}{q_{\infty} S} \frac{-F}{q_{\infty}^{2} S} \sigma_{F} \sigma_{q_{\infty}}+\left(\frac{-F}{q_{\infty}^{2} S}\right)^{2} \sigma_{q_{\infty}}^{2}
$$

Similarly, the formulation for the generic moment is given by

$$
\sigma_{C_{M}}^{2}=\left(\frac{1}{q_{\infty} S c}\right)^{2} \sigma_{M}{ }^{2}+2 \rho \frac{1}{q_{\infty} S c} \frac{-M}{q_{\infty}^{2} S c} \sigma_{M} \sigma_{q_{\infty}}+\left(\frac{-M}{q_{\infty}^{2} S c}\right)^{2} \sigma_{q_{\infty}}^{2}
$$

If the estimates of the variance generated by the REML procedure are normalized by Equation 80 , the attributable variance can effectively be removed from the analysis. If there was no other variance (other than that variance attributable to Equation 80 ) in the force measurements present, the resultant variance ratio would be under one for the entire design space, which is desirable. This would indicate that the vast majority of the variance in the force and moment coefficients was due to instrumentation; implying that there were no other factors inflating variance. The normalized REML estimate of the total normal force, axial force and pitching moment coefficients variances are given in Table 37. 


\begin{tabular}{|c|c|c|c|c|c|}
\hline \multicolumn{3}{|c|}{ Design Space Location } & \multicolumn{3}{|c|}{ Normalized Estimates of Total Variance } \\
\hline$q$ & $\alpha$ & $\phi$ & $\tilde{\boldsymbol{\sigma}}_{C_{N_{T}}}^{2}$ & $\widetilde{\sigma}_{C_{A_{T}}}^{2}$ & $\tilde{\sigma}_{C_{M_{T}}}^{2}$ \\
\hline 444 & -2 & Upright & 0.245033 & 0.016093 & 0.005238 \\
\hline 444 & -2 & Inverted & 0.074912 & 0.018182 & 0.005875 \\
\hline 750 & -2 & Upright & 0.132919 & 0.011231 & 0.004021 \\
\hline 750 & -2 & Inverted & 0.073403 & 0.009947 & 0.004393 \\
\hline 980 & -2 & Upright & 0.162033 & 0.016655 & 0.004846 \\
\hline 980 & -2 & Inverted & 0.080526 & 0.015186 & 0.003711 \\
\hline 444 & 2 & Upright & 0.239363 & 0.031588 & 0.006863 \\
\hline 444 & 2 & Inverted & 0.111429 & 0.016835 & 0.006029 \\
\hline 750 & 2 & Upright & 0.110197 & 0.014516 & 0.005491 \\
\hline 750 & 2 & Inverted & 0.064814 & 0.012859 & 0.003467 \\
\hline 980 & 2 & Upright & 0.167954 & 0.014535 & 0.004678 \\
\hline 980 & 2 & Inverted & 0.076788 & 0.008832 & 0.002369 \\
\hline 444 & 6 & Upright & 0.272815 & 0.023195 & 0.0021 \\
\hline 444 & 6 & Inverted & 0.175901 & 0.015525 & 0.004021 \\
\hline 750 & 6 & Upright & 0.172205 & 0.012927 & 0.001626 \\
\hline 750 & 6 & Inverted & 0.14949 & 0.010945 & 0.00176 \\
\hline 980 & 6 & Upright & 0.228027 & 0.014477 & 0.001669 \\
\hline 980 & 6 & Inverted & 0.17654 & 0.013734 & 0.000843 \\
\hline \multicolumn{3}{|c|}{ Mean } & 0.150797 & 0.015403 & 0.003833 \\
\hline
\end{tabular}

Table 37 Normalized Estimates of Total Variance

While none of the values in Table 37 appear to be near unity, confidence intervals are placed on the normalized REML estimates to ensure that statistically rigorous conclusions are drawn regarding the areas of higher variance. Assuming that the estimates of variance derived by the uncertainty propagation are those of the virtual population, the test statistic for statistical significance on the normalized estimates of variance is given by an $\chi^{2}$ test in Equation 82

$$
\chi_{0}^{2}=\frac{(n-1) S^{2}}{\sigma_{0}^{2}}
$$

where $S^{2}$ is the normalized estimate of the total variance from REML, and $\sigma_{0}^{2}$ is the equivalent normalized estimate of total variance from the uncertainty propagation which is simply unity. For rejection of the null hypothesis that the normalized estimates of variance from REML are not statistically greater than the normalized variances of the uncertainty propagation, $\chi_{0}^{2}>\chi_{a, n-1}^{2}$ 
where $\chi_{a, n-1}^{2}$ is the chi-squared distribution, ${ }^{54} n$ is the degrees of freedom, in this case 20 (for the 20 baseline tests), $S^{2}$ is the estimate of the standard deviation, in this case the REML estimate and $a$ is the significance level, in this case 0.05 . After applying the statistical test in Equation 82 to the data in it Table 37 was concluded, as expected, that the null hypothesis could not be rejected. Therefore it could be concluded that the estimates of REML total variance were not larger than those from the uncertainty propagation estimates.

Because the estimates of the REML total variance were normalized, the mean values could also be compared for the different responses. To compare the normalized estimates of variance across the responses, an $\mathrm{F}$ test for equal variances could be used with the test statistic: ${ }^{9}$

$$
F_{0}=\frac{S_{1}^{2}}{S_{2}^{2}}
$$

For rejection of the null hypothesis that the mean of the variance of each of the responses is equal, $F_{0}>F_{a / 2, n_{1}-1, n_{2}-1}$ or $F_{0}<F_{1-a / 2, n_{1}-1, n_{2}-1}$. Where the degrees of freedom of the mean variance are equal to the 20 tests multiplied by the 18 unique design point locations giving a total of 360 . After applying this statistical test, it is seen again as expected from inspection of the mean values in Table 37, the mean normalized estimate of the total variance of the pitching moment was statistically lower that the normal force and axial force coefficients.

\subsection{Balance Tare Charting}

It is common during the process of wind tunnel testing to monitor the balance voltage output under a zero applied load condition. This gives researchers a method to monitor the balance health throughout the course of the testing. Conventionally, the zeros are obtained after the tare process at a "wind off" condition, hence yielding a value of zero nominally. It is important to note that this condition is not unloaded strictly speaking as the balance is supporting the weight of the model (the weight of the model is removed via the tare process). The absolute tare values better serves as a metric for monitoring balance health. Presented in Figure 102, Figure 103 and Figure 104 are the tare values at 2.2 degrees angle of attack and 0.5 degrees roll prior to each test. The limits on the charts are derived from the same formulation of the across-test control charts presented previously in Equations 45 and 46 . Withintest tares were also taken for new tares but are not presented. The attitude of the model is not at zero 
degrees angle of attack and roll due to the nature of the test matrix and the need for the tare values to "bracket" the measured value. The values obtained for the tares are also confounded with the positioning error of the model support system.

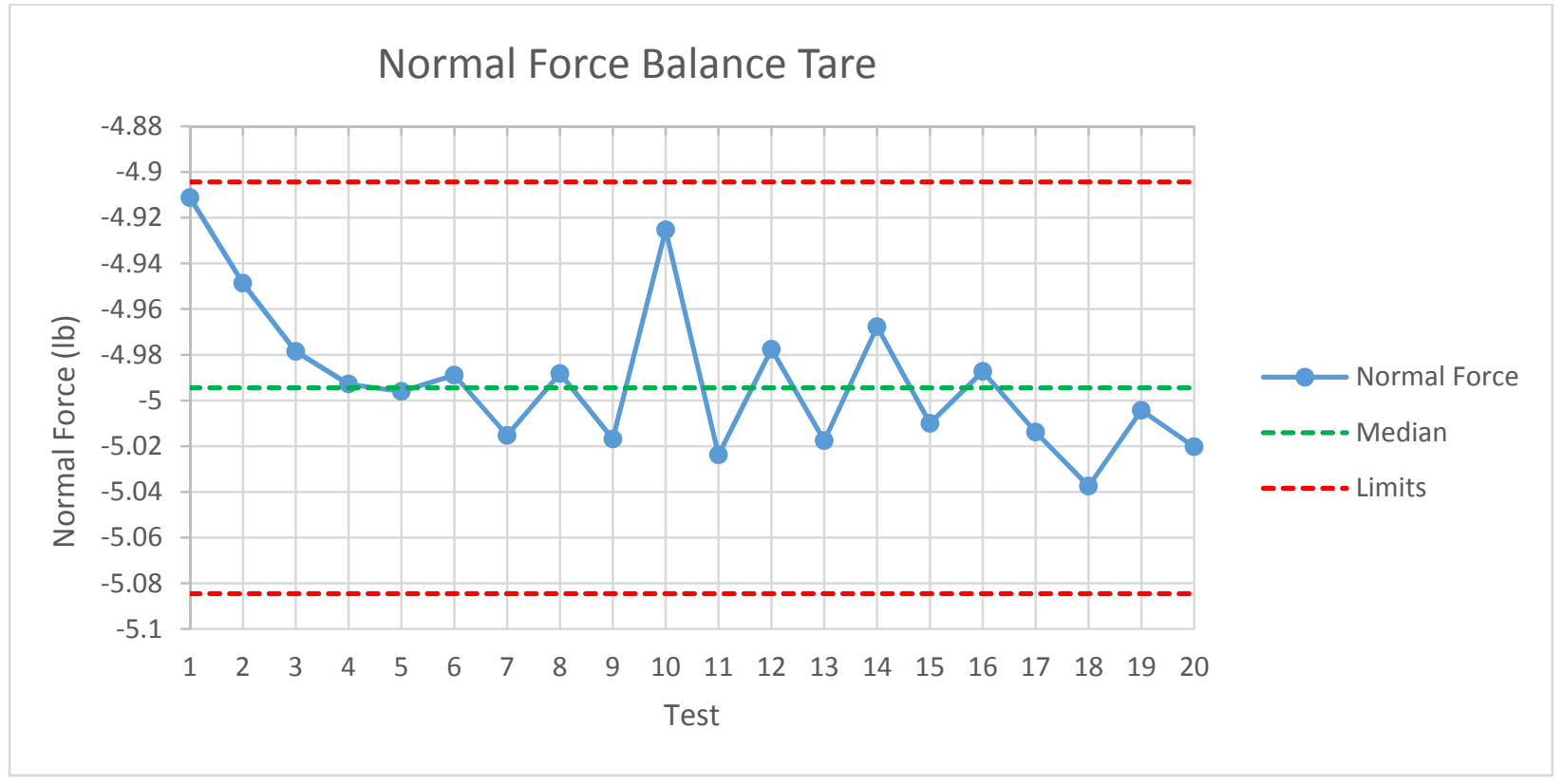

Figure 102 Normal Force Balance Tare 


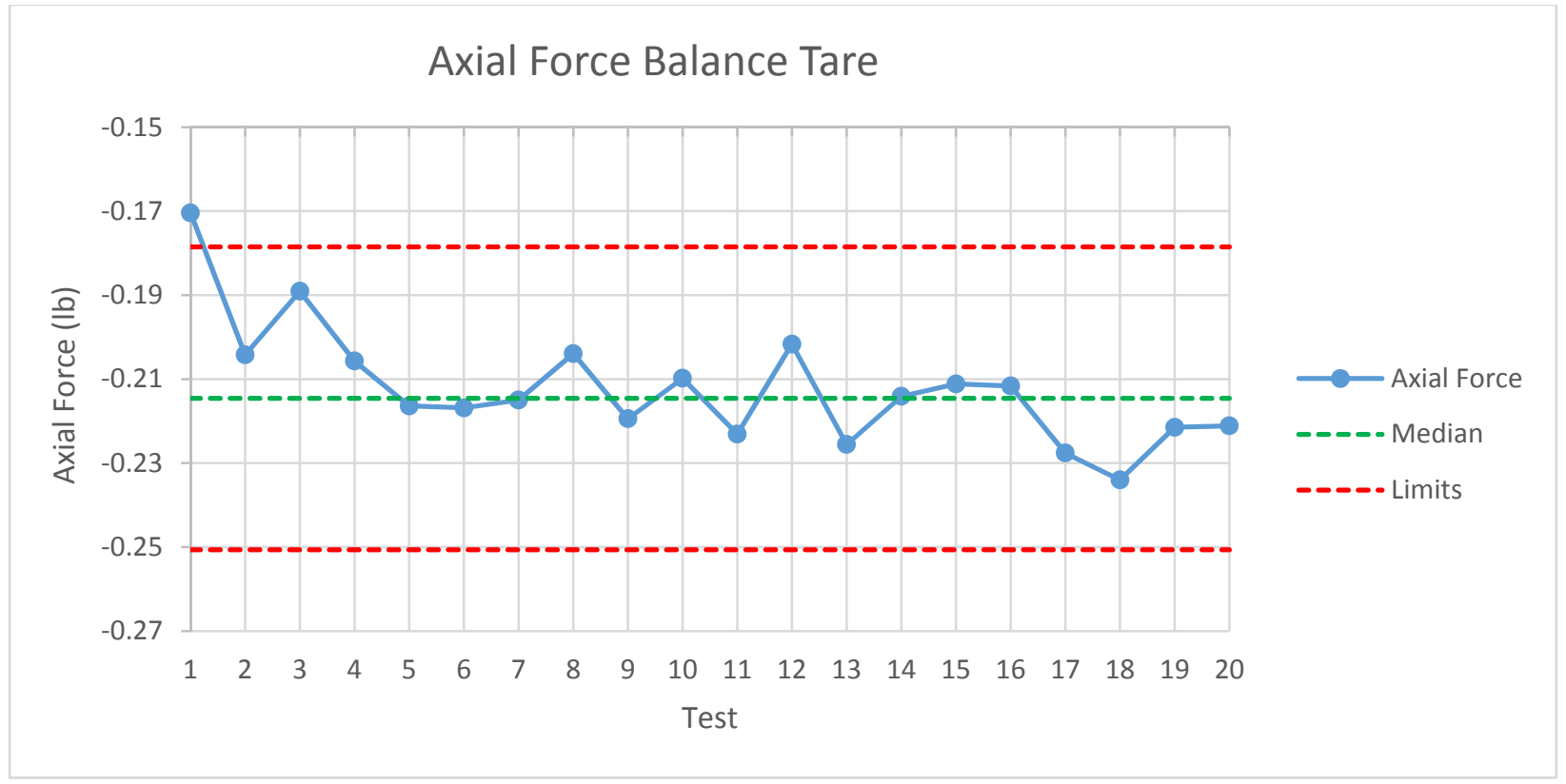

Figure 103 Axial Force Balance Tare

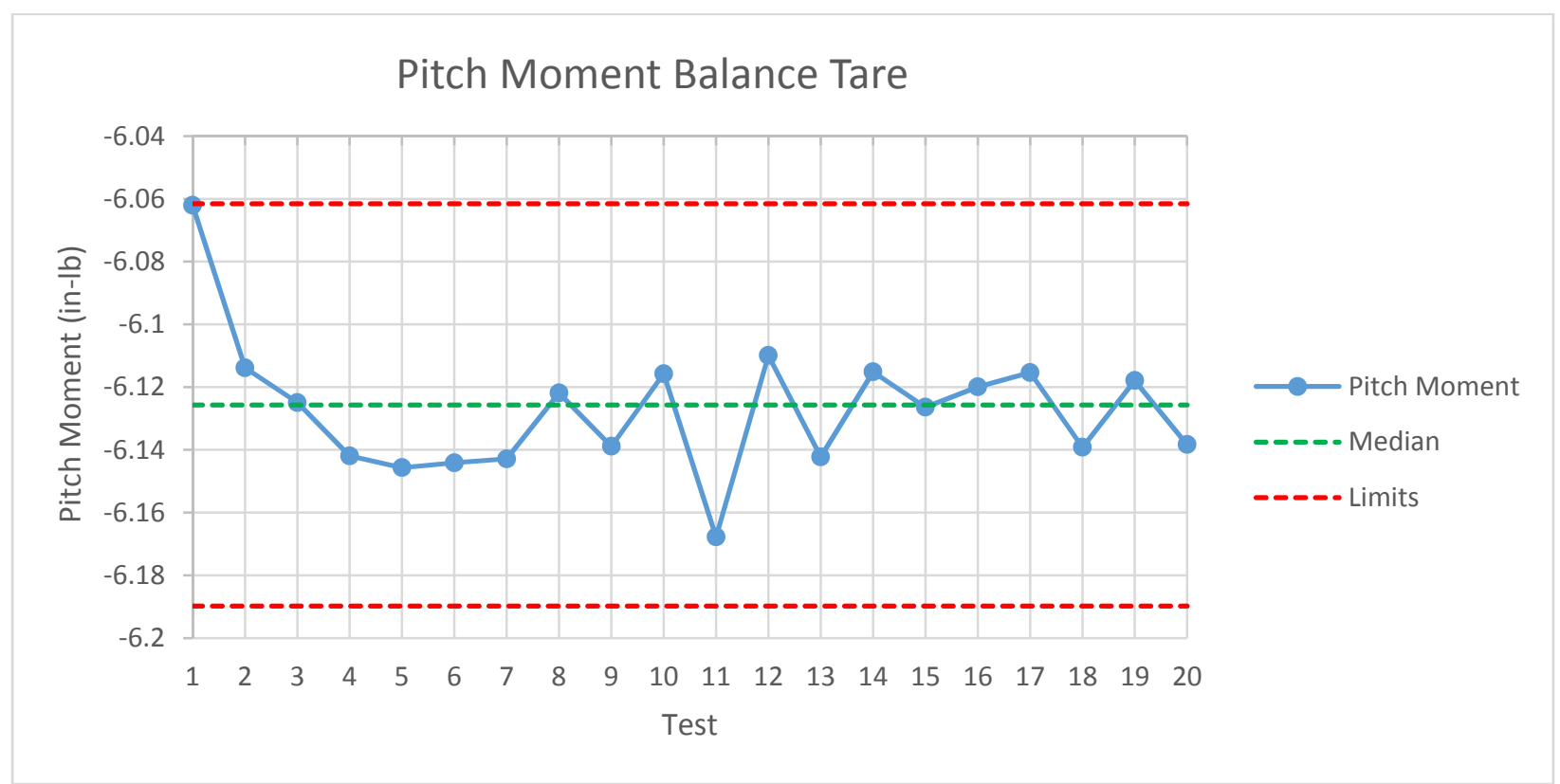

Figure 104 Pitch Moment Balance Tare 
While there appears to be a similar trend across each of the responses for the first 3 tests, it is not of the magnitude to cause any concern in the health of the balance or overall quality of the data. Given these charts, there can be a high level of confidence that the balance is performing as it should.

\subsection{Flow Angularity}

A separate response in addition to the three balance force coefficients acquired during the airframe testing was the tunnel flow angularity. The test design allows for the calculation of both pitch flow and cross flow angularity. It is important to note that for the testing completed and the processes outlined, the flow angularities obtained are not strictly the aerodynamic flow angles. They are a combination of aerodynamic flow angularity, model misalignment and other process driven factors. Both flow angularity responses should be viewed as quality control variables that represent the actual aerodynamic flow angularities. The flow angularity was obtained through an adaptation of the framework developed by Hemsch ${ }^{36}$

\subsubsection{Pitch Flow Angularity}

At each dynamic pressure tested, both upright $\left(\phi=0^{\circ}\right)$ and inverted $\left(\phi=180^{\circ}\right)$, the normal force coefficient was fit as a function of angle of attack across the range of angle of attack tested $\left(-2^{\circ}\right.$ to $\left.6^{\circ}\right)$. Thus Equations 84 and 85 would be obtained for each level of dynamic pressure which would result in six total equations. Due to the nature of the aerodynamics of the model tested, a second order model in angle of attack was necessary to properly characterize the normal force coefficient.

$$
\begin{aligned}
& C_{N_{U P}}=C_{N_{0}}+C_{N_{\alpha}} \alpha+C_{N_{\alpha^{2}}} \alpha^{2} \\
& C_{N_{I N V}}=C_{N_{0}}+C_{N_{\alpha}} \alpha+C_{N_{\alpha^{2}}} \alpha^{2}
\end{aligned}
$$

For each curve fit at a given dynamic pressure, the normal force coefficient at zero angle of attack, $C_{N_{0}}$ is found for the upright and inverted case, $C_{N_{0} U P}$ and $C_{N_{0} I N V}$ respectively and then averaged to obtain $\overline{C_{N_{0}}}$. Then for each level of dynamic pressure, Equations 84 and 85 are solved for the angle of 
attack, $\alpha^{*}$ that gives the averaged zero angle of attack normal force coefficient. Finally, the pitch flow angularity is found by Equation 86 below.

$$
\text { Pitch }_{F A}=0.5\left(\alpha_{I N V}^{*}-\alpha_{U P}^{*}\right)
$$

The set of calculations for Test 9 is shown as an example in Table 38.

\begin{tabular}{|l|c|c|c|l|l|l|}
\hline $\boldsymbol{q}(\mathbf{P a})$ & $\boldsymbol{C}_{\boldsymbol{N}_{\mathbf{0}} \boldsymbol{U P}}$ & $\boldsymbol{C}_{\boldsymbol{N}_{\mathbf{0}}}$ & $\overline{\boldsymbol{C}_{\boldsymbol{N}_{\mathbf{0}}}}$ & $\boldsymbol{\alpha}_{\boldsymbol{U} \boldsymbol{P}}^{*}$ & $\boldsymbol{\alpha}_{\boldsymbol{I N V}}^{*}$ & Pitch $_{\boldsymbol{F A}}$ \\
\hline $\mathbf{4 4 4}$ & -0.03617 & -0.01245 & -0.02431 & 0.2165 & -0.2236 & -0.22005 \\
\hline $\mathbf{7 5 0}$ & -0.03414 & -0.01474 & -0.02444 & 0.1718 & -0.1766 & -0.1742 \\
\hline $\mathbf{9 8 0}$ & -0.03261 & -0.01647 & -0.02454 & 0.14 & -0.1434 & -0.1417 \\
\hline
\end{tabular}

Table 38 Test 9 Pitch Flow Angularity Calculations

Both pitch and cross flow angularity were obtained for each level of dynamic pressure for each of the twenty airframe tests. These values were then charted using the standard SPC practice for across-test charts where the limits are given by Equations 58 and 59 . The pitch flow angularity SPC charts are given in Figure 105, Figure 106 and Figure 107.

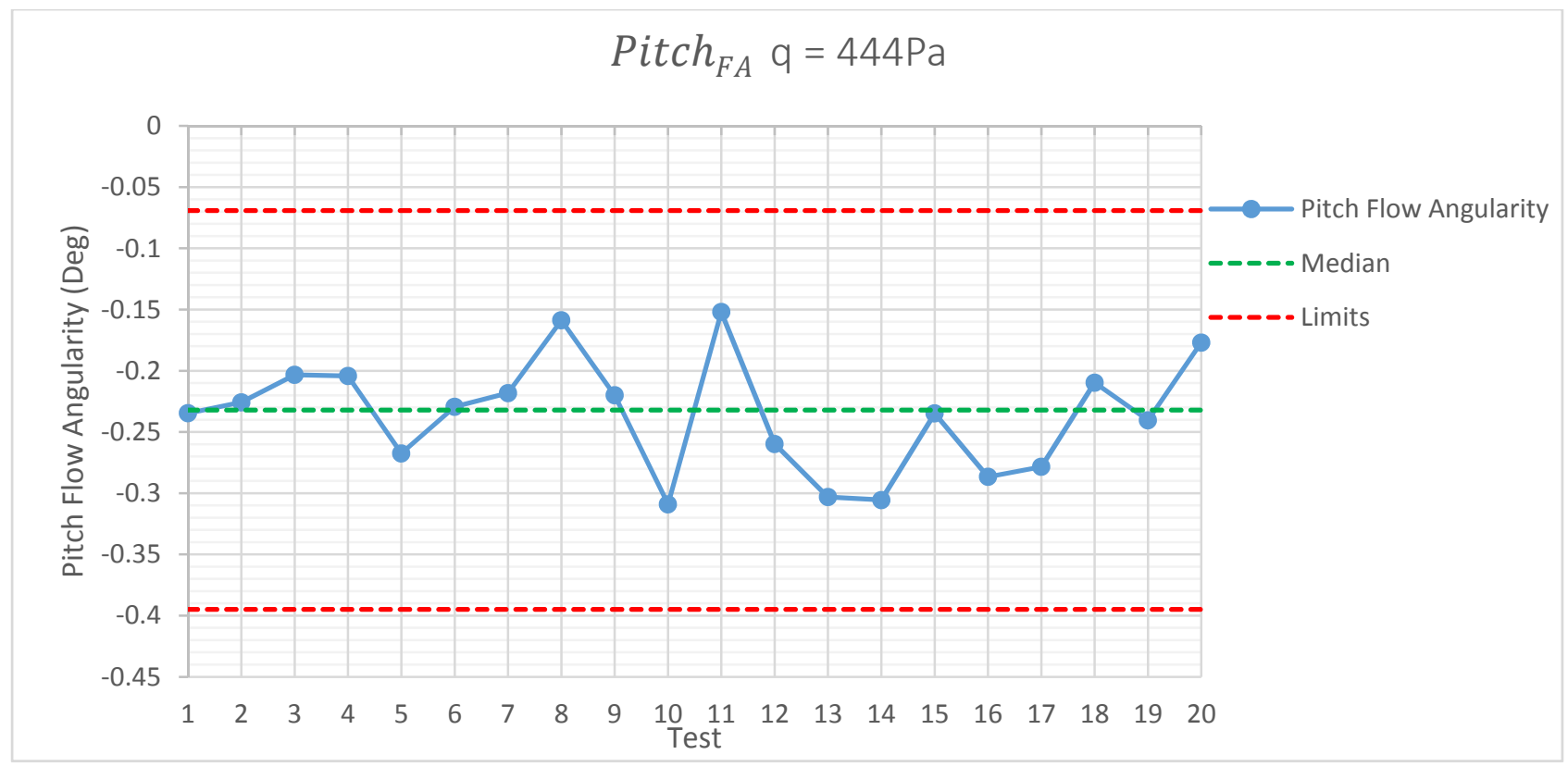

Figure 105 Pitch Flow Angularity Control Chart q=444Pa 


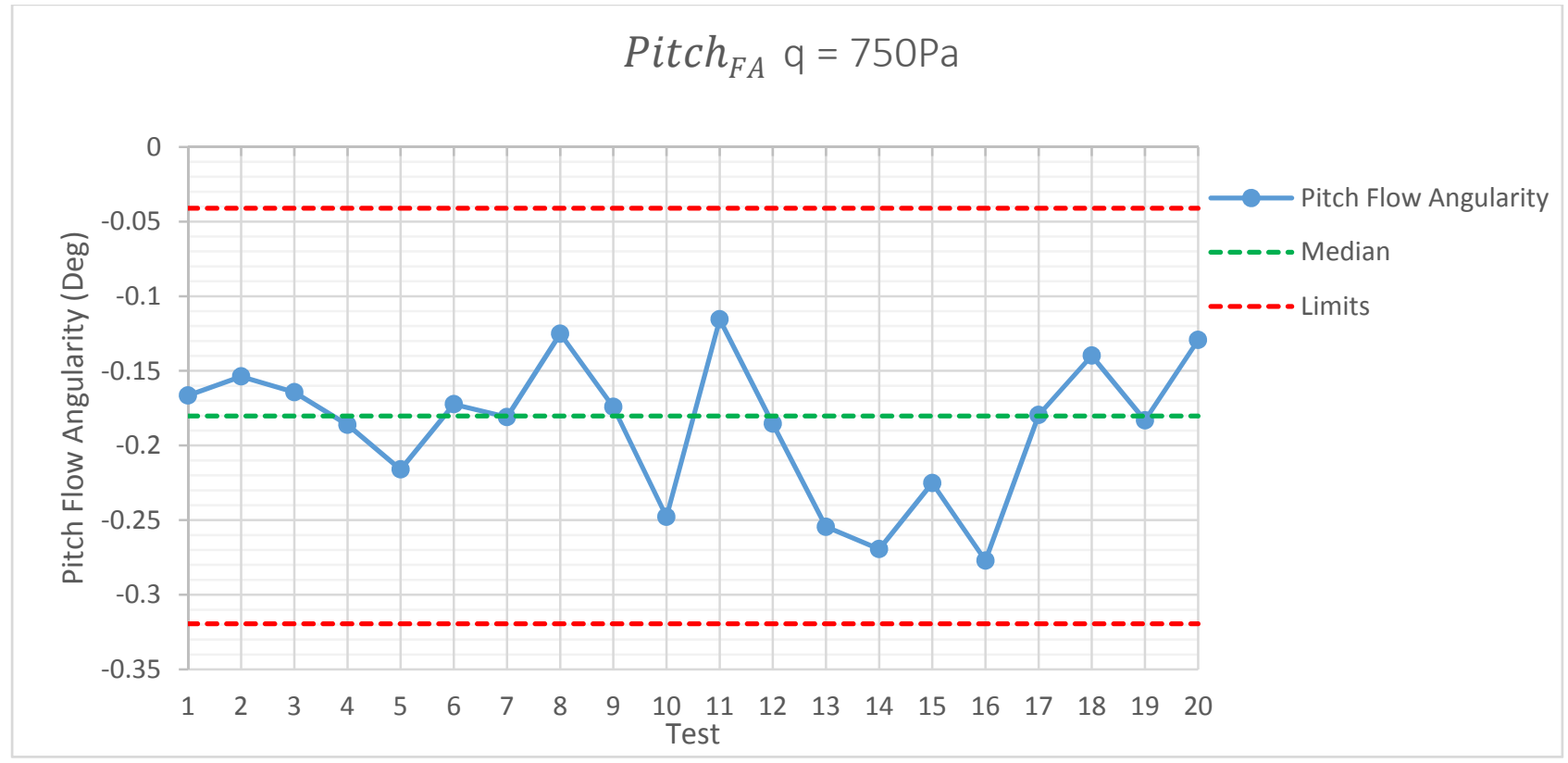

Figure 106 Pitch Flow Angularity Control Chart q=750Pa

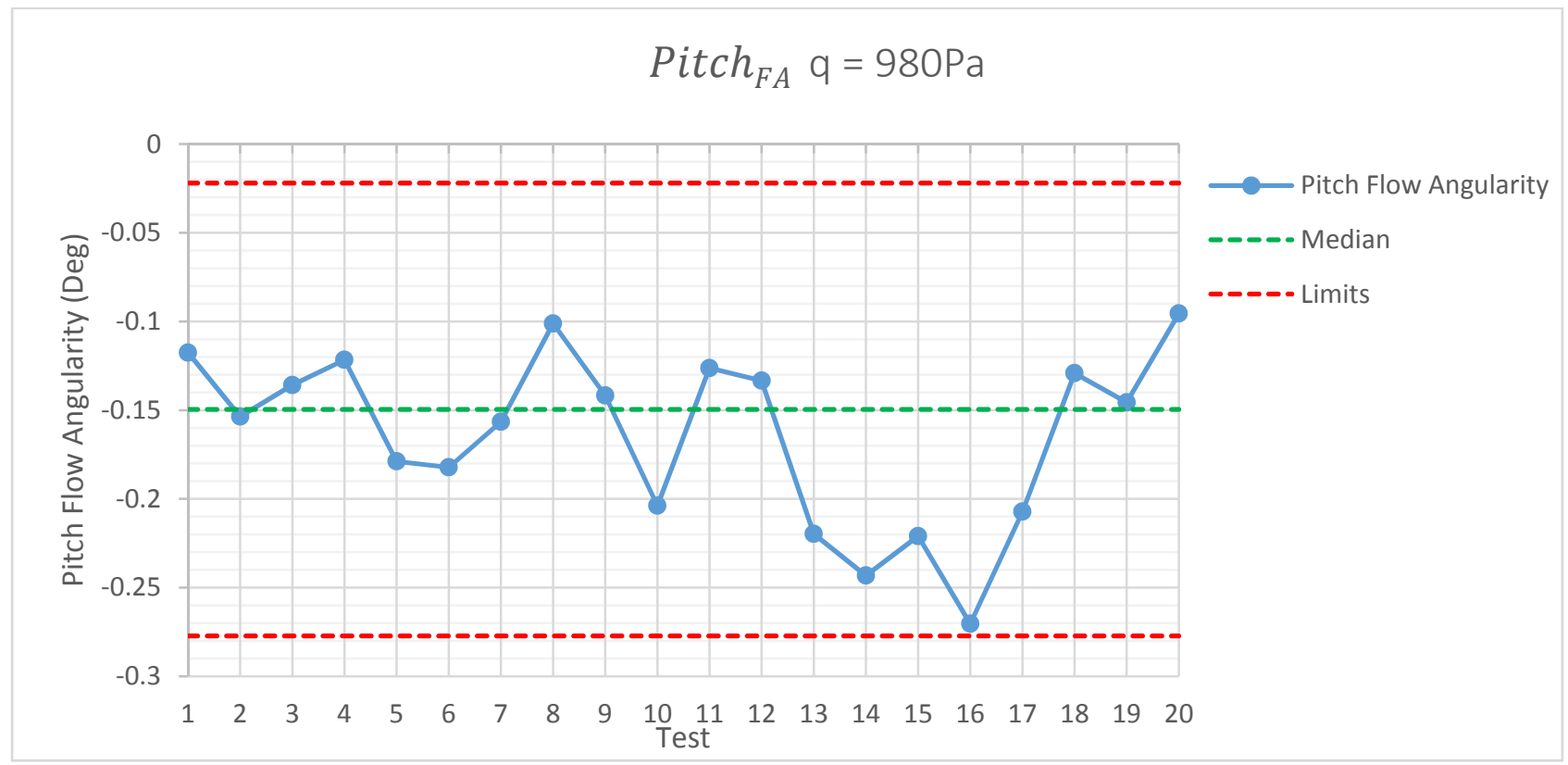

Figure 107 Pitch Flow Angularity Control Chart q=980Pa 
From inspecting the pitch flow angularity charts above it is seen that the pitch flow angularity response remains in control for all levels of dynamic pressure.

\subsubsection{Cross Flow Angularity}

The added runs for the calculation of cross flow angularity are shown as an example from test 12's run schedule in Table 36. The order of the runs was fully randomized within the whole plots (levels of dynamic pressure) and the ordering of the whole plots was randomized. At each level of dynamic pressure three data points at each of $\phi=90$ (Right) and $\phi=-90$ (Left) were gathered (green points in Figure 75). With the added runs for the calculation of cross flow angularity, the total number of runs for each airframe test was 120 .

\begin{tabular}{|c|c|c|c|c|c|c|c|}
\hline Run & $q$ & $\alpha$ & $\phi$ & Run & $q$ & $\alpha$ & $\phi$ \\
\hline 1 & 980 & 0 & Right & 61 & 865 & 0 & Right \\
\hline 5 & 980 & 0 & Right & 66 & 865 & 0 & Left \\
\hline 9 & 980 & 0 & Left & 71 & 444 & 0 & Right \\
\hline 12 & 980 & 0 & Left & 77 & 444 & 0 & Right \\
\hline 13 & 980 & 0 & Left & 79 & 444 & 0 & Left \\
\hline 14 & 980 & 0 & Right & 80 & 444 & 0 & Right \\
\hline 21 & 600 & 0 & Left & 81 & 444 & 0 & Left \\
\hline 24 & 600 & 0 & Right & 82 & 444 & 0 & Left \\
\hline 25 & 980 & 0 & Left & 85 & 750 & 0 & Left \\
\hline 26 & 980 & 0 & Right & 87 & 750 & 0 & Left \\
\hline 28 & 980 & 0 & Right & 88 & 750 & 0 & Right \\
\hline 31 & 980 & 0 & Left & 95 & 750 & 0 & Left \\
\hline 34 & 980 & 0 & Left & 97 & 750 & 0 & Right \\
\hline 42 & 980 & 0 & Right & 98 & 750 & 0 & Right \\
\hline 53 & 750 & 0 & Right & 103 & 444 & 0 & Left \\
\hline 54 & 750 & 0 & Right & 104 & 444 & 0 & Right \\
\hline 55 & 750 & 0 & Right & 105 & 444 & 0 & Right \\
\hline 56 & 750 & 0 & Left & 107 & 444 & 0 & Left \\
\hline 57 & 750 & 0 & Left & 108 & 444 & 0 & Right \\
\hline 59 & 750 & 0 & Left & 112 & 444 & 0 & Left \\
\hline
\end{tabular}

Table 39 Example Cross Flow Angularity Run Schedule

The procedure to calculate the cross flow angularity was similar to the calculation of the pitch flow angularity but without a separate $C_{N}$ versus alpha curve. The values of normal force coefficients were averaged at each dynamic pressure and each roll angle to give ${\overline{C_{N}}}_{\text {Right }}$ and ${\overline{C_{N}}}_{\text {Left }}$. Then, these values 
were substituted into the average of Equations 84 and 85 to give the "true" value of the $C_{N}$ versus alpha curve. Next the angle of attack that resulted in the averaged normal force coefficient was solved to give $\alpha_{\text {Right }}^{*}$ and $\alpha_{\text {Left }}^{*}$. Finally the cross flow angularity is found by

$$
\operatorname{Cross}_{F A}=0.5\left(\alpha_{R i g h t}^{*}-\alpha_{\text {Left }}^{*}\right)
$$

\begin{tabular}{|c|c|c|c|c|c|}
\hline$q(\mathrm{~Pa})$ & ${\overline{C_{N}}}_{R i g h t}$ & ${\overline{C_{N}}}_{L e f t}$ & $\alpha_{\text {Right }}^{*}$ & $\alpha_{\text {Left }}^{*}$ & $\operatorname{Cross}_{F A}$ \\
\hline 444 & 0.012659 & -0.07313 & 0.84425 & -0.74045 & 0.79235 \\
\hline 600 & 0.012554 & -0.06962 & 0.7722 & -0.72705 & 0.749625 \\
\hline 750 & 0.010587 & -0.05334 & 0.6726 & -0.46985 & 0.571225 \\
\hline 865 & 0.00657 & -0.04881 & 0.5548 & -0.42765 & 0.491225 \\
\hline 980 & 0.009502 & -0.04684 & 0.55585 & -0.43335 & 0.4946 \\
\hline
\end{tabular}

Table 40 Test 12 Cross Flow Angularity Calculations

The calculation of cross flow angularity in this method gives an estimate which does not have the same statistical power as the pitch flow angularity due to the fact that only single points at zero degrees angle of attack were obtained instead of building another set of $C_{N}$ curves. It is possible to build separate curves of the normal force coefficient which would give more insight into the cross flow angularity however the increase in the number of runs per test put this method out of reach for this initial research. To find the "true" cross flow angularities with the airframe model, four distinct pitch polars would be necessary. As was discussed earlier in this section, both flow angularities should be treated as quality variables with more importance placed on the variation in the values over time, not necessarily the relation to the "true" aerodynamic flow angles.

Similar to the pitch flow angularities, the cross flow angularities are charted in the standard SPC test control fashion. The cross flow angularity control charts are given in Figure 108, Figure 109 and Figure 110. 


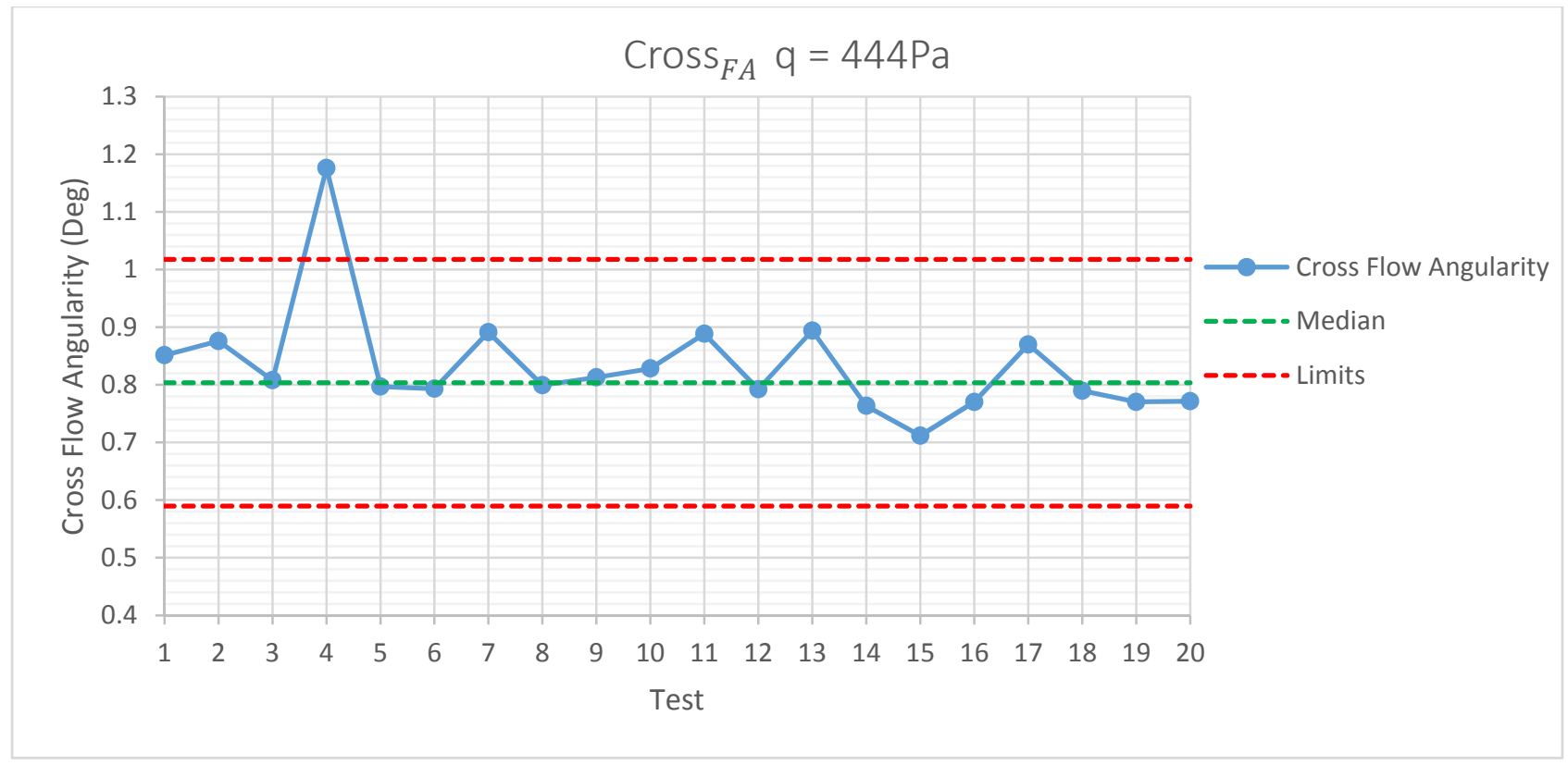

Figure 108 Cross Flow Angularity Control Chart $q=444 \mathrm{~Pa}$

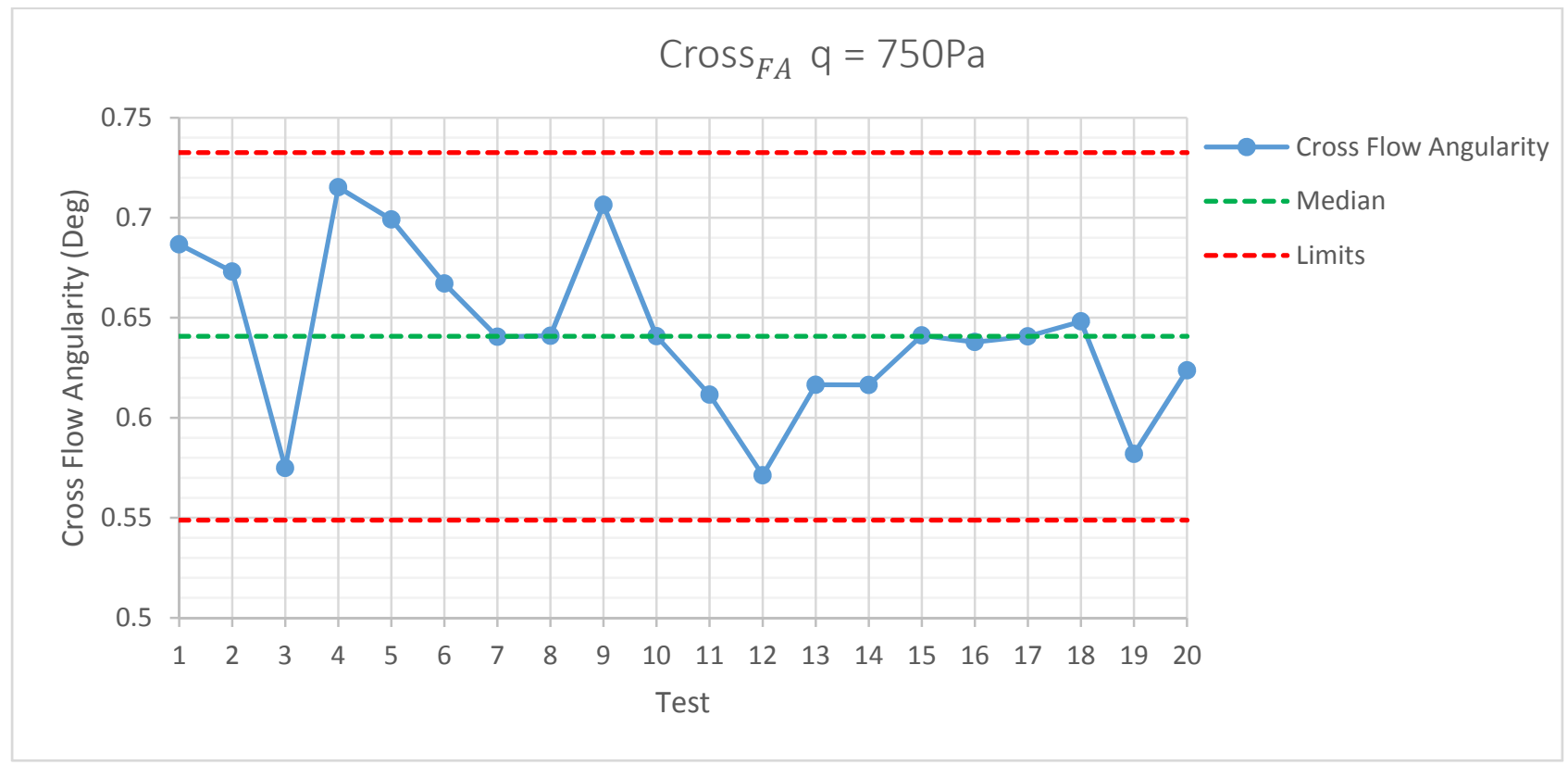

Figure 109 Cross Flow Angularity Control Chart $q=750 P a$ 


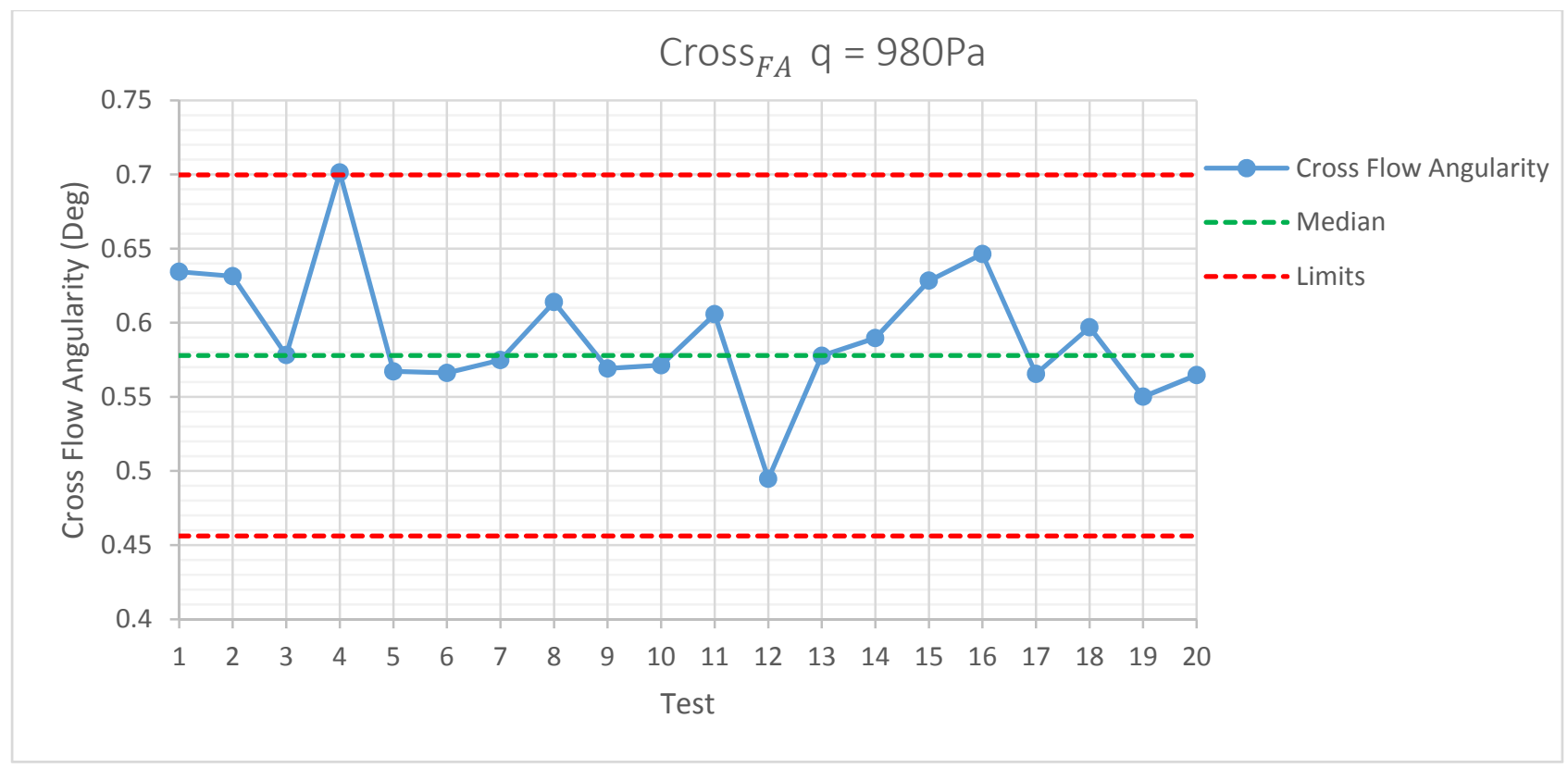

Figure 110 Cross Flow Angularity Control Chart q=980Pa

From inspection of the cross flow angularity charts above, the cross flow angularity could be considered sufficiently close to statistical control such that the testing completed could serve as the baseline for further research.

\subsection{Use of DOE and Regression Modeling}

This section will detail the development of the regression models for both the flow angularities and balance force coefficients. Both the local and global regression model builds for the balance force coefficients will be explored. The methodology and use of the prediction interval generated from the balance force coefficients global regression models will be described.

\subsubsection{Flow Angularity Regression Modeling}

The goal in obtaining flow angularities at three discrete levels was to build a regression model as a function of dynamic pressure as shown in Equations 88 and 89 below. 


$$
\begin{aligned}
& \operatorname{Pitch}_{F A}=f(q) \\
& \operatorname{Cross}_{F A}=f(q)
\end{aligned}
$$

To build the regression model for both flow angularities the data set from all twenty baseline tests was utilized. For example, each test used three data points, one for each level of dynamic pressure, giving a total of 60 data points. The method for building the model was a simple least squares regression as there was only one factor involved. The results from the regression model in engineering units of degrees and Pascals are shown below for both the pitch and cross flow angularities

$$
\begin{aligned}
& \operatorname{Pitch}_{F A}=0.000135 q-0.0294 \\
& \operatorname{Cross}_{F A}=-0.000460 q+1.02
\end{aligned}
$$

If the responses were treated as true aerodynamic flow angularities, both angles could now be identified throughout the entire design space (in this case dynamic pressure range). A second benefit to the development of a regression model for flow angularity and the initial split-plot design is the adaptability of the methodology. Any factor of interest thought to have an effect on flow angularity could be explored.

\subsubsection{Balance Force Coefficients: Local Regression Models}

A regression model was built at the local level (each test) for all three responses using the data from the split-plot design and REML analysis, where only the statistically significant terms were used to build the regression model. The fitted regression model for the normal force coefficient is given as Equation 92. In each of the responses, the dynamic pressure term $\beta_{1}$ was retained to maintain hierarchy. For all responses, this term was either not significant or marginally significant at the $5 \%$ level of significance. For example in test 8 , for the normal force coefficient response, the dynamic pressure term was not significant, however in test 9 the same term was marginally significant. 


$$
C_{N}=\beta_{0}+\beta_{1} q+\beta_{2} \alpha+\beta_{3} \phi+\beta_{13} q \alpha+\beta_{11} \alpha^{2}
$$

An example output from the normal force coefficient REML analysis from Test 18 is given in Table 41. It is seen that the dynamic pressure terms are not significant but interactions with the dynamic pressure and other factors are. The last column in the table, labeled " $\mathrm{p}$-value Prob $>\mathrm{F}$ " is a measure of model term significance (based on an F-test at a significance level of 0.05 ), the smaller the value, the more statistically significant. In this particular local test, some reduced cubic terms were statistically significant. This was not the case for the majority of other local tests. As expected for the normal force coefficient, the angle of attack term is highly significant. Investigating the ratio of variance components of whole plot to the sub plot in Table 41 shows that the variance ratio is approximately 0.9 . From the split-plot analysis ${ }^{10}$, if the variance ratio is less than one, the design can be analyzed as a completely randomized design. Due to the fact that on a test-to-test basis, this variance ratio varied above and below one, each local regression model was analyzed using REML for consistency. An advantage to the REML analysis in JMP for split-plot designs is the ability to have confidence intervals placed on the variance components. With so few degrees of freedom for the whole plot in this analysis, the interval on the whole plot variance contains zero. Also note for this test, the very high R-Squared and Adjusted R-Squared values indicating a very good model fit. A third variance component, labeled Tare in Table 41, is the variance associated with the three different tares for this test, treated as a blocking effect. For a model having no differences in the individual tares, this variance component should be low. 


\begin{tabular}{|c|c|c|c|c|}
\hline \multicolumn{5}{|c|}{ REML Analysis for selected model } \\
\hline \multicolumn{5}{|l|}{ Fixed Effects } \\
\hline & Term & Error & $\mathrm{F}$ & $p$-value \\
\hline Source & df & df & Value & Prob $>$ F \\
\hline Whole-plot & 2 & 0.998618 & 0.859524 & 0.6066 \\
\hline$q$ & 1 & 1.684183 & 1.727491 & 0.339398 \\
\hline$q^{2}$ & 1 & 3.015873 & 1.444098 & 0.315281 \\
\hline Subplot & 9 & 56.01674 & 82541.96 & 0 \\
\hline$\alpha$ & 1 & 56.04541 & 221818.2 & 0 \\
\hline$\phi$ & 1 & 56.03381 & 298.1321 & 0 \\
\hline$q \alpha$ & 1 & 56.00869 & 247.0389 & 0 \\
\hline$q \phi$ & 1 & 56.00869 & 25.59761 & $4.84 \mathrm{E}-06$ \\
\hline$\alpha \phi$ & 1 & 56.0215 & 7.0145 & 0.010484 \\
\hline$\alpha^{2}$ & 1 & 56.01311 & 4961.427 & 0 \\
\hline$q \alpha \phi$ & 1 & 56.00887 & 6.814053 & 0.011582 \\
\hline$q^{2} \alpha$ & 1 & 56.03389 & 5.827838 & 0.019066 \\
\hline$q^{2} \phi$ & 1 & 56.02578 & 5.332264 & 0.024645 \\
\hline \multicolumn{5}{|c|}{ Variance Components } \\
\hline Source & Variance & StdErr & $\begin{array}{l}95 \% \mathrm{Cl} \\
\text { Low }\end{array}$ & $\begin{array}{l}95 \% \mathrm{Cl} \\
\text { High }\end{array}$ \\
\hline Tare & $-1.7 \mathrm{E}-08$ & $3.6 \mathrm{E}-06$ & $-7.1 \mathrm{E}-06$ & $7.04 \mathrm{E}-06$ \\
\hline Whole Plot & $3.82 \mathrm{E}-06$ & 4.17E-06 & $-4.4 \mathrm{E}-06$ & $1.2 \mathrm{E}-05$ \\
\hline Sub Plot & 4.26E-06 & 8.06E-07 & 3.04E-06 & $6.42 \mathrm{E}-06$ \\
\hline Total & 8.06E-06 & & & \\
\hline $\begin{array}{l}-2 \text { Log } \\
\text { Likelihood }\end{array}$ & -532.726 & & $\mathrm{BIC}$ & -468.786 \\
\hline R-Squared & 0.999906 & & $\mathrm{AIC}$ & -502.726 \\
\hline Adj R-Squared & 0.999887 & & $\mathrm{AlCc}$ & -493.999 \\
\hline
\end{tabular}

Table 41 JMP REML Analysis for the Normal Force Coefficient from Test 18

An advantage in displaying the model in terms of coded factors (centered and scaled) is that the coefficients are non-dimensional and their relative magnitudes may be compared directly. From this comparison the most influential factors on the response can be seen. Later it is shown by observing Equation 95 (the global model), as expected, the most influential factors on the normal force coefficient 
are the angle of attack and angle of attack squared $\left(\beta_{1}\right.$ and $\left.\beta_{11}\right)$. Although all significant model terms can be charted, only the most influential model terms will be shown in the standard SPC chart format.

The $\beta_{0}$ coefficient control chart shown in Figure 111 represents the intercept of the regression model. This chart is the most directly relatable to the single point values of the traditional SPC charts currently utilized to track the balance force coefficients as it represents the design point where all factors are at their center (or pseudo center for the categorical roll factor) point. Therefore, the chart below could be viewed as the normal force coefficient response at the average of the upright and inverted roll conditions at the design point $\alpha=2^{\circ}, q=712 \mathrm{~Pa}$.

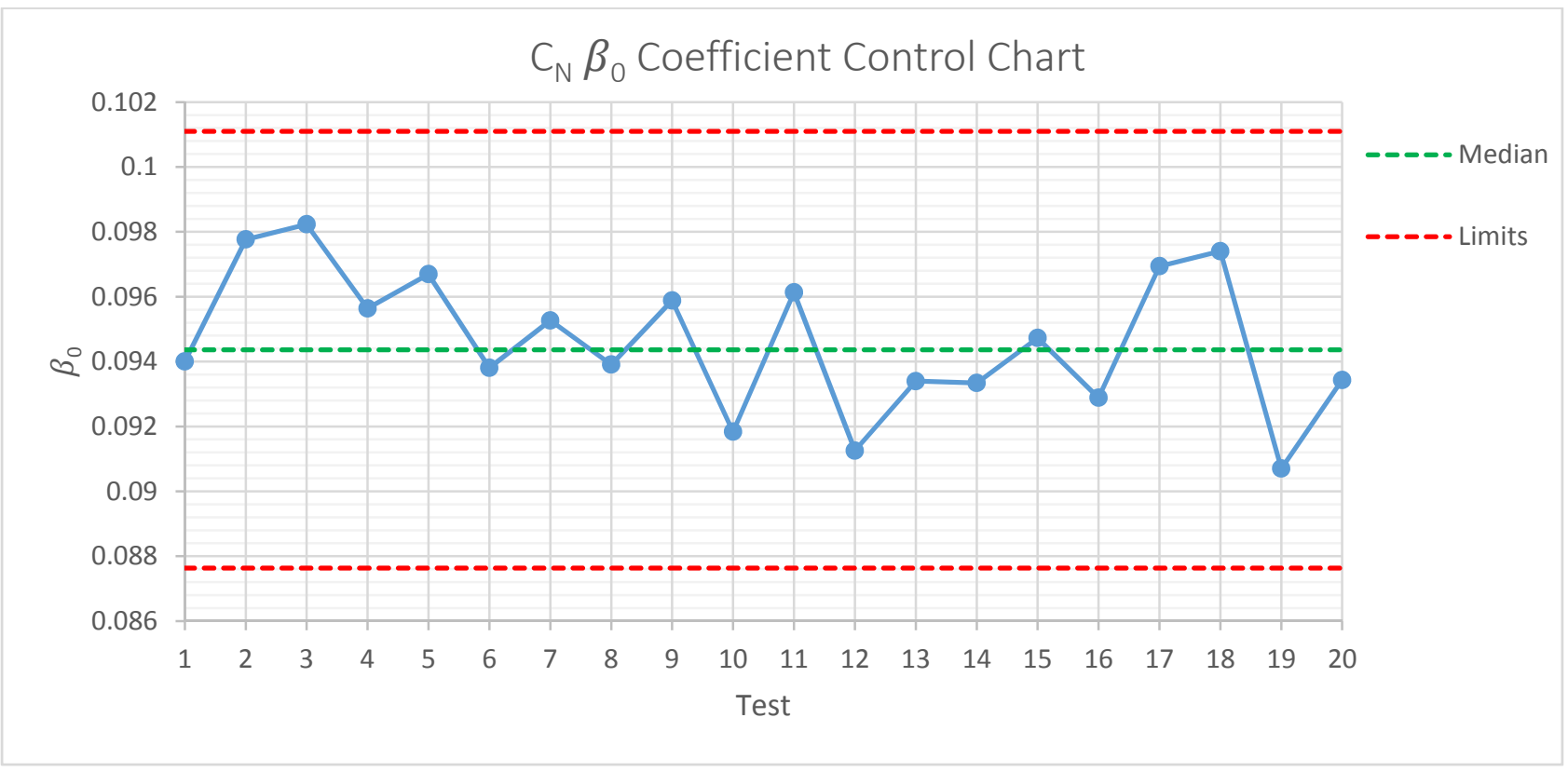

Figure $111 C_{N} \beta_{0}$ Coefficient Control Chart 


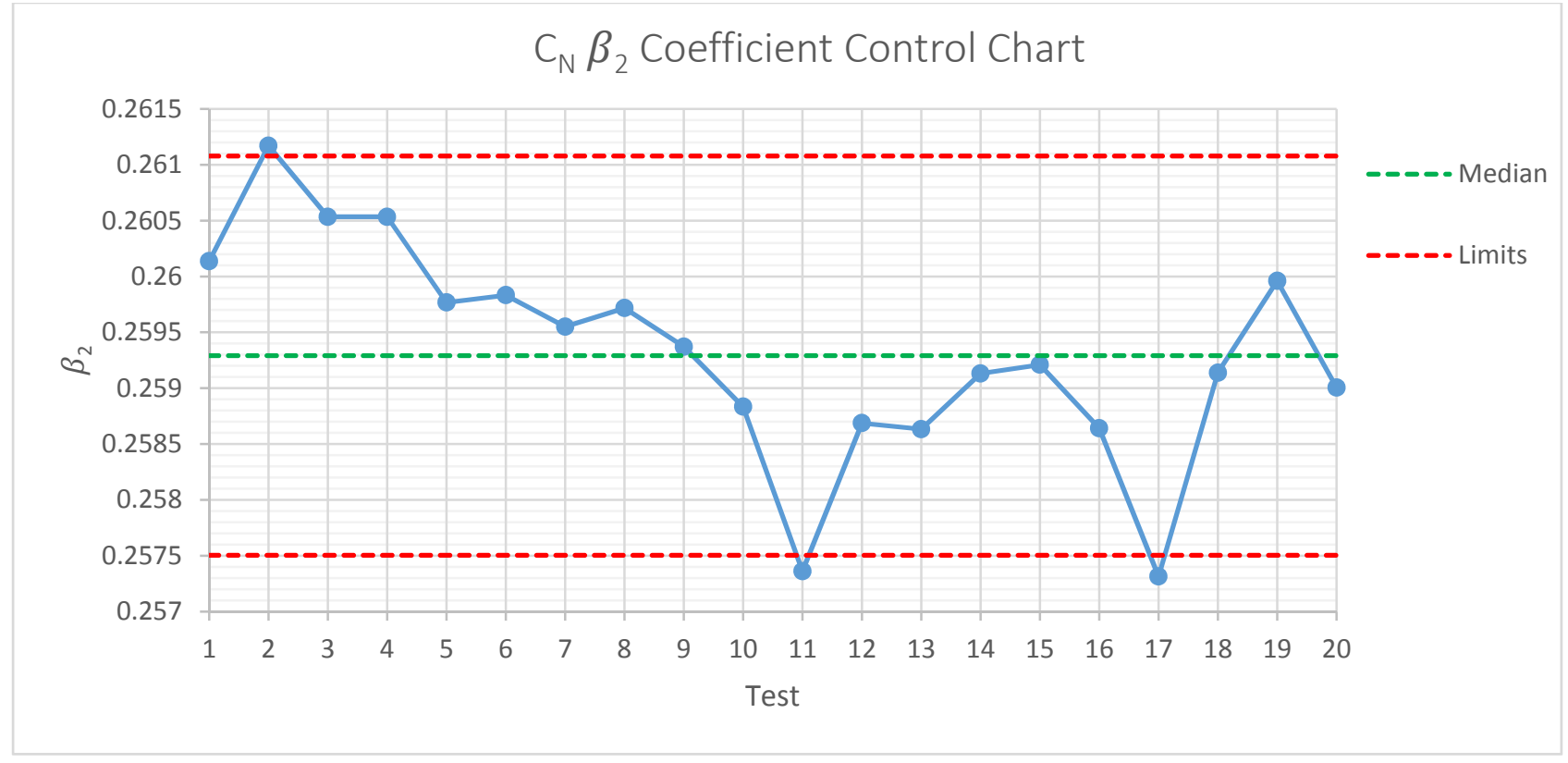

Figure $112 C_{N} \beta_{2}$ Coefficient Control Chart

The above control chart for $\beta_{2}$ is similar to a lift curve slope coefficient. The downward trend in the chart suggests a problem associated with setting or measuring angle of attack. The process of tracking the regression model coefficients proves its value with the above figure. The individual point value statistical process control charts may indicate that the normal force coefficients are in statistical control, but by charting the regression model coefficients, the components of the overall value can be scrutinized. For example, Equation 92 can be viewed as a summation of the contributions to the overall normal force coefficient from the individual factors (or interaction of factors). Therefore, it is possible that the overall variation in $\mathrm{C}_{\mathrm{N}}$ may be relatively constant but the underlying contributions of individual factors may be varying greatly. Understanding the variation in the contributing factors of the overall force coefficient values will allow for a better understanding of the variation of the force coefficient itself.

The $\beta_{3}$ coefficient control chart shown in Figure 113 could be viewed as a quality control variable related to tunnel pitch flow angularity. This chart shows the effect of the roll position of the model (Upright/Inverted) on the overall normal force coefficient. The magnitude of this regression model coefficient directly relates to the magnitude of the overall flow angularity. 


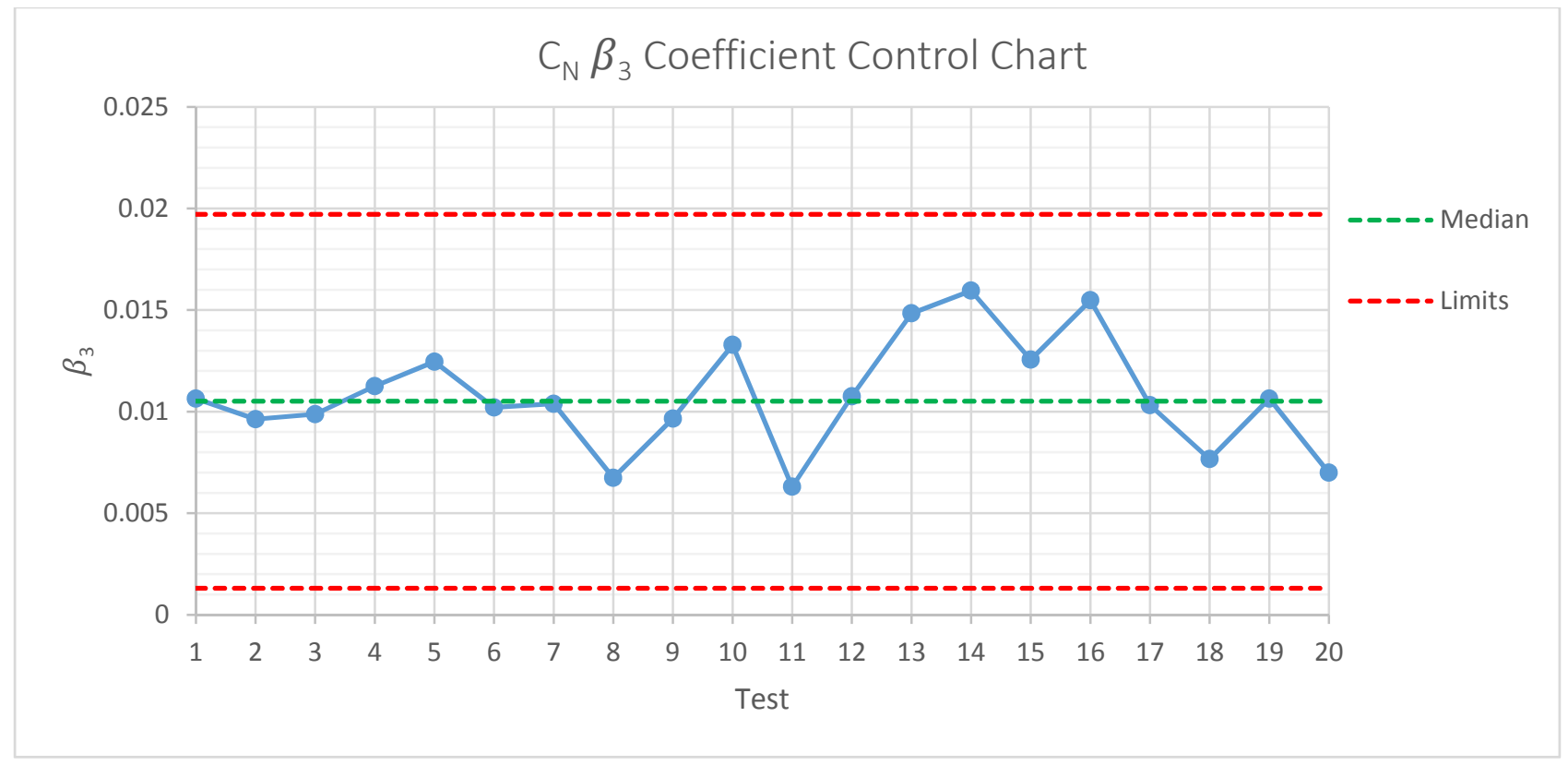

Figure $113 C_{N} \beta_{3}$ Coefficient Control Chart

The control chart in Figure 114 tracks the angle of attack squared coefficient which is most probably attributed to the wing leading edge delta-like sweep. Tracking the value is important due to the influence of the term on the overall response. 


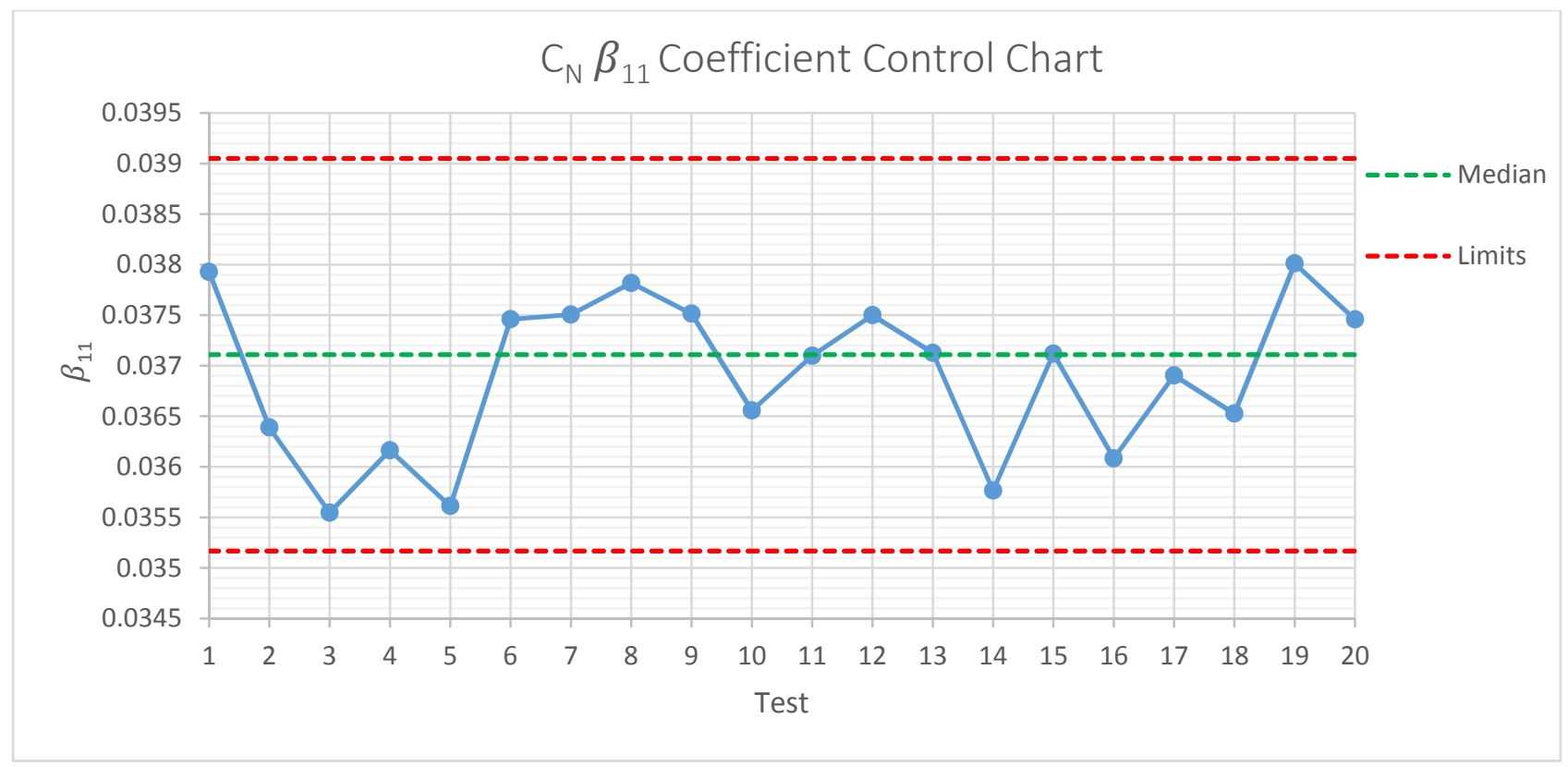

Figure $114 C_{N} \beta_{11}$ Coefficient Control Chart

The next three figures will show the regression coefficient charts associated with the axial force coefficient response. The axial force coefficient was previously defined in Equation 7 in Chapter 2 as the normal force divided by the reference area multiplied by the dynamic pressure. 


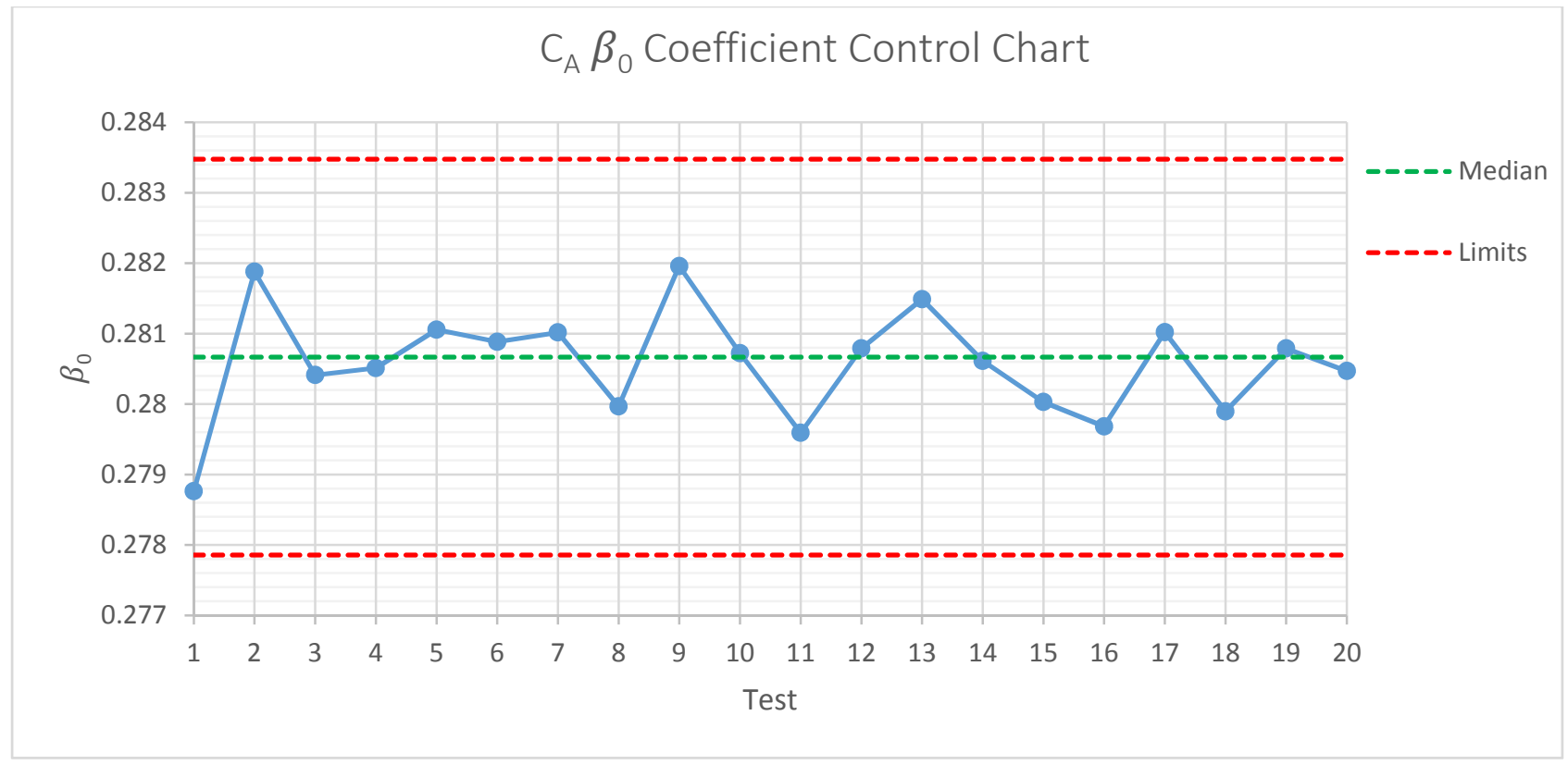

Figure $115 C_{A} \beta_{0}$ Coefficient Control Chart

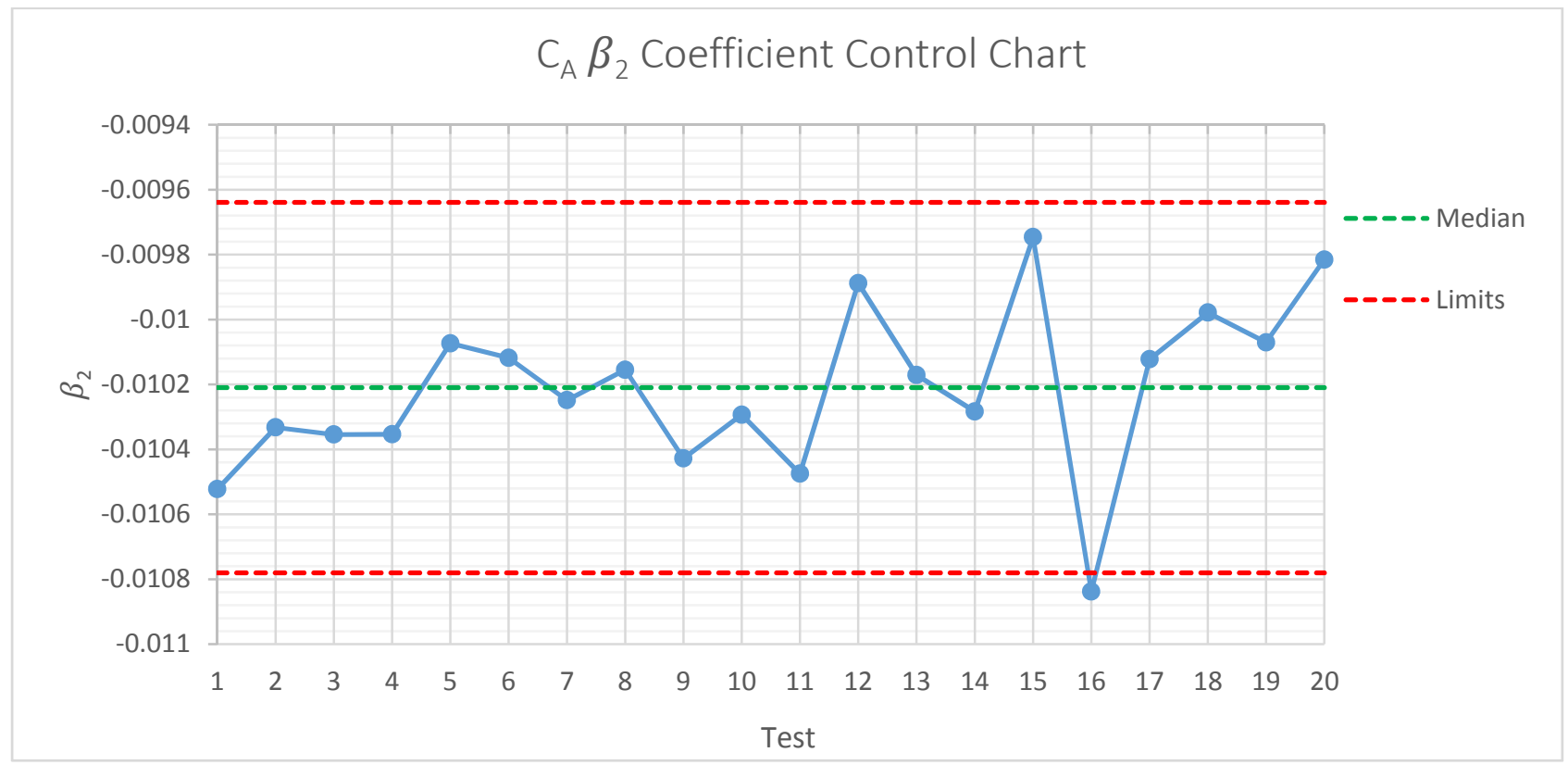

Figure $116 C_{A} \beta_{2}$ Coefficient Control Chart 


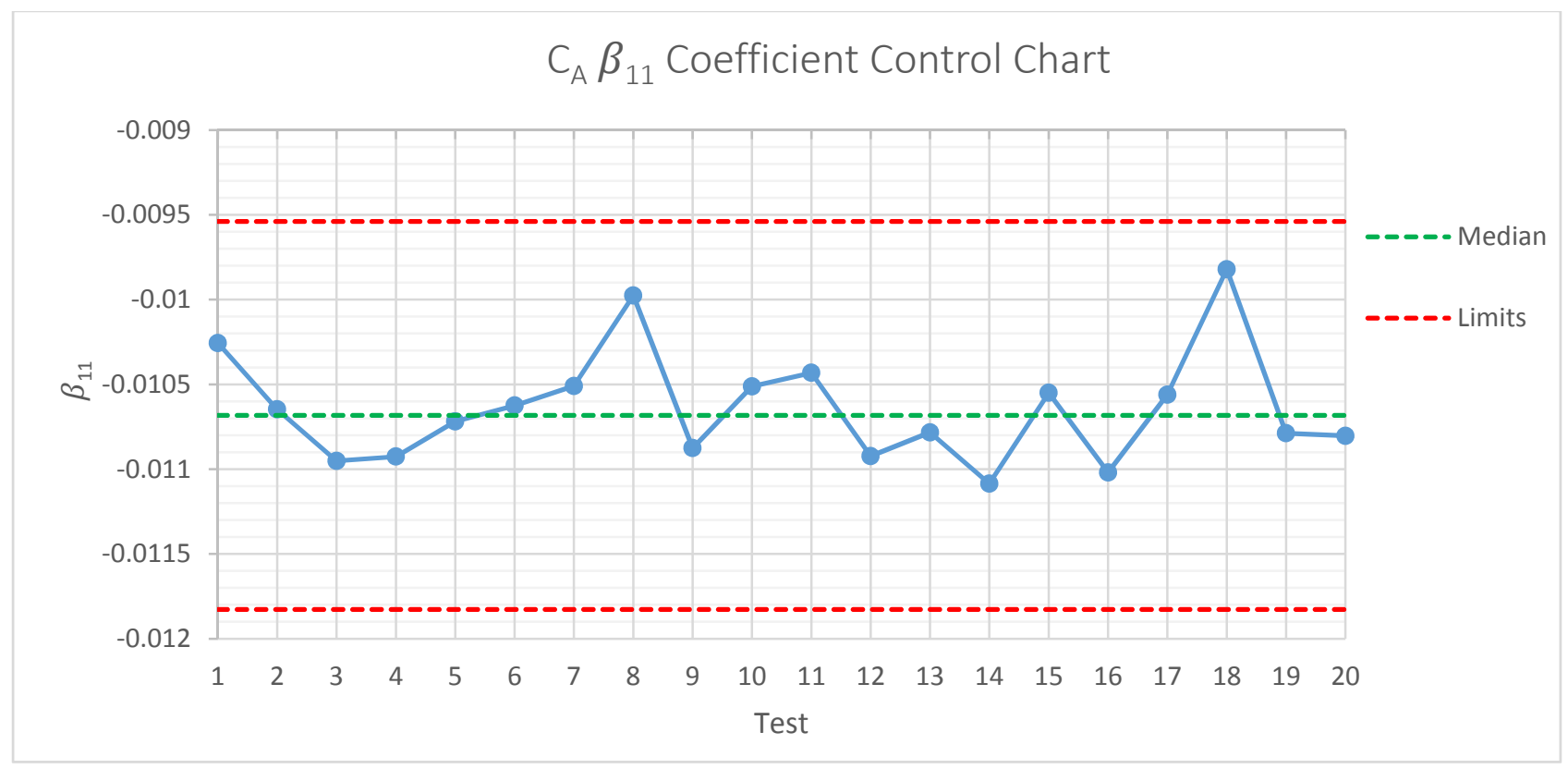

Figure $117 C_{A} \beta_{11}$ Coefficient Control Chart

The next three figures will detail the regression coefficient charts associated with the pitching moment coefficient response. The pitching moment coefficient was previously defined in Equation 7 in Chapter 2. 


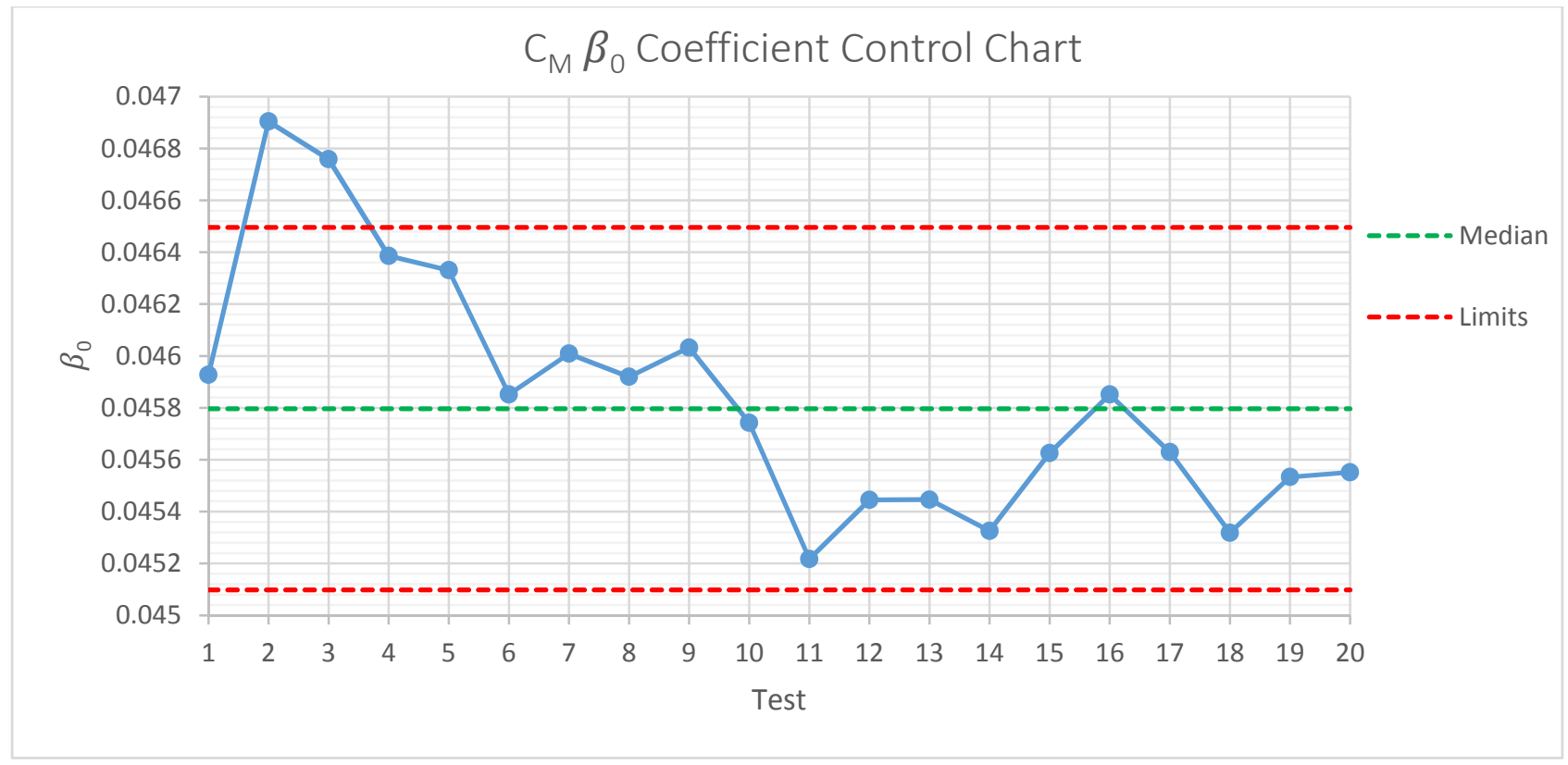

Figure $118 C_{M} \beta_{0}$ Coefficient Control Chart

As was seen in the individual control charts, the pitching moment coefficient was least likely to remain in statistical control which is again reinforced by Figure 118.

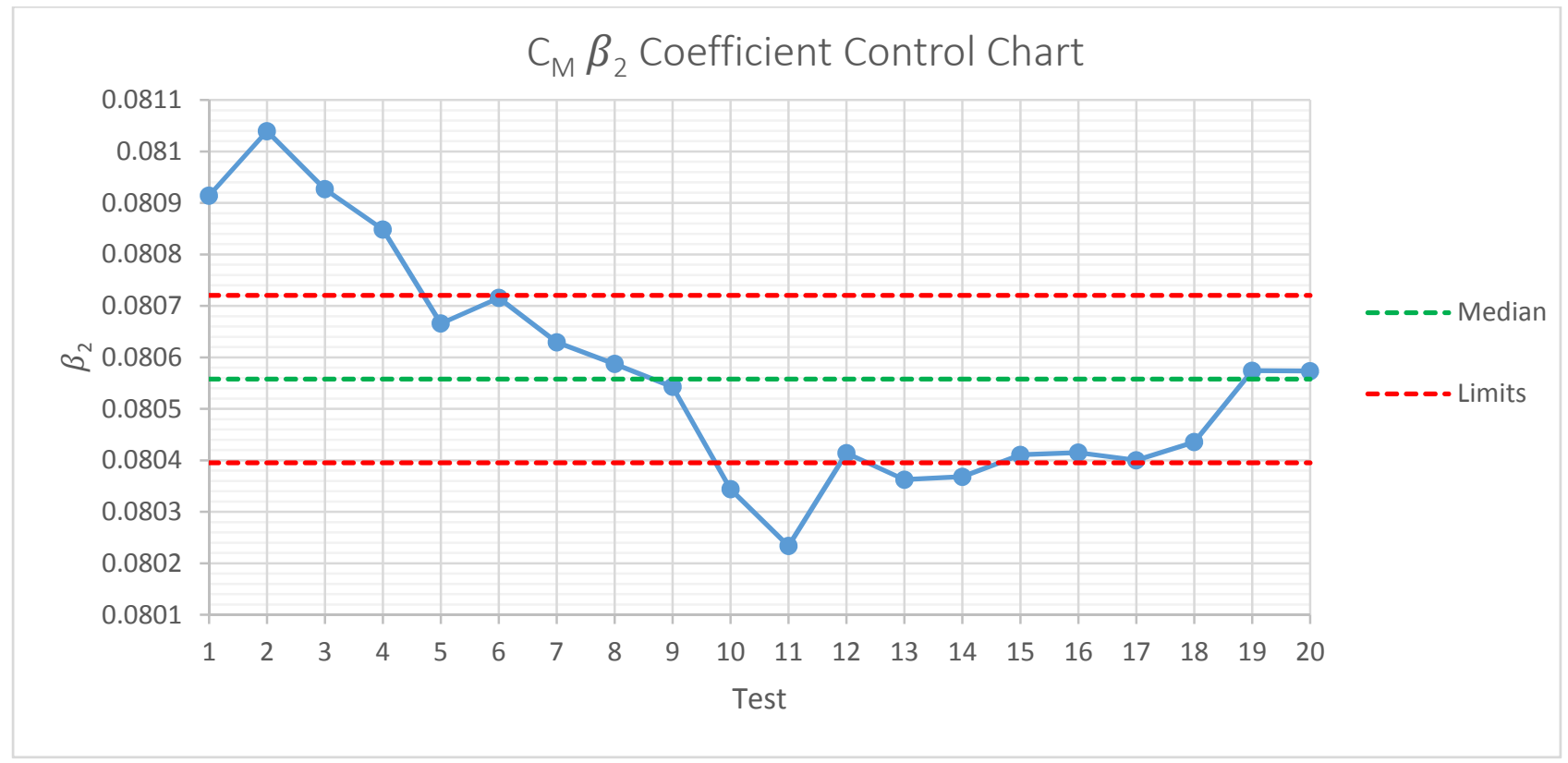

Figure $119 C_{M} \beta_{2}$ Coefficient Control Chart 
In Figure 119, the downward trend again points to a problem associated with setting or measuring angle of attack. In Figure 120 it is seen that the roll effect is in control.

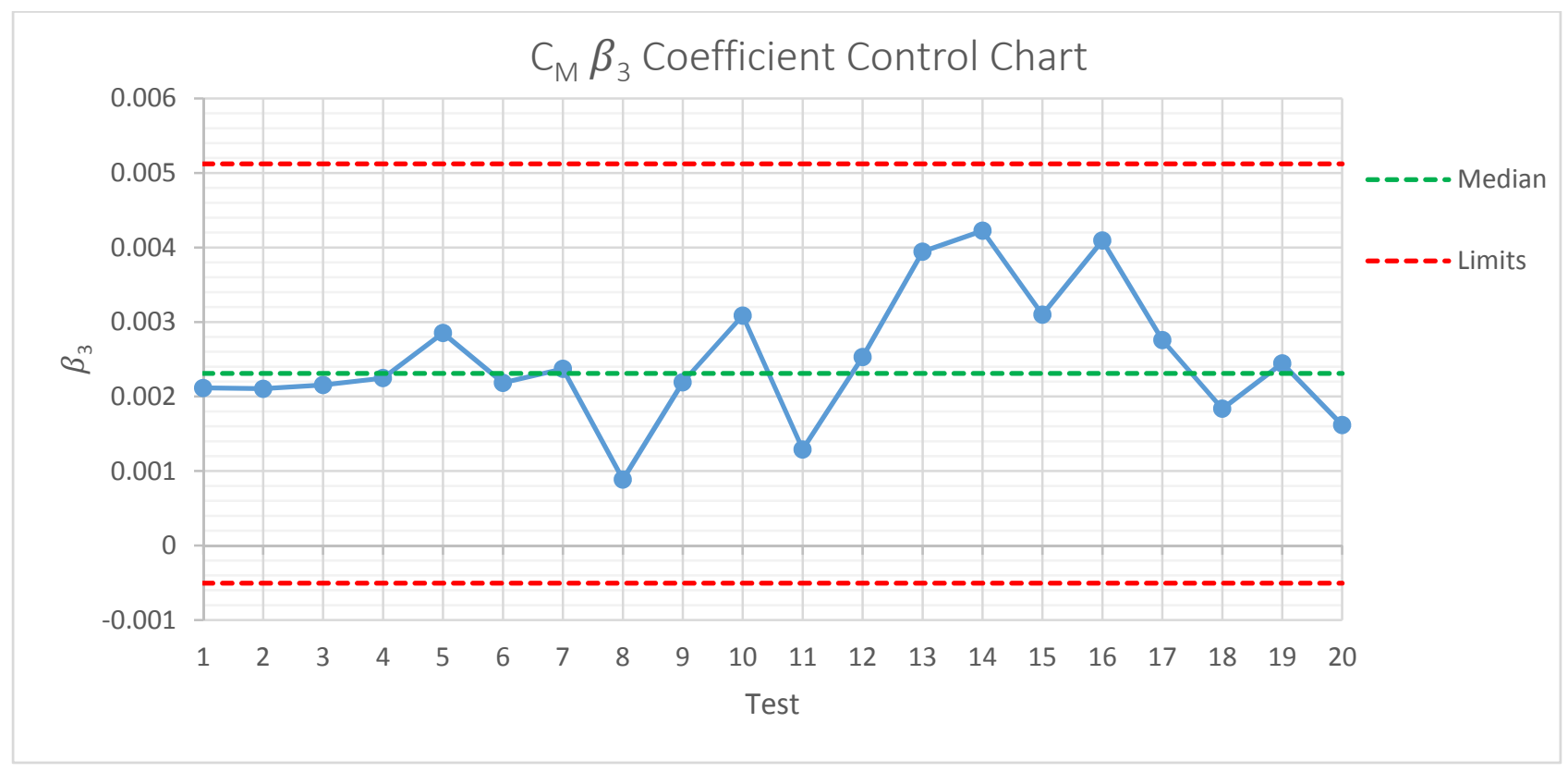

Figure $120 C_{M} \beta_{3}$ Coefficient Control Chart

\subsubsection{Validation of Local Test Regression Models}

In applying design of experiments methodology to test data, the researcher must validate the models by investigating multiple diagnostic criteria. One of the diagnostics investigated is the ability of the model to predict the confirmation points. For this, testing confirmation points were taken during each test however the number and location of the points varied within the design space. To evaluate each of the local regression models a metric was developed based on a normalized residual. The normal force coefficient normalized root mean square error for confirmation points is given as

$$
C N_{R M S E C}=\sqrt{\frac{1}{n} \sum\left(\frac{e_{i}}{S_{\hat{y}(x)_{i}}}\right)^{2}}
$$

Where $e_{i}$ is the residual (measured minus predicted) of the $i^{\text {th }}$ confirmation point and $S_{\hat{y}(x)_{i}}$ is the standard error of the prediction of the $\mathrm{i}^{\text {th }}$ confirmation point given by ${ }^{10}$ 


$$
S_{\hat{y}(x)}=(\sqrt{M S E}) \sqrt{\boldsymbol{x}_{0}{ }^{\prime}\left(\boldsymbol{X}^{\prime} \boldsymbol{X}\right)^{-1} \boldsymbol{x}_{0}}
$$

Where MSE is the mean square error of the regression, $\boldsymbol{x}_{0}$ is the vector in coded units representing the location of the $\mathrm{i}^{\text {th }}$ confirmation point within the design space and $\boldsymbol{X}$ is the model matrix (See Table 49 in Section 6.5.4.1 for an example). Normalizing the residuals in this manner allows the comparison of the predictive ability of all the local tests to each other regardless if the confirmation points are identical. The normal force coefficient normalized root mean square error for confirmation points is shown for each of the 20 tests in Figure 121.

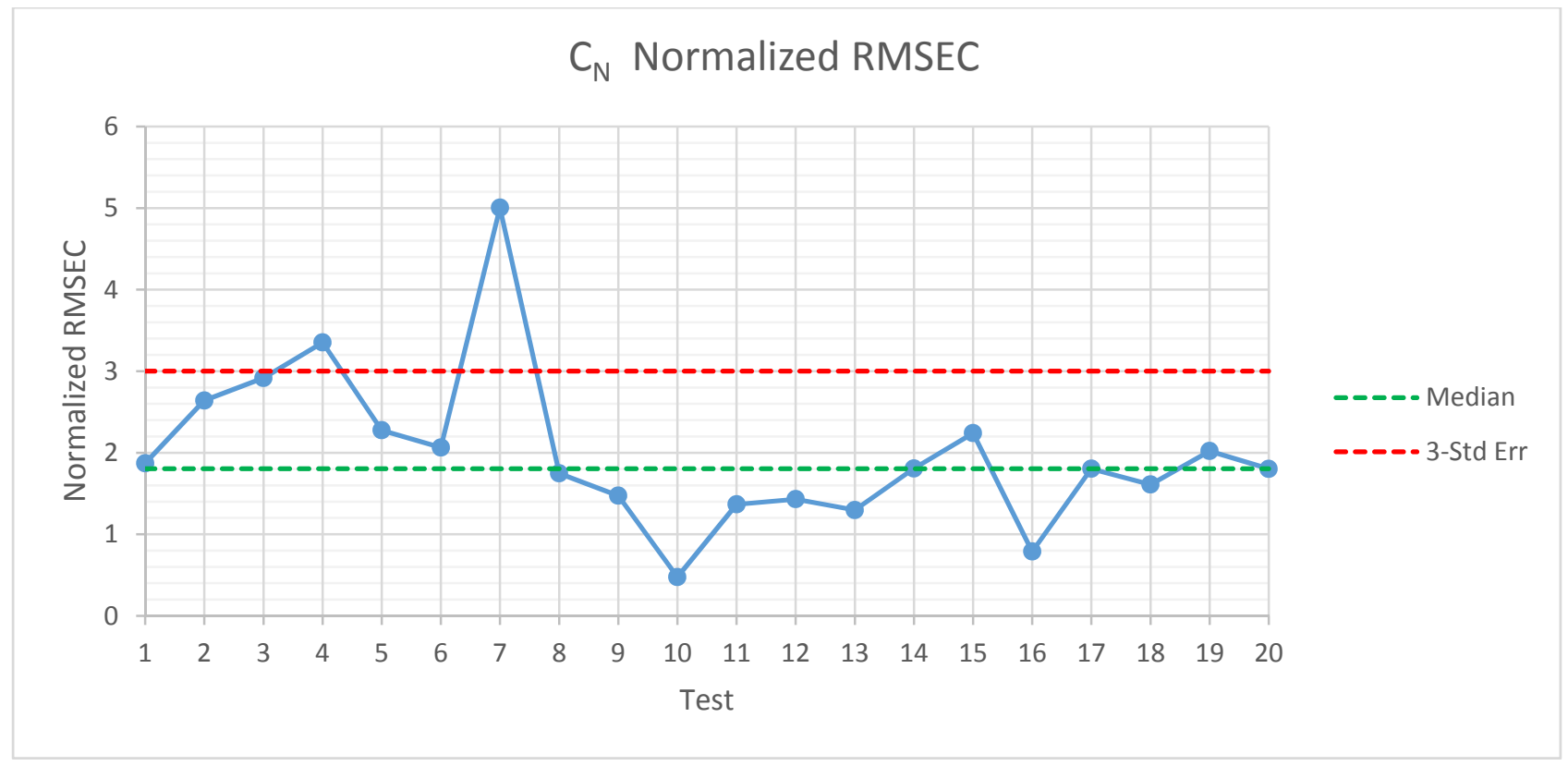

Figure 121 Normal Force Root Mean Square Error Confirmation Points

The limiting value in Figure 121 is three times the normalized standard error, which is simply three. From the DOE practitioners perspective, if the predictive ability of the model is not good, i.e. does not pass the diagnostics of the confirmation point test, other models or characterizations would need to be explored. From inspection of Figure 121, it is seen that the regression model built from the data in Test 7 did not predict the confirmation points well and would not be a suitable model to use in building the global regression model. From the DOE practitioner's perspective, this test should not be used in the global regression model build. However, omitting Test 7 does not significantly alter the resulting model 
(See Section 6.5.4). For the predicted value of the normal force coefficient from the global regression model at the center of the design space, only a $0.07 \%$ difference was observed from omitting Test 7. Therefore, Test 7 was not omitted from the global regression model build.

Another diagnostic tool utilized to validate the local regression model built is the evaluation of residuals (of data points used to build the model unlike the discussion above of confirmation points which are not used to build the model). There should not be any trends in the residuals versus time, factors, or predicted values and they should be reasonably ${ }^{\ddagger}$ normally distributed. Examples of these diagnostic tests form the regression model for the normal force coefficient from Test 18 are shown in Figure 122 through Figure 126. By inspecting each of these figures, no significant departures from normality, or trends in residuals were observed. There are some slight trends with the residuals in the dynamic pressure and angle of attack plots (Figure 122 and Figure 123) which allowed the variance models to be built in Section 6.2.8. However these trends are not significant enough to prevent the regression analysis from being modified. It is also seen there was a single "outlier", a data point at $\alpha=2^{\circ}, q=$ $712 \mathrm{~Pa}$. There are procedures to statistically determine whether or not a point can be classified as an outlier and can be rejected from the data set. ${ }^{54}$ However, this point was only marginally outside of the three sigma limits and was retained in the data set.

\footnotetext{
‡ DOE methodology is robust to small departures from normality
} 


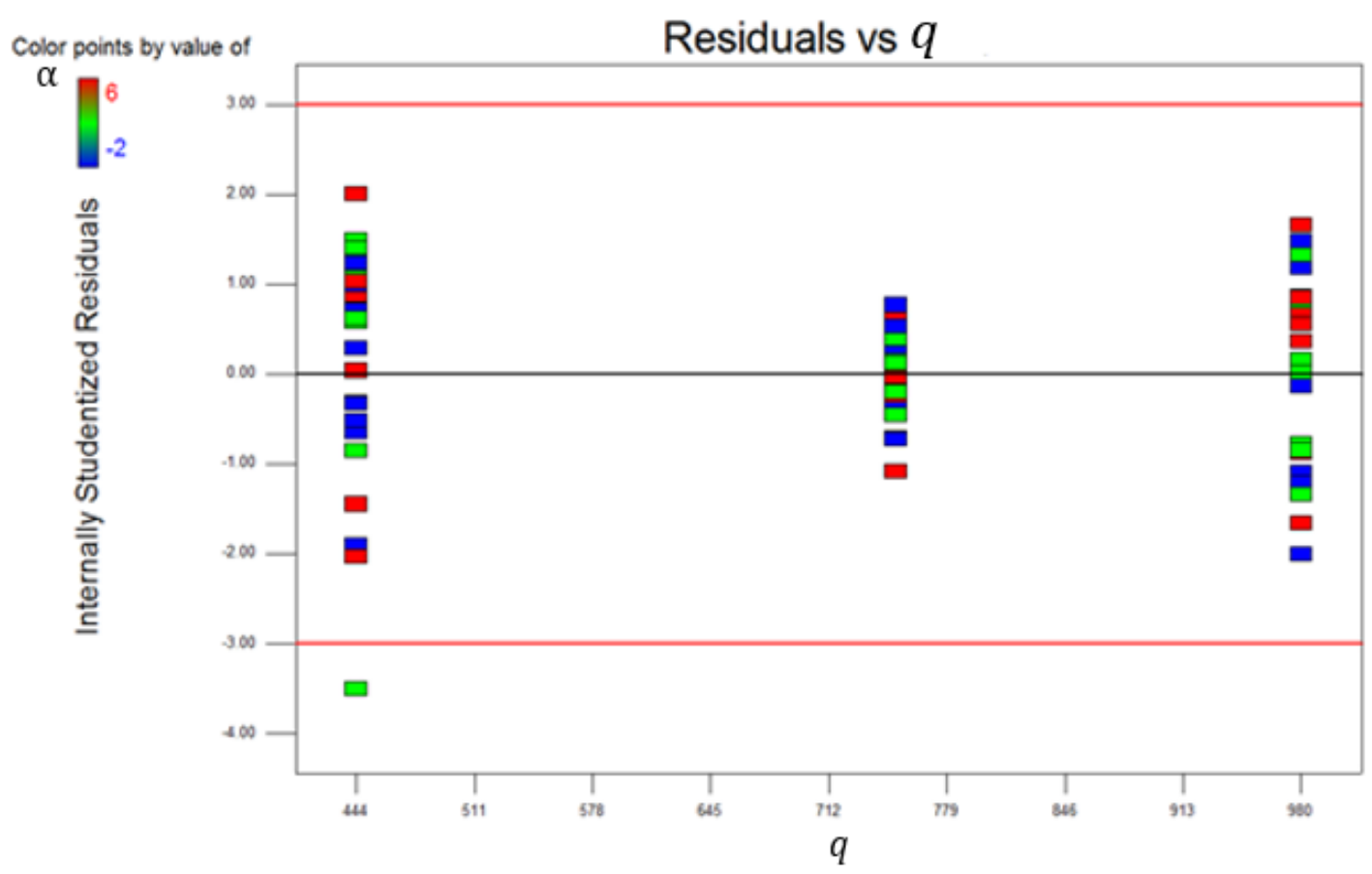

Figure 122 Test 18 Normal Force Coefficient Regression Model Residuals vs Dynamic Pressure

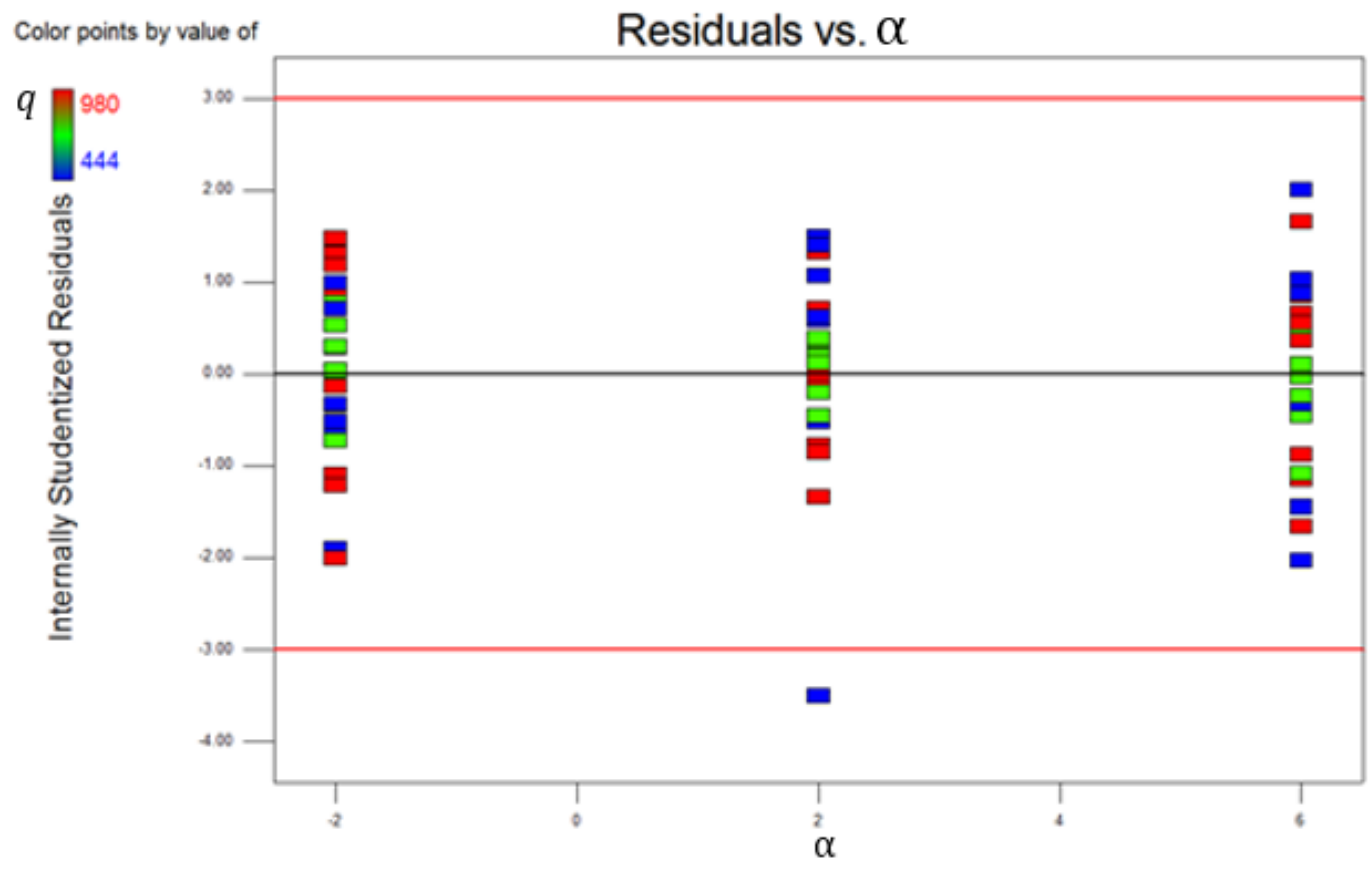

Figure 123 Test 18 Normal Force Coefficient Regression Model Residuals vs Angle of Attack 


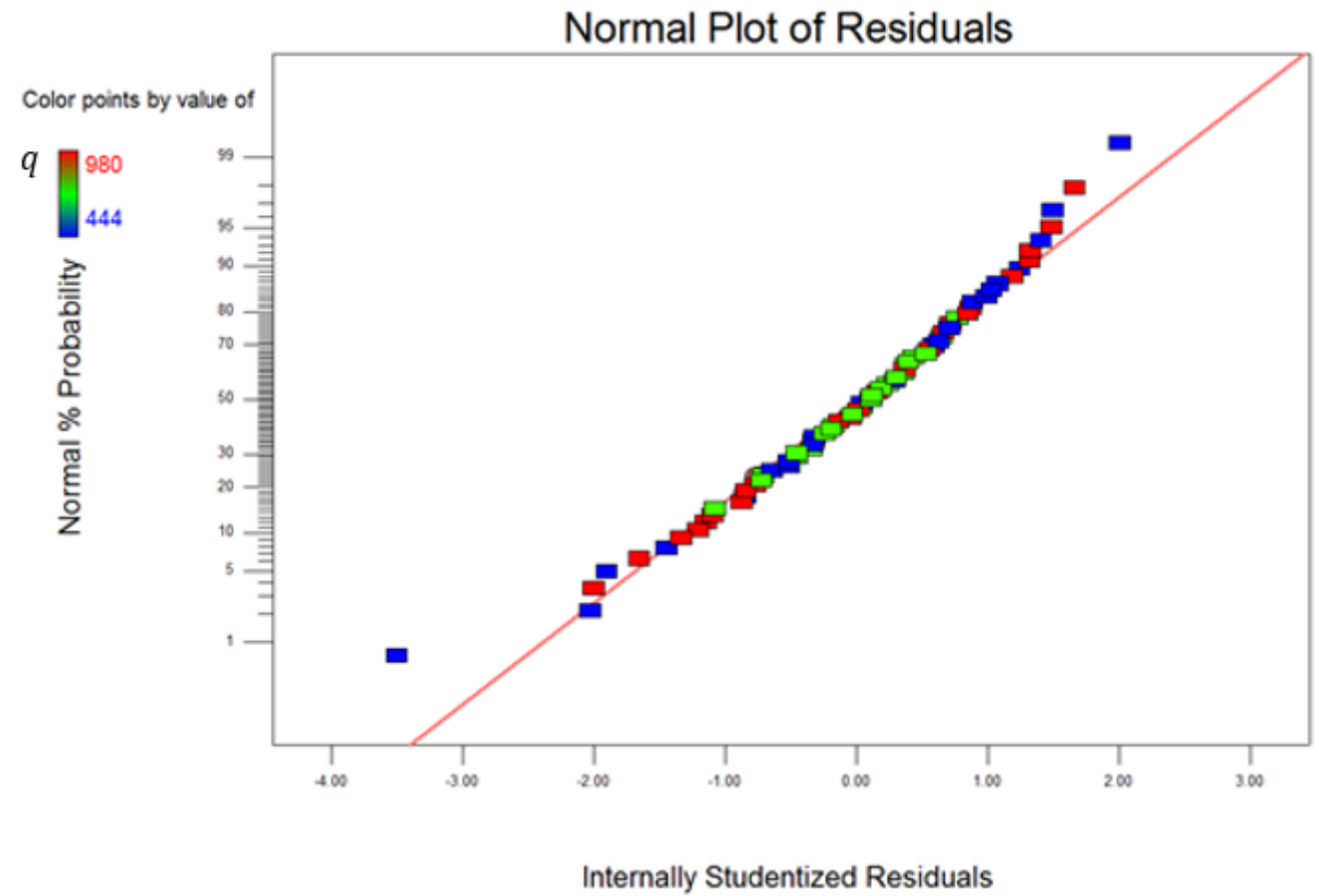

Figure 124 Test 18 Normal Force Coefficient Regression Model Normal Plot of Residuals

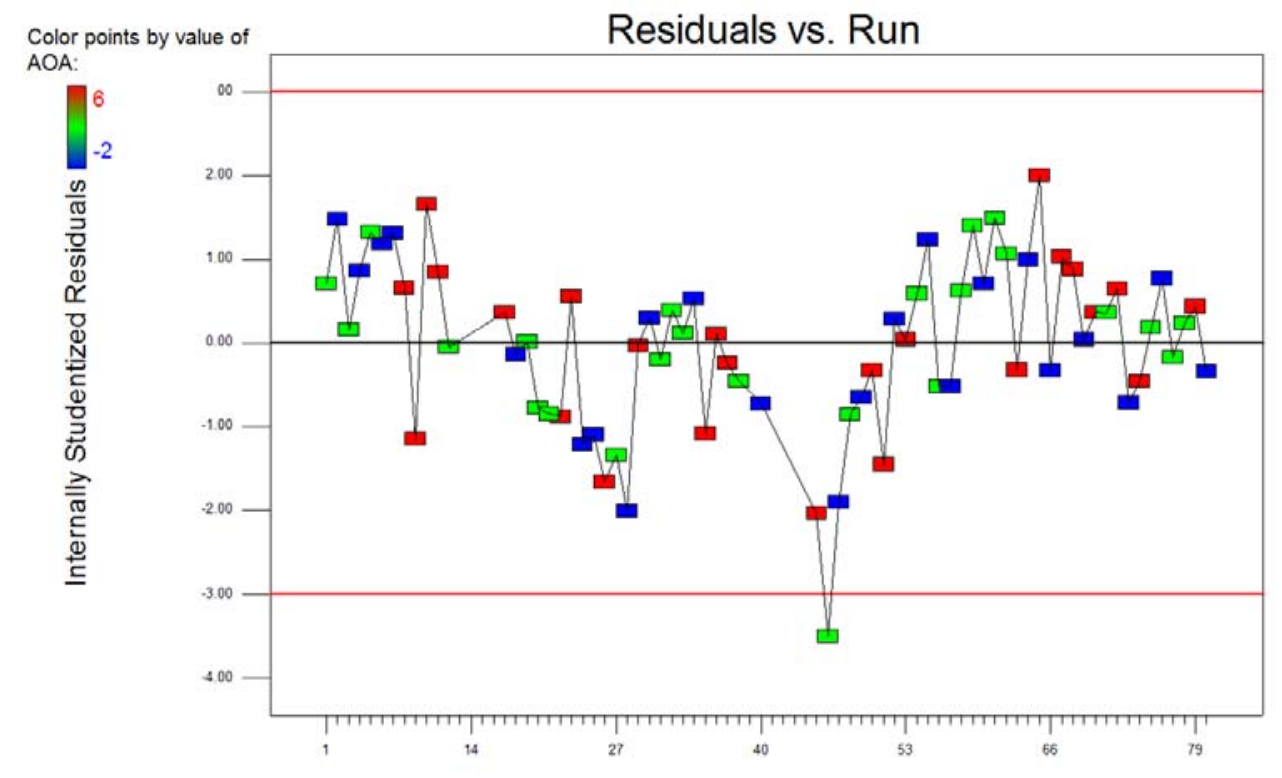

Run Number

Figure 125 Test 18 Normal Force Coefficient Regression Model Residuals vs Run 


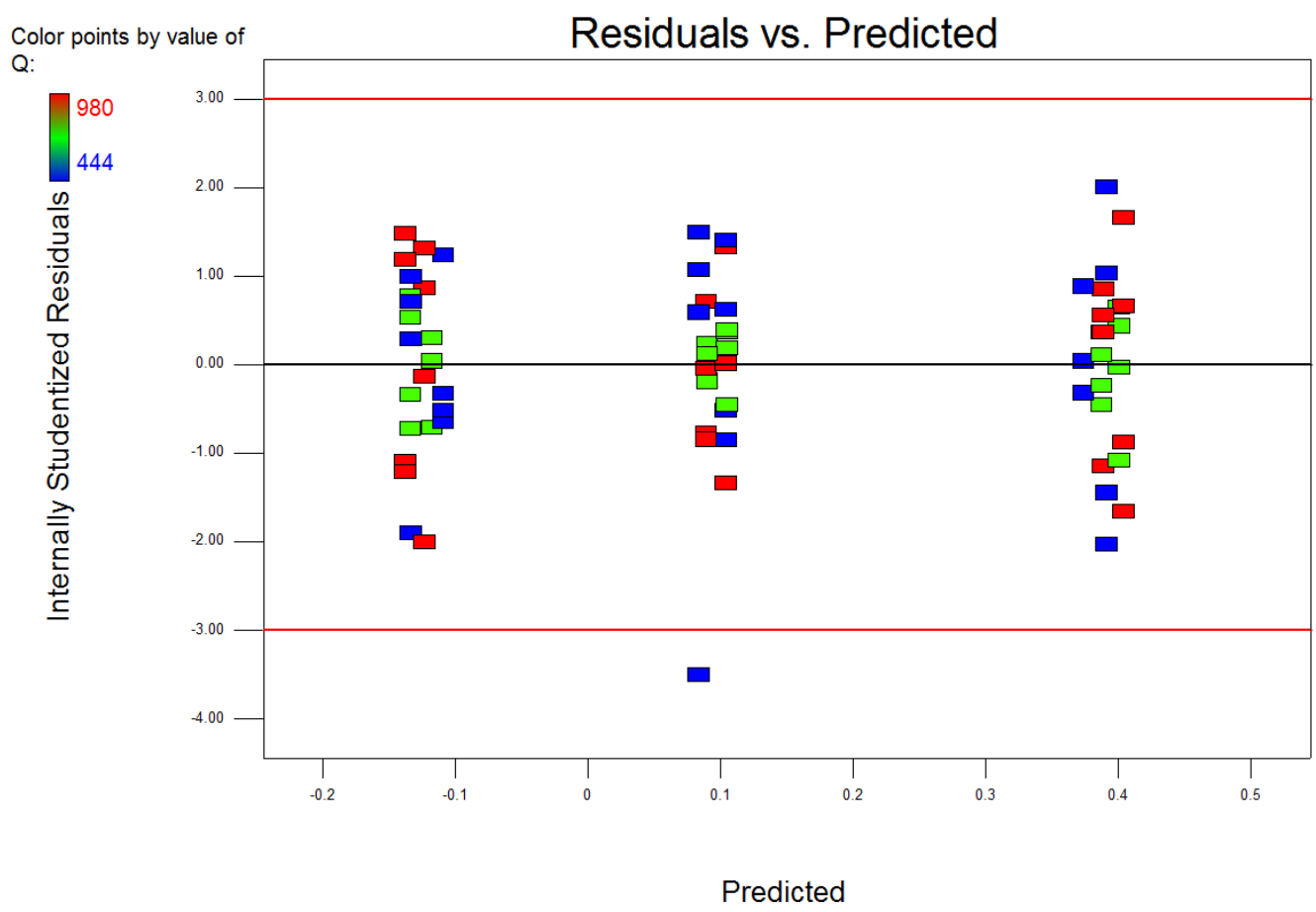

Figure 126 Test 18 Normal Force Coefficient Regression Model Residuals vs Predicted

\subsubsection{Balance Force Coefficients: Global Regression Model}

At the completion of the 20 baseline tests, a global regression model was built to serve as the baseline check standard model for the entire design space. Three different model analysis techniques were explored to build the global model. First a composite global model was built by using the medians from each of the individual regression coefficient control charts as the coefficients for the global model (coded units). The individual regression coefficients from each test for the normal force coefficient are shown along with their respective medians in Table 42. 


\begin{tabular}{|l|c|c|c|c|c|c|c|c|}
\hline Test & $\boldsymbol{\beta}_{\mathbf{0}}$ & $\boldsymbol{\beta}_{\mathbf{1}}$ & $\boldsymbol{\beta}_{\mathbf{2}}$ & $\boldsymbol{\beta}_{\mathbf{3}}$ & $\boldsymbol{\beta}_{\mathbf{1 2}}$ & $\boldsymbol{\beta}_{\mathbf{1 3}}$ & $\boldsymbol{\beta}_{\mathbf{2 3}}$ & $\boldsymbol{\beta}_{\mathbf{1 1}}$ \\
\hline $\mathbf{1}$ & 0.09400 & 0.00447 & 0.26014 & 0.01064 & 0.00509 & -0.00303 & 0.00186 & 0.03793 \\
\hline $\mathbf{2}$ & 0.09777 & 0.00282 & 0.26117 & 0.00963 & 0.00426 & -0.00132 & 0.00218 & 0.03639 \\
\hline $\mathbf{3}$ & 0.09823 & 0.00034 & 0.26053 & 0.00987 & 0.00488 & -0.00175 & 0.00150 & 0.03555 \\
\hline $\mathbf{4}$ & 0.09564 & 0.00362 & 0.26053 & 0.01125 & 0.00553 & -0.00209 & 0.00089 & 0.03616 \\
\hline $\mathbf{5}$ & 0.09669 & 0.00359 & 0.25977 & 0.01246 & 0.00454 & -0.00223 & & 0.03561 \\
\hline $\mathbf{6}$ & 0.09381 & 0.00357 & 0.25983 & 0.01021 & 0.00544 & -0.00102 & 0.00095 & 0.03746 \\
\hline $\mathbf{7}$ & 0.09527 & 0.00357 & 0.25955 & 0.01038 & 0.00590 & -0.00149 & 0.00069 & 0.03751 \\
\hline $\mathbf{8}$ & 0.09391 & 0.00289 & 0.25972 & 0.00674 & 0.00569 & -0.00142 & -0.00086 & 0.03782 \\
\hline $\mathbf{9}$ & 0.09588 & 0.00292 & 0.25937 & 0.00966 & 0.00607 & -0.00190 & 0.00020 & 0.03751 \\
\hline $\mathbf{1 0}$ & 0.09184 & 0.00612 & 0.25883 & 0.01329 & 0.00514 & -0.00258 & -0.00063 & 0.03656 \\
\hline $\mathbf{1 1}$ & 0.09613 & 0.00240 & 0.25736 & 0.00630 & 0.00545 & -0.00002 & -0.00036 & 0.03710 \\
\hline $\mathbf{1 2}$ & 0.09125 & 0.00871 & 0.25869 & 0.01075 & 0.00551 & -0.00325 & & 0.03750 \\
\hline $\mathbf{1 3}$ & 0.09339 & 0.00587 & 0.25863 & 0.01484 & 0.00543 & -0.00195 & 0.00191 & 0.03713 \\
\hline $\mathbf{1 4}$ & 0.09334 & 0.00342 & 0.25913 & 0.01595 & 0.00569 & -0.00150 & 0.00211 & 0.03577 \\
\hline $\mathbf{1 5}$ & 0.09472 & 0.00177 & 0.25921 & 0.01255 & 0.00668 & & & 0.03712 \\
\hline $\mathbf{1 6}$ & 0.09289 & 0.00341 & 0.25864 & 0.01548 & 0.00649 & & & 0.03608 \\
\hline $\mathbf{1 7}$ & 0.09694 & 0.00645 & 0.25731 & 0.01032 & 0.00533 & -0.00164 & 0.00121 & 0.03690 \\
\hline $\mathbf{1 8}$ & 0.09740 & 0.00134 & 0.25914 & 0.00766 & 0.00574 & -0.00151 & -0.00080 & 0.03653 \\
\hline $\mathbf{1 9}$ & 0.09070 & 0.00611 & 0.25996 & 0.01064 & 0.00543 & -0.00178 & 0.00022 & 0.03801 \\
\hline $\mathbf{2 0}$ & 0.09343 & 0.00471 & 0.25900 & 0.00699 & 0.00612 & -0.00148 & 0.00000 & 0.03746 \\
\hline Medians & 0.09436 & 0.00357 & 0.25929 & 0.01051 & 0.00548 & -0.00169 & 0.00079 & 0.03711 \\
\hline
\end{tabular}

Table 42 Normal Force Coefficient Regression Local Coefficients

In this technique, the model built directly uses the medians of the regression coefficients. Note as previously discussed some of the higher order terms were marginally significant in the local tests. Only regression coefficients that were significant in $75 \%$ or 15 out of 20 local tests were used for this model. The resultant model for the normal force coefficient in terms of coded factors is given in Equation 95. Of the three techniques explored, this technique to assemble the global model is the simplest analytically.

$$
\begin{gathered}
C_{N}=0.09436+0.00357 q+0.2593 \alpha+0.01051 \phi+0.00548 q \alpha-0.001695 q \phi \\
+0.0007872 \alpha \phi+0.03711 \alpha^{2}
\end{gathered}
$$

Following the same procedure gives the global regression models for the axial force coefficient and the pitching moment coefficient in Equations 96 and 97 respectively. 


$$
\begin{gathered}
C_{M}=0.04584+0.0005074 q+0.08057 \alpha+0.002502 \phi+0.0008750 q \alpha \\
-0.0006420 q \phi-0.0009021 \alpha \phi+0.0003054 \alpha^{2} \\
C_{A}=0.2807+0.0002803 q-0.01021 \alpha-0.001358 \phi-0.0003701 q \alpha-0.002311 \alpha \phi \\
-0.01068 \alpha^{2}
\end{gathered}
$$

The second technique to build the global regression model was to use all of the baseline test data (1440 data points, 72 per test times 20 tests) and follow the same split-plot REML analysis that was done on the local level. This option is the most relatable to "conventional" DOE methodology. This technique is the most direct in the analysis and gives the following global regression models:

$$
\begin{gathered}
C_{N}=0.09491+0.00355 q+0.2595 \alpha+0.01090 \phi+0.00554 q \alpha-0.001702 q \phi \\
+0.0006808 \alpha \phi+0.03686 \alpha^{2}+0.001001 \alpha^{2} \phi \\
C_{M}=0.04582+0.0003780 q+0.08052 \alpha+0.002530 \phi+0.0008920 q \alpha \\
-0.0006426 q \phi-0.0009054 \alpha \phi+0.0002885 \alpha^{2}-0.0003679 \alpha^{2} \phi \\
C_{A}=0.2802+0.0005907 q-0.01021 \alpha-0.001264 \phi-0.0002755 q \alpha+0.000083 q \phi \\
-0.002379 \alpha \phi-0.01064 \alpha^{2}-0.0001413 \alpha^{2} \phi
\end{gathered}
$$

In each of the three global models, the mixed cubic term, $\alpha^{2} \phi$ was significant. This term was significant at the local level for some of the tests for each of the responses but it did not meet the threshold of inclusion into the first technique's model of being significant for 15 out of the 20 tests.

The third technique explored to build the global model is to use the baseline test medians as data points (18 unique data points per test, giving 18 medians, totaling 360 data points) and follow the same splitplot REML analysis that was done at the local level. Using the medians of the test values gives the analysis robustness against outliers in the data set similar to the method of establishing the bounds on the traditional SPC charts. This model technique gives the following regression models: 


$$
\begin{gathered}
C_{N}=0.09442+0.004072 q+0.2591 \alpha+0.01086 \phi+0.005573 q \alpha-0.001667 q \phi \\
+0.0006792 \alpha \phi+0.03688 \alpha^{2}+0.001020 \alpha^{2} \phi \\
C_{M}=0.04580+0.0005359 q+0.08053 \alpha+0.002485 \phi+0.0008706 q \alpha \\
-0.0006338 q \phi-0.0009005 \alpha \phi+0.0002696 \alpha^{2}-0.0003445 \alpha^{2} \phi \\
C_{A}=0.2802+0.0006020 q-0.01019 \alpha-0.001348 \phi-0.0002929 q \alpha+0.0001040 q \phi \\
-0.002369 \alpha \phi-0.01064 \alpha^{2}-0.0004273 \alpha^{2} \phi
\end{gathered}
$$

A comparison of the most significant regression coefficients (angle of attack and intercept) for the three techniques is shown in Table 43 and Table 44. For clarity, Technique 1 is the analysis using the medians of the regression coefficients, Technique 2 is the analysis using the full data set and Technique 3 is the analysis using the medians of the test data points.

\begin{tabular}{|c|c|c|c|}
\hline & \multicolumn{3}{|c|}{ Regression } \\
\hline Technique & 1 & 2 & 3 \\
\hline $\boldsymbol{C}_{\boldsymbol{N}}$ & 0.09436 & 0.09491 & 0.09442 \\
\hline $\boldsymbol{C}_{\boldsymbol{M}}$ & 0.04584 & 0.04582 & 0.04580 \\
\hline $\boldsymbol{C}_{\boldsymbol{A}}$ & 0.2807 & 0.2802 & 0.2802 \\
\hline
\end{tabular}

Table 43 Comparison of Model Building Technique's Regression Intercepts

\begin{tabular}{|c|c|c|c|}
\hline & \multicolumn{3}{|c|}{ Regression Angle of Attack Coefficient Comparison } \\
\hline Technique & 1 & 2 & 3 \\
\hline $\boldsymbol{C}_{\boldsymbol{N}}$ & 0.2593 & 0.2595 & 0.2591 \\
\hline $\boldsymbol{C}_{\boldsymbol{M}}$ & 0.08057 & 0.08052 & 0.08053 \\
\hline $\boldsymbol{C}_{\boldsymbol{A}}$ & -0.01021 & -0.01021 & -0.01019 \\
\hline
\end{tabular}

Table 44 Comparison of Model Building Technique's Angle of Attack Regression Coefficient

It is seen that there is very little difference between the estimates of coefficients between the three techniques. To statistically compare the regression coefficients, a confidence interval can be placed on them by 


$$
\widehat{\beta}_{J}-t_{a / 2, n-p} \sqrt{\hat{\sigma}^{2} C_{j j}} \leq \beta \leq \widehat{\beta}_{J}+t_{a / 2, n-p} \sqrt{\hat{\sigma}^{2} C_{j j}}
$$

where $\beta$ is the "true" value of the regression coefficient, $\widehat{\beta}_{J}$ is the estimate of the $j^{t h}$ regression coefficient (for example the values in Table 43 and Table 44) and $C_{j j}$ is the $j j^{\text {th }}$ element of the $\left(\boldsymbol{X}^{\prime} \boldsymbol{X}\right)^{-1}$ matrix ( $\boldsymbol{X}$ is the model matrix, see section 6.5.4.1) and $\hat{\sigma}^{2}$ is the estimate of variance given by the regression MSE. After applying the statistical test in Equation 106, it was seen that for each of the highly significant model terms in Table 44 are not statistically different. It should be noted that for some of the less significant model terms, there were statistical differences.

\subsubsection{Prediction Interval Development}

Once each of the global regression models were established for each of the responses, a prediction interval was developed to allow for the regression models to serve as the check standard baseline model. The prediction interval allows future testing using confirmation points for the established global model. The prediction interval is defined as a bound on a future individual observation and is based on Equation $105^{9}$. It should be noted that the prediction interval is for a single test average which must be consistent with the baseline test's degrees of freedom per data point. For example, during the baseline tests each data point was obtained four times, therefore the prediction interval is valid for an average of four samples. The primary advantage to this approach is that the future tests need not be in the same region of the design space as there is now a continuous global model covering the design space.

$$
\begin{gathered}
\hat{y}\left(\boldsymbol{x}_{0}\right)-t_{a / 2, n-p} \sqrt{\hat{\sigma}^{2}\left(1+\boldsymbol{x}_{0}{ }^{\prime}\left(\boldsymbol{X}^{\prime} \boldsymbol{X}\right)^{-1} \boldsymbol{x}_{0}\right)} \leq y_{0} \leq \hat{y}\left(\boldsymbol{x}_{0}\right)+ \\
t_{a / 2, n-p} \sqrt{\hat{\sigma}^{2}\left(1+\boldsymbol{x}_{0}{ }^{\prime}\left(\boldsymbol{X}^{\prime} \boldsymbol{X}\right)^{-1} \boldsymbol{x}_{0}\right)}
\end{gathered}
$$

In Equation 105, $\boldsymbol{X}$ is the model matrix, $\hat{\sigma}^{2}$ is the mean square error (MSE), $\boldsymbol{x}_{0}$ is the design space vector representing one point within the design space (eg. the confirmation point), $n$ is the number of unique design point locations, $p$ is the number of model terms, $a$ is the significance level and $\hat{y}$ is the regression model predicted value. A benefit to this approach of generating the prediction interval is that statistically, the number of degrees of freedom necessary to construct the interval are independent of the number of baseline tests. As long as the number of unique design points $(n)$ exceeds the number of 
model terms $(p)$, the interval expression is valid. However, the mean square error (MSE) will be dependent on the number of baseline tests completed. A comparison of the three techniques' predicted values and confirmation points is given in Table 45 and Table 46. Because each of these confirmation points was only obtained once, an average value was obtained from four baseline tests to maintain continuity with the degrees of freedom of the global models. Therefore in Table 45, the measured values are an average of the confirmation points obtained in tests 10-13. Similarly, for Table 46, the measured values are an average of the confirmation points obtained in tests 14-17.

\begin{tabular}{|r|r|l|r|r|r|r|r|c|c|}
\hline & & & \multicolumn{1}{|c|}{$\mathbf{T}$} & \multicolumn{1}{c|}{$\mathbf{2}$} & \multicolumn{1}{c|}{$\mathbf{3}$} & $\mathbf{1}$ & $\mathbf{2}$ & $\mathbf{3}$ \\
\hline $\mathbf{q}$ & $\boldsymbol{\alpha}$ & $\boldsymbol{\phi}$ & Measured & \multicolumn{3}{|c|}{ Predicted } & \multicolumn{3}{c|}{ Residual } \\
\hline $\mathbf{8 6 5}$ & 1 & Upright & 0.0276 & 0.0238 & 0.0237 & 0.0237 & 0.0039 & 0.0040 & 0.0040 \\
\hline $\mathbf{8 6 5}$ & -1 & Upright & -0.0851 & -0.0885 & -0.0890 & -0.0892 & 0.0034 & 0.0039 & 0.0041 \\
\hline $\mathbf{8 6 5}$ & 1 & Inverted & 0.0478 & 0.0425 & 0.0432 & 0.0433 & 0.0054 & 0.0046 & 0.0046 \\
\hline $\mathbf{8 6 5}$ & -1 & Inverted & -0.0634 & -0.0706 & -0.0693 & -0.0692 & 0.0071 & 0.0059 & 0.0058 \\
\hline $\mathbf{6 0 0}$ & 1 & Upright & 0.0229 & 0.0199 & 0.0198 & 0.0198 & 0.0030 & 0.0031 & 0.0031 \\
\hline $\mathbf{6 0 0}$ & -1 & Upright & -0.0875 & -0.0896 & -0.0907 & -0.0907 & 0.0021 & 0.0032 & 0.0033 \\
\hline $\mathbf{6 0 0}$ & 1 & Inverted & 0.0463 & 0.0420 & 0.0429 & 0.0423 & 0.0043 & 0.0034 & 0.0040 \\
\hline $\mathbf{6 0 0}$ & -1 & Inverted & -0.0642 & -0.0684 & -0.0671 & -0.0675 & 0.0042 & 0.0029 & 0.0033 \\
\hline
\end{tabular}

Table 45 Comparison of Model Predicted Values of Confirmation Points 10-13

\begin{tabular}{|r|r|l|r|r|r|r|r|c|c|}
\hline & & & Technique & \multicolumn{1}{|c|}{$\mathbf{1}$} & \multicolumn{2}{|c|}{$\mathbf{2}$} & $\mathbf{3}$ & $\mathbf{1}$ & $\mathbf{2}$ \\
\hline $\mathbf{q}$ & $\boldsymbol{\alpha}$ & $\boldsymbol{\phi}$ & Measured & \multicolumn{3}{|c|}{ Predicted } \\
\hline $\mathbf{8 6 5}$ & 1 & Upright & 0.0254 & 0.0238 & 0.0237 & 0.0237 & 0.0017 & 0.0018 & 0.0018 \\
\hline $\mathbf{8 6 5}$ & -1 & Upright & -0.0864 & -0.0885 & -0.0890 & -0.0892 & 0.0021 & 0.0026 & 0.0028 \\
\hline $\mathbf{8 6 5}$ & 1 & Inverted & 0.0502 & 0.0425 & 0.0432 & 0.0433 & 0.0078 & 0.0070 & 0.0070 \\
\hline $\mathbf{8 6 5}$ & -1 & Inverted & -0.0620 & -0.0706 & -0.0693 & -0.0692 & 0.0086 & 0.0074 & 0.0073 \\
\hline $\mathbf{6 0 0}$ & 1 & Upright & 0.0217 & 0.0199 & 0.0198 & 0.0198 & 0.0018 & 0.0019 & 0.0019 \\
\hline $\mathbf{6 0 0}$ & -1 & Upright & -0.0883 & -0.0896 & -0.0907 & -0.0907 & 0.0014 & 0.0024 & 0.0025 \\
\hline $\mathbf{6 0 0}$ & 1 & Inverted & 0.0488 & 0.0420 & 0.0429 & 0.0423 & 0.0069 & 0.0060 & 0.0066 \\
\hline $\mathbf{6 0 0}$ & -1 & Inverted & -0.0596 & -0.0684 & -0.0671 & -0.0675 & 0.0088 & 0.0076 & 0.0079 \\
\hline
\end{tabular}

Table 46 Comparison of Model Predicted Values of Confirmation Points from Tests 14-17

In Table 47, the prediction intervals for each of the model techniques along with the status (In/Out) of the confirmation points from tests $10-13$ are given. Similar data are given in Table 48 for the confirmation points from tests 14-17. 


\begin{tabular}{|c|r|c|c|c|c|c|c|c|c|c|c|}
\hline \multicolumn{3}{|c|}{ Technique } & \multicolumn{3}{c}{$\mathbf{1}$} & \multicolumn{3}{c|}{$\mathbf{2}$} & \multicolumn{3}{|c|}{$\mathbf{3}$} \\
\hline $\mathbf{q}$ & $\boldsymbol{\alpha}$ & $\boldsymbol{\phi}$ & Pi low & Pi high & IN? & Pi low & Pi high & IN? & Pi low & Pi high & IN? \\
\hline $\mathbf{8 6 5}$ & 1 & Upright & 0.0195 & 0.0281 & $\mathbf{Y}$ & 0.0155 & 0.0319 & $\mathbf{Y}$ & 0.0104 & 0.0369 & $\mathbf{Y}$ \\
\hline $\mathbf{8 6 5}$ & -1 & Upright & -0.0928 & -0.0842 & $\mathbf{Y}$ & -0.0972 & -0.0808 & $\mathbf{Y}$ & -0.1025 & -0.0759 & $\mathbf{Y}$ \\
\hline $\mathbf{8 6 5}$ & 1 & Inverted & 0.0382 & 0.0468 & $\mathbf{N}$ & 0.0350 & 0.0514 & $\mathbf{Y}$ & 0.0300 & 0.0565 & $\mathbf{Y}$ \\
\hline $\mathbf{8 6 5}$ & -1 & Inverted & -0.0749 & -0.0663 & $\mathbf{N}$ & -0.0775 & -0.0612 & $\mathbf{Y}$ & -0.0825 & -0.0560 & $\mathbf{Y}$ \\
\hline $\mathbf{6 0 0}$ & 1 & Upright & 0.0156 & 0.0242 & $\mathbf{Y}$ & 0.0116 & 0.0280 & $\mathbf{Y}$ & 0.0061 & 0.0326 & $\mathbf{Y}$ \\
\hline $\mathbf{6 0 0}$ & -1 & Upright & -0.0939 & -0.0853 & $\mathbf{Y}$ & -0.0989 & -0.0824 & $\mathbf{Y}$ & -0.1040 & -0.0775 & $\mathbf{Y}$ \\
\hline $\mathbf{6 0 0}$ & 1 & Inverted & 0.0377 & 0.0463 & $\mathbf{N}$ & 0.0346 & 0.0511 & $\mathbf{Y}$ & 0.0290 & 0.0555 & $\mathbf{Y}$ \\
\hline $\mathbf{6 0 0}$ & -1 & Inverted & -0.0727 & -0.0641 & $\mathbf{Y}$ & -0.0754 & -0.0589 & $\mathbf{Y}$ & -0.0808 & -0.0542 & $\mathbf{Y}$ \\
\hline
\end{tabular}

Table 47 Prediction Interval Comparison for Confirmation Points from Tests 10-13

\begin{tabular}{|r|r|l|l|l|l|l|l|l|l|l|l|}
\hline \multicolumn{3}{|c|}{ Technique } & \multicolumn{3}{|c|}{$\mathbf{1}$} & \multicolumn{3}{|c|}{$\mathbf{2}$} \\
\hline $\mathbf{q}$ & $\boldsymbol{\alpha}$ & $\boldsymbol{\phi}$ & Pi low & Pi high & IN? & Pi low & Pi high & IN? & Pi low & Pi high & IN? \\
\hline $\mathbf{8 6 5}$ & 1 & Upright & 0.0195 & 0.0281 & $\mathbf{Y}$ & 0.0155 & 0.0319 & $\mathbf{Y}$ & 0.0104 & 0.0369 & $\mathbf{Y}$ \\
\hline $\mathbf{8 6 5}$ & -1 & Upright & -0.0928 & -0.0842 & $\mathbf{Y}$ & -0.0972 & -0.0808 & $\mathbf{Y}$ & -0.1025 & -0.0759 & $\mathbf{Y}$ \\
\hline $\mathbf{8 6 5}$ & $\mathbf{1}$ & Inverted & 0.0382 & 0.0468 & $\mathbf{N}$ & 0.0350 & 0.0514 & $\mathbf{Y}$ & 0.0300 & 0.0565 & $\mathbf{Y}$ \\
\hline $\mathbf{8 6 5}$ & -1 & Inverted & -0.0749 & -0.0663 & $\mathbf{N}$ & -0.0775 & -0.0612 & $\mathbf{Y}$ & -0.0825 & -0.0560 & $\mathbf{Y}$ \\
\hline $\mathbf{6 0 0}$ & 1 & Upright & 0.0156 & 0.0242 & $\mathbf{Y}$ & 0.0116 & 0.0280 & $\mathbf{Y}$ & 0.0061 & 0.0326 & $\mathbf{Y}$ \\
\hline $\mathbf{6 0 0}$ & -1 & Upright & -0.0939 & -0.0853 & $\mathbf{Y}$ & -0.0989 & -0.0824 & $\mathbf{Y}$ & -0.1040 & -0.0775 & $\mathbf{Y}$ \\
\hline $\mathbf{6 0 0}$ & $\mathbf{1}$ & Inverted & 0.0377 & 0.0463 & $\mathbf{N}$ & 0.0346 & 0.0511 & $\mathbf{Y}$ & 0.0290 & 0.0555 & $\mathbf{Y}$ \\
\hline $\mathbf{6 0 0}$ & -1 & Inverted & -0.0727 & -0.0641 & $\mathbf{N}$ & -0.0754 & -0.0589 & $\mathbf{Y}$ & -0.0808 & -0.0542 & $\mathbf{Y}$ \\
\hline
\end{tabular}

Table 48 Prediction Interval Comparison for Confirmation Points from Tests test 14-17

From inspection, it is seen that the first model technique, building the global model from the medians of the test regression coefficients performs very poorly when predicting the confirmation points. In contrast the second and third techniques to build the global regression model perform very well in the prediction of confirmation points. Although this is the case, the width of the prediction intervals for each of the techniques should be explored. From inspection of Table 48, is it seen that on average the first technique produces the smallest prediction interval and third technique produces the widest prediction interval.

The graph in Figure 127 shows the example prediction intervals from all three techniques superimposed over a control chart at that design location to give a visualization of how the global regression model's prediction interval compares with the SPC derived limits. Similar charts are shown for the coefficient of 
axial force and pitching moment coefficient in Figure 128 and Figure 129. An example of the variables used in Equation 105 to generate the charts are given in Table 49 for the third technique of assembling the global model.

\begin{tabular}{|c|l|l|l|}
\hline \multicolumn{2}{|c|}{ Desired Point } & \multicolumn{2}{|c|}{ Parameters } \\
\hline $\boldsymbol{\alpha}$ & $2^{\circ}$ & $\boldsymbol{X}$ & Model Matrix \\
\hline $\boldsymbol{\phi}$ & Upright & $\hat{\sigma}^{2}$ & $1.34 \mathrm{E}-05$ \\
\hline $\boldsymbol{q}$ & $750 \mathrm{~Pa}$ & $n$ & 18 \\
\hline & & $p$ & 9 \\
\hline & & $a$ & 0.003 \\
\hline & & $\boldsymbol{x}_{0}$ & $\begin{array}{l}{\left[1, x_{1}, x_{2}, x_{3}, x_{1} x_{2}, x_{1} x_{3}, x_{2} x_{3}, x_{2}^{2}, x_{2}^{2} x_{3}\right.} \\
=[1,0.14,0,-1,0,-0.14,0,0,0,]\end{array}$ \\
\hline
\end{tabular}

Table 49 Global Prediction Interval Sample Calculation 


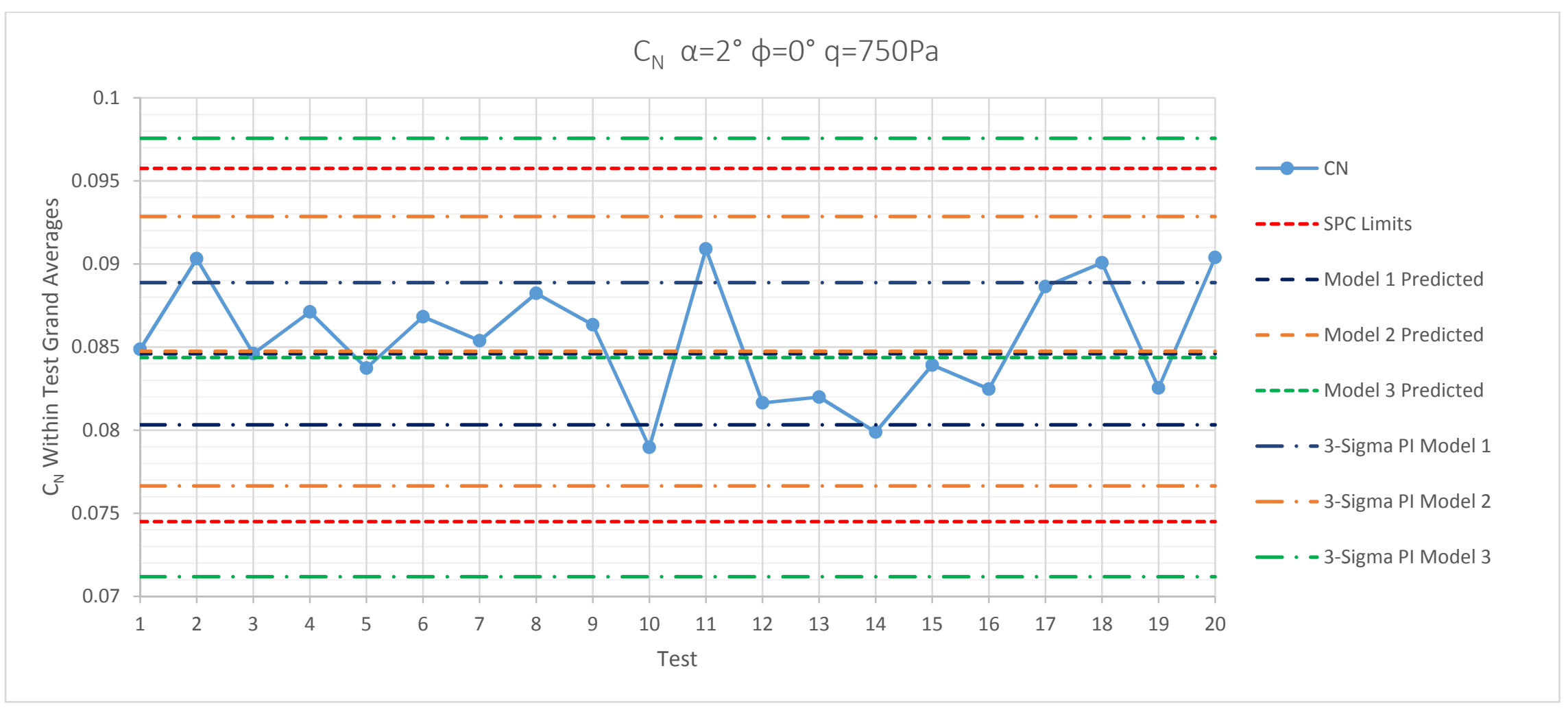

Figure 127 Global Prediction Interval Sample Graph for $C_{N}$ 


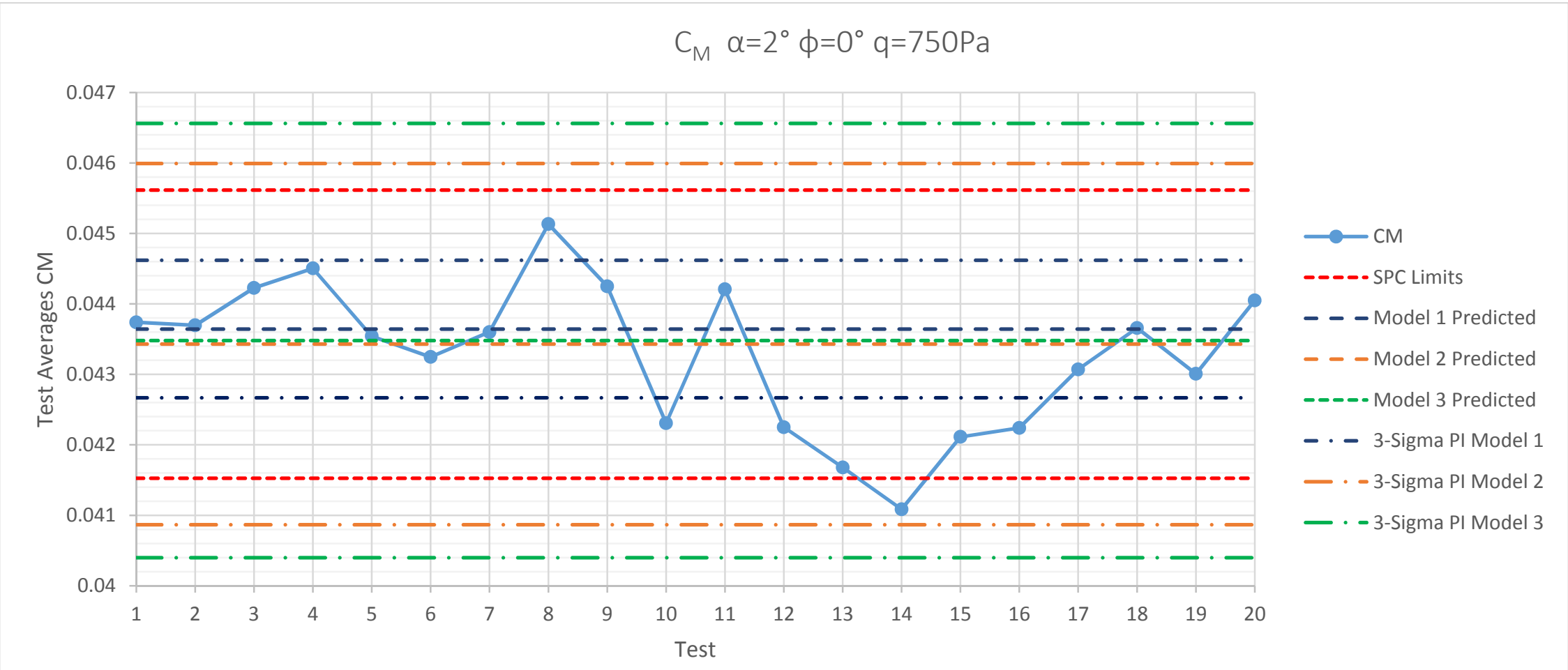

Figure 128 Global Prediction Interval Sample Graph for $C_{M}$ 


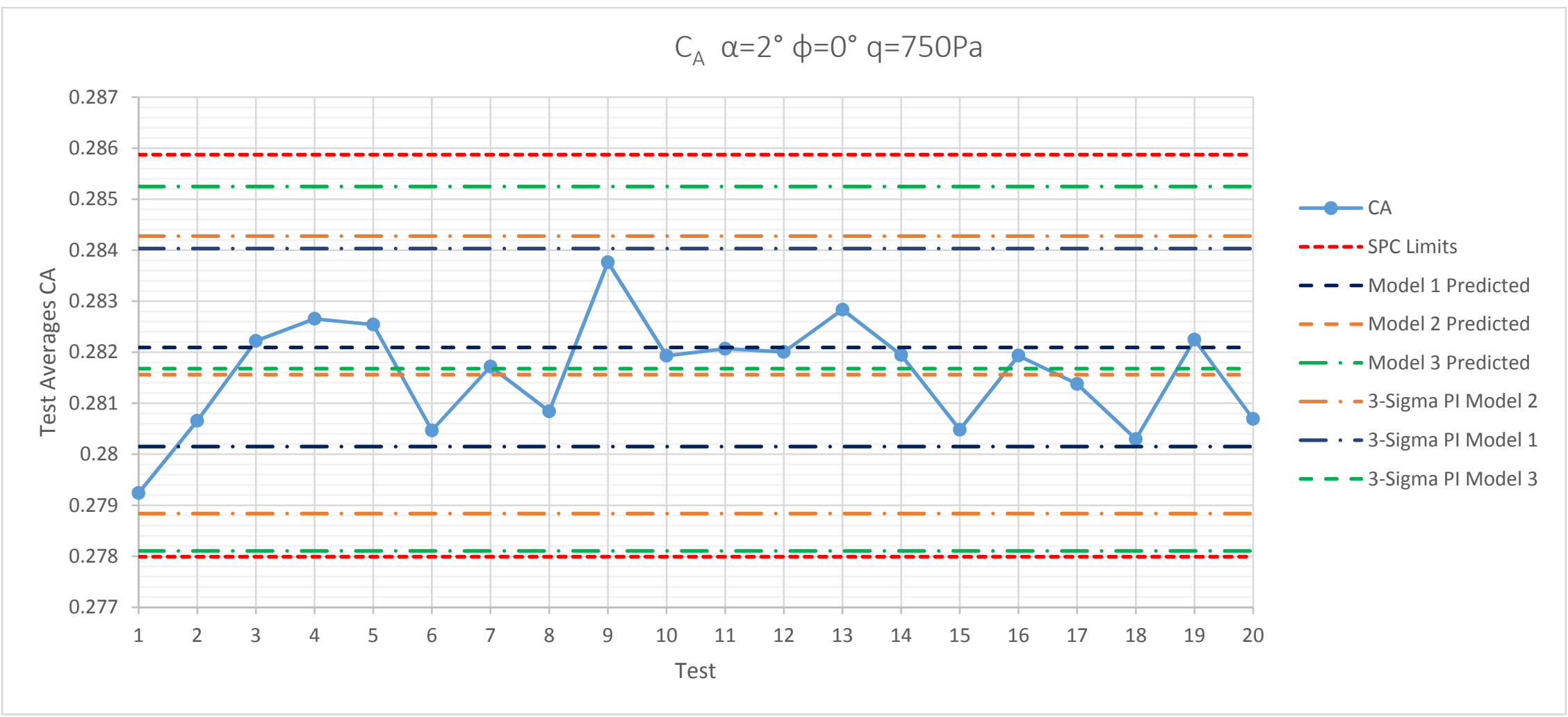

Figure 129 Global Prediction Interval Sample Graph for $C_{A}$ 
From inspection of the global prediction interval sample graphs it is seen that the limits on the predicted values are in fair agreement with the SPC derived chart limits. Although each of the three techniques to build the global models only varied slightly in the predicted values, there was much more variation in the prediction intervals. The second and third model techniques are in better agreement with the SPC limits than the first technique. This is most likely do to the choice of MSE for the first technique. The MSE was chosen as the median of the baseline tests' MSE which was significantly less than the other two models. Also, for the second and third model building techniques, all of the baseline test averages were within the prediction interval. While each of the models were fair agreement, the most statistically robust technique to assemble the global regression model is the third option, using the medians of the individual test values. This method allows for the integration of the SPC and DOE methodology, taking advantage of the benefits of each. The choice of medians instead of means of the tests baseline data is keeping within the current SPC practices and allows for the robustness against outliners. The regression diagnostics will be completed using, the third technique the global regression model assembled using the medians of the individual test values.

\subsubsection{Global Regression Model Diagnostics}

The same evaluation of the local regression models will be utilized to evaluate the validity of the global regression models. The same basic diagnostic tests are applicable, normal distribution of residuals, no trends of residuals with factors, time or prediction. Again as was the case with the local regression model example, there are some slight trends with the residuals in the dynamic pressure and angle of attack plots which allowed the variance models to be built in Section 6.2.8. However these trends are not significant enough to prevent the regression analysis from being modified. The analysis of the validity of the axial force and pitching moment coefficients was also completed (not shown) and no trends in residuals or any other diagnostic tests significantly deviated from the assumptions. 


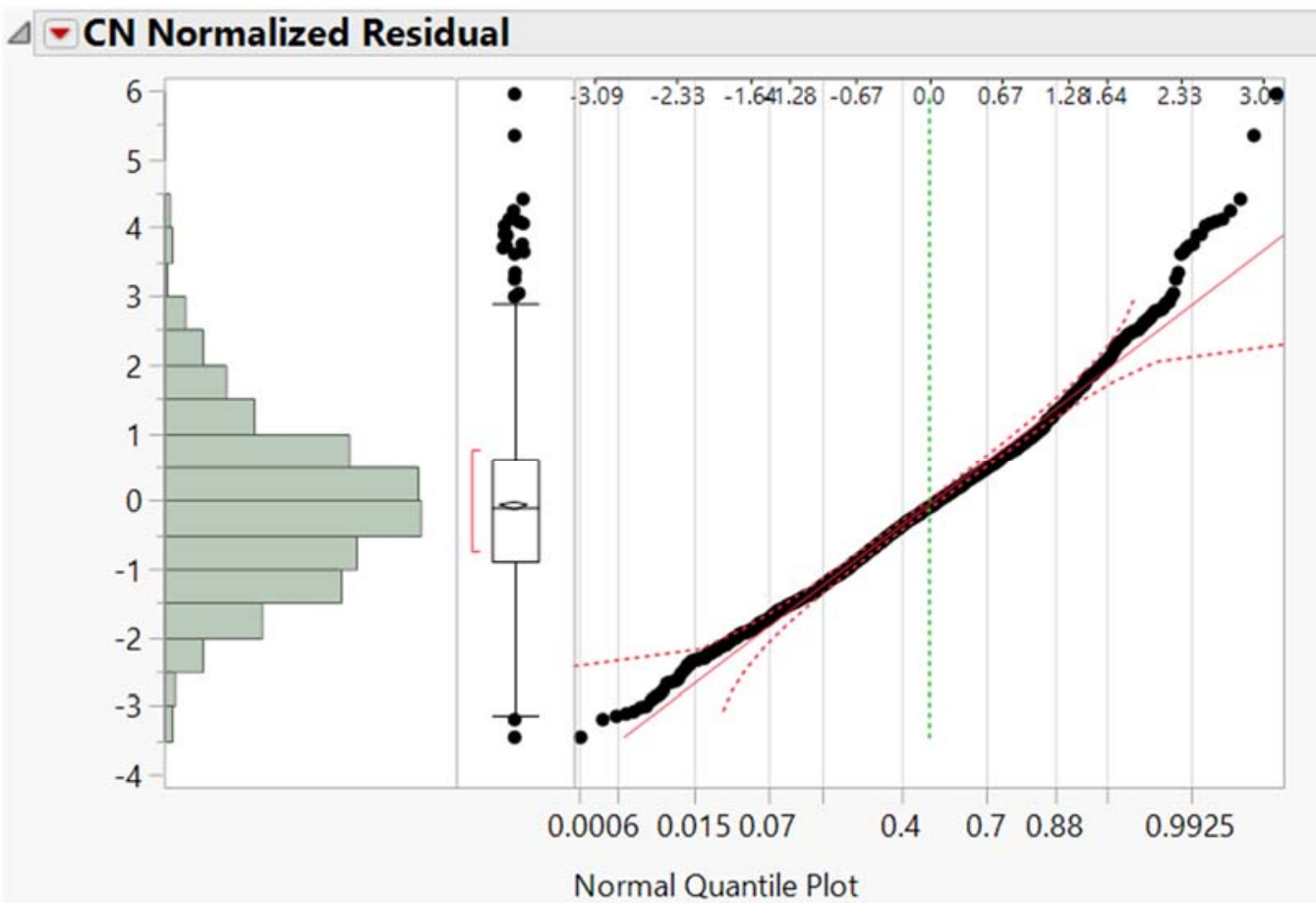

Figure 130 Normal Force Coefficient Global Regression Model Normalized Residuals

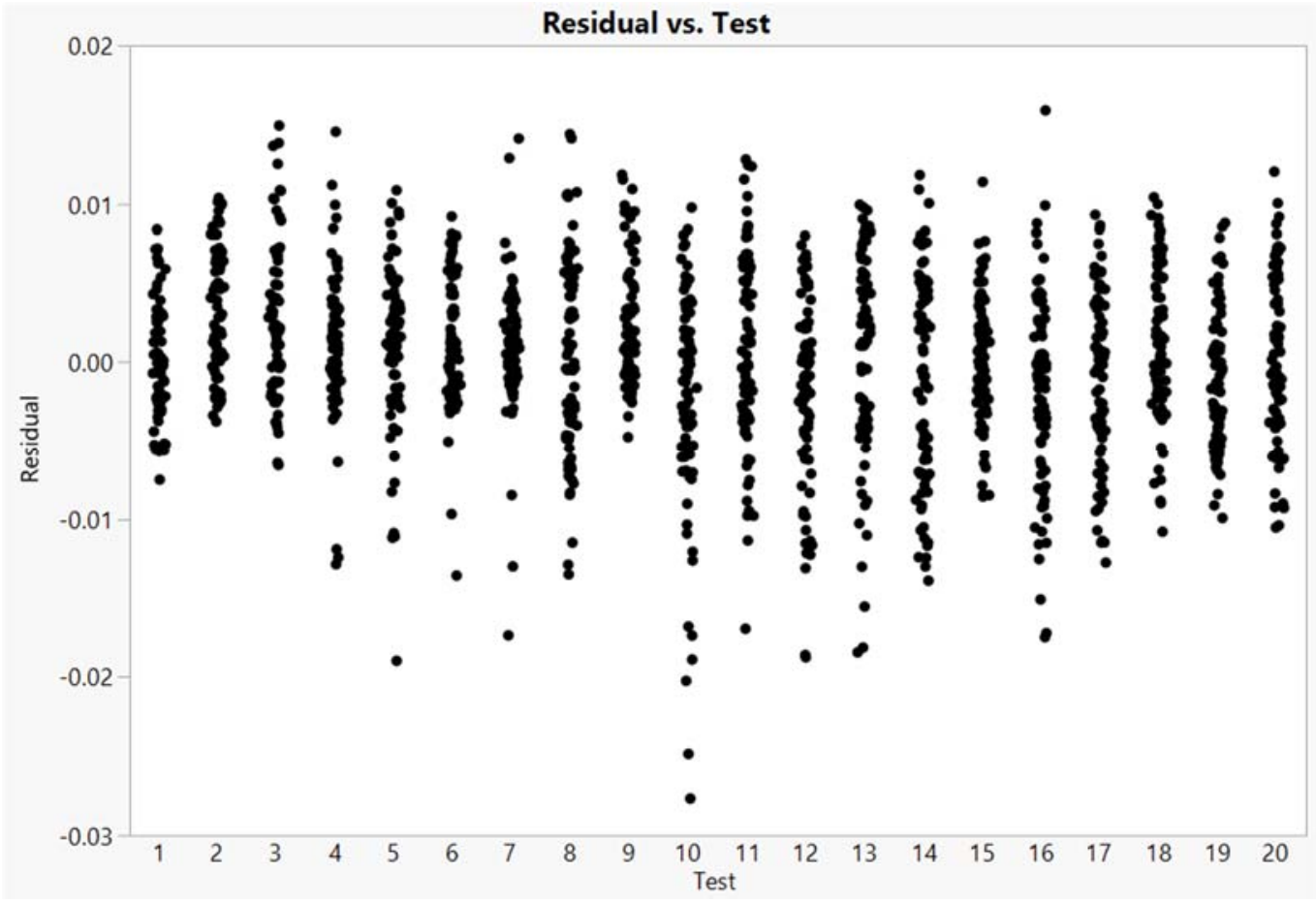

Figure 131 Normal Force Coefficient Global Regression Model Residuals vs Test 


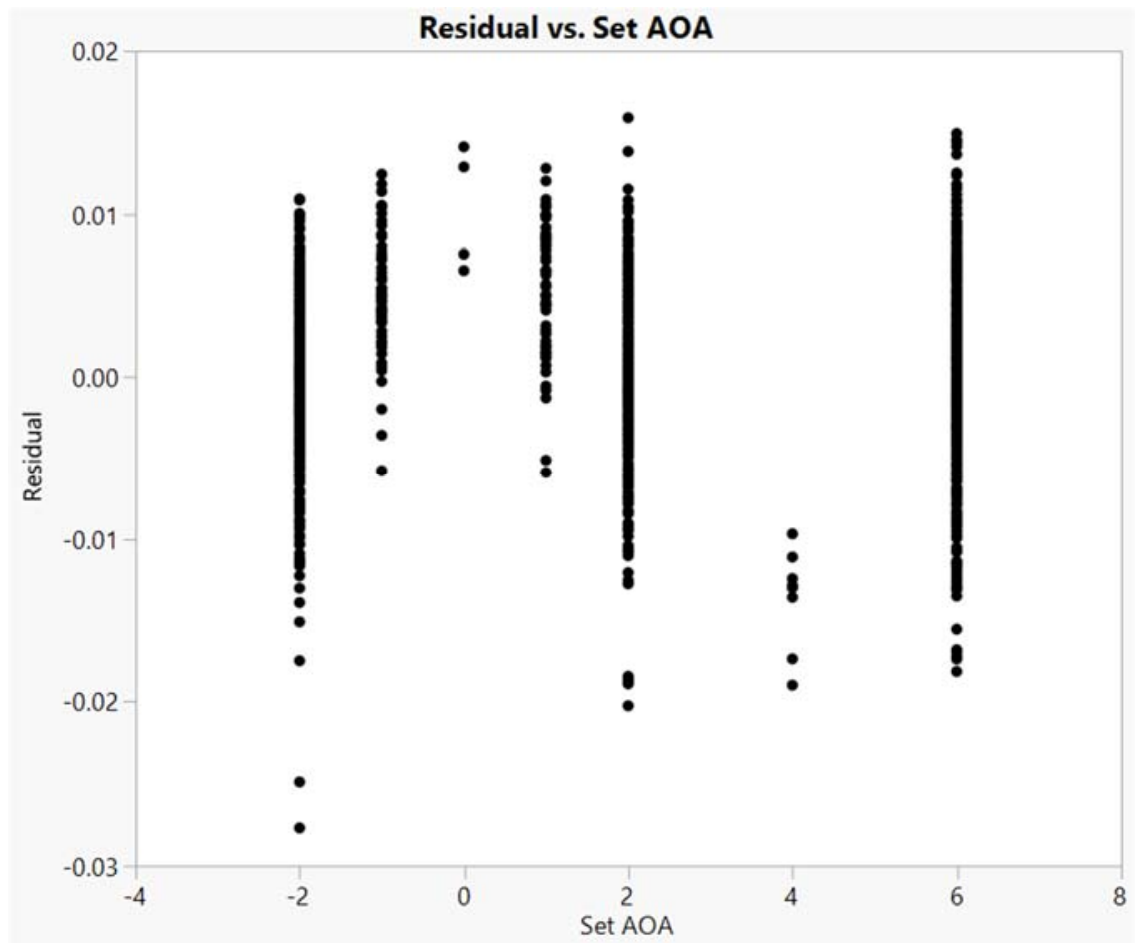

Figure 132 Normal Force Coefficient Global Regression Model Residuals vs Angle of Attack

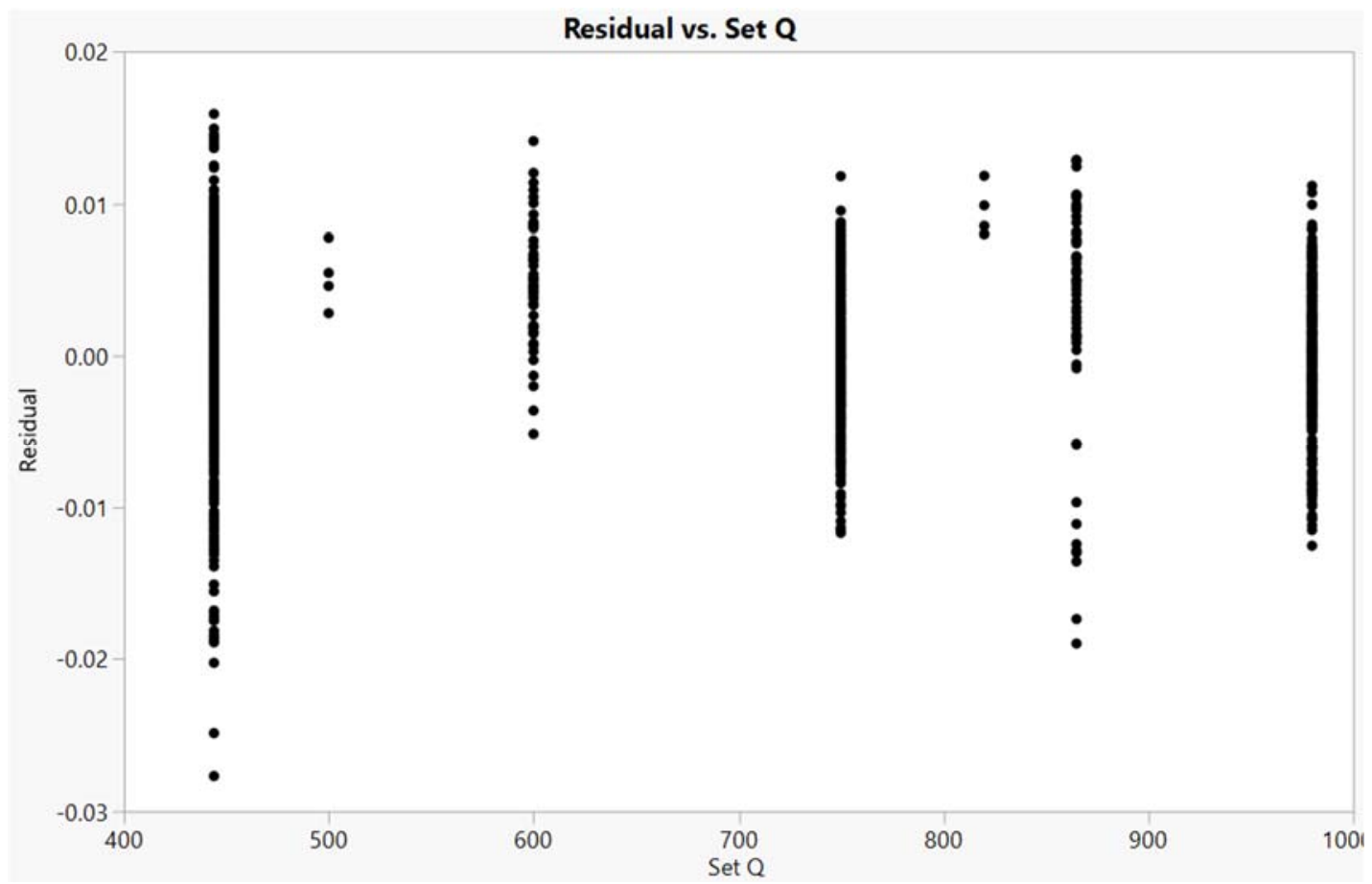

Figure 133 Normal Force Coefficient Global Regression Model Residuals vs Dynamic Pressure 


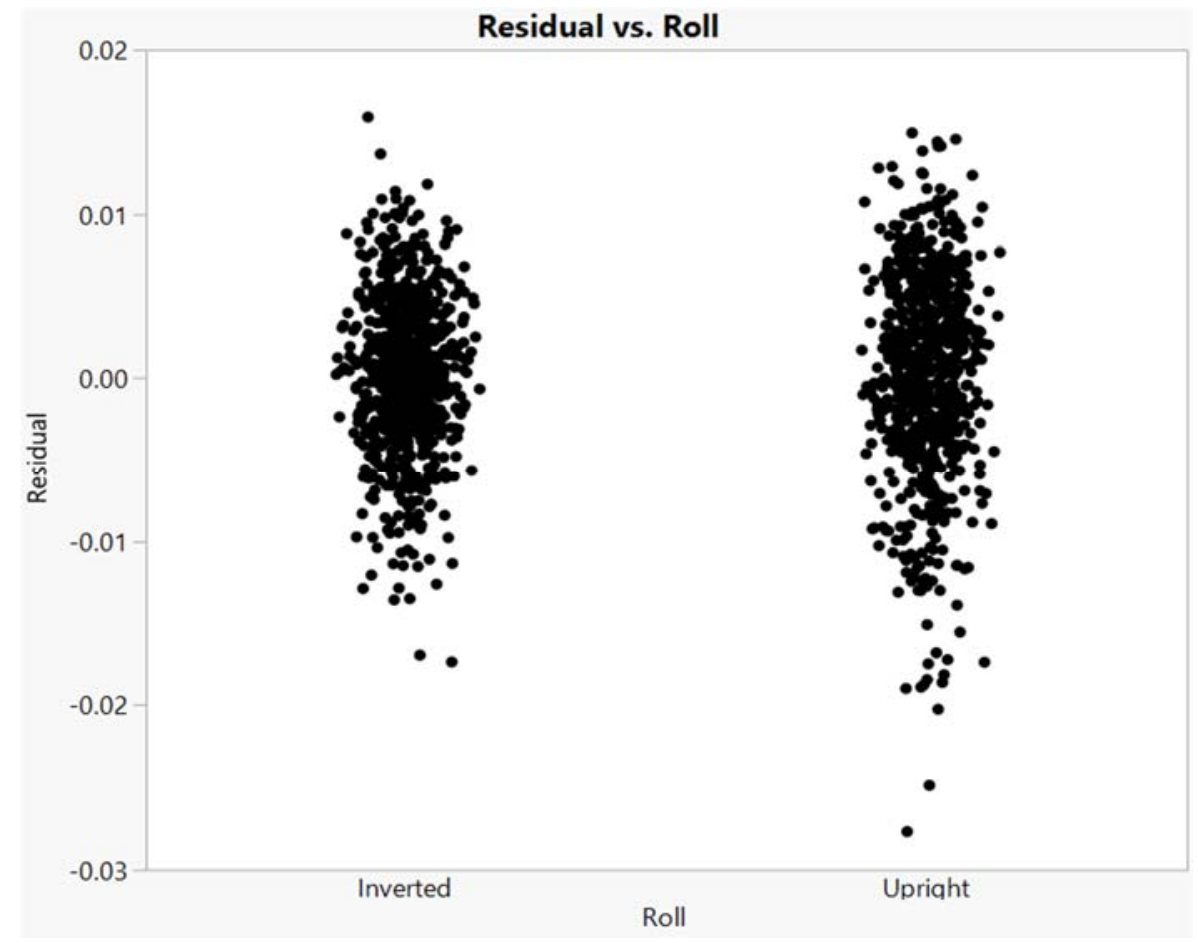

Figure 134 Normal Force Coefficient Global Regression Model Residuals vs Roll

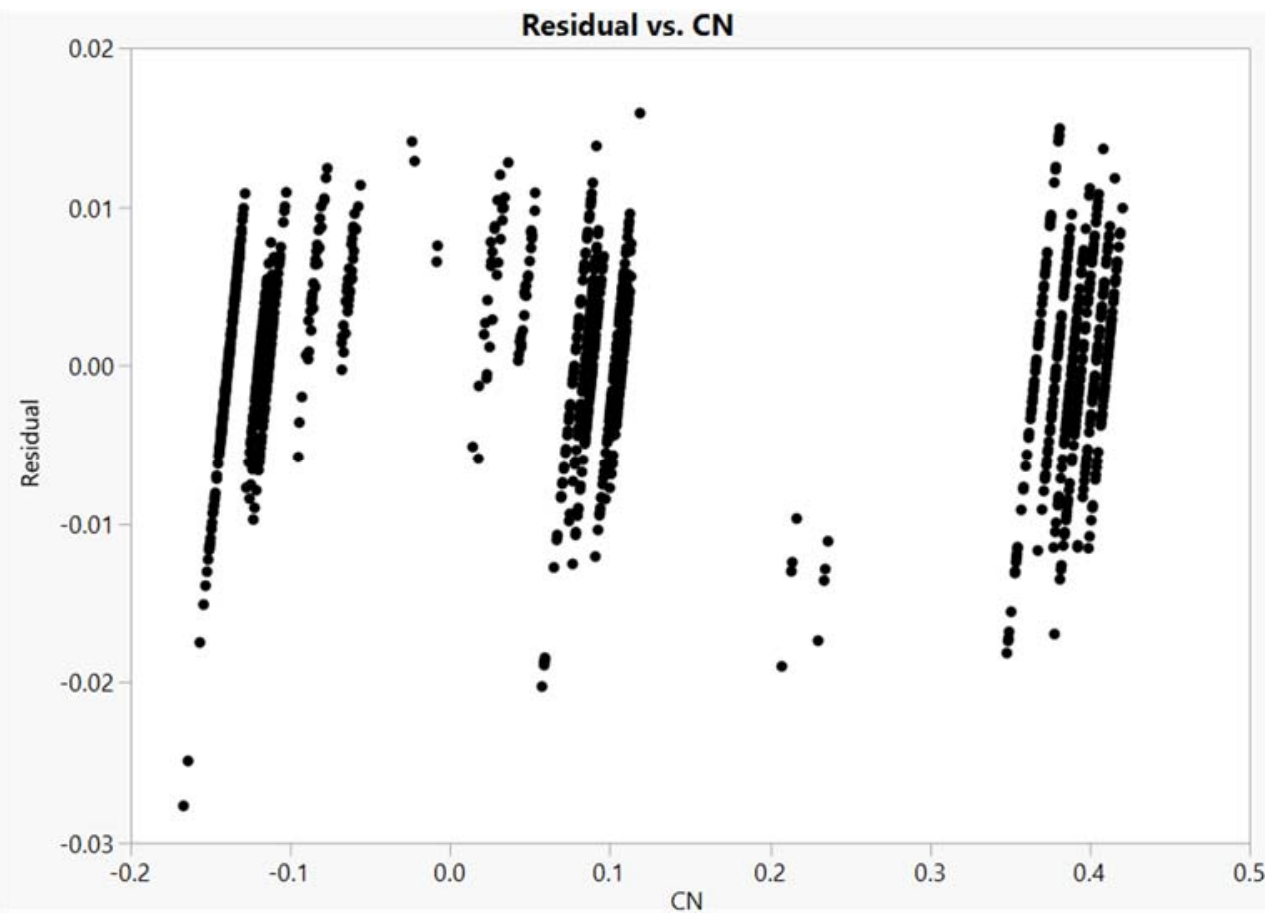

Figure 135 Normal Force Coefficient Global Regression Model Residuals vs Roll 
Similar diagnostic procedures were completed for the evaluation of the axial force and pitching moment coefficients global regression models and no substantial deviations from normality or trends with factors, time or prediction were encountered.

\subsubsection{Error Detection}

To further evaluate the methodology of tracking the regression model coefficients in addition to the traditional statistical process control charts, a simulated error was injected into the data set. The thought process behind this exercise was to investigate the ability of the regression model charts to identify possible problems in the data set that would not be identifiable in the traditional SPC charting methodology. The injected error was represented by a simulated angle of attack offset (bias). This would be encountered in tunnel testing if the reference angle of attack was incorrectly measured or recorded.

For the demonstration of the injected error, the normal force coefficient response was studied, as it is most sensitive to changes in angle of attack. Test 9 was utilized for this exercise due to the fact that both the test average and the regression coefficient associated with angle of attack, $\beta_{2}$ were near the median values of all 20 tests. First the difference in normal force coefficient due to an injected angle of attack offset, $C_{N_{\Delta \alpha}}$ (Equation 106) was found by evaluating the established global regression model (Equation 95) for a condition of $q=750 \mathrm{~Pa}, \alpha=2^{\circ}, \phi=0^{\circ}$ and angle of attack offsets, $\Delta \alpha=$ $-0.1,-0.05,0.05,0.1$. The results are shown superimposed on the across-test control chart for the normal force coefficient of Test 9 in Figure 136. From inspection, it is seen that the injection of the angle of attack offset in the standard SPC control charts would not indicate a process that is out of statistical control.

$$
C_{N_{\Delta \alpha}}=C_{N}(q, \alpha+\Delta \alpha, \phi)
$$




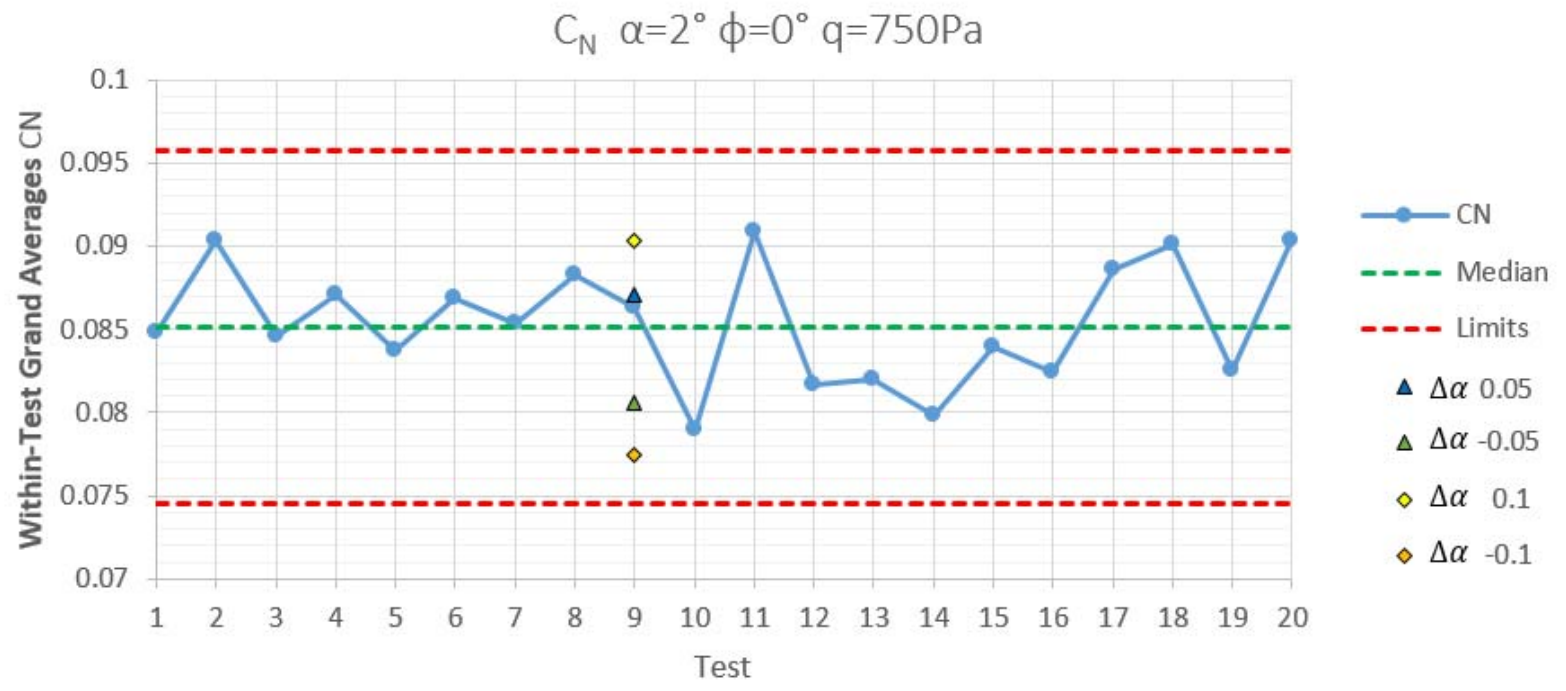

Figure 136 SPC Chart with Injected Angle of Attack Offset

To determine the new regression coefficient $\beta_{2}$ for Test 9 due to the injected angle of attack offset, the entire (local) regression model was rebuilt in the form of Equation 92. The model was built using the actual recorded response for normal force coefficient, but with the new angle of attack due to the injection of the bias. The same procedure was followed as was used to originally build the model (FCD split-plot, REML). The new regression coefficient, $\beta_{2}$ is shown superimposed over the regression control chart for all 20 Tests in Figure 137. 


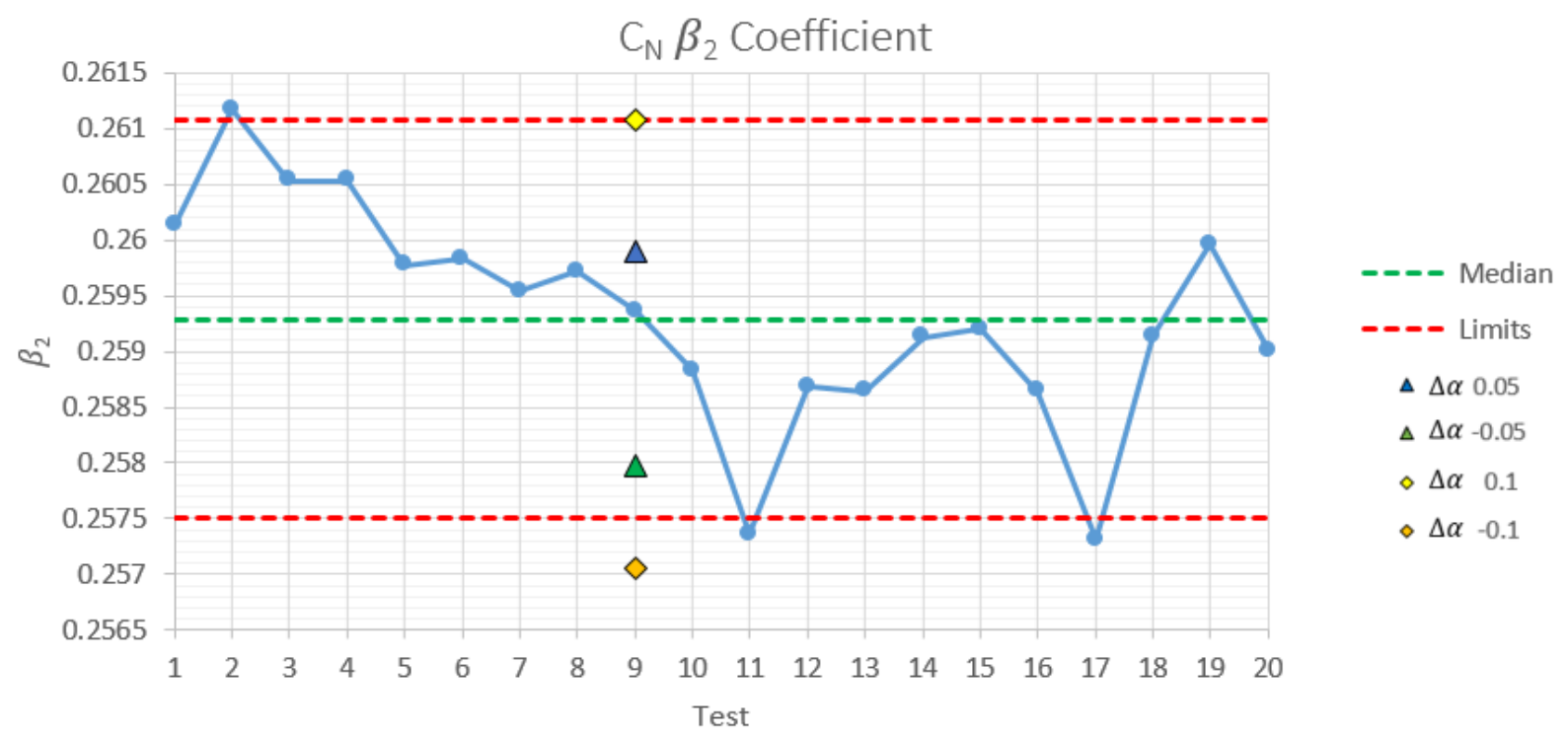

Figure 137 Regression Coefficient Control Chart with Injected Angle of attack Offset

From inspection it is seen that the injected angle of attack bias into the regression coefficient control charts presents a much greater relative change in $\beta_{2}$ verses the standard control chart for $C_{N}$. In Table 50 the normalized residual calculated for each of the charts is presented to compare the relative sensitivities. For the regression coefficient chart the normalized residual is defined as

$$
e_{\beta_{2}}=\frac{\Delta \alpha \beta_{2}-\beta_{2}}{\hat{\sigma}_{\beta_{2}}}
$$

where $\Delta \alpha \beta_{2}$ is the new regression coefficient due to the injected error and $\hat{\sigma}_{\beta_{2}}$ is the estimate of the standard deviation from the charting procedure. The normalized residual for the standard SPC chart is given as

$$
e_{C_{N}}=\frac{C_{N_{\Delta \alpha}}-C_{N}}{\hat{\sigma}_{C_{N}}}
$$

where $\hat{\sigma}_{C_{N}}$ is the estimate of the standard deviation from the charting procedure. 


\begin{tabular}{|l|l|l|}
\hline \multicolumn{1}{|c|}{$\Delta \boldsymbol{\alpha}$} & \multicolumn{1}{c|}{$\boldsymbol{e}_{\boldsymbol{C}_{\boldsymbol{N}}}$} & \multicolumn{1}{c|}{$\boldsymbol{e}_{\boldsymbol{\beta}_{\mathbf{2}}}$} \\
\hline $\mathbf{0 . 1}$ & 1.517 & 3.283 \\
\hline $\mathbf{0 . 0 5}$ & 0.529 & 1.709 \\
\hline$-\mathbf{0 . 0 5}$ & -1.306 & -1.439 \\
\hline $\mathbf{- 0 . 1}$ & -2.153 & -3.013 \\
\hline
\end{tabular}

Table 50 Normalized Residuals from Angle of Attack Error Injection

This sensitivity metric is presented in Table 50 and it is shown that for each of the angle of attack errors, the regression coefficient control chart is more sensitive to detecting errors. Injecting an error simulated by an angle of attack offset into the check standard data gives an objective metric for the efficacy of the regression coefficient charts. This exercise demonstrated that the regression coefficient control charts are able to identify potential underlying problems in the check standard data that may not be identifiable in the standard SPC charting methods. In these cases, the standard SPC charting methods would have indicated no problems and no investigations into the angle of attack measurement process would have been undertaken. This could potentially lead to entire tests being run with faulty measurement systems in place. 


\section{CONCLUSIONS}

The current check standard testing program at NASA Langley Research Center is focused on increasing data quality, uncertainty quantification and overall control and improvement of wind tunnel measurement processes. The statistical process control methodology employed in the check standard testing program allows for the tracking of variations in measurements over time. While the SPC approach can and does provide researchers with valuable information, it has certain limitations in the areas of process improvement and uncertainty quantification. It has been shown that in using design of experiments methodology in conjunction with the current SPC practices, that one can efficiently and more robustly characterize uncertainties and develop enhanced process improvement procedures.

The developed methodology provides a continuous regression model for force balance coefficients as a function of dynamic pressure, angle of attack, and roll angle across the entire tunnel operating envelope. This is a marked improvement over the traditional approach which only yields multiple discrete points from pitch polars. Similarly for the tunnel conditions testing, a continuous regression model for the calibration coefficient is built as a function of dynamic pressure and temperature across the entire tunnel operating envelope. This, again, is a marked improvement over current practices in which discrete points are obtained. Likewise, the flow angularities obtained throughout the airframe testing are also modeled continuously throughout the design space which is an improvement over current practices. The general procedure outlined in this research for the combination of DOE and SPC methodologies is adaptable to any factors of interest that are thought to have an influence on the tunnel calibration, flow angularities, or balance force and moment coefficients.

The demonstrated methodology of tracking the regression model coefficient in standard SPC charts can provide a higher level of understanding of the overall variation in the balance force coefficients as well as an aid in identifying assignable causes of the control status. The ability of the regression model control chart to identify underlying problems within the check standard testing data was explored. It was shown that the regression model charts can be more sensitive to these underlying problems and can potentially detect these problems when the standard application of the statistical process control charts may not have indicated any problems. 
The established global regression models and associated prediction intervals from the baseline testing for the tunnel calibration coefficient and balance force and moment coefficients allow future check standard testing to be treated as regression model confirmation points, drastically reducing the required test time and resources. The developed prediction interval can be applied with relatively few degrees of freedom (baseline tests). The vast improvement over current SPC practices is the ability of the global regression model to generate the prediction interval for locations within the design space where data were not gathered during check standard testing.

The outlined procedure of variance modeling is able to generate statistically defensible estimates of uncertainty throughout the entire design space. The models are also able to identify areas within the design space that exhibit higher relative variances through the implementation of uncertainty propagation models.

The use of design of experiments methodology can efficiently provide these enhanced models in addition to providing data which is directly relatable to current SPC and check standard practices. The benefits of design of experiments methodology over traditional methods as it relates to resources necessary to operate a wind tunnel facility for a check standard test scale with the number of factors studied. Therefore the more factors of interest identified, the more DOE can benefit check standard testing. Future guidelines and best practices will be developed to outline the procedures for the implementation of the methodology for facility managers.

It is recognized that the research performed as it relates to the potential benefits of the applications of design of experiments is still a point of contention within the ground test community. For many years, the traditional approach of one factor at a time testing has provided relatively reliable results. However, it is the hope of the author that as more research is conducted into the applications and benefits of the design of experiments methodology in check standard testing and ground testing more broadly, that more facilities and researchers consider its implementation. 


\section{REFERENCES}

1. Hemsch, M.J. and Holden, H.P., Repeatability Modeling for Wind-Tunnel Measurements: Results for Three Langley Facilities, in 52nd Aerospace Sciences Meeting. 2014: National Harbor, Maryland.

2. Iyer, V., Kuhl, D.D., and Walker, E.L., Improvements to Wall Corrections at the NASA Langley 14x22-Ft Subsonic Tunnel. AIAA, 2003. 2003-3950.

3. Wheeler, D.J., Advanced Topics in Statistical Process Control. 2 ed. 2004: SPC Press.

4. DeLoach, R., Check-Standard Testing Across Multiple Transonic Wind Tunnels with the Modern Design of Experiments, in 28th Aerodynamic Measurement Technology Conference. 2012: New Orleans, Louisiana.

5. Campbell, J.F., The National Transonic Facility - A Research Perspective, in AIAA 2nd Applied Aerodynamics Conference. 1984: Seattle, WA.

6. Fisher, R.A., The Design of Experiments. 1935: Hafner Publishing Company.

7. Box, G.P. and Wison, K.B., On the Experimental Attainment of Optimum Conditions. Journal of the Royal Statistical Society, 1951. 23(1): p. 45.

8. Taguchi, G., The System of Experimental Design: Engineering Methods to Optimize Quality and Minimize Costs. 1 ed. 1987: Quality Resources.

9. Montgomery, D.C., Design and Analysis of Experiments. 8th ed. 2013: John Wiley \& Sons, Inc.

10. Myers, R.H., Montgomery, D.C., and Anderson-Cook, C.M., Response Surface Methodology. 3rd ed. 2009: John Wiley \& Sons Inc.

11. Shewhart, W., A., Economic Control of Quality of a Manufactured Product. 1931: Van Nostrand.

12. Montgomery, D.C., Introduction to Statistical Quality Control. 6th ed. 2009: John Wiley \& Sons, Inc.

13. AIAA, Assessment of Experimental Uncertainty with Application to Wind Tunnel Testing, in Technical Standard: S-071A. 1999.

14. AGARD, A Review of Measurements on AGARD Calibration Models. 1964.

15. Starmer, C., Experiments in Economics... (Should We Trust the Dismal Scientists in White Coats?), U.N. School of Economic and Social Studies, UK, Editor. 1997.

16. Aldrich, J., Information and Economics in Fisher's Design of Experiments. International Statistical Review, 2007. 75(2).

17. Muller, W.G. and Ponce de Leon, A., Optimal Design of an Experiment in Economics. The Economic Journal, 1996. 106(434).

18. Brunswik, E. Systematic and Representative Design of Psychological Experiments with Results in Physical and Social Perception. in Proceedings of the First Berkeley Symposium on Mathematical Statistics and Probability. 1949. Berkeley, CA: University of California Press.

19. Brunswik, E., Perception and the Representative Design of Psychological Experiments. 1956: University of California Press. 
20. Campbell, D.T., Factors Relevant to the Validity of Experiments in Social Settings. Psychological Bulletin, 1957. 54(4).

21. Rosnow, R.L. and Rosenthal, R., Statistical Procedures and the Justification of Knowledge in Psychological Science. American Psychologist, 1989. 44(10).

22. Collins, L.M. and Dziak, J.J., Design of Experiments with Multiple Independent Variables: A Resource Management Perspective on Complete Reduced Factorial Designs. Psychol Methods, 2009. 14(3).

23. George, B.J., et al., Common Scientific and Statistical Errors in Obesity Research. Obesity, 2016. 24.

24. Sonnaert, M., Kerckhofs, G., and Papantoniou, I., Multifactorial Optimization of ContrastEnhanced Nanofocus Computed Tomography for Quantitative Analysis of Neo-Tissue Formation in Tissue Engineering Constructs. PLoS ONE, 2015. 10(6).

25. Knight, K.L., Study/Experimental/Research Design: Much More Than Statistics. Journal of Athletic Training, 2010. 45(1).

26. Elskens, M., de Brauwere, A., and Beucher, C., Statistical Process Control in Assessing Production and Dissolution Rates of Biogenic Silica in Marine Environments. Marine Chemistry, 2007. 106.

27. Bartfai, A., Markovic, G., and Sargenius, K., The Protocol and Design of Randomised Controlled Study on Training Attention Within the First Year After Acquired Brain Injury. 14, 2014. 102.

28. Rutman, L., Migita, R., and Spencer, S., Standardized Asthma Admission Criteria Reduce Length of Stay in a Pediatric Emergency Department. Academic Emergency Medicine, 2016. 23.

29. Kang, L. and Albin, S.L., On-line Monitoring When the Process Yields a Linear Profile. Journal of Quality Technology, 2000. 32(4).

30. Bisgaard, S., Must a Process Be in Statistical Control before Conducting Designed Experiments? Quality Engineering, 2008. 20.

31. Callahan, R.J., et al., Long-Term Study of Wind-Tunnel Balance Calibration History using Statistical Process Control, in 31st AIAA Aerodynamic Measurement Technology and Ground Testing Conference. 2015: Dallas TX.

32. Noorossana, R., Eyvazian, M., and Amiri, A., Statistical Monitoring of Multivariate Multiple Linear Regression Profiles in Phase I with Calibration Application. Quality and Reliability Engineering International, 2010. 26.

33. Zhang, Y., et al., Directed Control Charts for Detecting the Shape Change from Linear Profiles to Quadratic Profiles. International Journal of Production Research, 2014. 52(11).

34. Nikoo, M. and Noorossana, R., Phase II Monitoring of Nonlinear Profile Variance Using Wavelet. Quality and Reliability Engineering International, 2012. 29.

35. Kammeyer, M.E., Wind Tunnel Facility Calibrations and Experimental Uncertainty, in 20th AIAA Advanced Measurement and Ground Testing Conference. 1998: Albuquerque, NM.

36. Hemsch, M.J., Analysis of Flow Angularity Repeatability Tests in the NTF, in 44th AIAA Aerospace Sciences Meeting. 2006: Reno, NV.

37. Bertin, J.J. and Cummings, R.M., Aerodynamics for Engineers. 5 ed. 2009: Pearson Prentice-Hall. 
38. Coleman, H.W. and Glenn, S.W., Experimentation and Uncertainty Analysis for Engineers. 1999: John Wiley \& Sons.

39. Steinle, F., Wind Tunnel Flow Quality and Data Accuracy Requirements. AGARD Advisory Report, 1982. 184.

40. Lawrence, W., Snead, A., and Wignal, T.J., Standard Dynamics Model, in Mechanical Engineering. 2011, Old Dominion University.

41. Beyers, M.E., Subsonic Roll Oscillation Experiments on the Standard Dynamics Model. AIAA, 1983. 83-2134.

42. H. Davis, F.N.K., A Resume of Aerodynamic Data on Air Brakes, A.R. Council, Editor. 1951, His Majesty's Stationery Office: London.

43. Buehrle, R.D., et al., Experimental Study of Dynamic Interaction Between model Support Structure and a High Speed Research Model in the National Transonic Facility. AIAA, 1994. 941623-CP.

44. Cole, S.R., et al., Test Activities in the Langley Transonic Dynamics Tunnel and a Summary of Recent Facility Improvements. 44th Structres, Structural Dynamics, and Materials Conference, 2003. 2003-1958.

45. Velmex. UniSlide Assemblies - Engineering Information. Retrieved 2015.

46. Velmex, NF90 Series User's Guide One, Two, and Three-axis Stepping Motor Controller/Drivers. Retrieved 2015.

47. Level Developments, LSR: Closed Loop Single Axis Servo Inclinometer. 2015: www.leveldevelopments.com.

48. Hogg, S., What is LabVIEW. 2013: http://www.ni.com/newsletter/51141/en/.

49. Using JMP Version 12. 2015: www.jmp.com/support/downloads/pdf/jmp8/jmp_user_guide.pdf.

50. Parker, P., Kowalski, S.M., and Vining, G.G., Construction of Balanced Equivalent Estimation Second Order Split Plot Designs. Technometrics, 2007: p. 9.

51. Parker, P., Kowalski, S.M., and Vining, G.G., Classes of Split-Plot Response Surface Designs for Equivalent Estimation. Quality and Reliability Engineering International, 2006a. 22: p. 14.

52. Coleman, H.W. and Steele, W.G., Experimentation and Uncertainty Analysis for Engineers. 2nd ed. 1999: John Wiley and Sons.

53. Haldar, A. and Mahadevan, S., Probability, Reliability and Statistical Methods in Engineering Design. 1st ed. 2000: John Wiley and Sons.

54. Montgomery, D.C. and Runger, G.C., Applied Statistics and Probability for Engineers. 4th ed. 2007: John Wiley \& Sons, Inc. 


\section{APPENDIX A: FORCE BALANCE SPECIFICATIONS}

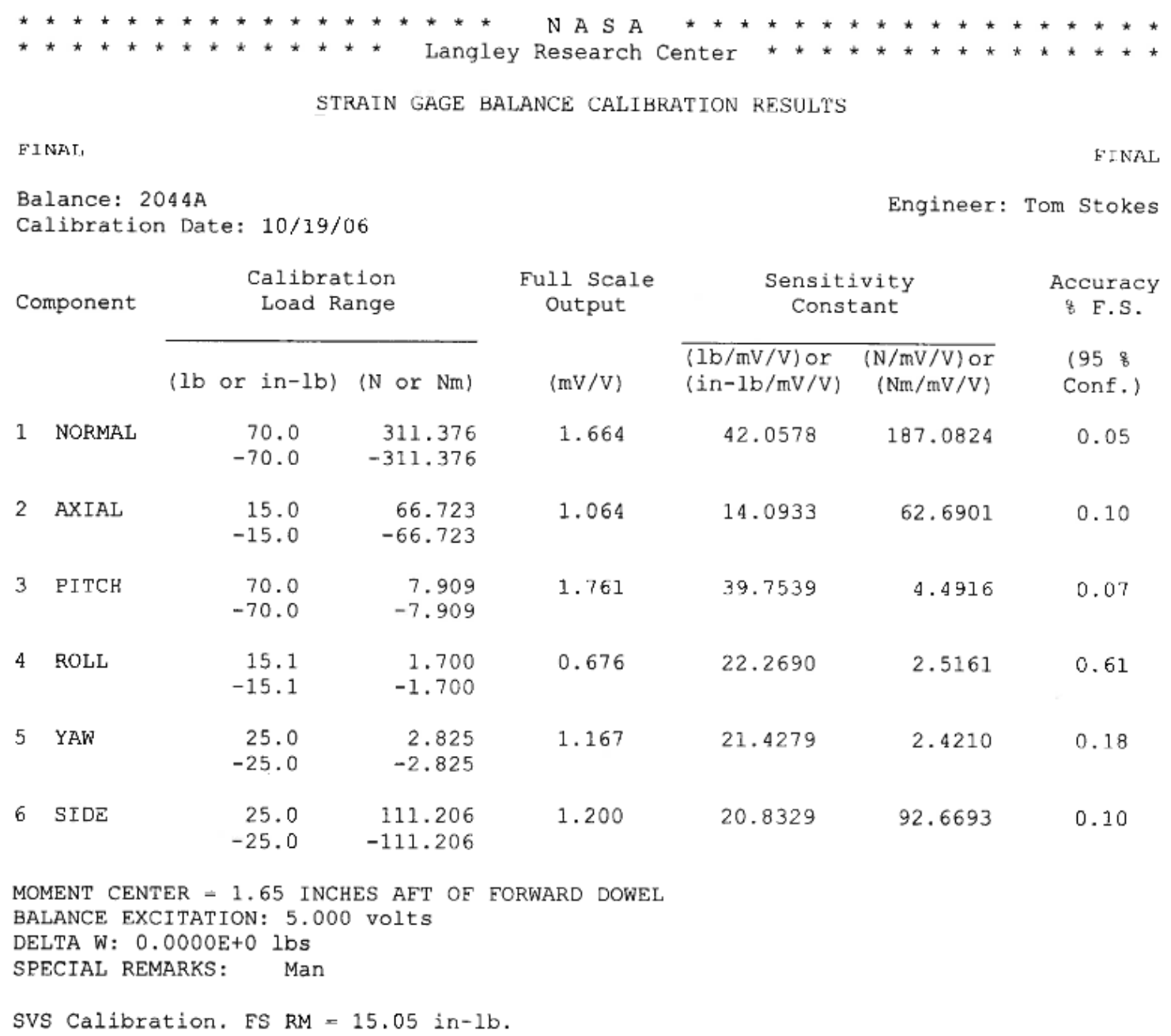

MOMENT CENTER $=1.65$ INCHES AFT OF FORWARD DOWEL BALANCE EXCITATION: 5.000 volts 

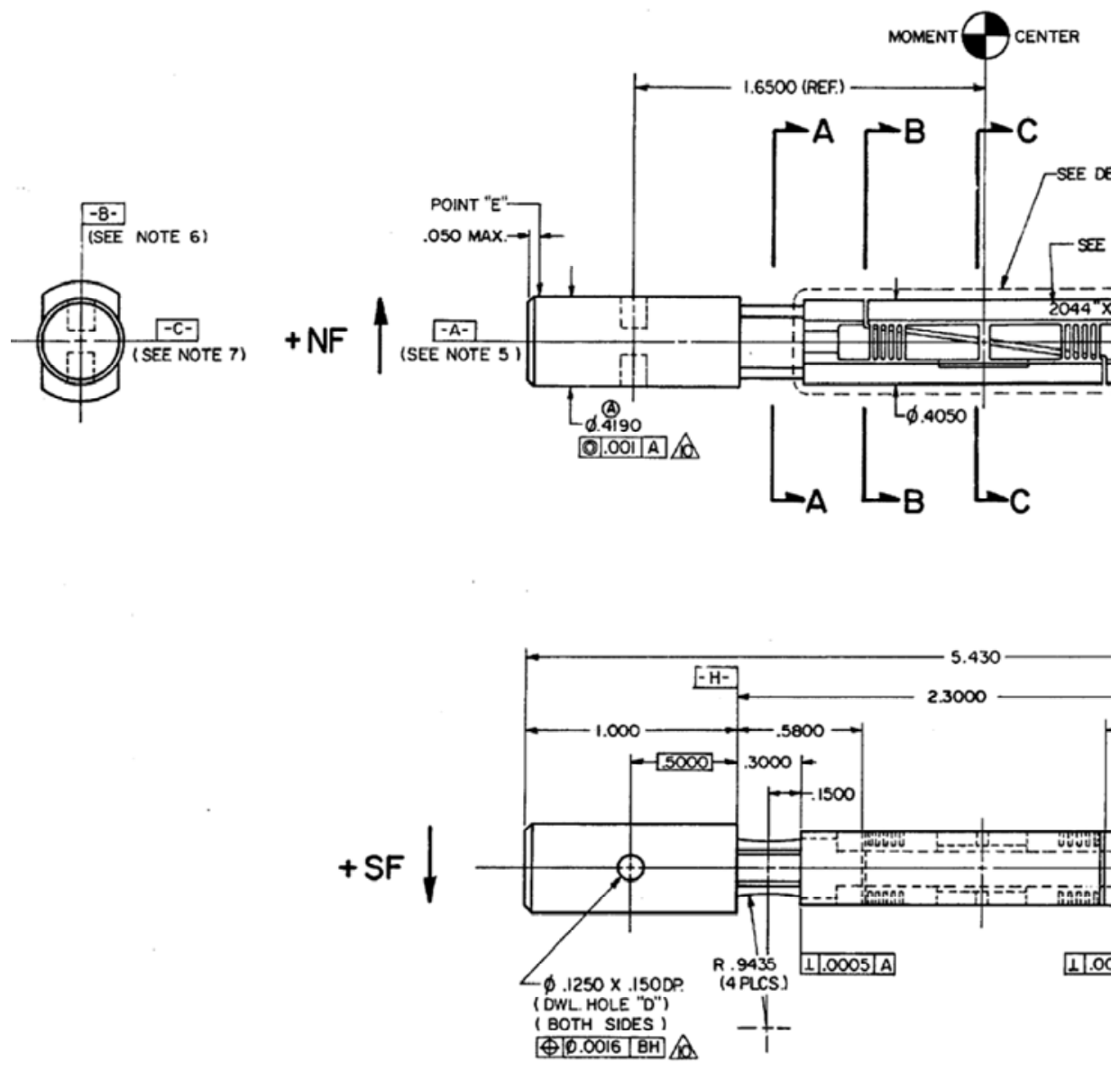


\section{APPENDIX B: INCLINOMETER SPECIFICATIONS}

\section{Environmental Characteristics}

Operating Temperature Range

SurvivalTemperature Range

Constant Acceleration Overload

Shock Survival

Endurance

Environmental Sealing
${ }^{\circ} \mathrm{C}$

${ }^{\circ} \mathrm{C}$

g

C

-18 to 70

-40 to 70

50

$1500 \mathrm{~g}, 0.5 \mathrm{msec}, 1 / 2$ sine

$35 \mathrm{~g} \mathrm{rms}, 20 \mathrm{~Hz}$ to $2000 \mathrm{~Hz}$ sinusoidal

IP65

\section{Specifications by Range @ $20^{\circ} \mathrm{C}$}

Range
Excitation Voltage
Current Consumption
Full Range Output (FRO) (see note 1)
Output Standardisation
Output Impedance
Output Noise
Non-Linearity (see note 2)
Non-Repeatability
Resolution
-3 dB Frequency
Sensitive Axis-to-Case Misalignment
Cross-axis sensitivity (see note 3)
Zero Offset (see note 4)
Thermal Zero Shift
Thermal Sensitivity

\begin{tabular}{cccccc} 
& $\pm 1^{\circ}$ & $\pm 3^{\circ}$ & $\pm 14.5^{\circ}$ & $\pm 30^{\circ}$ & $\pm 90^{\circ}$ \\
Volts dc & & \multicolumn{5}{c}{ \pm 12 to \pm 18} & \\
mA (nom) & \pm 15 & \pm 15 & \pm 15 & \pm 15 & \pm 15 \\
Volts dc & & & \pm 5 & & \\
$\%$ FRO (max) & & & \pm 1 & & \\
Ohm & & & $<10$ & & \\
V rms (max) & & & 0.002 & & 0.05 \\
$\%$ FRO (max) & 0.08 & 0.05 & 0.02 & 0.02 & 0.0005 \\
$\%$ FRO (max) & 0.02 & 0.01 & 0.002 & 0.001 & 4.0 \\
arc seconds & 0.1 & 0.2 & 1.0 & 2.0 & 55 \\
Hz & 10 & 15 & 30 & 40 & \pm 1.0 \\
deg (max) & \pm 0.15 & \pm 0.15 & \pm 0.25 & \pm 0.5 & \\
$\%$ FRO (max) & & & 0.1 & & \pm 0.02 \\
Volts dc (max) & \pm 0.08 & \pm 0.04 & \pm 0.04 & \pm 0.02 & \pm 0.003 \\
$\%$ FRO $/{ }^{\circ} \mathrm{C}$ (max) & \pm 0.05 & \pm 0.03 & \pm 0.01 & \pm 0.005 & \pm 0.006 \\
$\%$ Reading $/{ }^{\circ} \mathrm{C}$ (max) & \pm 0.05 & \pm 0.03 & \pm 0.01 & \pm 0.006 & \pm 0.006
\end{tabular}




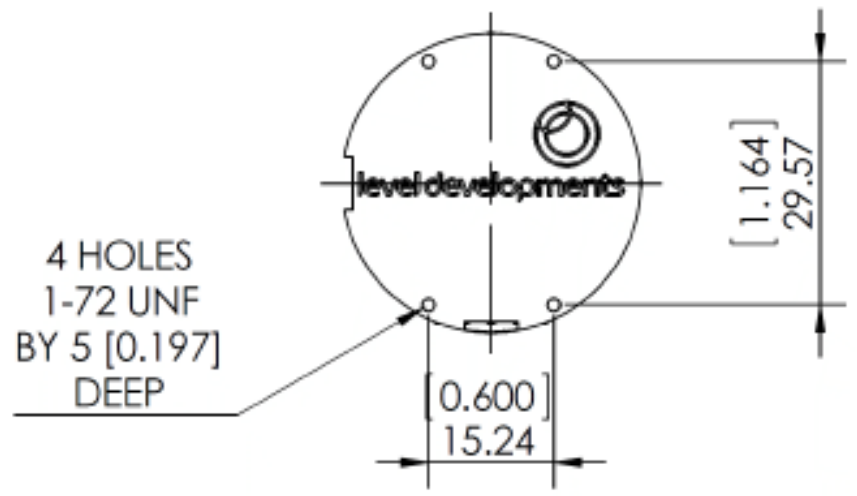

Pin Connection for LSR

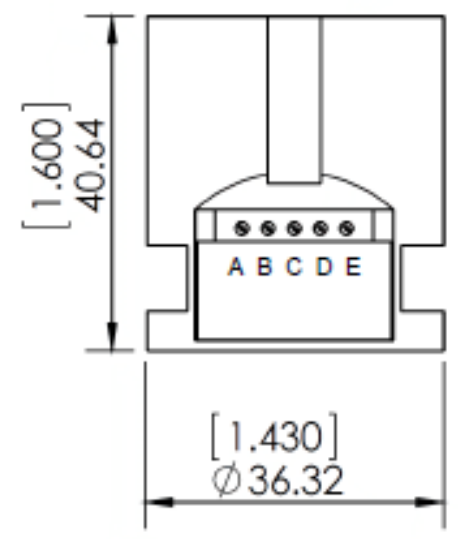

2 HOLES

Ø3 H7 [0.118]

BY 5 [0.197]

DEEP

2 HOLES

8-32 UNC

BY 5 [0.197]

DEEP

\begin{tabular}{|c|c|}
\hline Pin Ref & Function \\
\hline A & +12 to $+18 \mathrm{Vdc}$ \\
\hline B & GND \\
\hline C & -12 to $-18 \mathrm{Vdc}$ \\
\hline D & Output \\
\hline E & Self Test \\
\hline
\end{tabular}




\section{APPENDIX C: ROLL MOTOR SPECIFICATIONS}

Gearmotor Data, continued

\begin{tabular}{|c|c|c|c|c|c|c|c|c|c|c|c|c|c|c|c|}
\hline Line No. & Parameter & Symbol & Units & $5.9: 1$ & 115:1 & 19.71 & 38.31 & $65.5: 1$ & 127.8:1 & 218.4:1 & 425.9:1 & $728.1: 1$ & 1419.81 & 2426.91 & 4732.51 \\
\hline \multicolumn{16}{|c|}{ NO-LOAD SPEED (All Gears), continued } \\
\hline 28 & GM9X35 & $\mathrm{S}_{\mathrm{NL}}$ & $\underset{(\mathrm{rad} / \mathrm{s})}{\stackrel{\mathrm{rl} m}{2}}$ & $\begin{array}{c}1075 \\
(1125)\end{array}$ & $\begin{array}{c}552 \\
(57.8)\end{array}$ & $\begin{array}{c}322 \\
(33.7)\end{array}$ & $\begin{array}{c}166 \\
(17.4)\end{array}$ & $\begin{array}{c}96.9 \\
(10.1)\end{array}$ & $\begin{array}{l}49.7 \\
(5.2)\end{array}$ & $\begin{array}{l}29.1 \\
(3.0)\end{array}$ & $\begin{array}{l}14.9 \\
(1.6)\end{array}$ & $\begin{array}{c}8.7 \\
(.913)\end{array}$ & $\begin{array}{c}4.4 \\
(.468)\end{array}$ & $\begin{array}{c}2.6 \\
(.274)\end{array}$ & $\begin{array}{c}1.3 \\
(140)\end{array}$ \\
\hline 29 & GM9X36 & $\mathrm{S}_{\mathrm{NL}}$ & $\underset{(\mathrm{rad} / \mathrm{s})}{\mathrm{pm}}$ & $\begin{array}{c}834 \\
(87.3)\end{array}$ & $\begin{array}{c}427 \\
(44.7)\end{array}$ & $\begin{array}{c}250 \\
(26.2)\end{array}$ & $\begin{array}{c}128 \\
(13.4)\end{array}$ & $\begin{array}{c}75 \\
(7.85)\end{array}$ & $\begin{array}{c}38.5 \\
(4.03)\end{array}$ & $\begin{array}{l}22.5 \\
(2.36)\end{array}$ & $\begin{array}{c}11.5 \\
(1.20)\end{array}$ & $\begin{array}{c}6.8 \\
(.712)\end{array}$ & $\begin{array}{c}3.5 \\
(.367)\end{array}$ & $\begin{array}{c}2.0 \\
(.209)\end{array}$ & $\begin{array}{l}1.0 \\
(105)\end{array}$ \\
\hline
\end{tabular}

\section{Motor Data}

\begin{tabular}{|c|c|c|c|c|c|c|c|c|}
\hline Line No. & Parameter & Symbol & Units & GM9X32 & GM9X33 & GM9X34 & GM9X35 & GM9X36 \\
\hline 30 & $\begin{array}{c}\text { Continuous } \\
\text { Torqua }(\text { Max. })^{3}\end{array}$ & $\mathrm{~T}_{\mathrm{C}}$ & $\begin{array}{l}\text { or:in } \\
(\mathrm{N} \cdot \mathrm{m})\end{array}$ & $\begin{array}{c}2.3 \\
\left(16.2 \times 10^{-3}\right)\end{array}$ & $\begin{array}{c}4.7 \\
\left(33.2 \times 10^{-3}\right)\end{array}$ & $\begin{array}{c}6.1 \\
\left(43.1 \times 10^{-3}\right)\end{array}$ & $\begin{array}{c}6.9 \\
\left(48.7 \times 10^{-3}\right)\end{array}$ & $\begin{array}{c}9.5 \\
\left(67.1 \times 10^{-3}\right)\end{array}$ \\
\hline 31 & $\begin{array}{l}\text { Peak Torqua } \\
(\text { Stall })^{4}\end{array}$ & $T_{p K}$ & $\begin{array}{l}\text { oz-in } \\
(\mathrm{N} \cdot \mathrm{m})\end{array}$ & $\begin{array}{c}13.8 \\
\left(97.5 \times 10^{-3}\right)\end{array}$ & $\begin{array}{c}31.6 \\
\left(223.2 \times 10^{-3}\right)\end{array}$ & $\begin{array}{c}41.3 \\
\left(291.7 \times 10^{-3}\right)\end{array}$ & $\begin{array}{c}49.4 \\
\left(348.9 \times 10^{-3}\right)\end{array}$ & $\begin{array}{c}61.8 \\
\left(436.4 \times 10^{-3}\right)\end{array}$ \\
\hline 32 & Motor Constant & $K_{M}$ & $\begin{array}{l}o z-\mathrm{n} / \mathrm{WW} \\
(\mathrm{N} \cdot \mathrm{m} / \mathrm{WW})\end{array}$ & $\begin{array}{c}1.62 \\
\left(11.4 \times 10^{-3}\right)\end{array}$ & $\begin{array}{c}2.66 \\
\left(18.8 \times 10^{-3}\right)\end{array}$ & $\begin{array}{c}3.01 \\
\left(21.3 \times 10^{-3}\right)\end{array}$ & $\begin{array}{c}3.21 \\
\left(22.7 \times 10^{-3}\right)\end{array}$ & $\frac{4.11}{\left(29.0 \times 10^{-3}\right)}$ \\
\hline 33 & No-Loed Speed & $S_{0}$ & $\begin{array}{c}\mathrm{rpm} \\
(\mathrm{rad} / \mathrm{s})\end{array}$ & $\begin{array}{c}7015 \\
(734.6)\end{array}$ & $\begin{array}{c}5993 \\
(627.6)\end{array}$ & $\begin{array}{c}6151 \\
(644.2)\end{array}$ & $\begin{array}{c}6348 \\
(664.8)\end{array}$ & $\begin{array}{c}4916 \\
(514.8)\end{array}$ \\
\hline 34 & Friction Torqua & $\mathrm{T}_{\mathrm{F}}$ & $\begin{array}{l}o z \text { in } \\
(\mathbb{N} \cdot \mathrm{m})\end{array}$ & $\begin{array}{c}0.5 \\
\left(3.5 \times 10^{-3}\right)\end{array}$ & $\begin{array}{c}0.6 \\
\left(4.2 \times 10^{-3}\right)\end{array}$ & $\begin{array}{c}0.6 \\
\left(4.2 \times 10^{-3}\right)\end{array}$ & $\begin{array}{c}0.65 \\
\left(4.6 \times 10^{-3}\right)\end{array}$ & $\begin{array}{c}0.8 \\
\left(5.6 \times 10^{-3}\right)\end{array}$ \\
\hline 35 & Rotor Inertia & $\mathrm{J}_{\mathrm{M}}$ & $\begin{array}{l}o z-n s^{2} \\
\left(\lg -m^{2}\right)\end{array}$ & $\begin{array}{c}27 \times 10^{-4} \\
\left(1.91 \times 10^{-6}\right)\end{array}$ & $\begin{array}{c}4.6 \times 10^{-4} \\
\left(3.25 \times 10^{-6}\right)\end{array}$ & $\begin{array}{c}5.9 \times 10^{-4} \\
\left(4.17 \times 10^{-6}\right)\end{array}$ & $\begin{array}{c}7.9 \times 10^{-4} \\
\left(5.58 \times 10^{-6}\right)\end{array}$ & $\begin{array}{c}1.0 \times 10^{-3} \\
\left(7.06 \times 10^{-6}\right)\end{array}$ \\
\hline 36 & $\begin{array}{c}\text { Electrical Tima } \\
\text { Constant }\end{array}$ & $\tau_{E}$ & $\mathrm{~ms}$ & 0.63 & 0.84 & 0.85 & 0.89 & 106 \\
\hline 37 & $\begin{array}{l}\text { Mechanical Time } \\
\text { Constant }\end{array}$ & $\tau_{M}$ & $\mathrm{~ms}$ & 14.4 & 9.29 & 9.25 & 10.9 & 8.5 \\
\hline \multirow{2}{*}{38} & \multirow{2}{*}{$\begin{array}{l}\text { Viscous Damping- } \\
\text { Infinite Source } \\
\text { Impedance }\end{array}$} & \multirow{2}{*}{ D } & oz-in/kpm & 0.0272 & 0.0335 & 0.0387 & 0.0450 & 0.0525 \\
\hline & & & $(\mathrm{N} \cdot \mathrm{m} / \mathrm{rad} / \mathrm{s})$ & $\left(1.83 \times 10^{-6}\right)$ & $\left(2.25 \times 10^{-6}\right)$ & $\left(2.60 \times 10^{-6}\right)$ & $\left(3.03 \times 10^{-6}\right)$ & $\left(3.54 \times 10^{-6}\right)$ \\
\hline \multirow{2}{*}{39} & \multirow{2}{*}{$\begin{array}{c}\text { Viscous Damping- } \\
\text { Zero Source } \\
\text { Impedance }\end{array}$} & \multirow{2}{*}{$K_{D}$} & ozin/kpm & 1.94 & 5.23 & 6.68 & 7.6 & 12.5 \\
\hline & & & $(\mathrm{N} \cdot \mathrm{m} / \mathrm{rad} / \mathrm{s})$ & $\left(1.31 \times 10^{-4}\right)$ & $\left(3.52 \times 10^{-4}\right)$ & $\left(4.50 \times 10^{-4}\right)$ & $\left(5.12 \times 10^{-4}\right)$ & $\left(8.42 \times 10^{-4}\right)$ \\
\hline 40 & $\begin{array}{c}\text { Maximum } \\
\text { Winding Temp. }\end{array}$ & $\theta_{\operatorname{MAX}}$ & $\begin{array}{l}{ }^{\circ} \mathrm{F} \\
\left({ }^{\circ} \mathrm{C}\right)\end{array}$ & $\begin{array}{c}311 \\
(155)\end{array}$ & $\begin{array}{r}311 \\
(155)\end{array}$ & $\begin{array}{r}311 \\
(155) \\
\end{array}$ & $\begin{array}{c}311 \\
(155)\end{array}$ & $\begin{array}{r}311 \\
(155)\end{array}$ \\
\hline 41 & $\begin{array}{l}\text { Thermal } \\
\text { Impedance }\end{array}$ & $\mathbf{R}_{\mathrm{TH}}$ & $\begin{array}{l}{ }^{\circ} \mathrm{F} / \text { watt } \\
{ }^{\circ} \mathrm{C} / \text { watt. }\end{array}$ & $\begin{array}{c}72.9 \\
(22.7)\end{array}$ & $\begin{array}{c}66.4 \\
(19.1)\end{array}$ & $\begin{array}{c}62.8 \\
(17.1)\end{array}$ & $\begin{array}{c}58.5 \\
(14.7)\end{array}$ & $\begin{array}{c}56.3 \\
(13.5)\end{array}$ \\
\hline 42 & $\begin{array}{c}\text { Thermal Time } \\
\text { Constant }\end{array}$ & $\tau_{T H}$ & $\min$ & 7.21 & 11.1 & 12.0 & 12.9 & 13.5 \\
\hline 43 & $\begin{array}{l}\text { Motor Weight } \\
\text { (Mass) }\end{array}$ & $W_{M}$ & oz & $\begin{array}{c}6.98 \\
(197.9)\end{array}$ & $\begin{array}{c}8.90 \\
(252.3)\end{array}$ & $\begin{array}{c}10.1 \\
(286.3)\end{array}$ & $\begin{array}{c}0.0 \\
(\mathrm{TBD})\end{array}$ & $\begin{array}{c}13.8 \\
(391.2)\end{array}$ \\
\hline
\end{tabular}

Model GM9XX2 Winding Data (Other windings available upon request)

\begin{tabular}{|c|c|c|c|c|c|c|c|}
\hline Line $\mathrm{No}$. & Parameter & Symbol & Units & & & & \\
\hline 44 & Reference Voltage & $E$ & $\mathrm{v}$ & 12.0 & 19.1 & 24.0 & 30.3 \\
\hline 45 & Torque Constant & $K_{T}$ & $\begin{array}{l}0 z \cdot \operatorname{in} / \mathrm{A} \\
(\mathrm{N} \cdot \mathrm{m} / \mathrm{A})\end{array}$ & $\begin{array}{c}2.20 \\
\left(15.6 \times 10^{-3}\right)\end{array}$ & $\begin{array}{c}3.50 \\
\left(24.7 \times 10^{-3}\right)\end{array}$ & $\begin{array}{c}4.40 \\
\left(31.1 \times 10^{-3}\right)\end{array}$ & $\begin{array}{c}5.53 \\
\left(39.1 \times 10^{-3}\right)\end{array}$ \\
\hline 46 & BackEMF Constant & $K_{E}$ & $\begin{array}{l}\text { V/krpm } \\
\text { (V/rad/s) }\end{array}$ & $\begin{array}{c}1.63 \\
\left(15.6 \times 10^{-3}\right)\end{array}$ & $\begin{array}{c}2.59 \\
\left(24.7 \times 10^{-3}\right)\end{array}$ & $\begin{array}{c}3.25 \\
\left(31.1 \times 10^{-3}\right)\end{array}$ & $\begin{array}{c}4.09 \\
\left(39.1 \times 10^{-3}\right)\end{array}$ \\
\hline 47 & Resistance & $R_{T}$ & $\Omega$ & 1.93 & 4.70 & 7.38 & 11.6 \\
\hline 48 & Inductance & L & $\mathrm{mH}$ & 1.16 & 2.94 & 4.64 & 7.34 \\
\hline 49 & NoLload Current & $\mathrm{I}_{\mathrm{M}}$ & A & 0.32 & 0.20 & 0.16 & 0.13 \\
\hline 50 & Paak Current (Stal) & b & A & 6.22 & 4.06 & 3.25 & 2.60 \\
\hline
\end{tabular}

${ }^{3}$ Cortinuous torque specred at $25^{\circ} \mathrm{C}$ amblent temperature and without asoltional heat sink. 
GM94XX Motor
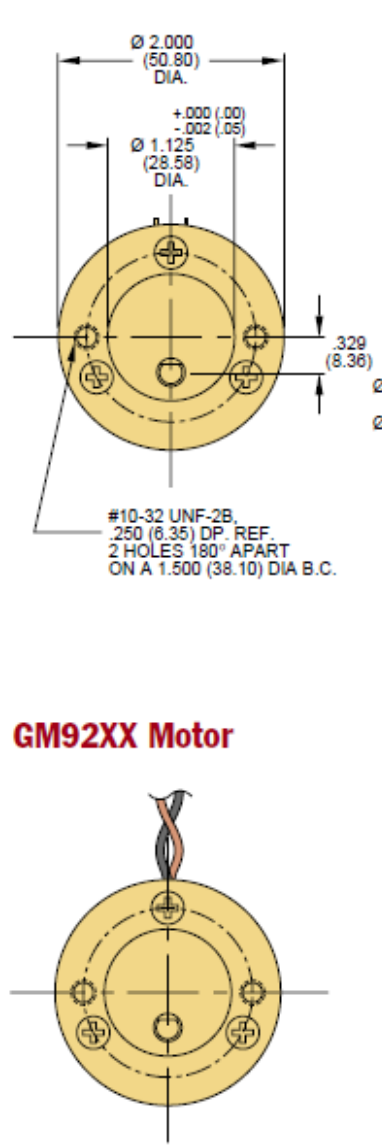
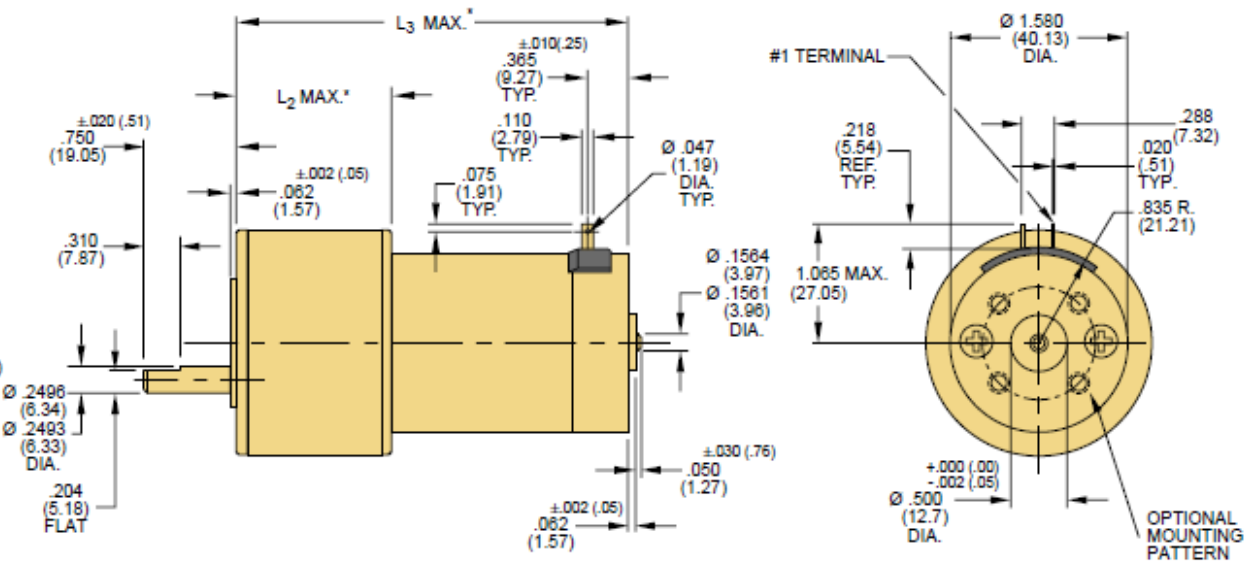

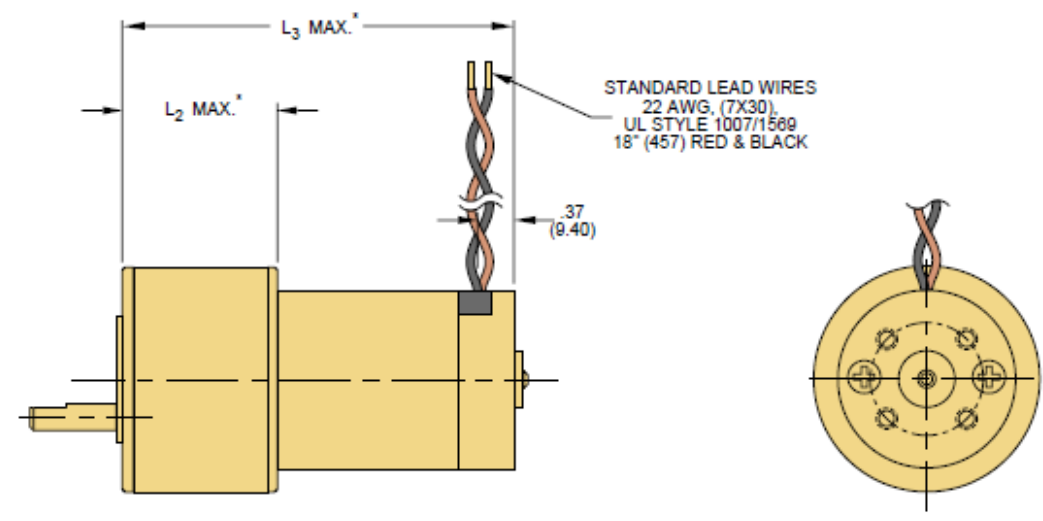


GM94XX Motor with 91XX Encoder

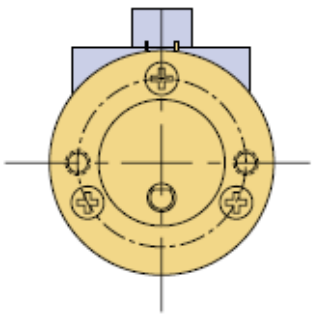

GM92XX Motor with 91XX Encoder

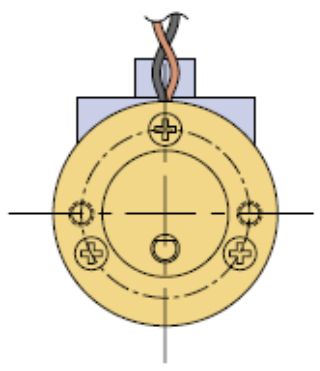

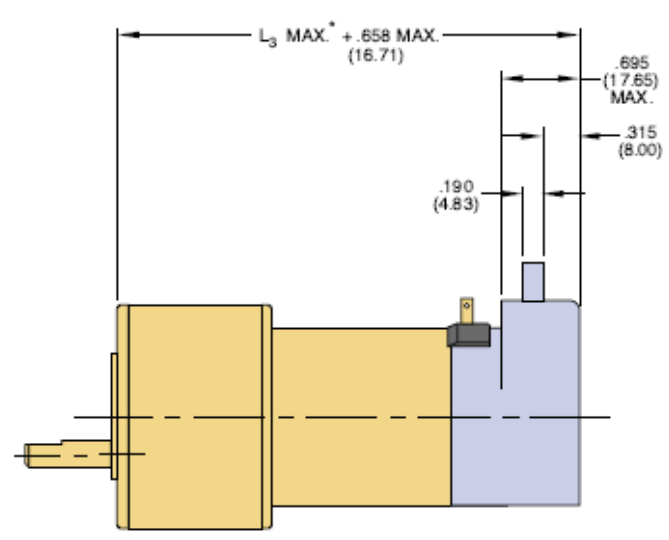
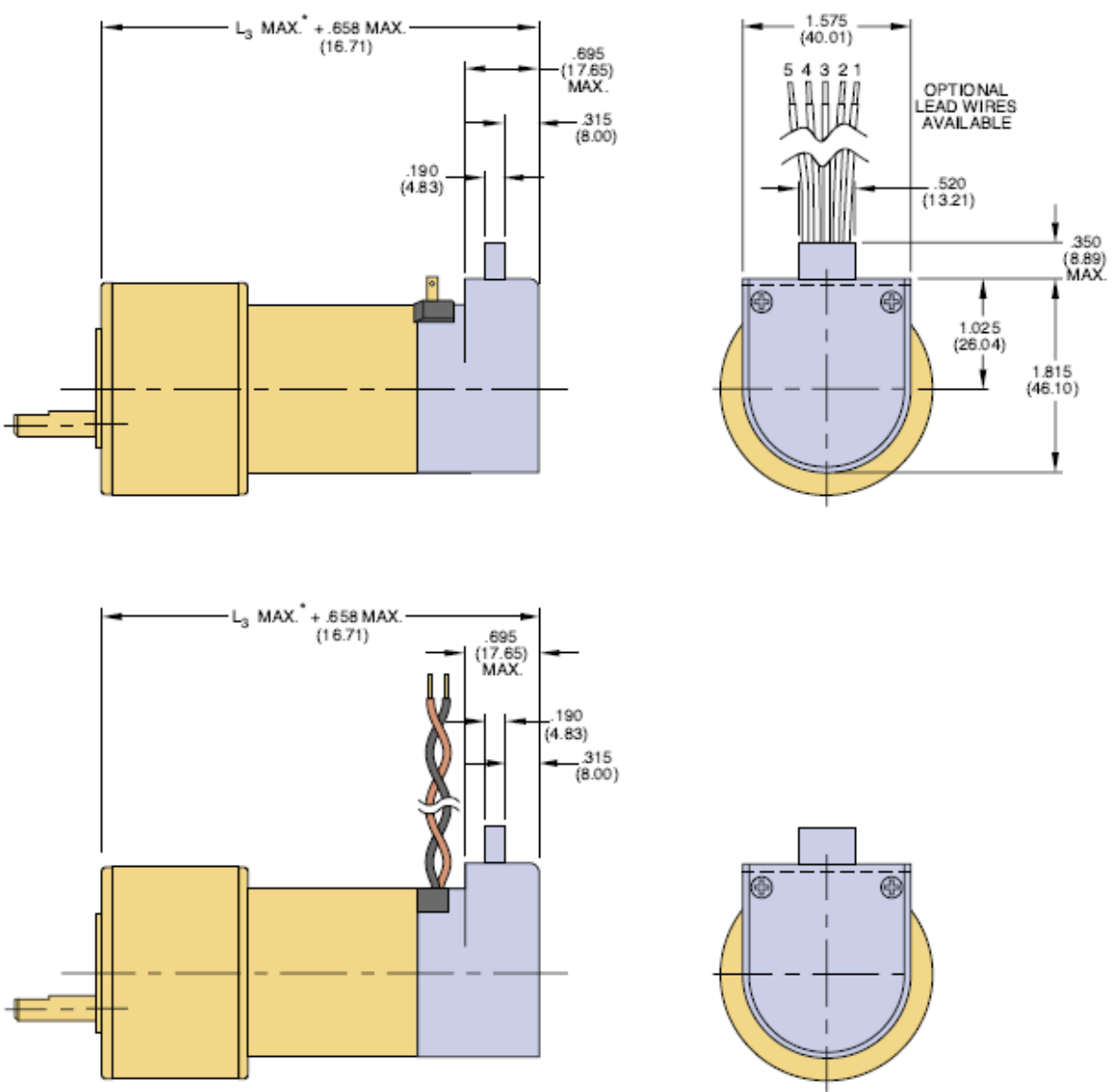

Encoder Connection Chart

\begin{tabular}{|c|c|c|}
\hline Pin No. & Color & Connection \\
\hline 1 & Black & Ground \\
\hline 2 & Green & Index/NC \\
\hline 3 & Yellow & Channel A \\
\hline 4 & Red & Voc \\
\hline 5 & Blue & Channel B \\
\hline
\end{tabular}

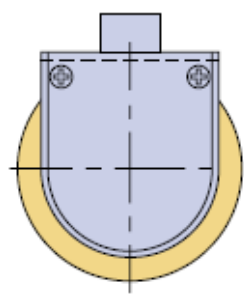




\section{APPENDIX D: PRESSURE TRANSDUCER SPECIFICATIONS}

\begin{tabular}{|c|c|}
\hline \multirow[t]{2}{*}{ Total Uncertainty } & $\begin{array}{l}0.01 \% \text { FS for ranges } \geq 1 \text { psig to } 6000 \mathrm{psig} \\
0.03 \% \text { FS for ranges }<1 \text { psig } \\
0.01 \% \text { FS for ranges } \geq 7.5 \text { psia to } 6015 \text { psia } \\
0.03 \% \text { FS for bi-directional spans } 0.36 \text { psi } \leq \text { span }<1 \mathrm{psi} \\
0.01 \% \text { FS for bi-directional spans } 1 \mathrm{psi} \leq \text { span } \leq 6015 \mathrm{psi}\end{array}$ \\
\hline & $\begin{array}{l}\text { Uncertainty statement includes all temperature, linearity, } \\
\text { hysteresis and repeatability effects. }\end{array}$ \\
\hline $\begin{array}{l}\text { Calibration Stability } \\
\text { (after warm-up) }\end{array}$ & $\begin{array}{l}\text { Better than } 0.010 \% \text { FS }(0.03 \% \text { for FS ranges }<1 \text { psig) for } 180 \\
\text { days with periodic re-zeroing. } \\
\text { Relative accuracy } 10.012 \% \text { I FS ( } 10.036 \% \text { for FS ranges }<1 \\
\text { psig) maximum, between any two pressure points. }\end{array}$ \\
\hline Calibration Interval & 180 days \\
\hline Calibration Adjustments & Zero and Span via the serial interface \\
\hline Pressure Ranges - Standard & $\begin{array}{l}\text { psia: } 0-7.5 \text { to } 0-6015 \max \\
\text { psig: } 0-0.36 \text { to } 0-6000 \text { max }\end{array}$ \\
\hline $\begin{array}{l}\text { Pressure Range - Bi-directional, } \\
\text { Vacuum }\end{array}$ & $\begin{array}{l}\text { psig: }-0.36 \text { to }+0.36 \mathrm{~min} \\
\text {-atm to } 6,000 \mathrm{max}\end{array}$ \\
\hline Pressure Units & $\begin{array}{l}\text { psi, inHg @ } 0^{\circ} \mathrm{C} \text { and } 60^{\circ} \mathrm{F}, \text { inH2O @ } 4^{\circ} \mathrm{C}, 20^{\circ} \mathrm{C} \text { and } 60^{\circ} \mathrm{F} \text {, } \\
\text { ftH2O @ } 4^{\circ} \mathrm{C}, 20^{\circ} \mathrm{C} \text { and } 60^{\circ} \mathrm{F}, \mathrm{mTorr}, \text { inSW } @ 0^{\circ} \mathrm{C} \text {, ftSW } @ \\
0^{\circ} \mathrm{C}, \mathrm{ATM}, \mathrm{bars}, \mathrm{mbars}, \mathrm{mmH} 2 \mathrm{O} @ 4^{\circ} \mathrm{C}, \mathrm{cmH} 2 \mathrm{O} @ 4^{\circ} \mathrm{C} \text {, } \\
\mathrm{MH} 2 \mathrm{O} @ 4^{\circ} \mathrm{C}, \mathrm{mmHg} @ 0^{\circ} \mathrm{C}, \mathrm{cmHg} @ 0^{\circ} \mathrm{C} \text {, Torr, hPa, mPa, } \\
\mathrm{kPa}, \mathrm{Pa}, \mathrm{D} / \mathrm{cmsq}, \mathrm{g} / \mathrm{cmsq}, \mathrm{kg} / \mathrm{cmsq}, \mathrm{mSW} @ 0^{\circ} \mathrm{C}, \mathrm{OSI}, \mathrm{PSF} \text {, } \\
\mathrm{TSF}, \mathrm{TSI}, \mathrm{mHg} @ 0^{\circ} \mathrm{C}, \% \mathrm{FS} \text {. All seawater units are } 3.5 \% \\
\text { salinity. }\end{array}$ \\
\hline Resolution & Up to $1 \mathrm{ppm}$, depending on measurement units and range \\
\hline Overpressure Limit & $150 \%$ FS or greater, depending on range \\
\hline
\end{tabular}




\begin{tabular}{|c|c|}
\hline Compensated Temp. Range & $15^{\circ} \mathrm{C}$ to $45^{\circ} \mathrm{C}$ \\
\hline Operating Temp. Range & $0^{\circ} \mathrm{C}$ to $50^{\circ} \mathrm{C}$ \\
\hline Storage Temperature Range & $0^{\circ} \mathrm{C}$ to $70^{\circ} \mathrm{C}$ \\
\hline Warm-up & 10 minutes to rated accuracy \\
\hline Reading Rate & $50 \mathrm{~Hz}$ \\
\hline \multirow[t]{3}{*}{ Noise } & $\begin{array}{l}\text { Filter set to } 90 \% \text { (factory default): } \\
20 \mathrm{ppm} \text { peak-to-peak and } 6.5 \mathrm{ppm} \text { rms. }\end{array}$ \\
\hline & $\begin{array}{l}\text { Filter set to } 0 \%: \\
53 \text { ppm p-p and } 12.5 \text { ppm rms. }\end{array}$ \\
\hline & $\begin{array}{l}\text { Filter set to } 99 \% \text { : } \\
13 \text { ppm p-p and } 4.2 \text { ppm rms. }\end{array}$ \\
\hline Orientation Effects & $<30 \mathrm{psi}$, orientation must be specified \\
\hline Communications & RS-232 or RS-485. From 9600 to $56 \mathrm{k}$ baud. \\
\hline Case Size & See "5.2 - Dimensions" \\
\hline Weight & Approximately 17.8 ounces (505 grams) \\
\hline Media Compatibility & $\begin{array}{l}\text { Clean, dry, non-corrosive gases for ranges }<15 \text { psi. } \\
\text { All other ranges compatible with aluminum, } 316 \text { stainless } \\
\text { steel, brass, Buna N, Viton, sealant, and silicone grease. } \\
\text { Not designed for oxygen use. }\end{array}$ \\
\hline Fittings & $\begin{array}{l}\text { Female } 7 / 16-20 \text { SAE/MS straight thread port. } \\
1 / 8 \text { inch female NPT adapter fitting is included. }\end{array}$ \\
\hline Power & 6-20 VDC, 55mA @ 12 VDC \\
\hline Option & Relief valves \\
\hline Mechanical Shock & $3 g$ max \\
\hline Multi-drop Capacity & $\begin{array}{l}\text { The maximum number of RS- } 485 \text { CPT } 6100 \text { transducers } \\
\text { which can be connected to a single host computer is } 31 \text {. }\end{array}$ \\
\hline Compliance & $\begin{array}{l}\text { The CPT6100 is compliant to the following CE Standards: } \\
\text { EN } 50081-1 \text {, EN } 50082-1 \text {, EN } 50081-2 \text {, EN } 50082-2 \text {. }\end{array}$ \\
\hline Optional Output & $\begin{array}{l}\text { Analog: } 0-1,0-5 \text {, and } 0-10 \text { VDC @ } 0.010 \% \text { FS accuracy } \\
(0.030 \% \text { FS for ranges }<1 \text { psig). }\end{array}$ \\
\hline
\end{tabular}




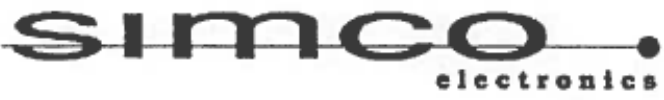

Mfr:

Mensor

Desc: Digital Pressure Transducer

Cert \#: 592245

Asset \#:

6547260 Serial \#.

$51817-2$

\section{As-Left:}

\section{Report Information}

Test Performed: Thursday, May 29, 2014 @ 1:5:8 PM

Data file:

C:IProgram FilesICOMPASS for PressurelDatalMensorl592245L20140529_002.dat

Procedure: COMPASS for Pressure Enhanced 4.2.0, Differential comm

\section{Device Under Test (DUT) Information}

\begin{tabular}{|c|l}
\hline Measurement Mode: Gauge & Nominal Uncertainty: $0.01 \% \mathrm{FS}$ \\
\hline Pressure Range: -10.000 to 10.000 inH2O@20C & Data Acq. Method: Manual \\
\hline Excitation: n/a & Ambient Pressure: 30.1213 in Hg \\
\hline Ambient Temperature: $71.0 \mathrm{~F}$ & Ambient Humidity: $49 \% \mathrm{RH}$ \\
\hline
\end{tabular}

\begin{tabular}{|c|c|c|c|c|c|}
\hline \multicolumn{6}{|l|}{ Test Data } \\
\hline $\begin{array}{c}\text { Standard } \\
\text { Indication } \\
\text { inH2O@20C }\end{array}$ & $\begin{array}{c}\text { DUT } \\
\text { Pressure } \\
\text { in } 20 @ 20 \mathrm{C}\end{array}$ & $\underset{\text { inH2O@20C }}{\frac{\text { Deviation }}{\text { Con }}}$ & $\frac{\text { Deviation }}{\% \text { of Span }}$ & $\begin{array}{c}\text { DUT } \\
\text { Tolerance } \\
\text { inH2O@20C }\end{array}$ & Result \\
\hline 0.00000 & 0.0000 & 0.0000 & 0.0000 & 0.0010 & Pass \\
\hline 5.53920 & 5.5394 & 0.0002 & 0.0010 & 0.0010 & Pass \\
\hline 10.04140 & 10.0412 & -0.0002 & -0.0010 & 0.0010 & Pass \\
\hline 5.53920 & 5.5395 & 0.0003 & 0.0014 & 0.0010 & Pass \\
\hline 0.00000 & 0.0000 & 0.0000 & 0.0000 & 0.0010 & Pass \\
\hline-5.53920 & -5.5385 & 0.0007 & 0.0036 & 0.0010 & Pass \\
\hline-10.04140 & -10.0408 & 0.0006 & 0.0031 & 0.0010 & Pass \\
\hline-5.53920 & -5.5390 & 0.0002 & 0.0011 & 0.0010 & Pass \\
\hline 0.00000 & -0.0003 & -0.0003 & -0.0015 & 0.0010 & Pass \\
\hline
\end{tabular}




\section{Calibration Certificate \\ Accredited Calibration}

Cal Cert ID: 112455

Cust. Name: OLD DOMINION UNIVERSITY

Address: DEPARTMENT OF MECHANICAL \&

AEROSPACE ENGINEERING

4750 ELKHORN AVE RM 1309

City: NORFOLK

State/Zip: VA 23529

Instr SN: $410005 \mathrm{YJ} / 824985$

Instr Descr: 6100

Sensor SN: 410005YJ/824985

Procedure ID: W102058
US

Out1 Min Range: Out1 Max Range:

Limit of Error:

Pressure Type:

Out2 Min Range:

Out2 Max Range:

Limit of Error:

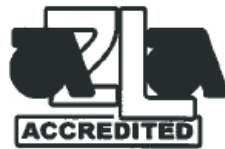

Callbration Lab Cert 2066.01

Page 1 of 2
$0.000000 \mathrm{PSI}$

$0.360000 \mathrm{PSI}$

$0.030 \%$ F.S.

Gauge

0.0000 VOLTS

10.0000 VOLTS

$0.030 \% \mathrm{~F} . \mathrm{S}$.

Test Points Values and Readings

\begin{tabular}{r|c|c|r|r|r|r|r|}
$\begin{array}{c}\text { Test } \\
\text { Point } \\
\#\end{array}$ & $\begin{array}{c}\text { Reference } \\
\text { Value } \\
\text { PSI }\end{array}$ & $\begin{array}{c}\text { Measured } \\
\text { Values } \\
\text { PSI }\end{array}$ & $\begin{array}{c}\text { Error } \\
\text { F F.S. }\end{array}$ & $\begin{array}{c}\text { Uncertainty } \\
\text { PSI }\end{array}$ & $\begin{array}{c}\text { Output 2 } \\
\text { Reading } \\
\text { non-accredited } \\
\text { VOLTS }\end{array}$ & $\begin{array}{c}\text { Deviation } \\
\% \text { F.S. }\end{array}$ & $\begin{array}{c}\text { Uncertainty } \\
\text { VOLTS }\end{array}$ \\
\hline 1 & -0.0000120 & -0.000012 & 0.000 & .0000021 & -0.0003 & 0.000 & .00005 \\
2 & 0.0719316 & 0.071935 & 0.001 & .0000027 & 1.9981 & 0.000 & .00011 \\
3 & 0.1438050 & 0.143818 & 0.004 & .0000038 & 3.9949 & 0.003 & .00017 \\
4 & 0.2157452 & 0.215752 & 0.002 & .0000051 & 5.9931 & 0.002 & .00023 \\
5 & 0.2876896 & 0.287692 & 0.001 & .0000065 & 7.9913 & -0.001 & .00029 \\
6 & 0.3595630 & 0.359566 & 0.001 & .0000079 & 9.9879 & 0.000 & .00035 \\
7 & 0.2876896 & 0.287691 & 0.000 & .0000065 & 7.9913 & -0.001 & .00029 \\
8 & 0.2157452 & 0.215753 & 0.002 & .0000051 & 5.9931 & 0.002 & .00023 \\
9 & 0.1438051 & 0.143817 & 0.003 & .0000038 & 3.9948 & 0.002 & .00017 \\
10 & 0.0719316 & 0.071931 & 0.000 & .0000027 & 1.9980 & -0.001 & .00011 \\
11 & -0.0000120 & -0.000020 & -0.002 & .0000021 & -0.0006 & -0.003 & .00005 \\
\hline
\end{tabular}


APPENDIX E: MODEL SUPPORT DRAWINGS

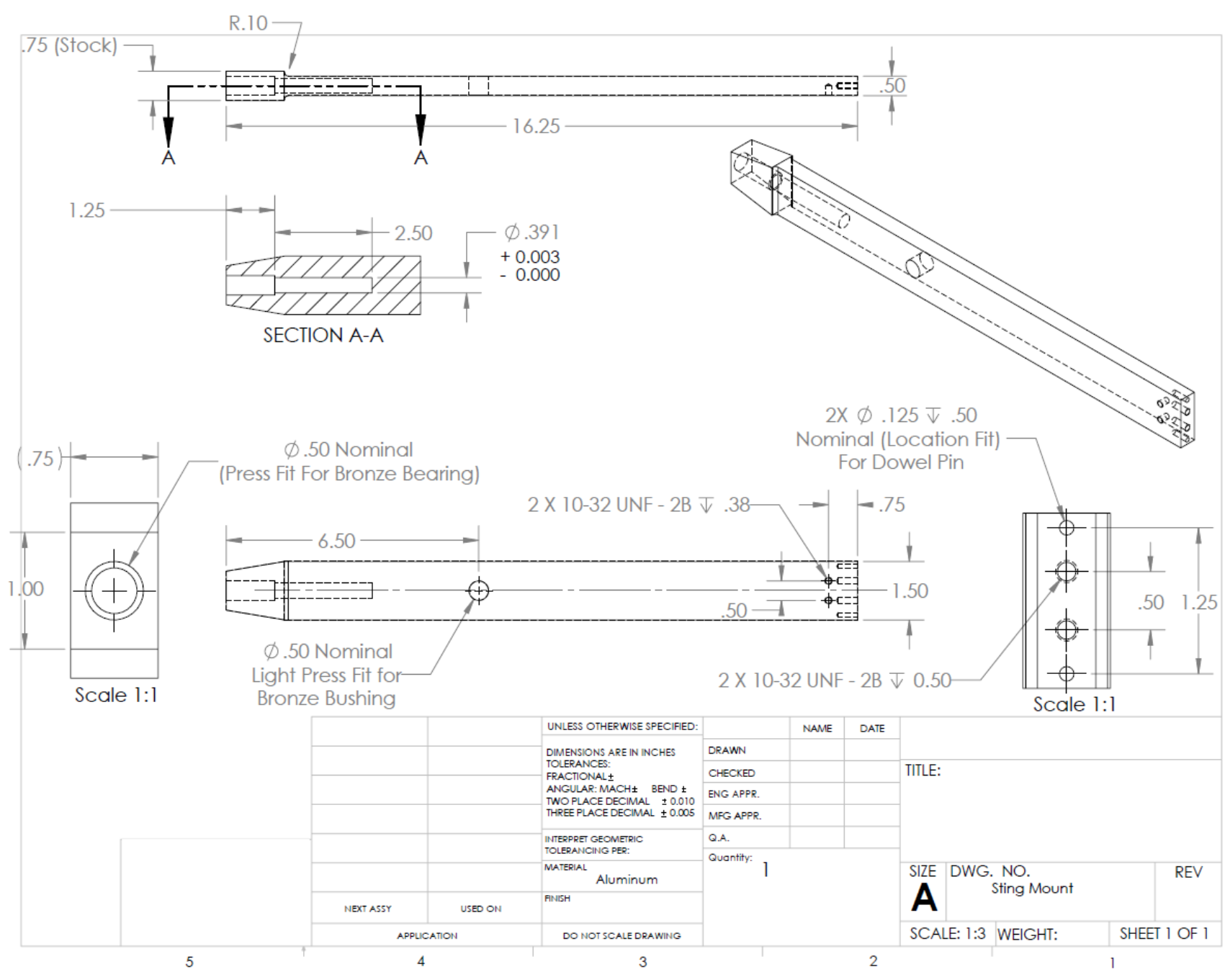




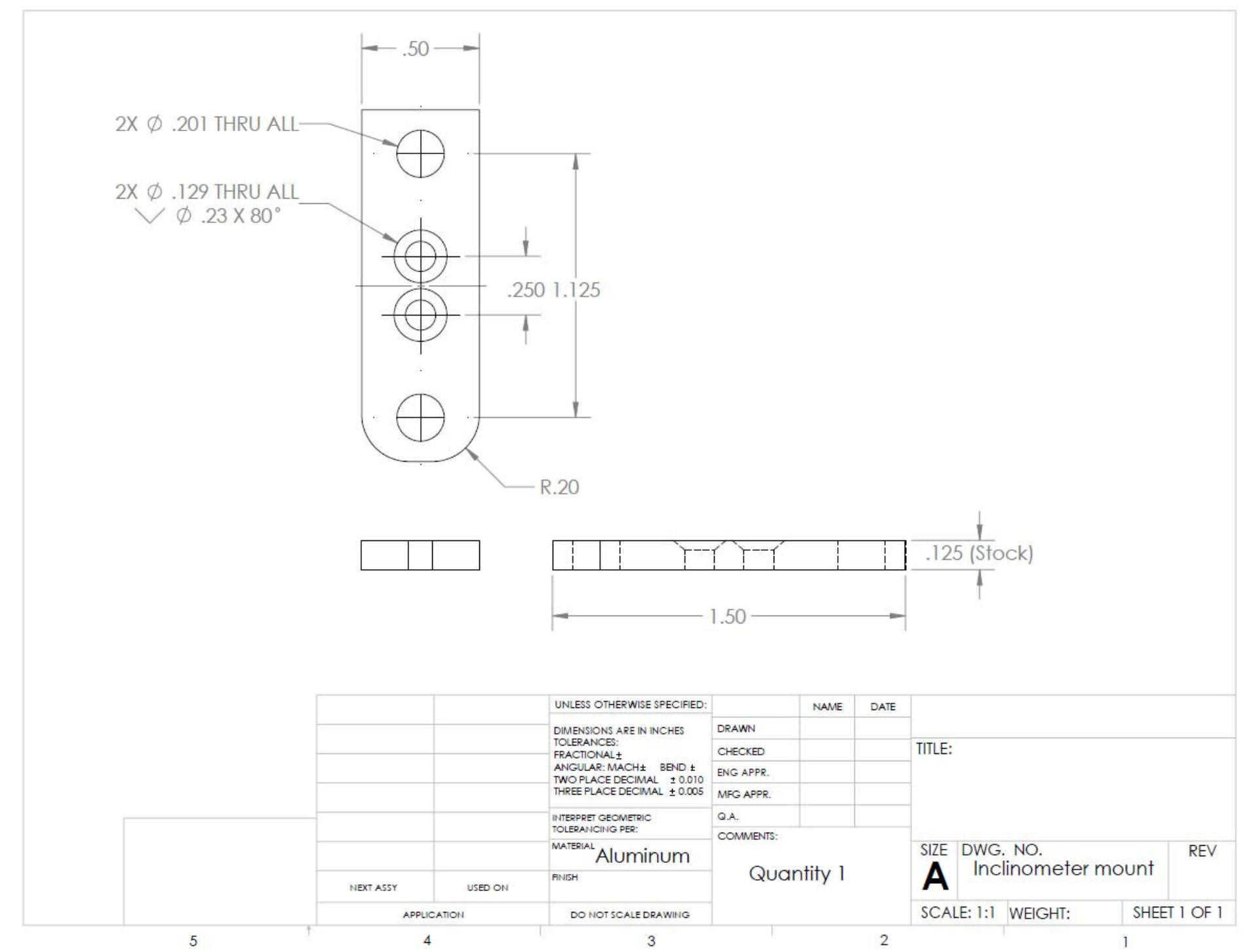




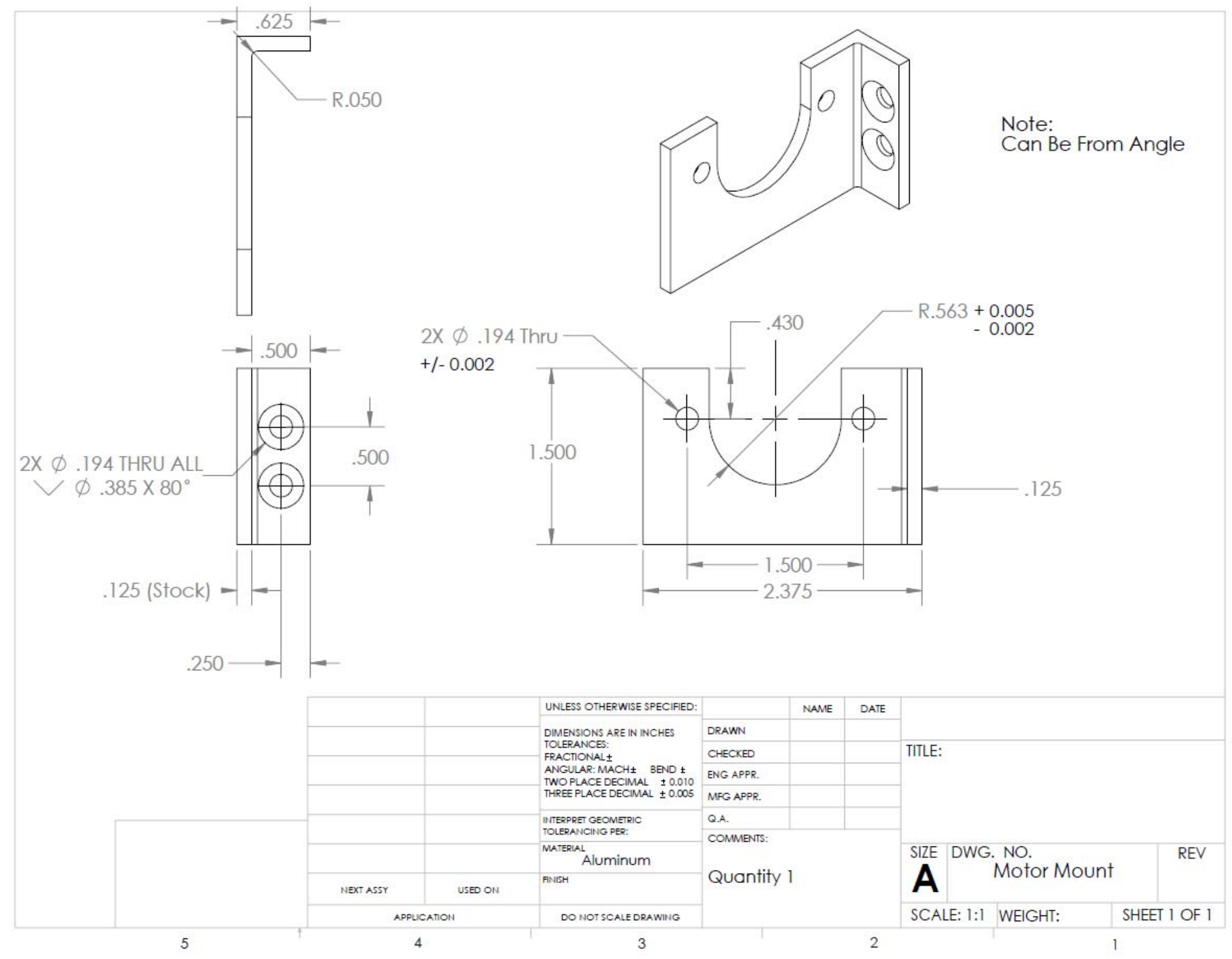




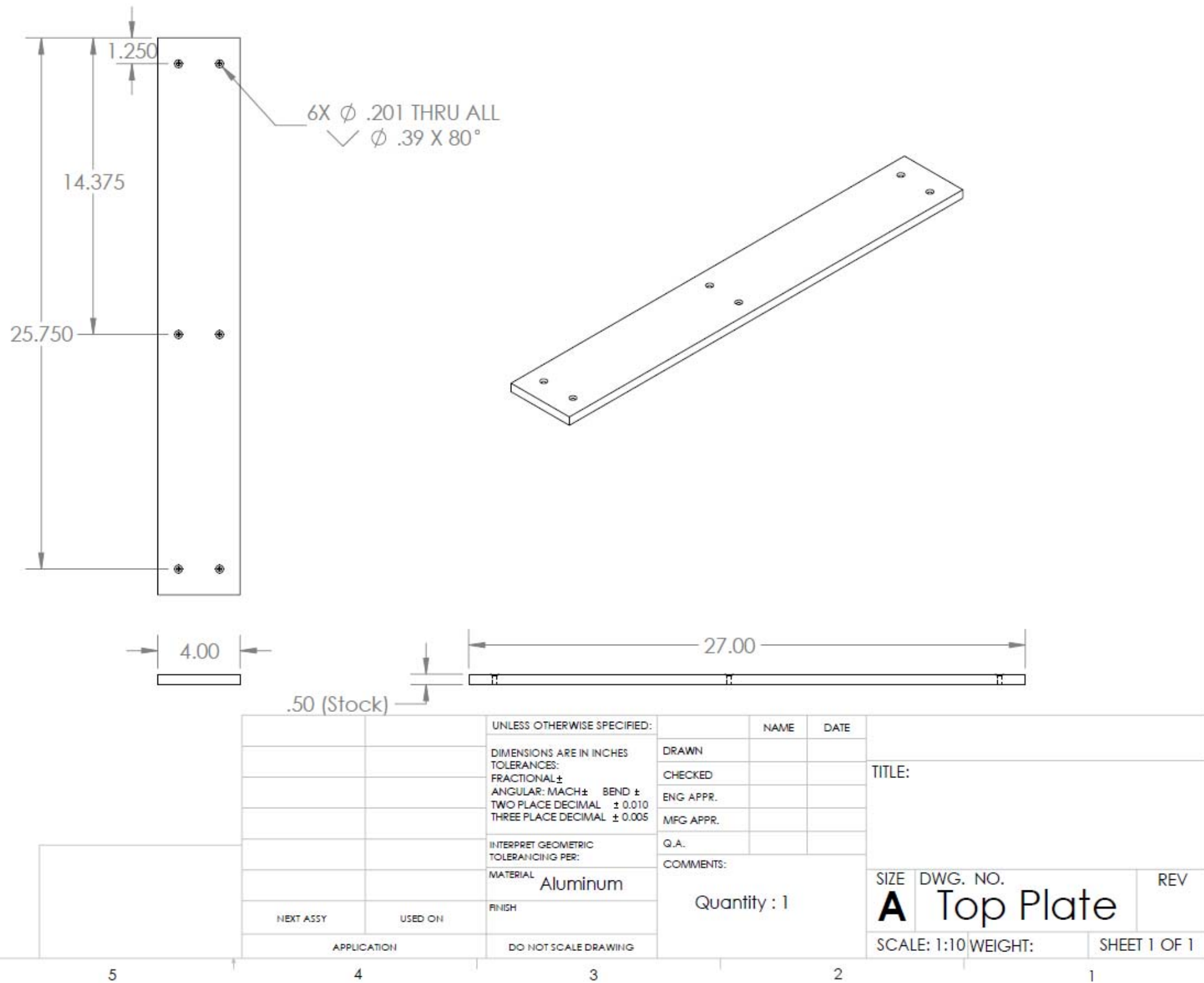



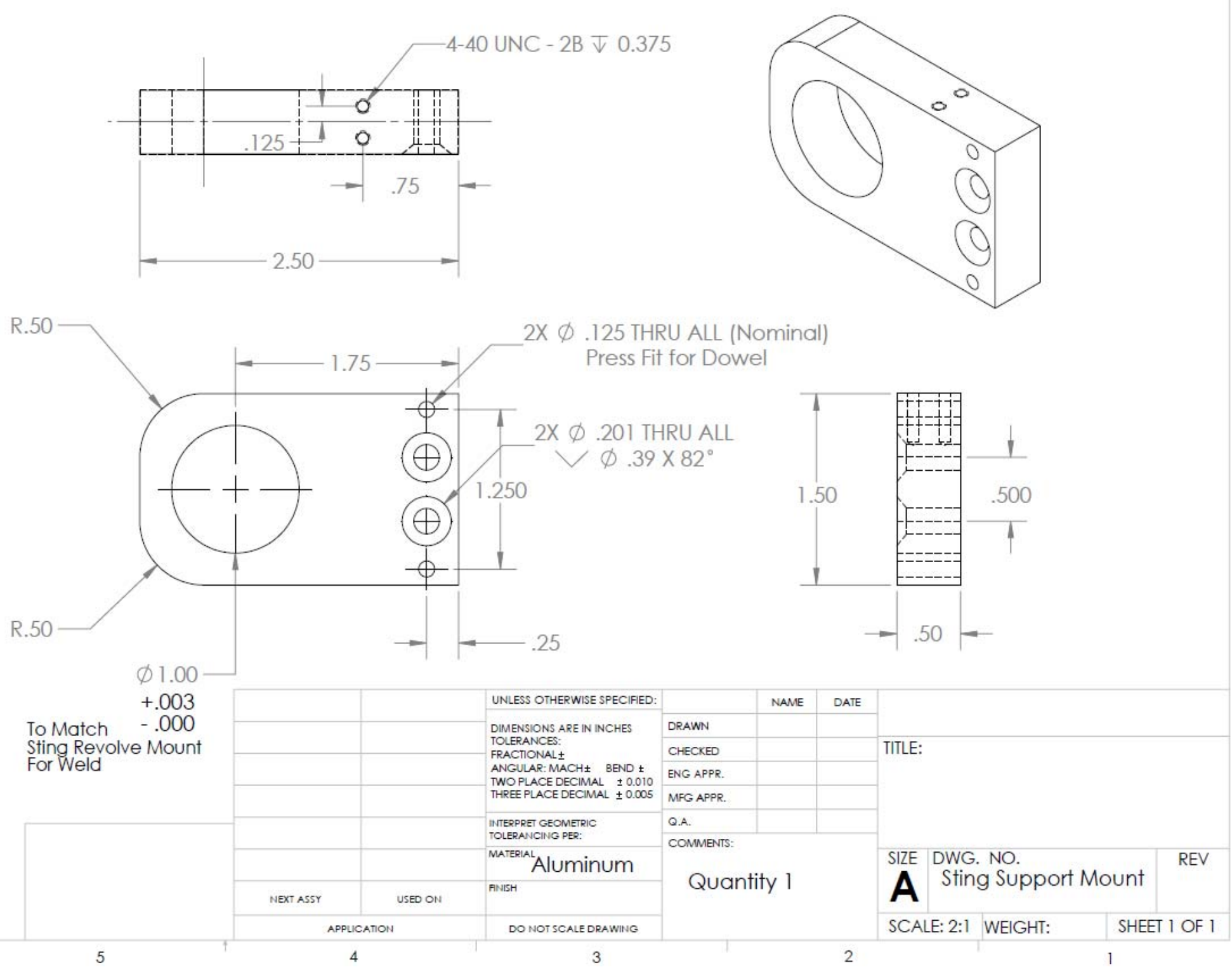


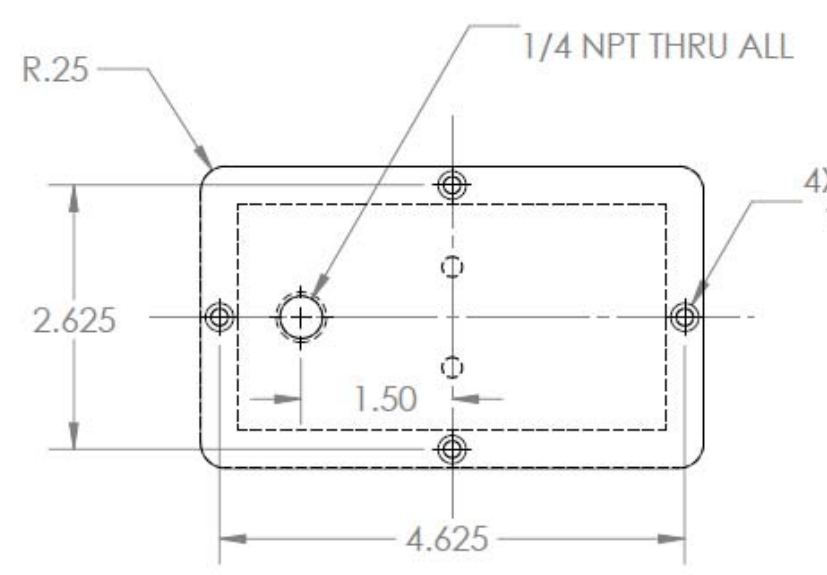

$4 X \varnothing .17$ THRU ALL
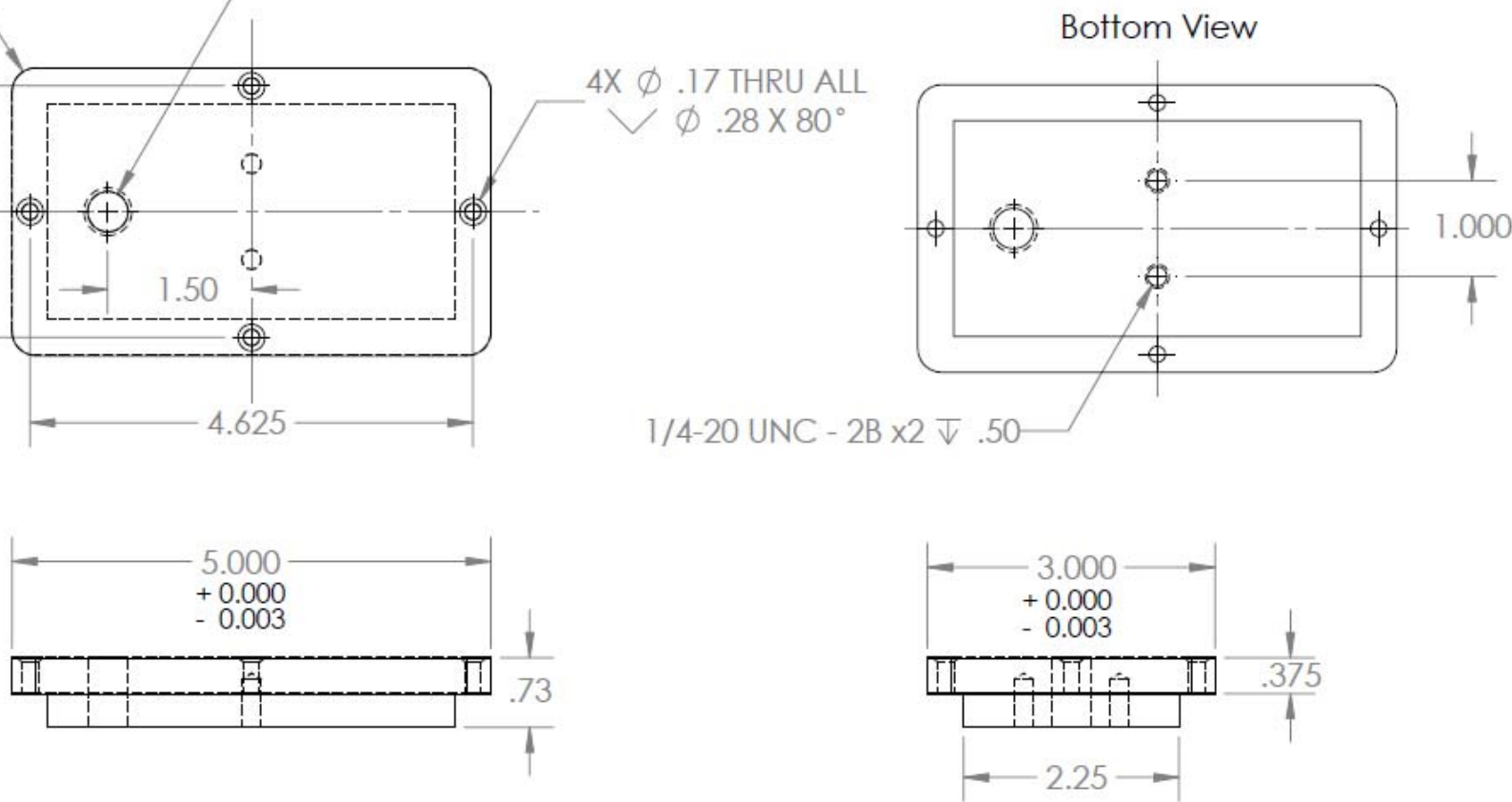

$1 / 4-20$ UNC - $2 B \times 2 \downarrow .50$

1

$=2.25-$
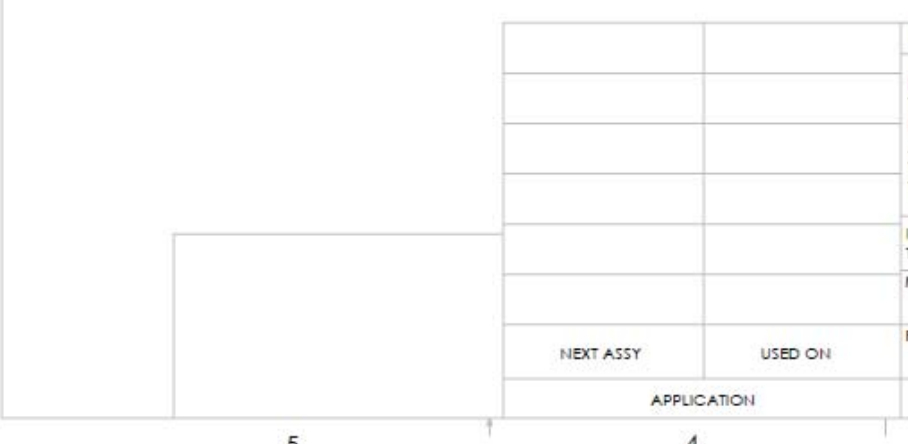

UNLESS OTHERWISE SPECIFIED:

DIMENSIONS ARE IN INCHES

TOLERANCES:

ENG APP

MEG APP

INTERPRET GEOMETRIC
TOLERANCING PEQ: Q.A.

COMMENTS

Aluminum

Quantity 1

DO NOT SCALE DRAWING

A Pitot Tube Filler Piece

REV \begin{tabular}{l|l|}
\hline SCALE: $1: 2$ WEIGHT: & SHEET 1 OF 1
\end{tabular} 


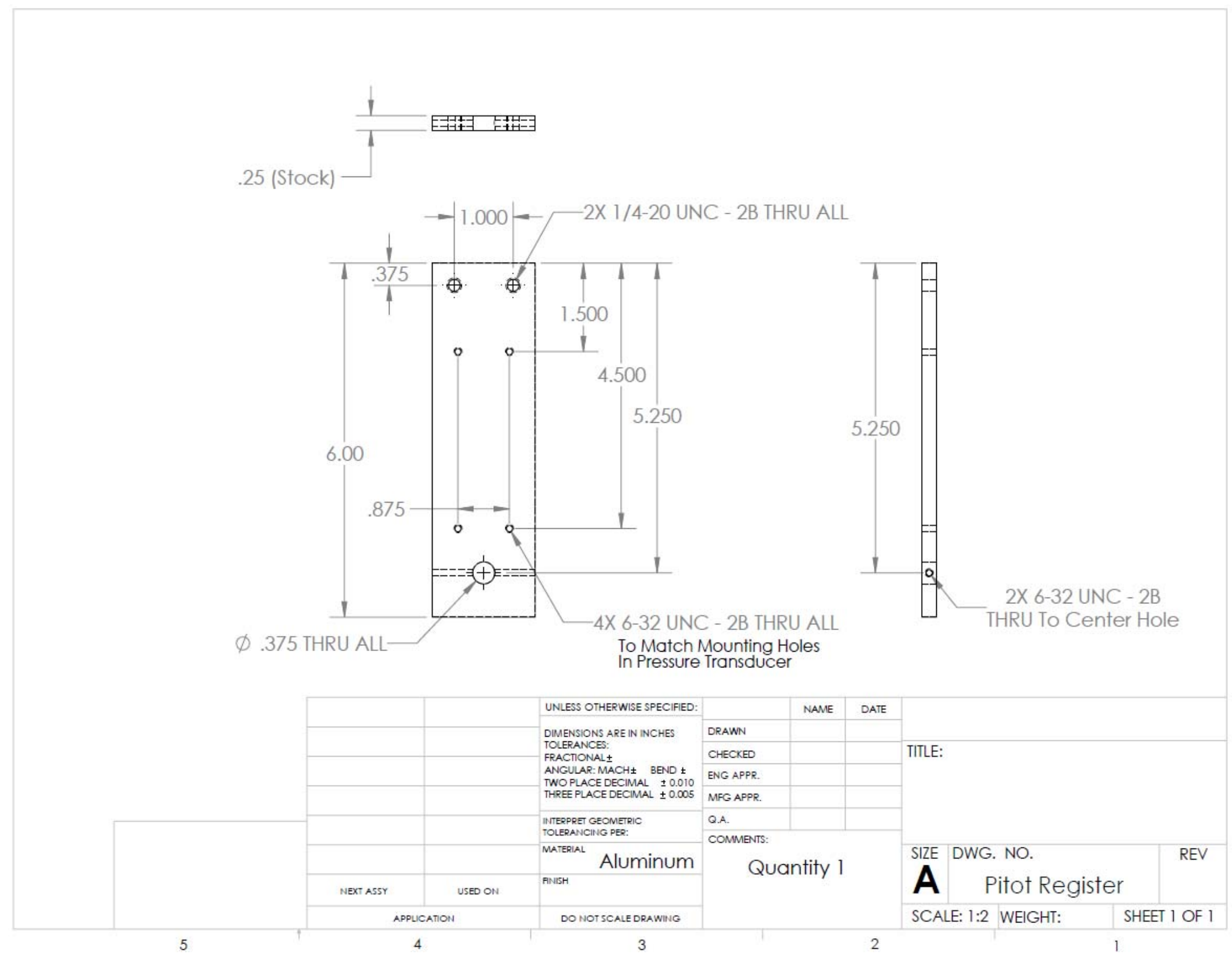




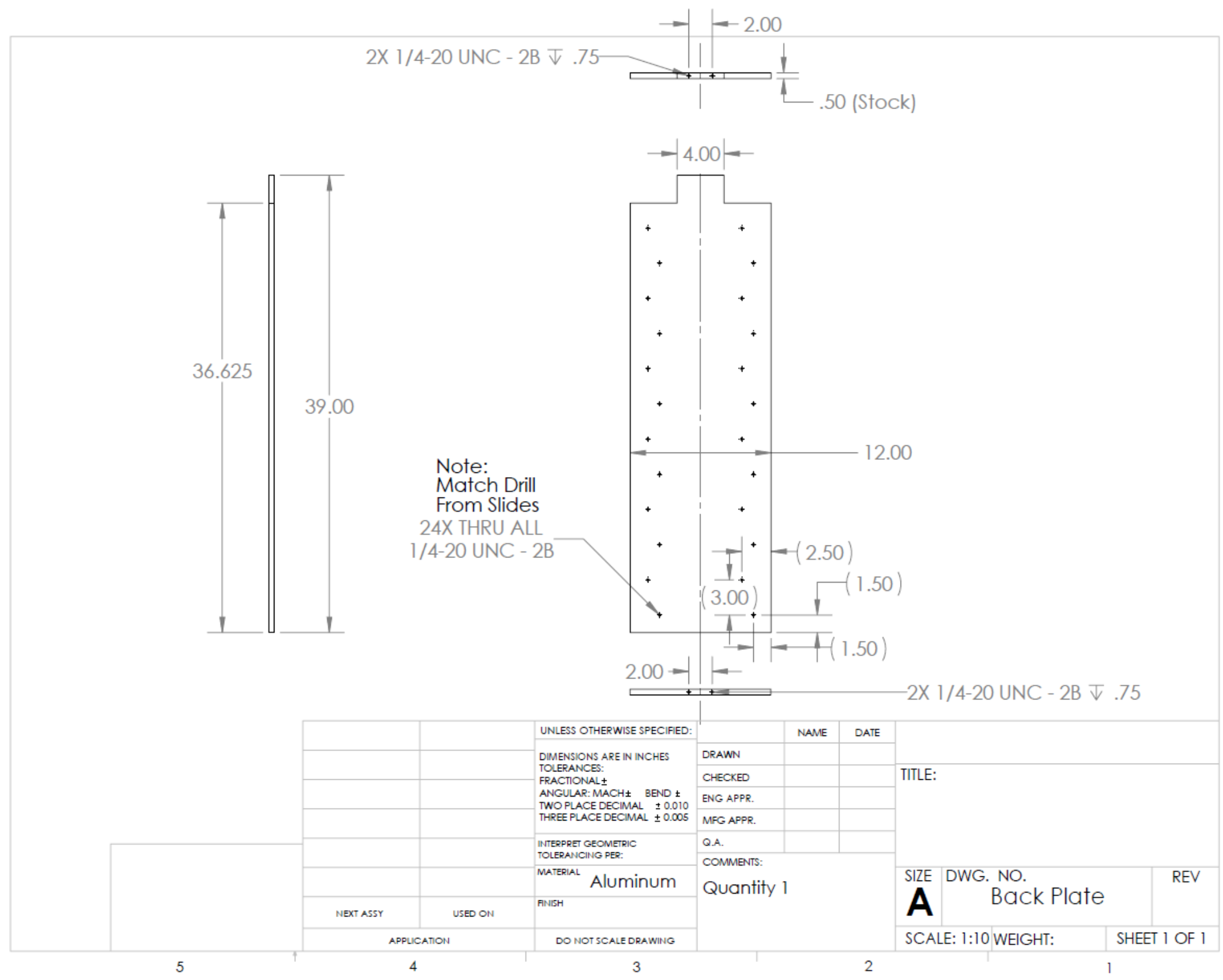



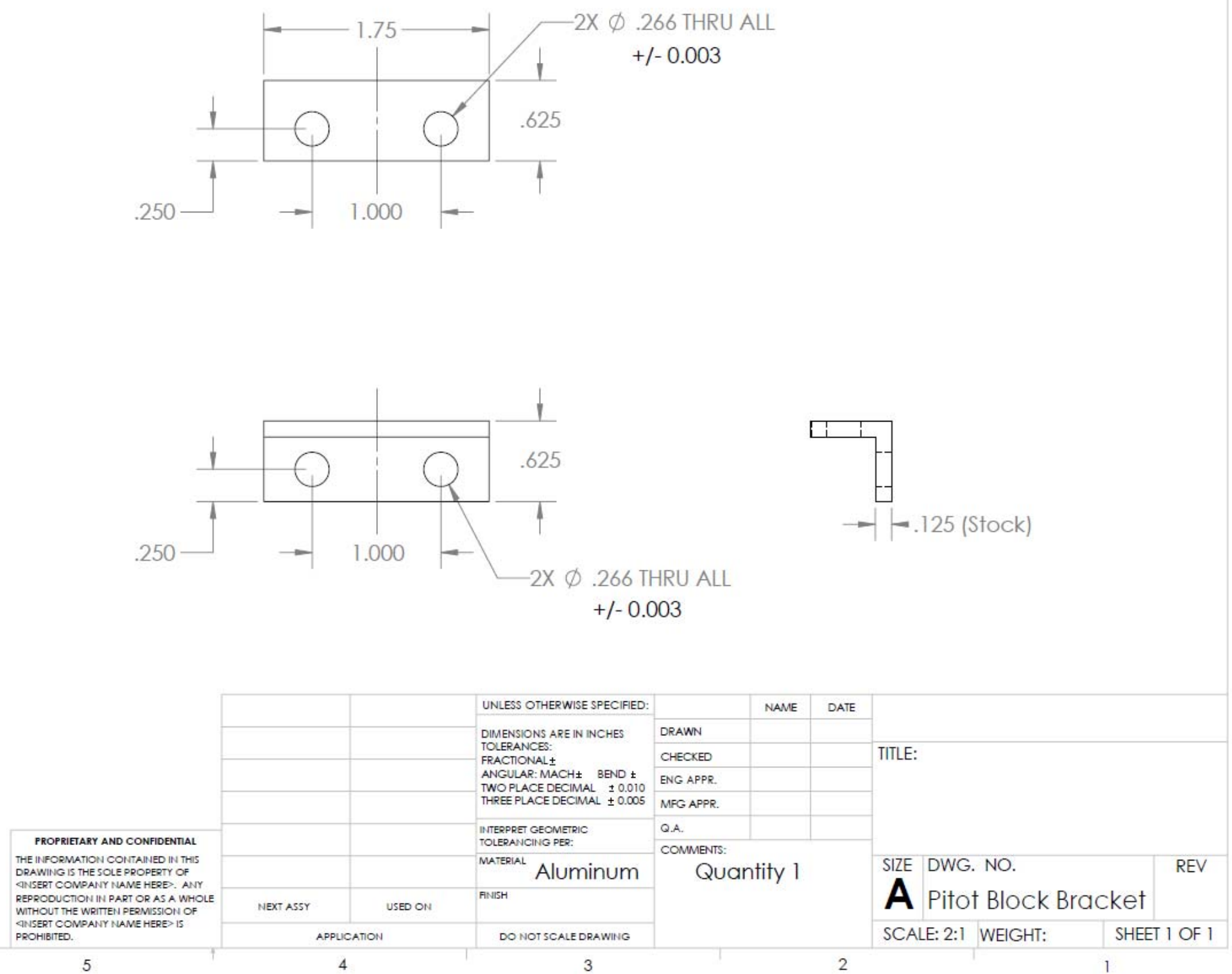


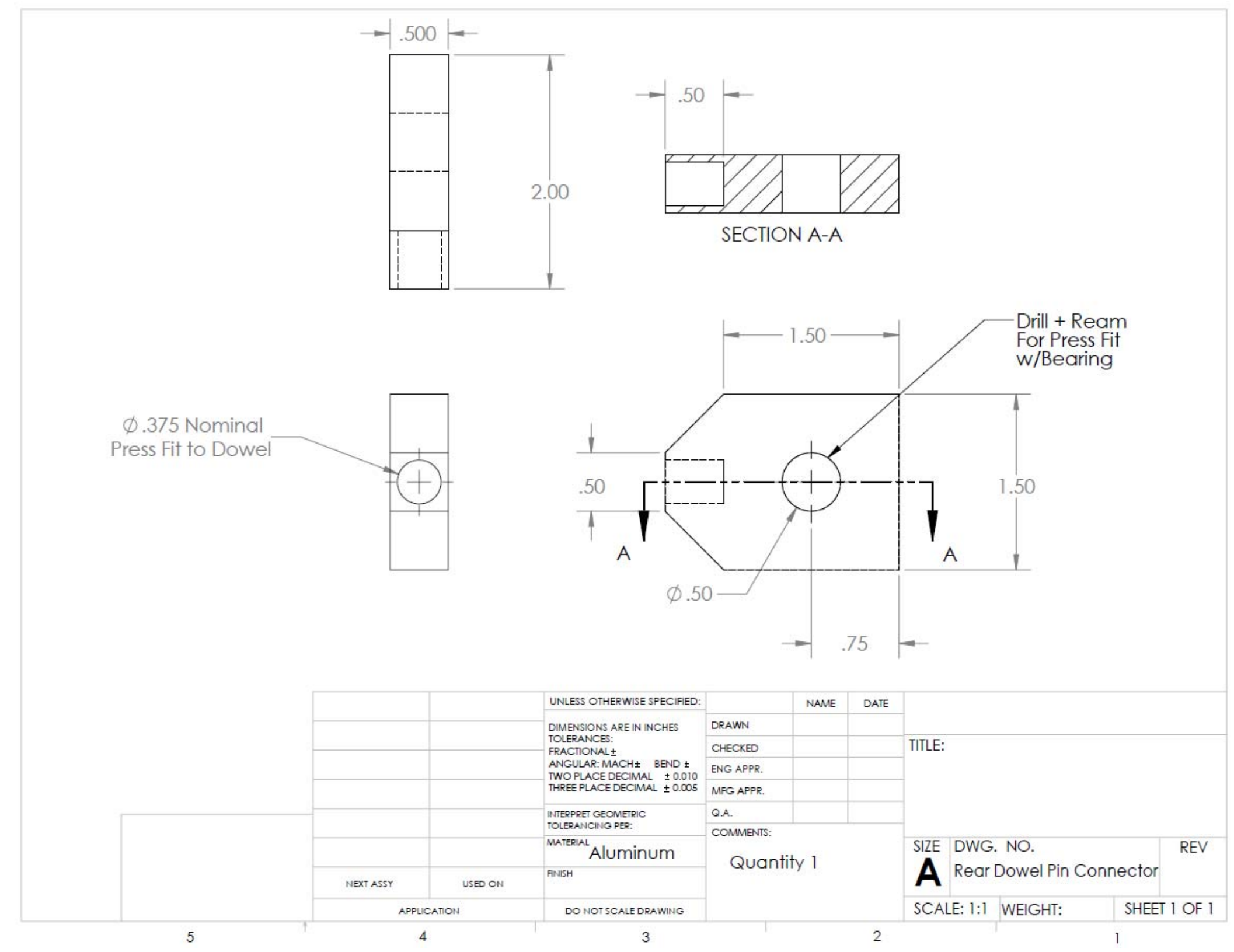




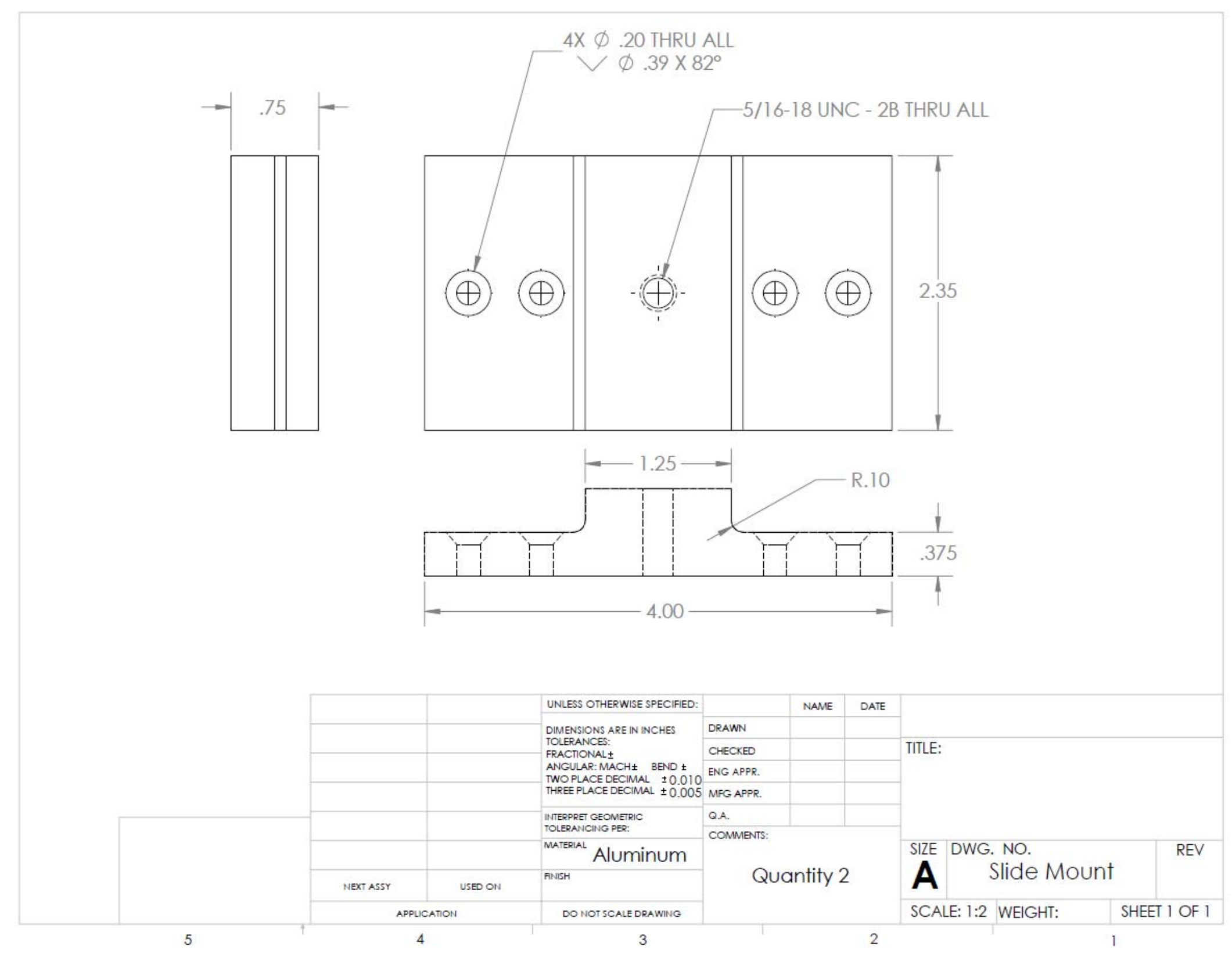




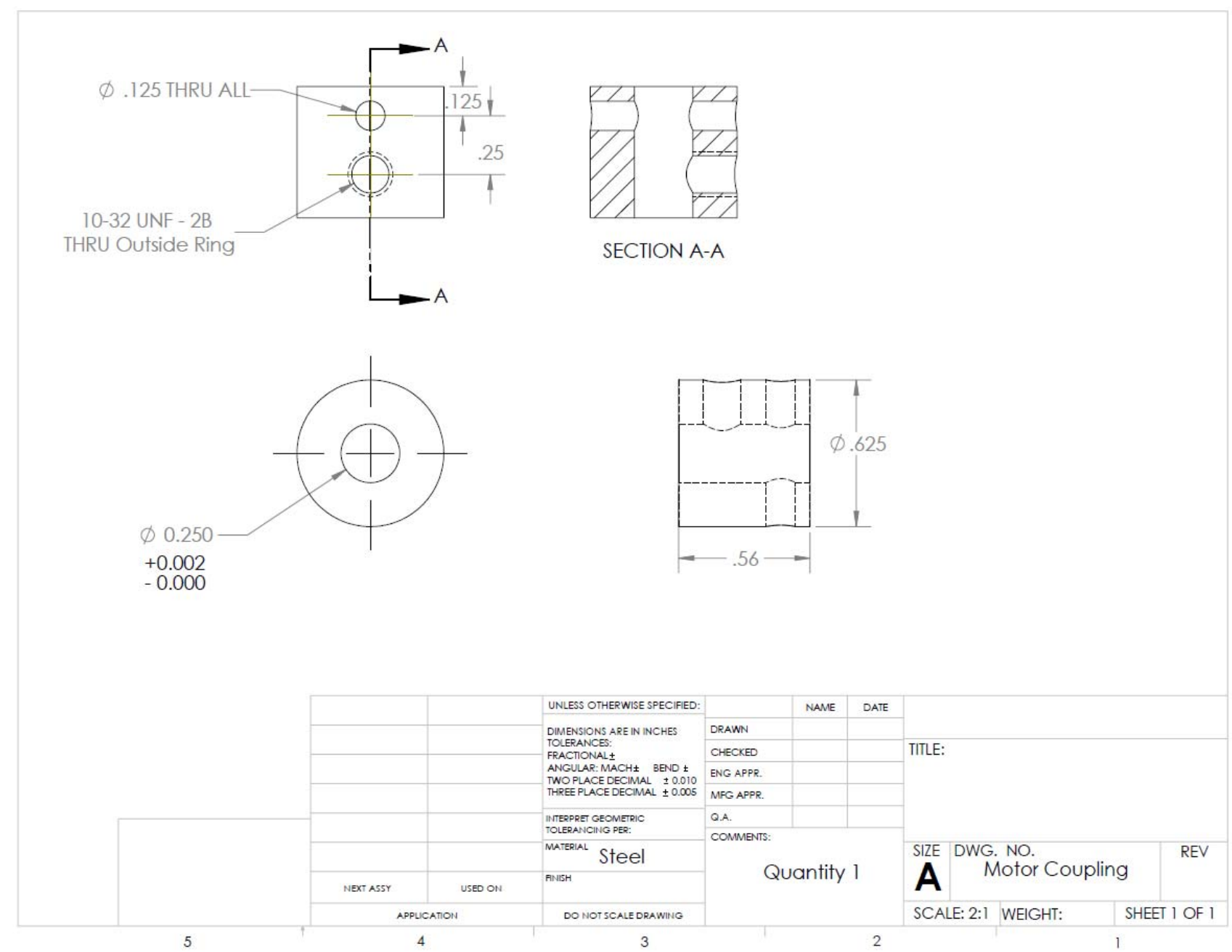




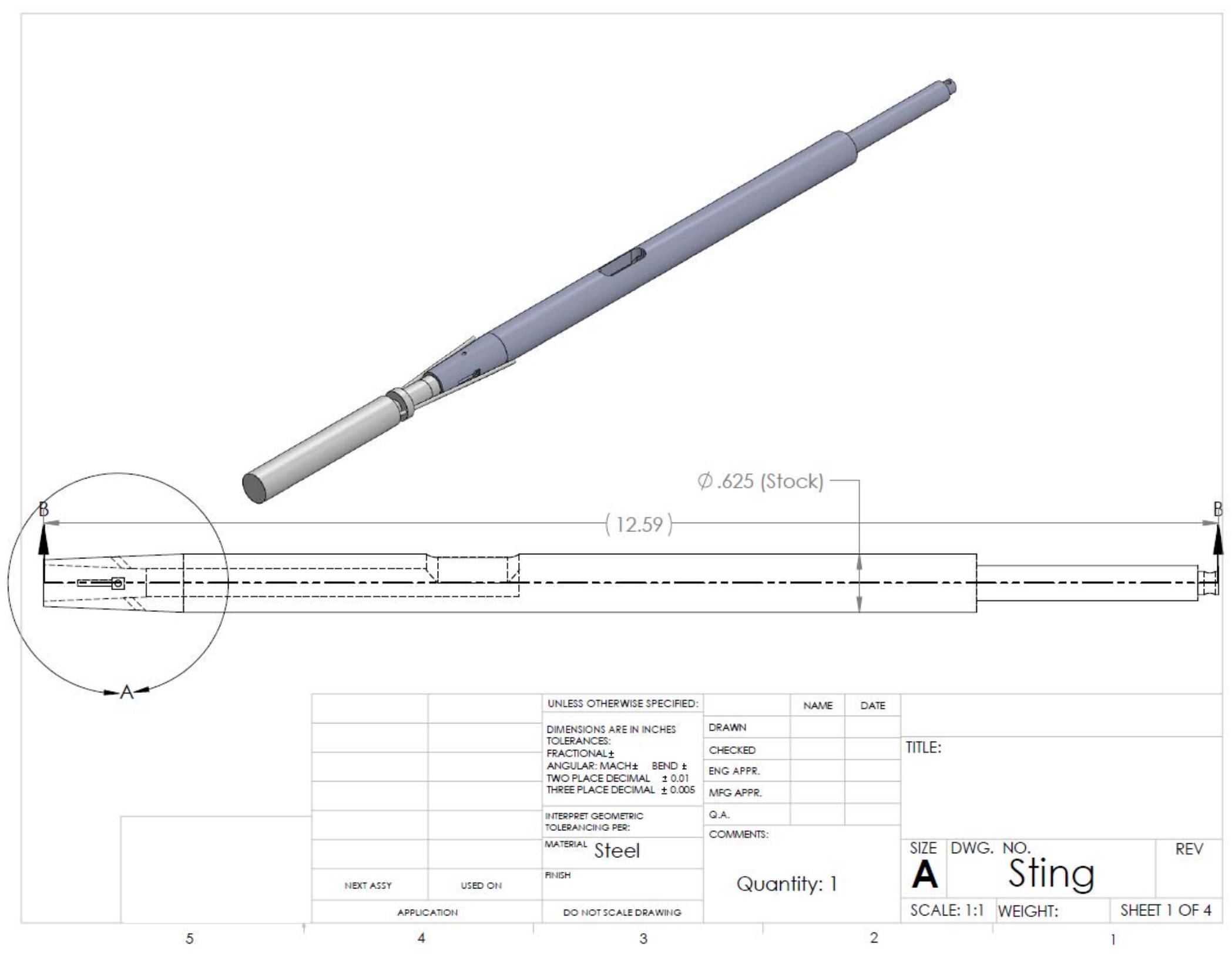




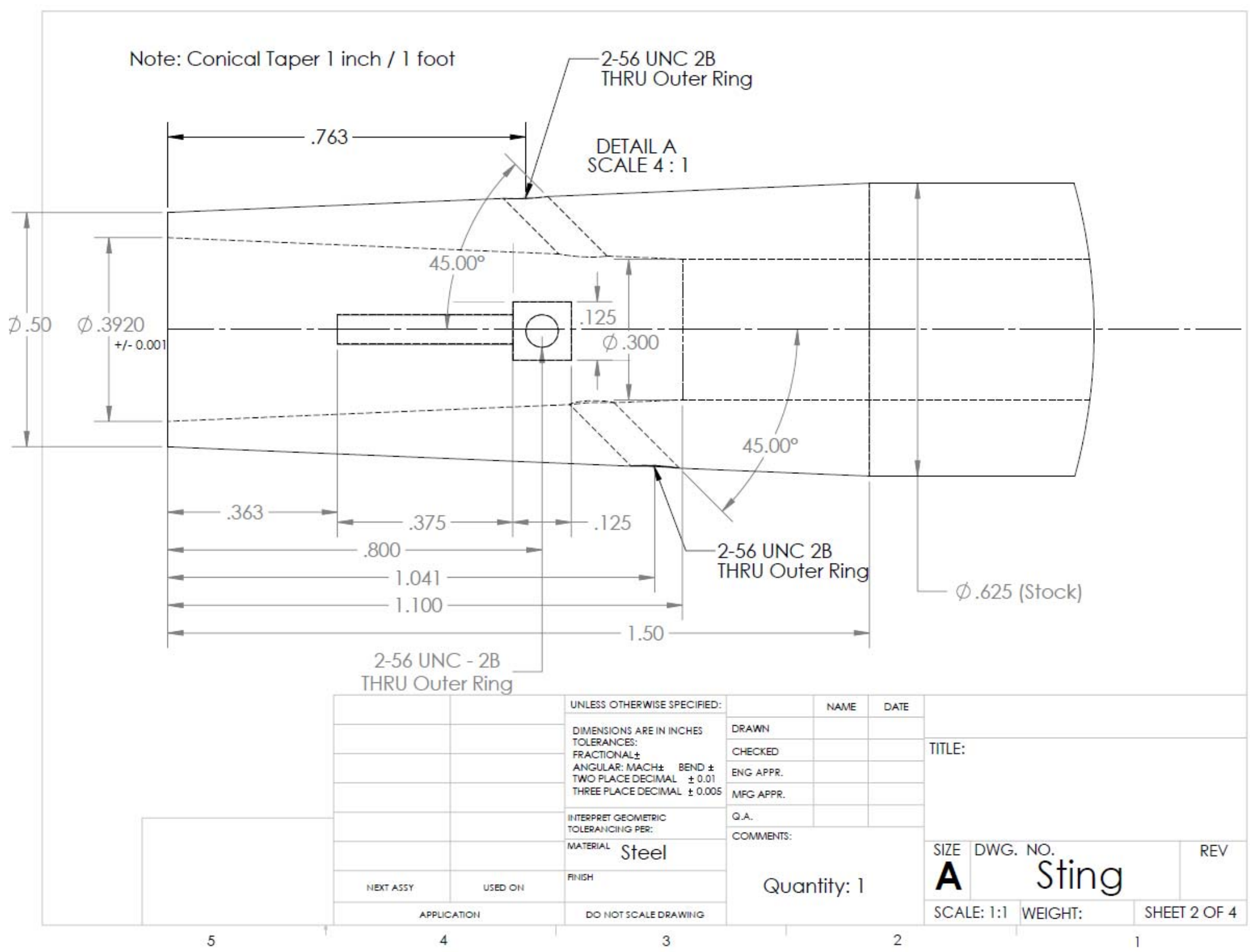



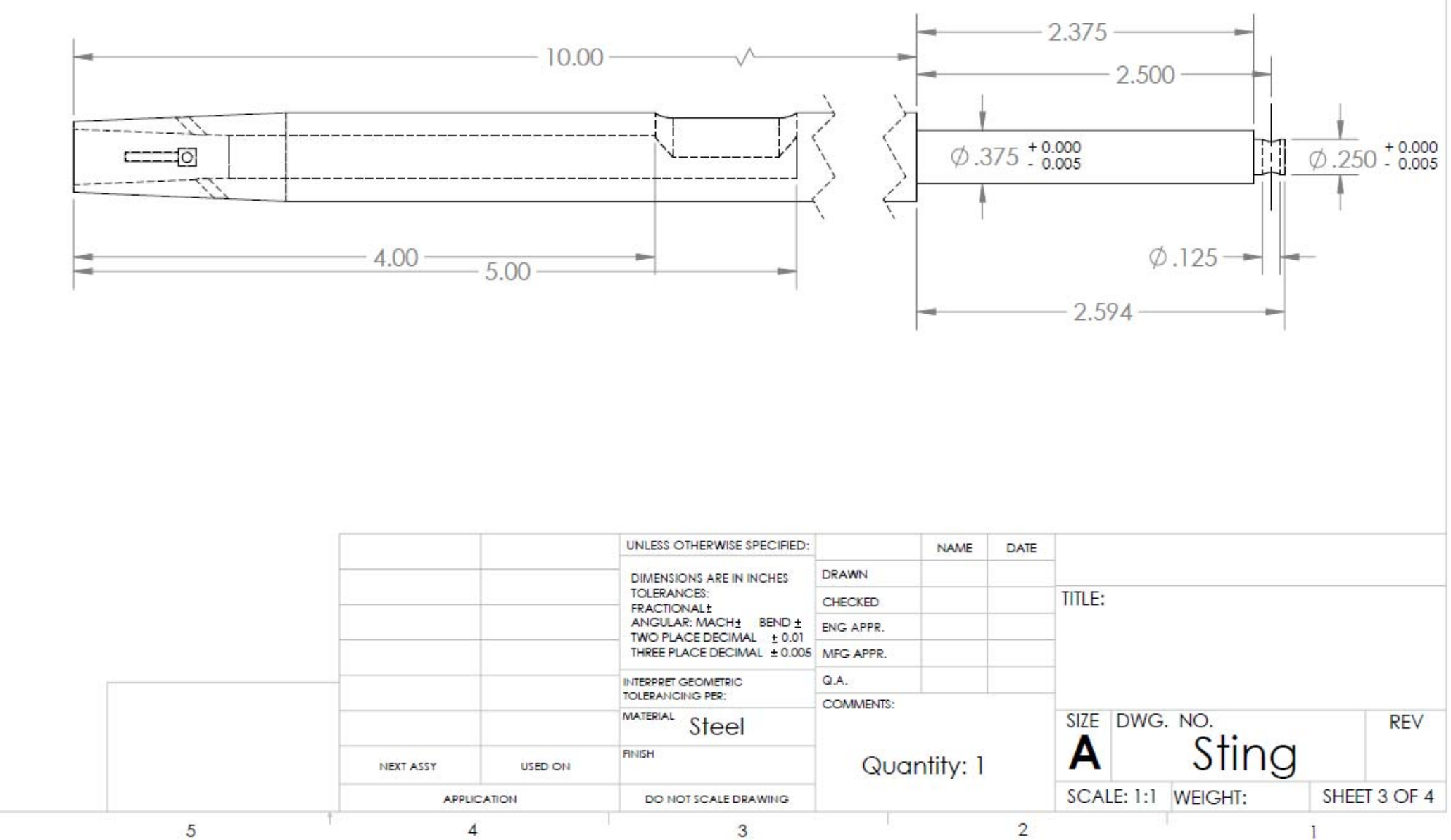


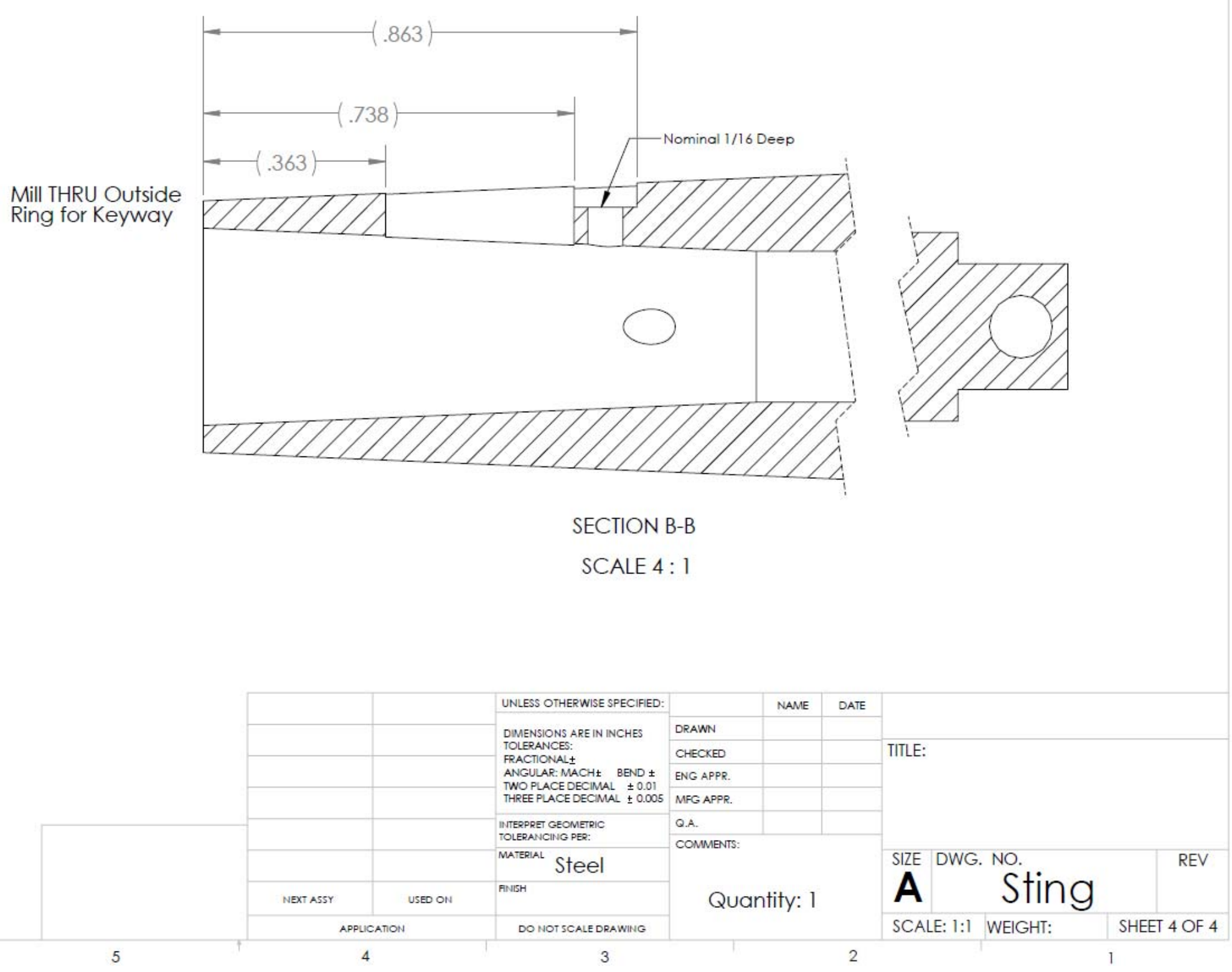




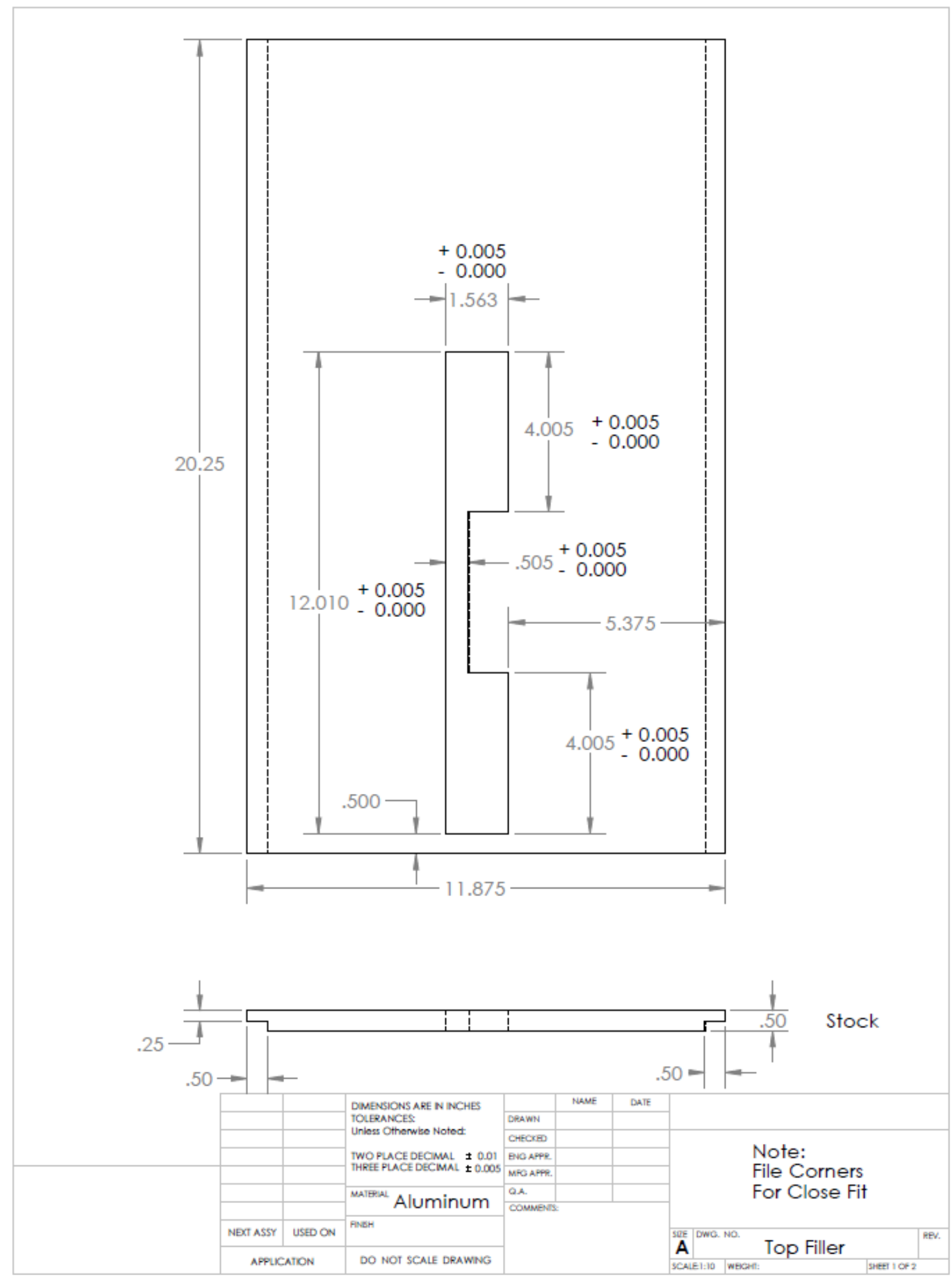




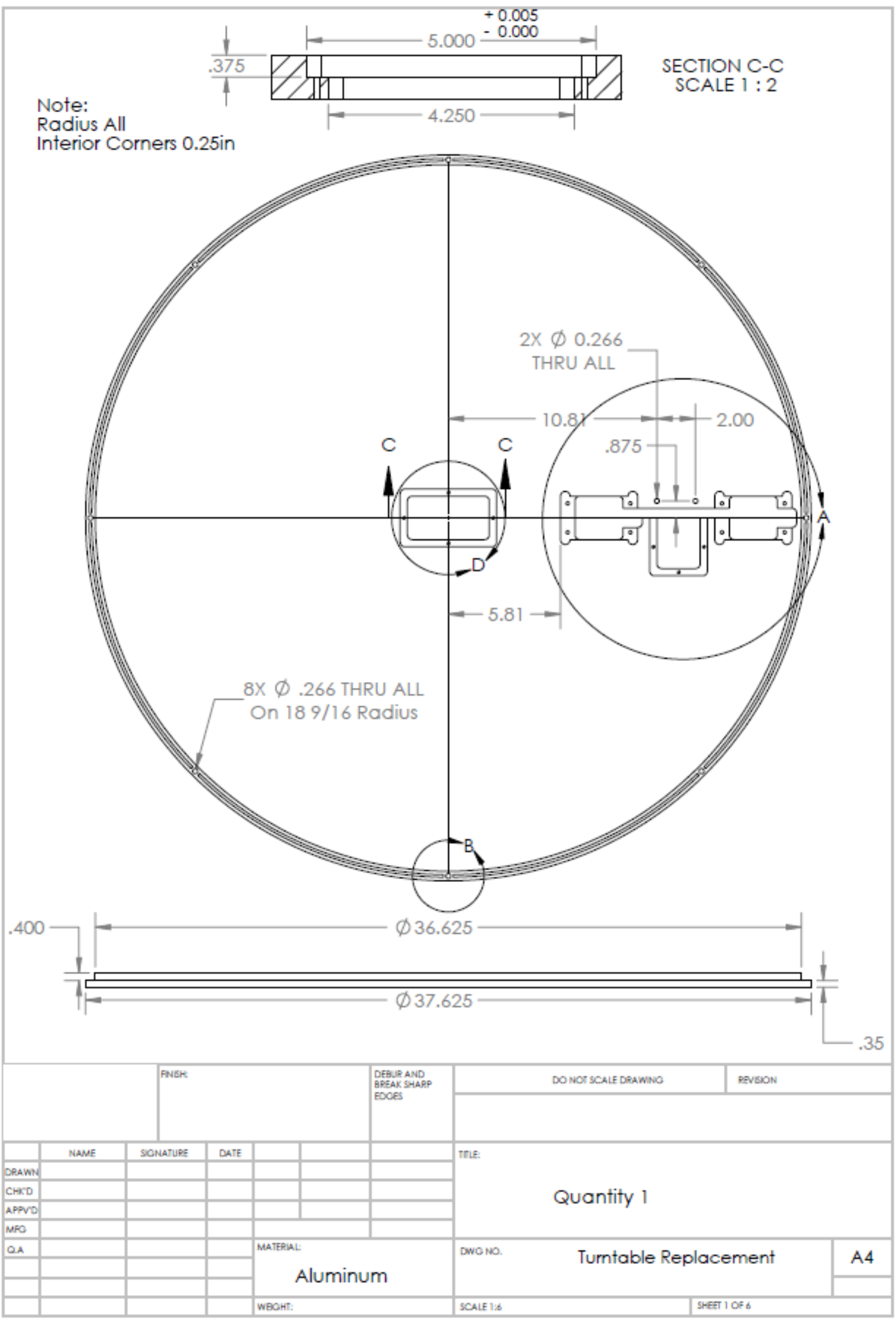




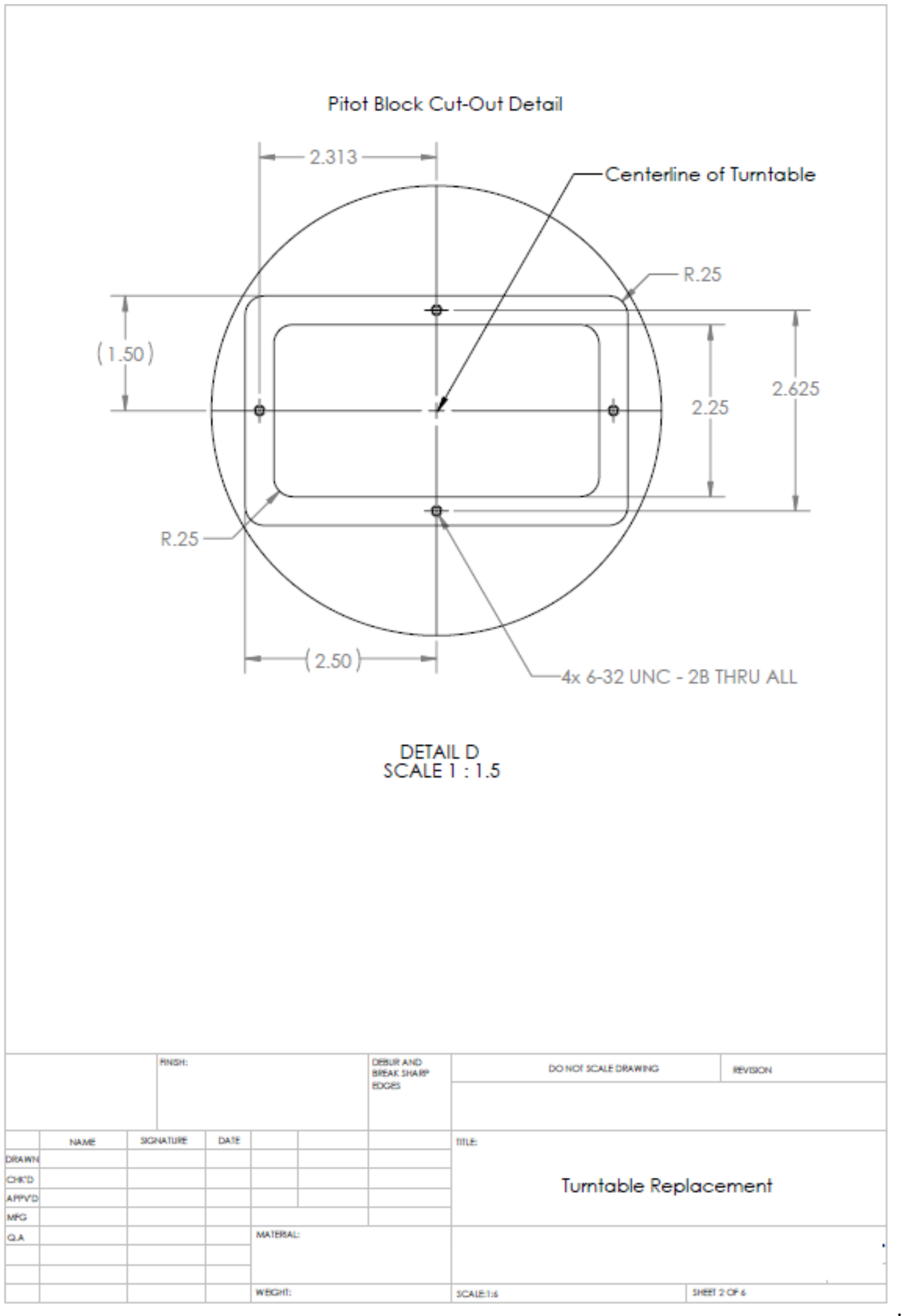




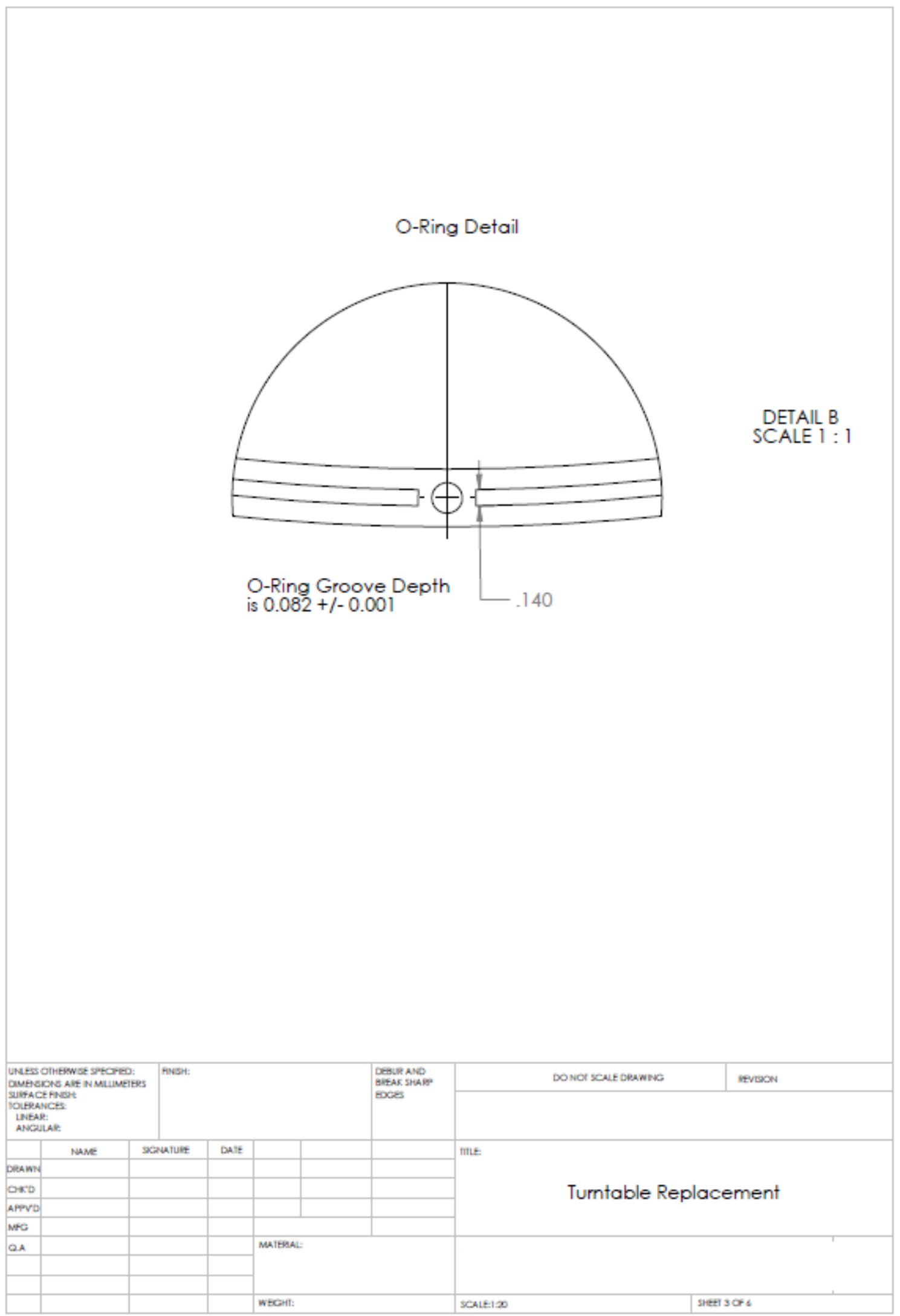




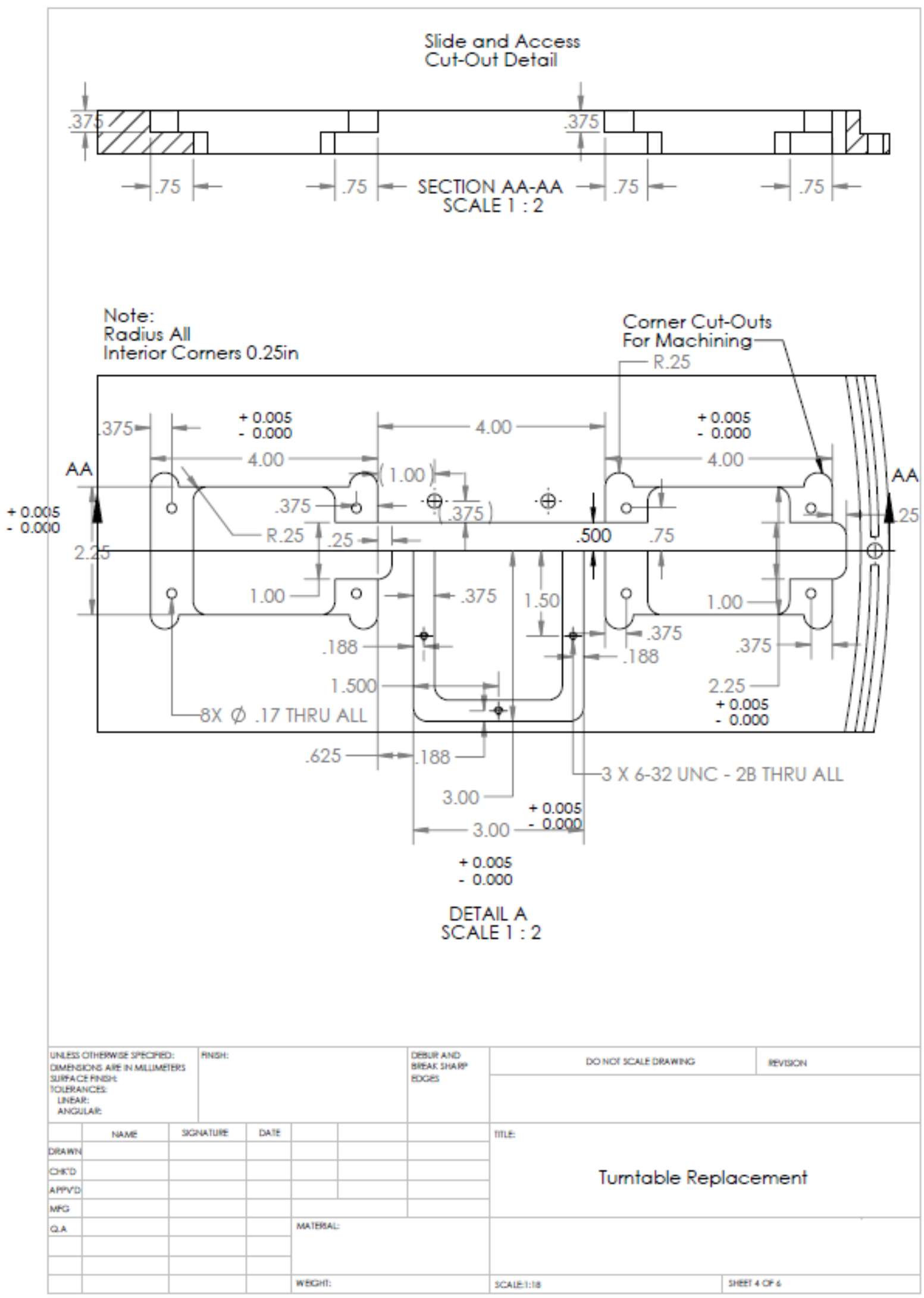




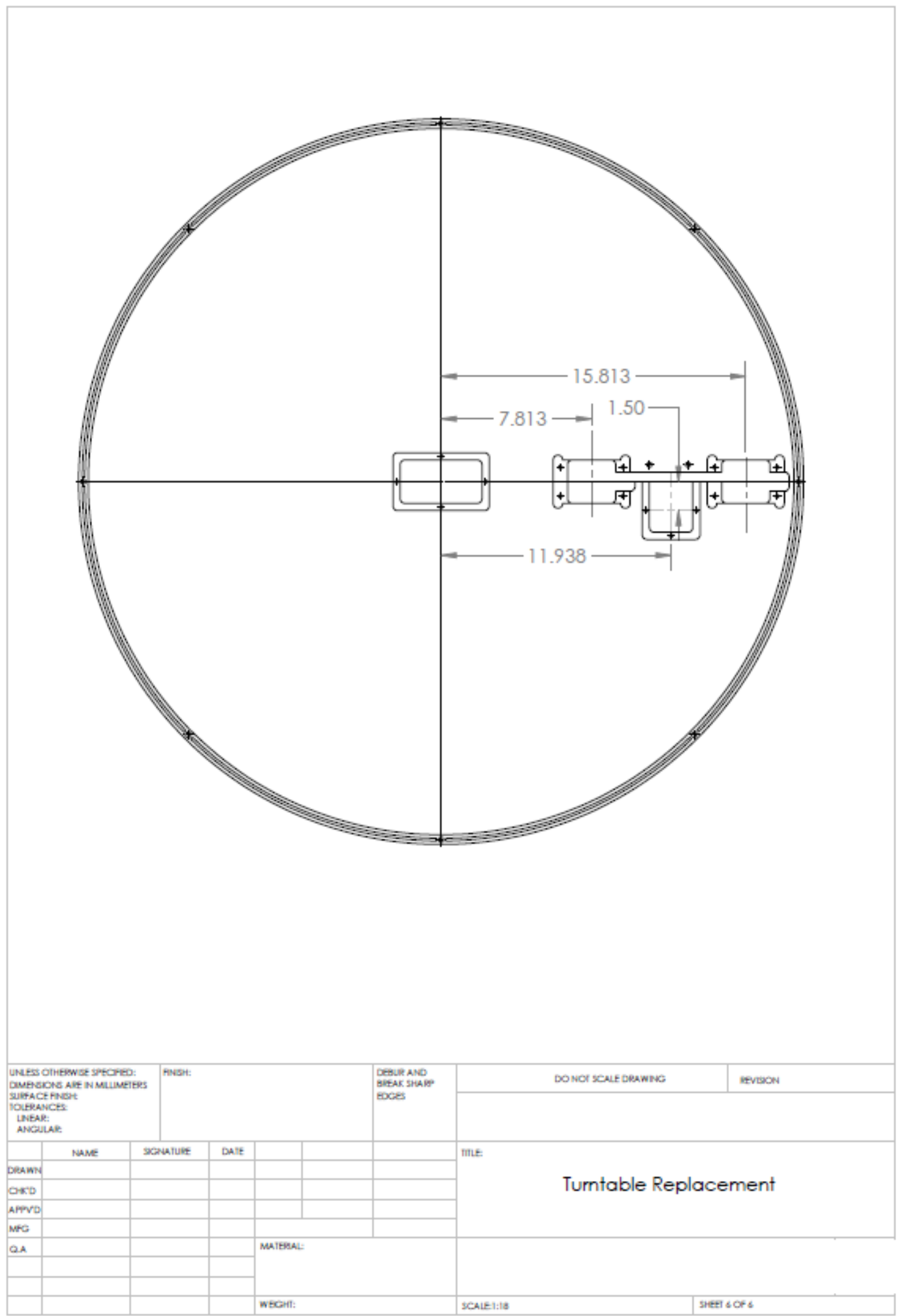


APPENDIX F: ESTIMATES OF THE STANDARD DEVIATION OF THE BALANCE FORCE AND MOMENT COEFFICIENTS

SPC Standard Deviation Estimates for $\boldsymbol{C}_{\boldsymbol{A}}$

\begin{tabular}{|c|c|c|c|c|c|c|}
\hline$q(P a)$ & $\alpha(\mathrm{deg})$ & $\phi$ & $\hat{\sigma}_{W G}$ & $\hat{\sigma}_{W T}$ & $\hat{\sigma}_{A T}$ & $\hat{\sigma}_{T}$ \\
\hline 444 & -2 & Upright & 0.00106 & 0.00225 & 0.00112 & 0.00272 \\
\hline 444 & -2 & Inverted & 0.00131 & 0.00198 & 0.00135 & 0.00273 \\
\hline 750 & -2 & Upright & 0.00103 & 0.00124 & 0.00056 & 0.00171 \\
\hline 750 & -2 & Inverted & 0.00107 & 0.00106 & 0.00052 & 0.00160 \\
\hline 980 & -2 & Upright & 0.00084 & 0.00116 & 0.00087 & 0.00168 \\
\hline 980 & -2 & Inverted & 0.00095 & 0.00117 & 0.00098 & 0.00180 \\
\hline 444 & 2 & Upright & 0.00141 & 0.00334 & 0.00081 & 0.00371 \\
\hline 444 & 2 & Inverted & 0.00124 & 0.00222 & 0.00132 & 0.00286 \\
\hline 750 & 2 & Upright & 0.00091 & 0.00123 & 0.00124 & 0.00197 \\
\hline 750 & 2 & Inverted & 0.00089 & 0.00146 & 0.00069 & 0.00185 \\
\hline 980 & 2 & Upright & 0.00104 & 0.00103 & 0.00084 & 0.00169 \\
\hline 980 & 2 & Inverted & 0.00078 & 0.00101 & 0.00029 & 0.00131 \\
\hline 444 & 6 & Upright & 0.00116 & 0.00253 & 0.00135 & 0.00310 \\
\hline 444 & 6 & Inverted & 0.00111 & 0.00220 & 0.00189 & 0.00310 \\
\hline 750 & 6 & Upright & 0.00089 & 0.00133 & 0.00127 & 0.00204 \\
\hline 750 & 6 & Inverted & 0.00084 & 0.00071 & 0.00071 & 0.00131 \\
\hline 980 & 6 & Upright & 0.00079 & 0.00113 & 0.00133 & 0.00191 \\
\hline 980 & 6 & Inverted & 0.00088 & 0.00089 & 0.00085 & 0.00152 \\
\hline
\end{tabular}


SPC Standard Deviation Estimates for $\boldsymbol{C}_{\boldsymbol{N}}$

\begin{tabular}{|c|c|c|c|c|c|c|}
\hline$q(P a)$ & $\alpha(\mathrm{deg})$ & $\phi$ & $\hat{\sigma}_{W G}$ & $\hat{\sigma}_{W T}$ & $\hat{\sigma}_{A T}$ & $\hat{\sigma}_{T}$ \\
\hline 444 & -2 & Upright & 0.00211 & 0.00581 & 0.00409 & 0.00741 \\
\hline 444 & -2 & Inverted & 0.00222 & 0.00357 & 0.00311 & 0.00523 \\
\hline 750 & -2 & Upright & 0.00141 & 0.00210 & 0.00320 & 0.00408 \\
\hline 750 & -2 & Inverted & 0.00148 & 0.00278 & 0.00171 & 0.00358 \\
\hline 980 & -2 & Upright & 0.00172 & 0.00392 & 0.00207 & 0.00475 \\
\hline 980 & -2 & Inverted & 0.00142 & 0.00195 & 0.00253 & 0.00349 \\
\hline 444 & 2 & Upright & 0.00235 & 0.00597 & 0.00571 & 0.00859 \\
\hline 444 & 2 & Inverted & 0.00216 & 0.00305 & 0.00341 & 0.00506 \\
\hline 750 & 2 & Upright & 0.00156 & 0.00266 & 0.00341 & 0.00460 \\
\hline 750 & 2 & Inverted & 0.00143 & 0.00197 & 0.00148 & 0.00285 \\
\hline 980 & 2 & Upright & 0.00272 & 0.00205 & 0.00136 & 0.00367 \\
\hline 980 & 2 & Inverted & 0.00109 & 0.00219 & 0.00072 & 0.00255 \\
\hline 444 & 6 & Upright & 0.00212 & 0.00637 & 0.00588 & 0.00893 \\
\hline 444 & 6 & Inverted & 0.00249 & 0.00565 & 0.00321 & 0.00696 \\
\hline 750 & 6 & Upright & 0.00157 & 0.00236 & 0.00574 & 0.00640 \\
\hline 750 & 6 & Inverted & 0.00184 & 0.00348 & 0.00406 & 0.00566 \\
\hline 980 & 6 & Upright & 0.00223 & 0.00250 & 0.00403 & 0.00524 \\
\hline 980 & 6 & Inverted & 0.00193 & 0.00344 & 0.00271 & 0.00478 \\
\hline
\end{tabular}


SPC Standard Deviation Estimates for $\boldsymbol{C}_{M}$

\begin{tabular}{|c|c|c|c|c|c|c|}
\hline$q(P a)$ & $\alpha($ deg $)$ & $\phi$ & $\hat{\sigma}_{W G}$ & $\hat{\sigma}_{W T}$ & $\hat{\sigma}_{A T}$ & $\hat{\sigma}_{T}$ \\
\hline 444 & -2 & Upright & 0.00045 & 0.00075 & 0.00085 & 0.00122 \\
\hline 444 & -2 & Inverted & 0.00042 & 0.00088 & 0.00126 & 0.00159 \\
\hline 750 & -2 & Upright & 0.00031 & 0.00067 & 0.00083 & 0.00111 \\
\hline 750 & -2 & Inverted & 0.00029 & 0.00063 & 0.00086 & 0.00110 \\
\hline 980 & -2 & Upright & 0.00027 & 0.00039 & 0.00099 & 0.00110 \\
\hline 980 & -2 & Inverted & 0.00022 & 0.00049 & 0.00057 & 0.00079 \\
\hline 444 & 2 & Upright & 0.00058 & 0.00104 & 0.00032 & 0.00123 \\
\hline 444 & 2 & Inverted & 0.00052 & 0.00112 & 0.00093 & 0.00155 \\
\hline 750 & 2 & Upright & 0.00037 & 0.00063 & 0.00064 & 0.00097 \\
\hline 750 & 2 & Inverted & 0.00032 & 0.00055 & 0.00042 & 0.00077 \\
\hline 980 & 2 & Upright & 0.00045 & 0.00057 & 0.00030 & 0.00079 \\
\hline 980 & 2 & Inverted & 0.00036 & 0.00047 & 0.00037 & 0.00070 \\
\hline 444 & 6 & Upright & 0.00051 & 0.00089 & 0.00045 & 0.00112 \\
\hline 444 & 6 & Inverted & 0.00053 & 0.00105 & 0.00040 & 0.00124 \\
\hline 750 & 6 & Upright & 0.00038 & 0.00051 & 0.00061 & 0.00088 \\
\hline 750 & 6 & Inverted & 0.00042 & 0.00088 & 0.00076 & 0.00124 \\
\hline 980 & 6 & Upright & 0.00046 & 0.00049 & 0.00116 & 0.00134 \\
\hline 980 & 6 & Inverted & 0.00042 & 0.00058 & 0.00028 & 0.00077 \\
\hline
\end{tabular}


REML Standard Deviation Estimates for $\boldsymbol{C}_{\boldsymbol{A}}$

\begin{tabular}{|c|c|c|c|c|c|c|}
\hline$q(P a)$ & $\alpha($ deg $)$ & $\phi$ & $\hat{\sigma}_{W G}$ & $\hat{\sigma}_{W T}$ & $\hat{\sigma}_{A T}$ & $\hat{\sigma}_{T}$ \\
\hline 444 & -2 & Upright & 0.00103 & 0.00173 & 0.00062 & 0.00211 \\
\hline 444 & -2 & Inverted & 0.00113 & 0.00182 & 0.00065 & 0.00224 \\
\hline 750 & -2 & Upright & 0.00088 & 0.00094 & 0.00042 & 0.00136 \\
\hline 750 & -2 & Inverted & 0.00089 & 0.00078 & 0.00048 & 0.00127 \\
\hline 980 & -2 & Upright & 0.00091 & 0.00085 & 0.00075 & 0.00145 \\
\hline 980 & -2 & Inverted & 0.00094 & 0.00069 & 0.00074 & 0.00138 \\
\hline 444 & 2 & Upright & 0.00154 & 0.00226 & 0.00095 & 0.00290 \\
\hline 444 & 2 & Inverted & 0.00153 & 0.00131 & 0.00063 & 0.00211 \\
\hline 750 & 2 & Upright & 0.00070 & 0.00131 & 0.00027 & 0.00151 \\
\hline 750 & 2 & Inverted & 0.00085 & 0.00099 & 0.00055 & 0.00142 \\
\hline 980 & 2 & Upright & 0.00100 & 0.00062 & 0.00060 & 0.00132 \\
\hline 980 & 2 & Inverted & 0.00086 & 0.00026 & 0.00049 & 0.00103 \\
\hline 444 & 6 & Upright & 0.00115 & 0.00200 & 0.00073 & 0.00242 \\
\hline 444 & 6 & Inverted & 0.00095 & 0.00158 & 0.00071 & 0.00198 \\
\hline 750 & 6 & Upright & 0.00093 & 0.00076 & 0.00068 & 0.00138 \\
\hline 750 & 6 & Inverted & 0.00082 & 0.00070 & 0.00067 & 0.00127 \\
\hline 980 & 6 & Upright & 0.00072 & 0.00076 & 0.00072 & 0.00127 \\
\hline 980 & 6 & Inverted & 0.00079 & 0.00027 & 0.00091 & 0.00124 \\
\hline
\end{tabular}


REML Standard Deviation Estimates for $C_{N}$

\begin{tabular}{|c|c|c|c|c|c|c|}
\hline$q(P a)$ & $\alpha(\mathrm{deg})$ & $\phi$ & $\hat{\sigma}_{W G}$ & $\hat{\sigma}_{W T}$ & $\hat{\sigma}_{A T}$ & $\hat{\sigma}_{T}$ \\
\hline 444 & -2 & Upright & 0.00230 & 0.00767 & 0.00150 & 0.00815 \\
\hline 444 & -2 & Inverted & 0.00209 & 0.00371 & 0.00146 & 0.00450 \\
\hline 750 & -2 & Upright & 0.00142 & 0.00268 & 0.00348 & 0.00462 \\
\hline 750 & -2 & Inverted & 0.00162 & 0.00229 & 0.00196 & 0.00343 \\
\hline 980 & -2 & Upright & 0.00178 & 0.00269 & 0.00308 & 0.00446 \\
\hline 980 & -2 & Inverted & 0.00155 & 0.00107 & 0.00251 & 0.00314 \\
\hline 444 & 2 & Upright & 0.00265 & 0.00677 & 0.00339 & 0.00802 \\
\hline 444 & 2 & Inverted & 0.00242 & 0.00367 & 0.00326 & 0.00547 \\
\hline 750 & 2 & Upright & 0.00173 & 0.00242 & 0.00295 & 0.00419 \\
\hline 750 & 2 & Inverted & 0.00125 & 0.00182 & 0.00233 & 0.00321 \\
\hline 980 & 2 & Upright & 0.00224 & 0.00173 & 0.00353 & 0.00452 \\
\hline 980 & 2 & Inverted & 0.00128 & 0.00152 & 0.00232 & 0.00306 \\
\hline 444 & 6 & Upright & 0.00223 & 0.00729 & 0.00384 & 0.00854 \\
\hline 444 & 6 & Inverted & 0.00284 & 0.00511 & 0.00357 & 0.00685 \\
\hline 750 & 6 & Upright & 0.00160 & 0.00246 & 0.00431 & 0.00522 \\
\hline 750 & 6 & Inverted & 0.00267 & 0.00171 & 0.00368 & 0.00486 \\
\hline 980 & 6 & Upright & 0.00192 & 0.00236 & 0.00428 & 0.00525 \\
\hline 980 & 6 & Inverted & 0.00248 & 0.00221 & 0.00320 & 0.00462 \\
\hline
\end{tabular}


REML Standard Deviation Estimates for $C_{M}$

\begin{tabular}{|c|c|c|c|c|c|c|}
\hline$q(P a)$ & $\alpha($ deg $)$ & $\phi$ & $\hat{\sigma}_{W G}$ & $\hat{\sigma}_{W T}$ & $\hat{\sigma}_{A T}$ & $\hat{\sigma}_{T}$ \\
\hline 444 & -2 & Upright & 0.00230 & 0.00767 & 0.00150 & 0.00815 \\
\hline 444 & -2 & Inverted & 0.00209 & 0.00371 & 0.00146 & 0.00450 \\
\hline 750 & -2 & Upright & 0.00142 & 0.00268 & 0.00348 & 0.00462 \\
\hline 750 & -2 & Inverted & 0.00162 & 0.00229 & 0.00196 & 0.00343 \\
\hline 980 & -2 & Upright & 0.00178 & 0.00269 & 0.00308 & 0.00446 \\
\hline 980 & -2 & Inverted & 0.00155 & 0.00107 & 0.00251 & 0.00314 \\
\hline 444 & 2 & Upright & 0.00265 & 0.00677 & 0.00339 & 0.00802 \\
\hline 444 & 2 & Inverted & 0.00242 & 0.00367 & 0.00326 & 0.00547 \\
\hline 750 & 2 & Upright & 0.00173 & 0.00242 & 0.00295 & 0.00419 \\
\hline 750 & 2 & Inverted & 0.00125 & 0.00182 & 0.00233 & 0.00321 \\
\hline 980 & 2 & Upright & 0.00224 & 0.00173 & 0.00353 & 0.00452 \\
\hline 980 & 2 & Inverted & 0.00128 & 0.00152 & 0.00232 & 0.00306 \\
\hline 444 & 6 & Upright & 0.00223 & 0.00729 & 0.00384 & 0.00854 \\
\hline 444 & 6 & Inverted & 0.00284 & 0.00511 & 0.00357 & 0.00685 \\
\hline 750 & 6 & Upright & 0.00160 & 0.00246 & 0.00431 & 0.00522 \\
\hline 750 & 6 & Inverted & 0.00267 & 0.00171 & 0.00368 & 0.00486 \\
\hline 980 & 6 & Upright & 0.00192 & 0.00236 & 0.00428 & 0.00525 \\
\hline 980 & 6 & Inverted & 0.00248 & 0.00221 & 0.00320 & 0.00462 \\
\hline
\end{tabular}




\section{APPENDIX G: STANDARD STATISTICAL PROCESS CONTROL CHARTS}
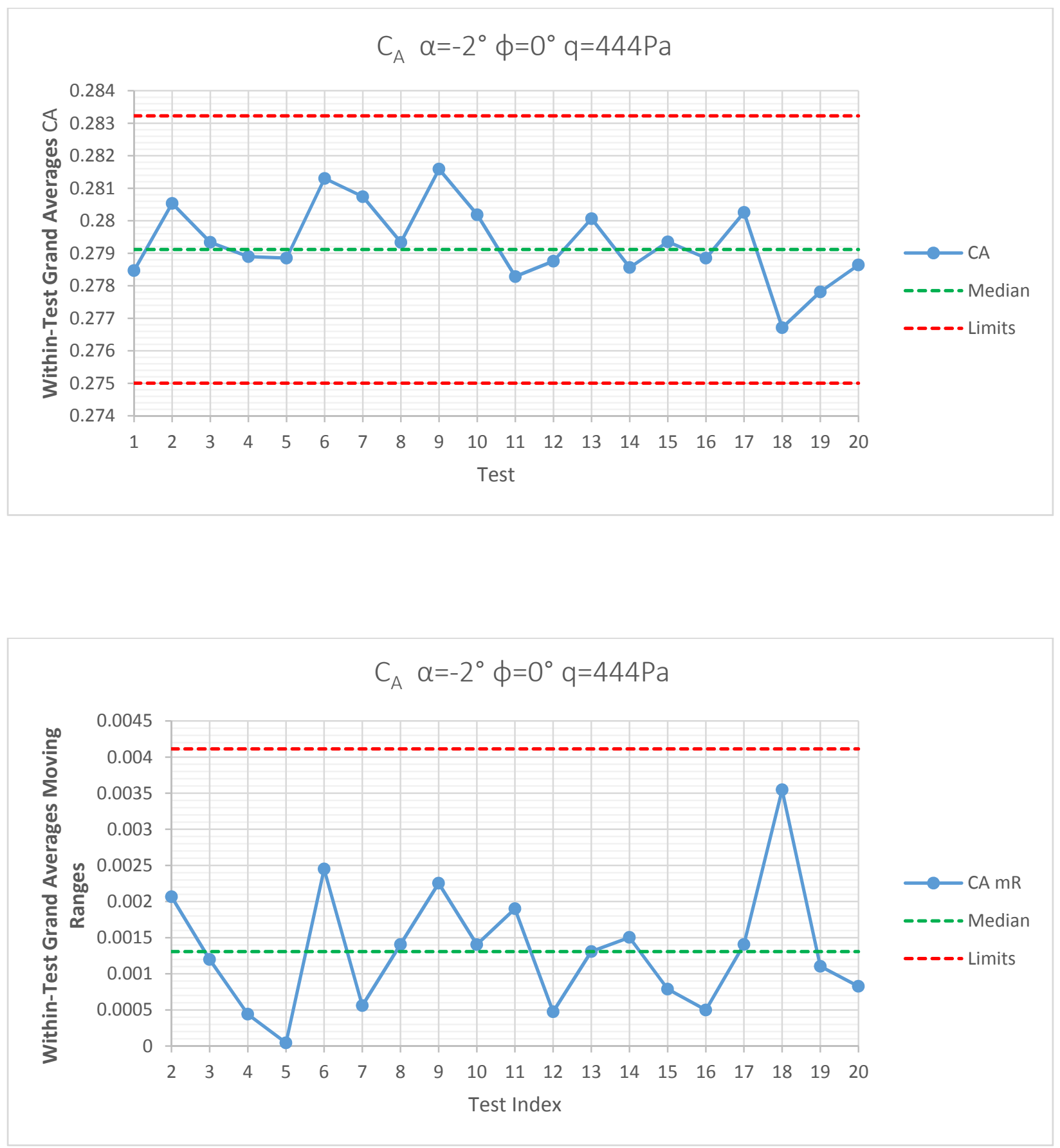

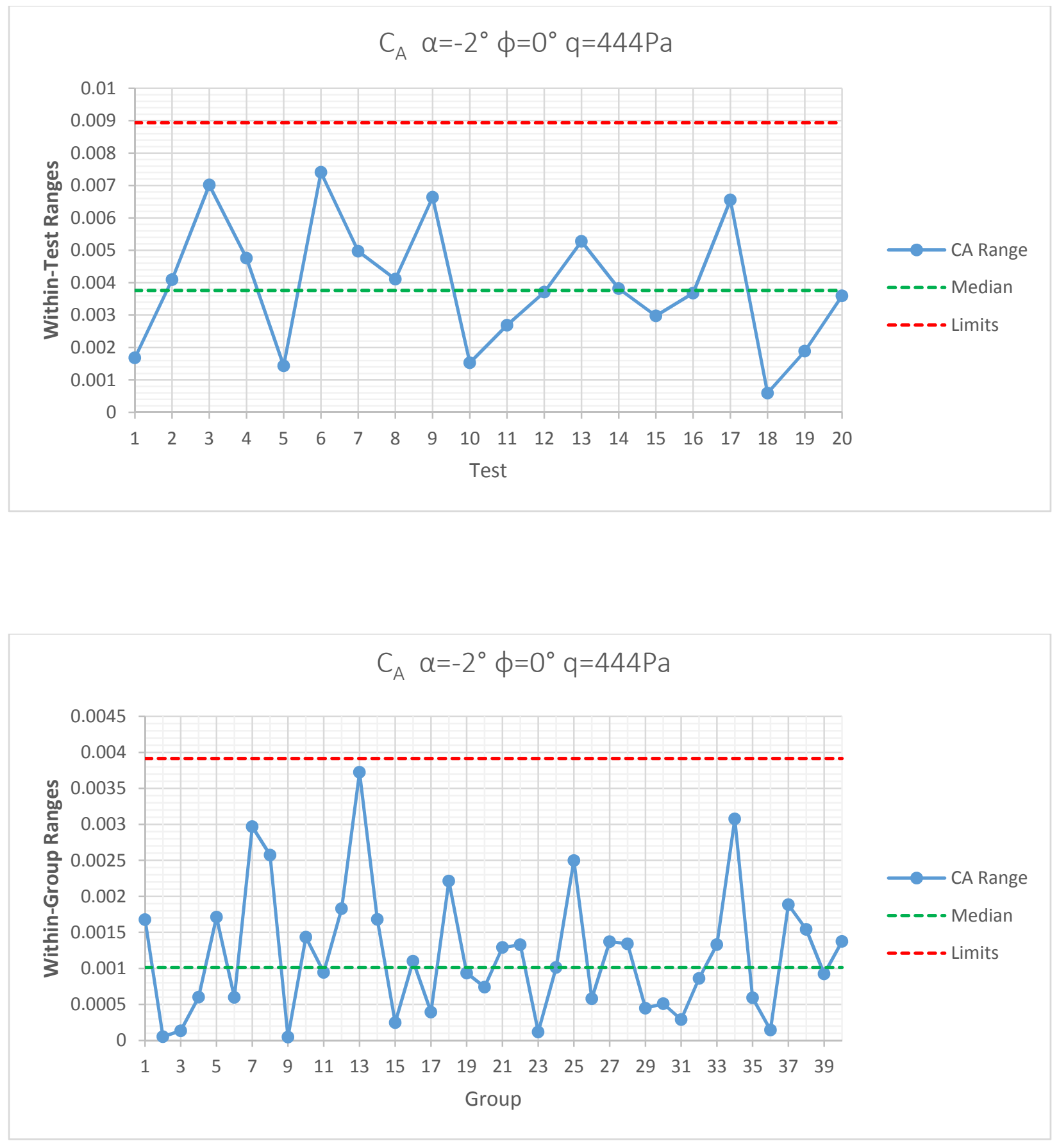

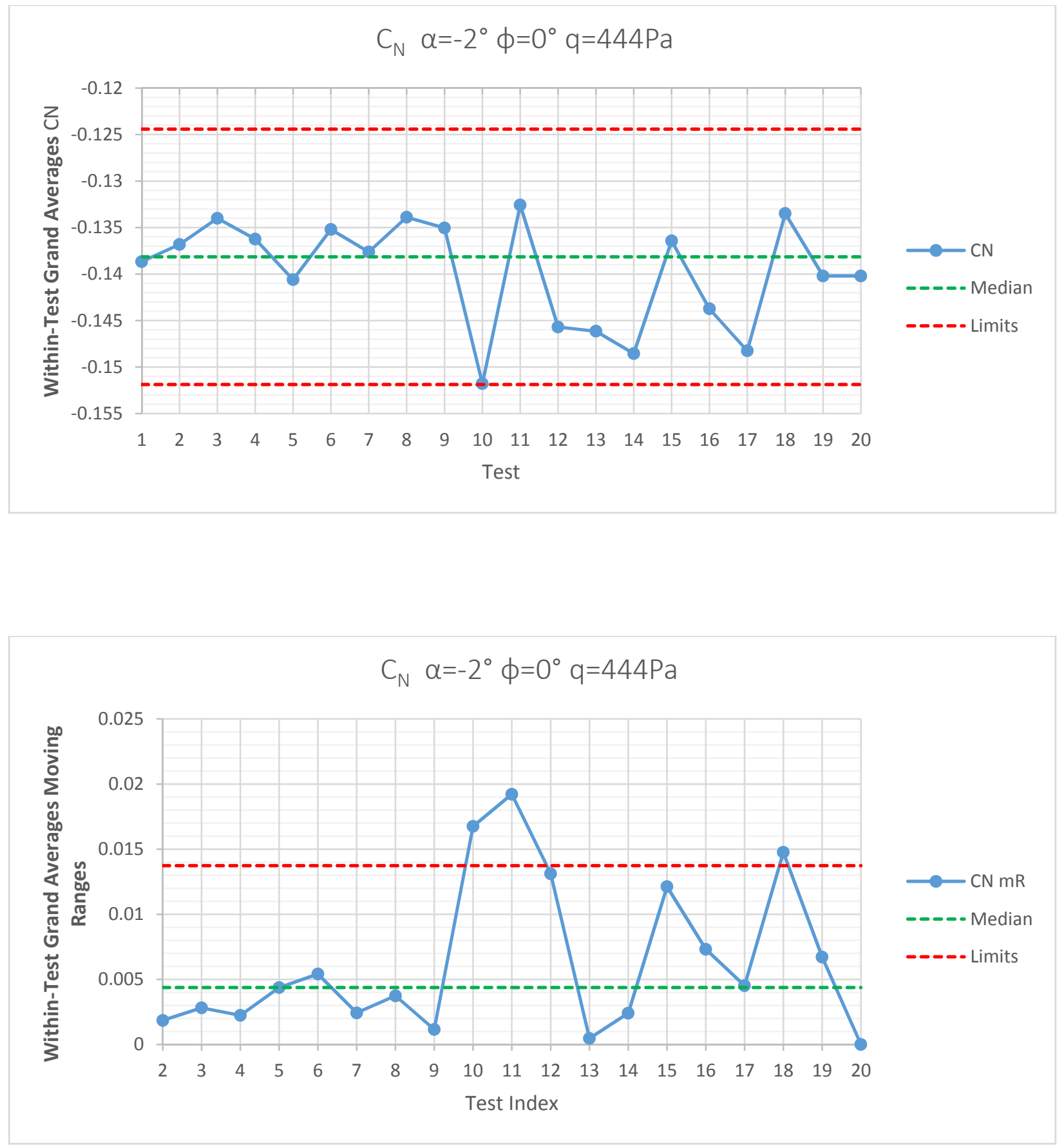

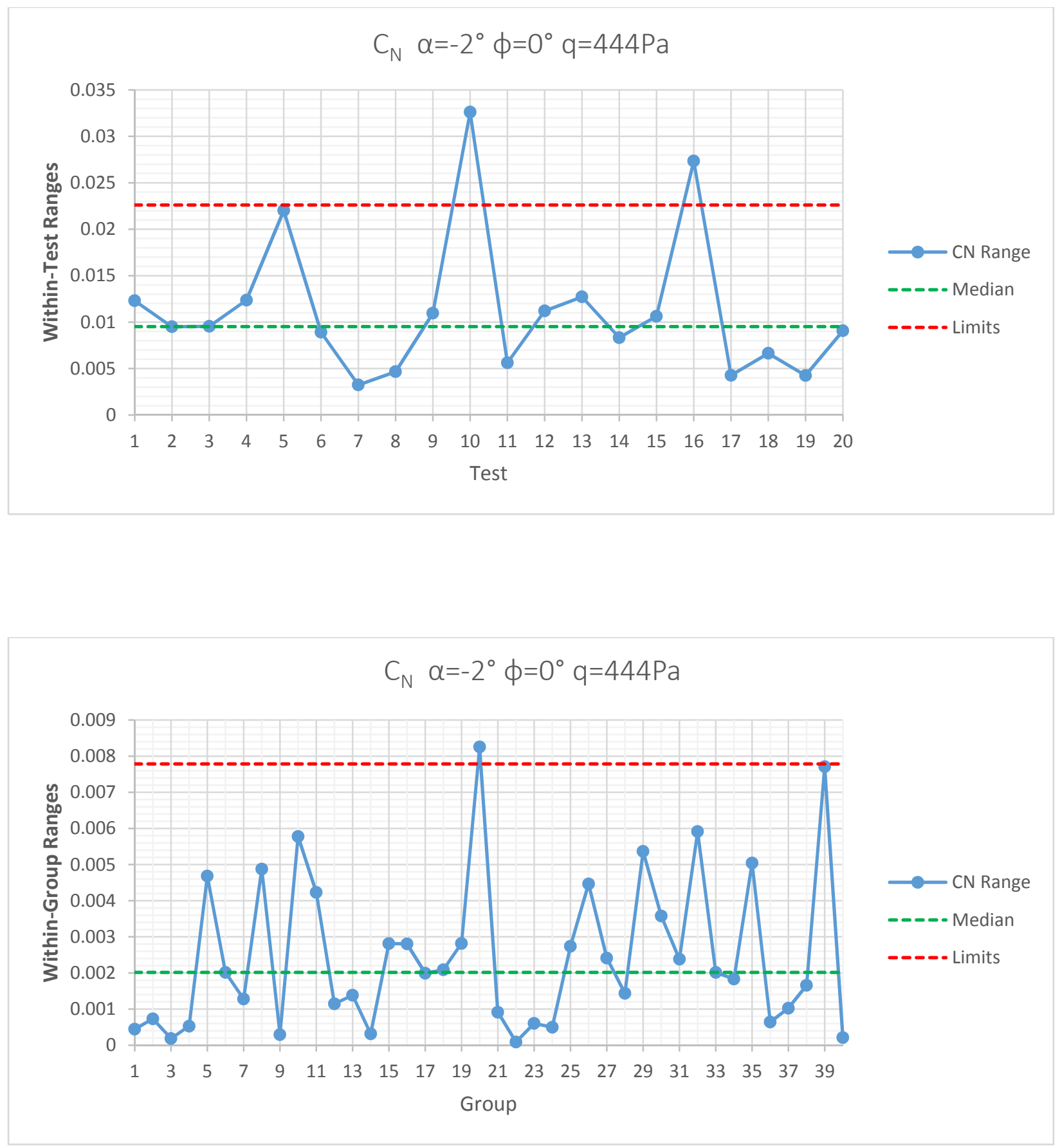

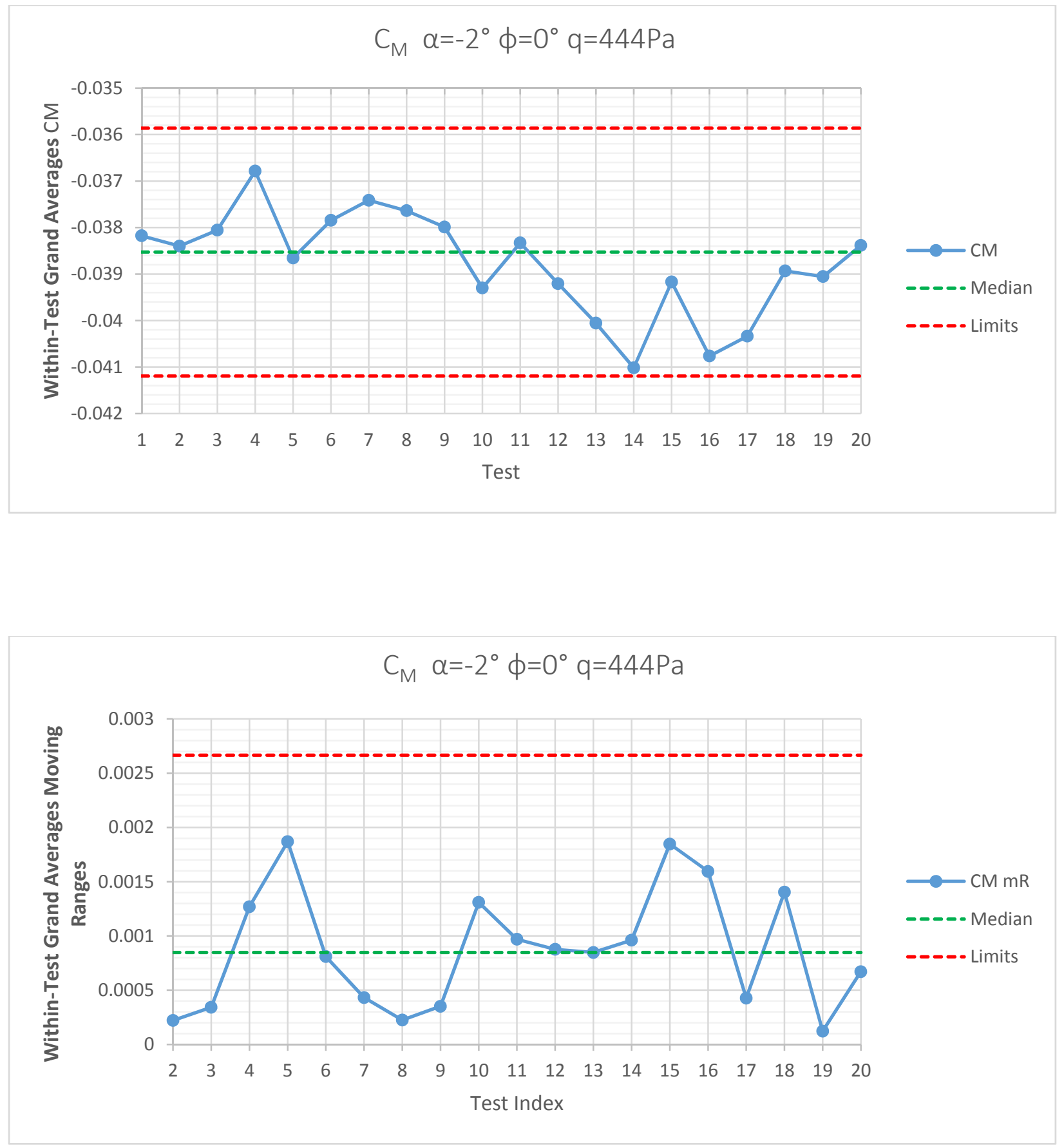

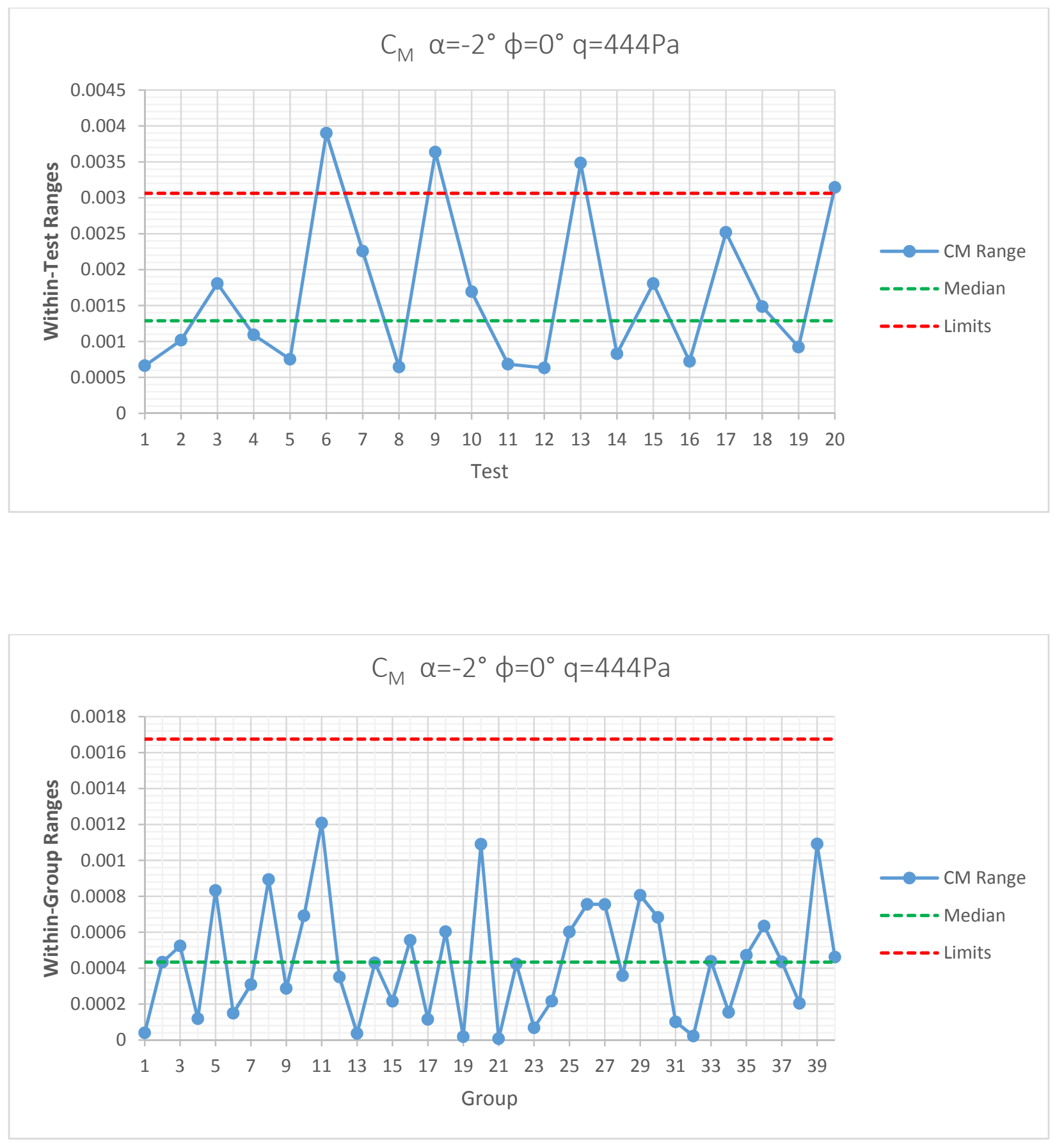

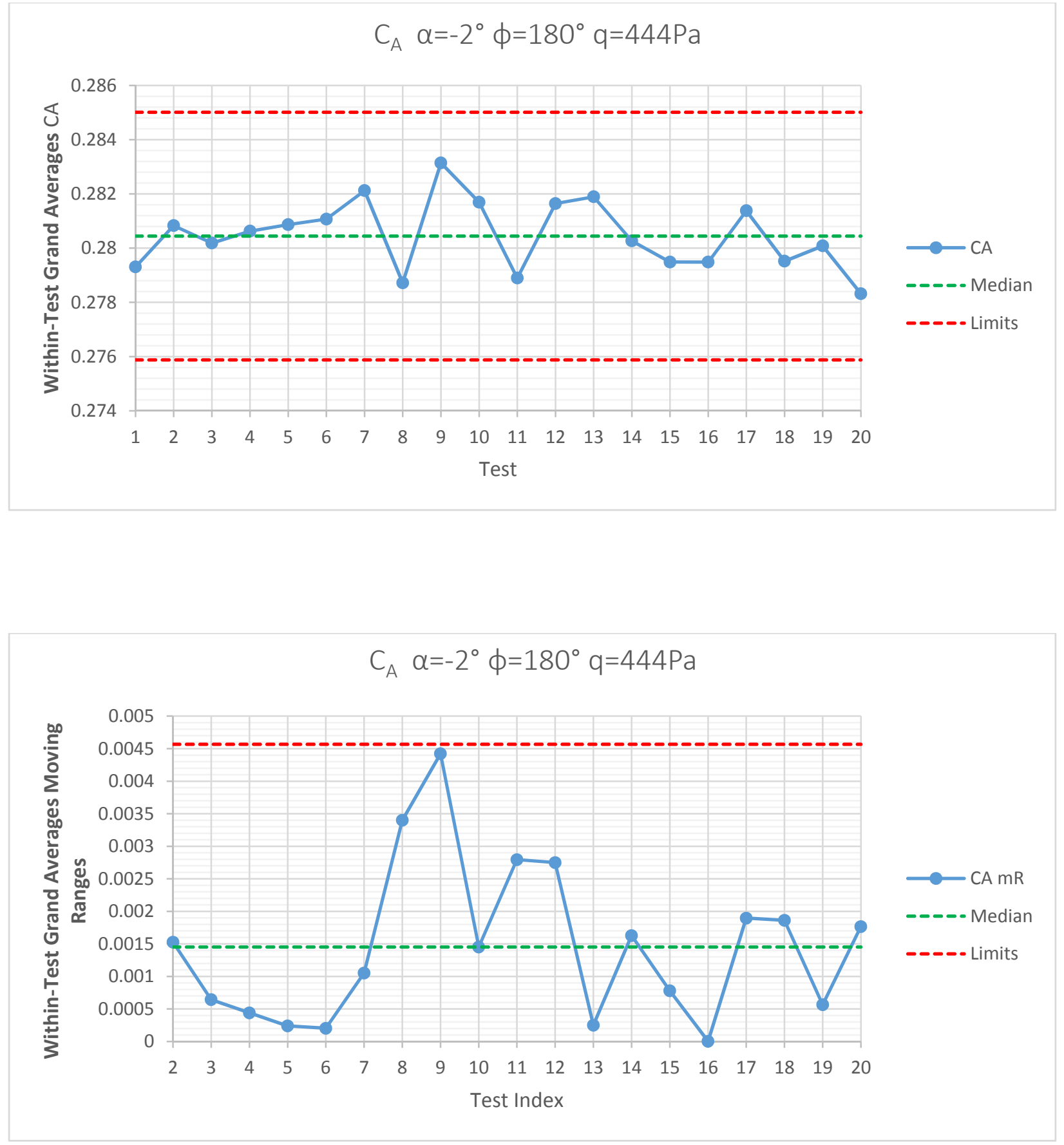

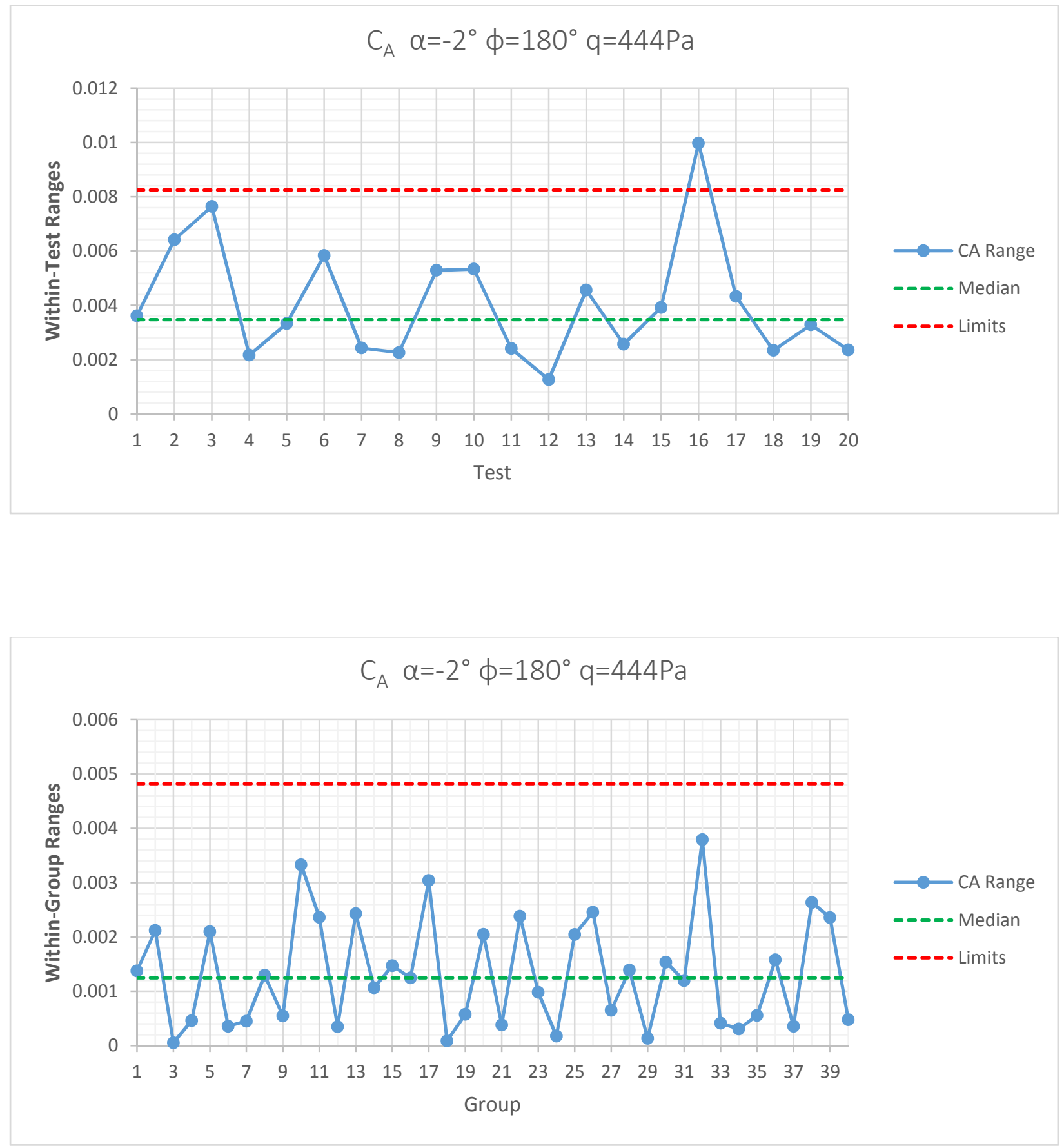

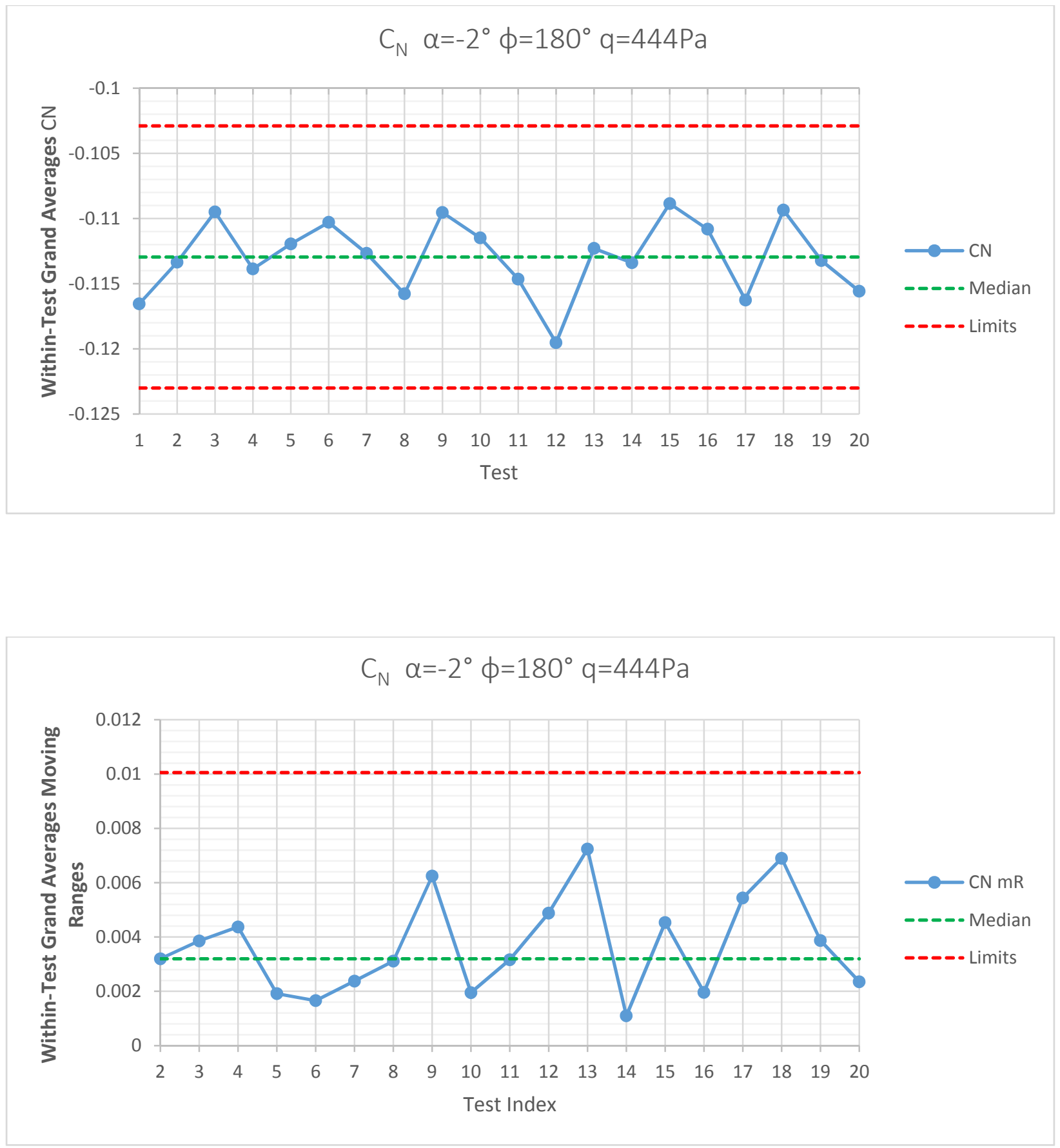

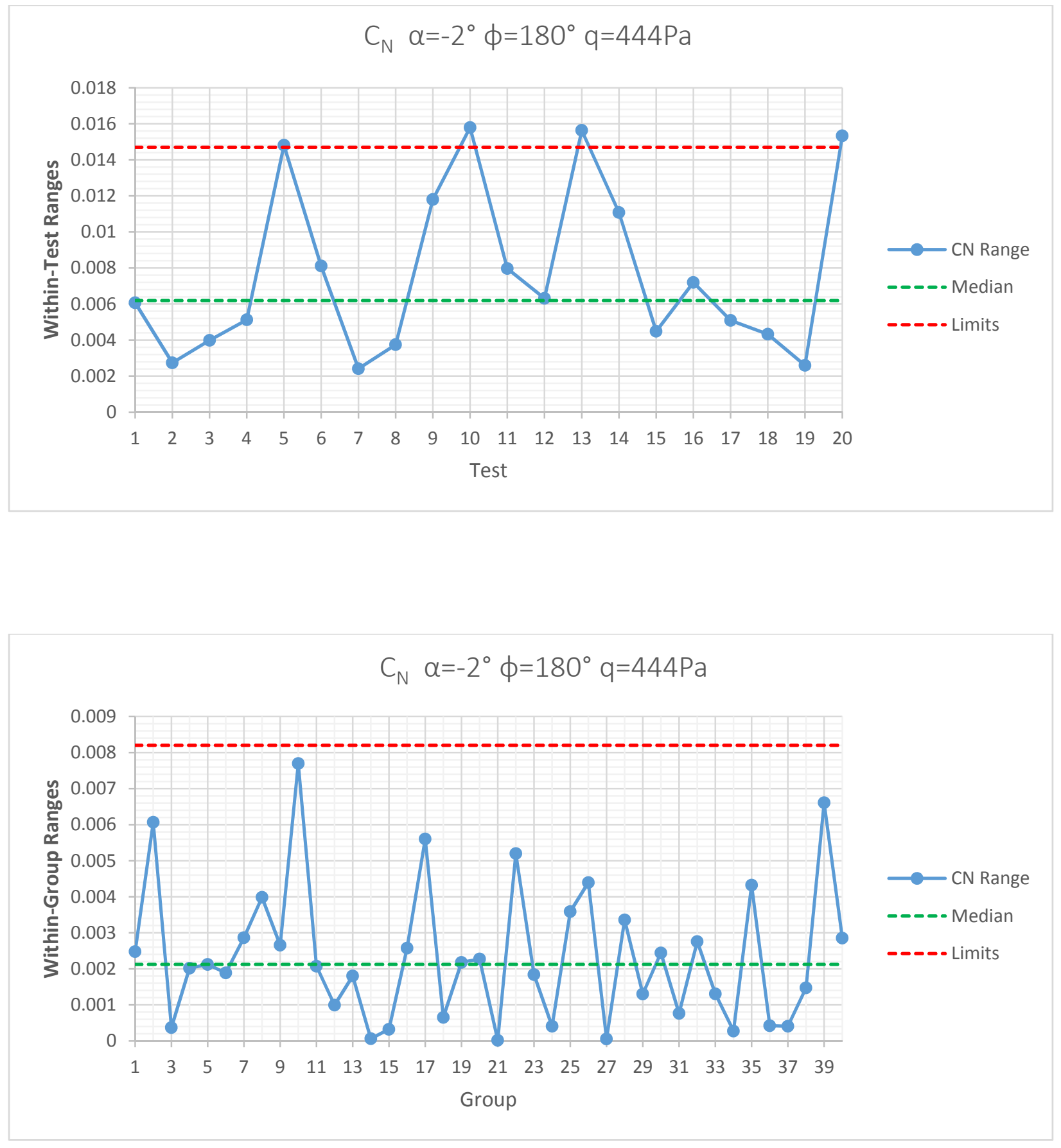

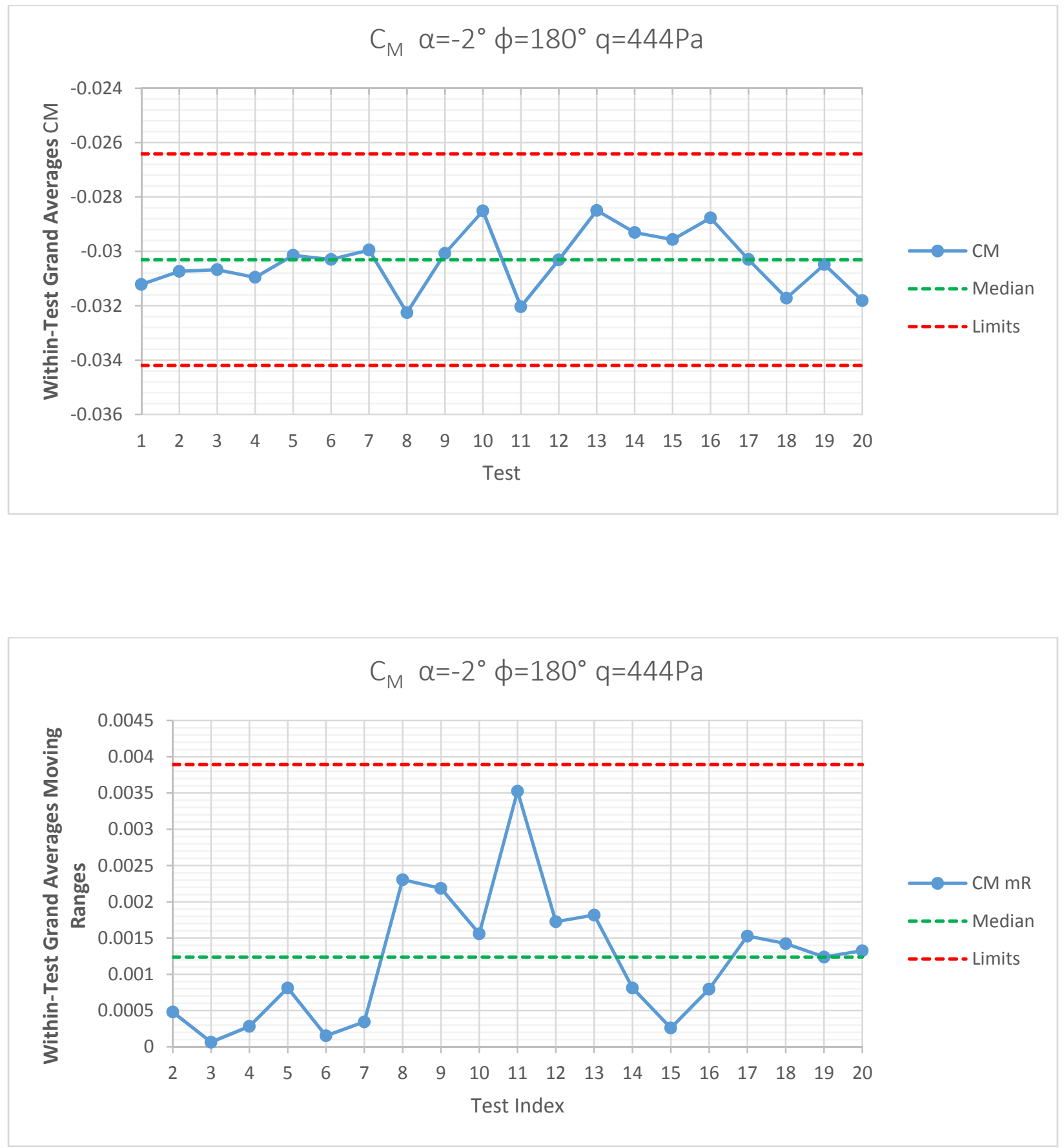

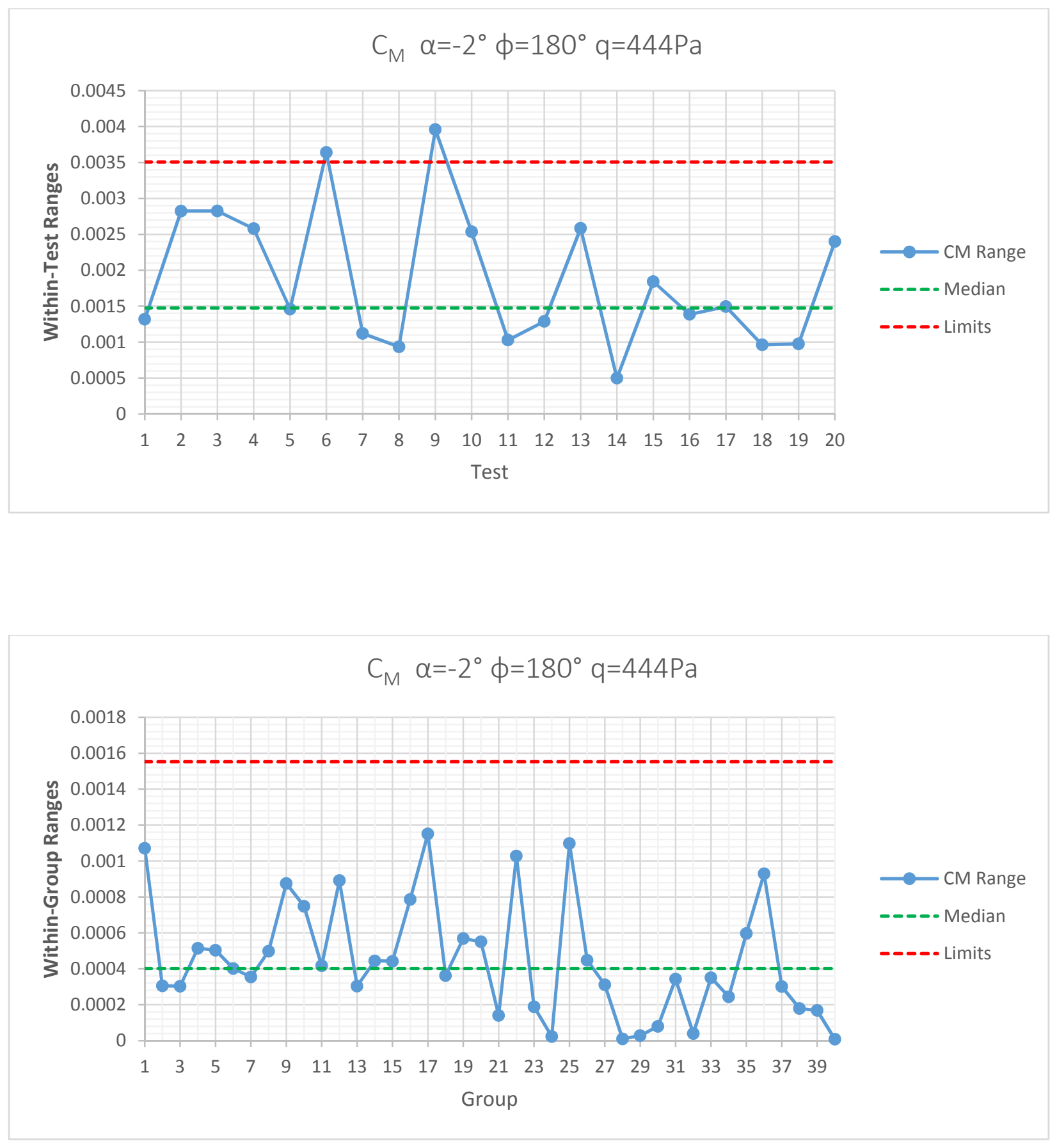

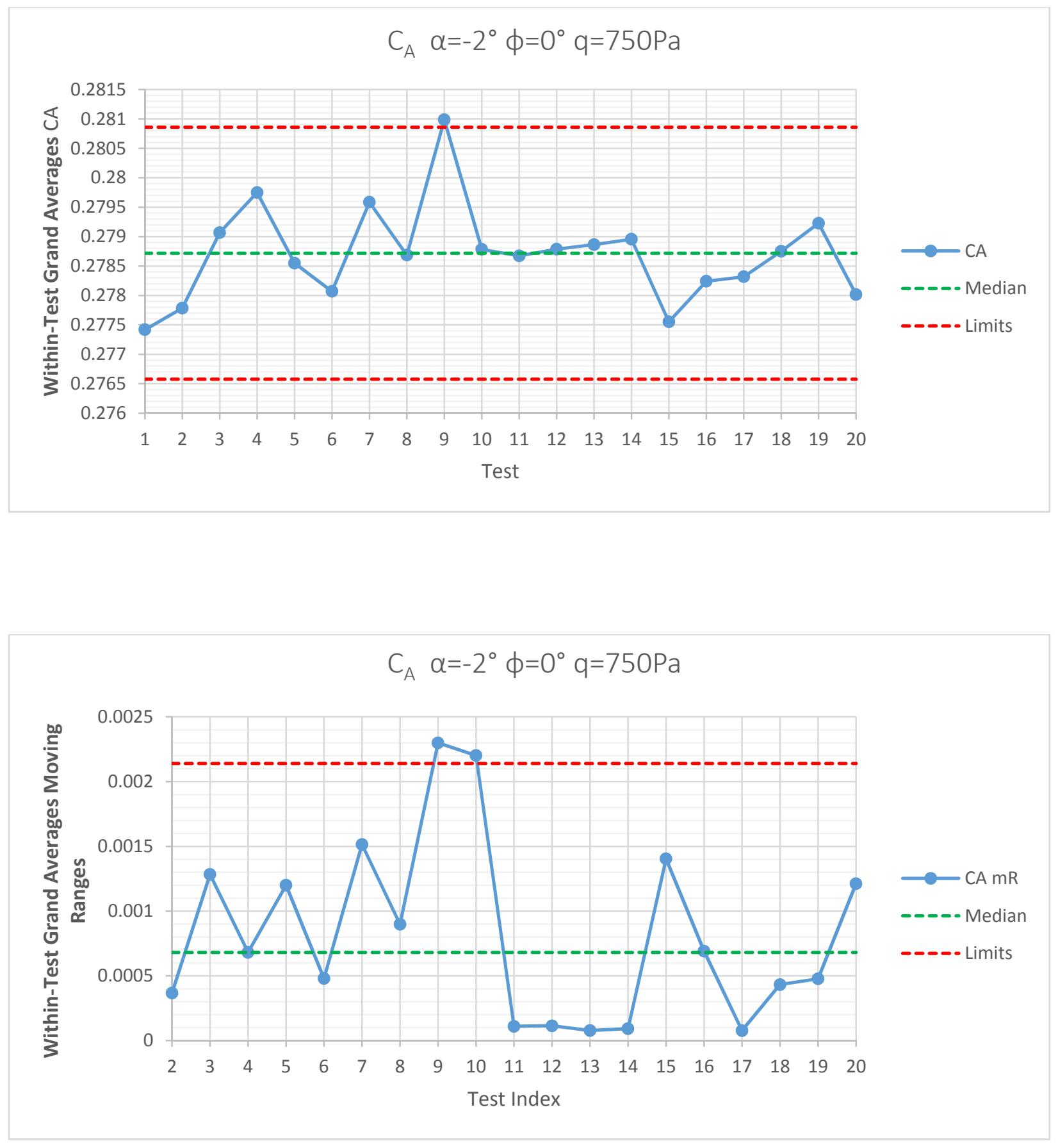

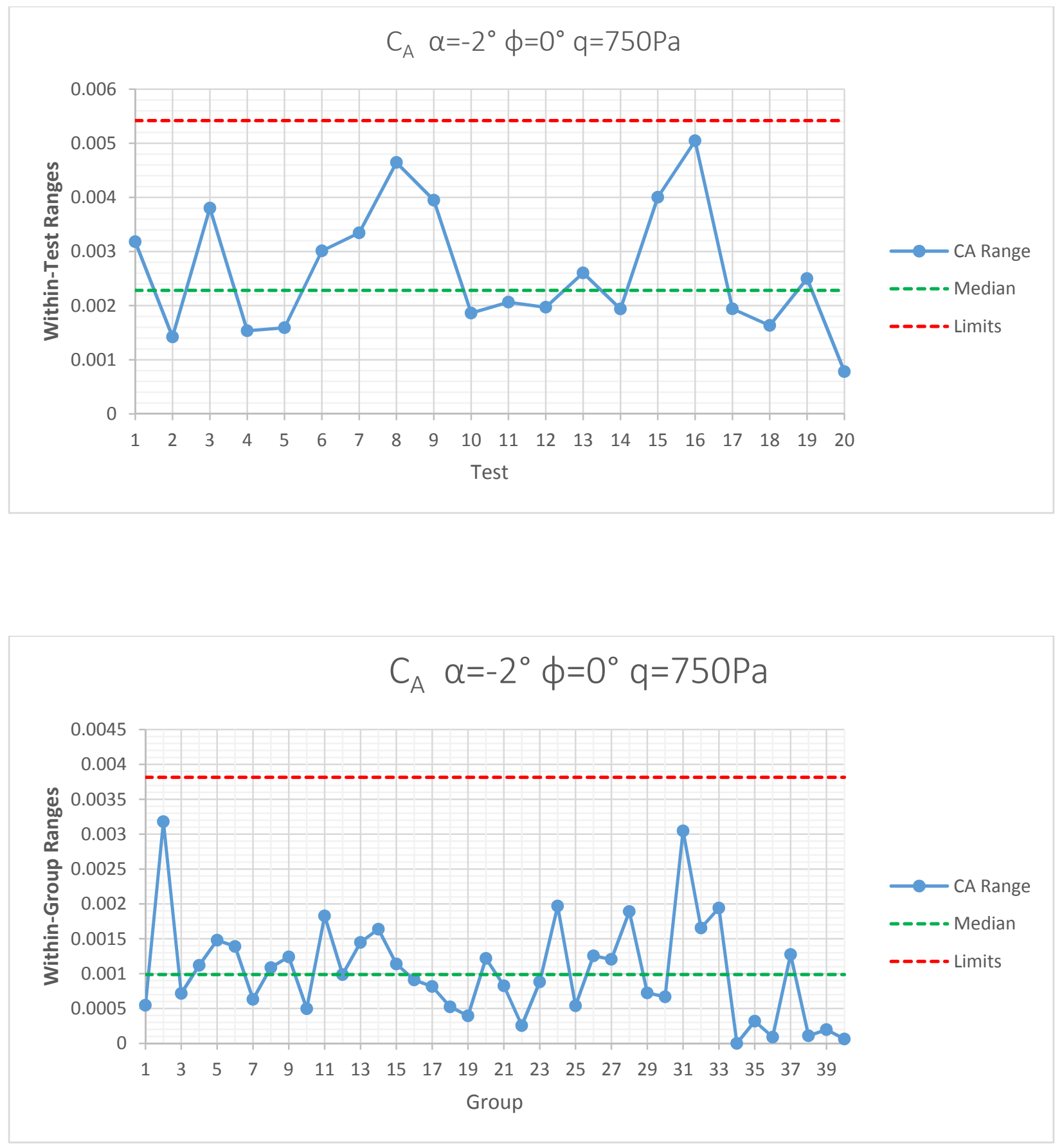

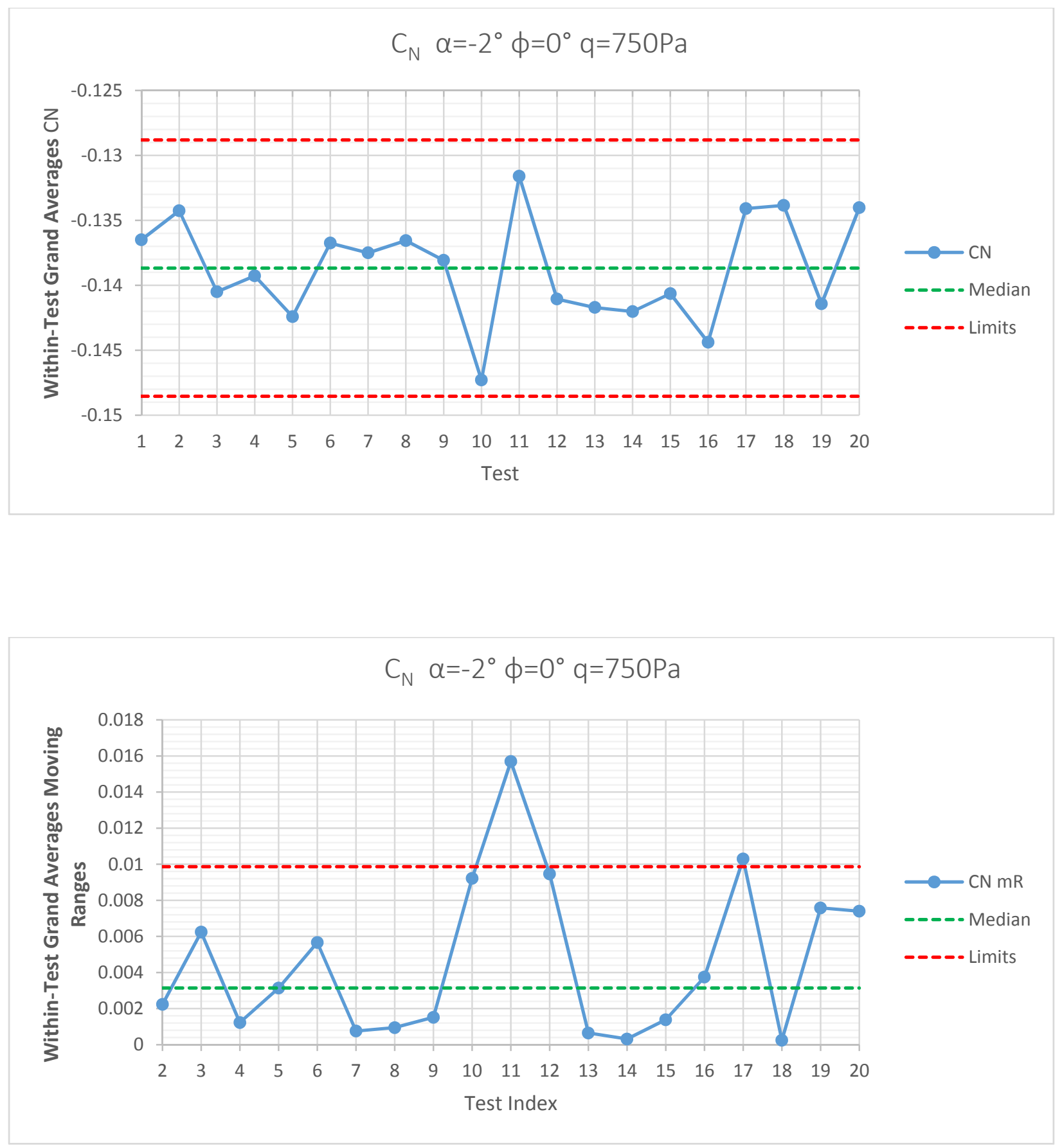

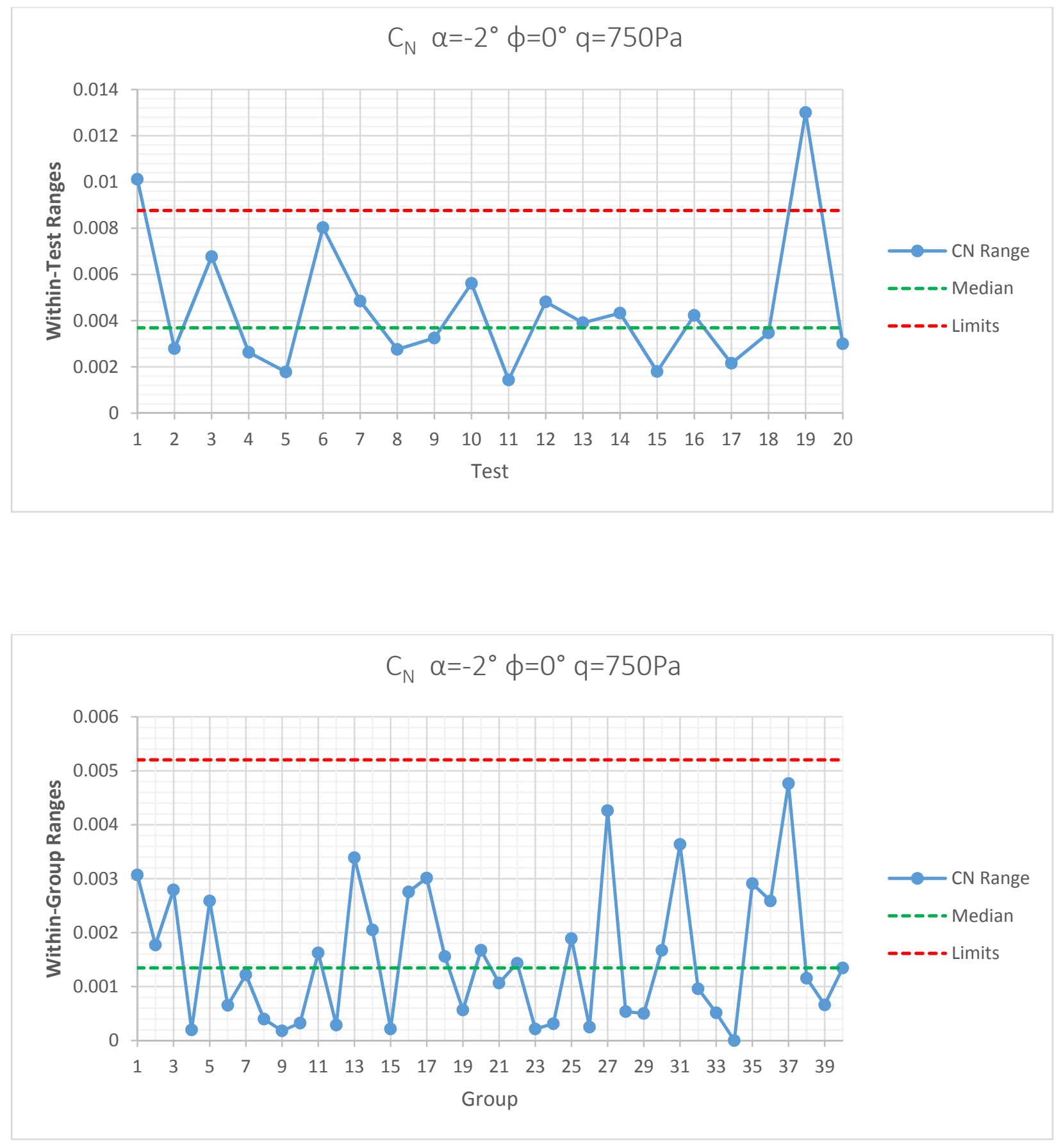

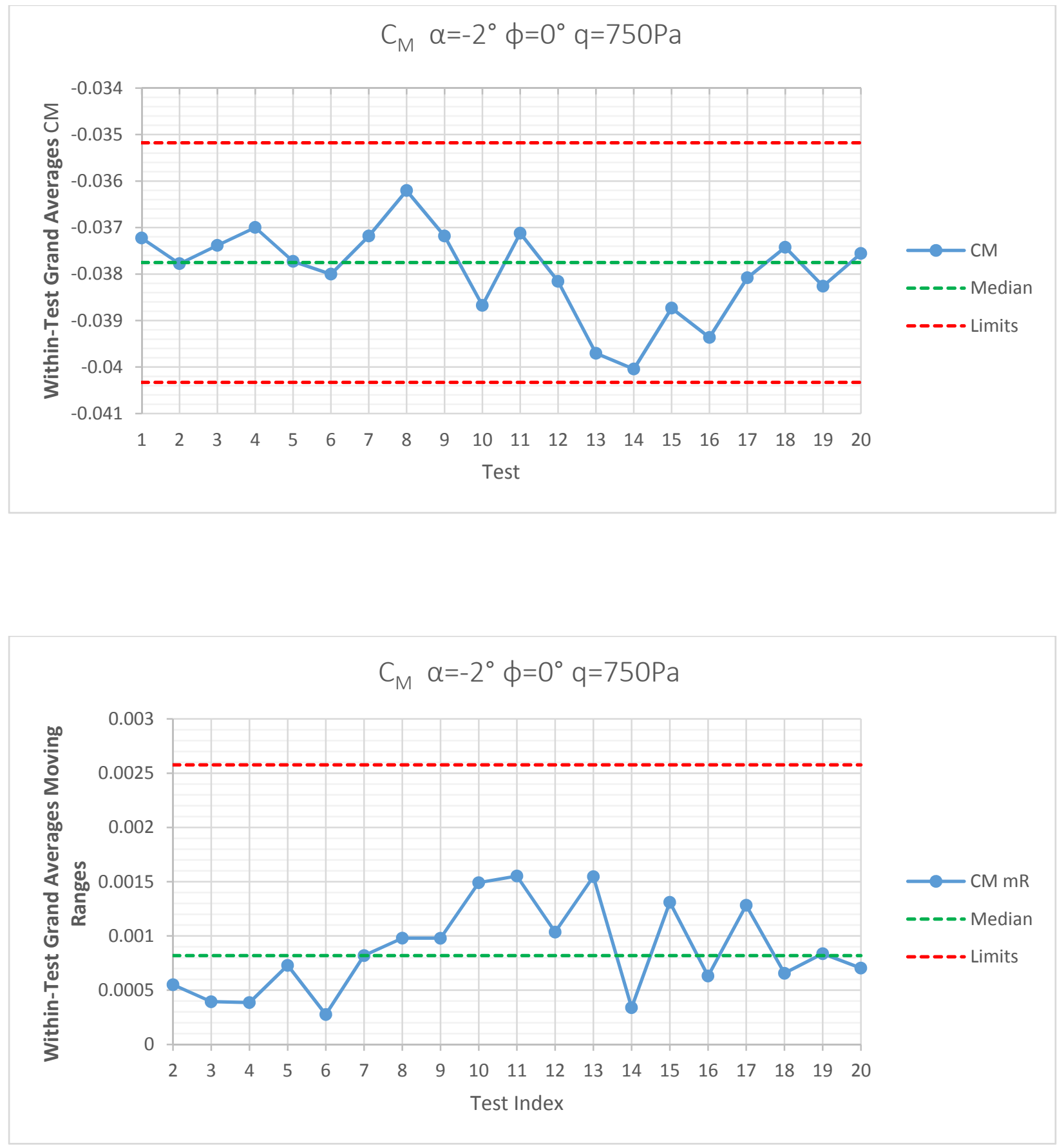

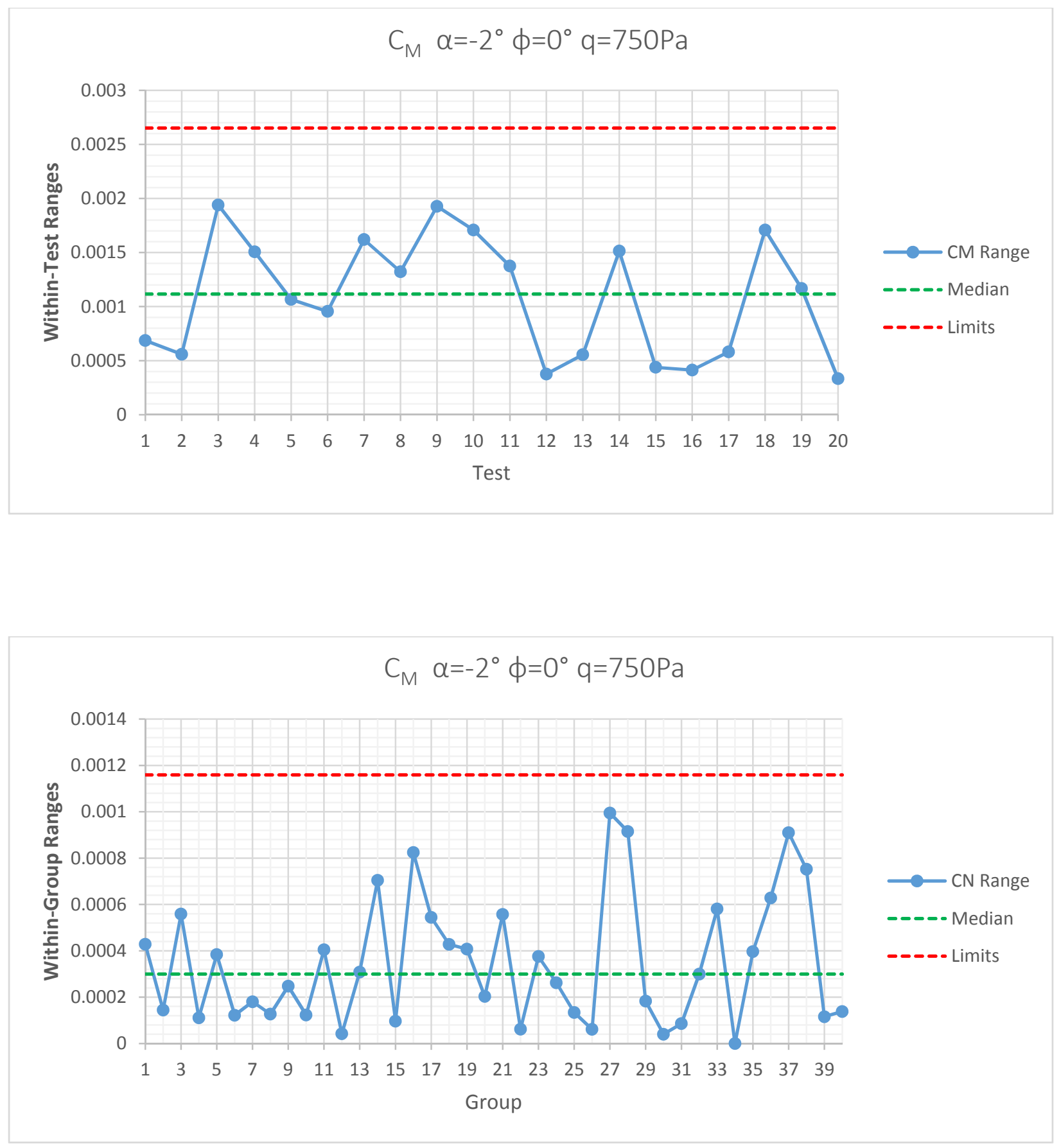

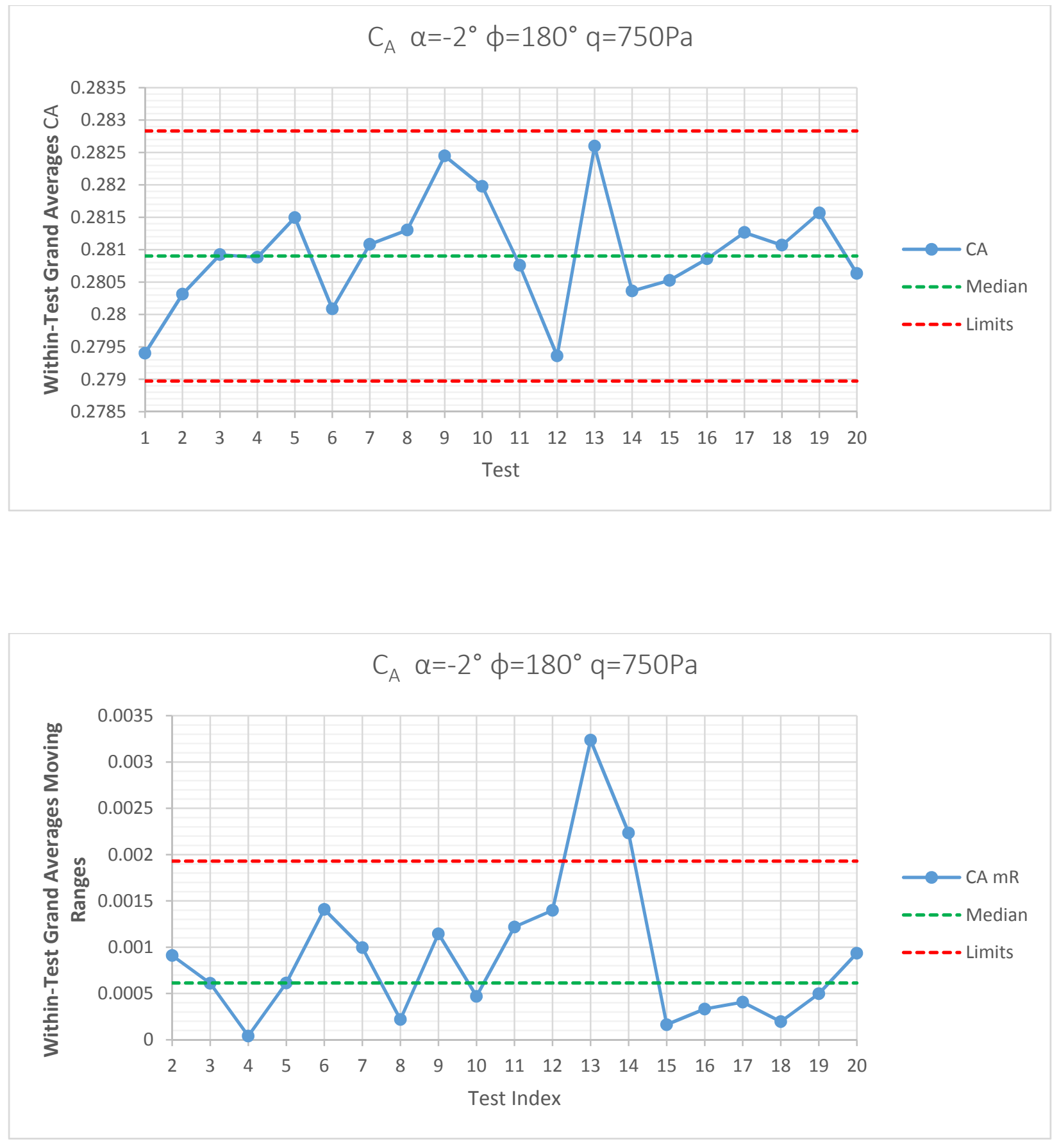

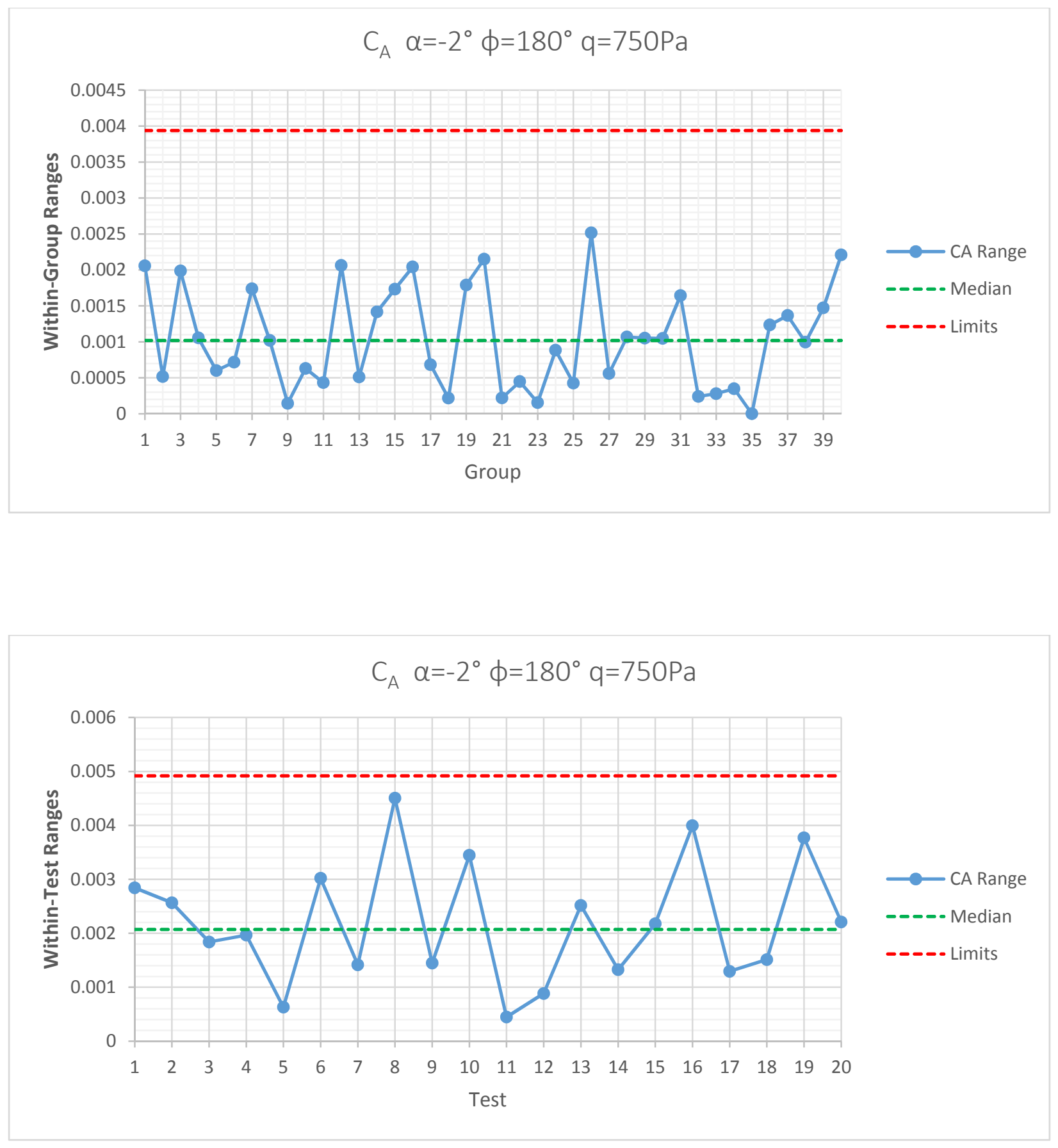

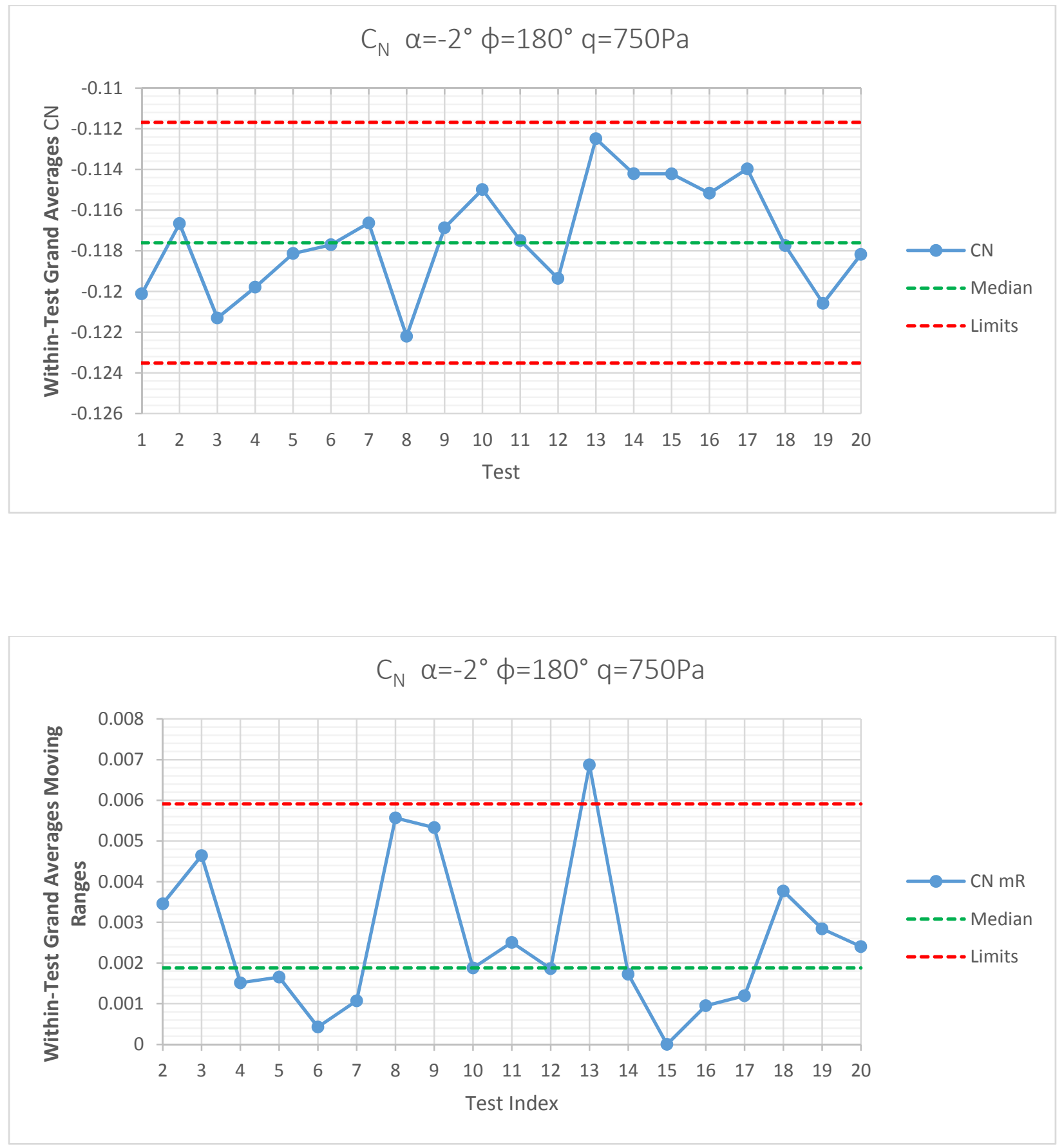

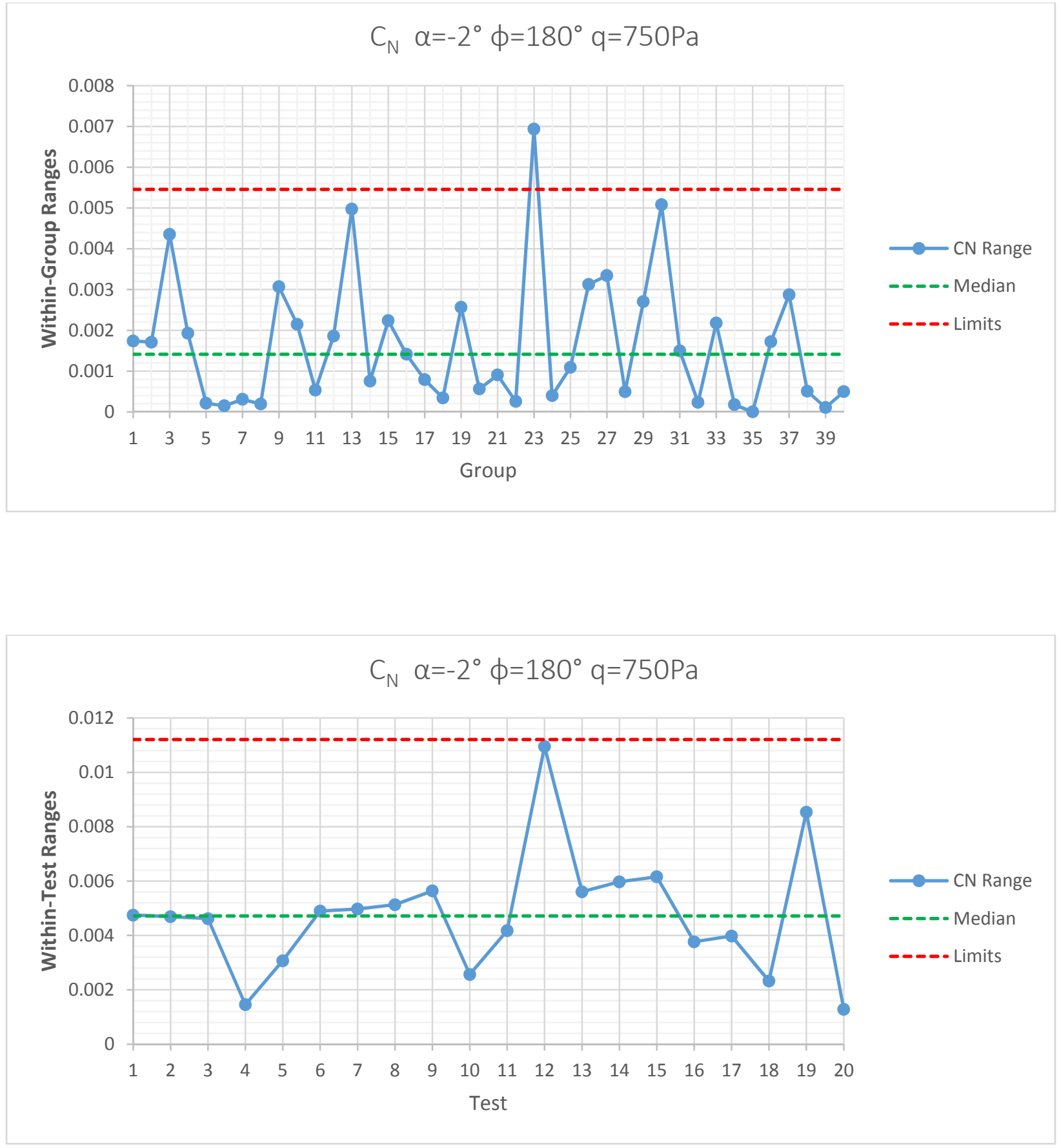

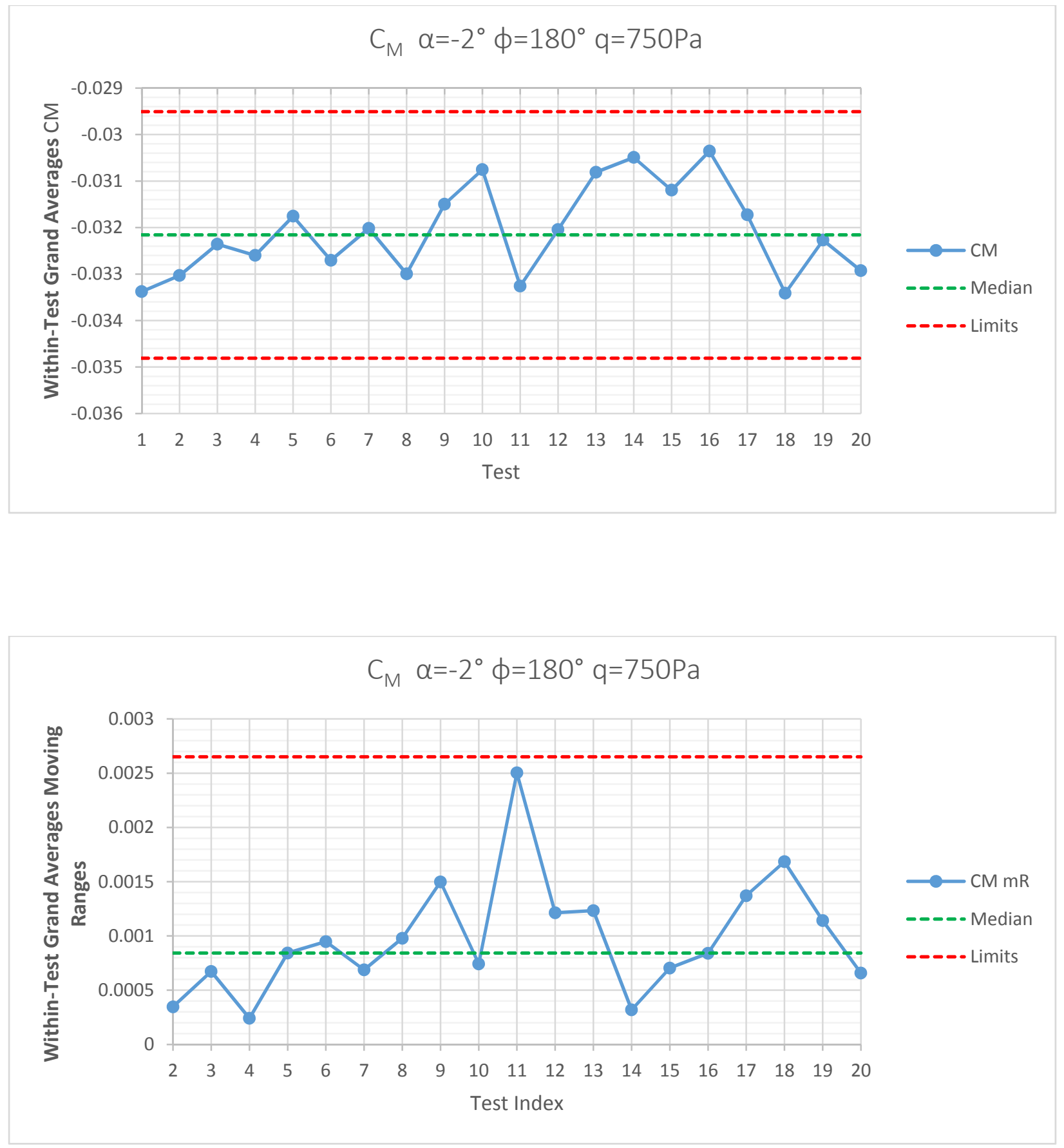

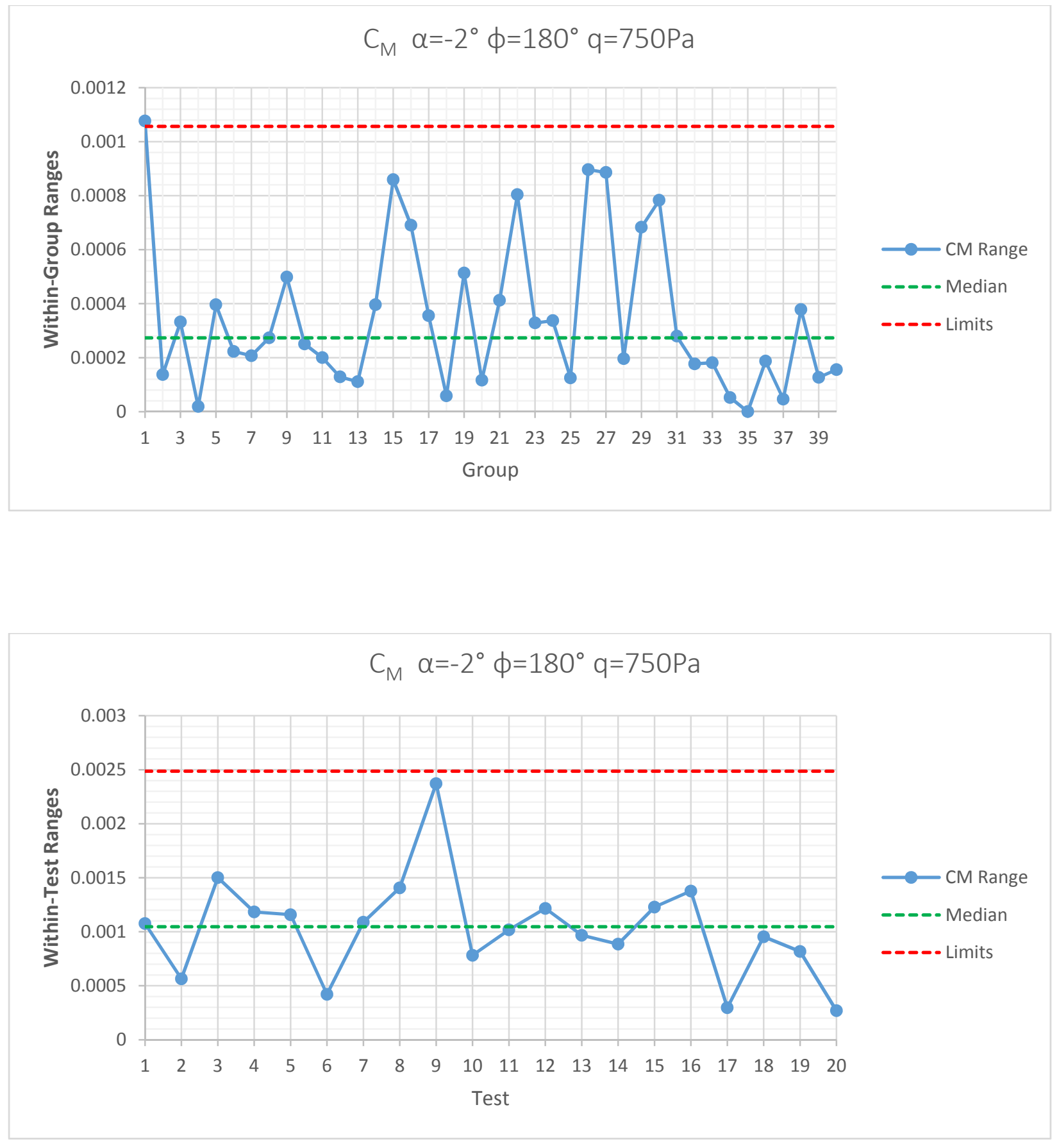

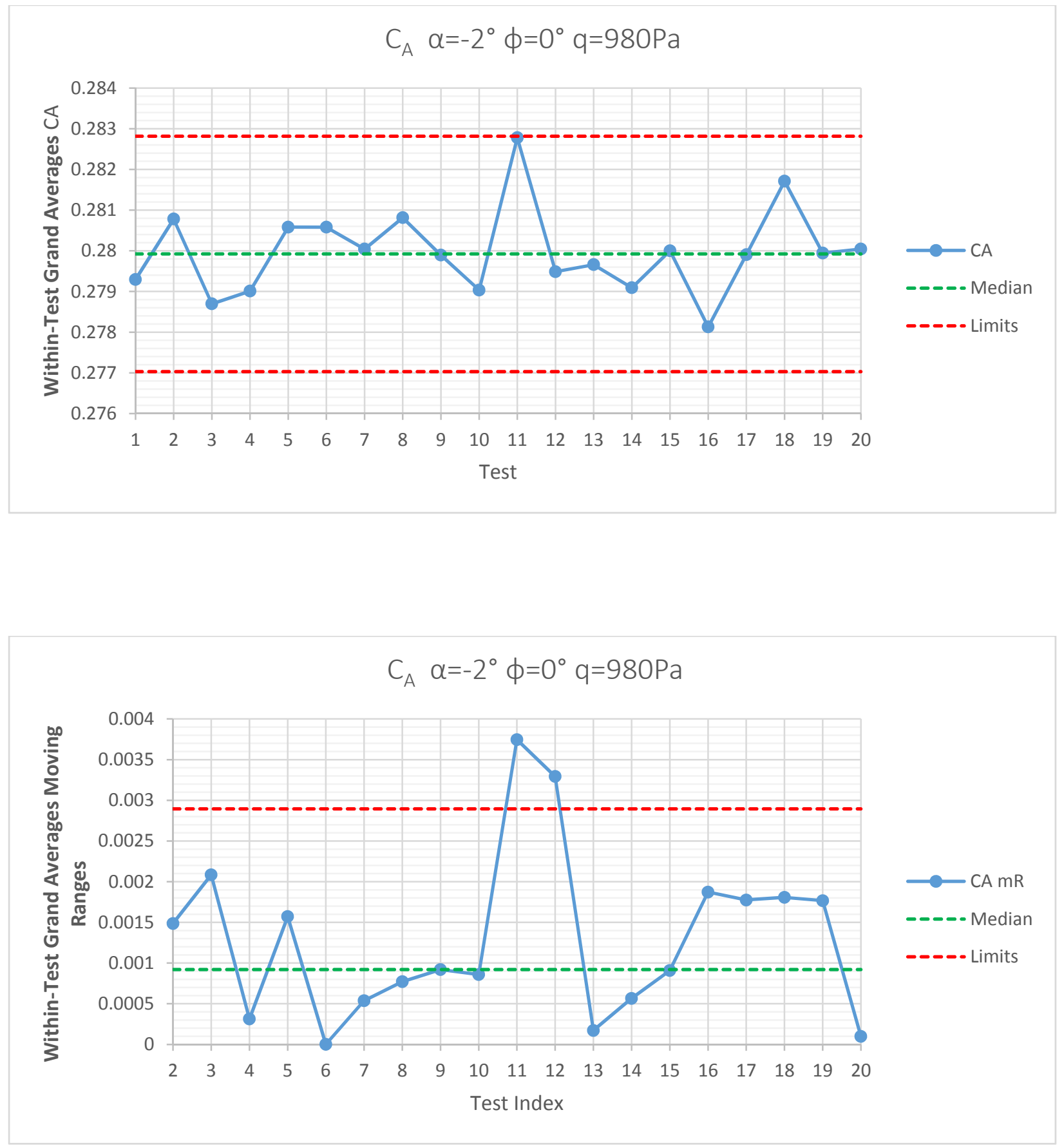

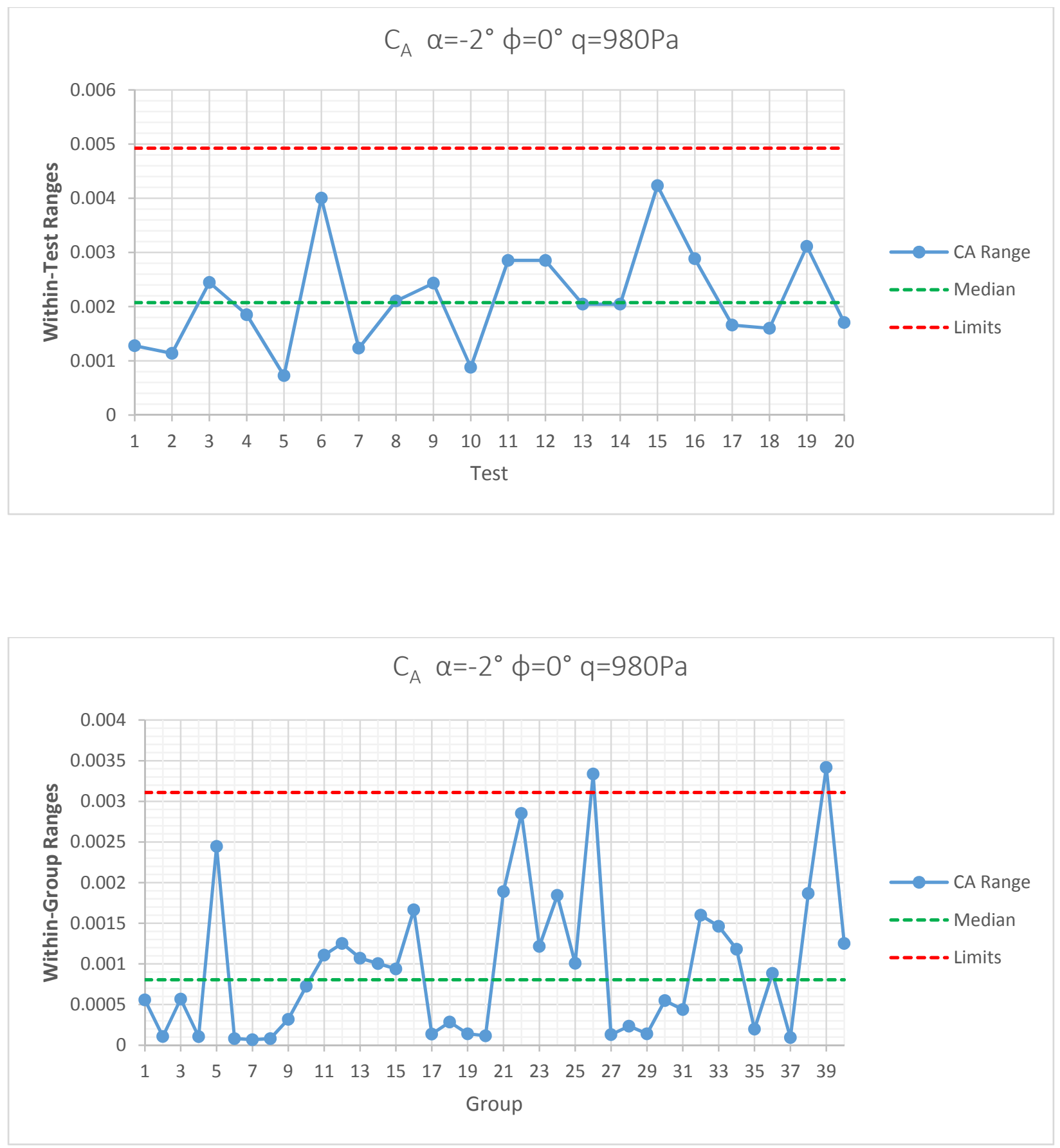

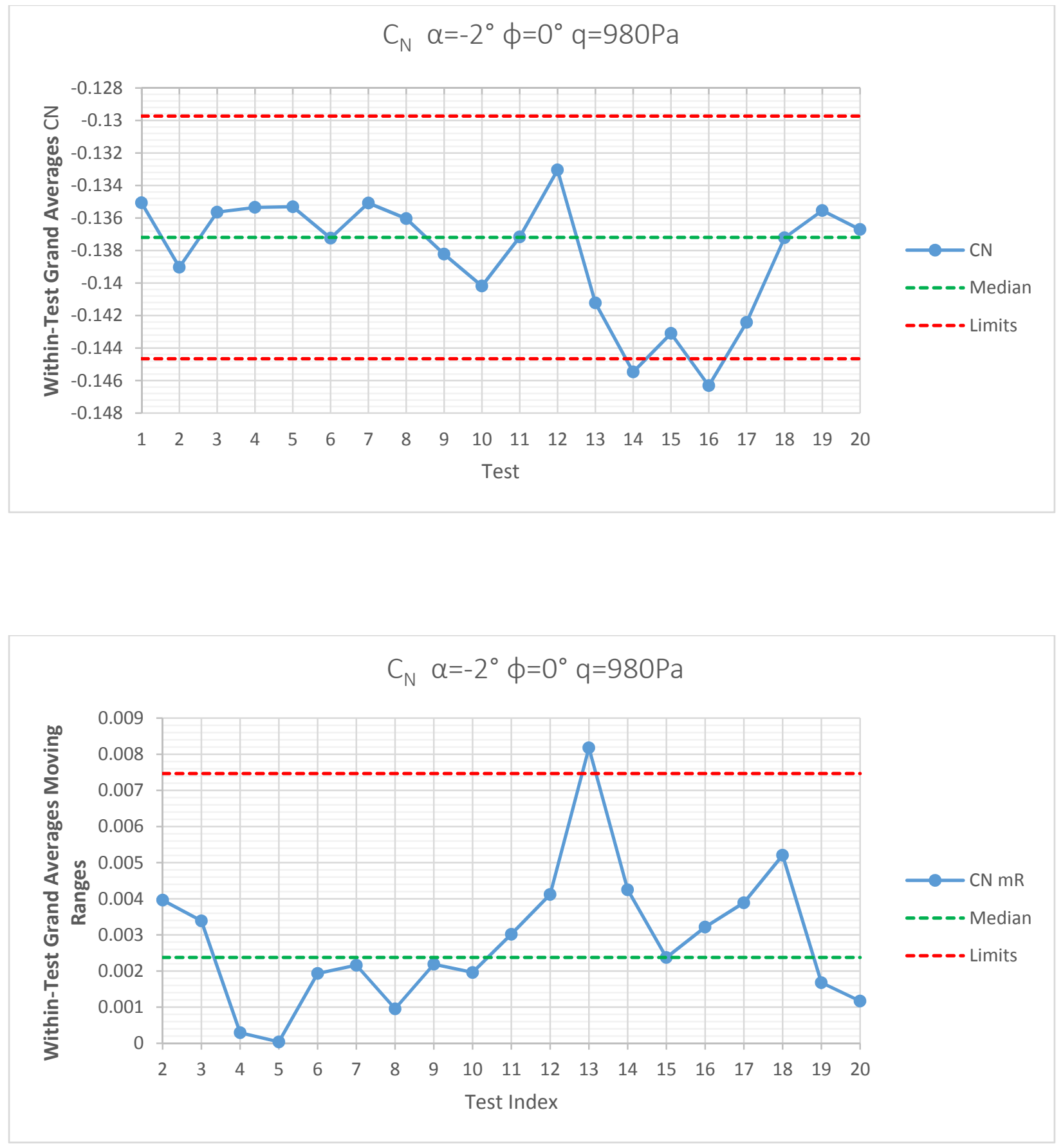

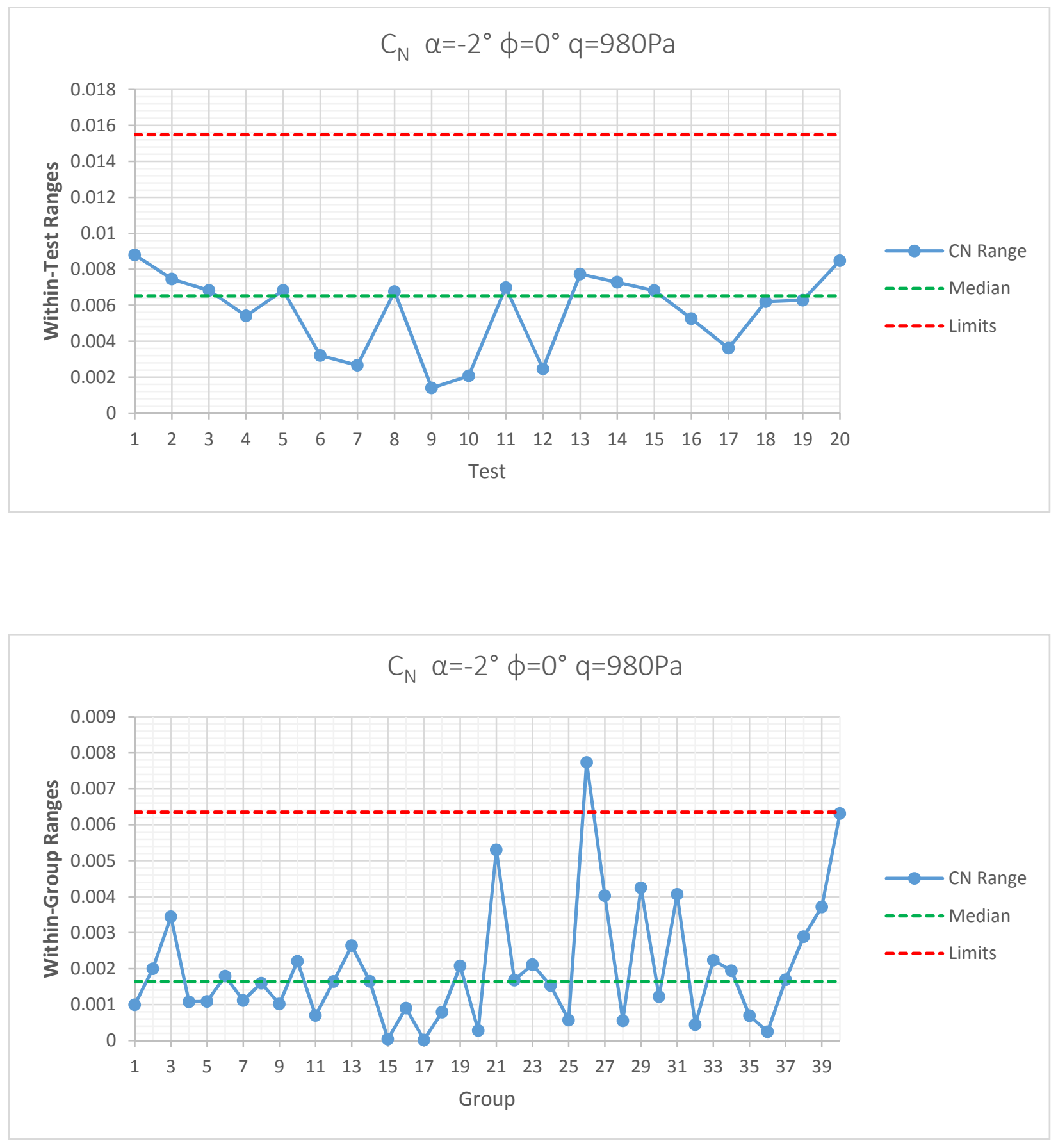

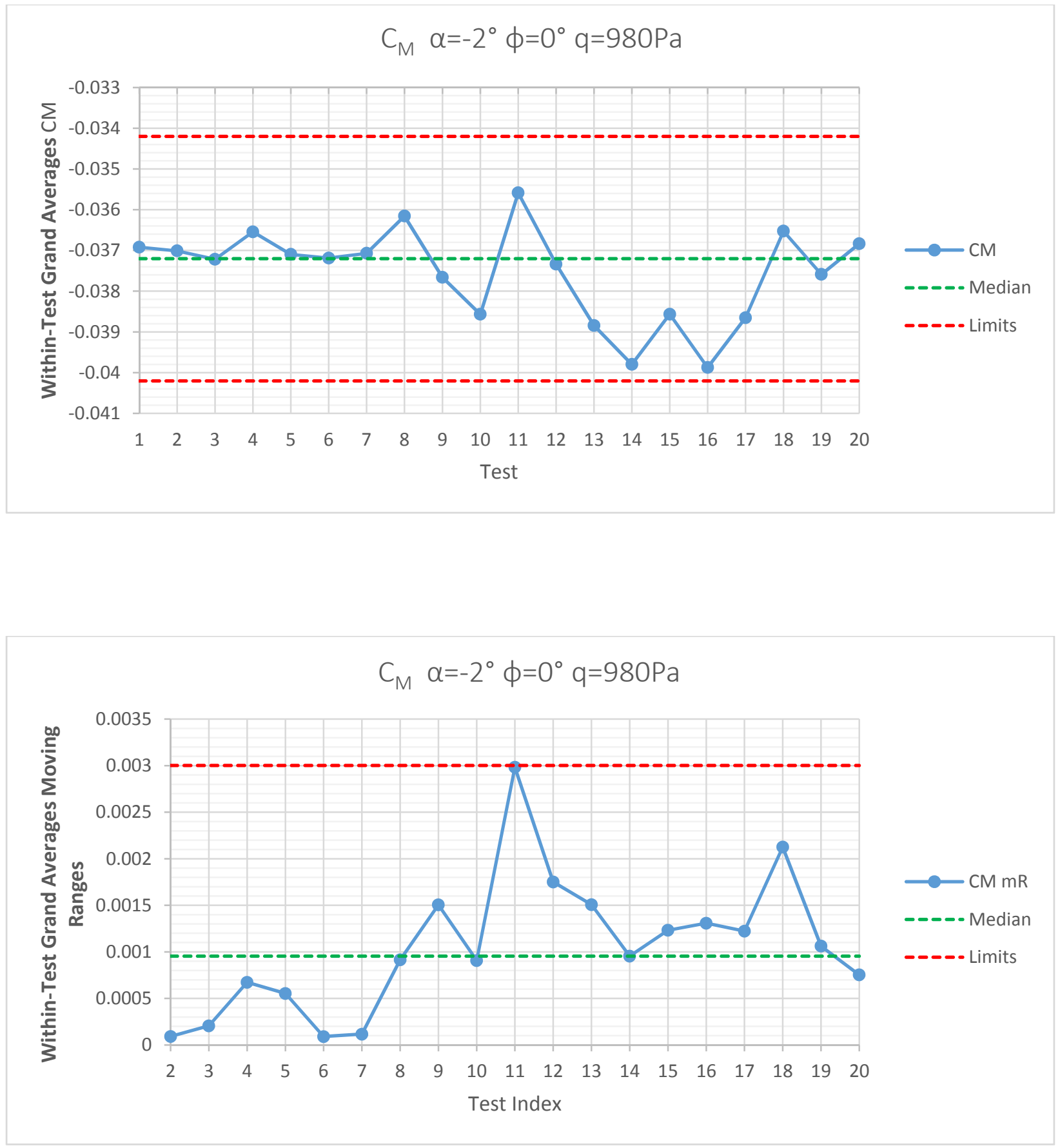

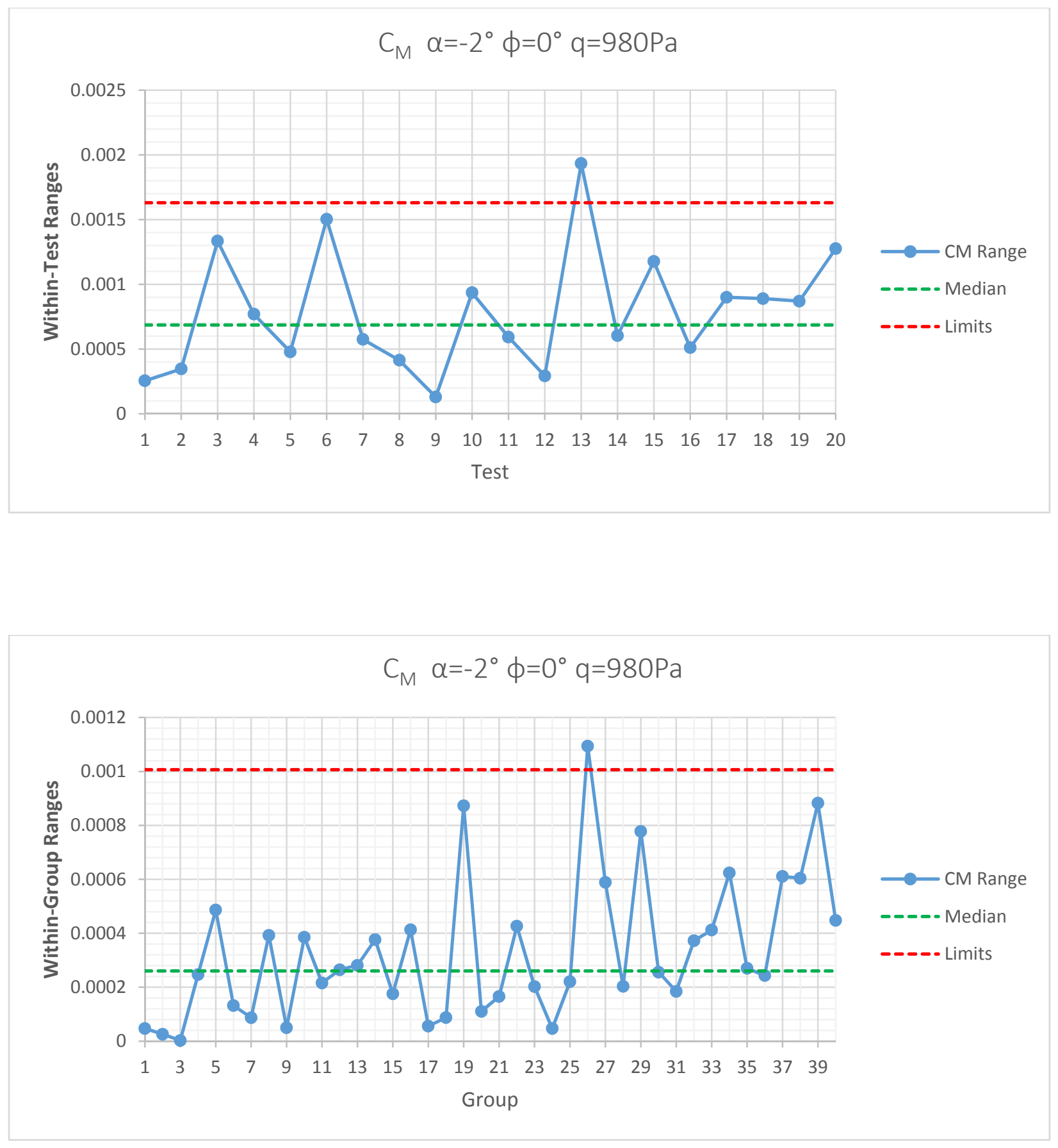

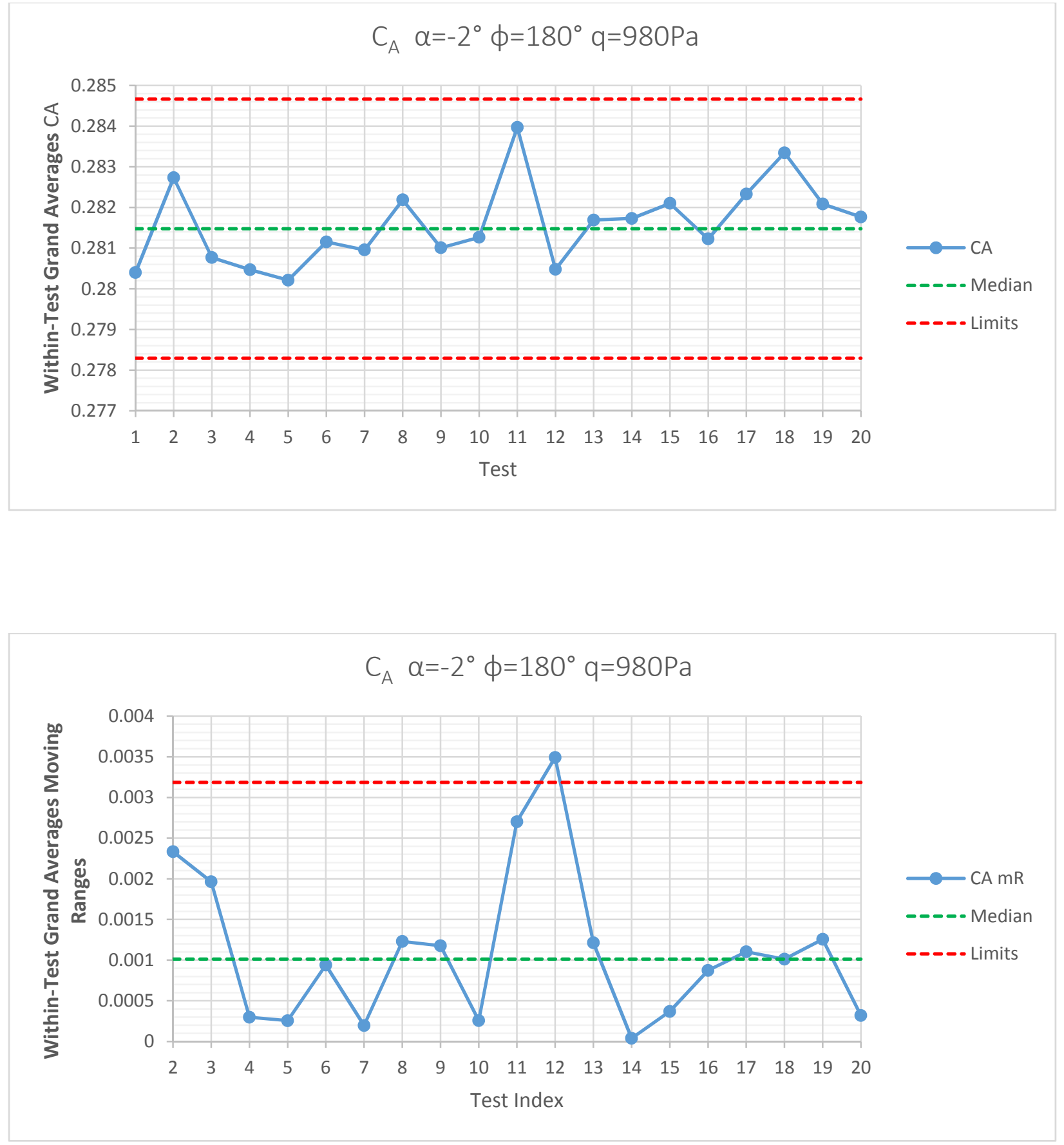

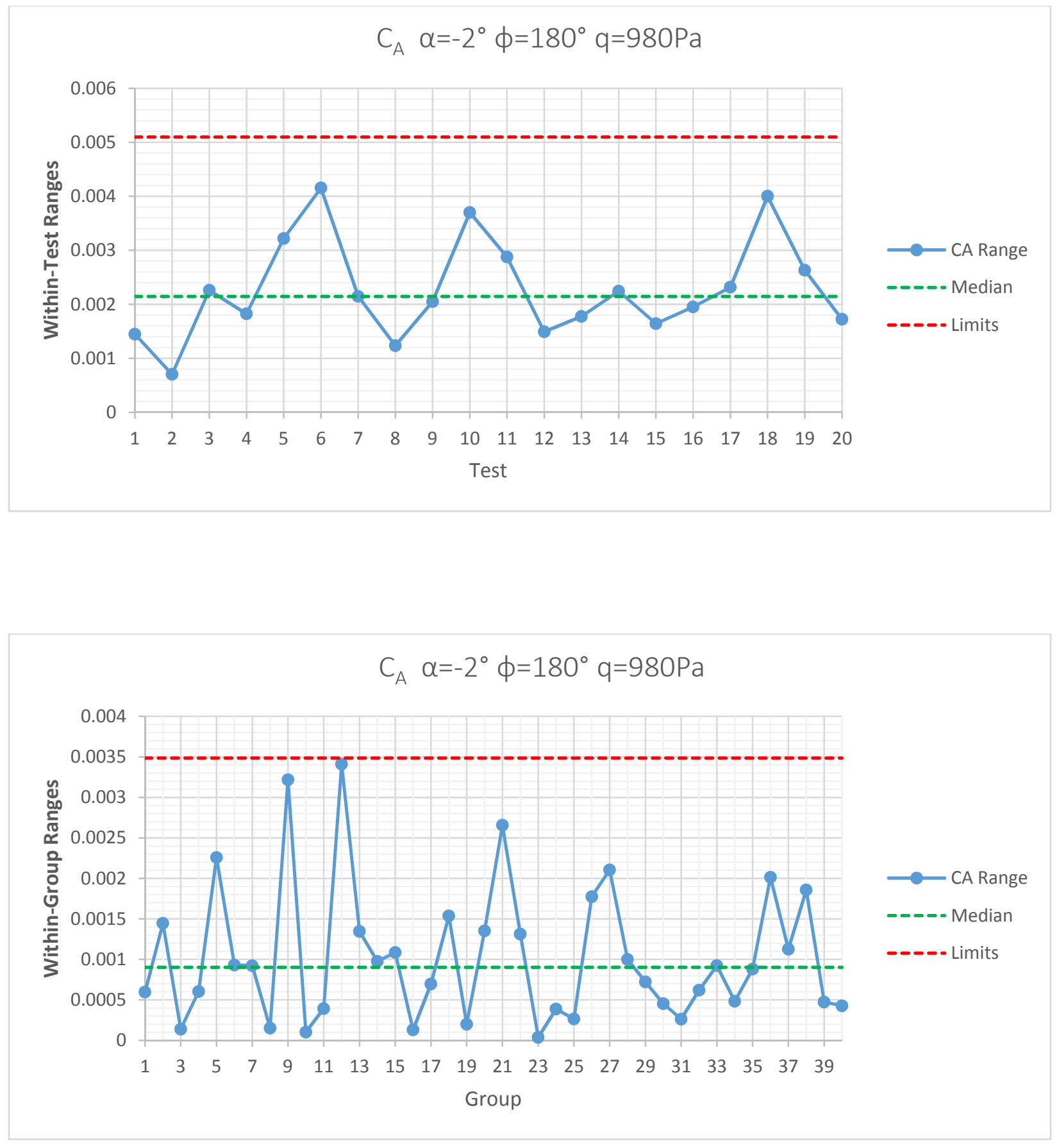

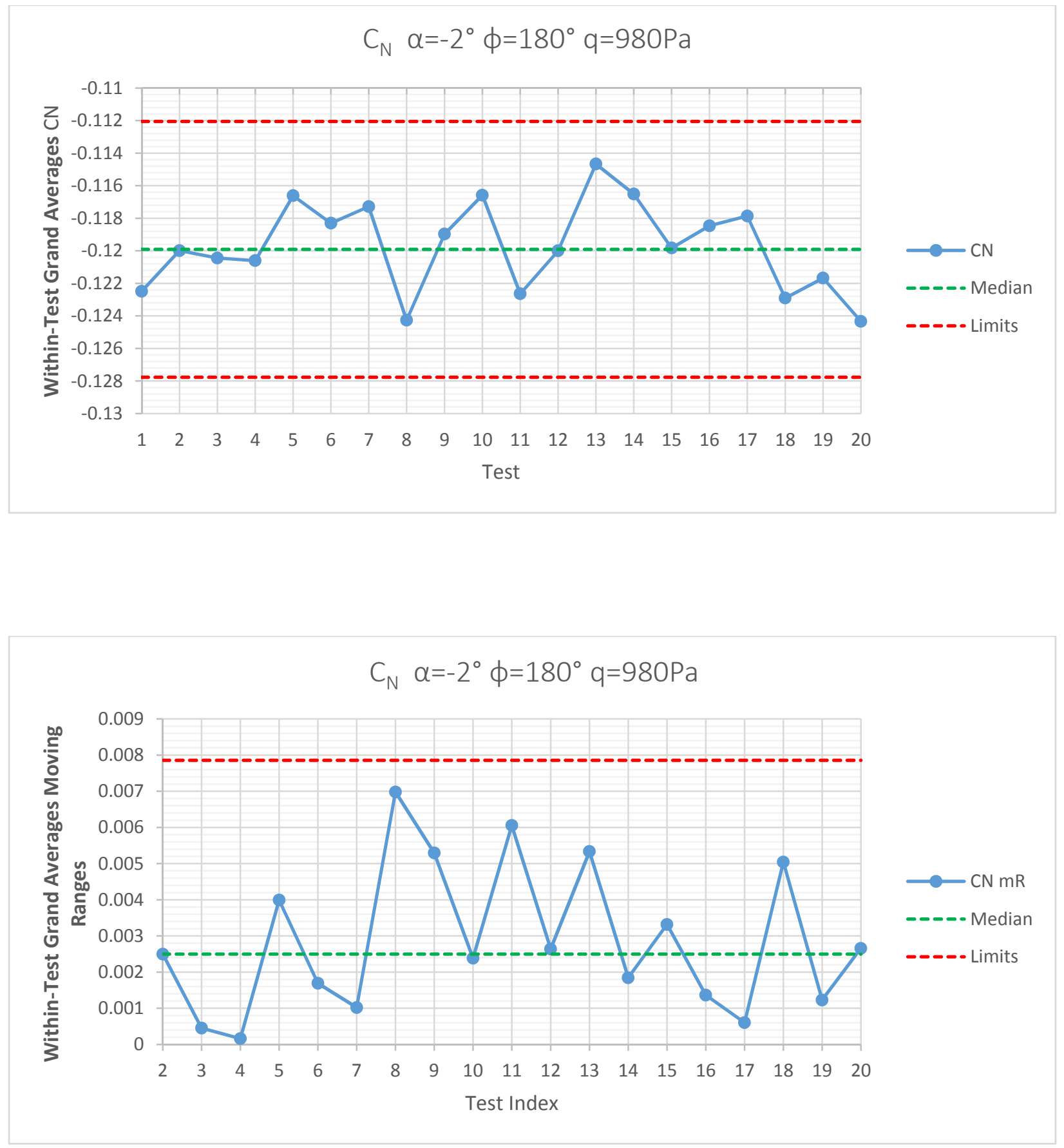

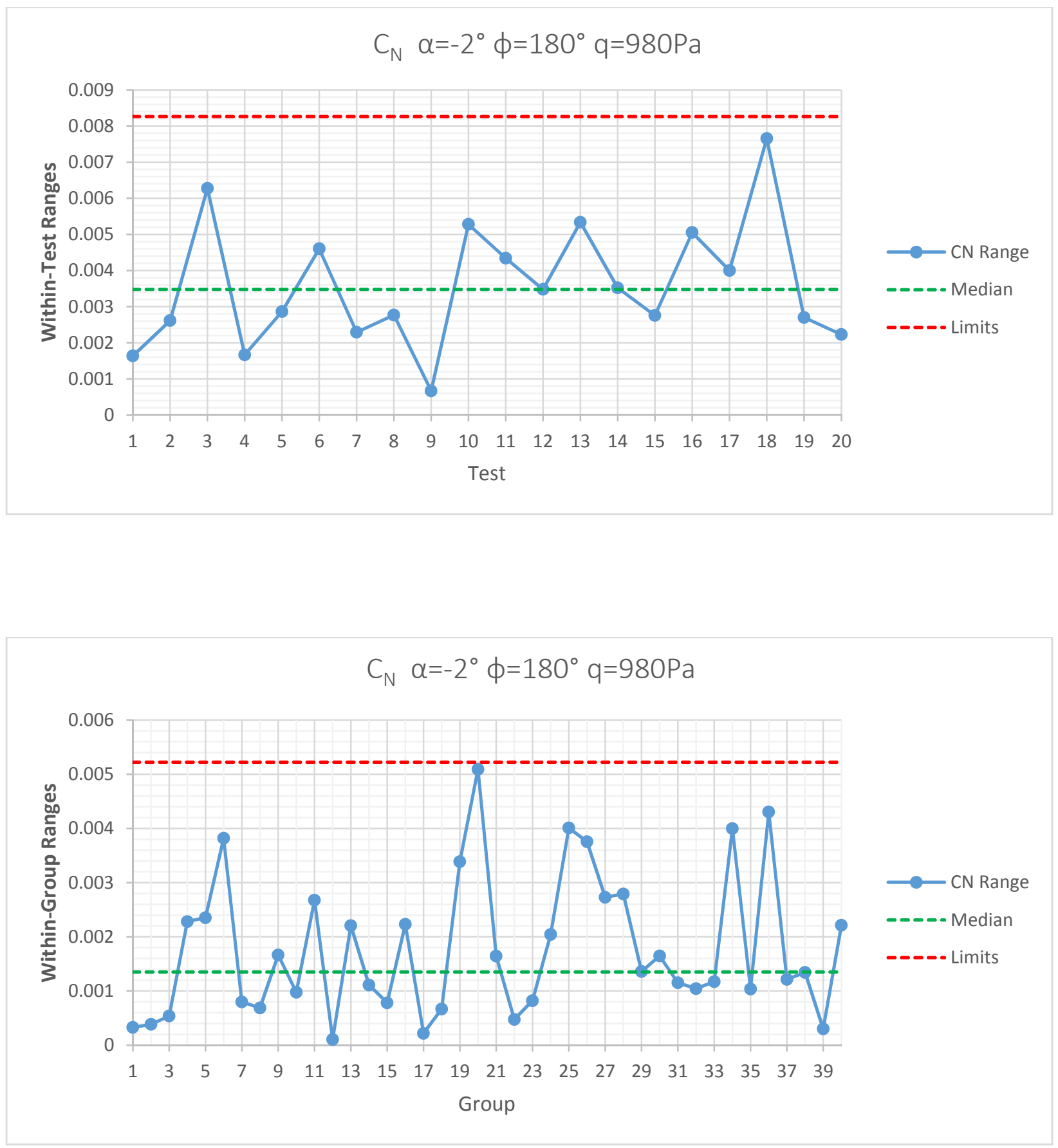

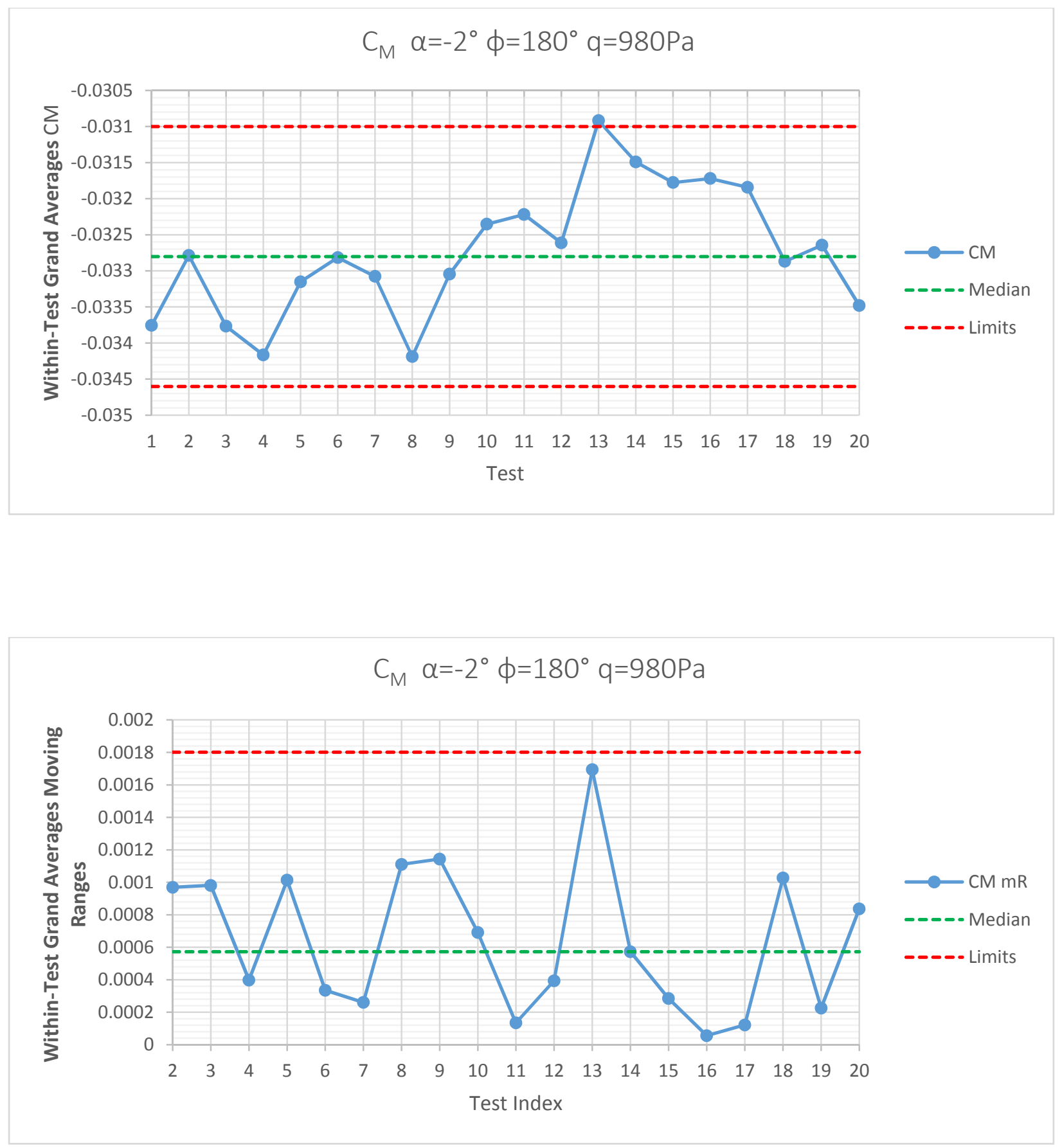

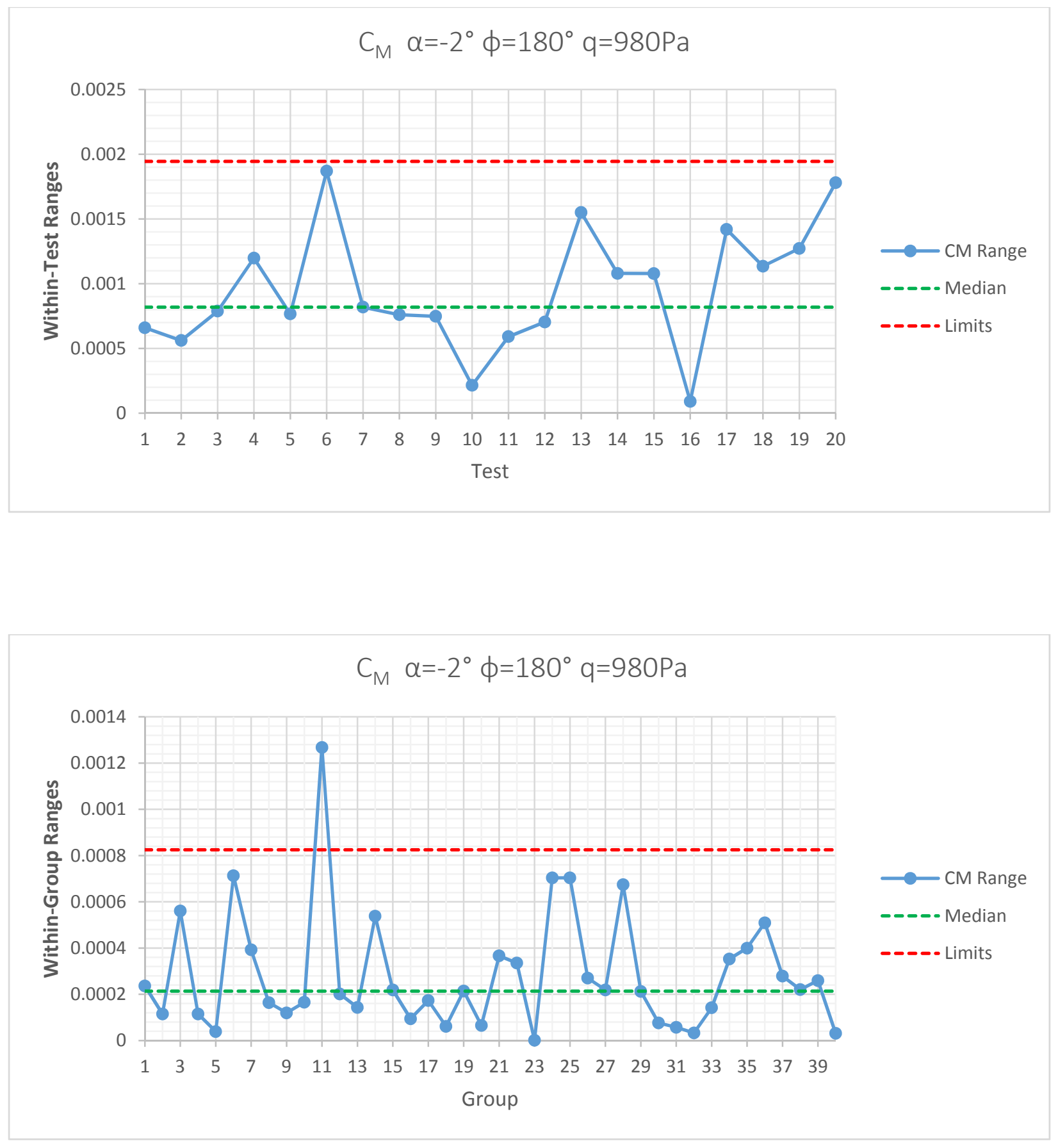

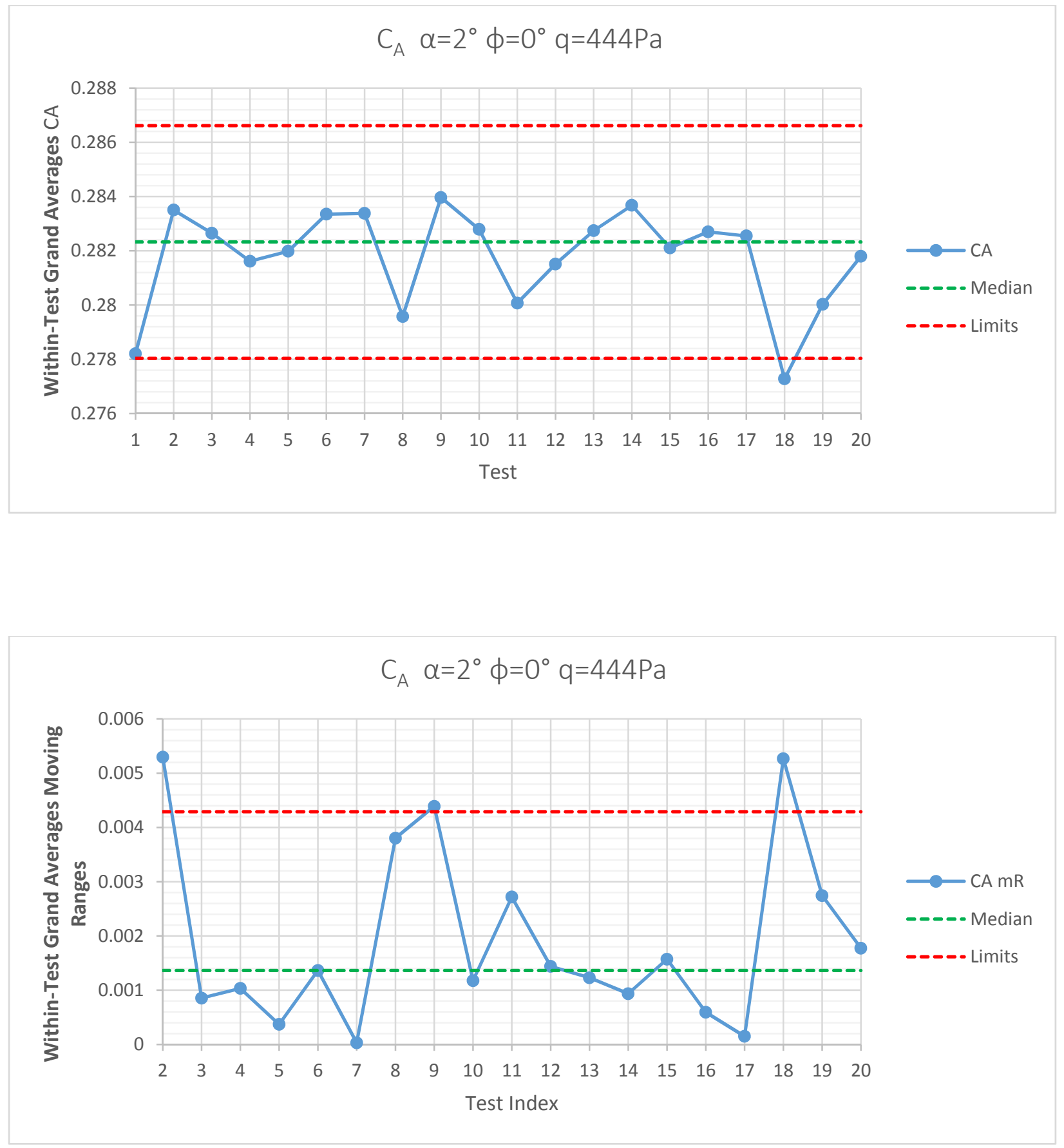

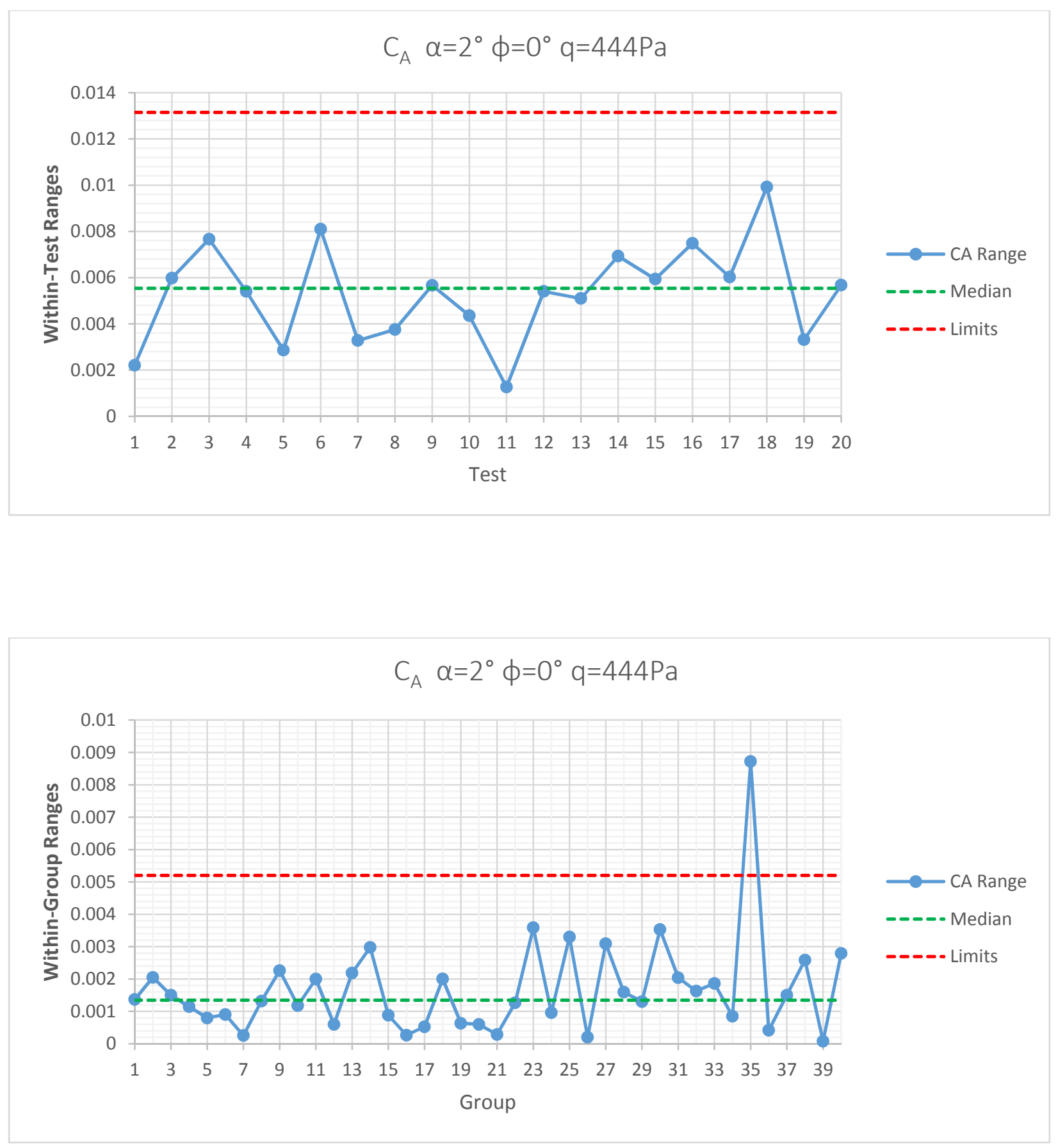

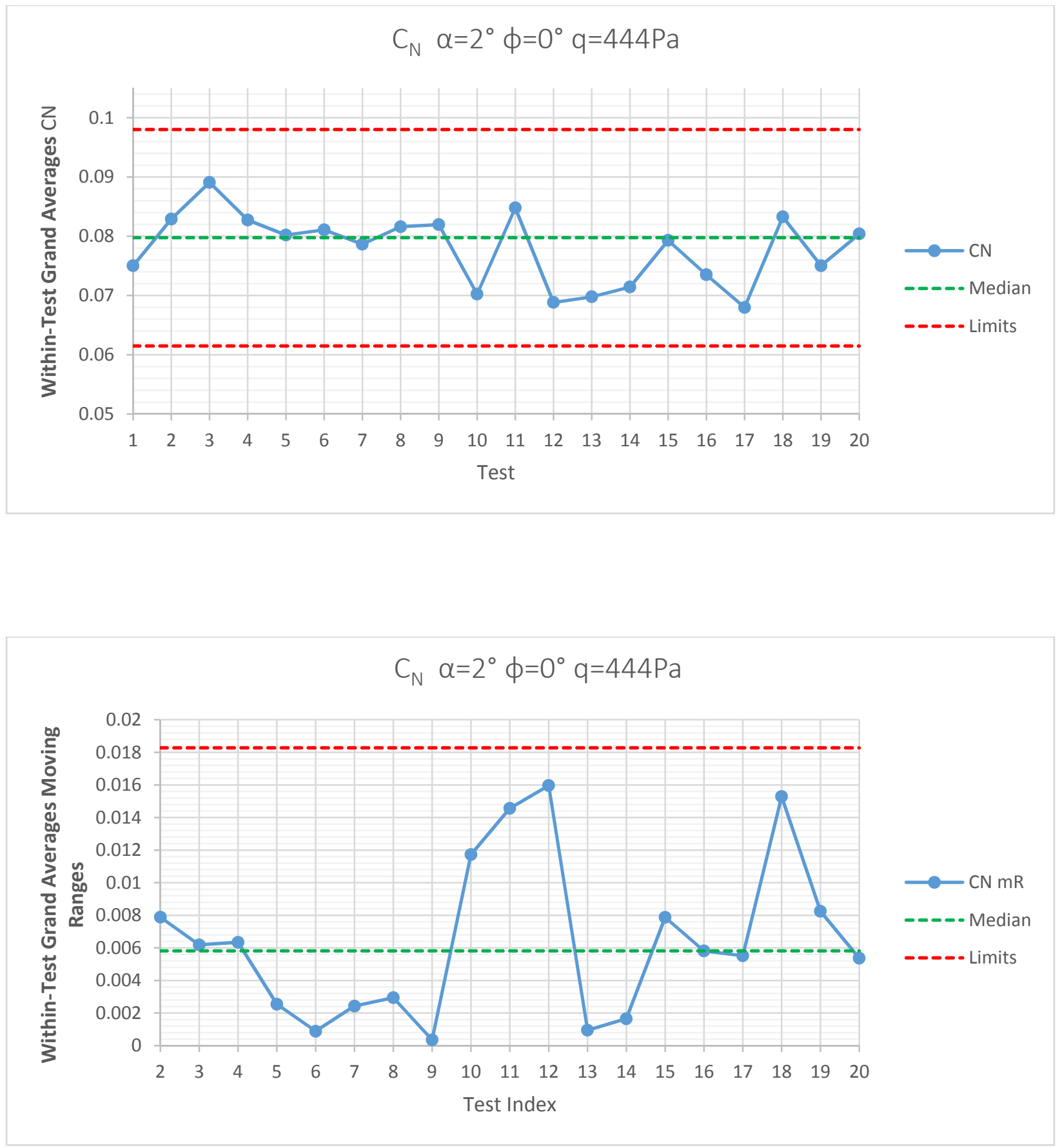

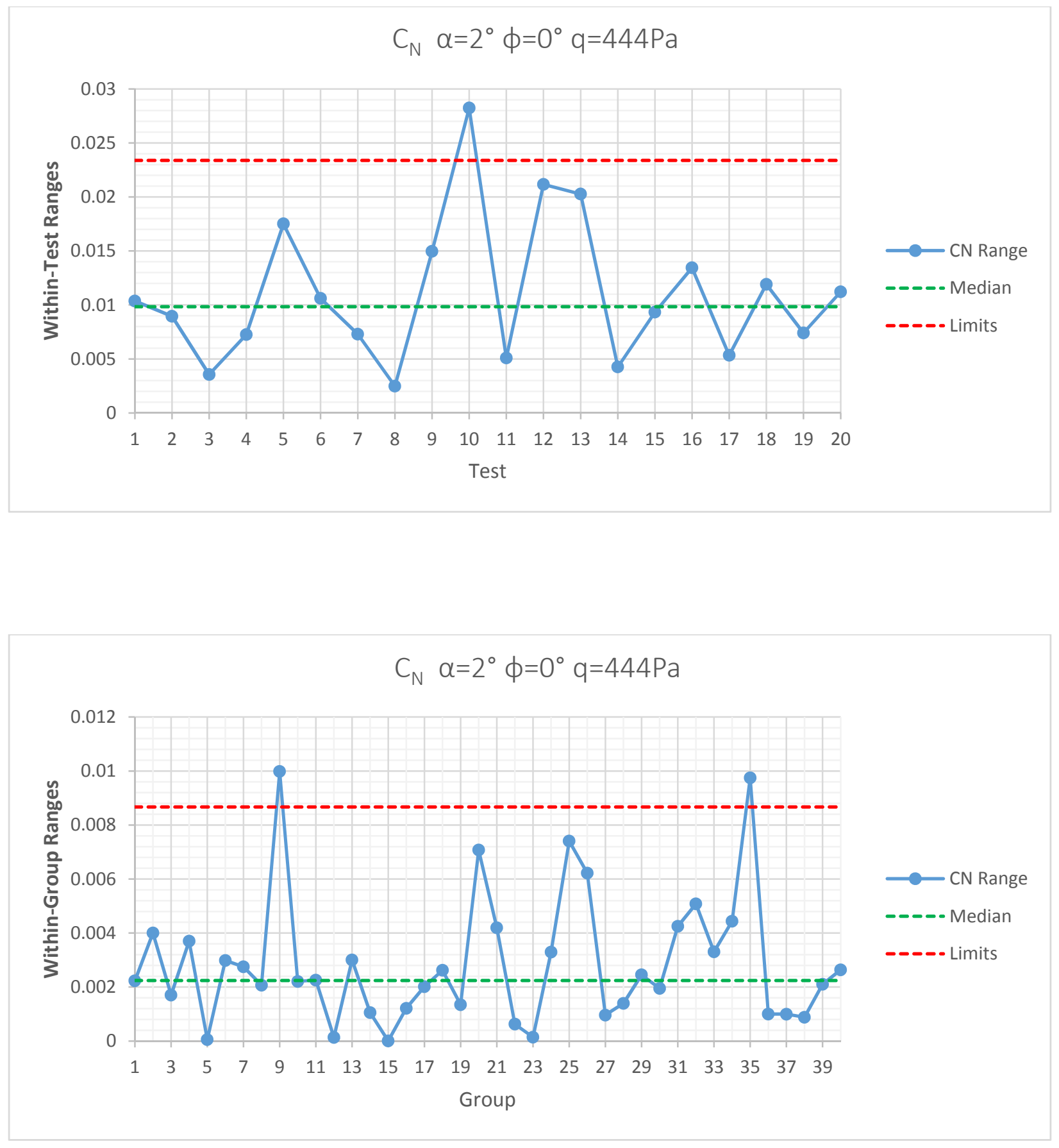

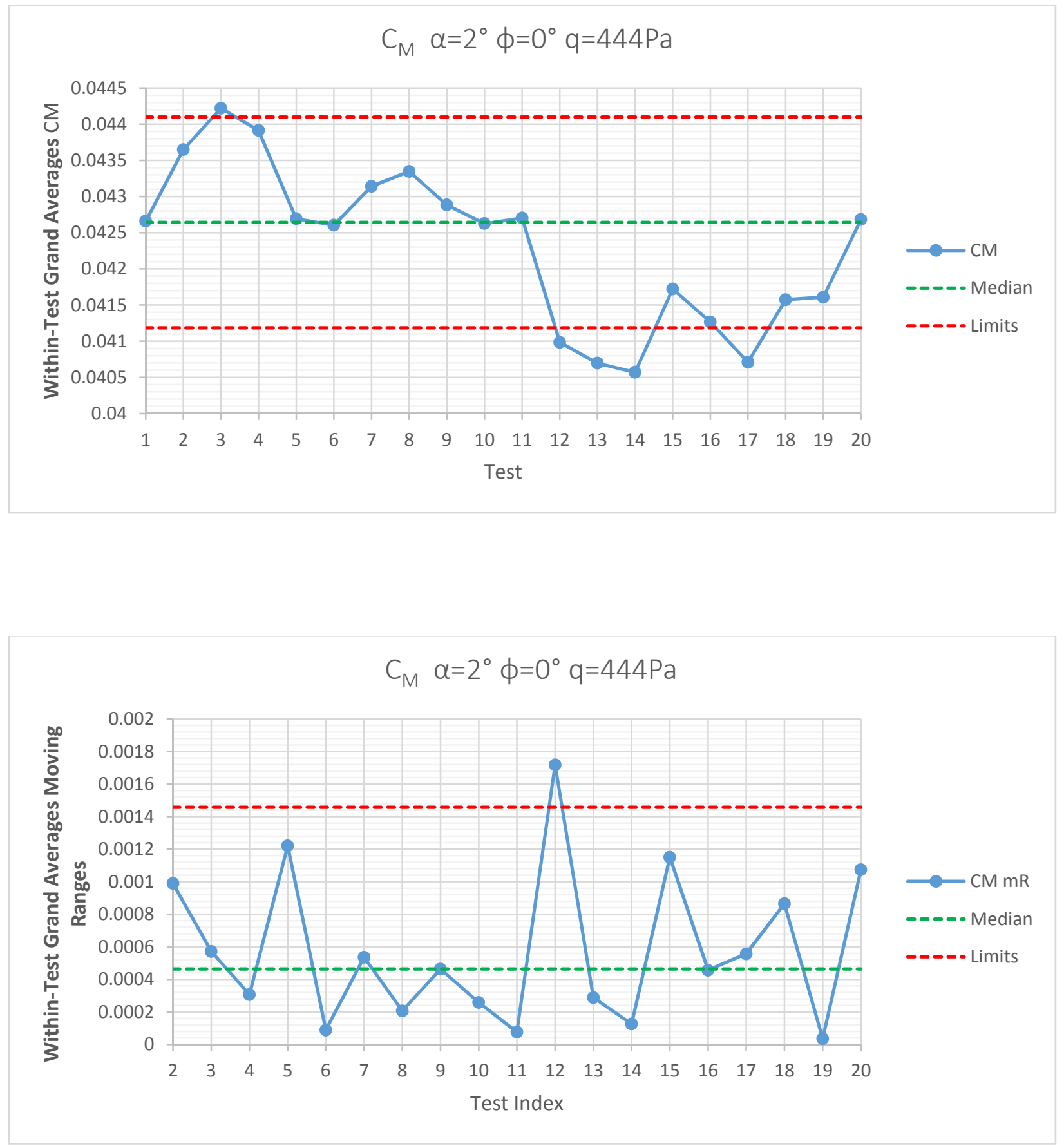

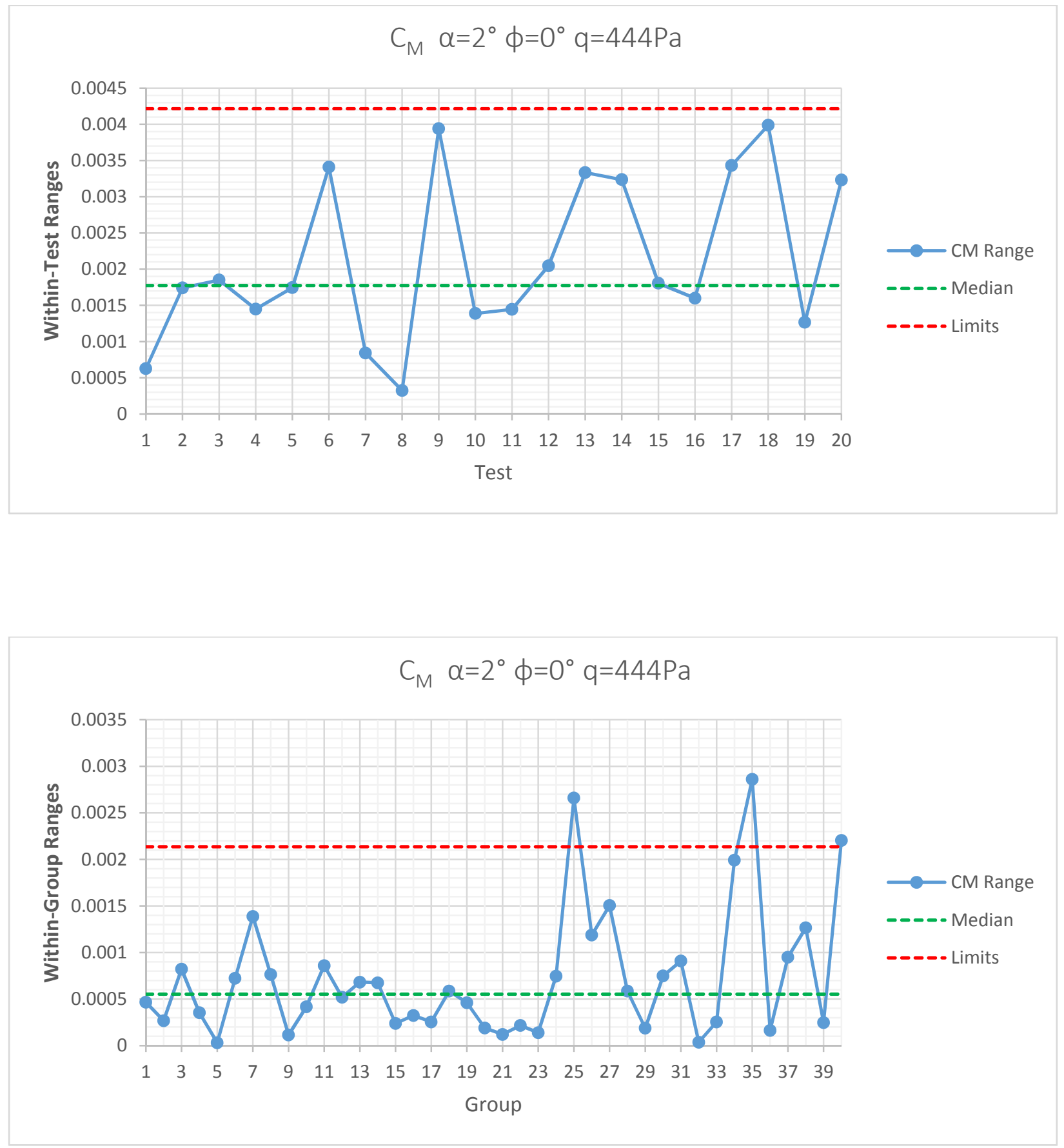

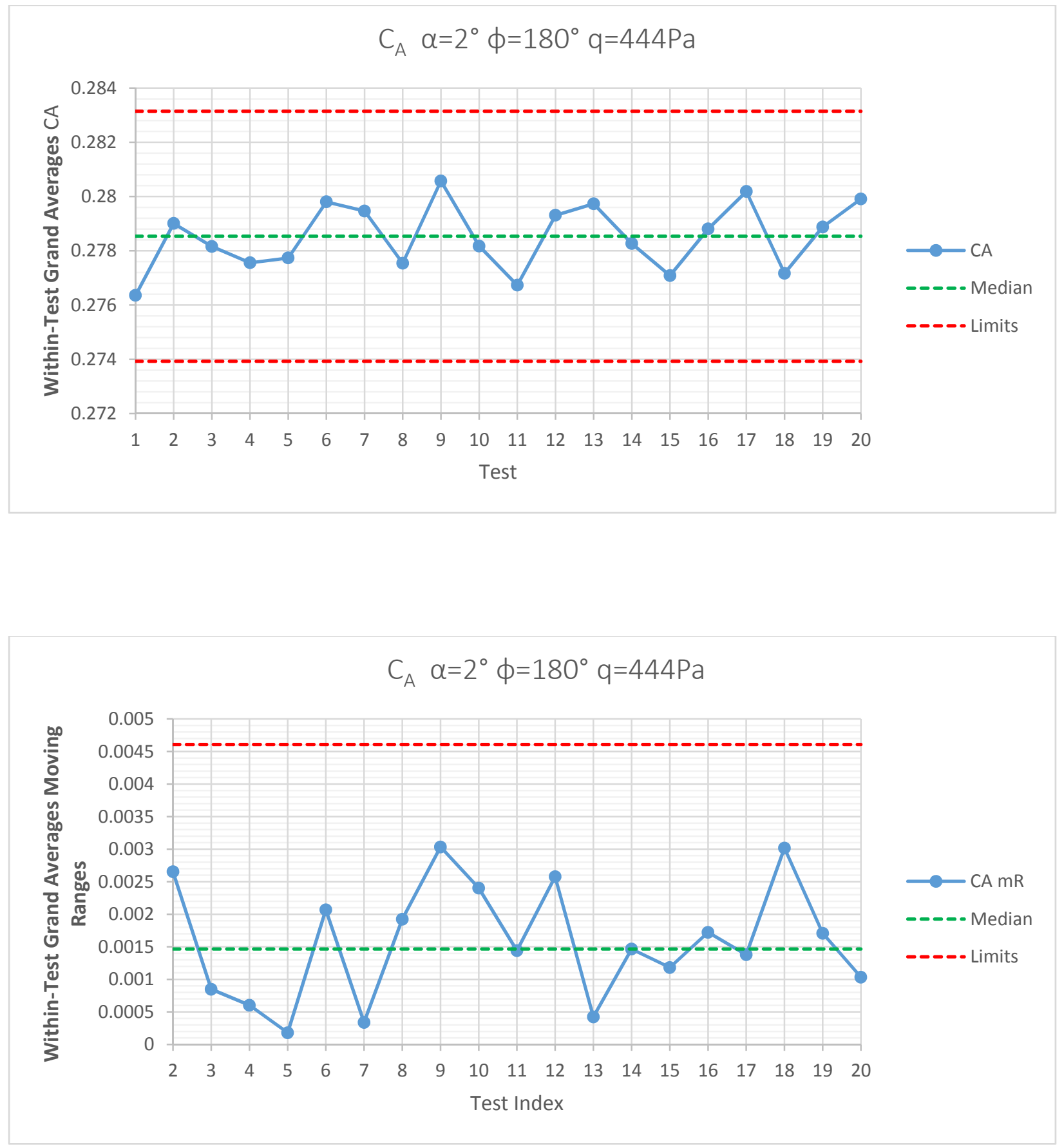

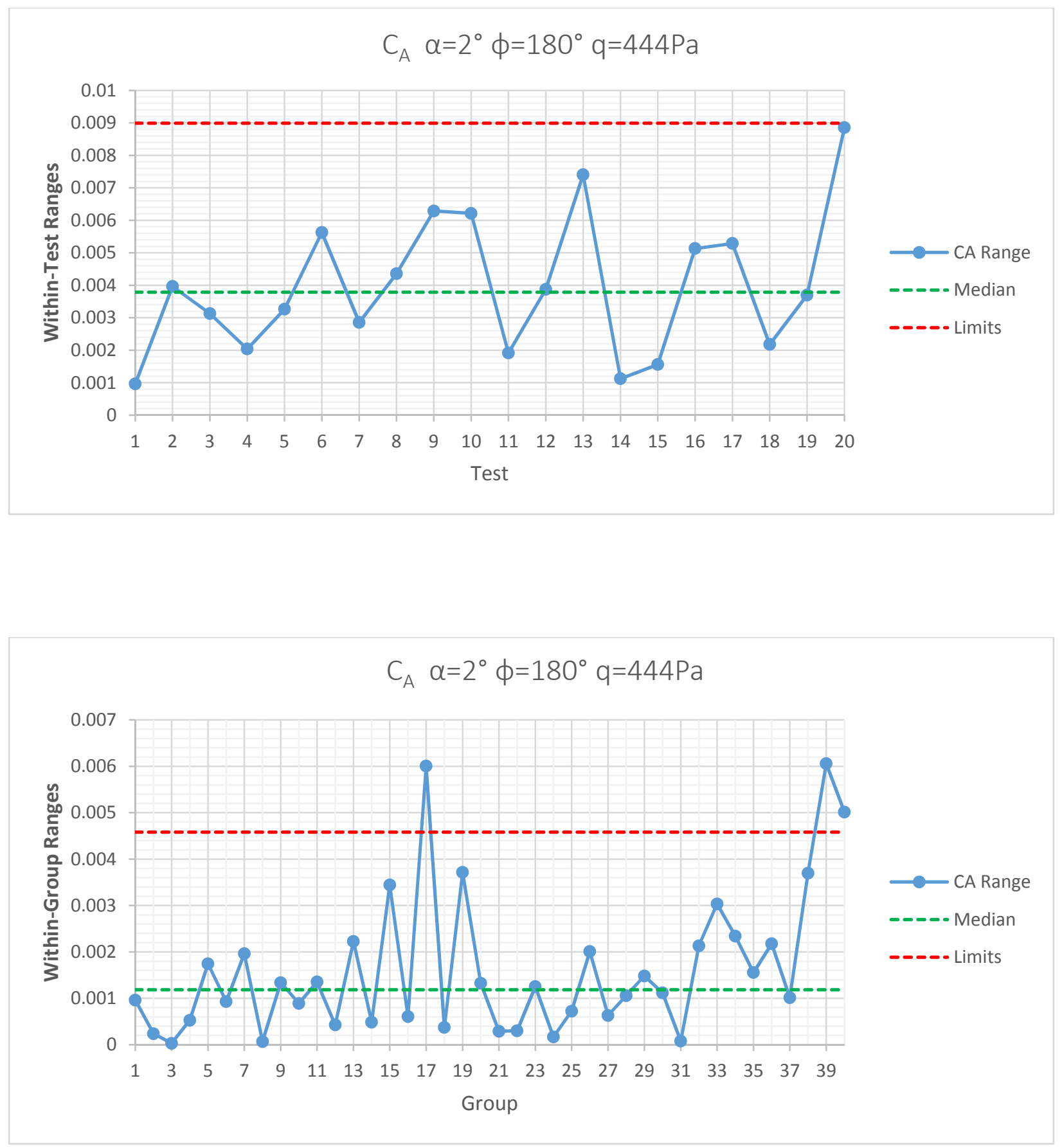

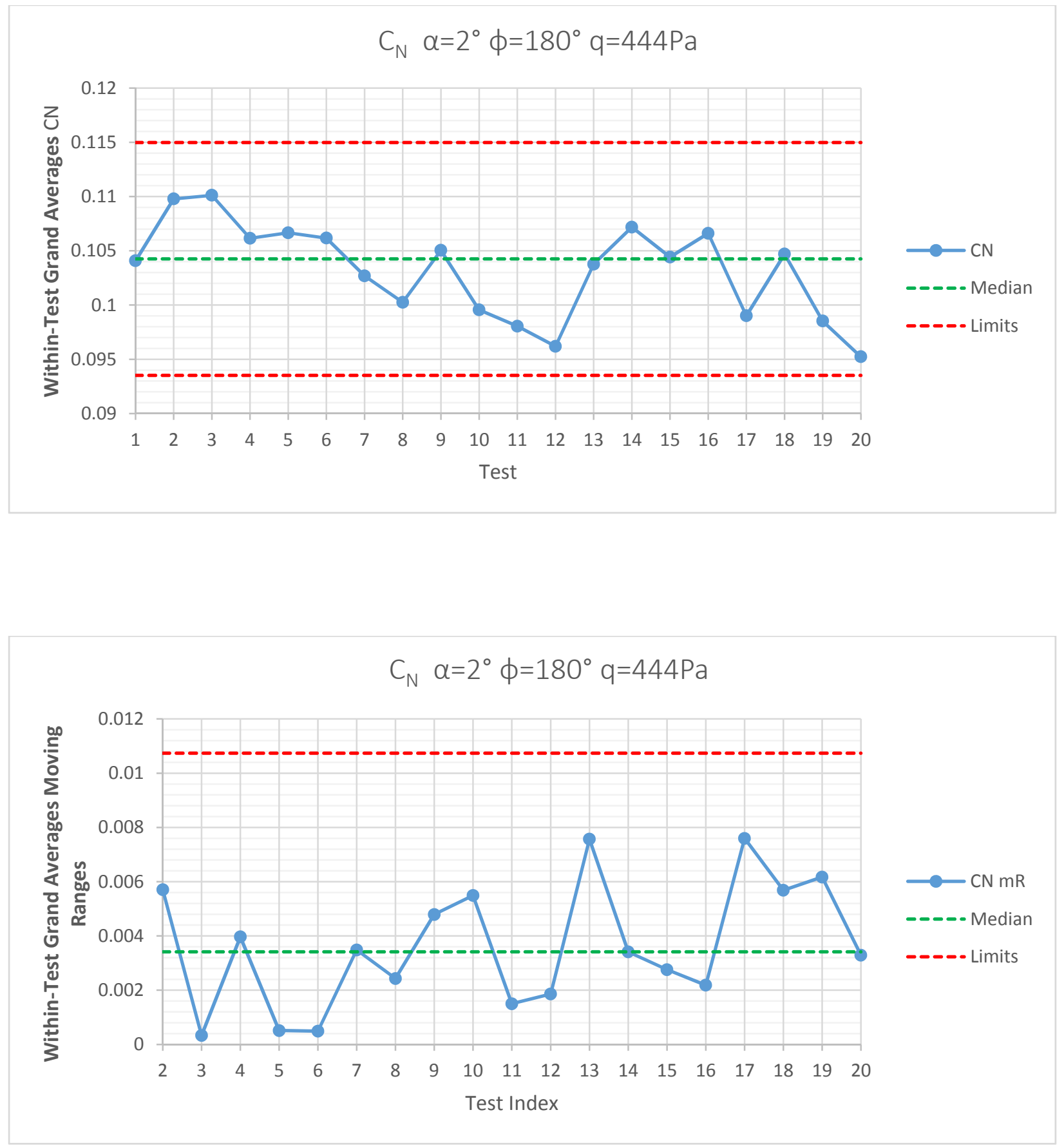

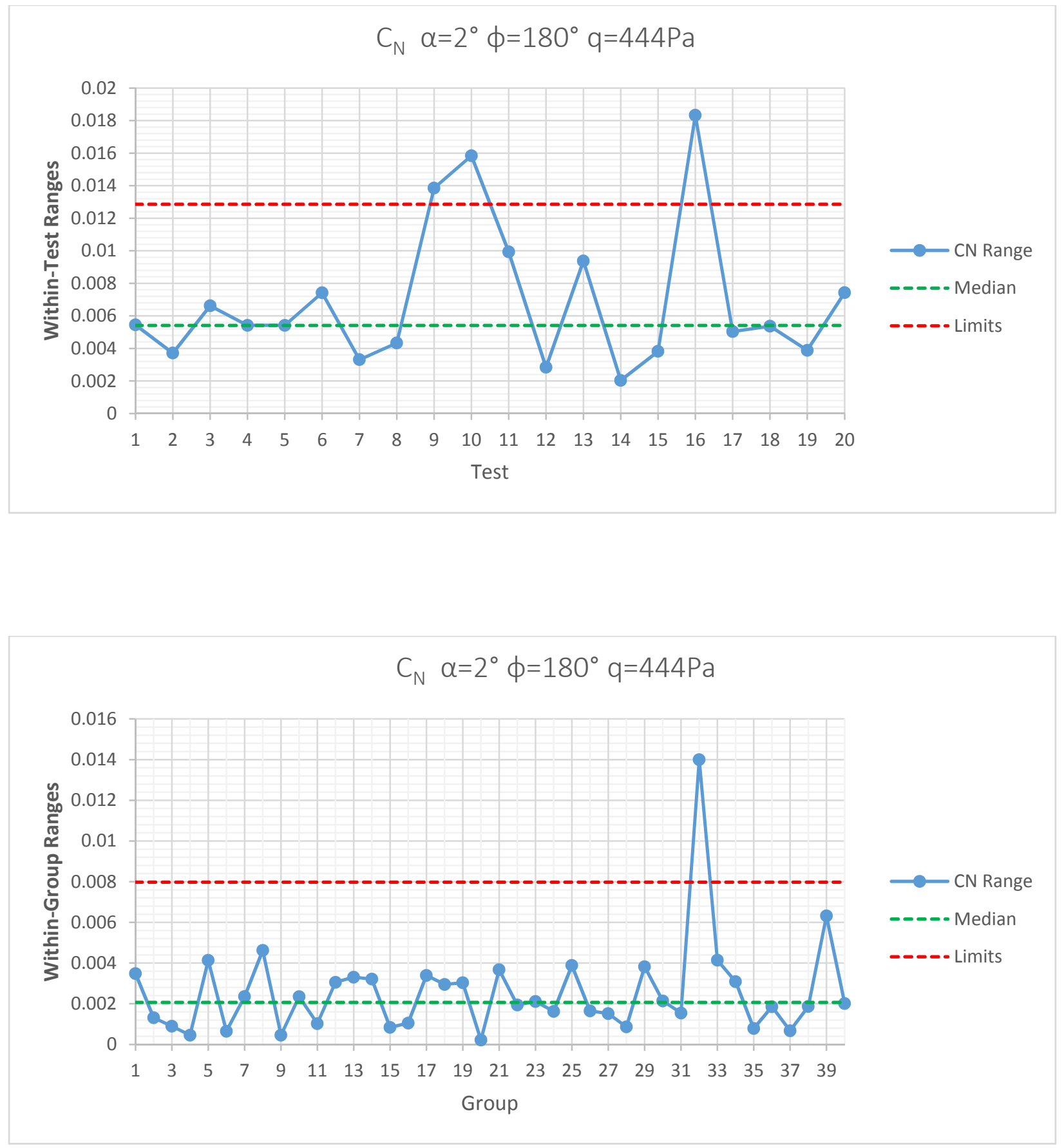

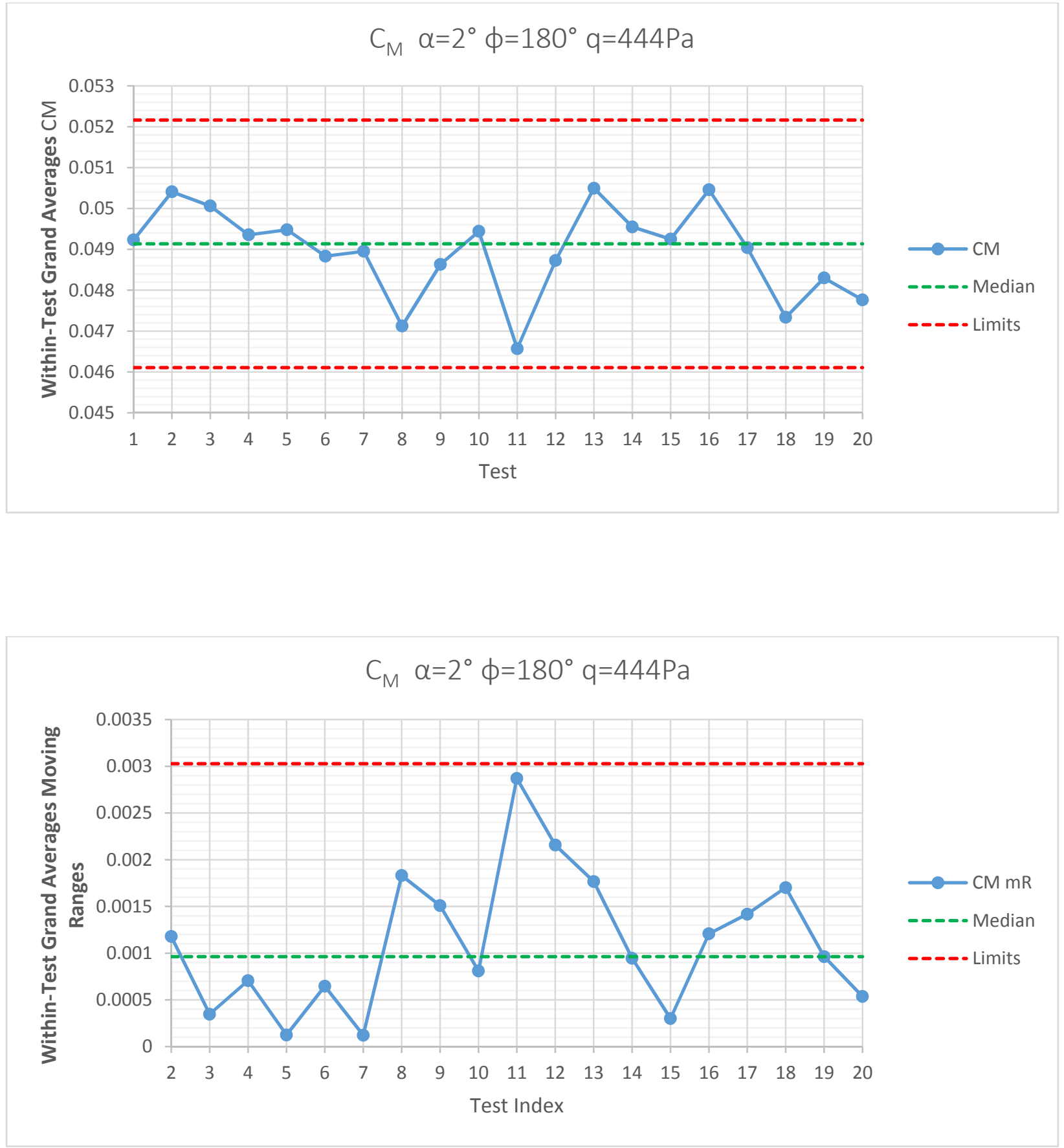

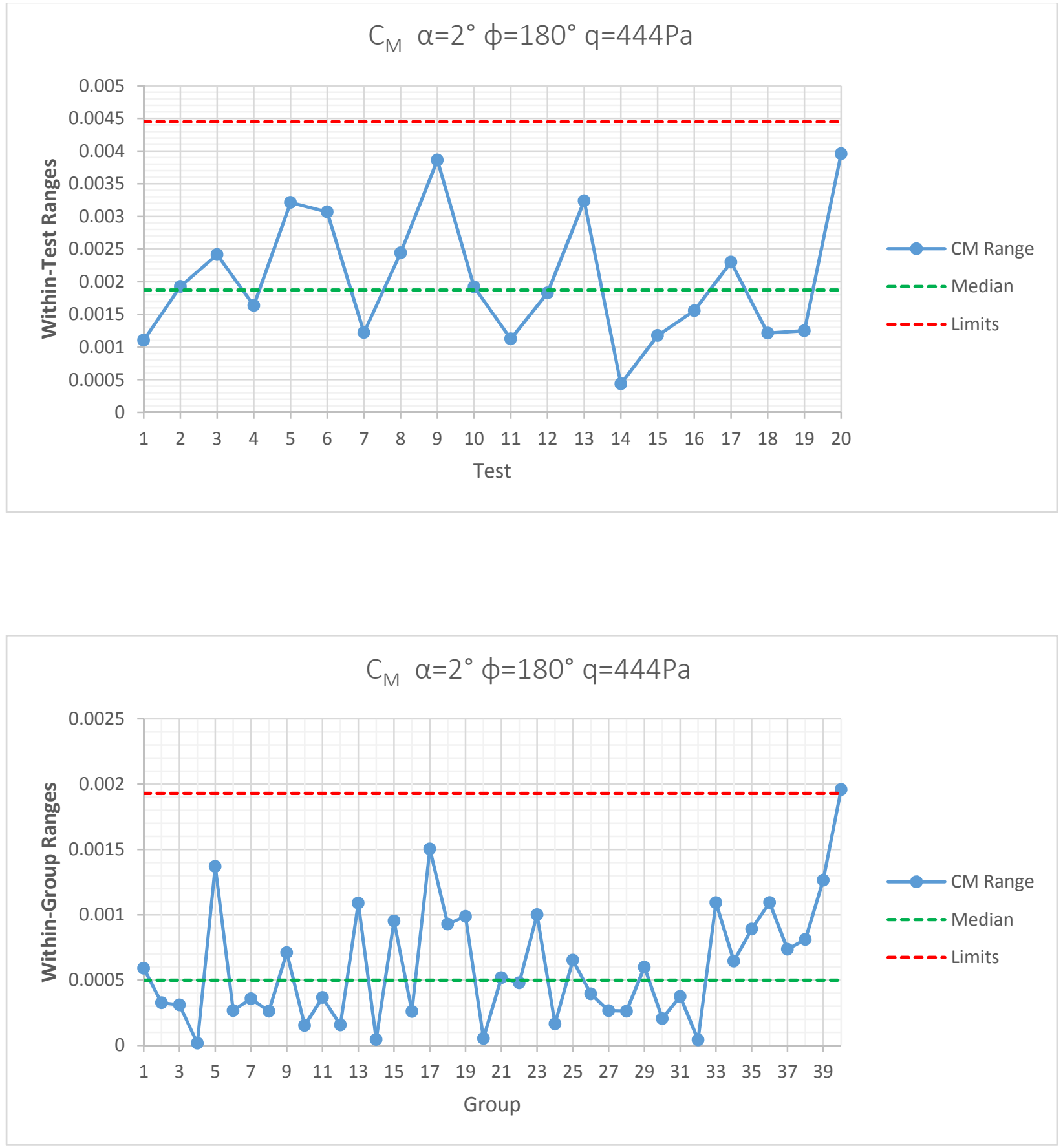

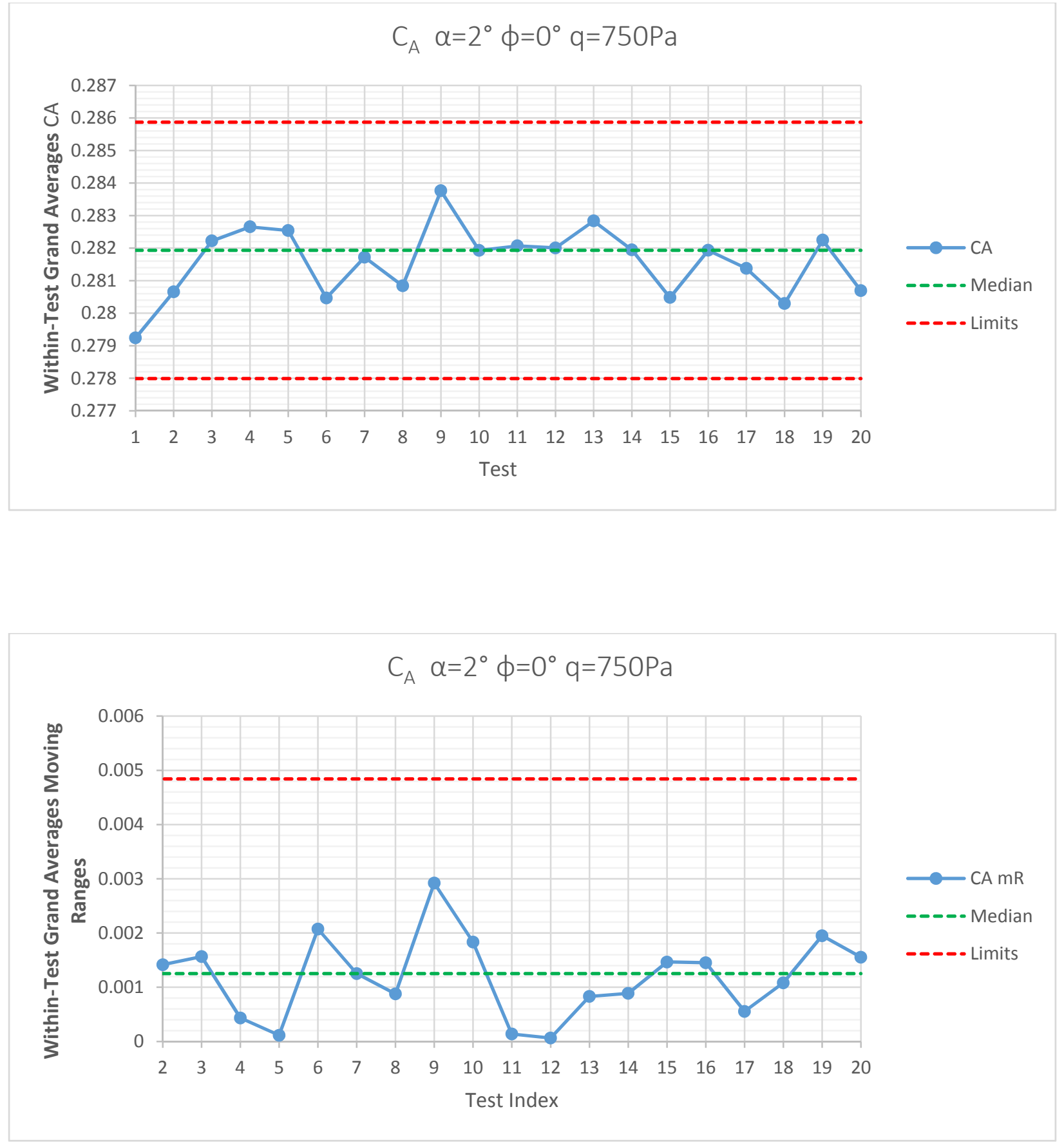

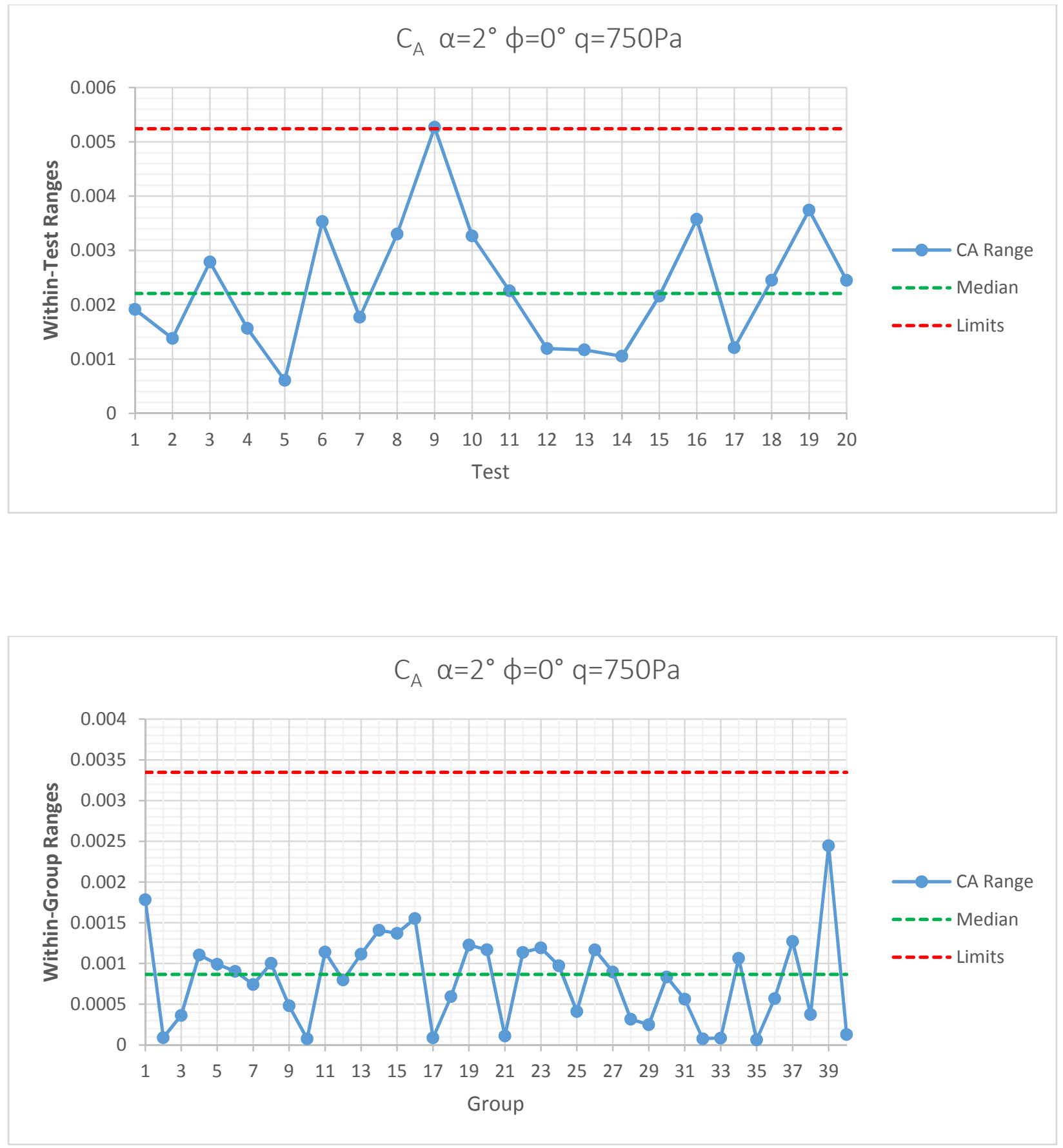

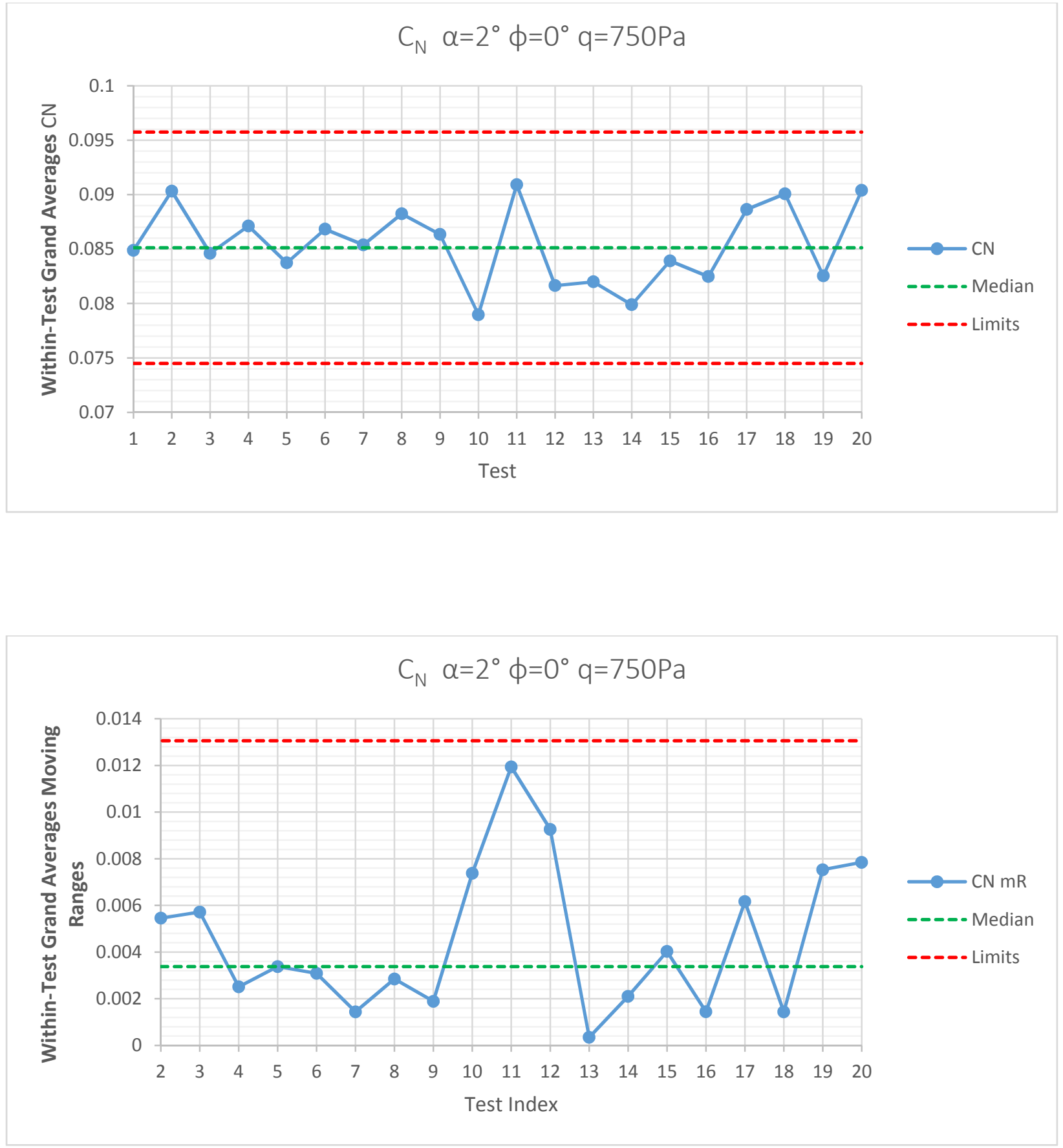

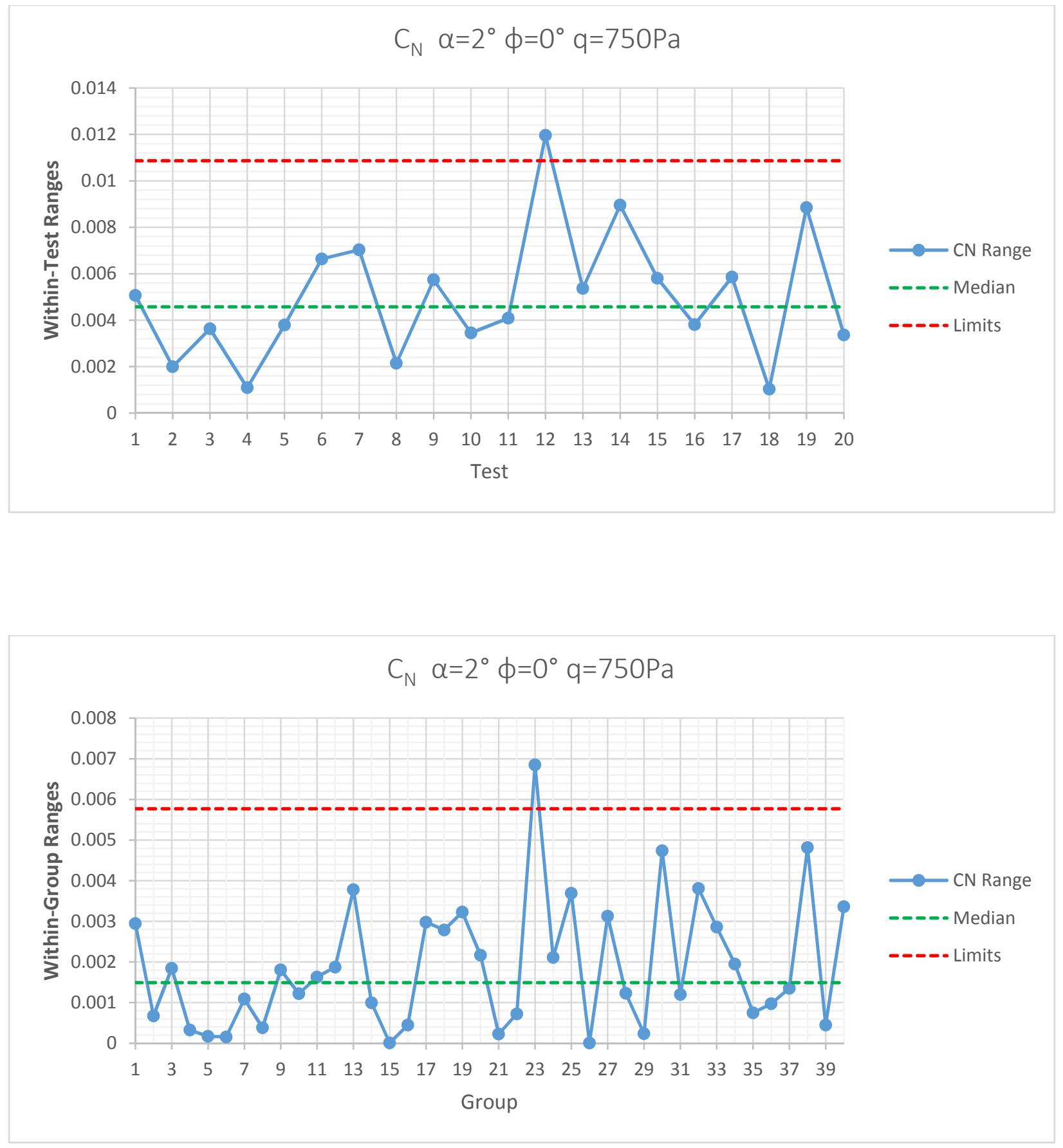

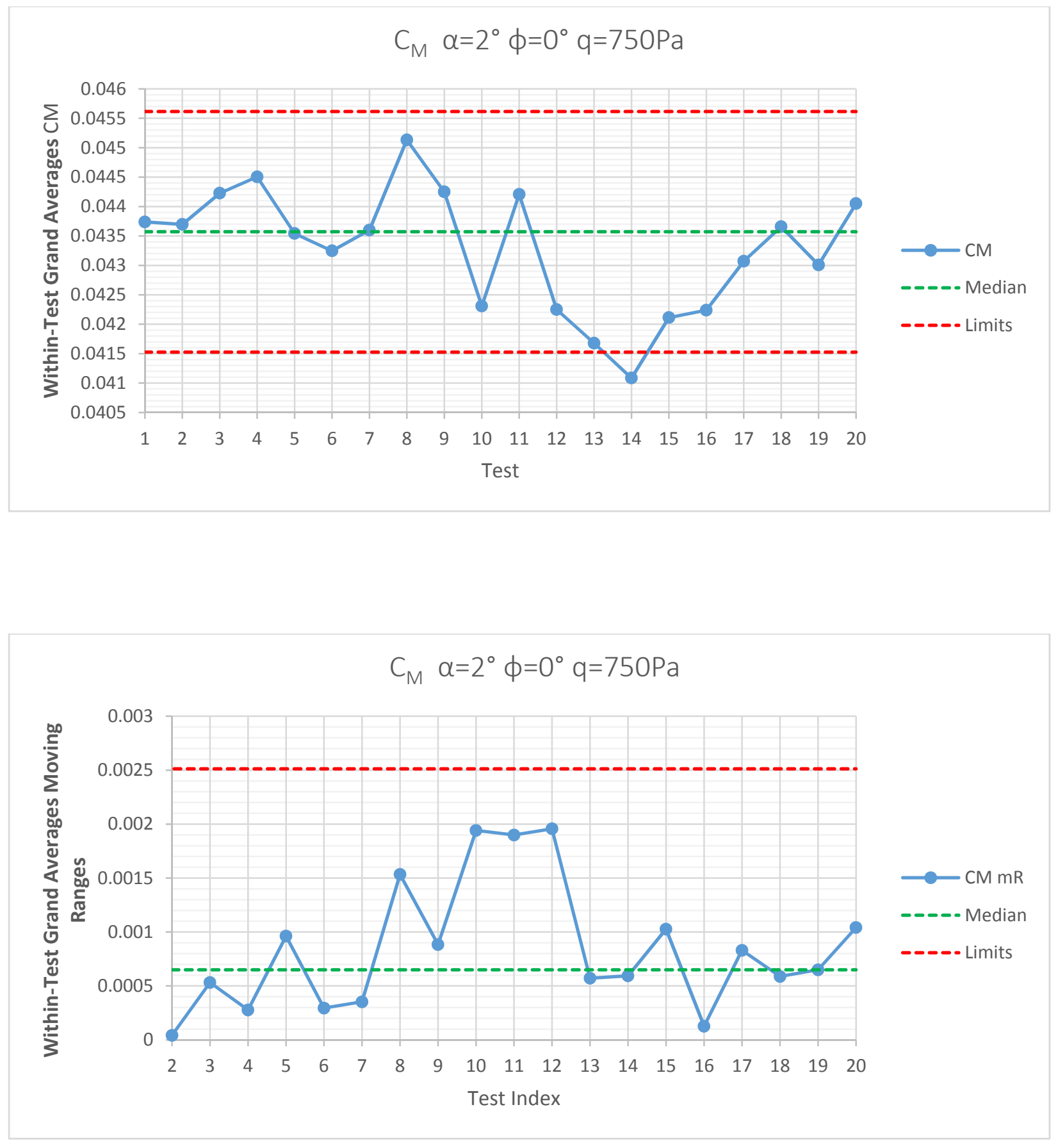

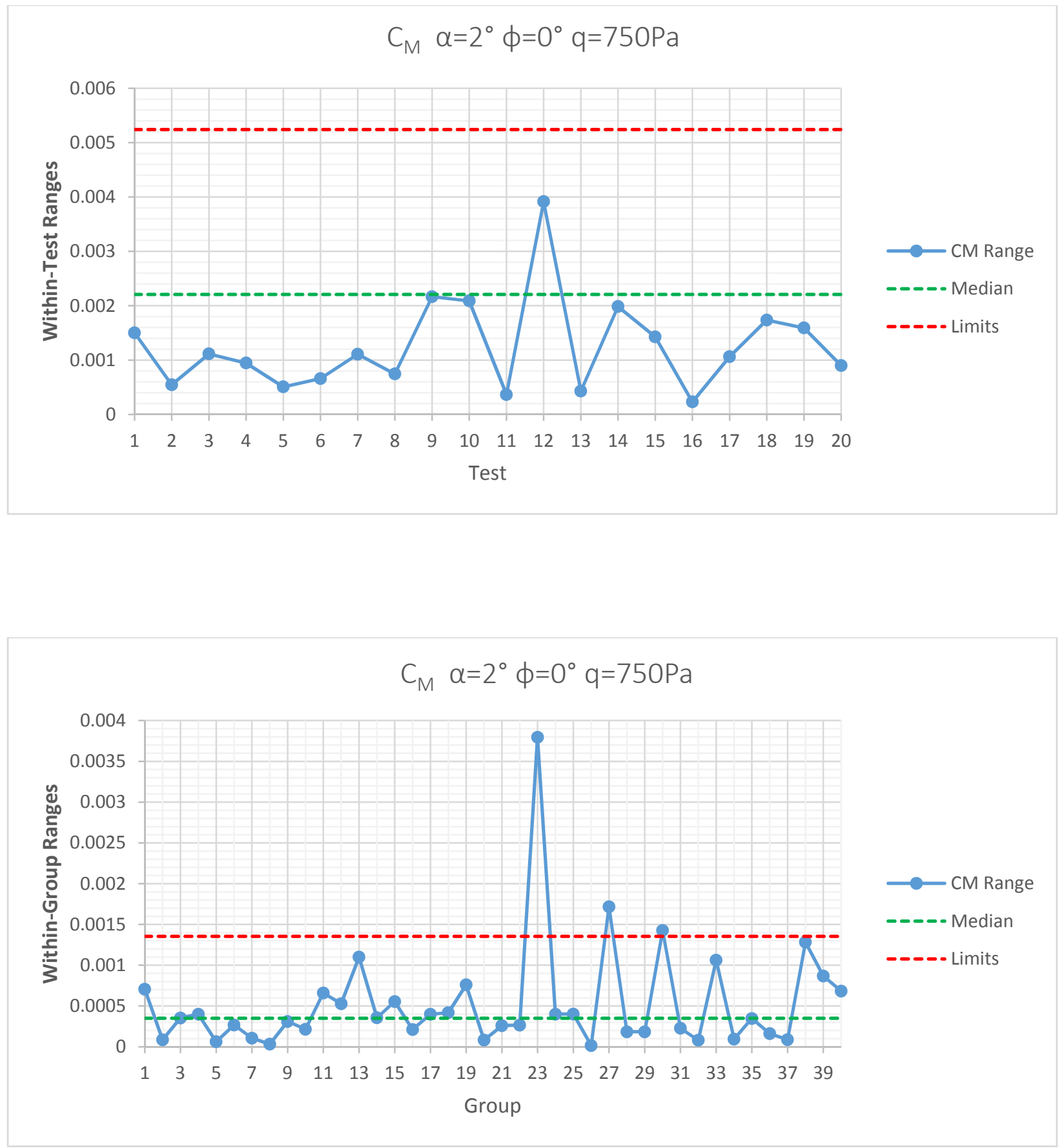

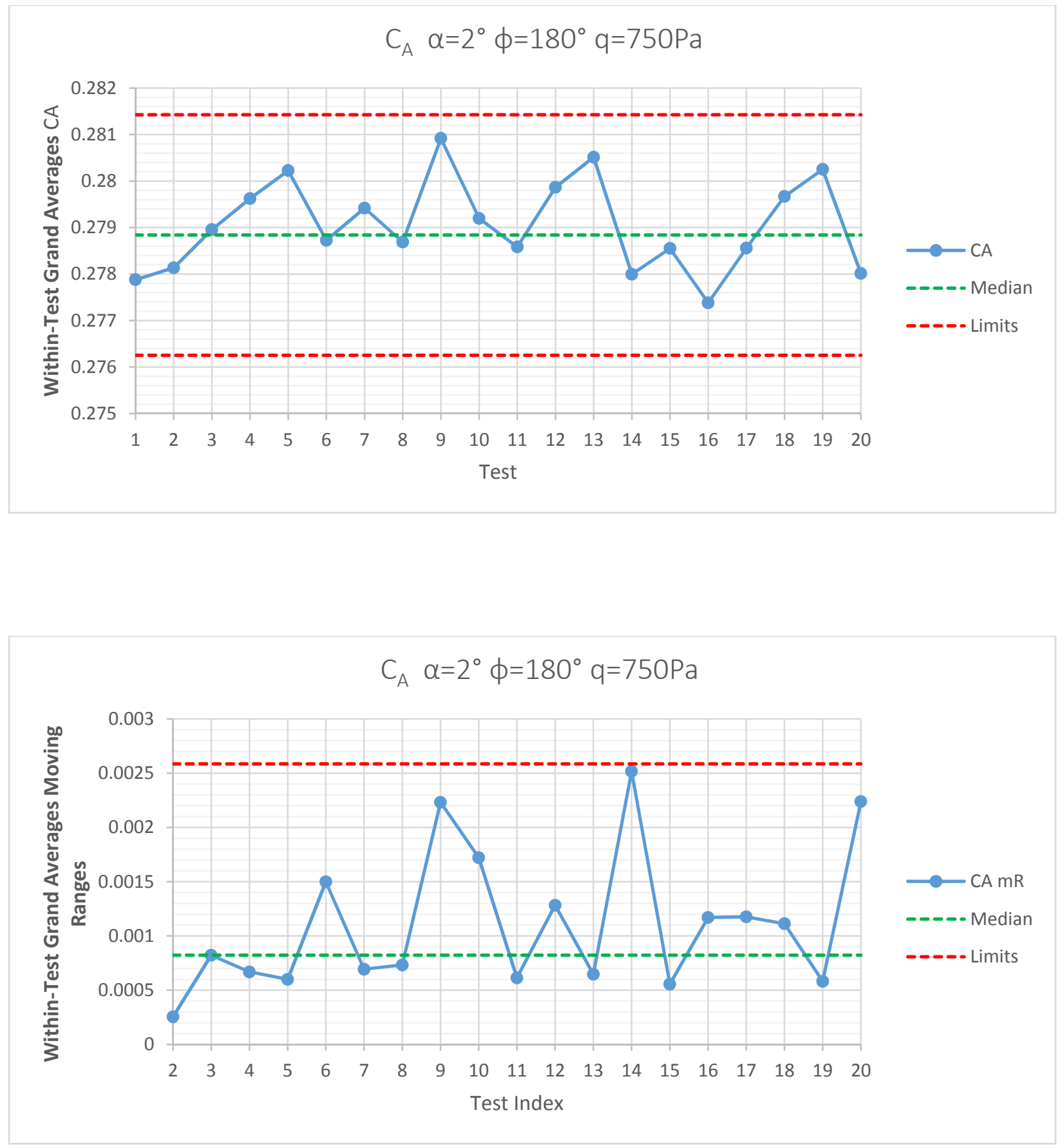

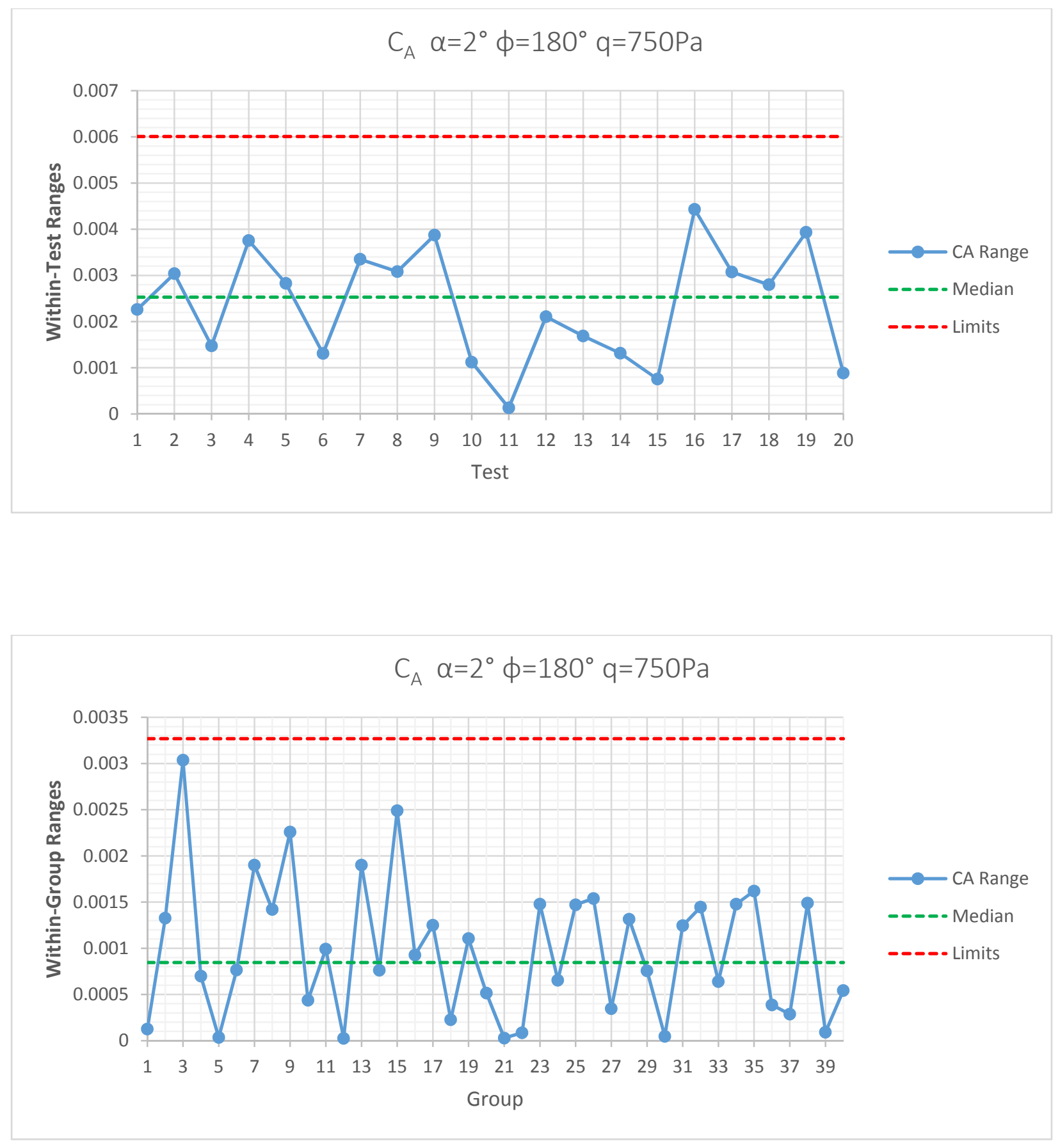

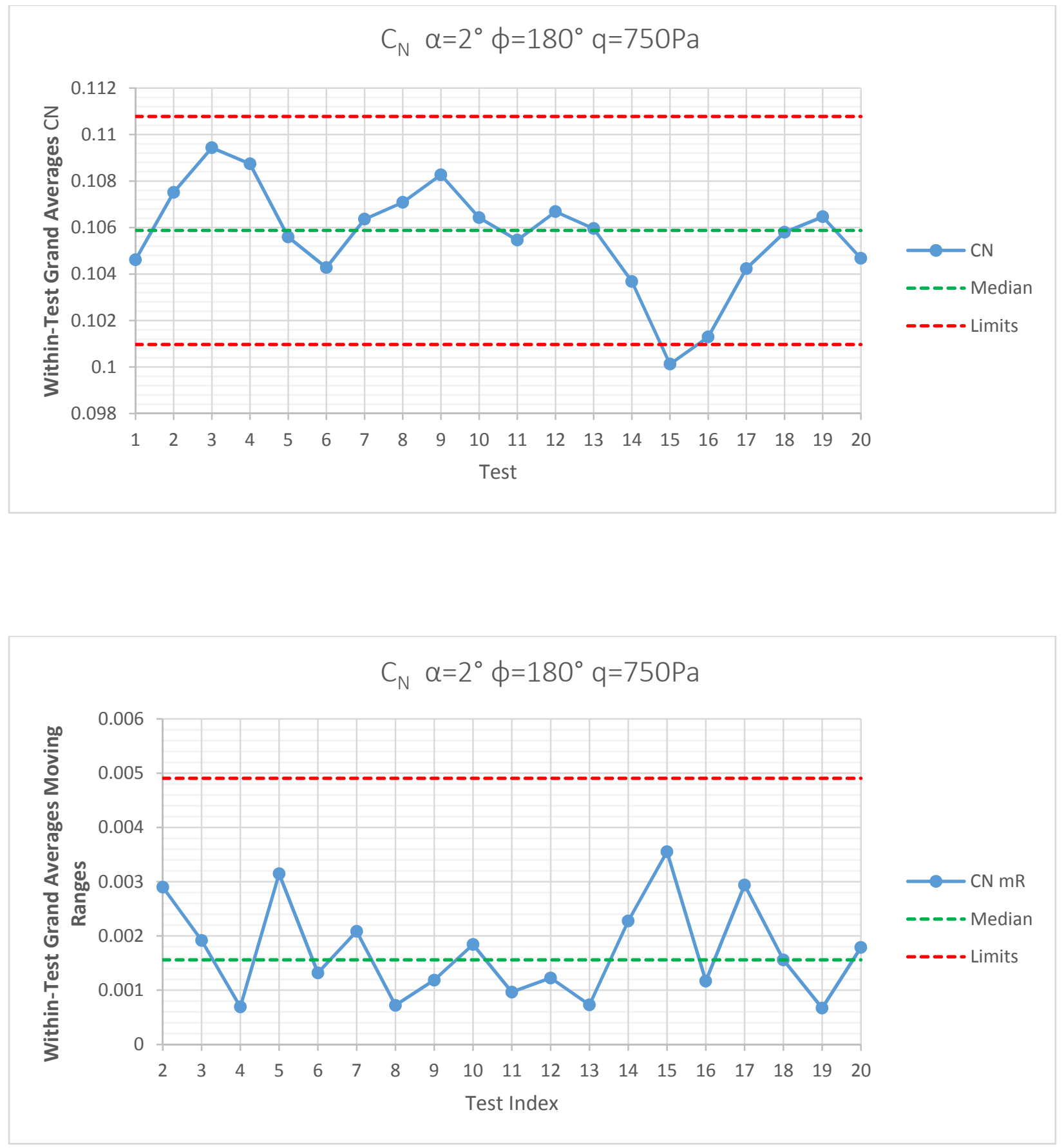

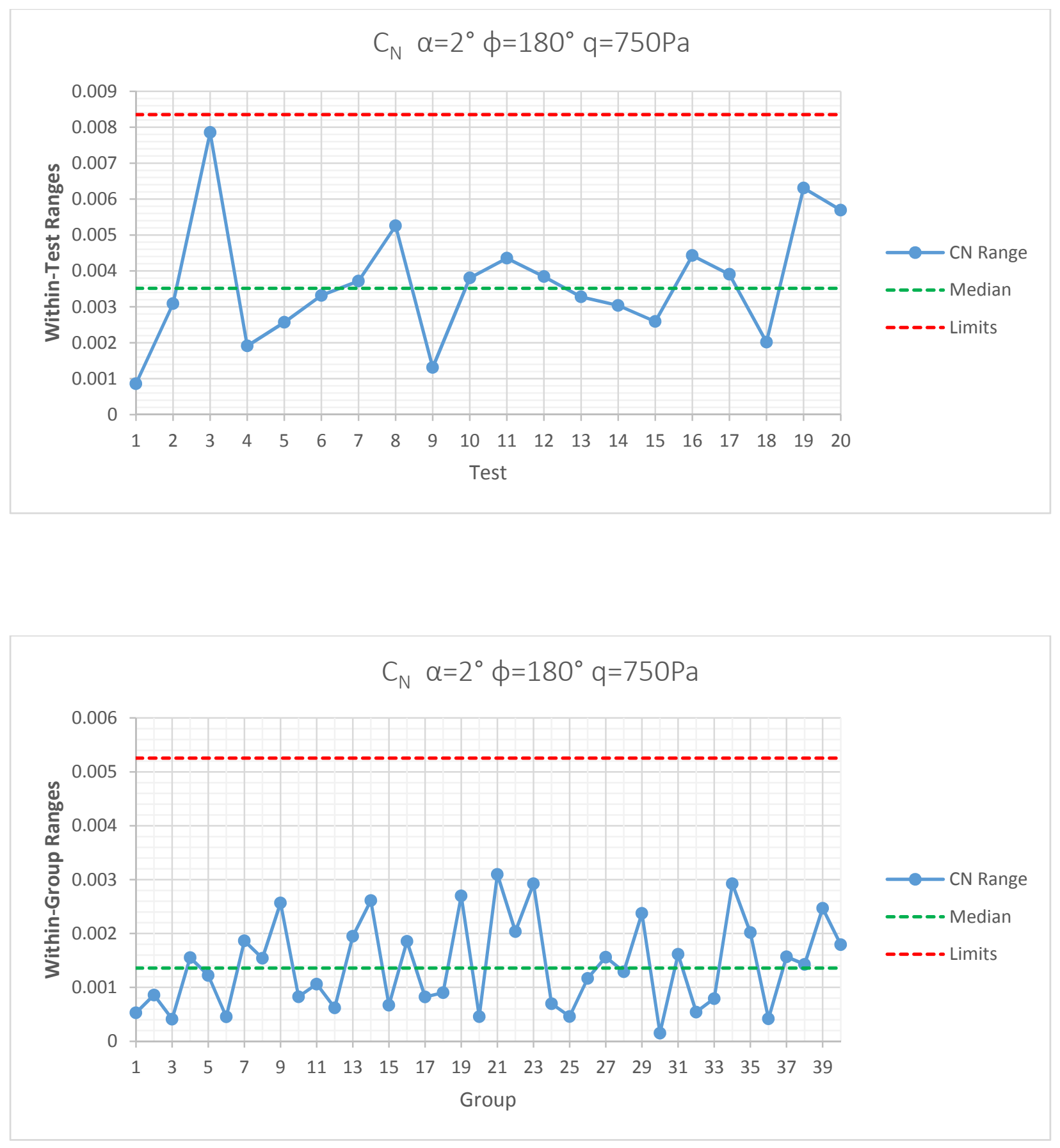

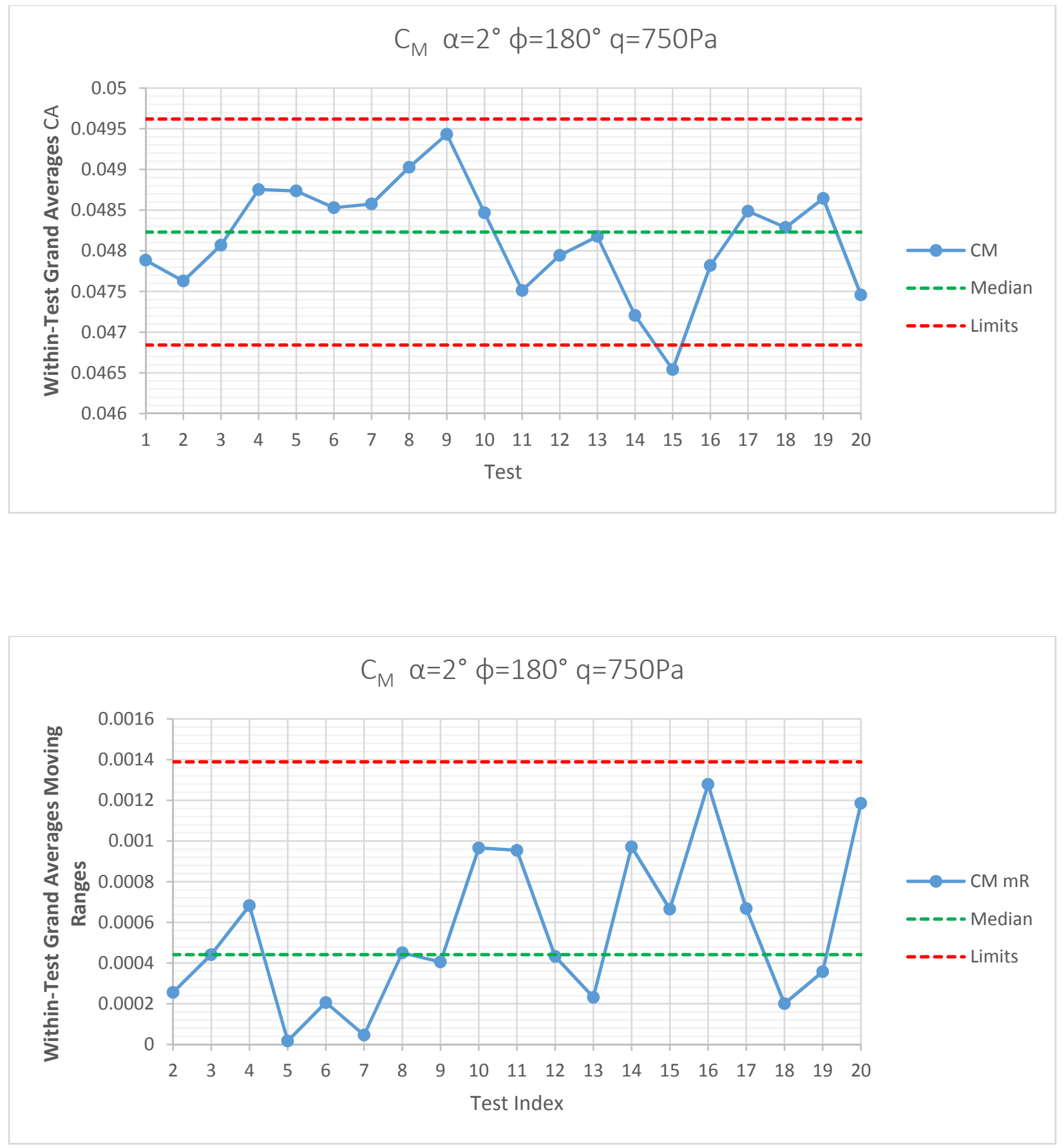

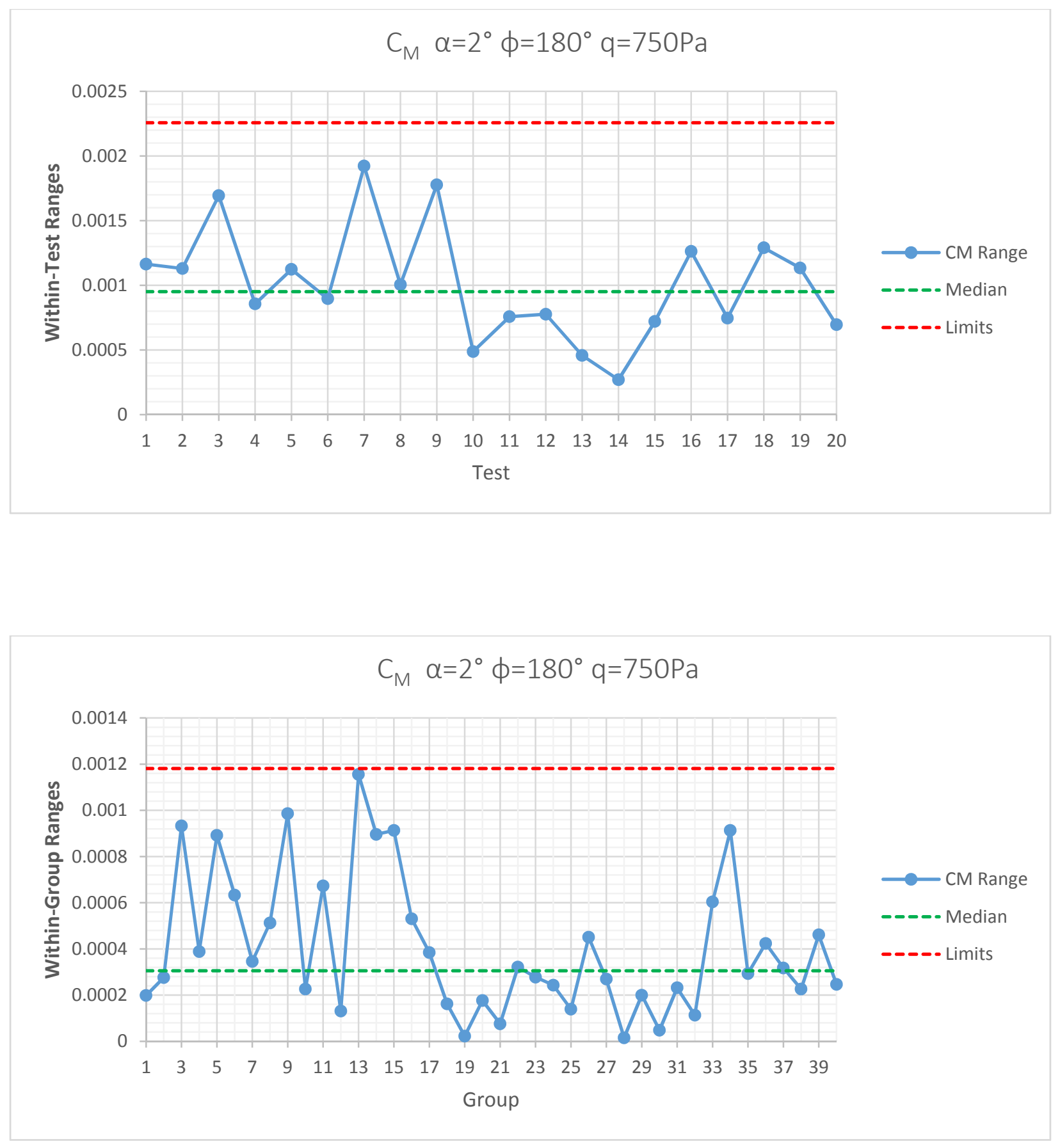

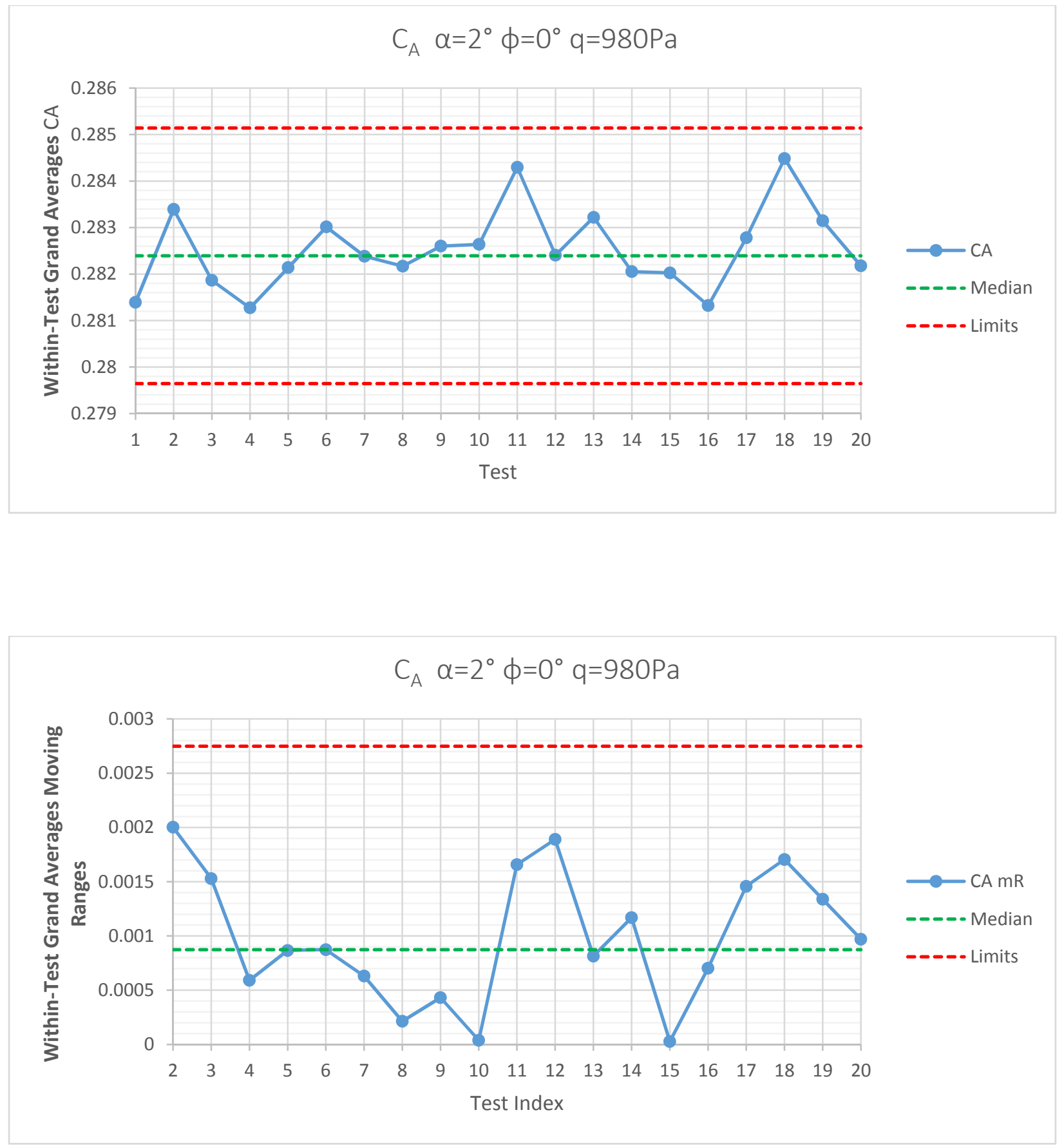

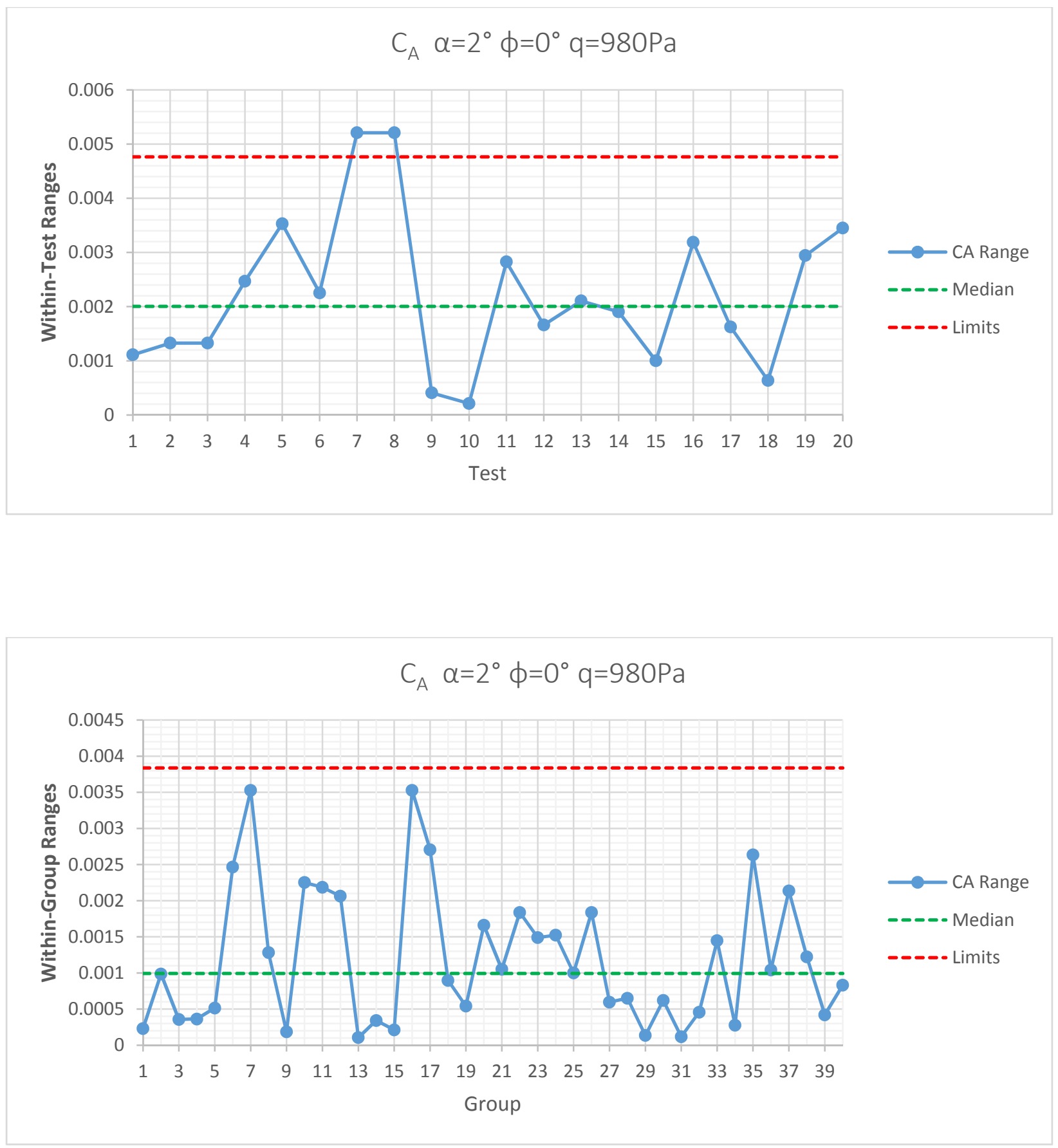

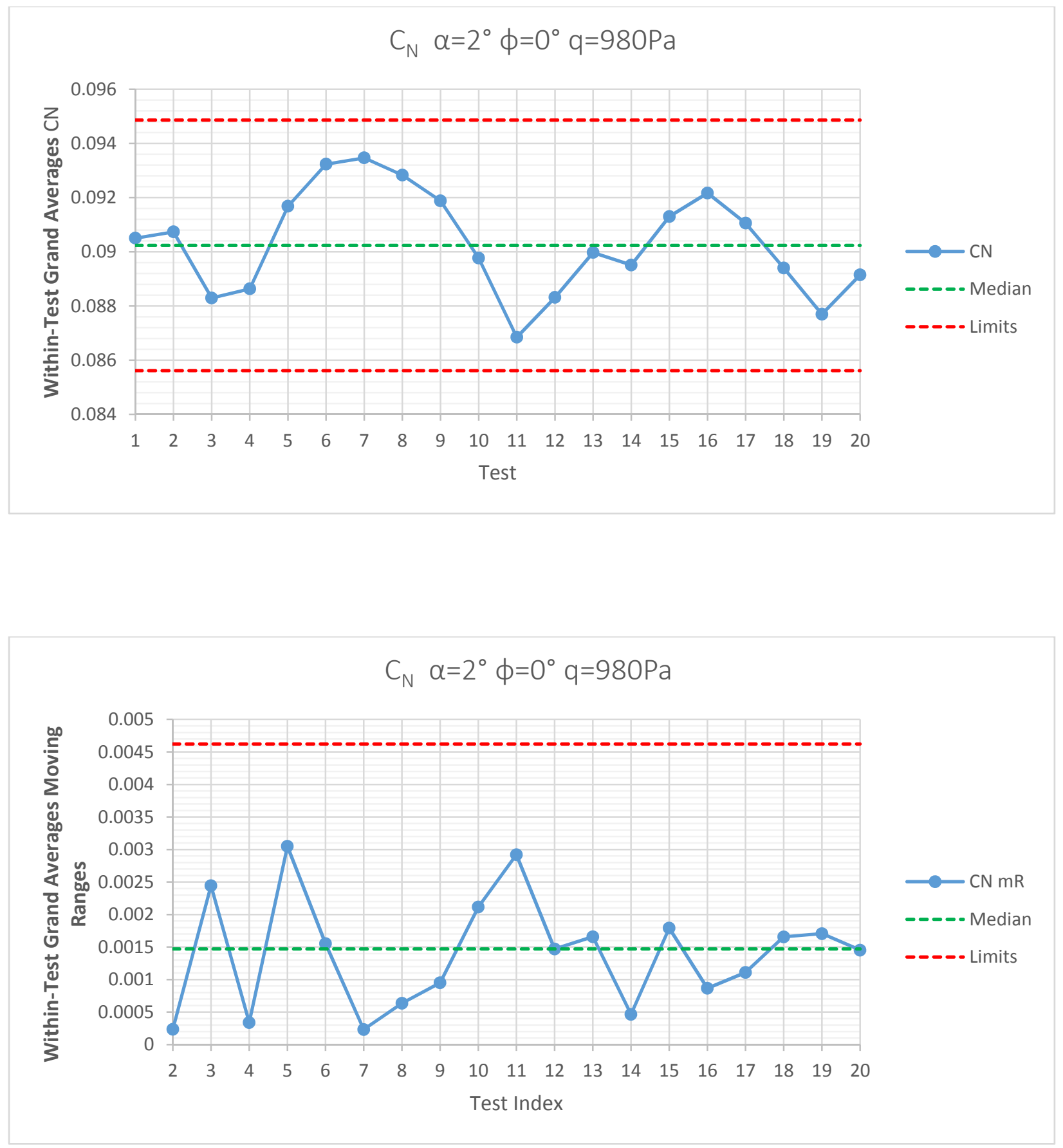

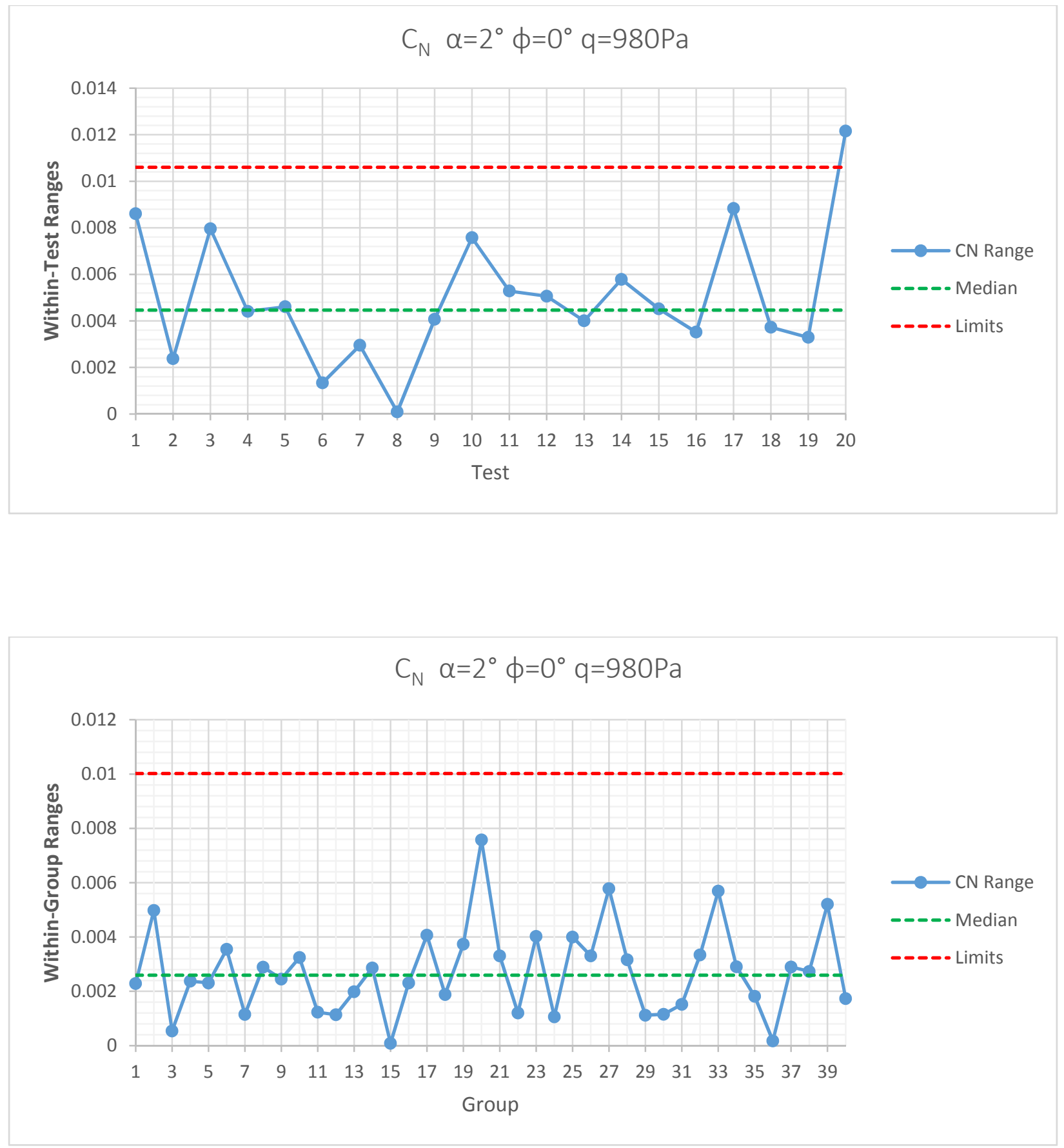

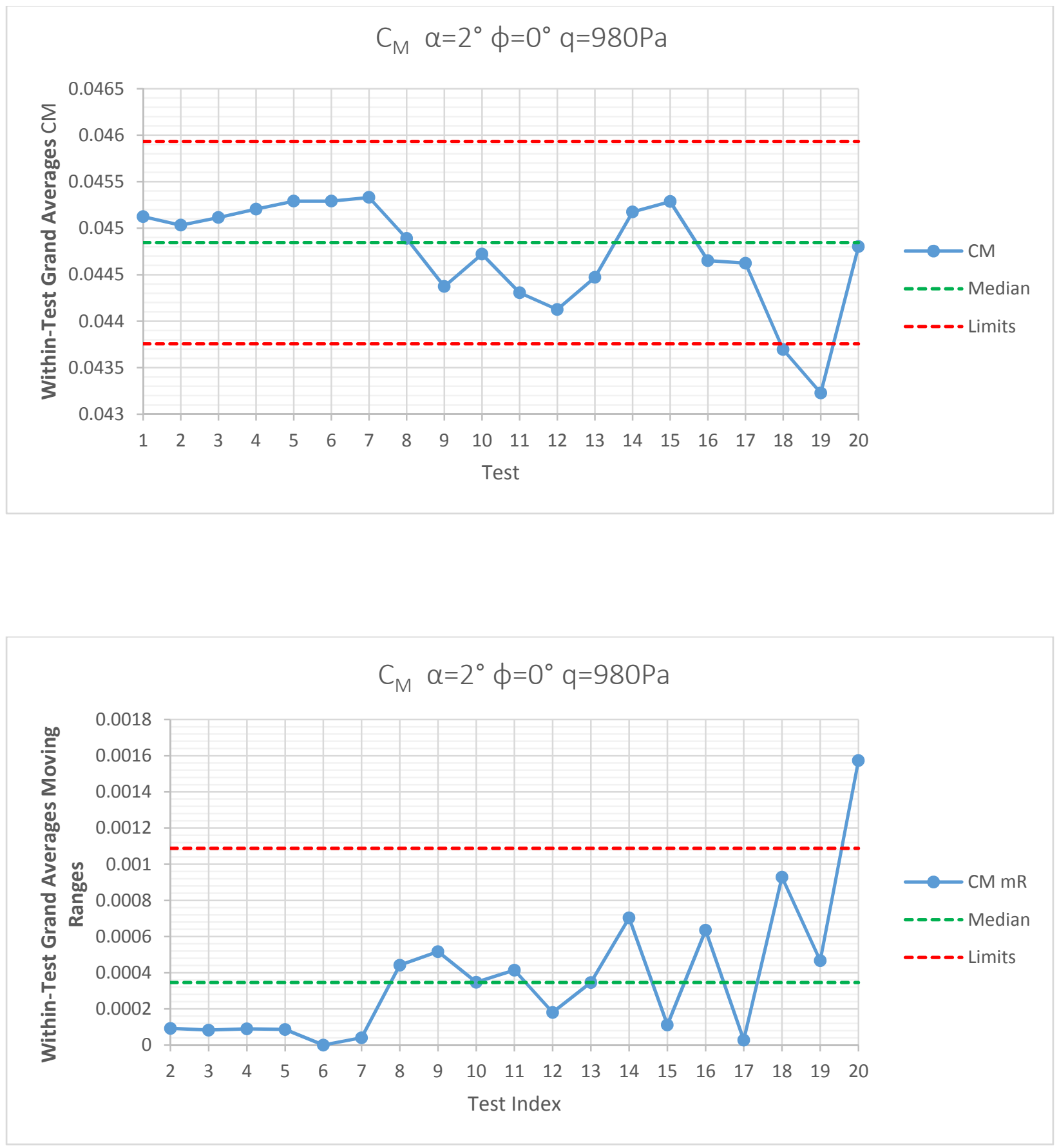

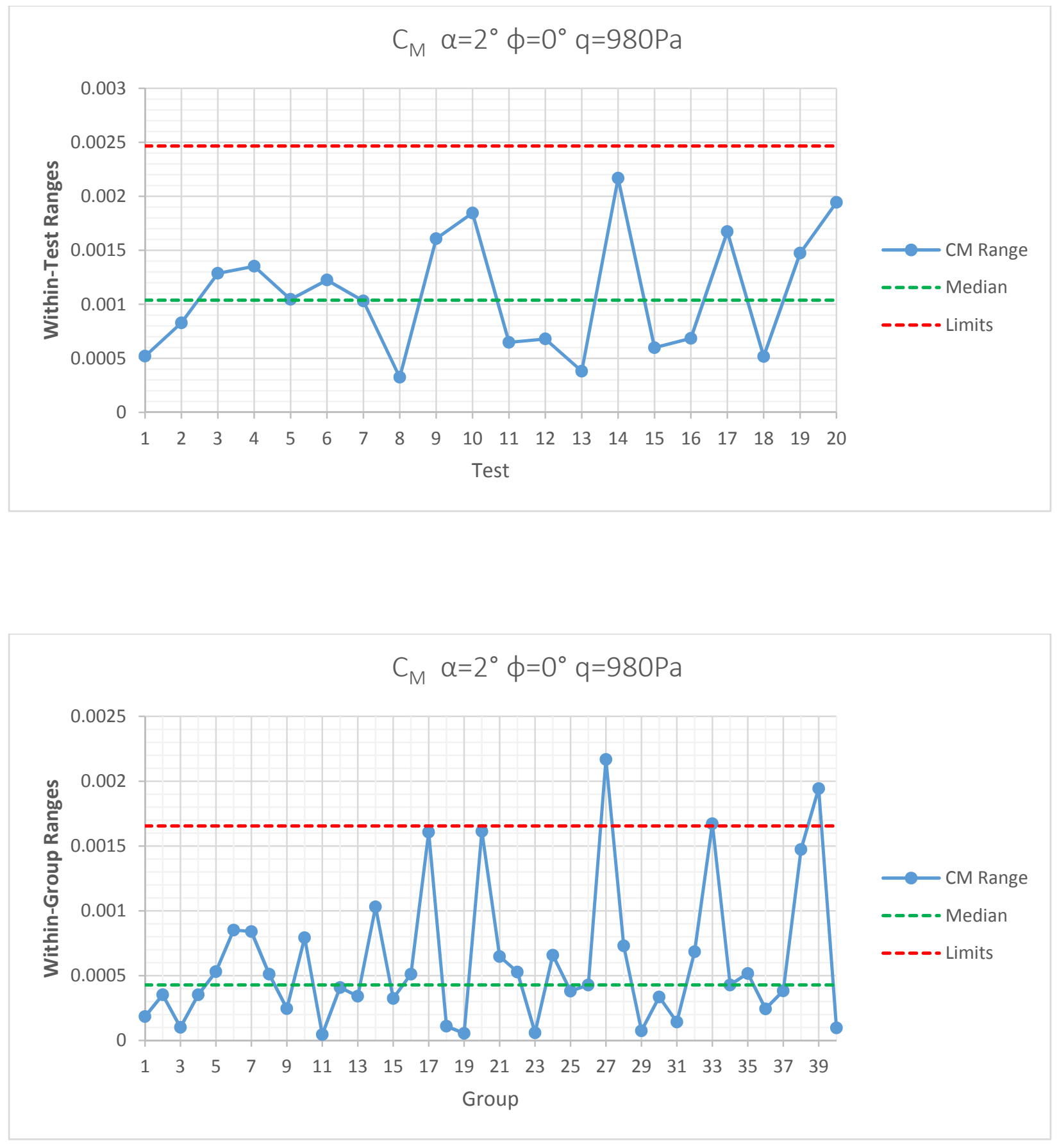

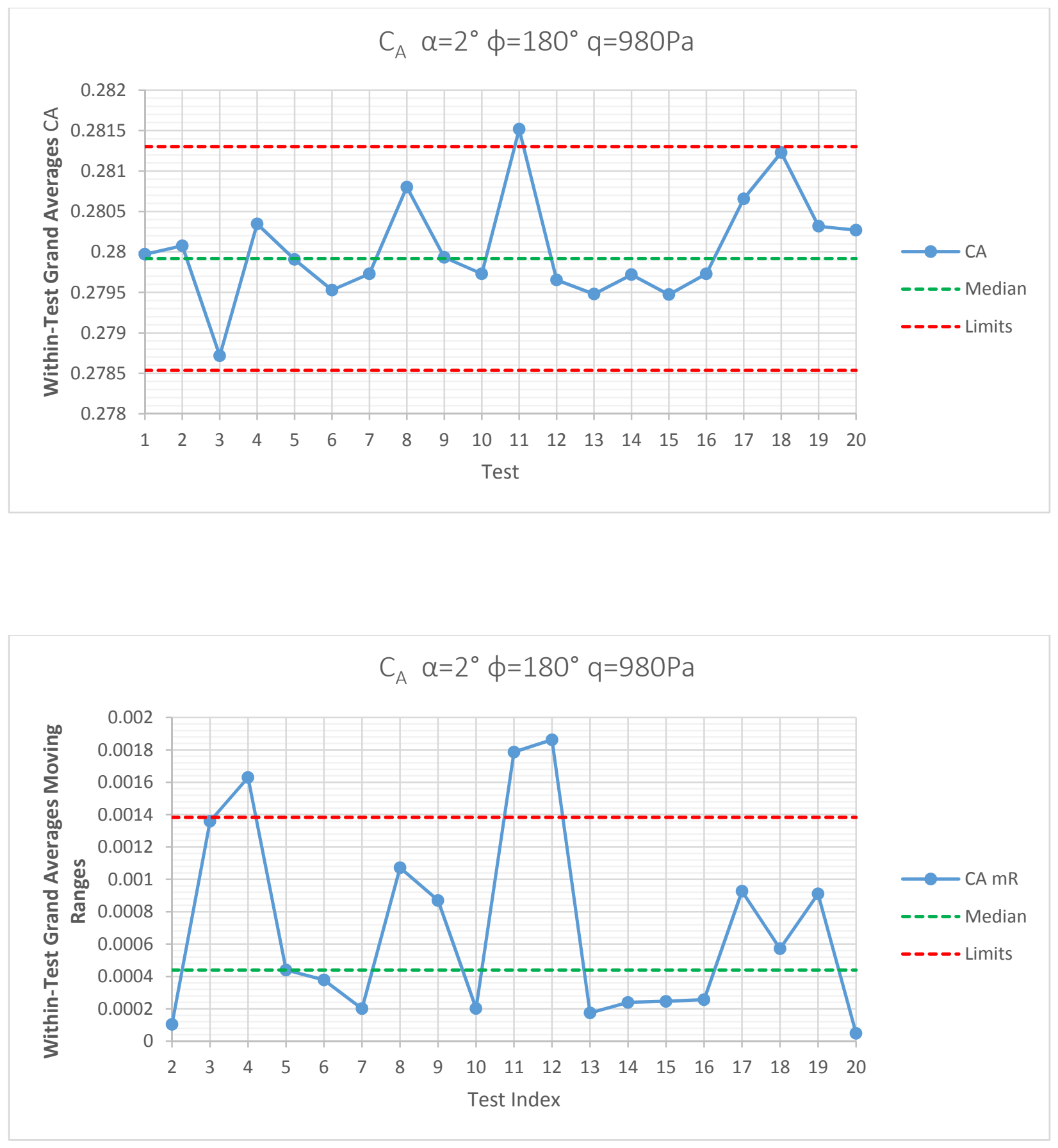

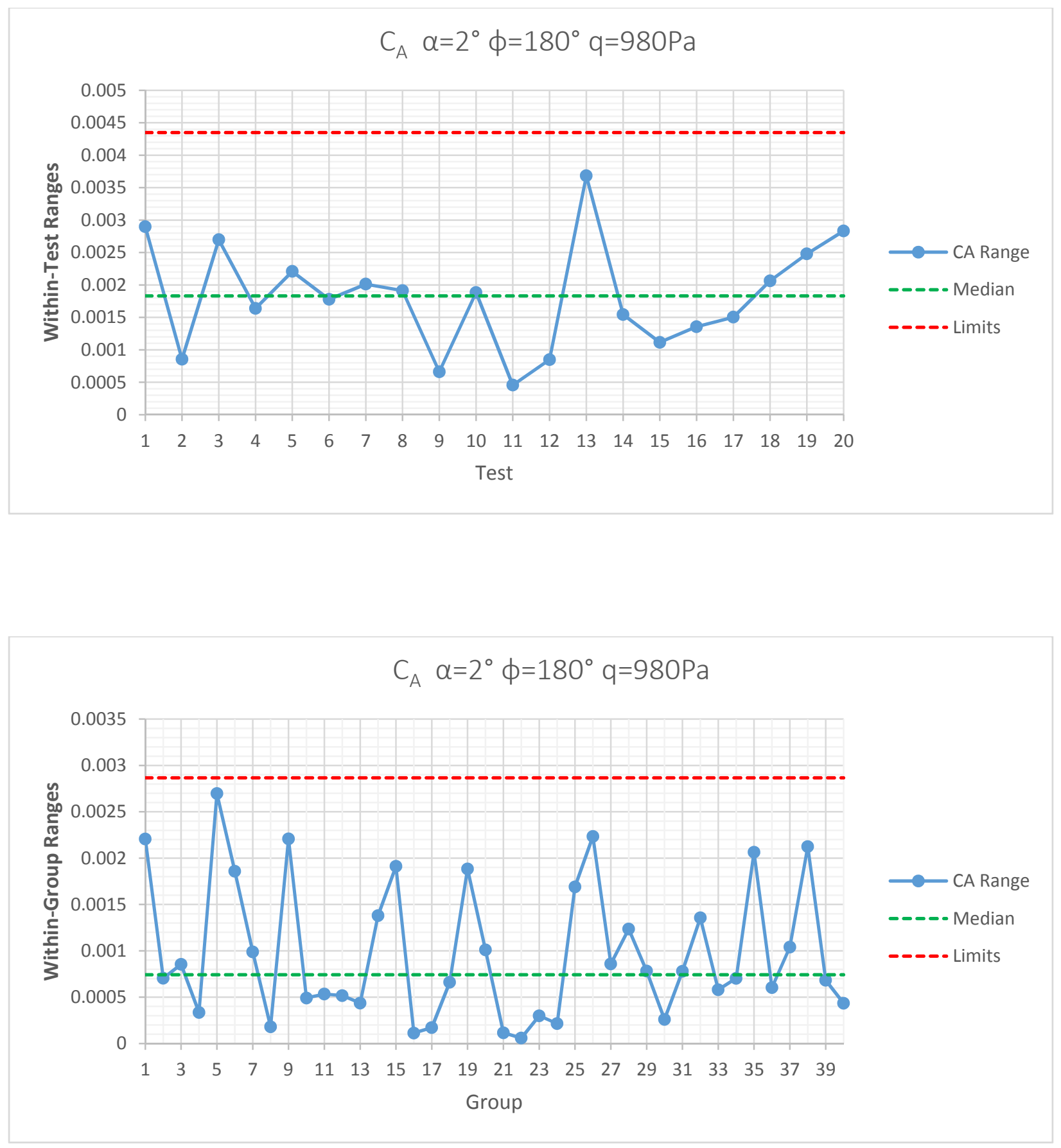

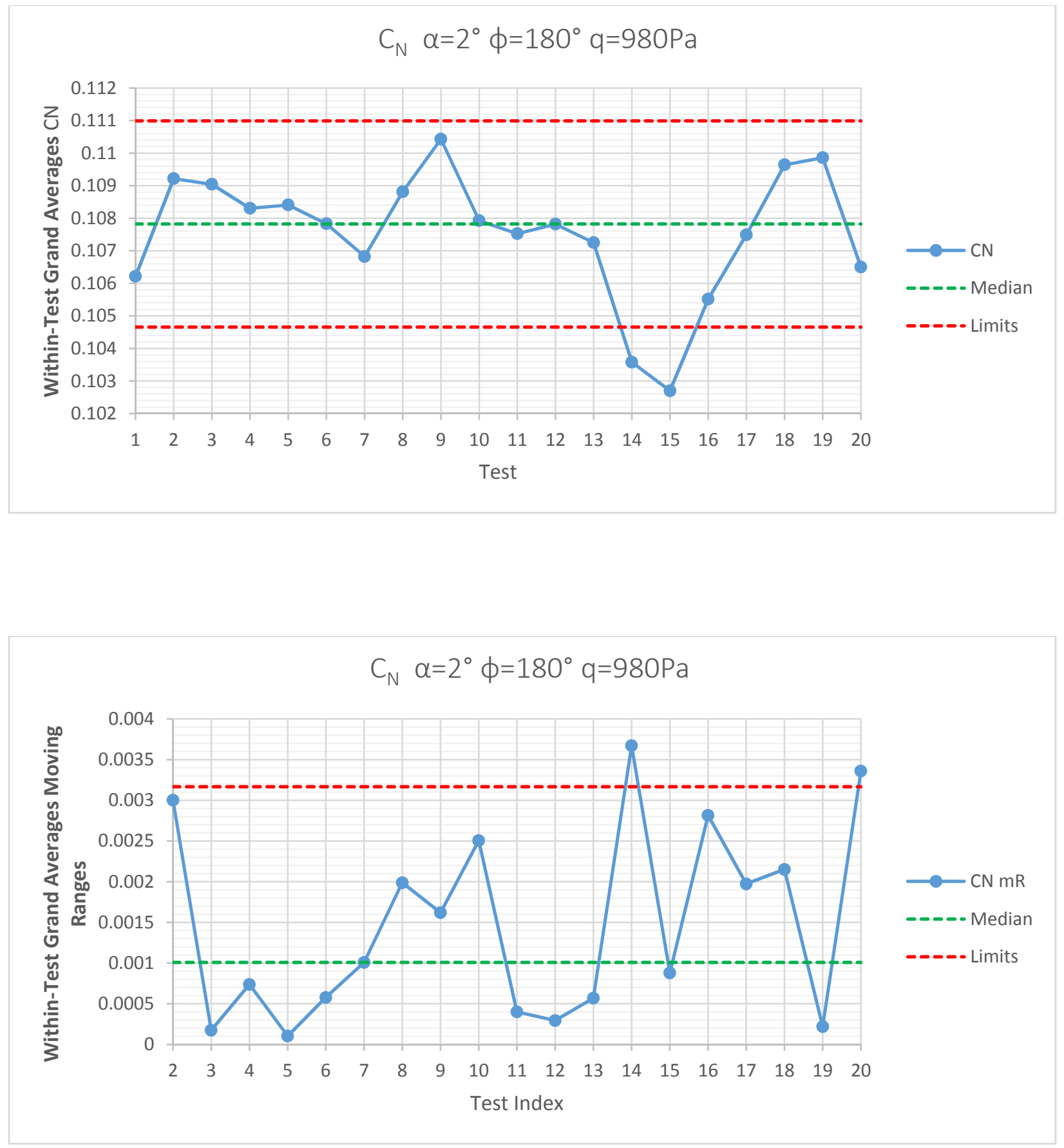

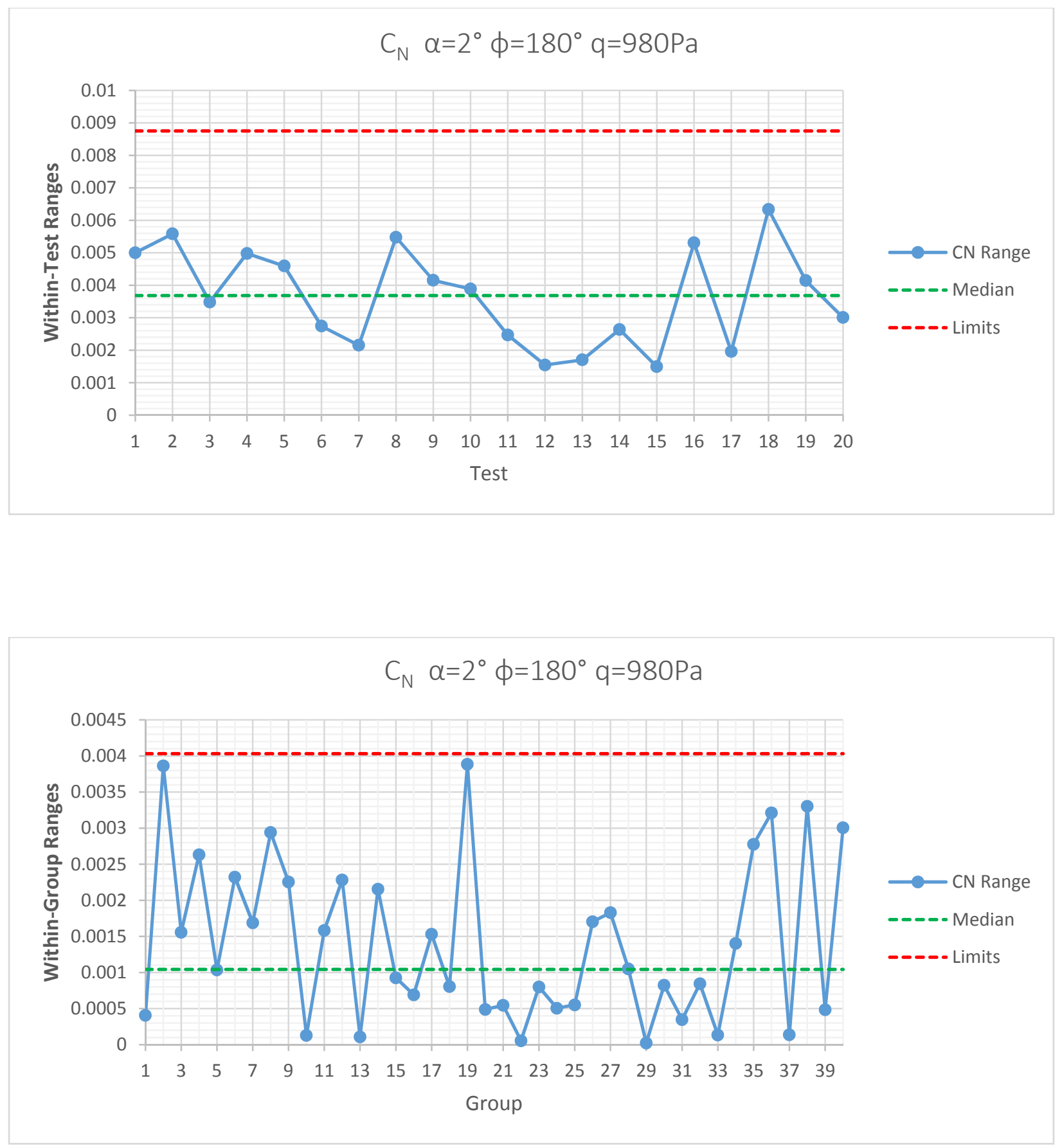

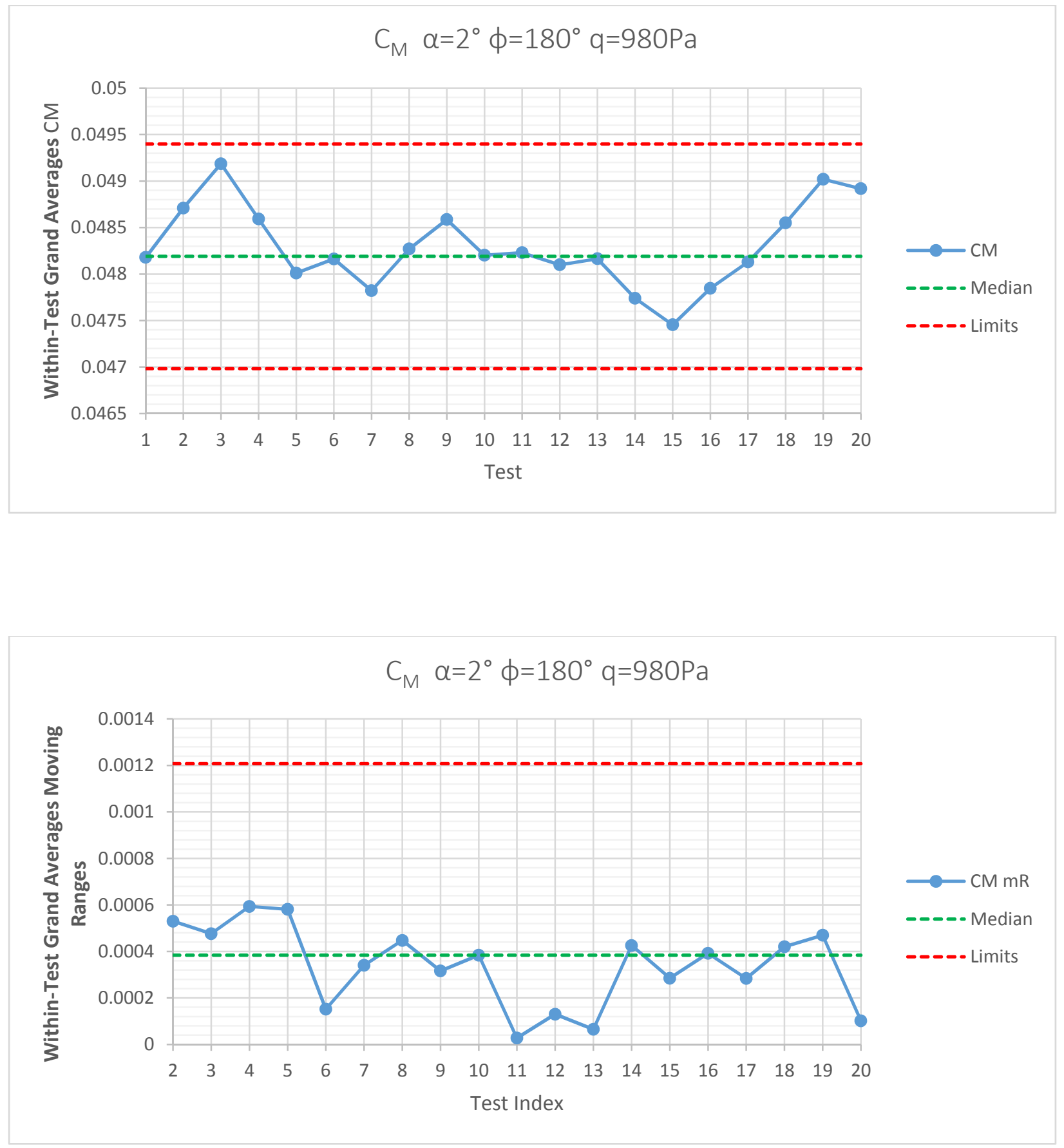

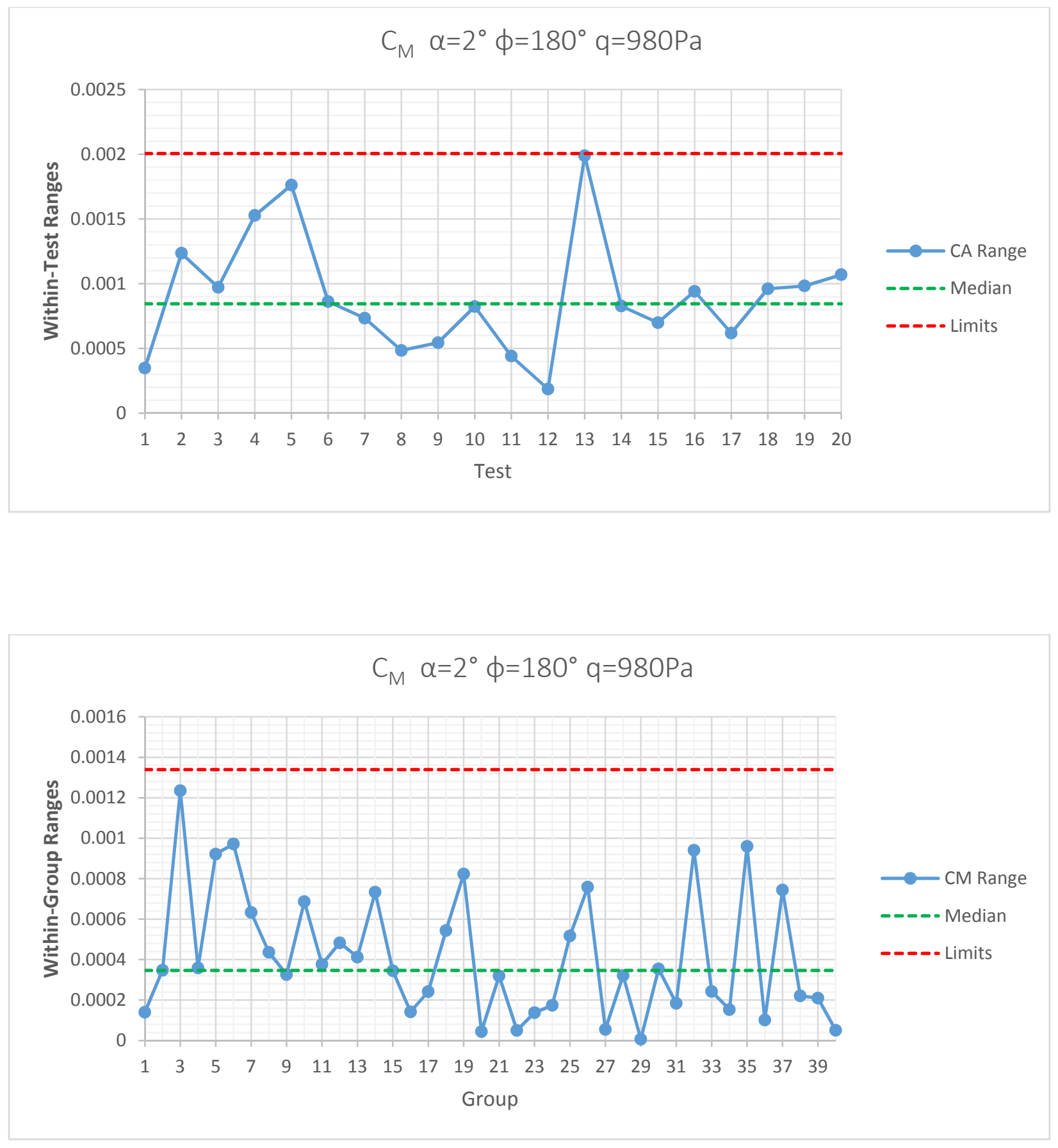

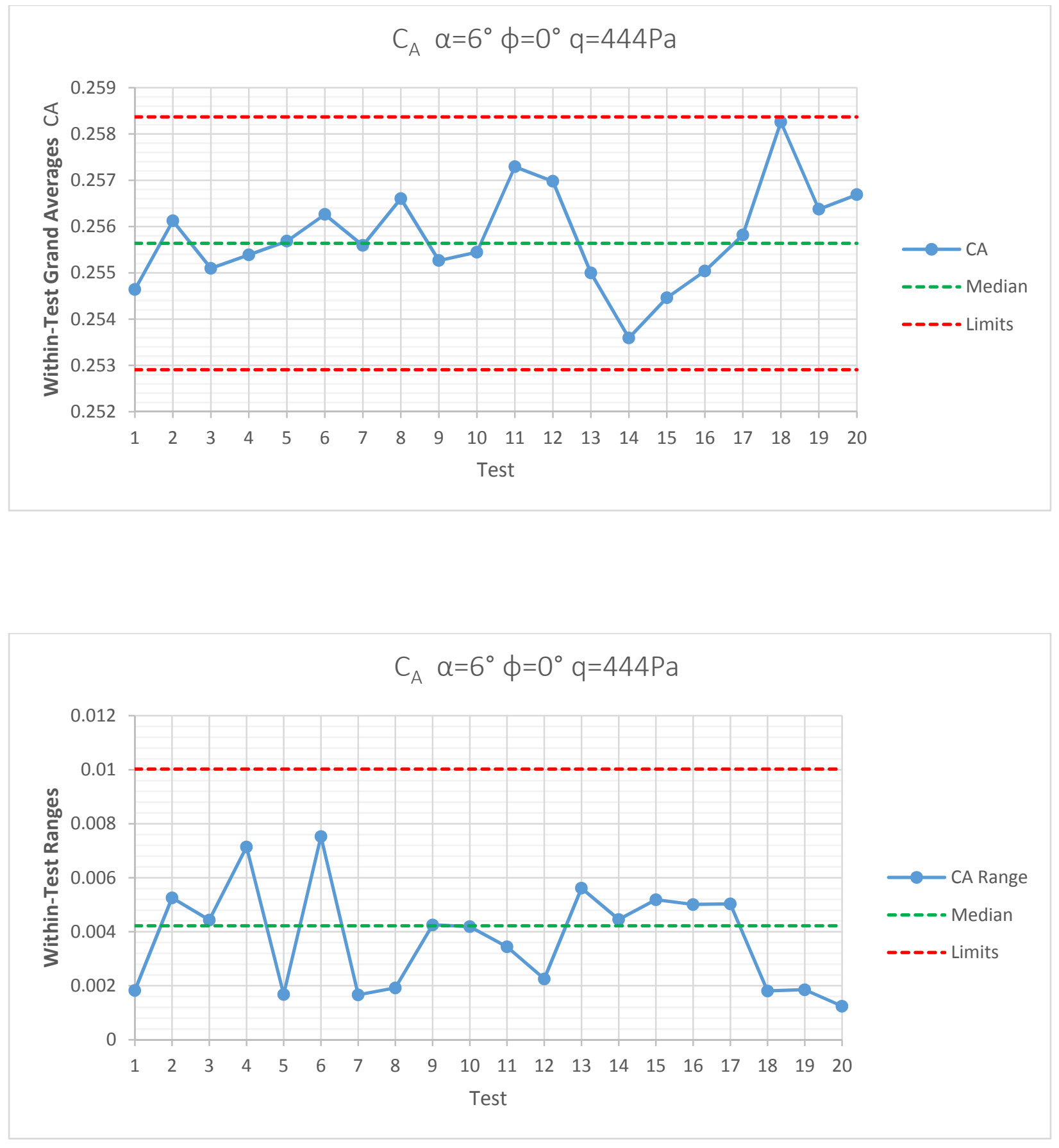

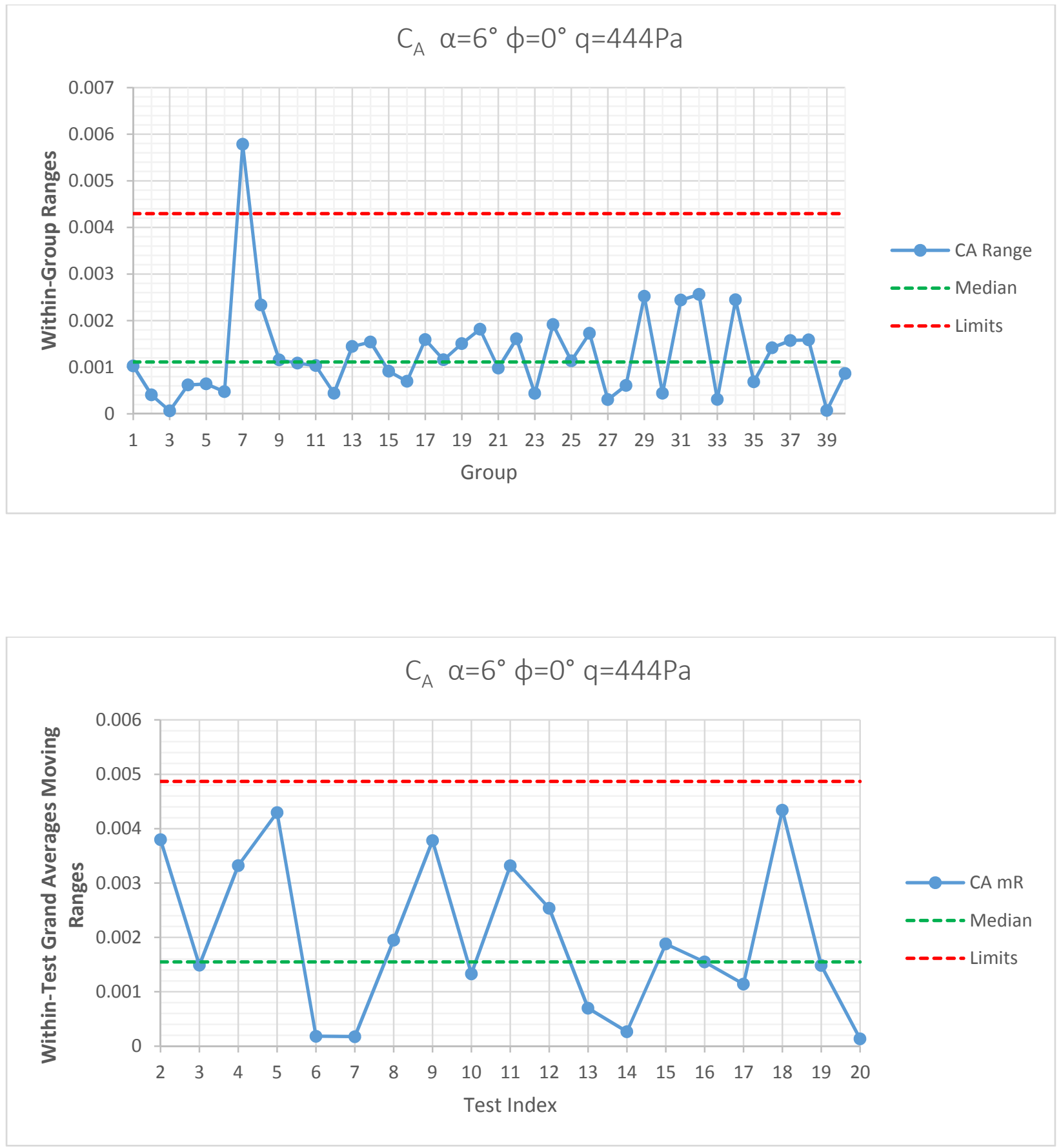

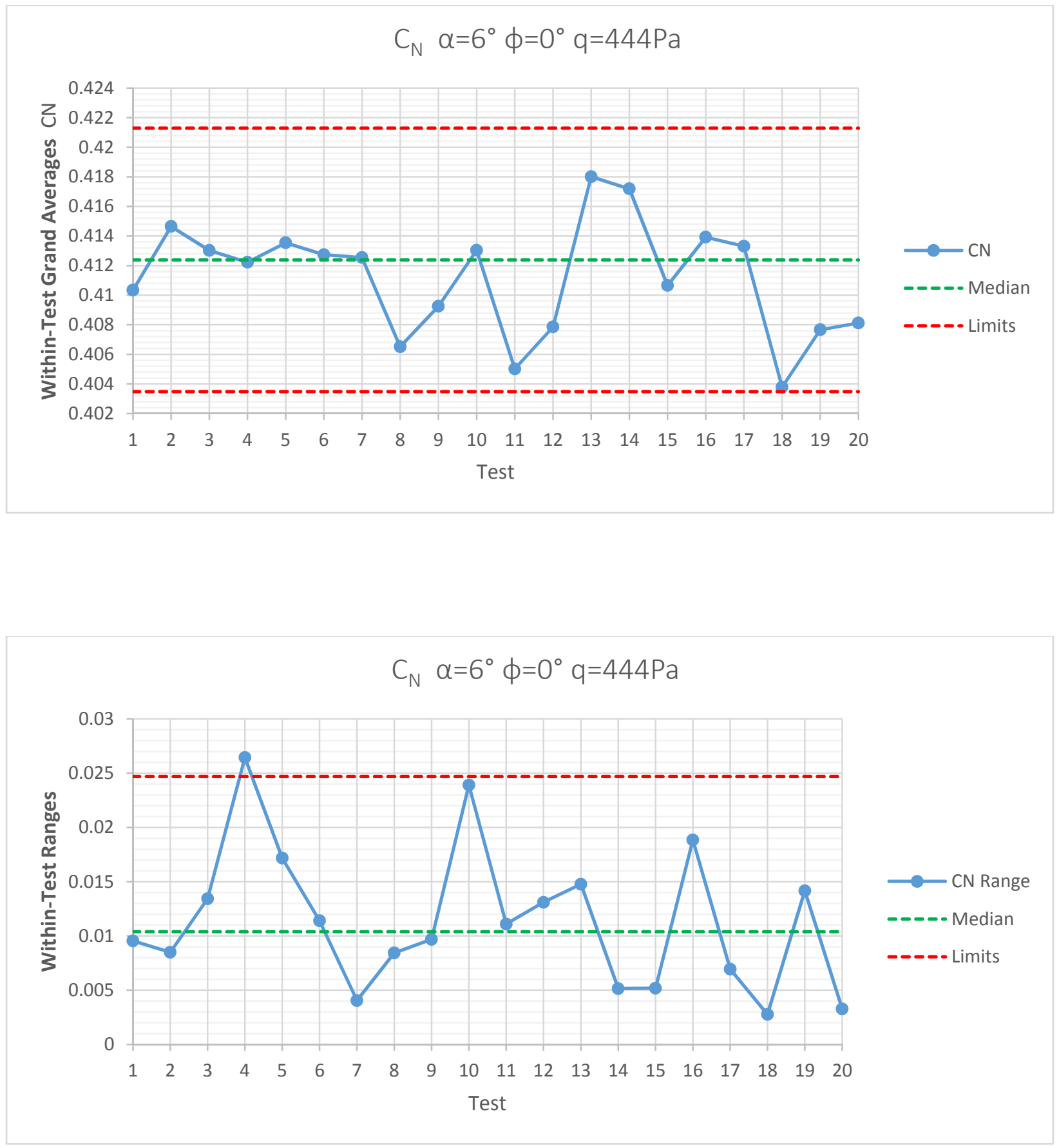

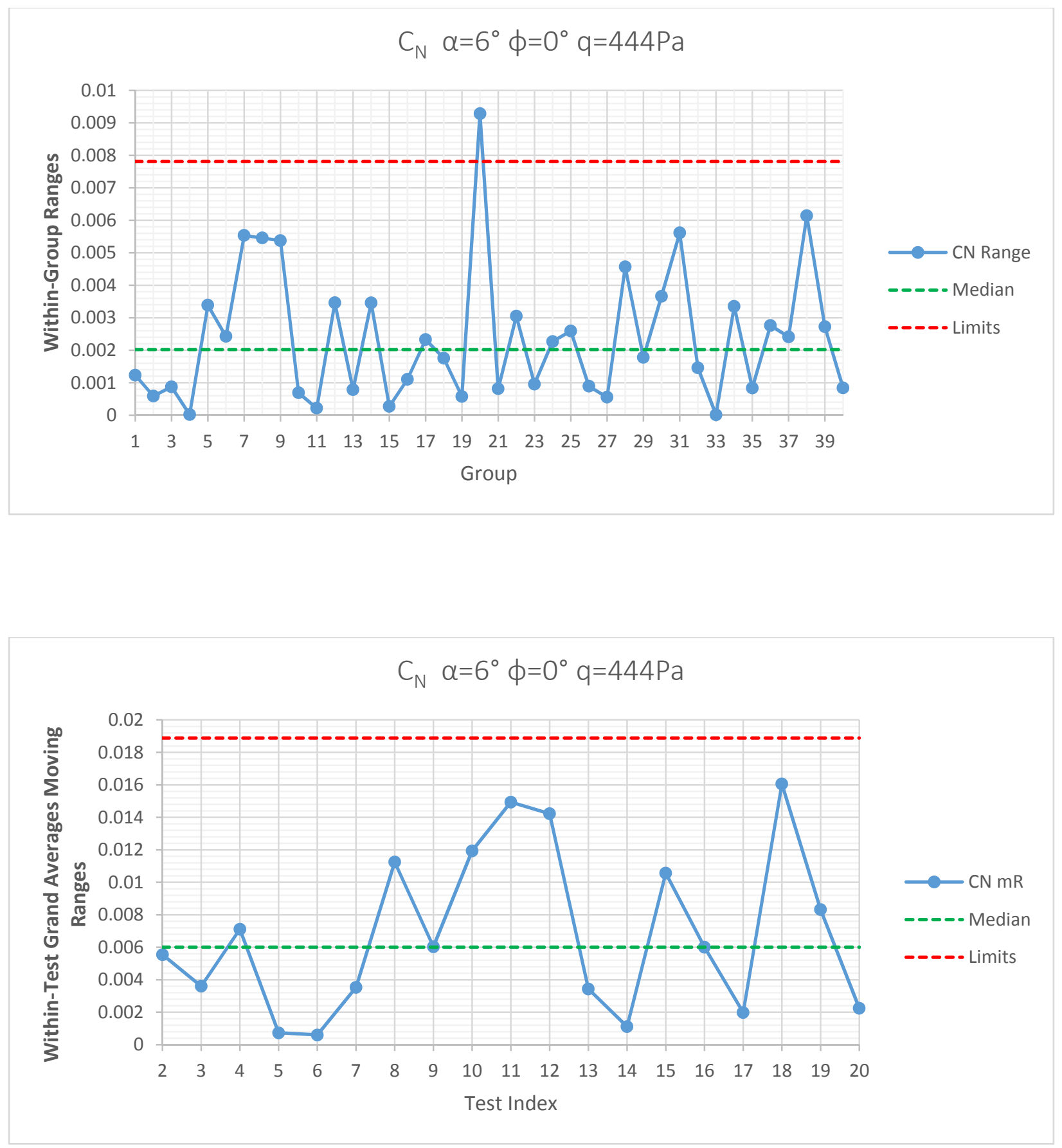

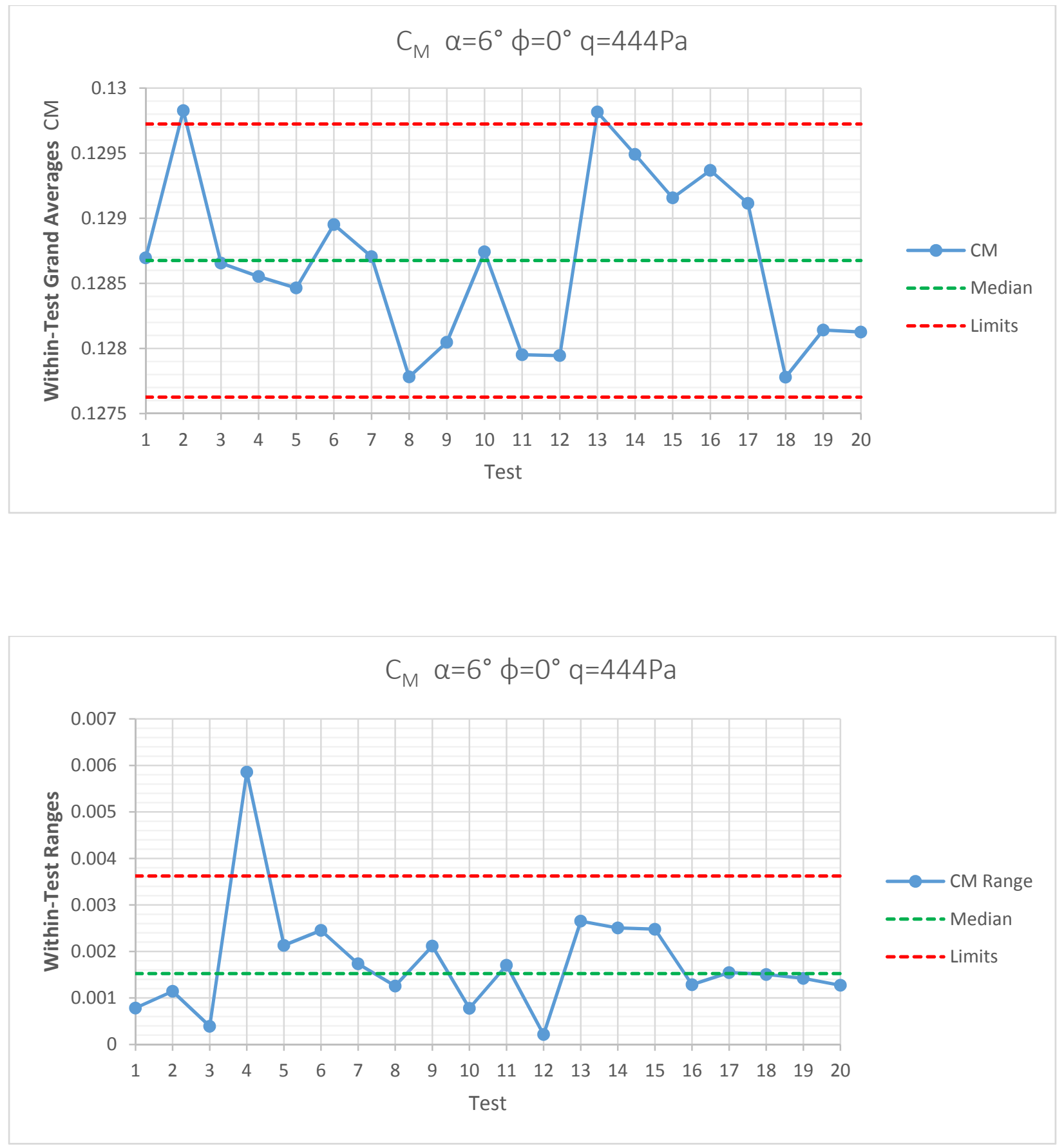

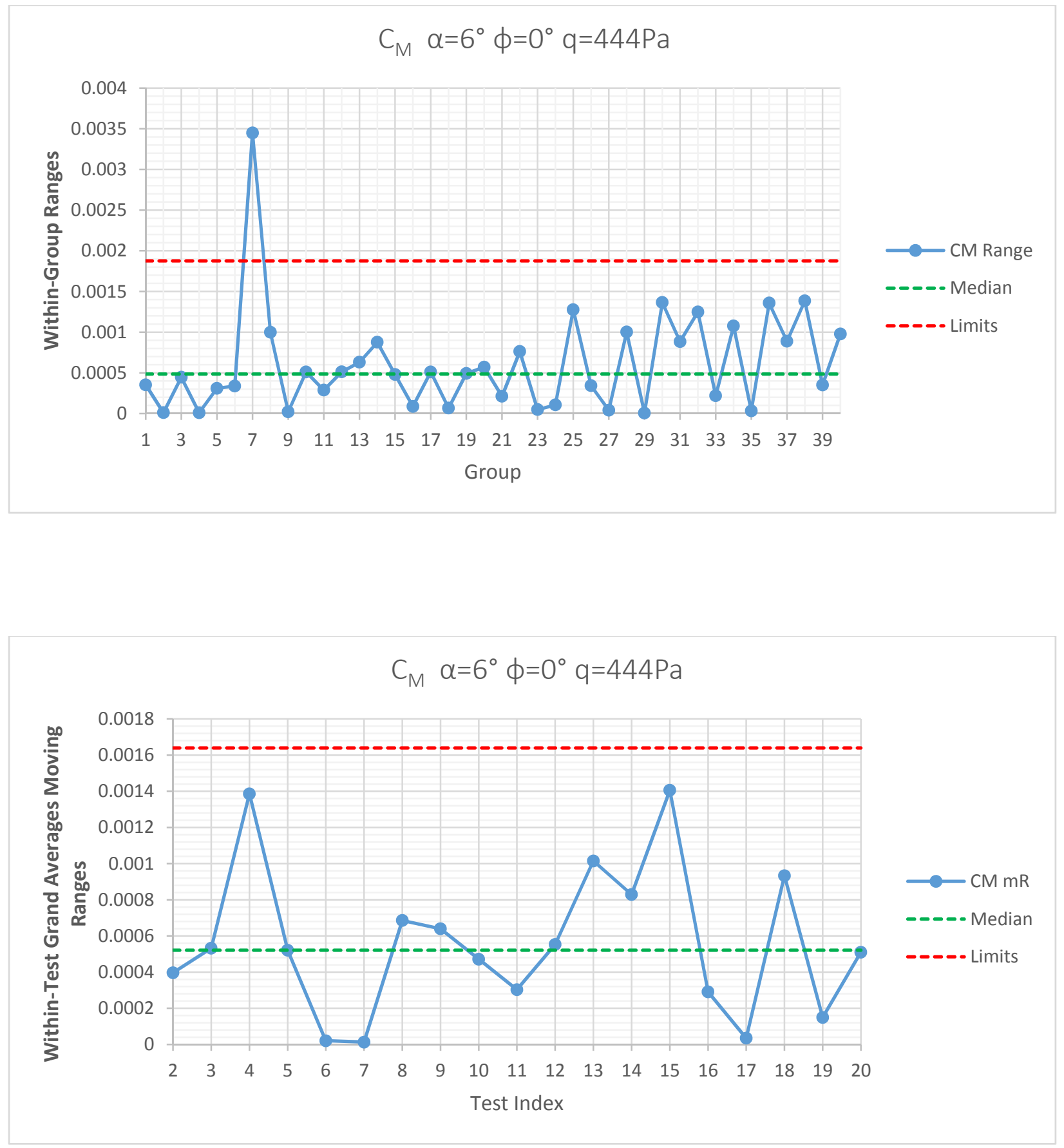

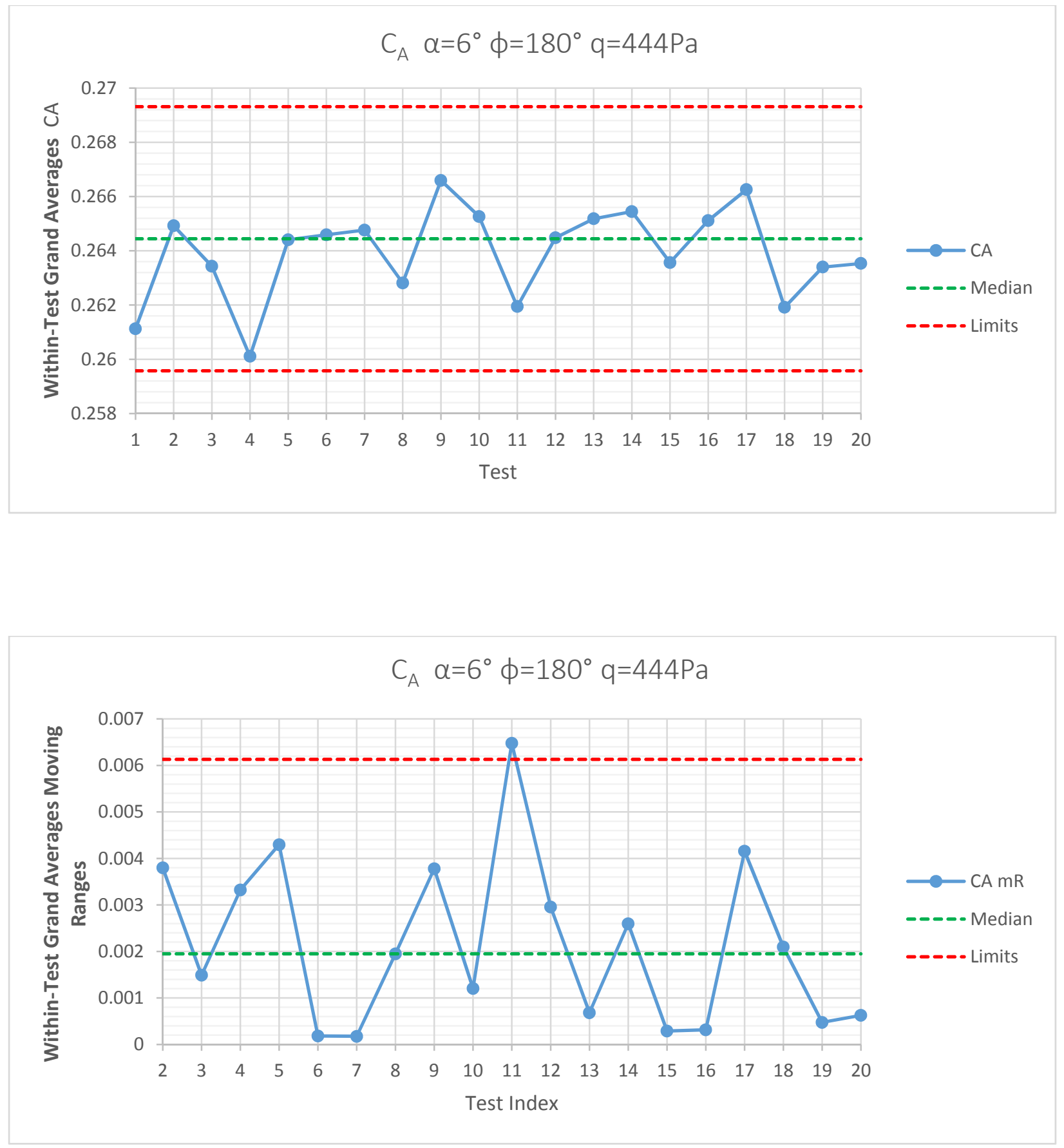

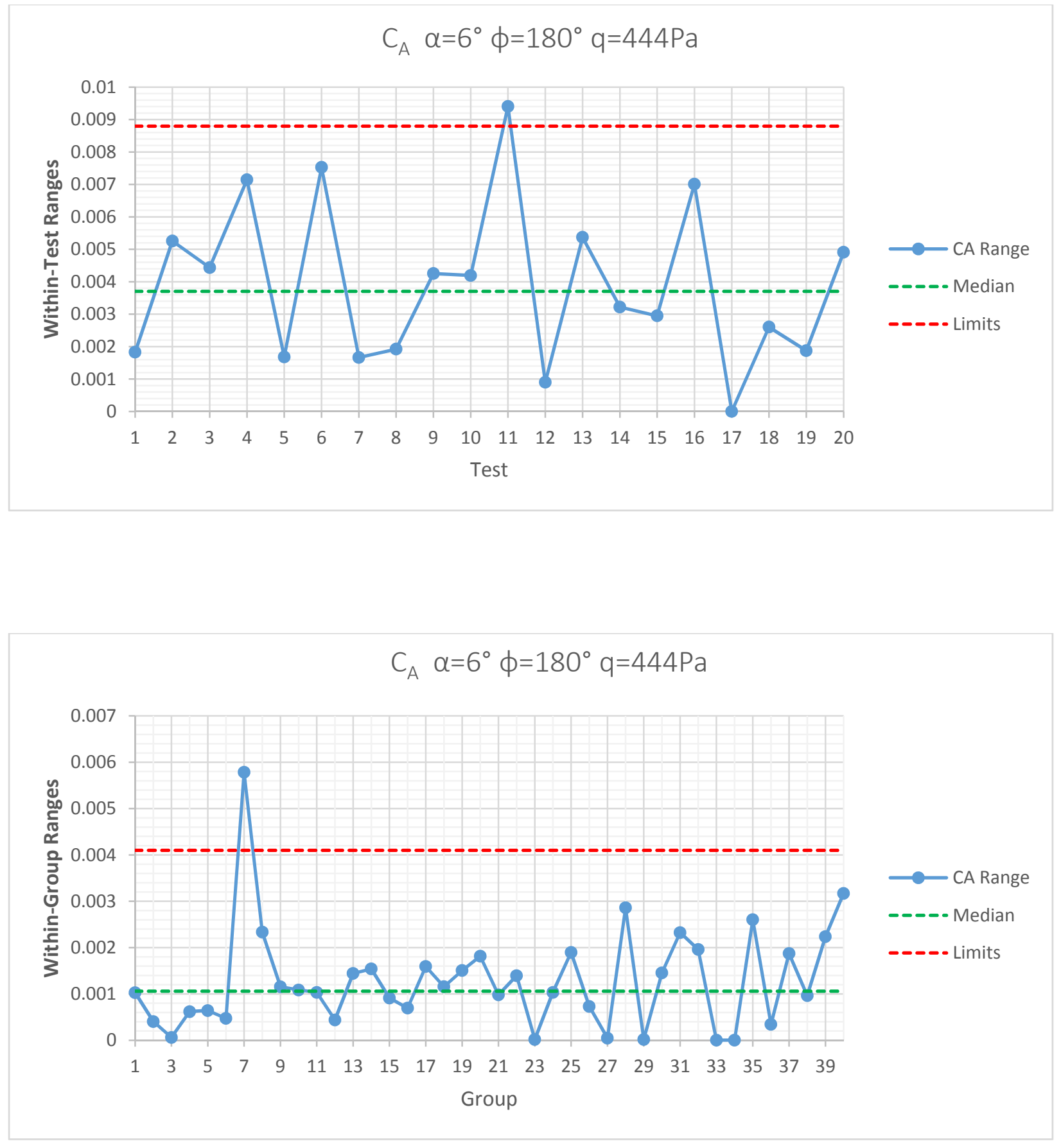

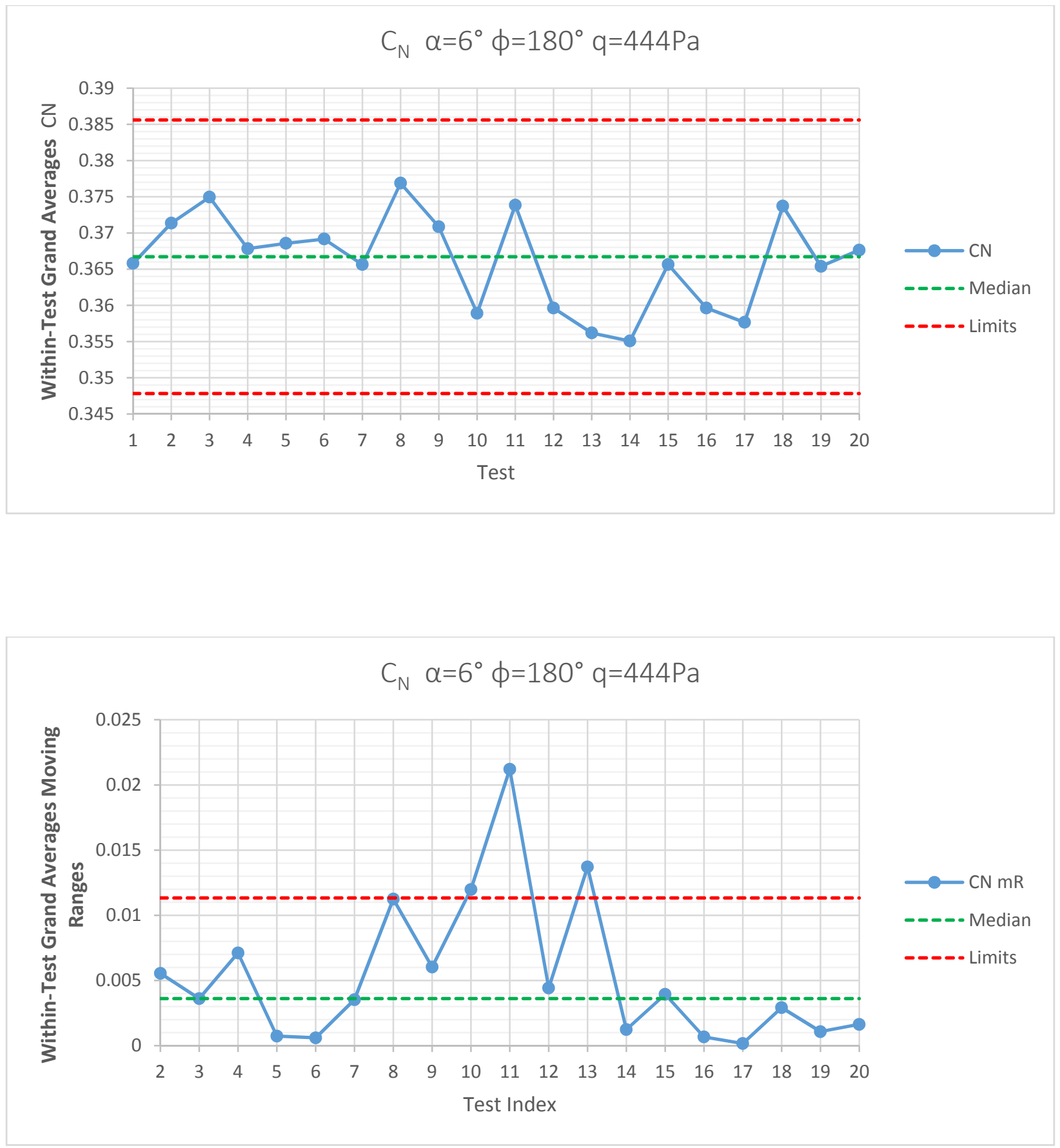

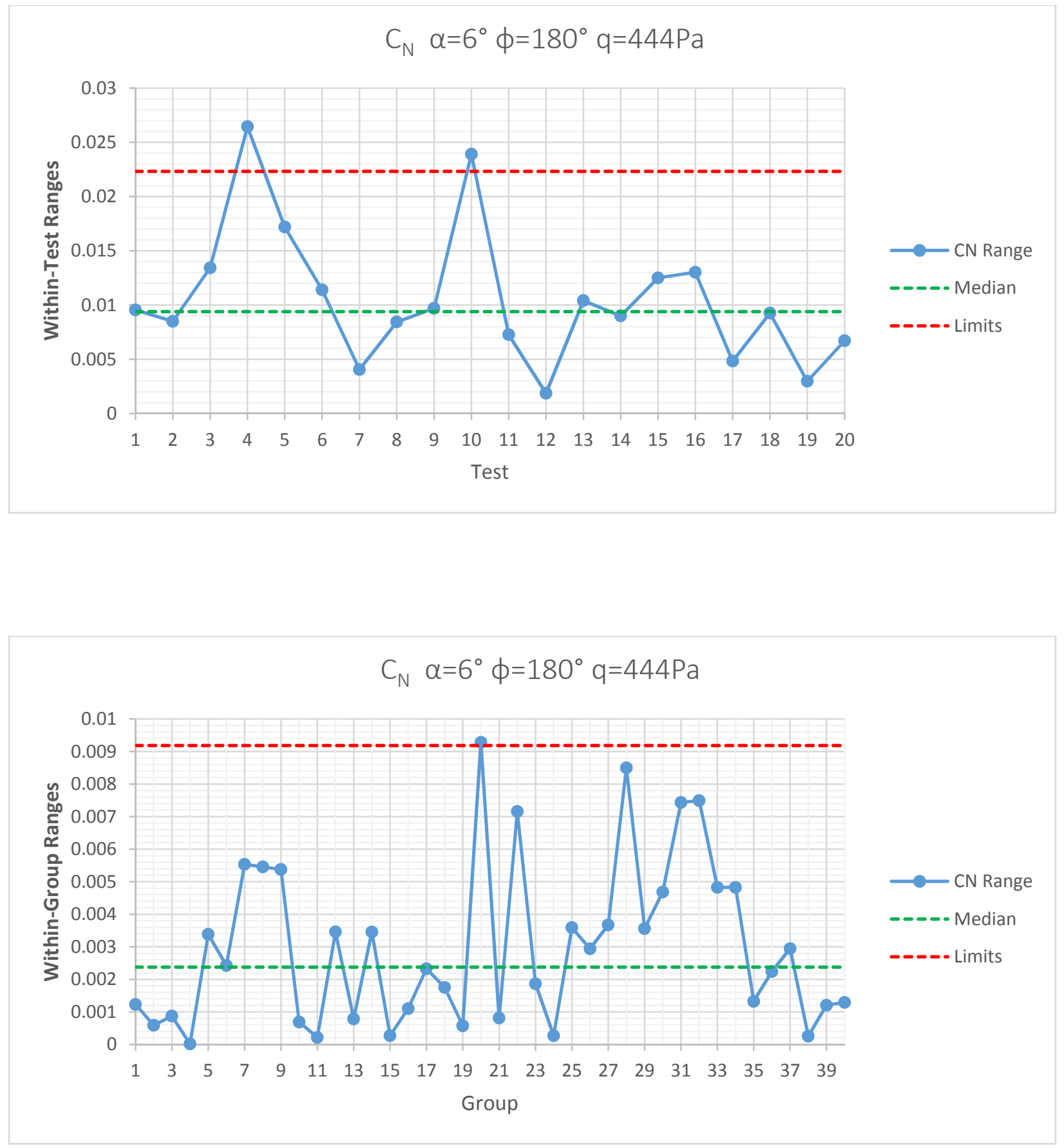

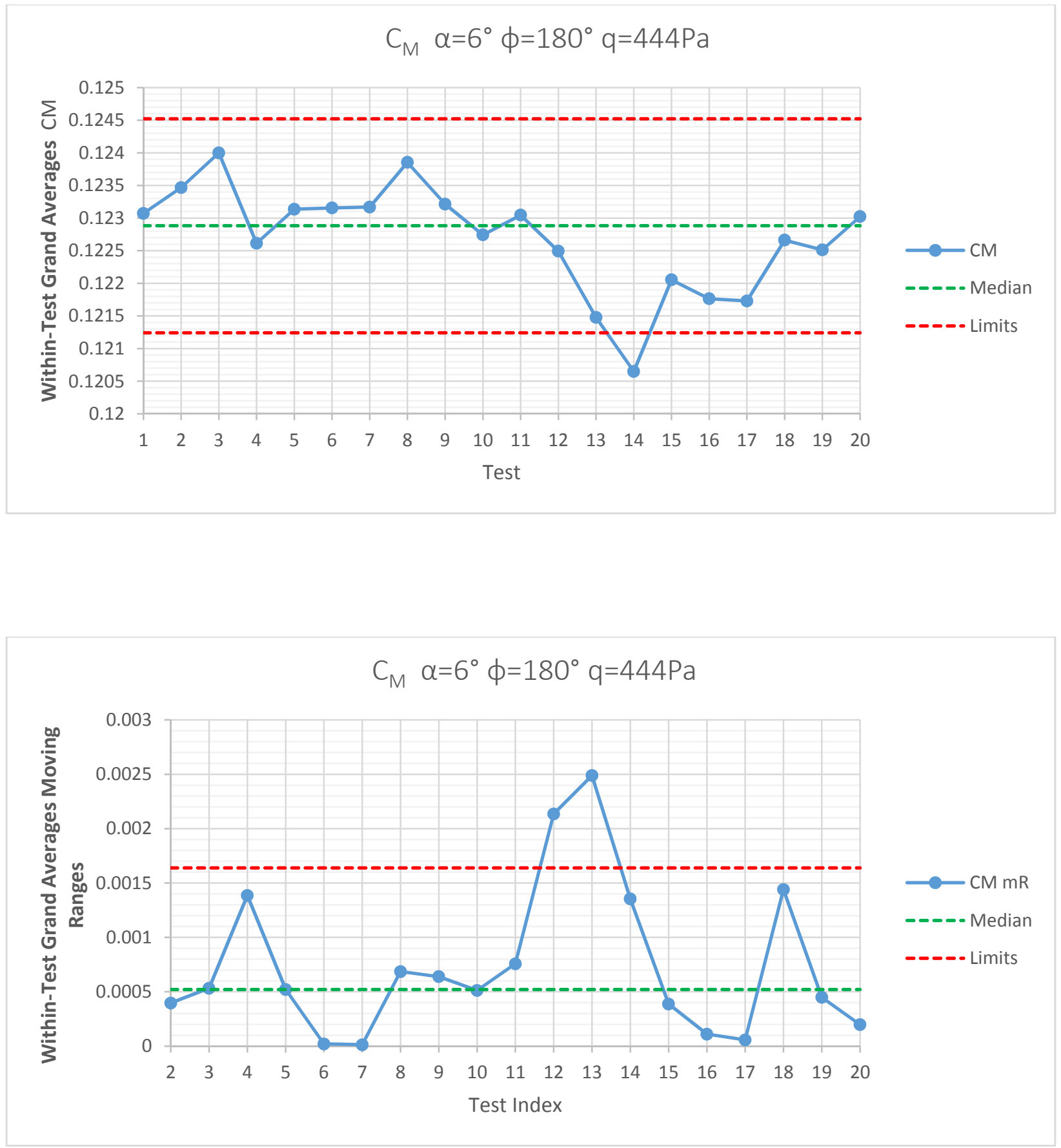

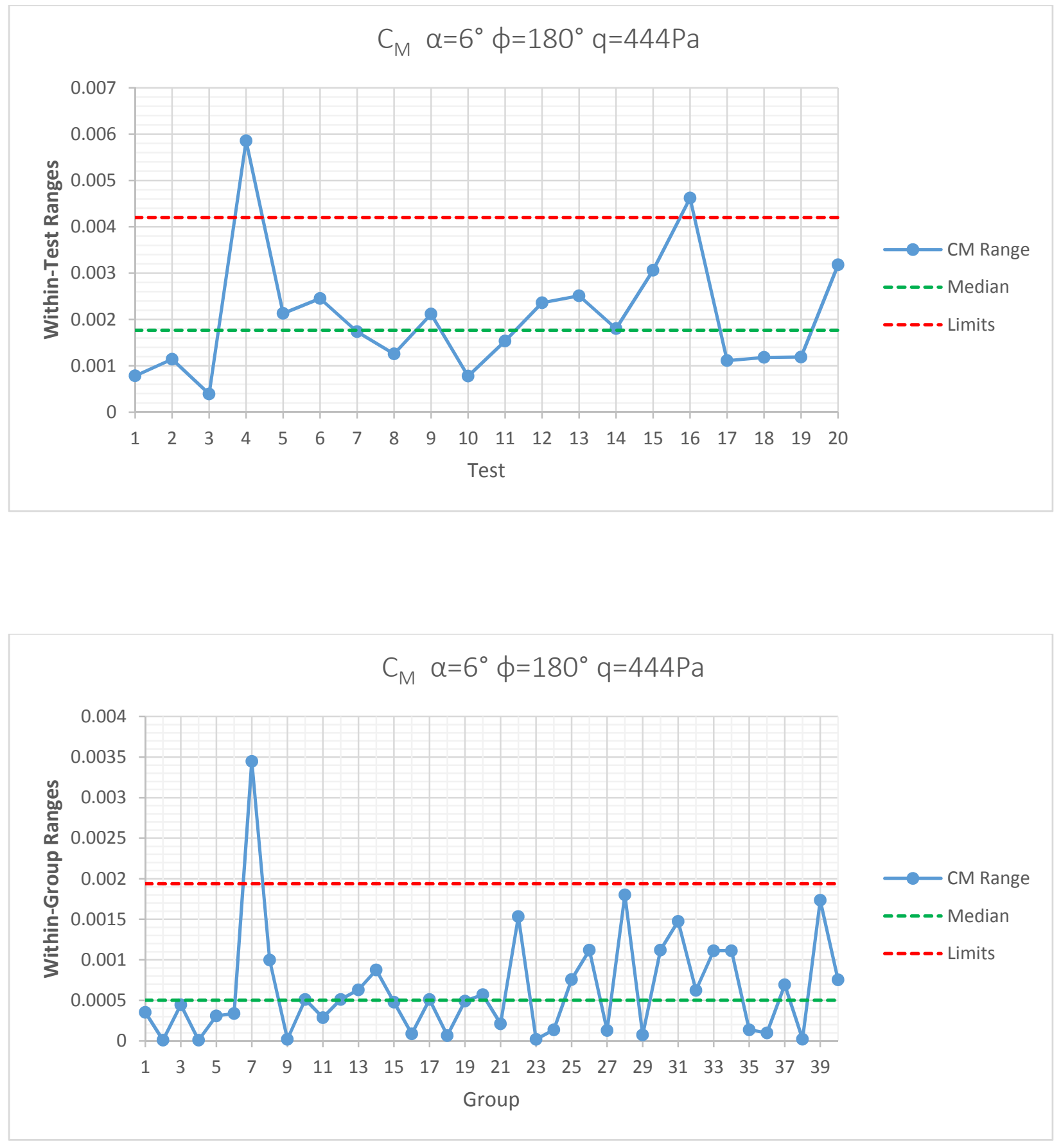

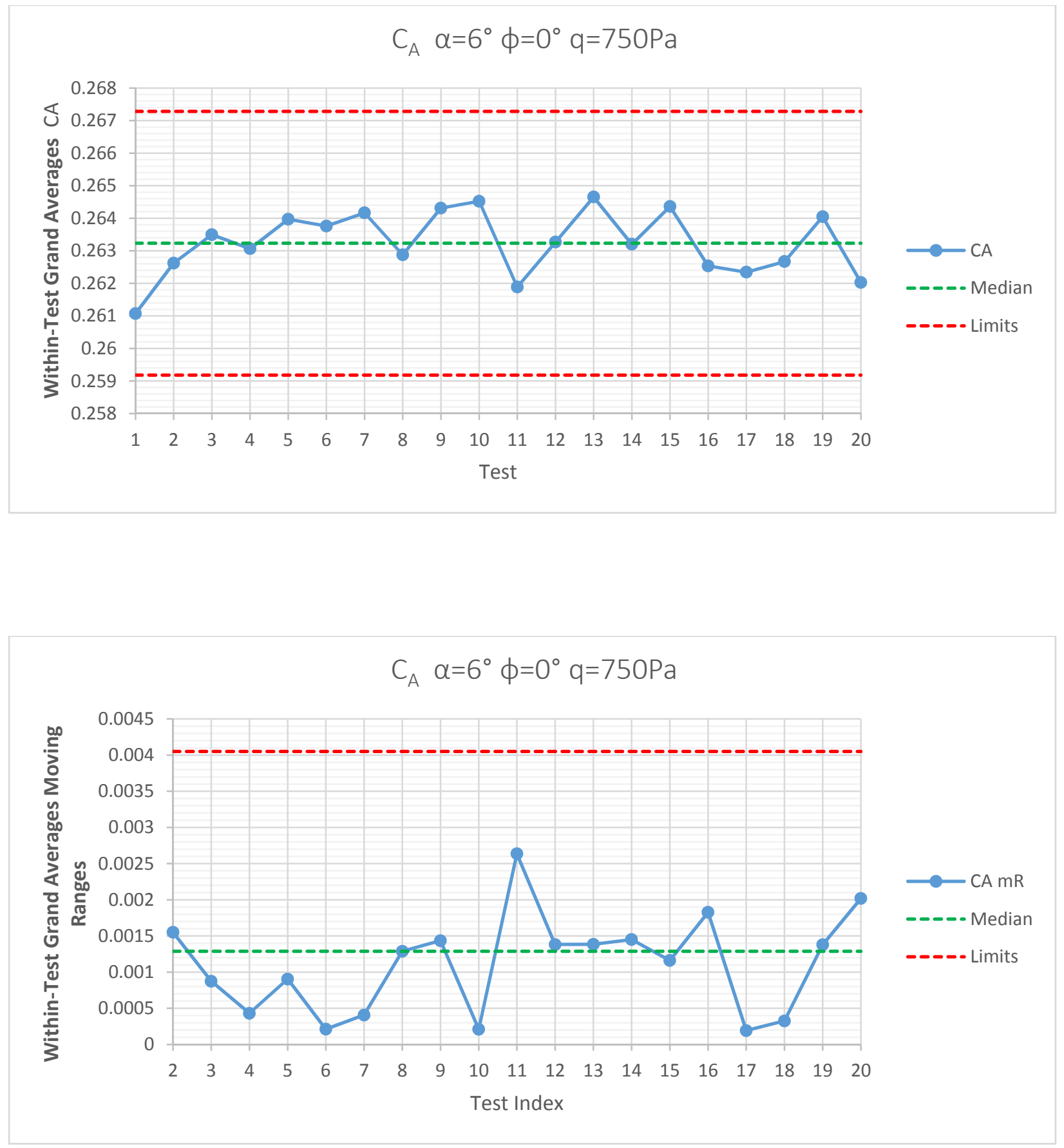

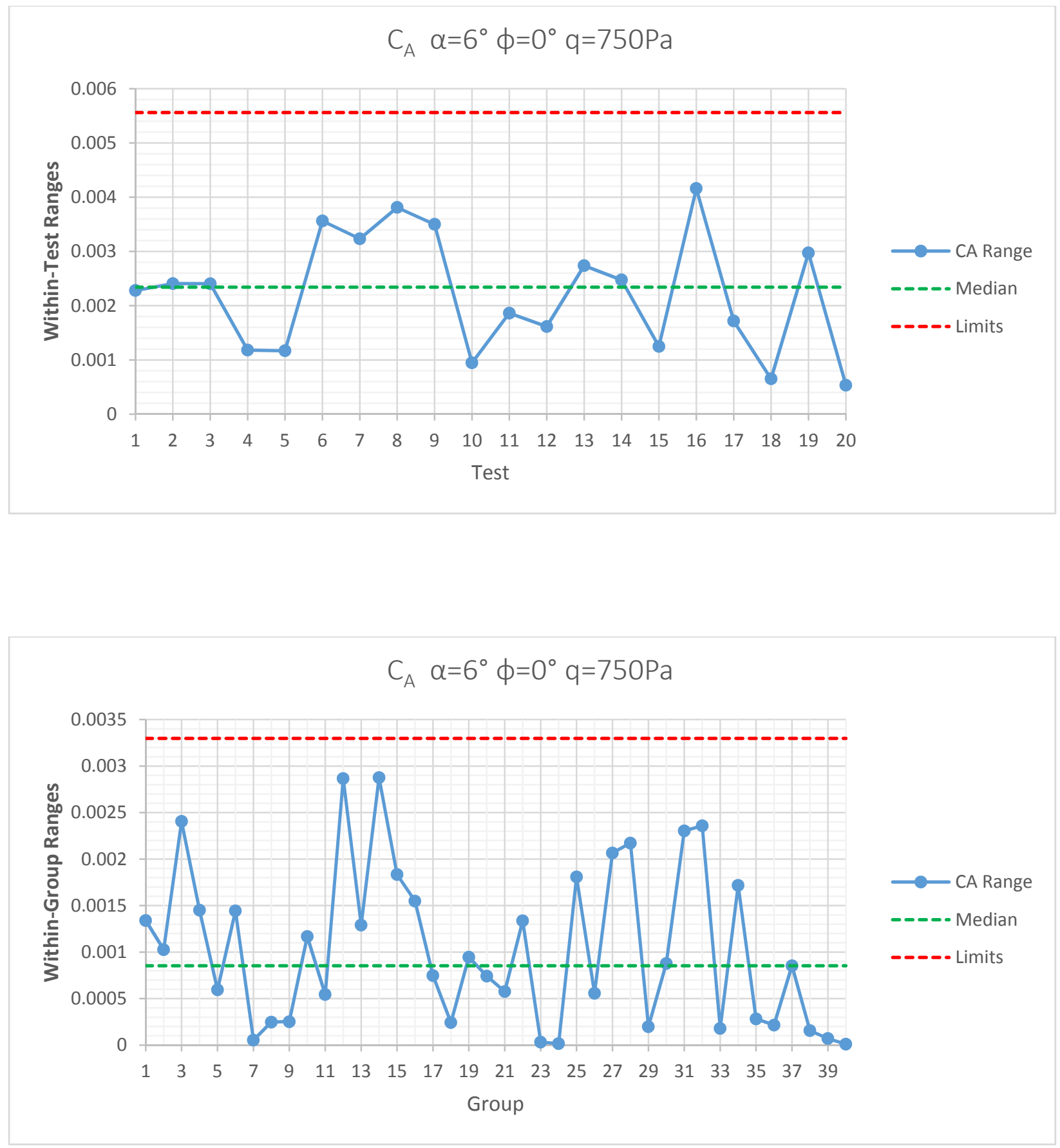

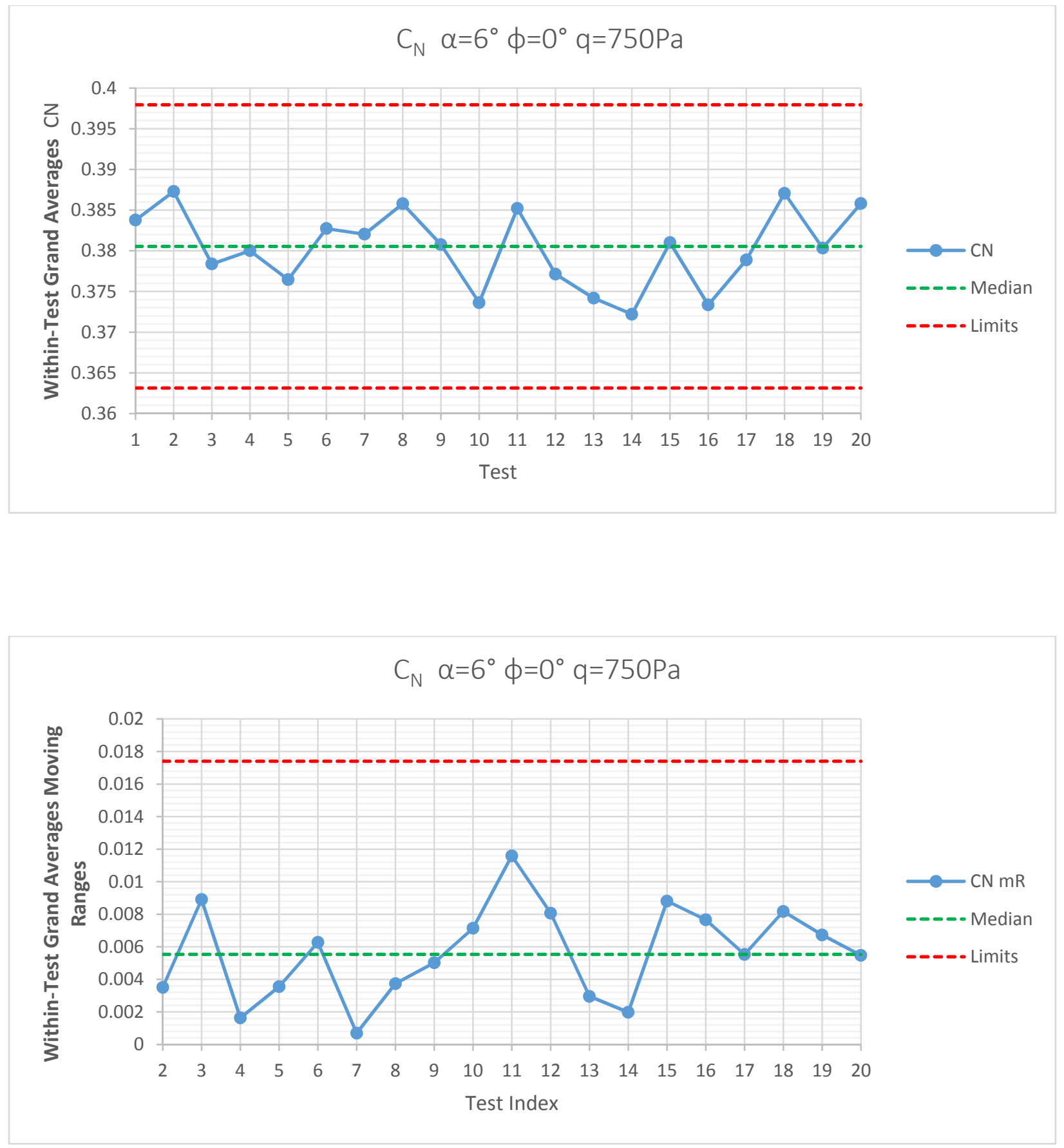

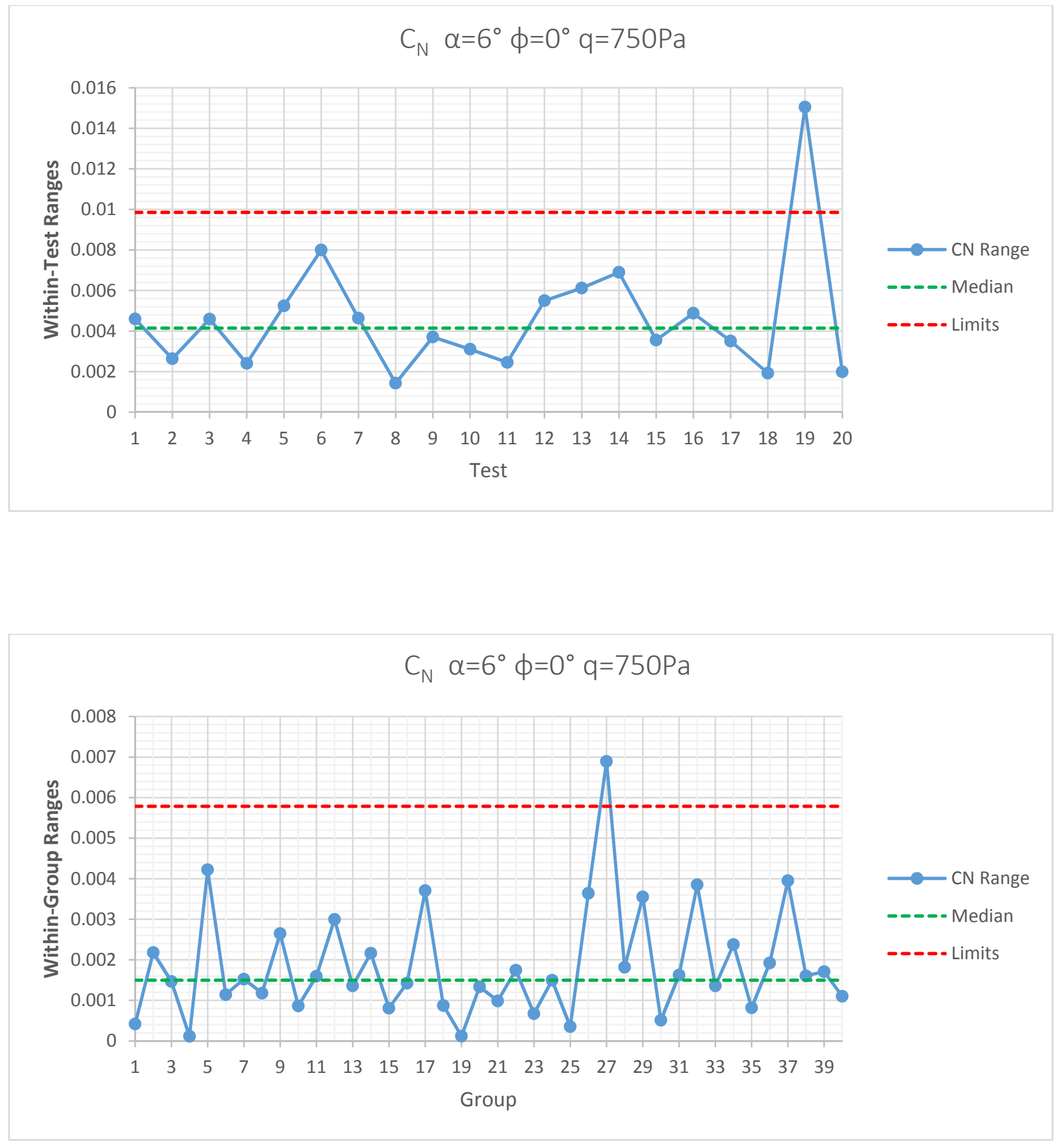

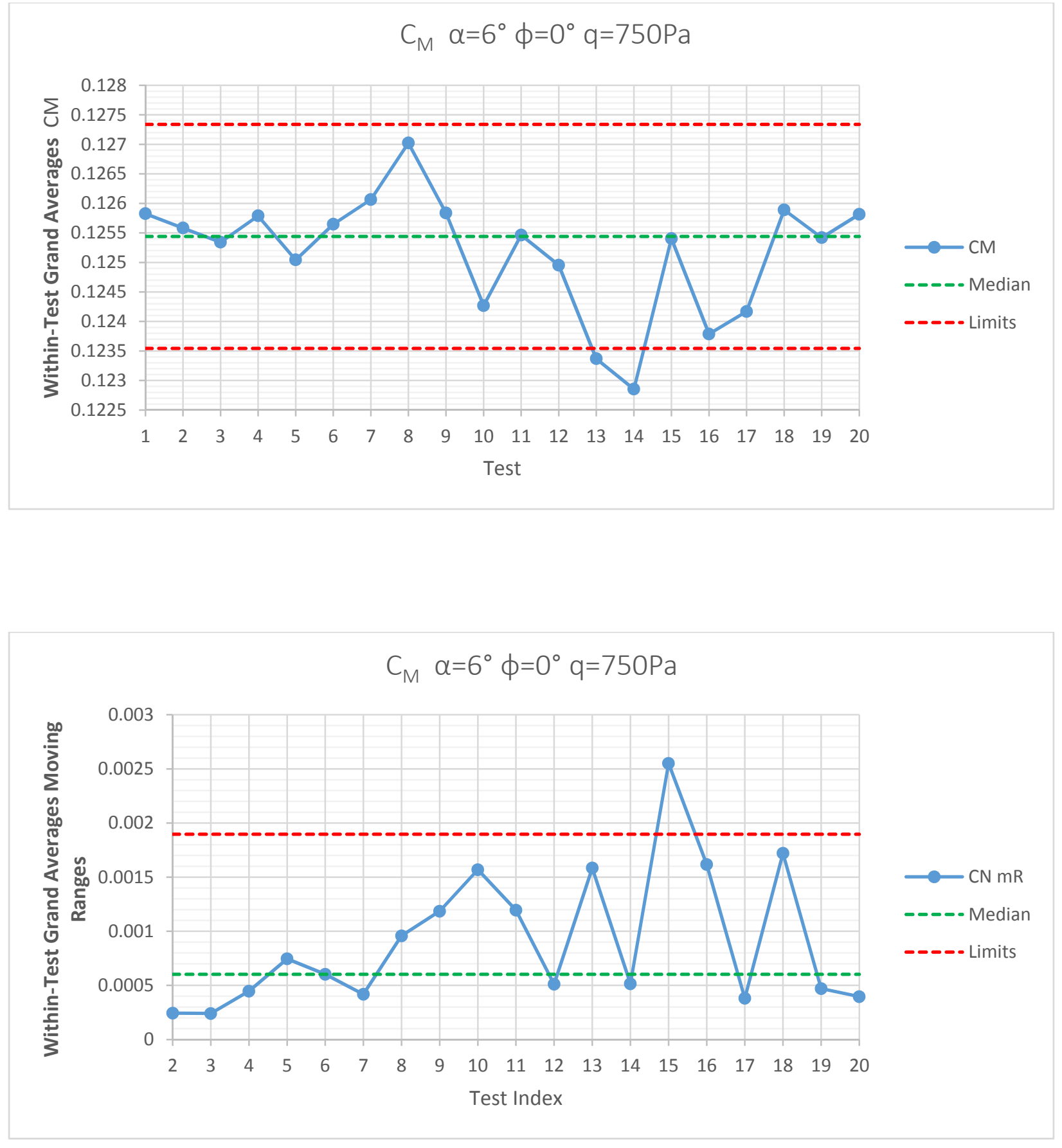

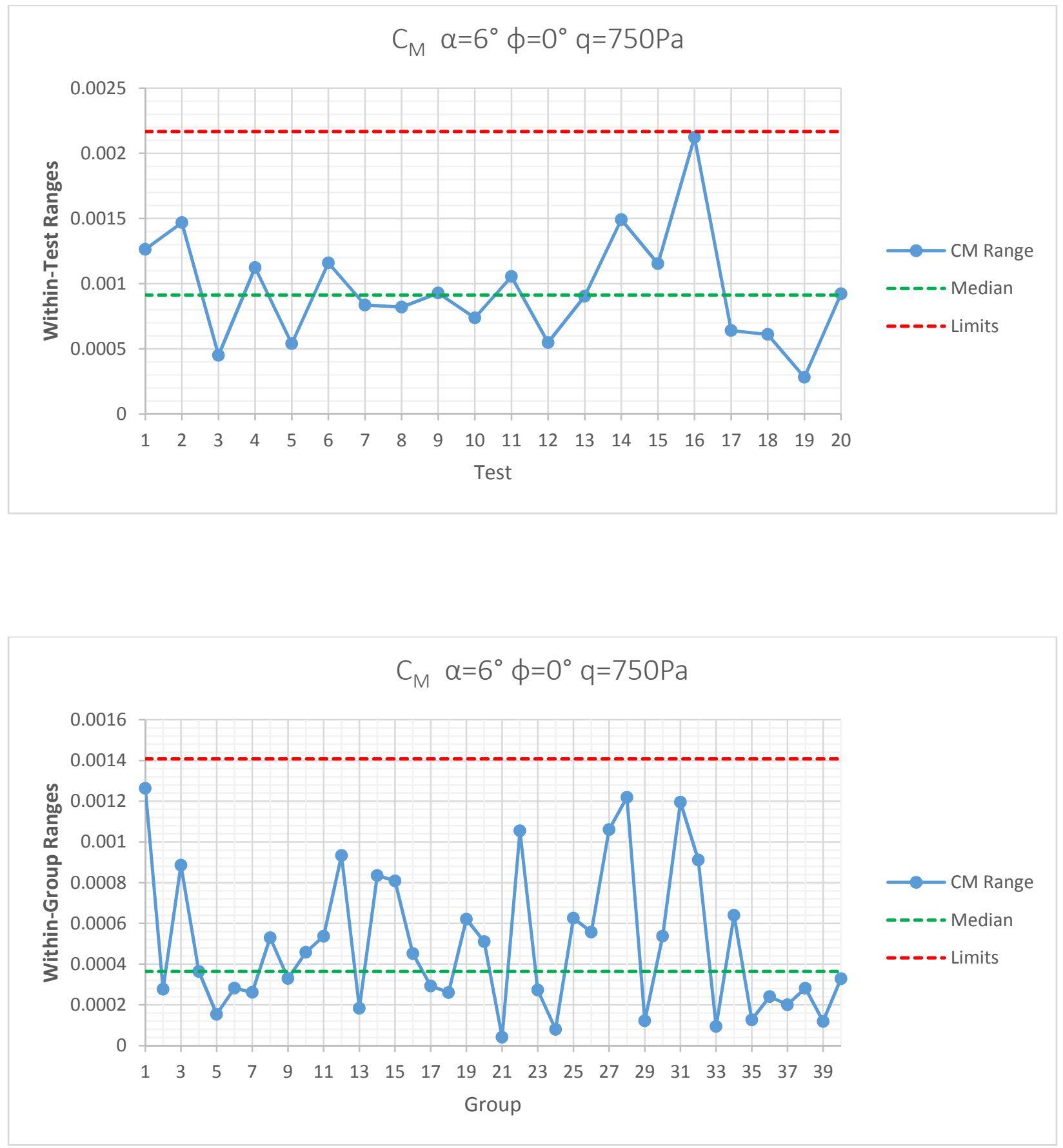

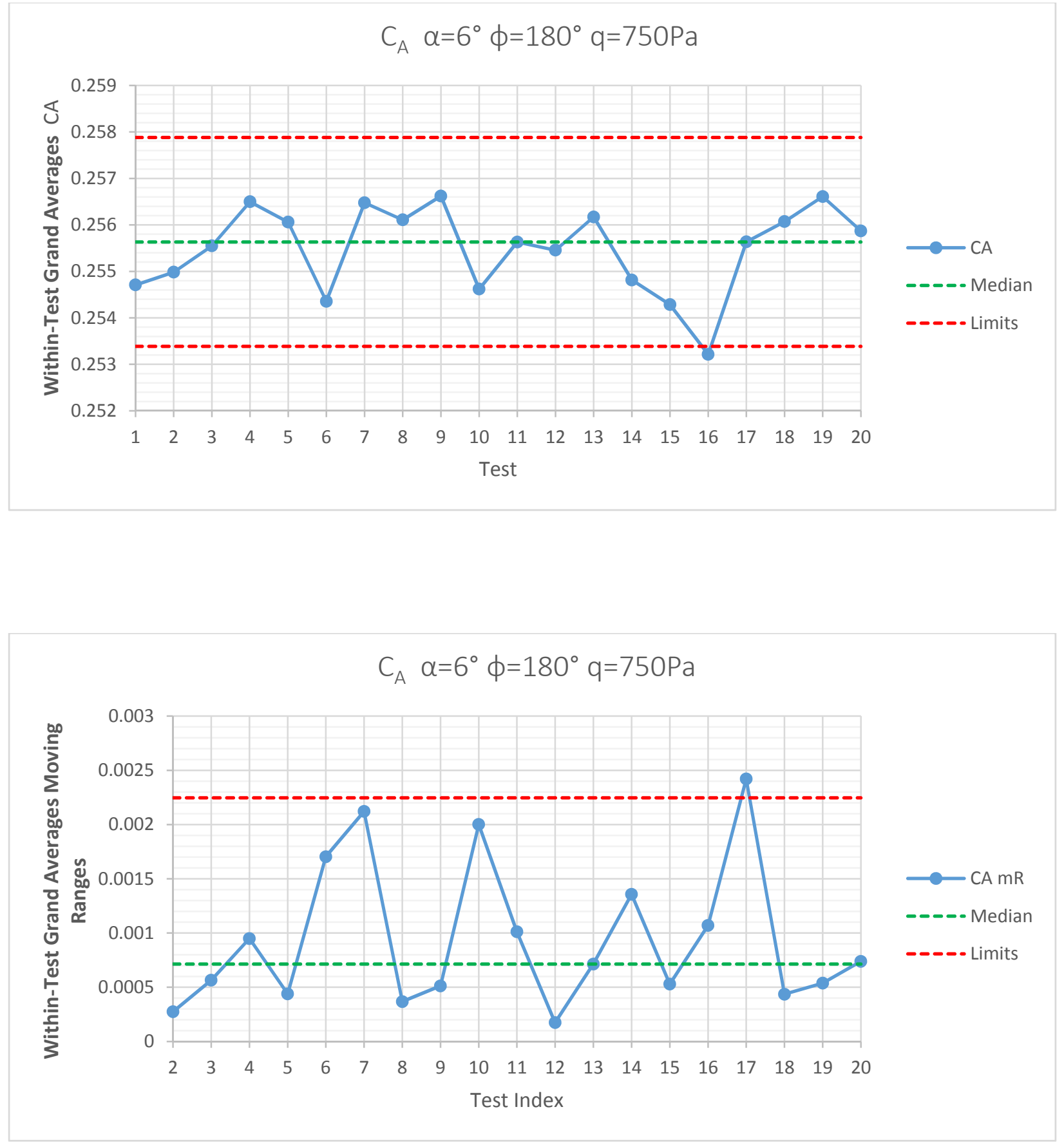

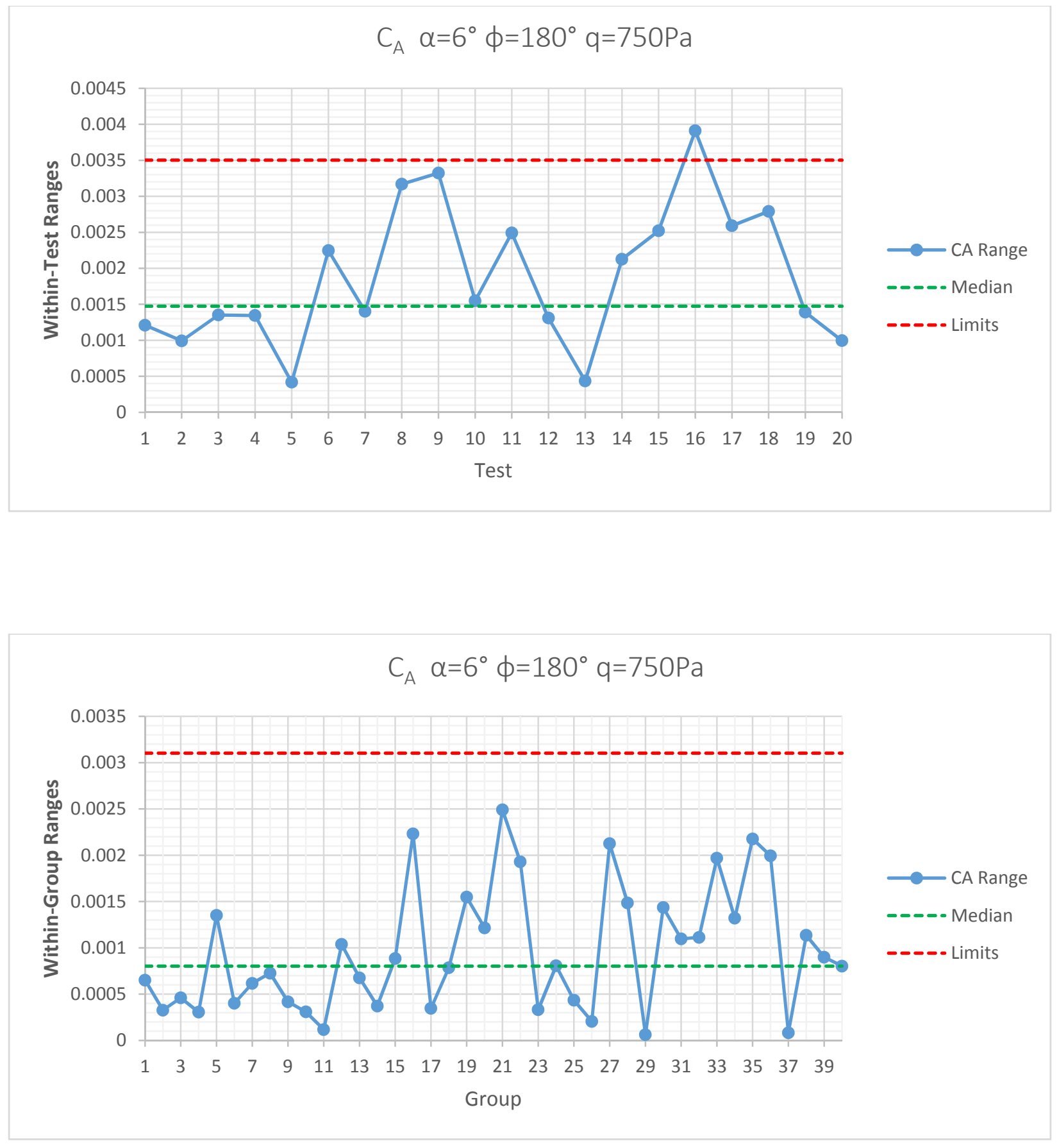

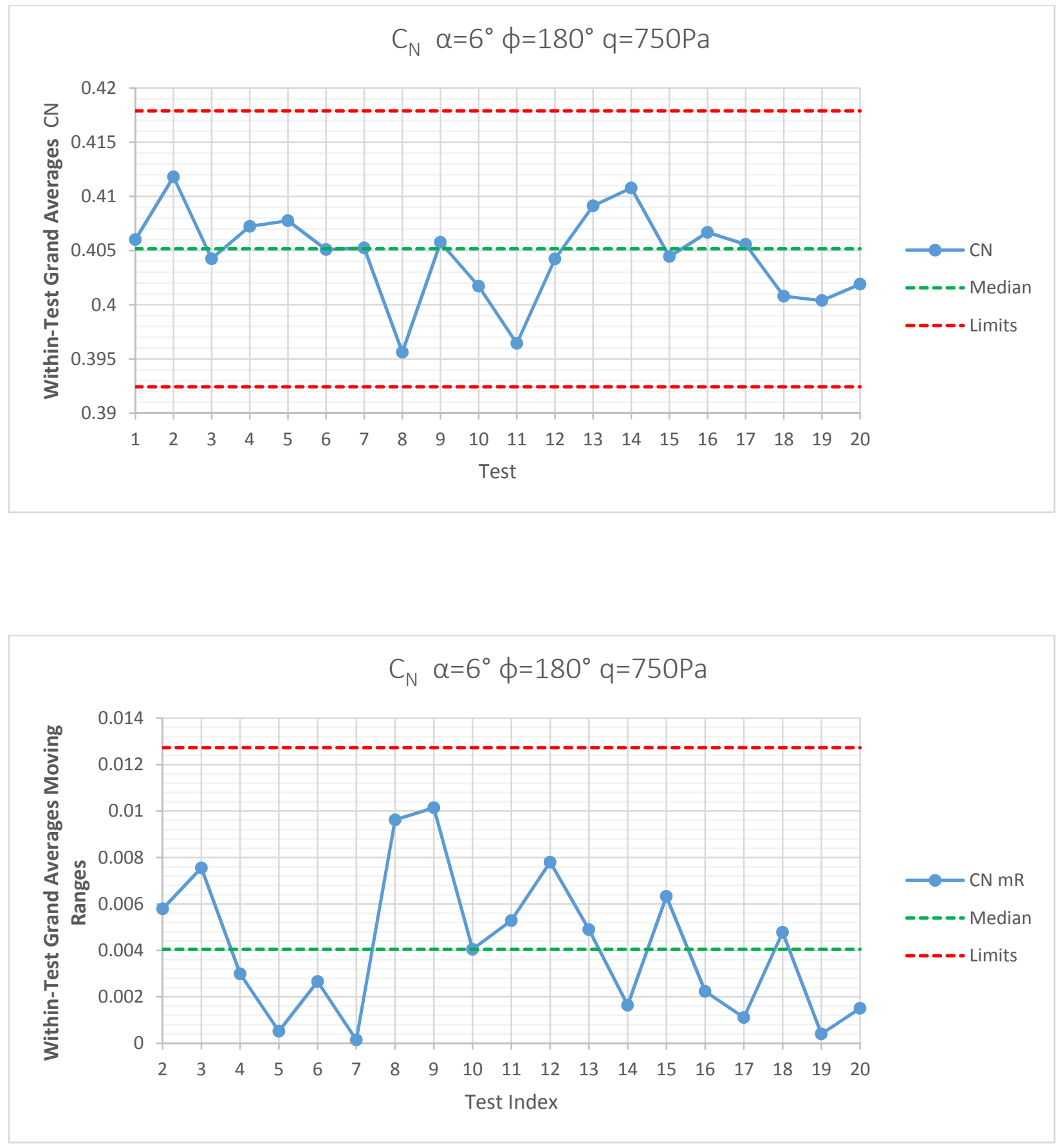

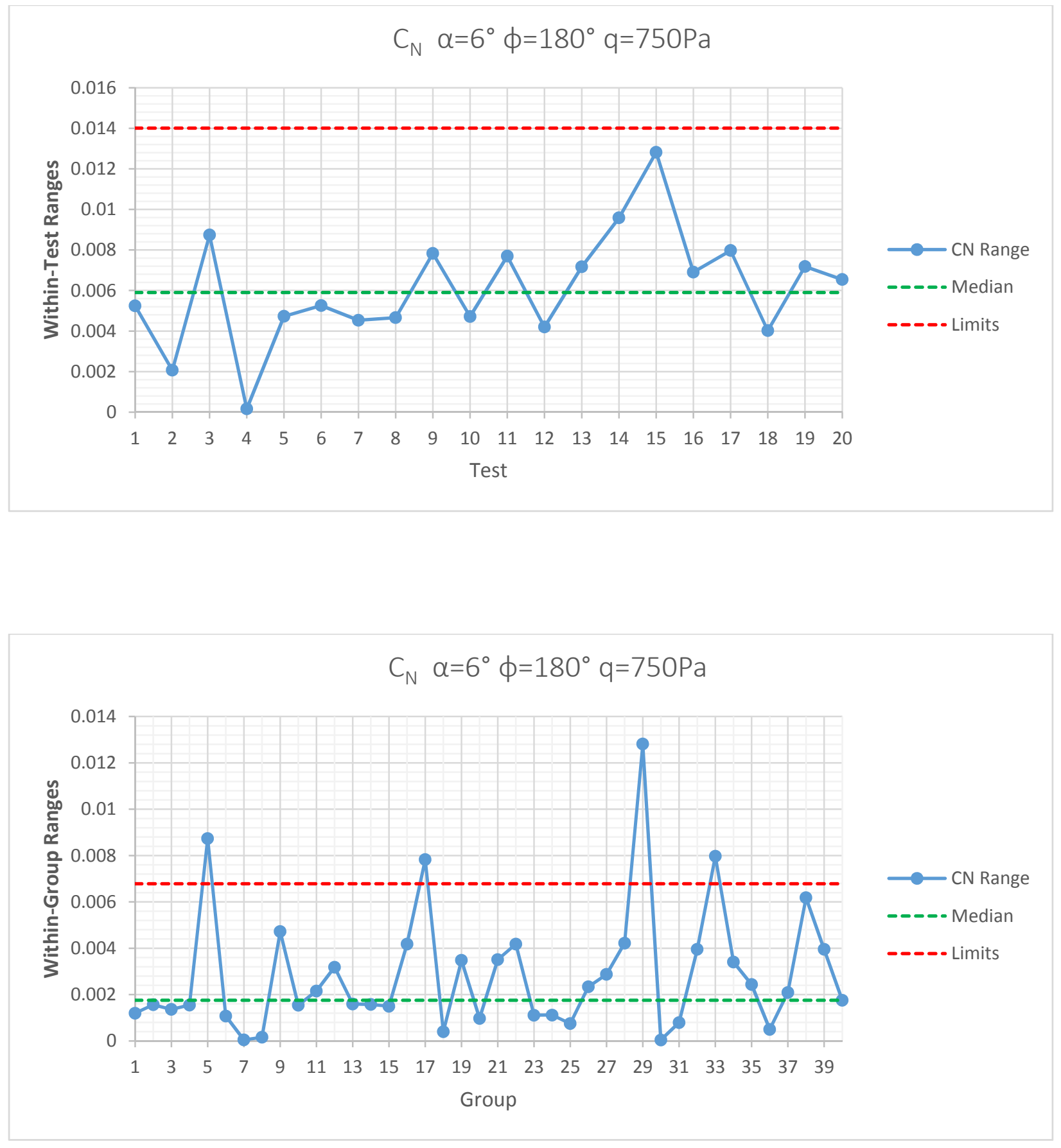

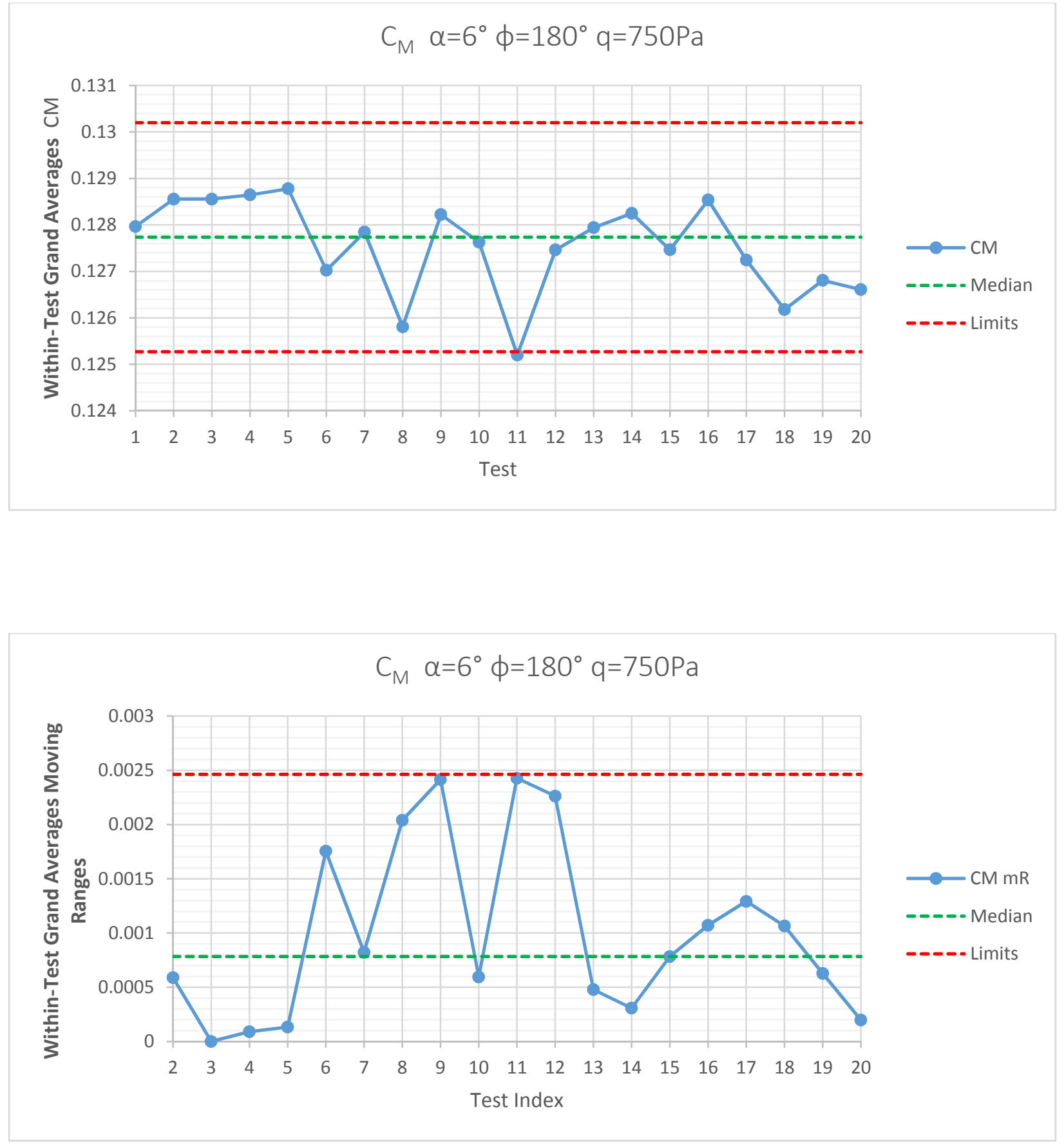

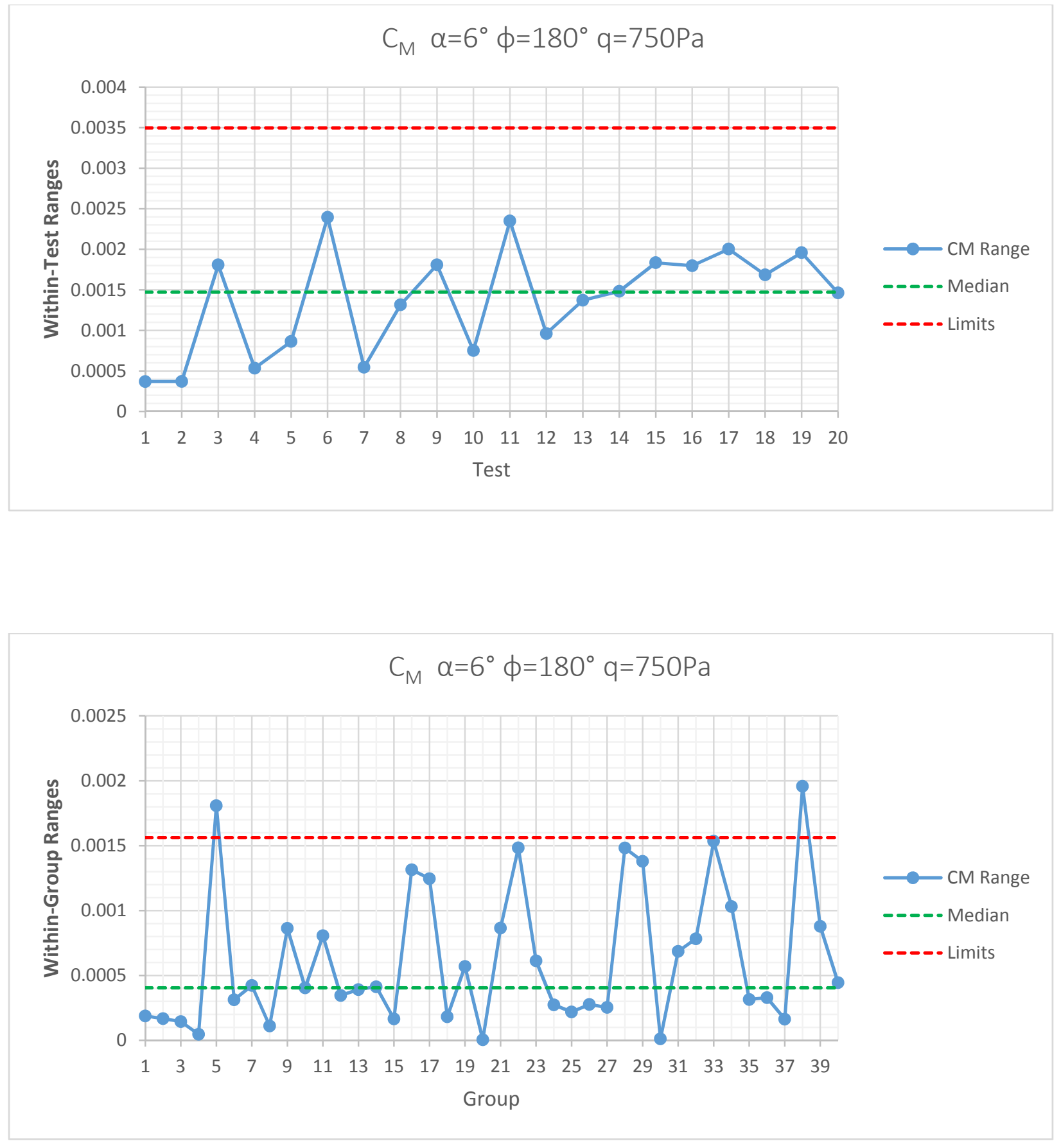

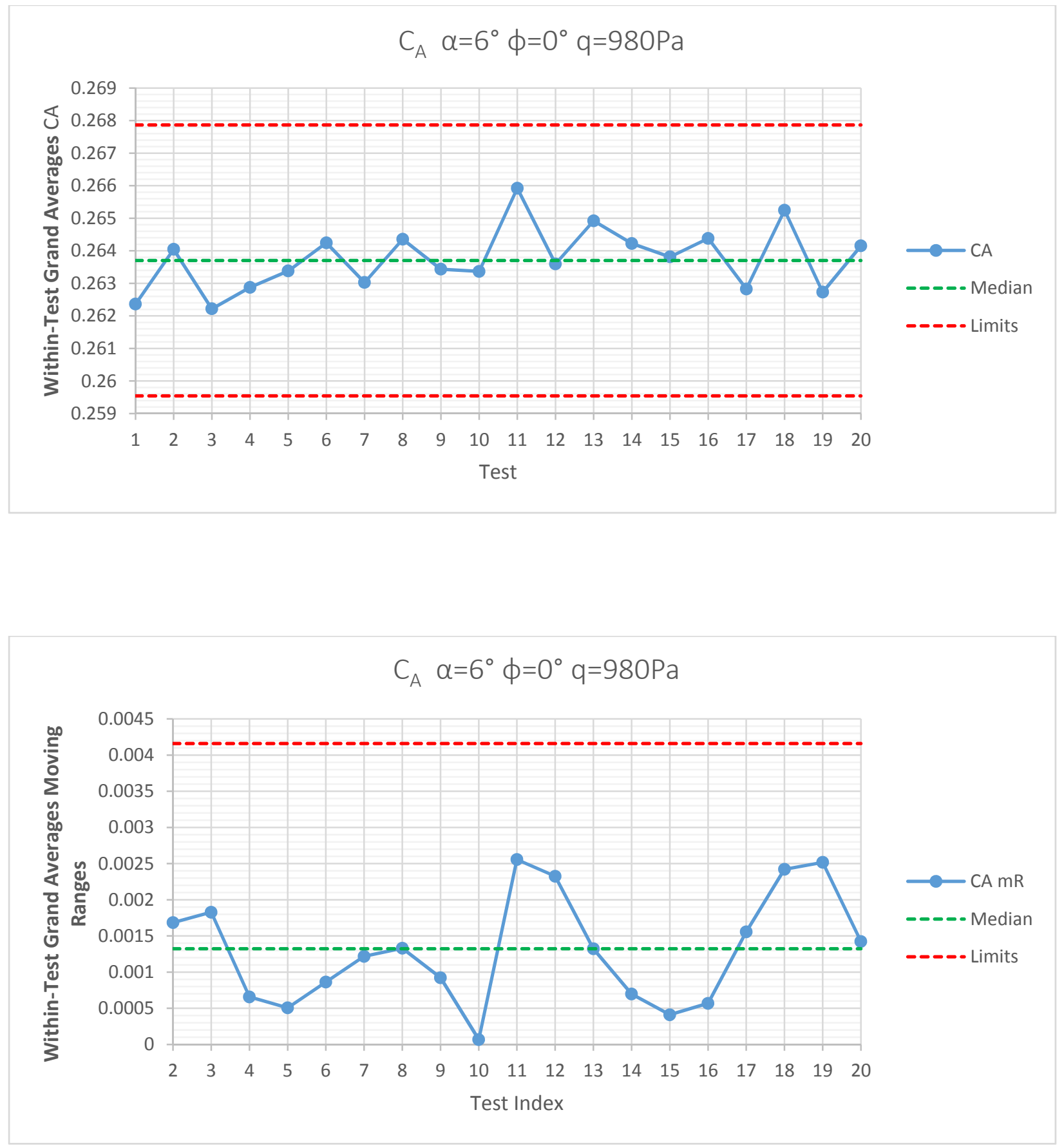

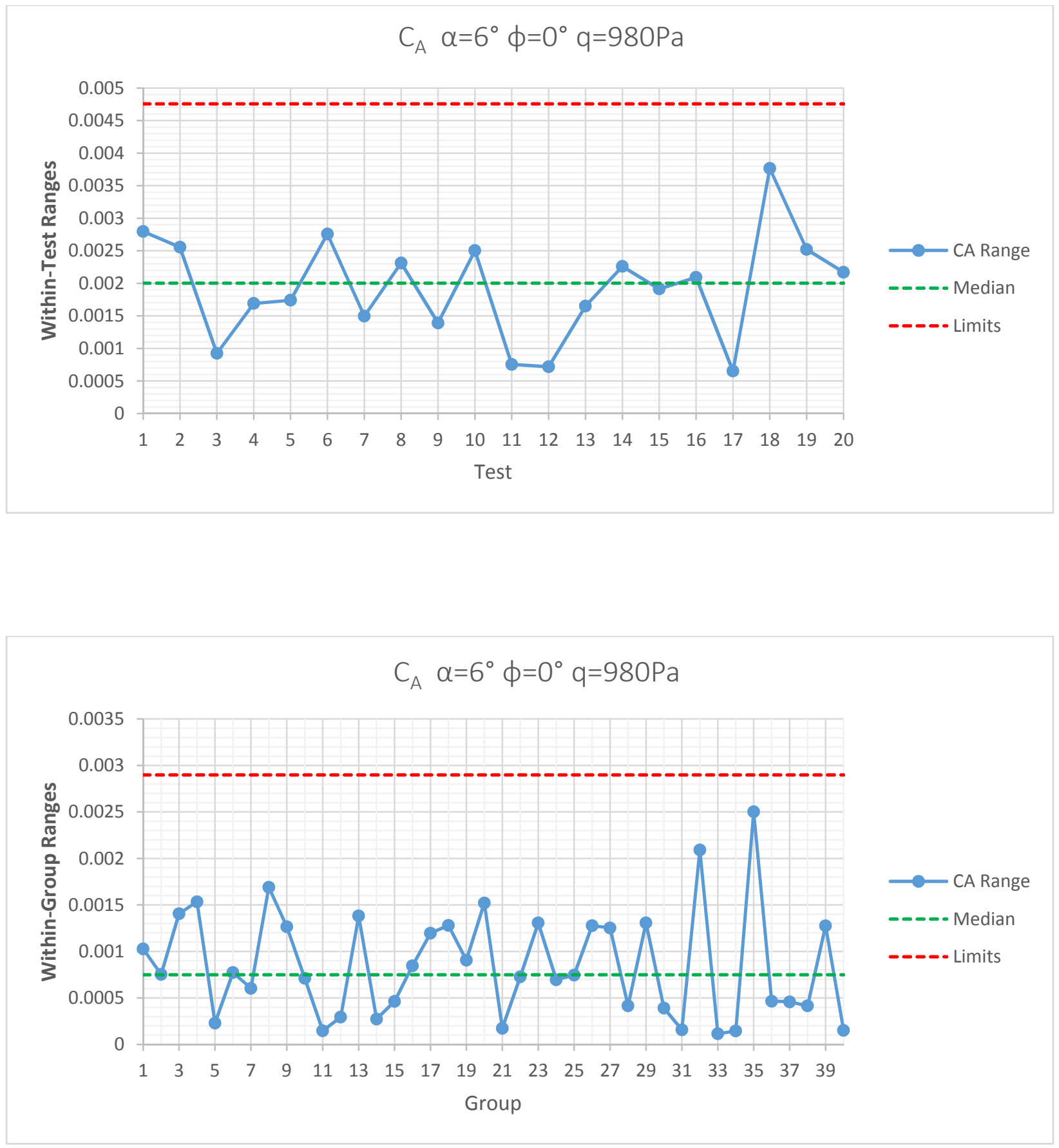

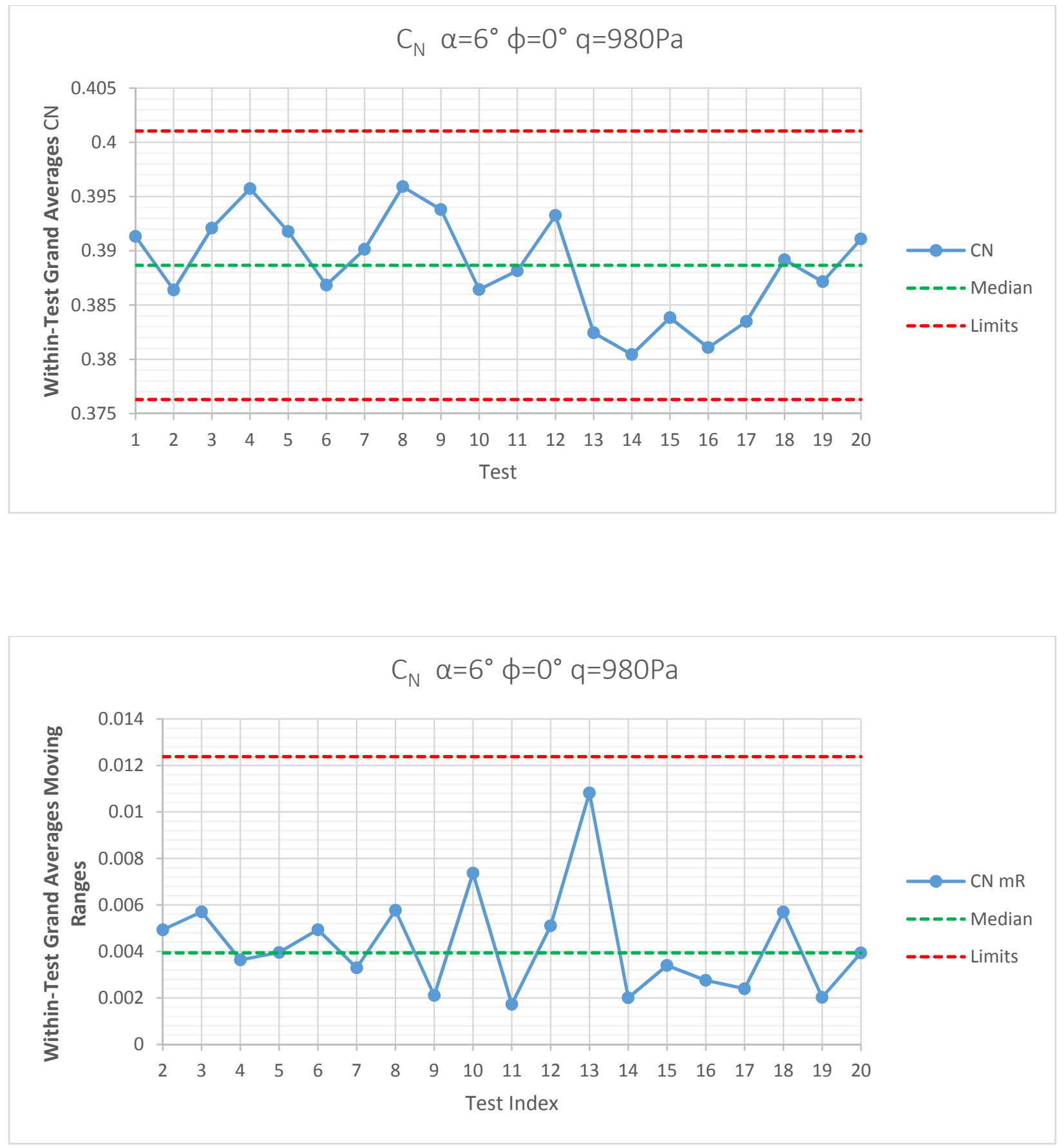

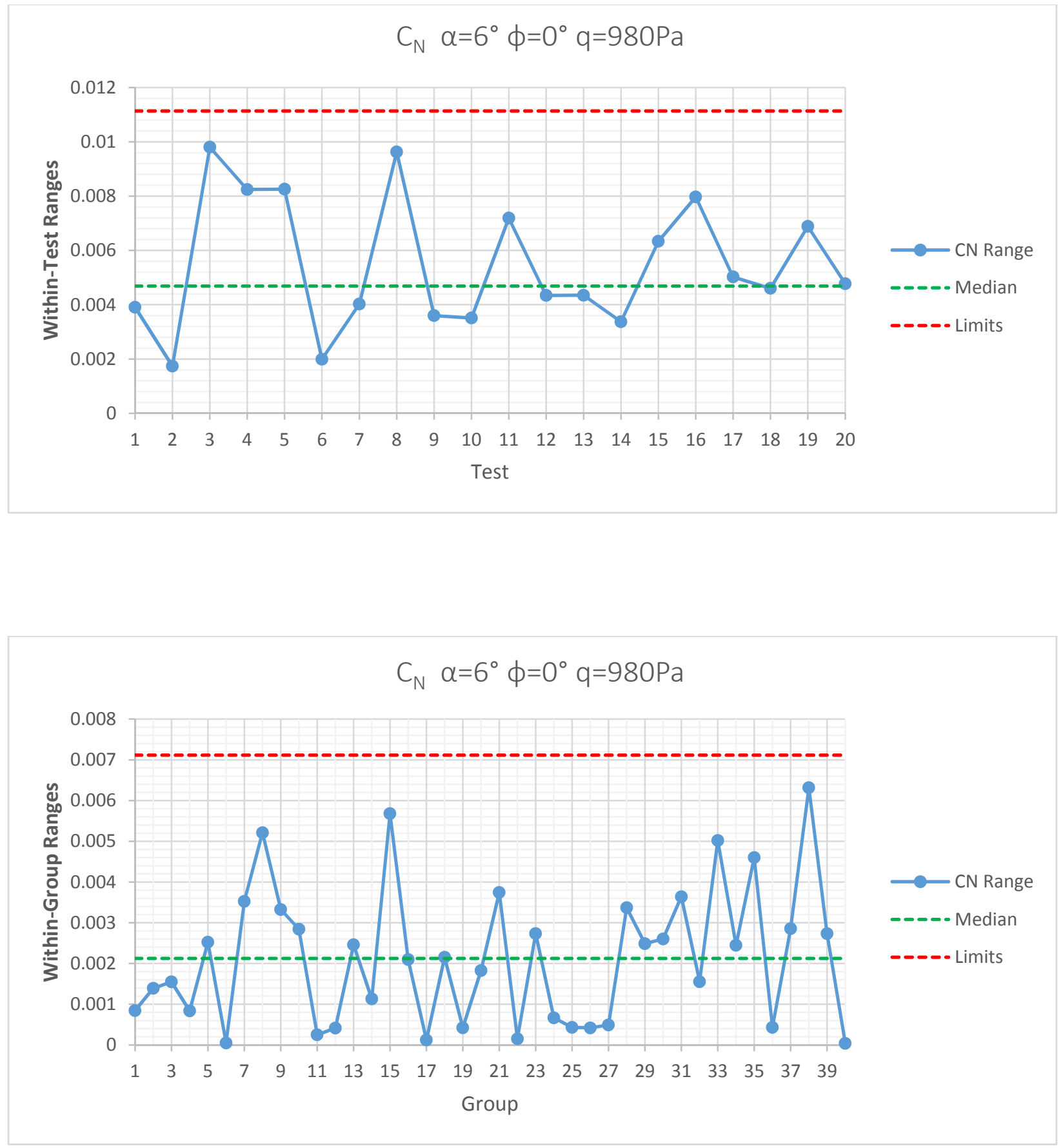

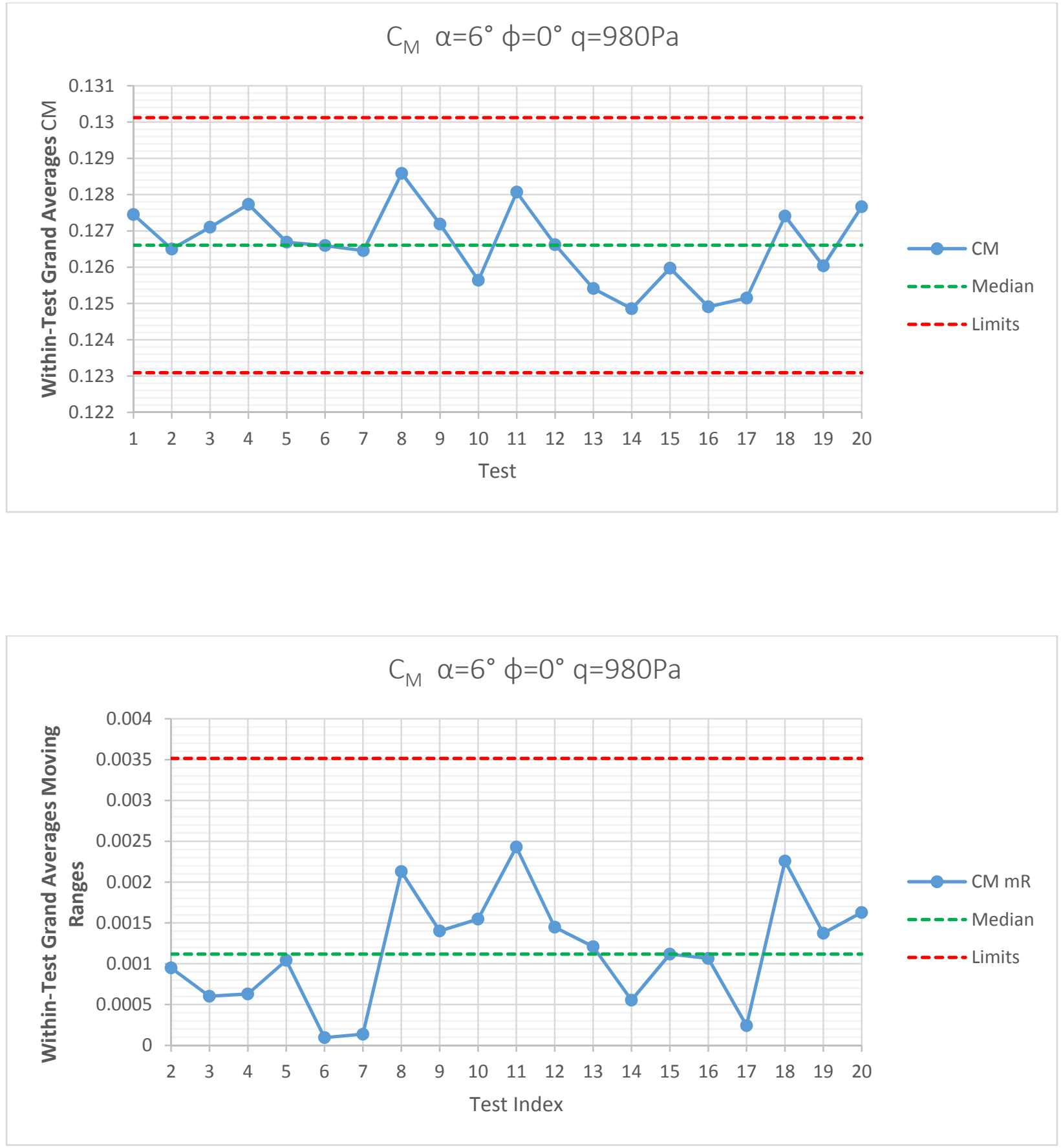

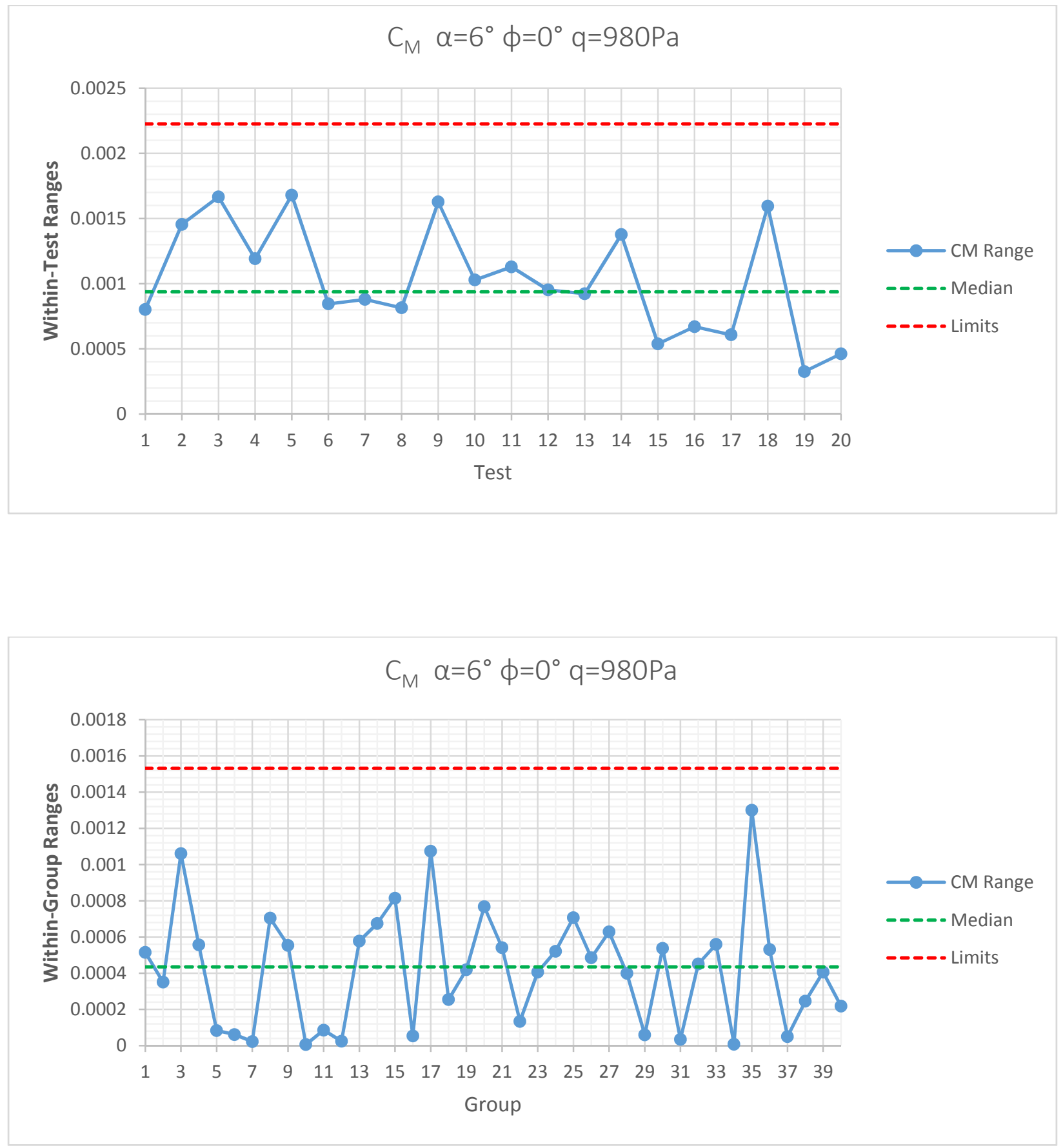

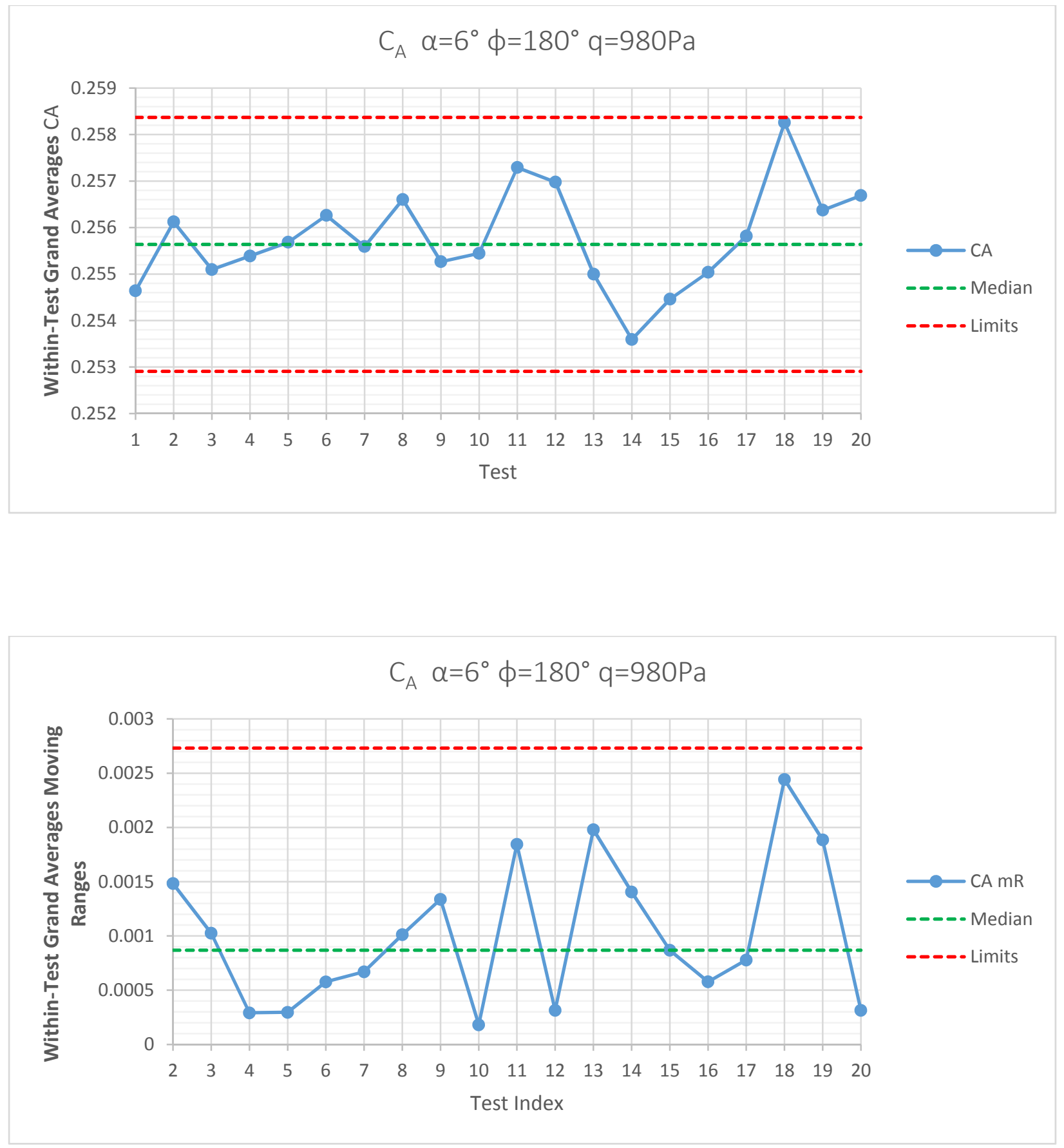

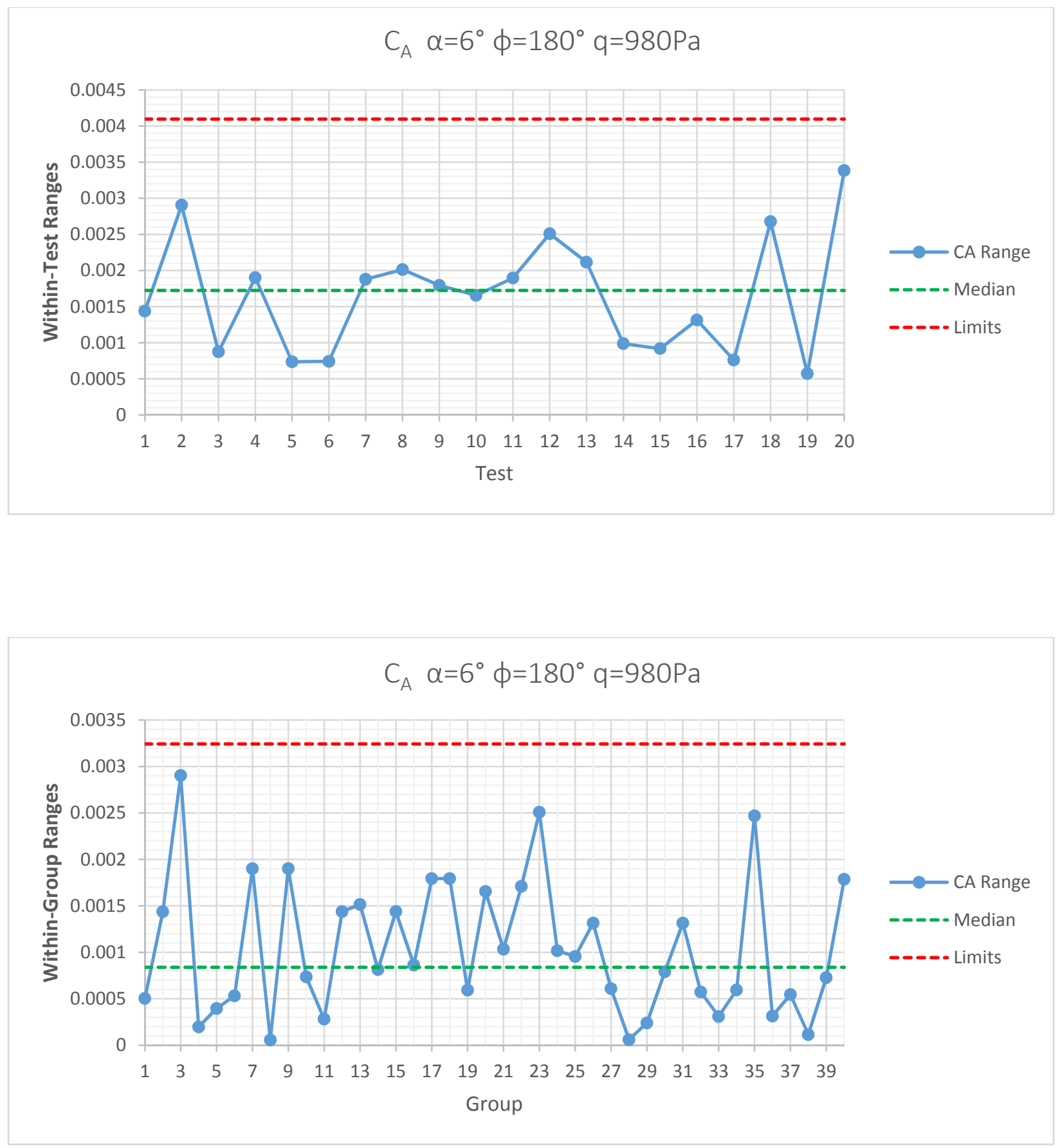

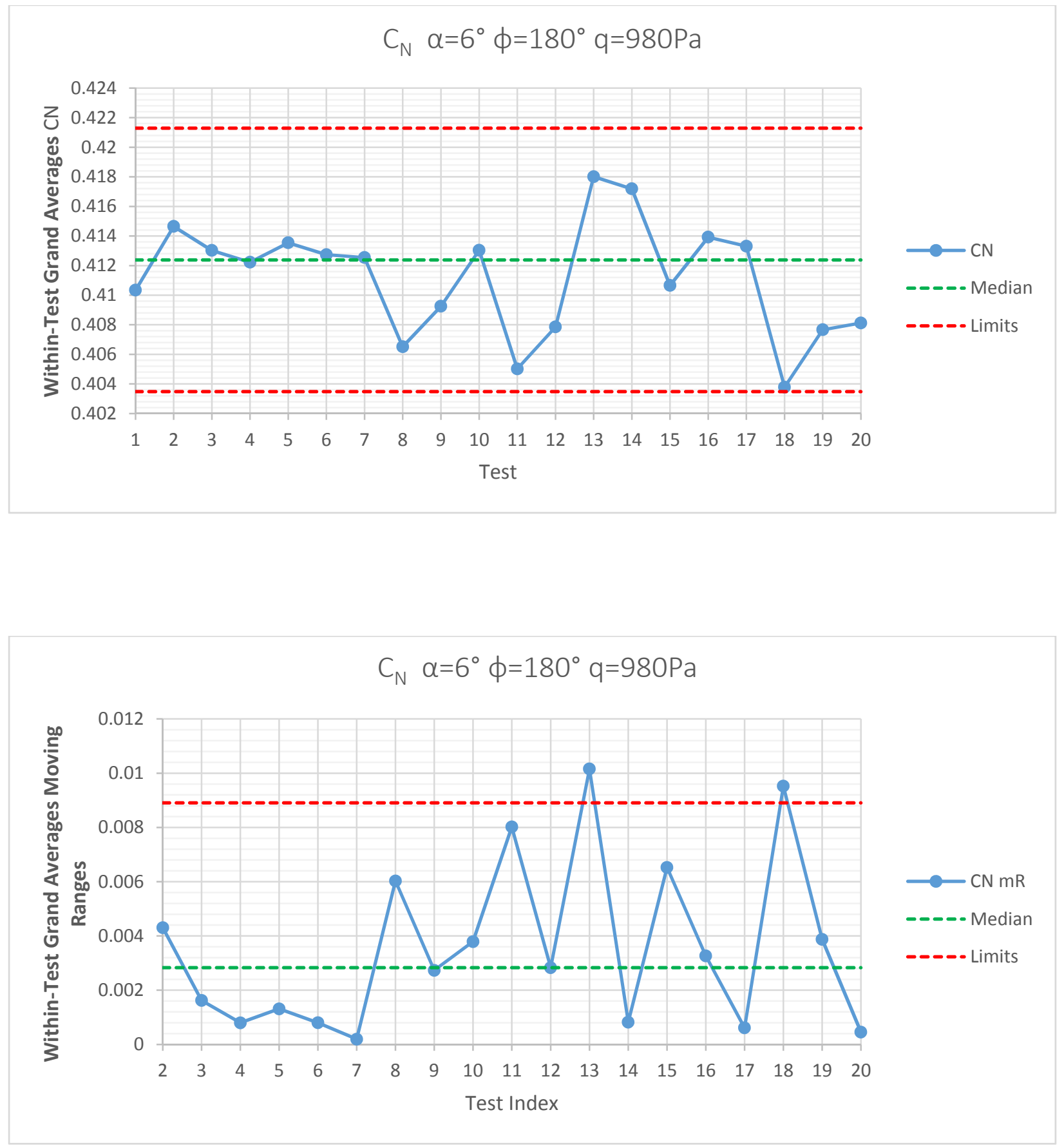

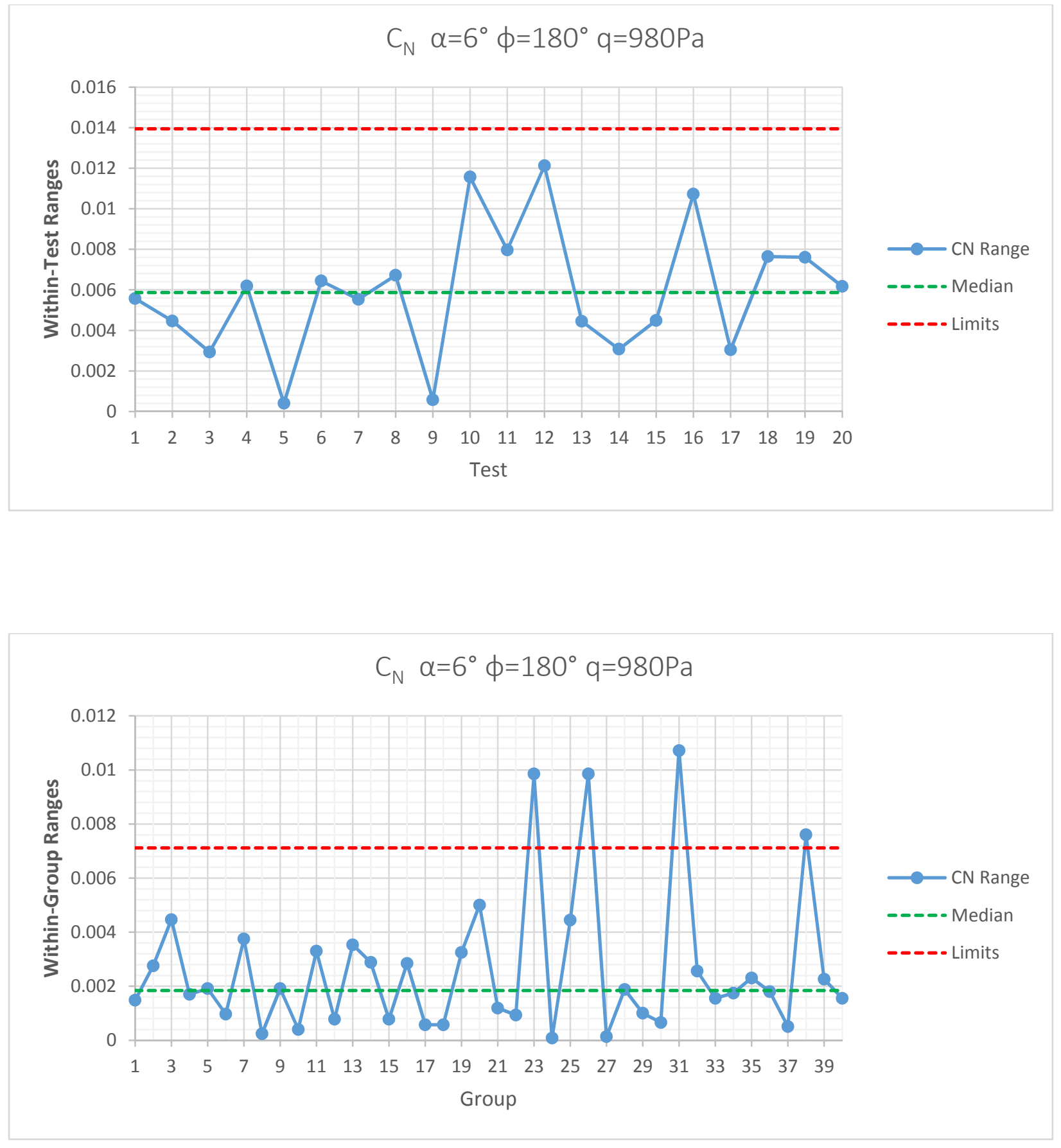

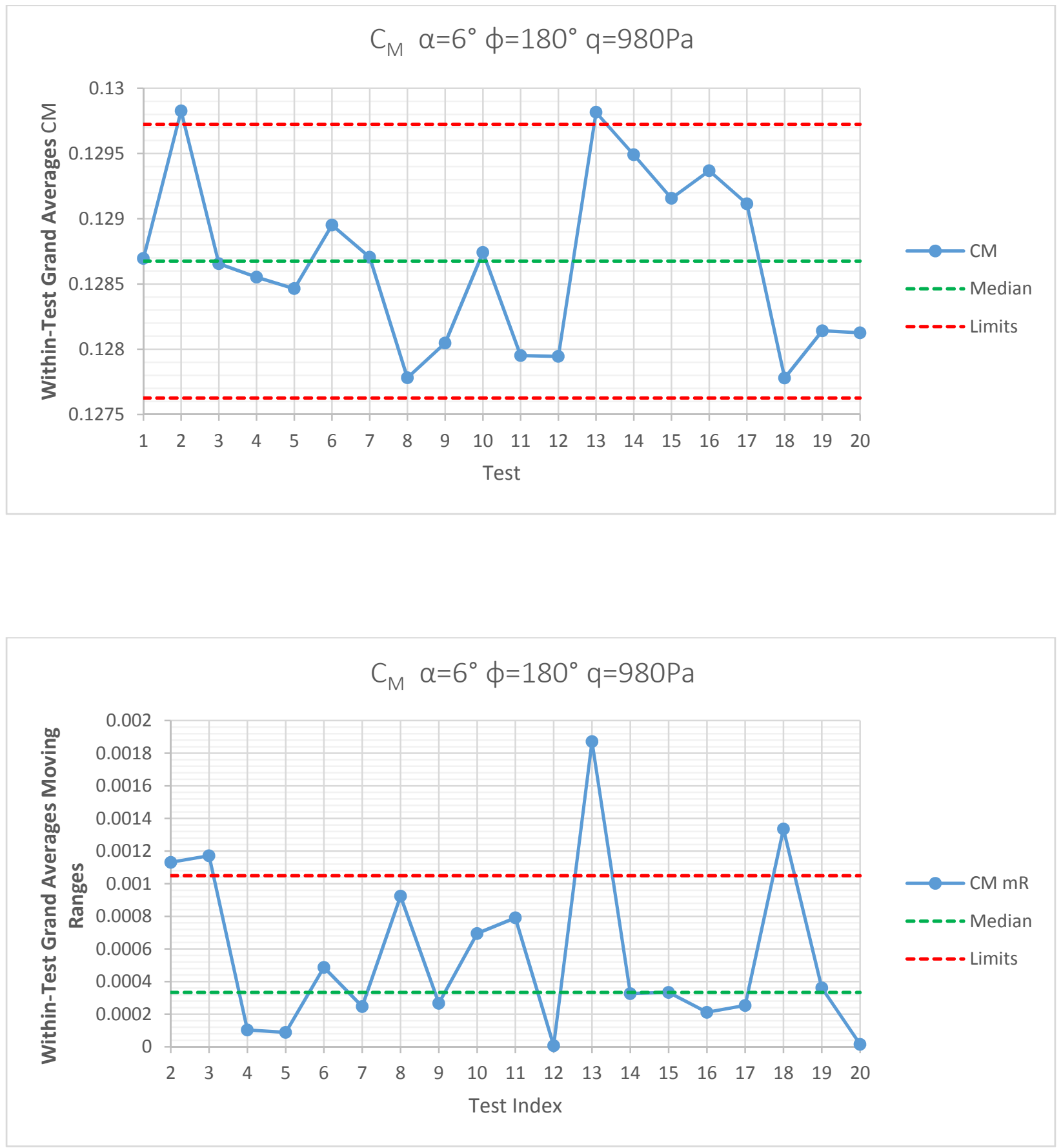

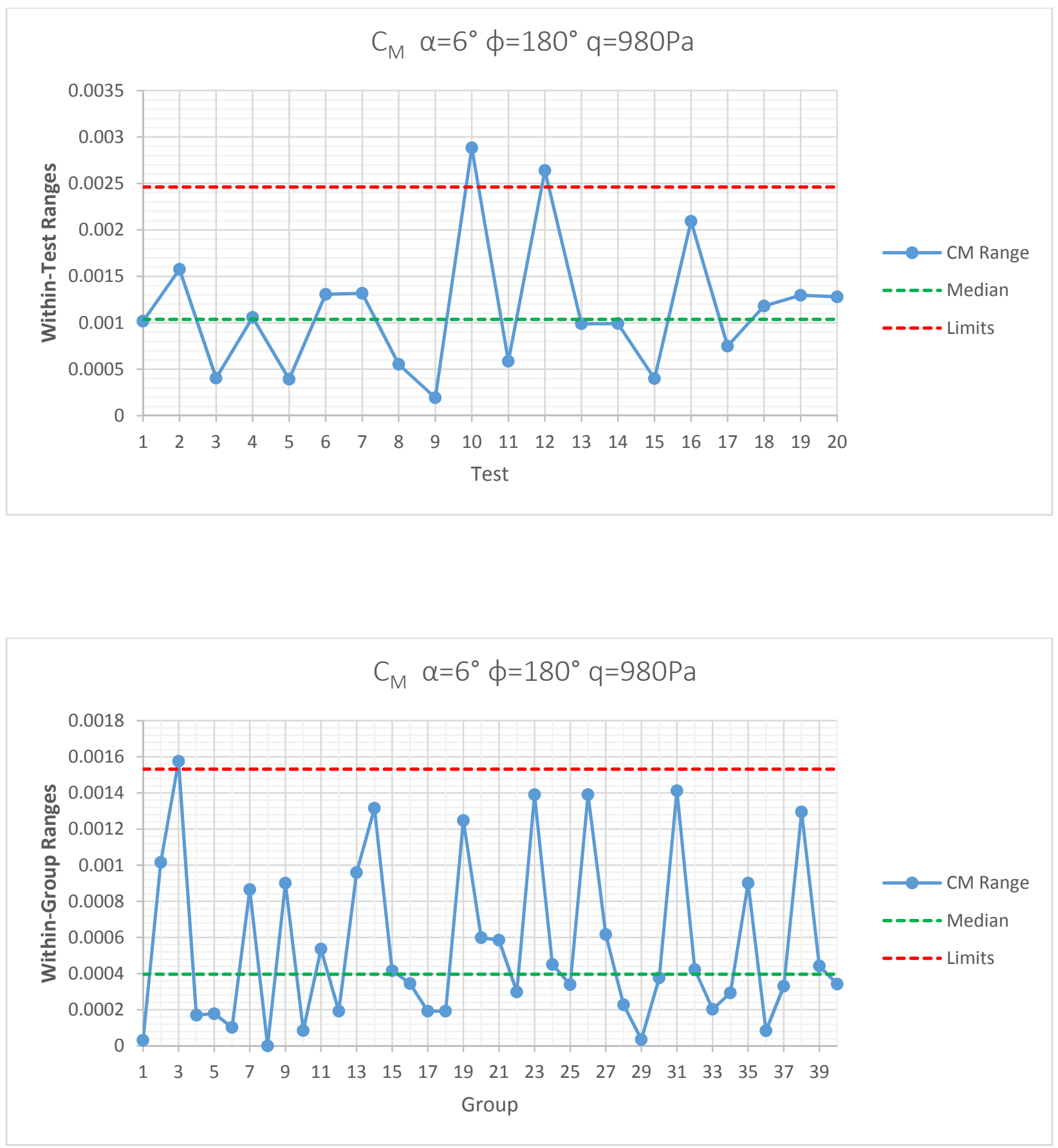


\section{VITA}

Ben D. Phillips was born in Salisbury, Maryland, on October $18^{\text {th }} 1989$. After finishing high school in 2007, he studied engineering at the University of Maryland Eastern Shore, where he received a B.S. in Engineering in 2011. Between 2011 and 2016 he studied aerospace engineering at Old Dominion University in Norfolk, Virginia, where he received a M.S. in 2013 and PhD in 2016. 